TITLE:

\title{
A CATALOGUE OF MOLLUSCS OF WAKAYAMA PREFECTURE, THE PROVINCE OF KII. -I. BIVALVIA, SCAPHOPODA and CEPHALOPODA-
}

AUTHOR(S):

HABE, Tadashige

CITATION:

HABE, Tadashige. A CATALOGUE OF MOLLUSCS OF WAKAYAMA PREFECTURE, THE PROVINCE OF KIII. -I. BIVALVIA, SCAPHOPODA and CEPHALOPODA-. Publications of the Seto Marine Biological Laboratory. Special Publication Series 1981, 7: xiii-264

\section{ISSUE DATE:}

1981-08-31

URL:

http://hdl.handle.net/2433/176470

RIGHT: 


\section{A CATALOGUE OF MOLLUSCS OF WAKAYAMA PREFECTURE, THE PROVINCE OF KII.}

I BIVALVIA, SCAPHOPODA and CEPHALOPODA

和 歌山県貝類目録 I

二枚具綱 掘足網 頭足綱

BASED ON

THE KURODA'S MANUSCRIPT

AND

SUPERVISED BY

TADASHIGE HABE

With 13 Plates

\section{CONTENTS 目 次}

A Brief Biography of Dr. Tokubei Kuroda 黒田德米博士略歷 …………..... v

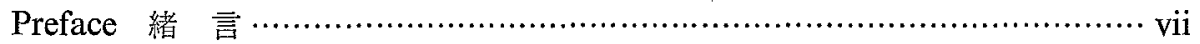

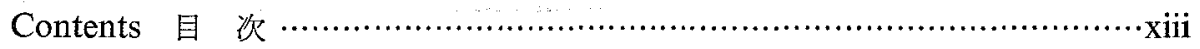

A History of Conchological Studies in Wakayama Prefecture 和歌山県

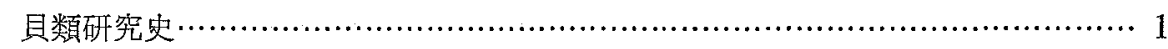

Molluscan Fauna of Wakayama Prefecture 和歌山県の貝類相 ………........ 7

Map of Wakayama Prefecture 和歌山県地図 ……................................

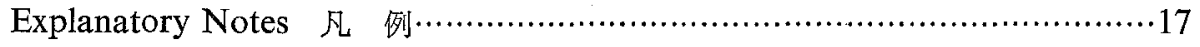

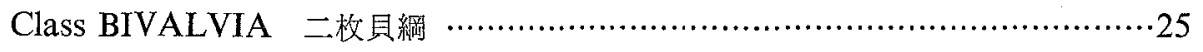

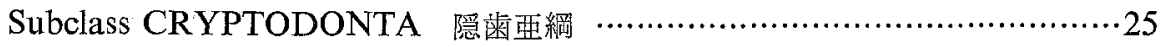

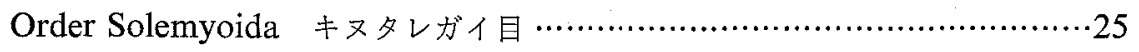

Superfamily Solemyacea キヌタレガイ超科 …….......................2

Family Solemyidae キヌタレガイ科……............................25

Superfamily Nucinellacea キビガラガイ超科………...................25

Family Nucinellidae キビガラガイ科 ……….......................25

Subclass PALAEOTAXODONTA 古多歯亜綱 ……...........................2.

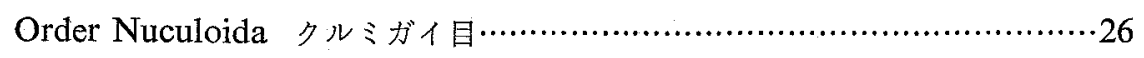

Superfamily Nuculacea クルミガイ超科…….........................2.

Family Nuculidae クルミガイ科 ……...............................26 
Superfamily Nuculanacea シワロウバイガイ超科……………...........27

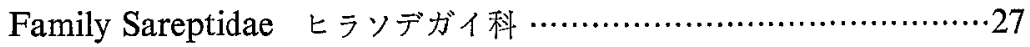

Subfamily Sareptinae ヒラソデガイ 亜科……….....................27

Family Malletiidae スミゾメソデガイ科…….........................2 28

Family Nuculanidae シワロウバイガイ科 …….........................28

Subfamily Nuculaninae シワロウバイガイ亜科…………………228

Subfamily Yoldiinae ナギナタソデガイ亜科……………...........3

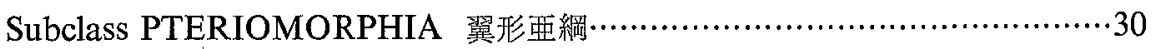

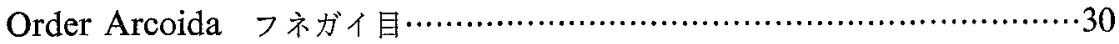

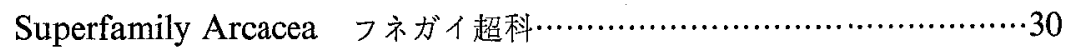

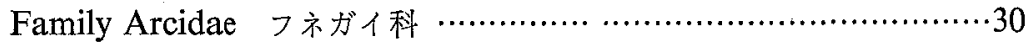

Subfamily Arcinae フネガイ㚊科…….............................3 30

Subfamily Anadarinae リュウキニウサルボウガイ两科 $\cdots \cdots \cdots \cdots \cdots \cdots \cdot \cdots$

Subfamily Striarcinae ミミエガイ亜科 ……………………....3

Family Parallelodontidae シコロエガイ科……..........................3

Subfamily Cucullarinaeシコロエガイ亚科 ……………….......39

Family Cucullaeidae ヌノメアカガイ科 ……............................4 40

Family Glycymerididae タマキガイ科……............................4.

Subfamily Glycymeridinae タマキガイ亜科 ……………............40

Superfamily Limopsacea オオシラスナガイ超科 …….......................42

Family Limopsidae オオシラスナガイ科…….............................42

Order Mytiloida イガイ目…….......................................................44

Superfamily Mytilacea イガイ超科 ………............................44

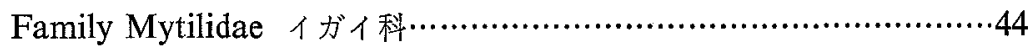

Subfamily Mytilinae イガイ亜科 …….................................44

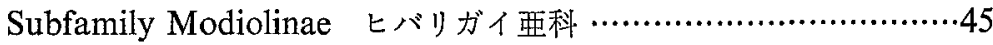

Subfamily Crenellinae キザミガイ亜科 ……...........................49

Subfamily Musculinae タマエガイ亜科 ………......................49

Subfamily Lithophaginae イシマテガイ亜科…………............5

Superfamily Pinnacea ハボウキガイ超科 ……............................5

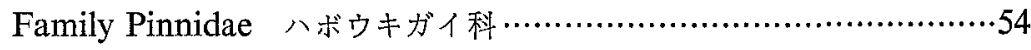

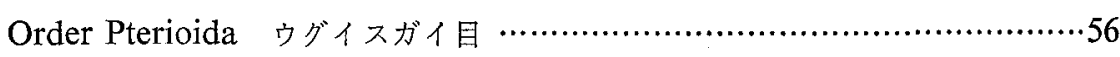

Suborder Pteriina ウグイスガイ亜目 ………...............................5

Superfamily Pteriacea ウグイスガイ超科 ………..........................5

Family Pteriidae ウグイスガイ科……….............................5

Family Isognomonidae マクガイ科………............................5

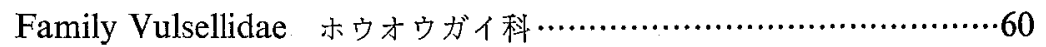

Family Malleidae シュモクガイ科 ………............................61

Superfamily Pectinacea イタヤガイ超科………..........................61

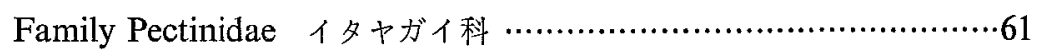


Subfamily Amusiinae ッキヒガイ塜科 ………………….......6.

Subfamily Propeamussinae ワタゾコッキヒガイ亜科………….....62

Subfamily Chlamydinae カミオニシキガイ亜科…….................63

Subfamily Camptonectinae ハリナデシコガイ巠科……………...69

Subfamily Hemipectininae イワオニシキガイ西科……………....69

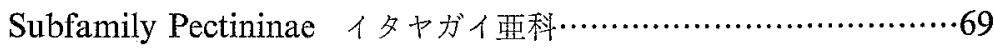

Subfamily Patinopectininae ホタテガイ亜科……………………71

Family Spondylidae ウミギクガイ科 ………..........................71

Family Plicatulidae ネズミノテガイ科 ……............................

Superfamily Dimyacea イシガキ超科…………….....................

Family Dimyidae イシガキ科 …….....................................75

Superfamily Limacea ミノガイ超科…………........................75

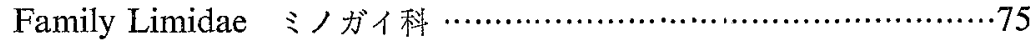

Superfamily Anomiacea ナミマガシワガイ超科 …………………....8

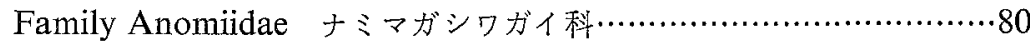

Subfamily Anomiinae ナミマガシワガイ亜科 …………............80

Subfamily Placunanomiinae ナミマガシワガイモドキ亜科 $\cdots \cdots \cdots . . . .81$

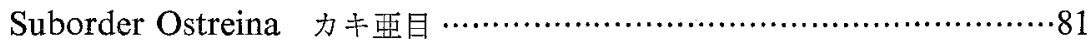

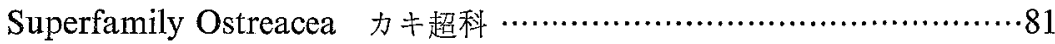

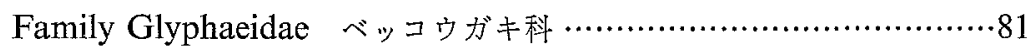

Subfamily Pycnodonteinae ベッコウガキ亜科 ……..................81

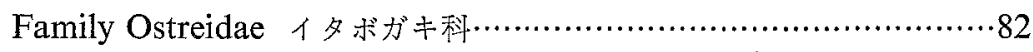

Subfamily Ostreinae イタボガキ亜科 ……........................

Subclass PALAEOHETERODONTA 古異歯亚綱……….....................

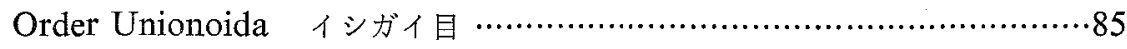

Superfamily Unionacea イシガイ超科 ……..............................

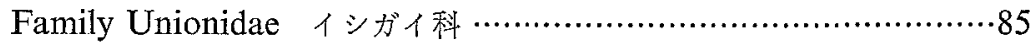

Subfamily Unioninae イシガイ亜科……...........................

Subfamily Anodontinae ドブガイ亜科 …….......................

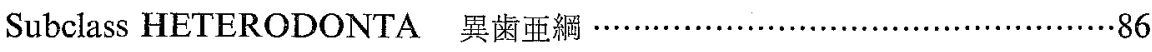

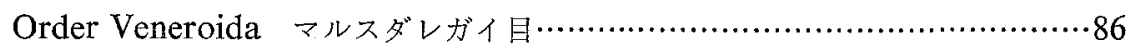

Superfamily Lucinacea ツキガイ超科…………........................86

Family Lucinidae ッキガイ科 …….................................86

Subfamily Lucininae ッキガイ互科……...........................

Subfamily Myrteinae ッキガイモドキ亜科…….......................8

Subfamily Milthinae カブラッキガイ巠科…….........................

Subfamily Divaricellinae セワケッキガイ亜科 …......................90

Family Thyasiridae ハナシガイ科 …................................

Family Ungulinidae フタバシラガイ科 …….......................92

Superfamily Chamacea キクザルガイ超科……............................94

Family Chamidae キクザルガイ科 ……..............................94 
Superfamily Galeommatacea ウロコガイ超科 ………....................96

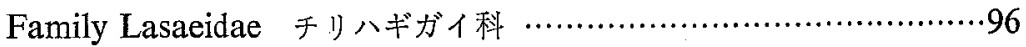

Subfamily Lasaeinae チリハギカイ亜科…………………........96

Subfamily Melliterycinae ハチミッガイ亜科…….....................98

Subfamily Borniolinae セワケガイ亜科 ………..........................98

Family Montacutidae ブンブクヤドリガイ科…….....................100

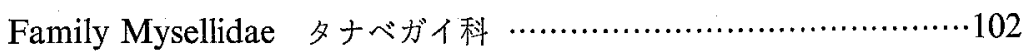

Family Galeommatidae ウロコガイ科 ……............................

Superfamily Cyamiacea ノミハマグリ超科 ………......................106

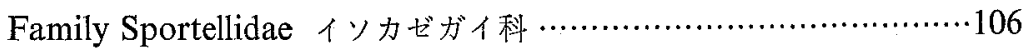

Superfamily Carditacea トマヤガイ超科……….......................106

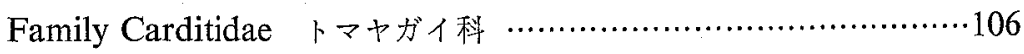

Subfamily Carditinae トマヤガイ亜科 ……………….............106

Subfamily Venericardiinae マルフミガイ亜科 …………….......107

Subfamily Carditellinae ケシザルガイ亜科 ………………......108

Superfamily Astartacea エゾシラオガイ超科 ………………..........109

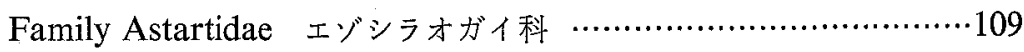

Superfamily Crassatellacea モシオガイ超科 ……........................109

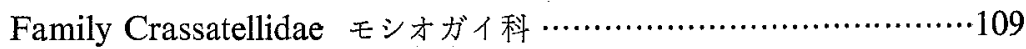

Subfamily Crassatellinae モシオガイ巠科 ………...................109

Superfamily Cardiacea ザルガイ超科 …….............................. 110

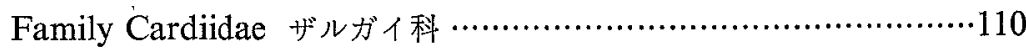

Subfamily Trachycardiinae ザルガイ亜科 ……....................110

Subfamily Fraginae オオヒシガイ 互科 …….........................111

Subfamily Protocardiinae キンギョガイ互科 ……..................112

Subfamily Laevicardiinae マクラザルガイ刺科………………...114

Superfamily Mactracea バカガイ超科 ………............................116

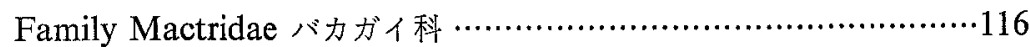

Subfamily Mactrinae バカガイ鱼科 …………...................116

Subfamily Lutrariinae オオトリガイ画科………………….....118

Subfamily Pteropsellinae ヤチョノハナガイ亜科 …………….....120

Family Mesodesmatidae チドリマスオガイ科 ………………...120

Subfamily Mesodesmatinae チドリマスオガイ亜科 ……………120

Subfamily Davilinae ナミノコマスオガイ亜科 ………………....121

Subfamily Ervilinae ハマチドリガイ亜科 ………...................121

Family Cardiliidae キサガイ科 ……............................... 122

Superfamily Tellinacea ニッコウガイ超科 …….......................122

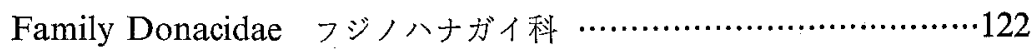

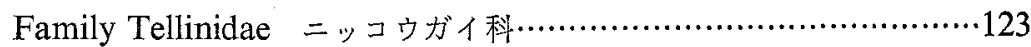

Subfamily Tellininae ニッコウガイ丳科 …….......................123 
Subfamily Arcopagiinae イチョウシラトリガイ亜科 …………126

Subfamily Strigillinae オガタザクラガイ亜科 ……...................132

Subfamily Macominae シラトリガイ無科 …….......................132

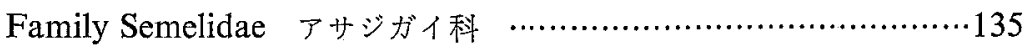

Family Psammobiidaeシオサザナミガイ科 ……..................138

Subfamily Psammobiinaeシオサザナミガイ亜科……………....138

Subfamily Sanguinolariinae ムラサキガイ亜科 ……………......141

Family Solecurtidae キヌタアゲマキガイ科 …….....................142

Superfamily Solenacea マテガイ超科 ……….......................... 144

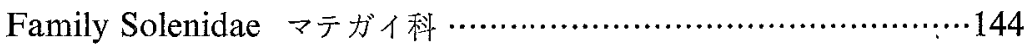

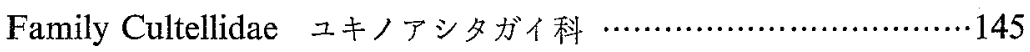

Superfamily Dreissenacea マゴコロガイ超科 …….......................146

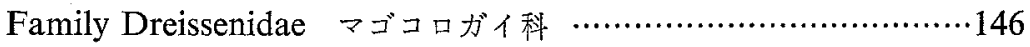

Superfamily Arcticacea アイスランドガイ超科 …………...............147

Family Kelliellidae ケシハマグリ科 ……...............................147

Family Trapeziidae フナガタガイ科 …………………............

Superfamily Glossacea コウホネガイ超科 ……......................... 148

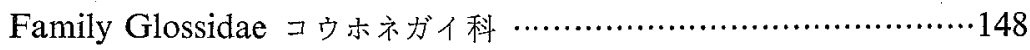

Family Vesicomyidae オトヒメハマグリ科 ……....................149

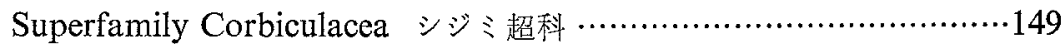

Family Corbiculidae シシミ科 …..................................149

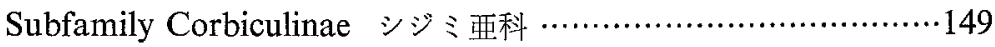

Family Pisidiidae マメシシミ科……............................... 150

Superfamily Veneracea マルスダレガイ超科 …………………..... 151

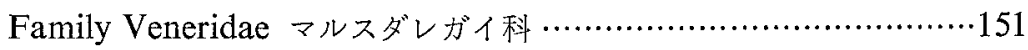

Subfamily Venerinae マルスダレガイ亜科 …….......................151

Subfamily Chioninae カノコアサリ亜科 ……........................ 153

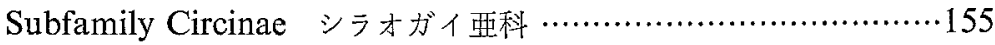

Subfamily Pitarinae ユゥカゲハマグリ刺科 …………………....157

Subfamily Lioconchinae マルオミナエシガイ亚科 …………........159

Subfamily Samarangiinae コンゴウハマグリ刺科 ………….......160

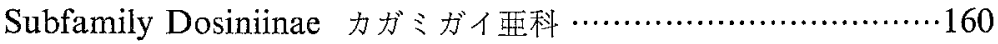

Subfamily Tapetinae リュゥキュウアサリ亜科 …………….......163

Subfamily Callistinae マッヤマワスレガイ亜科 ………………....166

Subfamily Sunettinae ワスレガイ亚科 ……………………...... 167

Subfamily Meretricinae ハマグリ亜科 ……………………..... 167

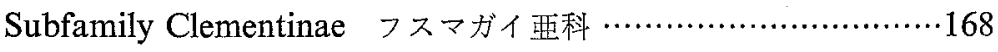

Subfamily Cyclininae オキシシミ巠科 ………........................ 168

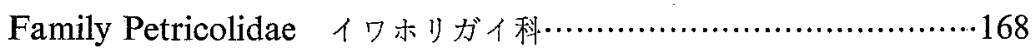

Order Myoida オオノガイ目 ……........................................... 170 
Suborder Myina オオノガイ亜目 ………………......................... 170

Superfamily Myacea オオノガイ超科 ……............................. 170

Family Myidae オオノガイ科………............................. 170

Subfamily Myinae オオノガイ亜科 ……........................... 170

Subfamily Cryptomyinae ヒメマスオガイ业科 ……………171

Family Corbulidae クチベニガイ科 ……..........................172

Subfamily Corbulinae クチベニガイ雨科…….......................172

Superfamily Hiatellacea キヌマトイガイ超科…………….............173

Family Hiatellidae キヌマトイガイ科 ……..........................

Superfamily Gastrochaenacea ツクエガイ超科 ……….................174

Family Gastrochaenidae ックエガイ科 ……........................ 174

Suborder Pholadina $=$ オガイ亜目……................................ 175

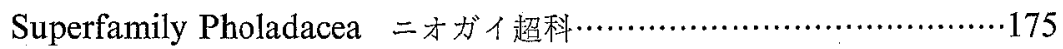

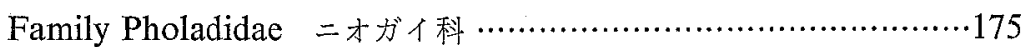

Subfamily Pholadinae ニオガイ東科…….........................175

Subfamily Martesinae カモメガイモドキ亚科 …….................176

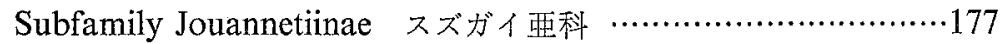

Family Xylophaginidae キクイガイ科 ……........................178

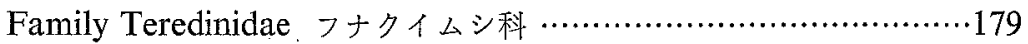

Subfamily Teredininae フナクイムシ亜科 …….....................179

Subfamily Bankiinae オオフナクイムシ亜科 ………………...181

Subfamily Zachsiinaeネムグリガイ刺科……...................... 182

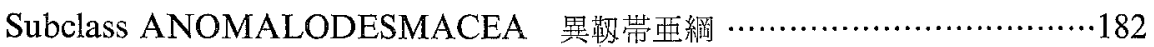

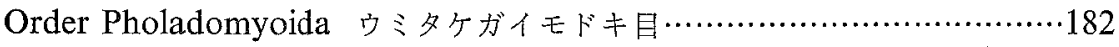

Superfamily Pholadomyacea ウミタケガイモドキ超科………............

Family Pholadomyidae ウミタケガイモドキ科 …………...........

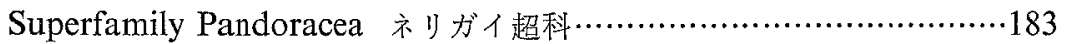

Family Lyonesiidae サザナミガイ科,……........................... 183

Family Pandoridae ネリガイ科…….................................. 184

Family Myochamidae ミッカドカタビラガイ科………...............

Family Periplomatidae リュウグウハゴロモガイ科 ……..............186

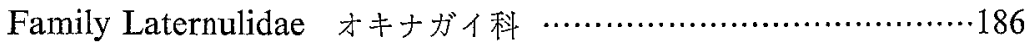

Family Thracidae スエモノガイ科…….............................. 187

Superfamily Clavagellacea ハマユウガイ超科…….....................18

Family Clavagellidae ハマユウガイ科 ……..........................

Superfamily Poromyacea スナメガイ超科 …….......................190

Family Verticordiidae オトヒメゴコロガイ科……...................190

Family Poromyidae スナメガイ科……............................191

Family Cuspidariidae シャクシガイ科…….........................192

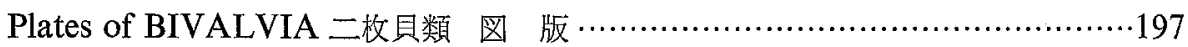




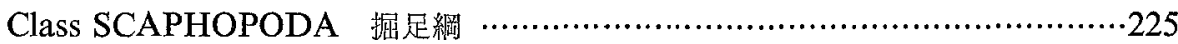

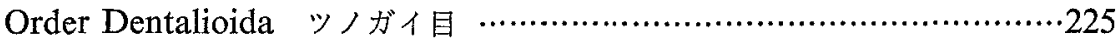

Family Dentaliidae ッノガイ科………............................2225

Subfamily Dentaliinae ゾウゲッノガイ亜科…….....................225

Subfamily Antaliinae ッノガイ亜科 ………........................227

Family Laevidentaliidae ミガキッノガイ科……......................228

Family Episiphonidae ロウソクッノガイ科…….......................229

Family Calliodentaliidae サフランッノガイ科 ……..................2229

Family Gadilinidae シラサャッノガイ科……......................229

Family Omniglyptidae ハリッノガイ科 ……......................230

Order Siphonodentalioida クチキレッノガイ目…….........................2230

Family Siphonodentaliidae クチキレッノガイ科 …...................2230

Family Cadulidae ハラブトッノガイ科……...........................231

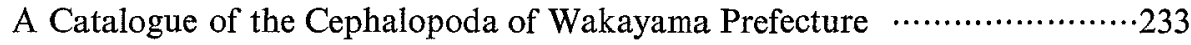

Class CEPHALOPODA 頭足綱 ………....................................2.

Subclass PROTOCEPHALOPODA 原始頭足亚綱………...................2.

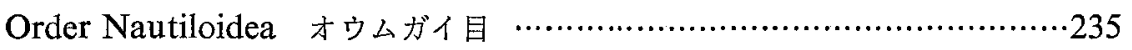

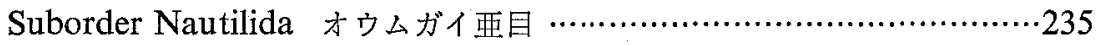

Family Nautilidae オウムガイ科 ……….........................235

Subclass METACEPHALOPODA 後生頭足亜綱 ……........................2235

Order Decembrachiata 十腕目 …….....................................236

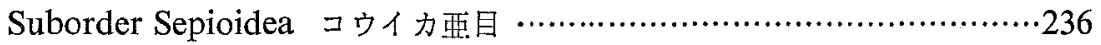

Superfamily Sepiacea コウイカ超科 ……..............................2236

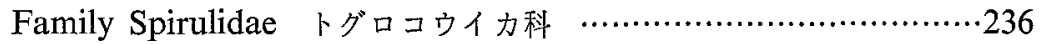

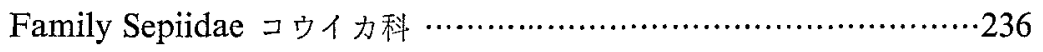

Family Sepiadariidae ミミイカダマシ科 ……........................239

Family Sepiolidae ダンゴイカ科……….............................2. 240

Subfamily Rossiinae ボウズイカ塜科……..........................240

Subfamily Sepiolinae ダンゴイカ亜科 ……….......................240

Family Idiosepiidae ヒナイカ科 ……..............................242

Superfamily Loliginacea ジンドウイカ超科 ……...................... 242

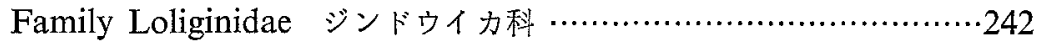

Superfamily Architeuthacea ダイオウイカ超科 …….....................2243

Family Enoploteuthidae ギンボシイカ科 …….....................2. 243

Subfamily Abraliinae ホタルイカモドキ亜科 ……..................24

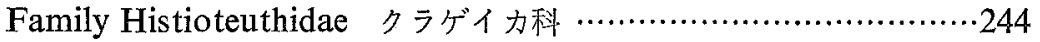

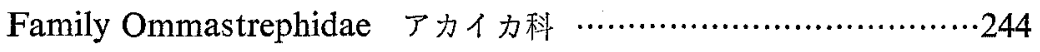

Subfamily Todarodinae スルメイカ亜科 …….......................2. 244

Subfamily Ommastrephinae アカイカ垔科 ……....................245 
Family Thysanoteuthidae ソデイカ科 ……...........................245

Family Chiroteuthidae ユゥレイイカ科 ……..........................246

Subfamily Chiroteuthinae ユウレイイカ巠科 ……………........246

Family Cranchiidae サメハダホウズキイカ科 …………………...246

Subfamily Cranchiinae サメハダホウズキイカ巠科 ………….....246

Family Bathothaumatidae メナガイカ科 …….......................247

Order Vampyromorphae コウモリダコ目 …….............................24

Family Vampyroteuthidae コウモリダコ科 ……....................24

Order Octobrachiata 八腕目 …................................................248

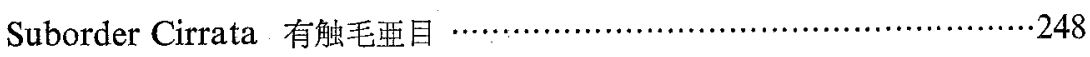

Superfamily Cirroteuthacea ヒゲダコ超科 …………...............2 248

Family Opisthoteuthidae メンダコ科 …….........................2. 248

Suborder Incirrata 無触毛西目 ………....................................248

Superfamily Bolitaenacea フクロダコ超科 ……........................248

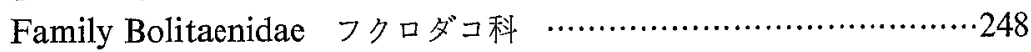

Superfamily Octopodacea マダコ超科 …….............................249

Family Octopodidae マダコ科 ……...............................24

Subfamily Octopodinae マダコ亜科 ………….....................2249

Subfamily Bathypolypodinae ワタゾコダコ刺科 ………….......254

Superfamily Argonautacea アオイガイ超科 ………….................25

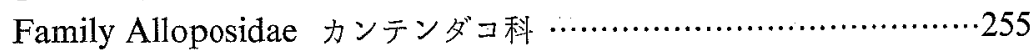

Family Tremoctopodidae ムラサキダコ科 ………...................2256

Family Ocythoidae アミダコ科 ……...............................256

Family Argonautidae アオイガイ科 ………….......................2256

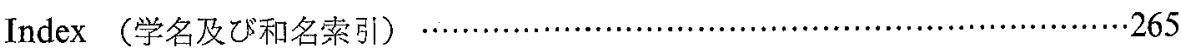

Postword 後書 


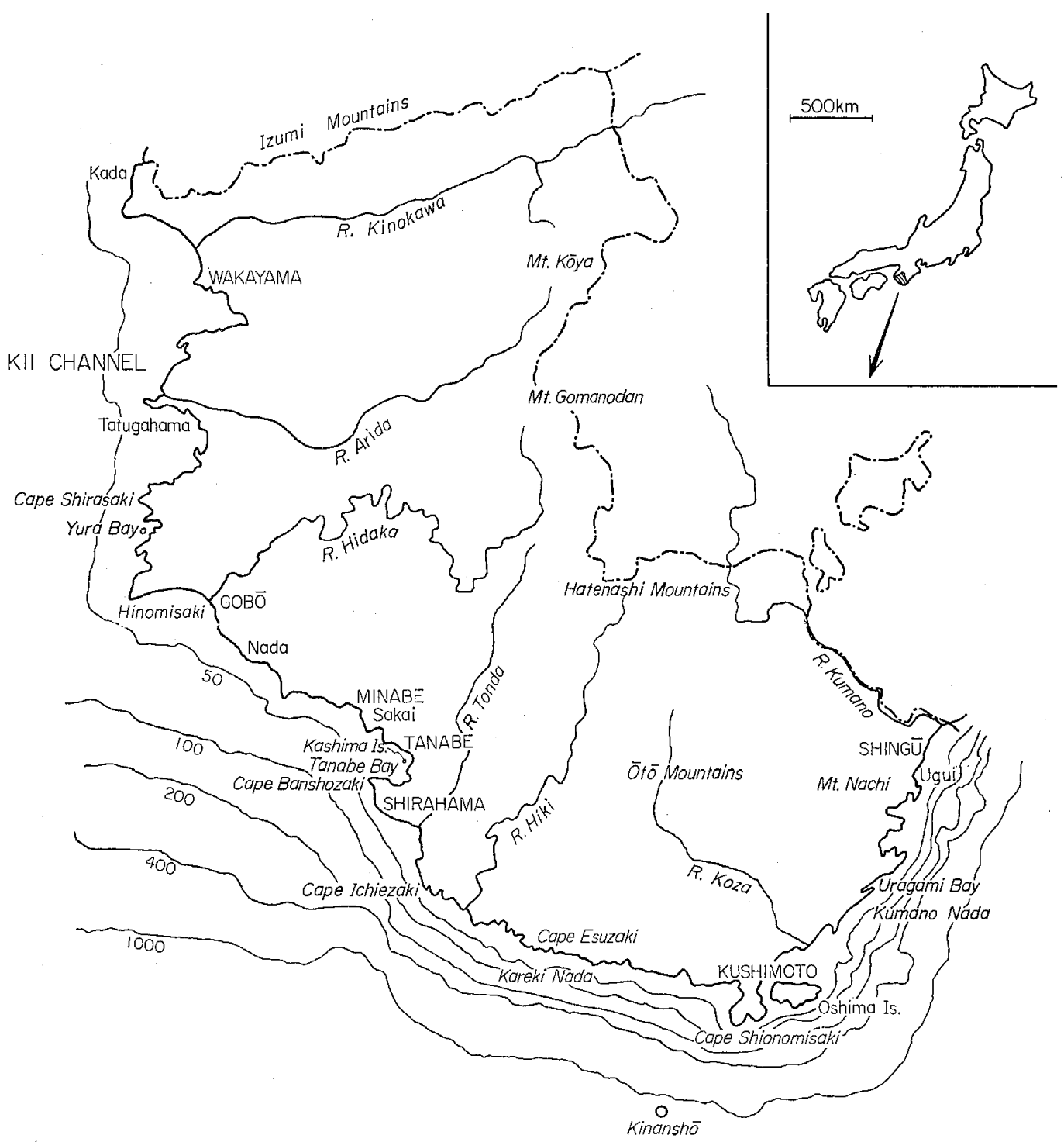

Map 1 Wakayama Prefecture 地図 1 和歌山眎地図

Cape Banshozaki 番所崎

Esuzaki 江須崎

Ichiezaki 市江㥓

Shionomisaki 潮岬

Cape Shirasaki 白崎

Gobō 御坊

Hatenashi mountains 果無山脤

Hinomisaki 日の版

Izumi Mountains 和泉山脈

Kada 加太

Kareki Nada 枯木灘

Kashima Is 神島

Kiichannel 紀伊水道
Kinanshō 紀南礁

Kumano-Nada 熊野灘

Kushimoto 审本

Minabe 南部

Mt. Gomanodan 護摩壇山

Mt. Kōya 高野山

Mt. Nachi 那智山

Nada 名田

Ōshima 大島

Otō Mountains 大塔山系

R. Arida 有田川

R. Hidaka 日高川

R. Hiki 日置川
R. Kinokawa 紀ノ川

R Kumano 熊野川

R. Koza 古座川

R. Tonda 富田川!

Sakai 堺

Shingū 新宮

Shirahama 白浜

Tanabe 田辺

Tanabe Bay 田辺湾

Tatugahama 辰厅浜

Ugui 宇久井

Uragami Bay 浦神湾

Wakayama 和歌山

Yura Bay 由良湾 


\section{凡例}

1 本目録所載種は黒田徳米博士が長年に亘って記録された“紀州貝類目録”(1929-1950) を基礎に1979年末迄に採集，報告されたものを收録し，これに下記11新種に関する波部 忠重の記載をつけ加えた。

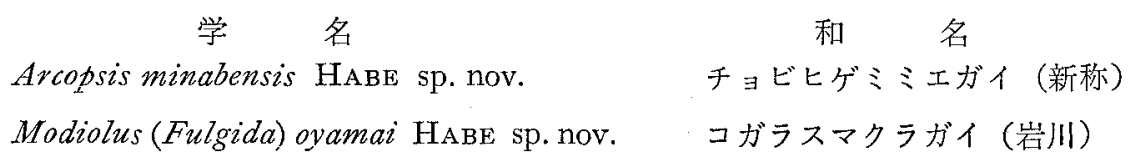

Limnoperna fortunei kikuchii HABE subsp. nov. コウロエンカワヒバリガイ（新称）

Cavatidens imajimai $\mathrm{HABE}$ sp. nov.

コカブラガイ (新称)

Conchocele koyamai HABE sp. nov. スマキオウナガイ（新称）

Mysella nipponica HABE sp. nov. ニホンタナベガイ（新称）

Galeomma purpurea HABE sp. nov. ウスムラサキキザミウロコガイ(新称)

Grammatomya kurodai HABE sp. nov.

クロダヨシガイ（新称）

Lyonsia tanakai HABE sp. nov.

シンテイサザナミガイ（新称）

Eximiothracia koyamai HABE sp. nov.

コヤマスエモノガイ（新称）

Clavagella japonica HABE sp. nov.

クビタテッツガキ (新称)

2 海棲種（頭足類 Cephalopoda を除く）は産地と産状を略記号で表示した。但し産地 及び産状のはっきりしないものは略記号表示を省略した。

a. 産地は北部，中部，南部の 3 海域に分け，それぞれ N.M.S. (大文字) の記号を市 って表示した．海域の区分は白浜番所崎（田辺湾口）以南を南部海域とし由良町白崎 以北を北部海域にその中間在中部海域とした。

b ．産状は主とする採集手段及び底質をそれぞれ略記昂で表示した。（小文字） 又共生，寄生種に就ては出来得るかざりその宿主を明らかにした。

1 産状略記号

$\begin{array}{ll}\text { int } & \text { 潮間帯 intertidal } \\ \text { gl } & \text { エビ刺網 gillnet for lobster fishing } \\ \text { dp } & \text { エビ漕網 (ドレッヂ採集を含む) dredge for prawn fishing } \\ \text { tr } & \text { トロール漁 trawl } \\ \text { sy } & \text { 共生 symbiotic } \\ \text { bo } & \text { 穿孔 boring } \\ \text { ep } & \text { 付着 (海草等) epiphytic } \\ \text { pl } & \text { 浮遊 pelagic }\end{array}$




$\begin{array}{cll}\text { 口 底質 (Substrutum) } & \\ \mathrm{r} & \text { 岩礁 } & \text { rocky } \\ \mathrm{st} & \text { 岩砅 } & \text { stony } \\ \mathrm{g} & \text { 砂礫 } & \text { gravely } \\ \mathrm{s} & \text { 砂 } & \text { sandy } \\ \mathrm{s}-\mathrm{m} & \text { 砂泥 } & \text { sandy muddy } \\ \mathrm{m} & \text { 泥 } & \text { muddy }\end{array}$

3 本県を模式産地とする種は種名の頭に*記号を附した.

4 引用文骫は略記号（19〜23ページ参照）にて示した。

\section{Explanatory Notes}

1. This catalogue is based on the unpublished manuscript of the "Catalogue of shells of the Province of Kii"'(1929-1950) compiled by Dr. Tokubei Kuroda for many years with the addition of all those species discovered by him and others between 1929 and 1979. In addition, descriptions of the following eleven new species, made by Tadashige Habe, are included.

Arcopsis minabensis HABE sp. nov.

Modiolus (Fulgida) oyamai HABE sp. nov.

Limnoperma fortunei kikuchii HABE subsp. nov.

Cavatidens imajimai HABE sp. nov.

Conchocele koyamai HABE sp. nov.

Mysella nipponica HaBE sp. nov.

Galeomma purpurea HABE sp. nov.

Grammatomya kurodai HABE sp. nov.

Lyonsia tanakai HABE sp. nov.

Eximiothracia koyamai HABE sp. nov.

Clavagella japonica HABE sp. nov.

2. In the marine species, with the exception of Cephalopoda localities and habitats are shown by abbreviations. However, for those species where such conditions have as yet to be clarified, these abbreviations have been deleted.

a. Localities are grouped into three regions: Northern, Middle and Southern, designated by the abbreviations N., M., and S., respectively. The Northern Region is described by the area north of Shirasaki, Yura-cho, the Middle Region by the area from Shirasaki to Banshozaki in Shirahama, the entrance to Tanabe Bay and the Southern Region by the region south of Banshozaki.

b. Natural habitats, substrata and collection methods are abbreviated with small letters. Symbiotic or parasitic species are listed with their host species, 
whenever possible.

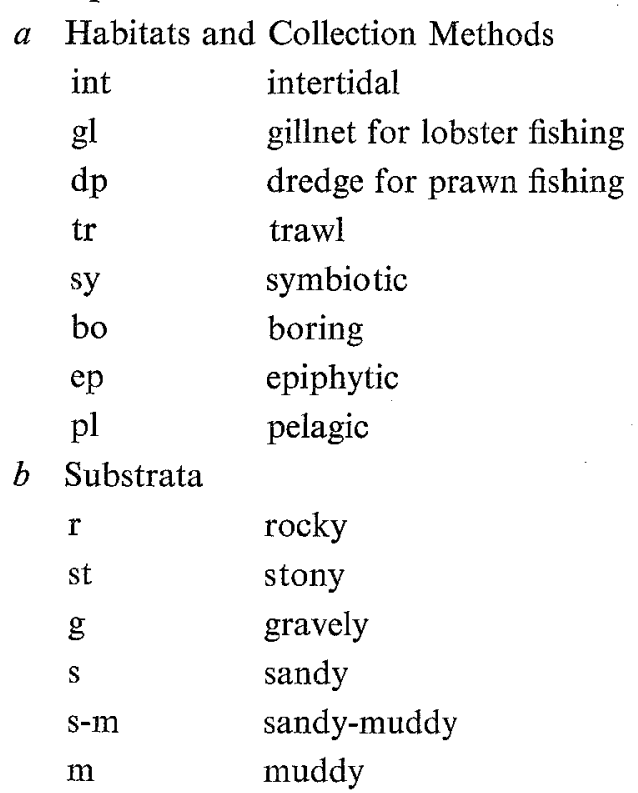

3. Those species whose type locality is in Wakayama prefecture have been denoted by an asterisk,*.

4. References have been abbreviated as seen in the following examples:

Abbild.

AfN

AfZ

AJC

AMHNP

AMNH

ASNG

ASNZ

Astrolabe

AsV

$\mathrm{AZ}$

AZJ

AZS

BHNLB

BMBSA

BMCZ

BMNHNP

BNSM

BNSUR

Bolten.
Abbild. Beschr. Conchyl.

Archiv. f. Naturg.

Archiv. f. Zool.

Amer. Jour. Conch.

Ann. Mus. Hist. Nat. Paris

Ann. Mag. Nat. Hist.

Abh. Senckenberg. Nat. Ges.

Ann. Sci. Nat. Zool.

Voy. Astrolabe Zool.

Anim. s. Vert.

Australian Zoologist.

Annot. Zool. Japan

Acta Zool. Sinica

Bull. Hist. Nat. Linn. Bordeaux

Bull. Mar. Biol. St. Asamushi

Bull. Mus. Comp. Zool.

Bull. Mus. Nat. Hist. Nat. Paris

Bull. Natn. Sci. Mus.

Beschr. Nat. Samml. Univ. Rostock

Mus. Bolten. 


\begin{tabular}{|c|c|}
\hline BPBMB & Bernice P. Bishop Mus. Bull. \\
\hline BSMI & Bull. Soc. Malac. Italia \\
\hline BTRFRL & Bull. Tokai Reg. Fisher. Res. Lab. \\
\hline $\mathrm{CA}$ & Conch. Asiatica \\
\hline $\mathrm{Cab}$. & Neues Conch. Cab. \\
\hline Cat. Bivalve & Illust. Descrip. Cat. Rec. Bivalve Shells \\
\hline Cat. Okinawa & Cat. Shell. Moll. Okinawa Islands \\
\hline Cat. Yamaguch & ii Cat. Moll. Yamaguchi Pref. \\
\hline Challenger & Rep. Sci. Res. Challenger Exped. \\
\hline CISJ & Color. Illust. Shells Japan \\
\hline CIP & Carnegie Inst. Publ. \\
\hline CMMJ & Cat. Mar. Moll. Japan \\
\hline DAWW & Denkschr. Akad. Wiss. Wien \\
\hline DCRS & Descrip. Cat. Rec. Shells \\
\hline DSN & Dict. Sci. Nat. \\
\hline EMHNV & Ency. Meth. Hist Nat. Vers. \\
\hline ENSVT & Essai Nouv. Syst. Vers Test. \\
\hline Extramar. & Fauna Moll. Extramar. Japon. \\
\hline FJ & Fauna Japonica \\
\hline GBRESR & Great Barrier Reef Exped 1928-29 Sci. Rep. \\
\hline GJS & Genera Jap. Shells. \\
\hline GNFBM & Ges. Nat. Fr. Berlin Mag. \\
\hline GRFS & Genera Rec. Fossil Shells \\
\hline GRM & Genera Rec. Moll. \\
\hline Hamburg & Mittl. Zool. Mus Hamburg \\
\hline HNEM & Hist Nat. Europe Merid. \\
\hline HNTB & Hist. Nat. Test. Brit. \\
\hline HsW & Handb. syst. Weicht. \\
\hline ICJS & Illust. Cat. Jap. Shells. \\
\hline Icon & Conch. Icon. \\
\hline $\mathrm{ICZN}$ & Intern. Comm. Zool. Nom. \\
\hline IMCV & Index Mus. Caes Vind. \\
\hline Index & Index Moll. Mar. Jap. \\
\hline IPM & Indo-Pacific Moll. \\
\hline JANSP & Jour. Acad. Nat. Sci. Philadelphia \\
\hline JCSIUT & Jour. Coll. Sci. Imp. Univ. Tokyo \\
\hline $\mathrm{J}$ de $\mathrm{C}$ & Journ. de Conchyl. \\
\hline JDMG & Jahrb. Deusch. Malak. Ges. \\
\hline JFSIUT & Jour. Fac. Sci. Imp. Univ. Tokyo \\
\hline JFSHU & Jour. Fac. Sci. Hokkaido Univ. \\
\hline
\end{tabular}




\begin{tabular}{|c|c|}
\hline $\mathrm{JJZ}$ & Jap. Jour. Zool. \\
\hline JGST & Jour. Geol. Soc. Tokyo \\
\hline JLSLZ & Jour. Linn. Soc. London Zool. \\
\hline $\mathrm{JMC}$ & Jap. Meer. Conchyl. \\
\hline JMSA & Jour. Malac. Soc. Australia \\
\hline $\mathrm{J}$ of $\mathrm{C}$ & Jour. of Conch. \\
\hline $\mathrm{J}$ of $\mathrm{M}$. & Jour. of Malac. \\
\hline $\mathrm{J}$ of $\mathrm{P}$ & Jour. of Paleont. \\
\hline JTUF & Jour. Tokyo Univ. Fisher. \\
\hline LBACBM & List Brit Anim. Coll. Brit. Mus. \\
\hline Le Nat. & Le Naturaliste \\
\hline MARSB & Mem. Acad. Roy. Soc. Belgique \\
\hline Mazatlan & Cat. Mazatlan Shells \\
\hline MBL & Malak. Blatte \\
\hline MCGB & Min. Conch. Great Brit. \\
\hline MCSKIU & Mem. Coll. Sci. Kyoto Imp. Univ. \\
\hline $\mathrm{M}$ de $\mathrm{C}$ & Man. de Conchyl. \\
\hline MFSATIU & Mem. Fac. Sci. Agr. Taihoku Imp. Univ. \\
\hline $\mathrm{MG}$ & Mineral \& Geol. \\
\hline MJ & Moll. Japonica \\
\hline MIRSNB & Mem. Inst. Roy. Soc. Sci. Nat. Belgique \\
\hline MMRHNB & Mem. Mus. Roy. Host. Nat. Belgique \\
\hline MMNHN & Mem. Mus. Natn. Hist. Nat. \\
\hline $\mathrm{M}$ of $\mathrm{C}$ & Man. of Conch. \\
\hline MQM & Mem. Queensland Mus. \\
\hline MSCKIU & Mem. Sci. Coll. Kyushu Imp. Univ. \\
\hline MSGF & Mem. Soc. Geol. France \\
\hline MSHNP & Mem. Soc. Hist. Nat. Paris. \\
\hline MSZF & Mem. Soc. Zool. France \\
\hline NAMHNP & Nouv. Arch. Mus. Hist. Nat. Paris \\
\hline Naut. & Nautilus \\
\hline NB1 & Nachr. Blatte \\
\hline $\mathrm{NCM}$ & Nov. Conch. Mar. \\
\hline NIEFJ & New Illust. Encycl. Fauna Japan \\
\hline NM & Nordjap. Moll. \\
\hline PANSP & Proc. Acad. Nat. Sci. Philadelphia \\
\hline PBSNH & Proc. Boston Soc. Nat. Hist. \\
\hline PBSW & Proc. Biol. Soc. Washington \\
\hline Peitaiho & Shells of Peitaiho \\
\hline Philippine & Philippine Jour. Sci. \\
\hline
\end{tabular}




\begin{tabular}{|c|c|}
\hline P. indica & Paleont. Indica \\
\hline PLSNSW & Proc. Linn. Soc. New South Wales \\
\hline PMSL & Proc. Malac. Soc. London \\
\hline PS & Pacific \\
\hline PSJSP & Palaeont. Soc. Japan Special Paper \\
\hline PSMBL & Proc. Seto Mar. Biol. Lab. \\
\hline PUSNM & Proc. U.S. Nat. Mus. \\
\hline PZSL & Proc. Zool. Soc. London \\
\hline RAM & Rec. Australian Mus. \\
\hline RAZM & Reise Amurland Zool. Moll. \\
\hline RBAAS & Rep. Brit. Assoc. Adv. Sci. \\
\hline RIM & Rec. Indian Mus. \\
\hline ROWJ & Rec. Oceanogr. Works Japan \\
\hline RZSC & Rev. Zool. Soc. Cuv. \\
\hline Samarang & Zool. Voy. Samarang Moll. \\
\hline San Diego & Trans. San Diego Soc. Nat. Hist. \\
\hline SBGNFB & Sitz-Ber. Ges. Nat. Fr. Berlin. \\
\hline SHKMRB & Saito Ho-on Kai Mus. Res. Bull. \\
\hline Siboga & Siboga Exped. Monogr. \\
\hline SMC & Smithsonian Inst. Misc. Coll. \\
\hline $\mathrm{SMJB} / \mathrm{S}$ & Syst. Moll. Japan Bivalvia Scaphopoda \\
\hline SMS & Studia Marina Sinica \\
\hline SNS & Skriv. Naturh. Selsk. Copenhagen. \\
\hline SRGSJ & Sci. Rep. Geol. Suv. Japan. \\
\hline SRTIU & Sci. Rep. Tohoku Imp. Univ. \\
\hline SRTU & Sci. Rep. Tohoku Univ. \\
\hline SRYNU & Sci. Rep. Yokohama National Univ. \\
\hline SSSB & Sea Shells of Sagami Bay \\
\hline St Petersburg & Bull. Acad. Sci. St Petersburg \\
\hline SWC & Shells World Col. \\
\hline Syn & Synopsis Moll. Great Brit. \\
\hline Syst. Nat. & Systema Naturae \\
\hline Tankerville & Cat. Tankerville \\
\hline TCAS & Trans Connecticut Acad. Sci. \\
\hline Thes & Thes. Conch. \\
\hline Tiefsee & Deutsch. Tiefsee Exped. \\
\hline TMCV & Test. Mus. Caes Vind. \\
\hline TNZI & Trans. Proc. New Zealand Inst. \\
\hline TPPSJ & Trans. Proc. Palaeont. Soc. Japan. \\
\hline Treat. & Treat. Malac. \\
\hline
\end{tabular}




$\begin{array}{ll}\text { TRSNZ } & \text { Trans. Roy. Soc. New Zealand } \\ \text { TWFIP } & \text { Trans. Wagner Free Inst. Philadelphia } \\ \text { USEE } & \text { U.S. Expl. Exped. } \\ \text { USNMB } & \text { U.S. Nat. Mus. Bull. } \\ \text { Vega } & \text { Vega Exped. } \\ \text { Venus } & \text { Jap. Jour. Malac. Venus } \\ \text { VVC } & \text { Vers. Vollst. Conchyl. } \\ \text { Yoldi } & \text { Cat. Conchyl. Yoldi } \\ \text { ZA } & \text { Zool. Analy. } \\ \text { ZfM } & \text { Zeitschr f. Malacoz. } \\ \text { ZfN } & \text { Zeitschr f. Naturg. } \\ \text { ZMT } & \text { Zool. Mag. Tokyo } \\ \text { ZJ } & \text { Zool. Jour. }\end{array}$




\section{B I V A L V I A}

prepared by Tadashige $\mathrm{H}_{\mathrm{ABE}}$

\section{Class Bivalvia Linnaeus 二枚貝綱}

\section{Subclass Cryptodonta NeumaYr 隠菌平綱}

Order Solemyoida DALL キヌタレガイ目

\section{Superfamily Solemyacea H. \& A. ADAMS キヌタレガイ超科}

Family Solemyidae H. \& A. ADAMS キヌタレガイ科

Genus Petrasma DALL キヌタレガイ属

1908 Petrasma Dall, Naut., 22, 2. Type (OD): Solemya borealis TotTEN

Petrasma pusilla (Gould) キヌタレガイ (黒田)

1861 Solemya pusilla GouLd, PBSNH, 8, 27

1950 Solemya (Petrasma) pussilla: HABE, ICJS, 1(10), 63, tf. 8

Loc. N. S. (s-m) dp

Dist. Southern Hokkaido to Kyushu.

Petrasma pervernicosa KURODA アブラキヌタレガイ（黒田）

1948 Solemya (Petrasma) pervernicosa KurodA, Venus, 15, 32, tf. 4, 5

1959 Solemya (Petrasma) pervernicosa: KIRA, CISJ, 1, 194, pl. 71, f. 3

Loc. $\mathrm{S}$ (s-m).

Dist. Honshu (South to Kii Peninsula) to Hokkaido and Japan Sea.

Genus Acharax DALL スエヒロキヌタレガイ属

1908 Acharax DaLl, Naut, 22, 2. Type (OD): Solemya johnsoni DaLL

Acharax japonicus (DUNKER) アサヒキヌタレガイ（波部）

1882 Solemya japonica DunKer, Index, 220, 261, pl. 14, f. 3

1927 Solemya yamakawai YoKoYAMA, JFSIUT, 20 1, 435, p1. 50, f. 10, 11

1959 Solemya (Acharax) japonica: KIRA, CISJ, 1, 106, pl. 41, f. 1

Loc. N. S. (s-m) dp.

Dist. Southern Hokkaido to Kyushu.

$$
\begin{array}{cc}
\begin{array}{c}
\text { Superfamily Nucinellacea VOKES } \\
\text { Family Nucinellidae VOKES }
\end{array} & \text { キビガラガイ超科 } \\
\text { Genus Huxleyia A. ADAMS } & \text { キビガラガイ属 }
\end{array}
$$

1860 (Apr.) Huxleyia A. Adams, AMNH, (3) 5, 303. Type (M): Huxleyia sulcata A. Adams 
Huxleyia sulcata A. ADAMS キビガラガイ (黑田)

1860 Huxleyia sulcata A. ADAMS, AMNH, (3) 5, 303

1965 Huxleyia sulcata: HABE, NIEFJ, 2, 228, No. 852

1971 Huxleyia sulcata: Kuroda \& HABE, SSSB, 514 (J.) 324 (E), pl. 117, f. 8, 9

Loc. S. (s) tr.

Dist. Honshu (north to Bōsō Peninsula) to Kyushu and the southern part of Japan Sea.

\section{Subclass Palaeotaxodonta KoroBKov 古多歯亜綱}

\section{Order Nuculoida DALLクルミガイ目 \\ Superfamily Nuculacea GRAY クルミガイ超科 \\ Family Nuculidae GRAY クルミガイ科 \\ Genus Nucula LAMARCK マメクルミガイ属}

1799 Nucula Lamarck, MSHNP, 1, 87. Type (OD): Arca nucleus Linnabus

Nucula paulula A. ADAMS マメクルミガイ (平瀬)

1856 Nucula paulula A. ADAMs, PZSL, 24, 52

1860 Nucula paulula: HANLEY, Thes., 3, 153, pl. 229, f. 131, 132

1961 Nucula paulula: HABE, CISJ, 2, 107, p.1 48, f. 2

Loc. N M S (s-m) int.

Dist. Honshu (north to Bōsō Peninsula) to Kyushu.

\section{Genus Lamellinucula SCHENCK ヨセナミクルミガイ属}

1944 Lamellinucula SCHENCK, J. of P., 18 (1), 97 . Type (OD): Nucula tamatavica ODHNER

Lamellinucula tokyoensis (YoKoYAMA)ヨセナミクルミガイ（黒田）

1920 Nucula tokyoensis YoKoYAMA, JCSIUT, 39 (6), 181, pl. 19, f. 10, 11

1961 Nucula (Lamellinucula) tokyoensis: HABE, CISJ, 2, 107, pl, 48, f. 3

Loc. $S$ (s) tr.

Dist. Honshu (north to Bōsō Peninsula) to Kyushu (Korean Strait).

Lamellinucula gemmulata (HABE) マメシジナリクルミガイ（吉良）

1953 Nucula (Lamellinucula) gemmulata HABE, ICIS, 1 (25), 215

1977 Nucula (Lamellinucula) gemmulata: HABE, Taxa. HABE, 44, pl. 1, f. 1

Loc. S (s) dp.

Dist. Honshu (Sagami Bay) to Shikoku.

Genus Acila H. \& A. AdAMS オオキララガイ属

1858 Acila H. \& A. ADAms, GRM, 2, 545. Type (SD, StoliczKA, 1871): Nucula divaricata HINDS

Subgenus Acila H. \& A. ADAMS オオキララガイ垔属

Acila (Acila) divaricata schencki KIRA オニキララガイ（吉良）

1843 Nucula divaricata HiNDs, PZSL, 11 (126), 97. 
1850 Nucula mirabilis A. ADAMS \& ReEVE, Samarang, 75, pl. 21, f. 8

1871 Nucula divaricata: SOWERBY, Icon., sp. 29

1959 Acila schencki KIRA, CISJ, 1, 107, pl. 41, f. 6

Loc. M S (s-m) g1.

Dist. Honshu (north to Enshu-nada) to Kyushu.

\section{Subgenus Truncacila GRANT \& GALEキララガイ車属}

1931 Trancacila Grant \& GaLe, San Diego 1, p. 115. Type (OD): Nucula castrensis HINDS, 1843

Acila (Truncacila) minutoides KuRODA \& HABE ツボミキララガイ（黒田）

1938 Acila (Truncacila) minutoides KURODA \& HABE in HABE, PSMBL, 6, 243

1971 Acila (Truncacila) minutoides: KURODA \& HABE, SSSB, 504 (J) 317 (E), pl. 66, f. 7

Loc. S (s-m) tr.

Dist. Honshu (Bōsō Peninsula to Kii Peninsula).

Genus Ennucula IREDALE クルミガイ属

1931 Ennucula Iredale, RAM, 18, 202. Type (OD): Nucula obliqua LAMARCK

Ennucula niponica (Sмгтн)クルミガイ（黒田）

1885 Nucula niponica SMTtH, Challenger, 13, 226, p1. 18, f. 8, 8a

1959 Ennucula niponica: KIRA, CISJ, 1, 107, pl. 41, f. 3

Loc. M S (s-m) tr.

Dist. Honshu (north to Bōsō Peninsula) to Kyushu.

Ennucula cyrenoides (KURODA)シジナリクルミガイ（黒田）

1929 Nucula cyrenoides Kuroda, Venus, 1 (3), App. 7, f. 8, 9

1961 Ennucula cyrenoides: HABE, CISJ, 2, 107, pl. 48, f. 6

Loc. S (s) tr.

Dist. Honshu to Kyushu.

Ennucula teramachii $\mathrm{HABE}$ ，テラマチクルミガイ（東）

1958 Ennucula teramachii $\mathrm{HABE}, \mathrm{PSMBL}, 6$ (3), 244

1961 Ennucula teramachii: HABE, CISJ, 2, 107, pl. 48, f. 5

Loc. S (s-m) tr.

Dist. Honshu (Enshu-nada) to Shikoku.

Superfamily Nuculanacea H. \& A. ADAMS シワロウバイガイ超科

Family Sareptidae A. ADAMS ヒラソデガイ科

Subfamily Sareptinae A. ADAMS ヒラソデガイ巫科

Genus Sarepta A. ADAMS ヒラソデガイ属

1860 Sarepta A. Adams, AMNH, (3) 5, 333. Type (M): Sarepta speciosa A. AdAms

Sarepta speciosa A. ADAMS ヒラソデガイ（黒田）

1860 Sarepta speciosa A. Adams, AMNH, (3) 5, 333

1868 Sarepta speciosa: A. AdAms, J. de C., 16, 41, pl. 4, f. 1

1965 Sarepta speciosa: HABE, CISJ, 2, 107, pl. 48, f. 22 
1971 Sarepta speciosa: Kuroda \& HABE, SSSB, 506 (J) 319 (E), pl. 66, f. 15, 16

Loc. M S (s-m) dp.

Dist. Honshu (north to Bōsō Peninsula and Noto Peninsula) to Kyushu.

\section{Family Malletiidae H. \& A. ADAMS スミゾメソデガイ科 \\ Genus Bathymalletia KURODA \& HABEスミゾメソデガイ属}

1971 Bathymalletia Kuroda \& Habe, SSSB, 504 (J) 318 (E). Type (OD): Malletia inaequilateralis HABE

Bathymalletia inaequilateralis (HABE)スミゾメソデガイ（黒田）

1951. Malletia inaequilateralis $\mathrm{HABE}, \mathrm{GJS}, 1,21$, f. 14, 15

1971 Bathymalletia inaequilateralis: KURODA \& HABE, SSSB, 505 (J) 318 (E), pl. 66, f. 1

Loc. $S$ (s) tr.

Dist. Honshu (north to Bōsō Peninsula) and Shikoku.

\section{Genus Carinineilo KURODA \& HABEオ゙ネダカソデガイ属}

1971 Carinineilo Kuroda \& HABE, SSSB, 505 (J) 318 (E). Type (OD): Malletia (Neilo) carinifera $\mathrm{HABE}$

Carinineilo carinifera (HABE) オネダカソデガイ (黒田)

1951 Malletia (Neilo) carinifera HABE, GJS, 1, 22, f. 17, 18

1961 Malletia (Neilo) carinifera: HABE, CISJ, 2, 108, pl. 48, f. 9

1971 Carinineilo carinifera: KuRODA \& HABE, SSSB, 505 (J) 318 (E), pl. 66, f. 2

Loc. M S (s-m) tr.

Dist. Honshu (north to Sagami Bay) and Shikoku.

\section{Genus Neilonella DALLハトムギソデガイ属}

1877 Saturnia Seguenza, Atti Accad. Lincei. Mem., (3) 1, 1178 (non SChrank, 1802)

1881 Neilonella Dall, BMCZ, 9, 125. Type (OD): Leda (Neilonella) corpulenta Dall

Neilonella dubia PRASHAD ハトムギソデガイ（波部）

1932 Neilonella dubia Prashad, Siboga, 53 C, 27, pl. 1, f. 48, 49

1951 Neilonella coix HABE, GJS, 1, 23, f. 12, 13

1961 Neilonella coix: HABE, CISJ, 2, 108, pl. 48, f. 10

Loc. S (s) tr.

Dist. Honshu (north to Bōsō Peninsula) to Kyushu and Indonesia.

Family Nuculanidae H. \& A. ADAMS シワロウバイガイ科

Subfamily Nuculaninae H. \& A. ADAMS シワロウバイガイ坐科

Genus Nuculana LIṆKシワロウバイガイ属

1807 Nuculana LiNK, BNSUR, 3, 155

1817 Leda SchumaCher, ENSVT, 55, 173. Type (OD): Arca pernula MÜLleR

Subgenus Thestyleda IREDALEアラボリロウバイガイ亜属

1929 The styleda Iredale, RAM, 17, 158, 187. Type (OD): Leda ramsayi SmitH 
Nuculana (Thestyleda) yokoyamai (KURODA)アラボリロウバイガイ（黒田）

1920 Leda ramseyi: Yokoyama, JCSIUT, 39 (6), 176, pl. 19, 3 (non SмrтH, 1885)

1934 Nuculana yokoyamai KuRodA: Venus, 4 (4), 204

1961 Nuculana (Thestyleda) yokoyamai: HABE, CISJ, 2, 108, pl. 48, 17

1971 Nuculana (Thestyleda) yokoyamai: KuRODA \& HABE, SSSB, 508 (J) 320 (E), pl. 66, f. 14

Loc. M S (s-m) dp.

Dist. Honshu to Kyushu.

Nuculana (Thestyleda) acinacea HABEシャクシロウバイガイ（吉良）

1938 Nucula (Thestyleda) acinacea HABE, PSMBL, 6 (3), 247

1959 Nuculana (Thestyleda) acinacea: KIRA, CISJ, 1, 107, pl. 41, f. 9

Loc. $\mathrm{S}$ (s) tr.

Dist. Honshu (Kii Peninsula) and Shikoku.

\section{Genus Saccella WoodRINGゲンロクソデガイ属}

1898 (Dec.) Ledina Sacco in Bellardi \& SACCO, Moll. Terr. Terz. Piemonte e Liguria, 26, 53 (non Dall, 1898 (Nov.)).

1925 Saccella Woodring, CIP, 366, 15 (new name for Ledina SACCo, 1898). Type (OD): Lembulus deltoides Risso

Saccella confusa (HANLEY) ゲンロクソデガイ（岩川）

1860 Leda confusa HANLEY, Thes., 3, 119, pl. 228, f. 85

1959 Saccella confusa: KIRA, CISJ, 1, 107, pl. 41, f. 10

1971 Saccella (Saccella) confusa: Kuroda \& HABE, SSSB, 510 (J) 322 (E), pl. 117, pf. 7

Loc. N M S (s) dp.

Dist. Honshu (north to Bōsō Peninsula) to Kyushu and the western Pacific.

Saccella gordonis (YoKOYAMA) ゴルドンソデガイ (大塚)

1920 Leda gordonis YокоYAMA, JCSIUT, 39 (6), 171, pl. 19, f. 4, 5

1961 Saccella gordonis: HABE, CISJ, 2, 108, p1. 48, f. 20

1971 Saccella (Saccella) gordonis: KuRodA \& HABE, SSSB, 509 (J) 321 (E), pl. 66, f. 20

Loc. S (s) tr.

Dist. Honshu (north to Bōsō Peninsula and Noto Peninsula) to Kyushu and East China Sea.

Saccella (Saccella) sematensis (SUzuKI \& ISHIZUKA)アラスジソデガイ (鈴木・石塚)

1943 Nuculana confusa sematensis Suzukr \& IsHrzukA, Venus, 13, 54, 61, pl. 2, f. 1-10, 12

1961 Saccella sematensis: HABE, CISJ, 2, 108, pl. 48, f. 21

1971 Saccella (Saccela) sematensis: Kunoda \& HABE, SSSB, 511 (J) 321 (E), pl. 117, f. 5, 6

Loc. M S (s-m) dp.

Dist. Honshu (north to Bōsō Peninsula) to Kyushu.

\section{Subgenus Costanuculana HABE スダレソデガイ亜属}

1951 Costanuculana HABE, GJS, 1, 25. Type (OD): Nuculana husamaru NomURA

\section{Saccella (Costanuculana) husamaru (NomURA) スダレソデガイ（波部）}

1940 Nuculana (Jupitaria) husamaru NomURA, ROWJ, 12, 89, pl. 1, f. 5a, b

1958 Saccella soyoae HABE, PSMBL, 6, 249, pl. 11, f. 18

1964 Saccella (Costanuculana) soyoae: HABE, CISJ, 2, 109, pl. 43, f. 23

1971 Saccella (Costanuculana) husamaru: KURODA \& HABE, SSSB, 509 (J) 321 (E), pl. 66, f. 17 
Loc. M S (s) dp.

Dist. Honshu (Bōsō Peninsula to Kii Peninsula).

$$
\begin{array}{lr}
\text { Subfamily Yoldiinae } & \text { ナギナタソデガイ亜科 } \\
\text { Genus Yoldia MöLLER } & \text { ナギナタソデガイ䲩 }
\end{array}
$$

1842 Yoldia MöLLeR, Index Moll. Grönl., 18. Type (SD, ICZN, Op. 679)：Yoldia hyperborea TORELI,

Yoldia glauca KuRODA \& HABEクモリソデガイ（黑田・波部）

1961 Yoldia glauca KURODA \& HABE, CISJ, 2, 108, App. 35, pl. 48, f. 13

1971 Yoldia glauca: KurodA \& HABE, SSSB, 513 (J) 323 (E), pl. 66, f. 18

Loc. S (s-m) tr.

Dist. Honshu (north to Bōsō Peninsula) to Kyushu.

Yoldia similis KURODA \& HABEナガソデガイ（黑田）

1929 Yoldia naganumana Kuroda, Venus, 1, App. 10, f. 19 (non Yokoyama, 1920)

1952 Yoldia similis KuRODA \& HABE, Check List, 35 (n.n.)

1961 Yoldia similis: KuRODA \& HABE, CISJ, 2, 108, pl. 48, f. 12

1971 Yoldia similis: Kuroda \& HABE, SSSB, 513 (J) 323 (E), pl. 66, f. 18

Loc. S (s-m) tr.

Dist. Honshu (north to Bōsō Peninsula) to Kyushu.

\section{Genus Portlandia MöRCHベッコウキララガイ属}

1857 Portlandia Mörch, Prodr. Fauna Moll. Grönland, 21. Type (SD, ICZN, OP. 769): Nucula arctica GraY

Portlandia japonica (A. ADAMS \& REEVE) ベッコウキララガイ（平瀬）

1850 Nucula japonica AdAMs \& ReEVE, Samarang, 75, pl. 21, f. 9

1959 Portlandia (Portlandella) japonica: KIRA, CISJ, 1, 108, pl. 41, f. 11

1971 Portlandia (Portlandella) japonica: KuRODA \& HABE, SSSB, 512 (J) 322 (E), pl. 66, f. 12

Loc. N M (s-m) dp.

Dist. Honshu (north to Bōsō Peninsula) to Kyushu.

\section{Subclass Pteriomorphia BEURLEN 翼形里網}

Order Arcoida StolicZKa フネガイ目

Superfamily Arcacea LAMARCK フネ分煺科

Family Arcidae LAMARCK フネガイ科

Subfamily Arcinae LAMARCKフネガイ亜科

Genus Arca LinNaEUs フネガイ属

1758 Arca Linnaeus, Syst. Nat., 10, 693

1825 Navicula BLAinVILLE, DSN. 34, 319

1833 (May) Byssoarca Swainson, PZSL, 16 (non SwaInson, 1833 (March)).

1853 Cibota Mörch, Yoldi (2), 39. Type (SD, Schmidt, 1818): Arca noae LINNAEus (ICZN Op. 189) 
Arca navicularis BRUGUIÈRE ワシノ八ガイ（六介）

1789 Arca navicularis BRUGUIÈRE, EMHNV, 1,99

1844 Arca navicularis: ReEve, Icon., sp. 70

1845 Arca linter JonAs, ZfM, 172

1847 Arca subquadrangula PhILIPPI, Abbild., 2, 211, pl. 3, f. 2

1959 Arca navicularis: KIRA, CISJ, 1, 110, pl. 43, f. 1

Loc. N M S (r) gl.

Dist. Honshu (north to Bōsō Peninsula) to Kyushu. Widely distributes in the tropic Pacific.

Arca ventricosa (LAMARCK) オオタカノハガイ (岩川)

1819 Arca ventricosa LAMARCK, AsV, 6 (1), 38

1844 Arca zebra REEVE, Icon., sp. 69, (non SwaInson, 1832)

1847 Arca ventricosa: PhILIPI, Abbild., 2, 211, pl. 3, f. 4, 5

1959 Arca ventricosa: KIRA, CISJ, 1, 110, pl. 43, f. 2

Loc. M S (r) gl.

Dist. Honshu (north to Kii Peninsula) to Kyushu.

Arca avellana LAMARCK フネガイ(六介)

1819 Arca avellana LAMARCK, AsV, 6 (1), 38

1844 Arca ocellata REEve, Icon., sp. 102

1847 Arca arabica Philippi, Abbild., 3, 28, pl. 4, f. 2

1895 Arca acuminata subnormalis PILSBRY, CMMJ, 148

1901 Arca bicarinata Sowerby, PMSL, 4, 211, pl. 22, f. 14 (non ReEve, 1844)

1959 Arca arabica: KIRA, CISJ, 1, 109, pl. 42, f. 7

1971 Arca ocellata Kuroda \& HABE, SSSB, 518 (J) 326 (E), pl. 67, f. 9

Loc. M S (r) int.

Dist. Honshu to Kyushu. Widely distributes in the Indo-Pacific region.

Arca boucardi JoUsSEAUMEコベルトフネガイ (平瀬)

1894 Arca boucardi JousSEAUME, Humming Bird, 4, 41

1904 Arca kobeltiana PILSBRY, PANSP, 56, 559, pl. 40, f. 14-19

1906 Arca rectangularis TOKUNAGA, JCSIUT, 21 (2), 61, pl. 3, f. 23a-c

1951 Arca miyatensis Oyama, MG, 4, 155

1959 Arca boucardi: KIRA CISJ, 1, 110, pl. 42, f. 15

$1966 \operatorname{Arca}$ (Arca) miuraensis NoDA, SRTU, (2) 38 (1), 61, pl. 3, f. 11, 12, 14, 15

Loc. N M S (r) int.

Dist. Hokkaido to Kyushu and Korea.

\section{Genus Barbatia GRAY エガイ属}

1842 Barbatia Gray, Synop. Conch. Brit. Mus, 44, 81. Type (SD, GraY, 1847): Arca barbata LINNAEUS

Subgenus Abarbatia DALL, BARTSCH \& REHDERエガイ亜属

1938 Abarbatia DALl, BARTSCh \& REHDER, BPBMB, 153, 29. Type (OD): Barbatia (Abarbatia) oahua DALL, BARTSCH \& REHDER

Barbatia (Abarbatia) velata (SOWERBY) エガイ (群品)

1833 Byssoarca velata SowERBY, PZSL, 18

1833 Byssoarca decussata Sowerby, PZSL, 18 (non Arca desussata LinnaEUs, 1758)

1844 Arca decussata: ReEve, Icon., sp. 81 
1844 Arca lima ReEve, Icon., sp. 101

1959 Barbatia lima: KIRA, CISJ, 1, 122, pl. 43, f. 12

Loc. N M S (r) int.

Dist. Honshu (north to Bōsō Peninsula) to Kyushu. Widely distributes in the Indo-Pacific region.

\section{Subgenus Savignyarca JoUSSEAUMEカリガネエガイ亜属}

1891 Savignyarca JousseAume, Le Nat., 5, 222. Type (T): Savignyarca savignyarca Jousseaume

Barbatia (Savignyarca) virescens (REEVE)カリガネエガイ (平瀬)

1844 Arca virescens ReEve, Icon., sp. 97

1844 Arca obtusa ReEve, Icon., sp. 77 (non KonINCK, 1842)

1848 Arca obrusoides NYST, MARSB, 22, 50 (new name for Arca obtusa REEVE, 1844)

1868 Arca decurvata LischKe, M BL, 16, 108

1959 Barbatia (Savignyarca) virescens obtusoides: KIRA, CISJ, 1, 109, pl. 42, f. 4

Loc. N M S (r) int.

Dist. Honshu to Kyushu. Widely distributes in the Indo-Pacific region.

Subgenus Barbatirus IREDALE トヤマエガイ巠属

1939 Barbatirus IreDALE, GBRESR, 5 (6), Moll. 1, 256. Type (OD): Barbatirus mimulus IREDALE

Barbatia (Barbatinus) cometa (REEVE) トヤマエガイ（吉良)

1844 Arca cometa REEVE, Icon., sp. 111

1959 Barbatia (Barbatirus) cometa: KIRA, CISJ, 1, 109, pl. 42, f. 9

Loc. S (r) int.

Dist. Honshu (Kii Peninsula) to Kyushu. Widely distributes in the tropic Pacific.

\section{Subgenus Ustularca IREDALE ベニエガイ亜属}

1839 Ustularca Iredale, GBRESR, 5 (6), Moll, 1, 268. Type (OD): Ustularca cruciata IREDALE

Barbatia (Ustularca) bicolorata (DILlwYN) ベニエガイ（目八）

1792 Arca fusca Bruguière, Encycl. Meth. Tabl. Vers., 1, 102 (non Lightroot, 1786)

1817 Arca bicolorata DillwyN, DCRS, 1, 230

1844 Arca fusca: ReEve, Icon., sp. 82

1959 Barbatia (Ustularca) bicolorata: KIRA, CISJ, 1, 110, pl. 42, f. 14

Loc. N M S (r) int.

Dist. Honshu (north to Kii Peninsula) to Kyushu. Widely distributes in the tropic Pacific.

Barbatia (Ustularca) stearnsi (PILSBRY) ハナエガイ (目八)

1895 Arca stearnsi Prlsbry, CMMJ, 148, pl. 3, f. 8-10

1961 Barbatia (Ustularca) stearnsi: HABE, CISJ, 2, 111, pl. 49, f. 16

1971 Barbatia (Ustularca) stearnsi: KurodA \& HABE, SSSB, 522 (J) 329 (E), pl. 67, f. 19, 20

Loc. N M S (r) int.

Dist. Honshu (north to Bōsō Peninsula) to Kyushu. 
Barbatia (Ustularca) hachijoensis Hatal, NinNo \& KoTAKAオオハナエガイ（黒田）

1952 Barbatia hachijoensis HaTar, Nino \& KotaKA, JTUF, 39 (1), 107 pl. 4, f. 1

1971 Barbatia (Ustularca) hachijoensis: KURODA \& HABE, SSSB, 522 (J) 329 (E), pl. 67, f. 14

Loc. M S (r) gl.

Dist. Honshu (north to Sagami Bay) to Kyushu.

Genus Barbarca DALL, BARTSCH \& REHDER ハブタエエガイ属

1938 Barbarca DALL, BARTSCH \& REHDER, BPBMB, 153, 23

1939 Opularca Iredale, GBRESR, 5 (6), Moll, 1, 269. Type (OD): Calloarca (Barbarca) hua DALL, BARTSCH \& REHDER, 1938

Barbarca tenella (REEVE) ハブタエエガイ（黒田）

1844 Arca tenella ReEve, Icon, sp. 91

1867 Barbatia mollis DUNKER, Nov. Conch., 92, pl. 31, f. 2-4

1938 Calloarca (Barbarca) hua DALL, BARTSCH \& REHDER, BPBMB, 153, 24, pl. 4, f. 5-8, tf. 8

1939 Opularca tenella egenora IREDALE, GBRESR, 5 (6), Moll. 1 269, pl. 3, f. 2, 2 a

1961 Barbatia (Barbarca) tenella: HABE, CISJ, 2, 111, pl. 49, f. 13

Loc. S (g) dp.

Dist. Honshu (north to Kii Peninsula). Widely ranging in the central and western Pacific.

\section{Genus Vitracar IREDALEニキエガイ属}

1939 Vitracar IREDALE, GBRESR, 5 (6), Moll. 1, 263. Type (OD): Vitracar laterosa (sic) IREDALE

Vitracar albidum SAKURAI \& HABEユキエガイ（桜井, 波部)

1966 Vitracar albida SAKURAI \& HABE, Venus, 24 (4), 293, 295

1977 Vitracar albida: HABE, Taxa HABE, 21, pl. 1, f. 7

Loc. M S (r) gl.

Dist. Honshu (Kii Peninsula) to Ryukyu Islands.

\section{Genus Acar GRAYコシロガイ属}

1857 Acar Gray, AMNH, (2) 19, 369. Type (SD, STOLICZKA, 1871): Arca divaricata Sowerby

Acar plicatum (DILlWYN) コシロガイ（目八）

1817 Acar plicatum DillwYN, DCRS, 1, 227

1930 Barbatia (Acar) reticulata: Kuroda, Venus 1 (6), App. 25, f. 34 (non GMELIN, 1791)

1959 Acar plicatum: KIRA, CISJ, 1, 109, pl. 42, f. 6

1966 Barbatia (Acar) numaensis NodA, SRTU, (2) 38(1), 68, pl. 5, f. 11, 12

Loc. N M S (r) int.

Dist. Honshu to Kyushu. Tropic Pacific.

Acar plicatum congenitum (SMITH) イトココシロガイ（波部）

1885 Acar congenita SMITH, Challenger, 264, pl. 17, f. 6, 6a

1961 Acar congenita: HABE, CISJ, 2, 110, pl: 49, f. 5

1971 Acar plicatum congenitum: KURODA, \& HABE, SSSB, 523 (J) 330 (E); pl. 67, f. 15, 16

Loc. M S (r) gl.

Dist. Honshu (north to Bōsō Peninsula) to Philippines. 
Genus Hawaiarca DALL, BARTSCH \& REHDER オトギノワシノ八ガイ属

1938 Hawaiarca DALL, BARTSCH \& REHDER, BPBMB, 153, 27. Type (OD): Hawaiarca rectangula DALL, BARTSCH \& REHDER

Hawaiarca uwaensis (YoKoYAMA)オトギノワシノハガイ（黒田）

1928 Arca uwaensis YokoYAMA, JFSIUT, (2) 2 (7), 349, pl. 67, f. 13, 14

1961 Hawaiarca uwaensis : HABE, CISJ, 2, 110, pl. 49, f. 10

Loc. M S (r) gl.

Dist. Honshu (north to Bōsō Peninsula) to Kyushu.

Hawaiarca yamamotoi (SAKuRAI \& HABE) サンゴエガイ（桜井, 波部)

1961 Barbatia yamamotoi SAKURAI \& HABE, CISJ, 2, 111, App. 35, pl. 44, f. 12

Loc. M (r) gl.

Dist. Honshu (Kii Peninsula) to Ryukyu Islands (Miyako Island).

$$
\text { Genus Nipponarca HABE ヒメエガイ属 }
$$

1951 Nipponarca Habe, GJS, 1, 34. Type (OD): Arca bistrigata DUNKER, 1866

Nipponarca bistrigata (DUNKER) ヒメエガイ（黒田）

1866 Arca bistrigata Dunker, Nov. Conch, 87, pl. 30, f. 4-6

1928 Arca (Barbatia) obtusa duplicostata GraBau \& KING, Peitaiho, 159, pl. 1, f. 7

1961 Nipponarca bistrigata: HABE, CISJ, 2, 110, pl. 49, f. 11

Loc. M (s-m) dp.

Dist. Honshu (north to Bōsō Peninsula) to Kyushu and the western Pacific.

$$
\text { Genus Mimarcaria IREDALE チゴワシノ八ガイ属 }
$$

1939 Mimarcaria Iredale, GBRESR 5(6), Moll. 1, 267. Type (OD): Mimarcaria saviolum IREDALE

Mimarcaria matsumotoi HABEチゴワシノ八ガイ（黑田）

1958 Mimarcaria matsumotoi HABE, Venus, 19 (3, 4), 177, 179, tf. 1,2

1961 Mimarcaria matsumotoi: HABE, CISJ, 2, 110, pl. 49, f. 2

1971 Mimarcaria matsumotoi: KuRodA \& HABE, SSSB, 520 (J) 327 (E), pl. 67, f. 5, 6

Loc. M S (r) gl.

Dist. Honshu (north to Bōsō Peninsula) to Kyushu.

\section{Genus Bentharea VERRILL \& BUSHクマサカヤドリエガイ属}

1898 Bentharca Verrill \& BUSH, PUSNM, 20, 842. Type (OD): Nacrodon asperula Dall, 1881

Bentharca rubrotincta KURODA \& HABEアカフクマサカヤドリエガイ（黑田・波部）

1958 Bentharca rubrotincta KURODA \& HABE in HABE, PSMBL, 6 (3), 252

1961 Bentharca rubrotincta: HABE, CISJ, 2, 110, pl. 49, f. 6

Loc. M (ep) gl.

Dist. Honshu (Kii Peninsula) to Ryukyu Islands.

Bentharca xenophoricola (KURODA) クマサカヤドリエガイ（黒田）

1930 Batharca xenophoricola KurodA, Venus, 2 (1), App. 33, f. 38, 39

1959 Bentharca xenophoricola: KIRA, CISJ, 1, 108, pl. 42, f. 2 
Loc. M S (ep) dp.

Dist. Honshu (north to Suruga Bay) to Kyushu.

Remark: This species may be a form of B. asperula (DALL).

Bentharca asperula (DALL) ワダッミエガイ (奥谷)

1881 Macrodon asperula DALL, BMCZ, 9 (2), 120

1885 Arca profundicola VerRILL, TCAS, 6, 439, pl. 44, f. 23

1885 Arca (Barbatia) pteroessa Sмітн, Challenger, 326, pl. 17, f. 4

1939 Bentharca hawaiensis DALL, Bartsch \& REHDER, BPBMB, 153, 20, p1. 3, f. 15-18

1939 Bentharca kauaiensis DALL, BARTSCH \& REHDER, BPBMB 153, 22, pl. 3, f. 5-6, tf. 7

1976 Bentharca asperula: OKuTANI, Chiribotan, 9 (3), 45, f. on p1. 5

Loc. S (g) tr.

Dist. Izu Islands to Amami Islands and Hawaii Islands. Also Indian and Atlantic Oceans.

\section{Genus Bathyarea KoBELT ワタゾコエガイ属}

1891 Bathyarca KoBeLt, Cab., 8 (2), 213

1929 Microcucullaea Iredale, RAM, 17, 159. Type (SD, Verrill \& Bush, 1898): Arca pectunculoides SACCHI

Bathyarca anaclima MelvILLワタゾコエガイ（黒田）

1906 Arca (Bathyarca) anaclima MeLVLL, PZSL, 794, pl. 54, f. 6, 6a

1932 Arca (Bathyarca) sibogai Prashad, Siboga., 53c, 55, pl. 2, f. 1-3

1940 Bathyarca (Microcucullaea) kyurokusimana NomURA \& HATAI, SHKMRB, 19, 75, pl. 4, f. 1a, b

1961 Bathyarca kyurokusimana: HABE, CISJ, 2, 110, pl. 49, f. 8

Loc. M S (s) dp.

Dist. Honshu to Kyushu. Widely distributes in the Pacific and Indian Oceans.

*Bathyarca japonica HABEダイコクワタゾコエガイ（波部）

1977 Bathyarca corpulenta HABE (non SмIтH, 1885), SMJB/S, 35

1977 Bathyarca japonica HABE, Venus, 36 (1), 2, tf. 1

Loc. S (s-m) tr.

Dist. Honshu (Kii Peninsula) and Indonesia.

\section{Genus Samacar IREDALE ミノエガイ属}

1936 Samacar IREDALE, RAM, 19 (3), 268

1940 Paragrammatodon Nomura \& NIINO, ROWJ, 12, 42. Type (OD): Arca strabo HedleY

Samacar strabo (HEDLEY) ミノェガイ（黒田）

1914 Arca strabo HeLdeY, PLSNSW 39, 697, pl. 78, f. 19, 20

1934 Bathyarca (Pseudogrammatodon) pacifica Nomura, NiIno \& Zinbo, SRTIU, (2) 16 (2), 53, pl. 5, f. 6a, b, 7a, b

1961 Samarcar pacifica: HABE, CISJ, 2, 111, pl. 49, f. 15

Loc. M S (r) gl.

Dist. Honshu (north to Bōsō Peninsula) to Kyushu and the east coast of Australia. 


\section{Subfamily Anadarinae REINHART リュウキュウサルボウガイ巠科 \\ Genus Scapharca GRAYサルボウガイ属}

1847 Scapharca GraY, PZSL, 15, 198 (as Scapharea). Type (OD): Arca inaequivalvis BRUGUIÈRE

Scapharca broughtonii (SCHRENCK)，アカガイ(和鈔)

1844 Arca inflata ReEve, Icon., sp. 30 (non ScHröter, 1802)

1848. Arca reeveana NyST, MARSB, 22, 70 (new name for Arca inflata ReEve, 1844) (non D'ORBIGNY, 1847)

1867 Arca broughtonii Schrenck, RAZM, 578, pl. 24, f. 1-3

1906 Arca tenuis ToKunAGA, JCSIUT, 21 (2), 58, pl. 4, f. 1a, b

1959 Anadara (Scapharca) broughtonii: KIRA, CISJ, 1, 111, pl. 43, f. 14

Loc. N M S (s-m) dp.

Dist. Southern Hokkaido to Kyushu and Korea.

Scapharca satowi DUNKERサトウガイ（岩川）

1882 Scapharca satowi Dunker, Index, 233, pl. 9, f. 1-3

1901 Arca nipponoensis PILSBRY, PANSP, 53, 209, pl. 19, f. 2

1959 Anadara (Scapharca) satowi: KIrA, CISJ, 1, 112, pl. 43, f. 13

Loc. N M S (s-m) dp.

Dist. Honshu (north to Bōsō Peninsula) to Kyushu.

Scapharca inaequivalvis (BRUGUÈRE)クイチガイサルボウガイ（岩川）

1789 Arca inaequivalvis BRUGUİ̀RE, EMHNV, 1, 106

1844 Arca rufescens REEVE, Icon., sp. 53

1844 Arca disparillis ReEve, Icon., sp. 59

1971 Scapharca inaequivalvis: Kuroda \& HABE, SSSB, 529 (J) 333 (E), pl. 69, f. 3, 4

Loc. N M S (s-m) dp.

Dist. Honshu (north to Bōsō Peninsula) to Kyushu. Widely ranging in the western Pacific.

Scapharca subcrenata (LISCHKE)サルボウガイ（目八)

1847 Anadra amygdalum PHILIPPI. Abbild., 2, pl. 2, f. 2 (non LiNk 1807)

1869 Arca subcrenata LiSCHKE, M BL, 16, 107

1869 Arca subcrenata: LISCHKE, JMC, 1, 146, pl. 9, f. 1-3 (as nodosocrenata on the plate)

1906 Arca kagoshimensis ToKunAGA, JCSIUT, 21 (2), 59, pl. 3, f. 21a, b

1928 Arca (Scapharca) peitaihoensis GrabaU \& King, Peitaiho, 159, pl. 1, f. 6

1959 Anadara (Scapharca) subcrenata: KIRA, CISJ, 1, 111, pl. 43, f. 7

Loc. N M (s-m) dp.

Dist. Honshu (north to Tokyo Bay) to Kyushu. Widely ranging in the western Pacific.

Scapharca troscheli (DUNKER)（シロクサルボウガイ（平瀬），ヒメアカガイ（岩川）

1882 Arca troscheli Dunker, Index, 234, pl. 1, f. 14, 15

1959 Anadara (Scapharca) troscheli: KIRA, CISJ, 1, 111, pl. 43, f. 5

1965 Scapharca troscheli: HABE, BNSM, 8 (1), 80, pl. 3, f. 5, 6

Loc. N M (s-m) int.

Dist. Honshu (north to Mutsu Bay) to Kyushu. 


\section{Genus Diluvarca WooDRINGハゴロモガイ属}

1825 Diluvarca Woodring, CIP, 366, 40. Type (OD): Arca diluvii Lamarck

Diluvarca ferruginea (REEVE) ハゴロモガイ（介志）

1844 Arca ferruginea ReEve, ICON., sp. 39

1844 Arca radiata REEVE, Icon., sp. 40 (non SOWERBY, 1840)

1848 Arca tricenicosta NysT, MARSB, 22, 52

1882 Scapharca philippiana DUNKER, Index, 235

1959 Anadara (Diluvarca) ferruginea: KIRA, CISJ, 1, 111, pl. 43, f. 11

1965 Anadara (Diluvarca) ferruginea: HABE, BNSM, 8 (1), 82, pl. 3, f. 1

1971 Diluvarca tricenicosta: KURODA \& HABE, SSSB, 530 (J) 335 (E), pl. 70, f. 4, 5

Loc. N M S (s-m) dp.

Dist. Honshu (north to Bōsō Peninsula) to Kyushu and the western Pacific.

Genus Kikaiarca NoDAキカイサルボウガイ属

1966 Kikaiarca NoDA, SRTU, (2) 38 (1), 127. Type (OD): Arca kikaizimana NomuRA \& ZiNBO

Kikaiarca kikaizimana (NomURA \& ZINBO)キカイサルボウガイ（鹿間）

1934 Arca kikaizimana Nomura \& ZinBo, SRTU, (2) 16, 152, pl. 5, f. 4a, b, 5a, b.

1965 Anadra kikaizimana: HABE, BNSM, 8 (1), 82, pl. 3, f. 8

Loc. M S (s-m) dp.

Dist. Honshu (Enshu-Nada) to Kyushu.

\section{Genus Tegillarca IREDALE 八イガイ属}

1853 Anomalocardia Mörch, Yoldi., 2, 41 (non SchumaCher, 1817)

1939 Tegillarca Iredale, GBRESR, 5 (6), Moll. 1, 281. Type (OD): Tegillarca (granosa) bessalis IREDALE

Tegillarca granosa (LinnaEUs) ハイガイ(目八)

1758 Arca granosa Linnaeus, Syst. Nat., 10, 694

1814 Arca granosa: ReEve, Icon., sp. 15

1938 Anadara bisenensis SCHRENCK \& ReINHART, MARSB, (2) 14, 44, pl. 4, f. 2; pl. 5, f. 1

1953 Tegillarca abessa KotAKA, TPPSJ, (n.s.) 10, 35, pl. 4, f. 1, 2, 6 (non Arca obesa Sowerby, 1833)

1959 Anadra (Telliarca) granosa bisenensis: KIRA, CISJ, 1, 111, pl. 43, f. 8

1966 Anadra (Tegillarca) granosa kamakurensis NODA, SRTU, (2) 38 (1), 125, pl. 14, f. 1-3

Loc. N M (s-m) int.

Dist. Seto-Naikai (Inland Sea of Japan) and Ariake Bay, Kyushu. Widely ranging in the Indo-Pacific region.

\section{Subfamily Striarcinae MacNEIL ミミエガイ亜稺 \\ Genus Arcopsis KOENEN ミミェガイ属}

1885 Arcopsis Koenen, Abh. Ges. Wiss. Göttingen, 32, 86. Type(OD): Arca limopsis Koenen

Arcopsis symmetrica (REEVE) ミミエガイ (平瀬)

1844 Arca symmetrica ReEve, Icon., sp. 117 (not of fig. 117)

1953 Striarca (Galactella) oyamai HABE, ICJS, 1 (25), 209, pl. 30, f. 20, 21

1959 Striarca (Galactella) oyamai: KrRA, CISJ, 1, 109, pl. 42, f. 5 
1961 Striarca (Galatella) symmetrica: HABE, CISJ, 2, 110, pl. 49, f. 1

Loc. N M S (r) int.

Dist. Honshu (north to Bōsō Peninsula) to Kyushu. Widely ranging in the tropic Pacific.

Arcopsis sculptilis (REEVE)ヤサガタミミエガイ（黒田）

1844 Arca sculptilis ReEve, Icon., sp. 118

1844 Arca zebuensis ReEve, Icon., sp. 120, f. 117 (not of f. 120)

1961 Striarca (Galactella) sculptilis: HABE, CISJ, 2, 110, p1. 49, f. 7

Loc. S (st) int.

Dist. Honshu (Kii Peninsula) to Ryukyu Islands. Widely ranging in the tropic Pacific.

Arcopsis interplicata (GRABAU \& KING) ヨコヤマミミエガイ（黑田）

1928 Arca (Barbatia) interplicata GrabaU \& KING, Peitaiho, 161, pl. 1, f. 9

1933 Arca (Barbatia) yokoyamai NomURA, SRTIU, (2) 16, (1), 41, pl. 1, f. 3a-b (new name for Arca symmetrica YoKoYAMA, 1920 non ReEve, 1844)

1959 Striarca (Spinearca) interplicata: KrRA, CISJ, 1, 108, pl. 42, f. 1

1971 Verilarca interplicata: Kuroda \& HABE, SSSB, 526 (J) 332 (E), pl. 67, f. 8

Loc. N M S (s-m) dp.

Dist. Honshu (north to Bōsō Peninsula) to Formosa and China.

*Arcopsis minabensis HaBE sp. nov. チョビヒゲミミエガイ（波部） pl. 2, fig. 1

Shell small, solid, inequilateral, transversely elongated ellipsoidal in shape, with the straight dorsal margin, roundly curved anterior margin, obliquely and widely truncated posterior margin and rather straight ventral margin, inflated at the umbonal portion. Umbo a little anterior from the middle of dorsal margin, directed forwards and beyond the dorsal margin. Surface covered with a light yellowish periostracum and sculputred with the growth riblets and radiating riblets forming the reticulated texture and moreover has the obtuse ridge running from the umbo to the postero-ventral corner and forming the triangular postro-dorsal area, and a weak depression medially cousing the weak ventral byssal sinuation. Diamond area between umbones narrow and long, flat and rather smooth except the very small black ligamental area below the umbo. Interior white. Hinge plate with distinct teeth, anterior row 8-10 and the posterior 15-20 in number. Inner margin crenulated corresponding with the radial riblets on the surface.

Length $6.5 \mathrm{~mm}$, height $4.5 \mathrm{~mm}$ and breadth $3.5 \mathrm{~mm}$. (Holotype conjoined valves preserved in the National Science Museum, NSMT-Mo 58956) Length $6.3 \mathrm{~mm}$, height 4.5 and breadth 3.4 . (Paratype conjoined vlaves No. 1, NSMT-Mo 58957)

Length $5.4 \mathrm{~mm}$, height $3.6 \mathrm{~mm}$ and breadth $3.0 \mathrm{~mm}$. (Paratype conjoined valves No. 2, NSMT-Mo 58958)

Type locality: off Minabe, Wakayama Pref., Honshu at the submarine reefs of about $20-30 \mathrm{~m}$ deep.

Remarks: This new species is similar to the intertidal species, Arcopsis symmetrica (REEVE) in general features but has the narrow and but long diamond with the very small ligamental area between umbones and the decidedly reticulated surface sculpture. 
Loc. M S (r) gl.

Dist. Wakayama Prefecture, Honshu.

Genus Spinearca IREDALE モエシマミミエガイ属

1939 Spinearca Iredale, GBRESR, 5 (6), Moll. 1, 286. Type (OD): Spinearca deliciosa IREDALE

Spinearca fausta (HABE) モエシマミミエガイ (波部)

1951 Striarca (Spinearca) fausta HABE, GJS, 1, 38

1961 Striarca (Spinearca) fausta: HABE, CISJ, 2, 110, pl. 49, f. 3

1971 Spinearca fausta: KURODA \& HABE, SSSB, 527 (J) 333 (E), pl. 117, f. 13, 14

Loc. M S (r) dp.

Dist. Honshu (north to Sagami Bay) to Kyushu.

\section{Genus Didimacar IREDALE マルミミエガイ属}

1939 Didimacar IreDALE, GBRESR, 5 (6), Moll. 1, 289. Type (OD): Didimacar repenta(sic) IREDALE

Didimacar tenebricum (REEVE) マルミミエガイ (岩川)

1844 Arca tenebrica ReEve, Icon., sp. 105

1952 Barbatia koshibensis HataI \& Nishryama, SRTU, (2) Sp. 3, 28

1959 Striarca (Didimacar) tenebrica: KIRA, CISJ, 1, 109, pl. 42, f. 8

Loc. N M S (s) dp.

Dist. Honshu (north to Bōsō Peninsula) to Kyushu. Widely ranging in the western Pacific.

Didimacar soyoae (HABE) ソウヨウミミエガイ (波部)

1958 Striarca (Spinearca) soyoae HABE, PSMBL, 6 (3), 255, pl. 11, f. 28, 29

1961 Striarca (Spinearca) soyoae: HABE, CISJ, 2, 110, pl. 49, f. 4

1971 Didimacar soyoae: KuRoda \& HabE, SSSB, 528 (J) 333 (E), pl. 67, f. 7

Loc. M S (r) dp.

Dist. Honshu (north to Bōsō Peninsula) to Kyushu and the southern part of Japan Sea.

\section{Family Parallelodontidae D ALL シコロエガイ科 \\ Subfamily Cucullarinae HABE シコロエガイ髙科 \\ Genus Porterius CLARK シコロエガイ属}

1925 Porterius Clark, Univ. Calif. Publ. Bull. Dept. Geol. Sci., 15 (4), 79

1931 Pseudogrammatodon ARKeLL, Geol. Mag. 67, 307. Type (OD): Barbatia andersoni von WINKLE, 1918

Porterius dalli (SMITH)シコロエガイ (河本)

1885 Arca (Macrodon) dalli Sмiтн, Challenger, 269, pl. 17, f. 10-10a, b

1920 Parallelodon obliquatus YоKоYAMA, JCSIUT, 39 (6), 170, pl. 18, f. 9-11

1922 Cucullaria orientalis YoKoyama, JCSIUT, 44 (1), 191, pl. 17, f. 8, 9

1959 Pseudogrammatodon dalli: KIRA, CISJ, 1, 109, pl. 42, f. 10

Loc. S (s) dp.

Dist. Honshu (south to Setonai-kai) to Southern Hokkaido and Korea. 


\section{Family Cucullaeidae STEWART ヌノメアカガイ科 \\ Genus Cucullaea LAMARCKヌノメアカガイ属}

1801 Cucullaea Lamarck, AsV, 116. Type (SD, Children, 1823): Cucullaea auriculifera LAMARCK

Cucullaea labiata granulosa (JoNAS)ヌノメアカガイ（岩川）

1786 Arca labiata Lightroot, Portland, 185

1846 Cucullaea granulosa JoNAS, PZSL, 36

1870 Cucullaea granulosa: SOWERBY, Icon., sp. 2

1959 Cucullaea granulosa: KIRA, CISJ, 1, 111, pl. 43, f. 9

Loc. N M S (s-m) dp.

Dist. Honshu (north to Bōsō Peninsula) to Kyushu. Widely distributes in the western Pacific.

\section{Family Glycymerididae NEWTON タマキガイ科 \\ Subfamily Glycymeridinae NEWTON タマキガイ互科 \\ Genus Glycymeris DA CosTA タマキガイ属}

1778 Glycymeris DA Costa, Brit. Conch., 168

1798 Tuceta RöDING, Bolten, 172

1799 Pectunculus Lamarck, MSHNP, 87. Type (T): Arca glycymeris LinNaEus

Glycymeris vestita (DUNKER) タマキガイ（目八)

1877 Pectunculus vestitus DUNKer, MBL., 24, 72

1877 Pectunculus fulguratus DUNKER, MBL., 24, 72

1882 Pectunculus vestitus: DUNKER, Index, 236, pl. 16, f. 7, 8

1882 Pectunculus fulguratus: DUNKER, Index, 236, pl. 14, f. 17, 18

1959 Glycymeris (Veletuceta) fulgurata: KIRA, CISJ, 1, 113, pl. 44, f. 9

1959 Glycymeris vestita: KIRA, CISJ, 1, 113, pl. 44, f. 11

Loc. M S (s) dp.

Dist. Honshu (north to Bōsō Peninsula) to Kyushu.

Glycymeris imperialis KURODA ミタマキガイ（黑田）

1934 Glycymeris imperialis Kuroda, Venus, 4 (4), 201, pl. 4, f. 1-5

1959 Glycymeris imperialis: KrRA, CISJ, 1, 113, pl. 44, f. 5

1971 Glycymeris imperialis: KURODA \& HABE, SSSB, 531 (1) 335 (E), pl. 71, f. 3-5

Loc. M S (s) dp.

Dist. Honshu (Sanriku to Kii Peninsula).

Glycymeris albolineata (LISCHKE) ベンケイガイ（目八）

1872 Pectunculus albolineatus LISCHKE, MBL, 19, 109

1874 Pectunculus albolineatus: LISCHKE, JMC, 3, 108, pl. 9, f. 11, 12

1959 Glycymeris (Veletuceta) albolineata: KIRA, CISJ, 1, 113, pl. 44, f. 12

Loc. N M S (s) dp.

Dist. Honshu (north to Bōsō Peninsula) to Kyushu.

Glycymeris rotunda (DUNKER) ベニグリガイ（目八）

1882 Pectunculus rotundus DUNKer, Index, 236, pl. 16, f. 9, 10

1920 Pectunculus nipponicus YokoYama, JCSIUT, 39 (6), 168, pl. 18, f. 3-7 
1922 Pectunculus yamakawai YokoYAmA, JCSIUT, 40 (1), 190, pl. 16, f. 4, 5

1959 Glycymeris rotunda: KIRA, CISJ, 1, 113, pl. 44, f. 8

1971 Glycymeris rotunda: KURODA, \& HABE, SSSB, 532 (J) 336 (E), pl. 71, f. 6

Loc. N M S (s) gl.

Dist. Tsugaru Strait to East China Sea.

Genus Tucetilla IREDALEビロウドタマキガイ属

1939 Tucetilla IrEDALE, GBRESR, 5 (6), Moll. 1, 300. Type (OD): Glycymeris capricornis HEDLEY

Tucetilla pilsbryi (YoKOYAMA)ビロウドタマキガイ（黑田）

1920 Pectunculus pilsbryi YoKоуамA, JCSIUT, 39 (6), 170, pl. 18, f. 8

1961 Glycymeris (Tucetilla) pilsbryi: HABE, CISJ, 2, 112, pl. 50,ff. 1

1971 Tucetilla pilsbryi: Kunoda \& HABE, SSSB, 533 (J) 336 (E), pl. 71, f. 7, 8

Loc. S (s) dp.

Dist. Tsugaru Strait to East China Sea.

Tucetilla amamiensis KURODAヤスリメタマキガイ（黒田）

1930 Glycymeris amamiensis KURODA, Venus, 1 (6), App. 2

1961 Glycymeris (Tucetilla) amamiensis: HABE, CISJ, 2, 112, pl. 50, f. 2

Loc. S (s) dp.

Dist. Honshu (Kii Peninsula) to Okinawa Island.

Remark: This may be as same as T. capricornea (HedLeY, 1906).

Genus Tucetonella HABE コギツネガイ属

1961 Tucetona (Tucetonella) planicostata HABE, CISJ, 2, app. p. 36. Type (OD): Pectunculus mundus SOWERBX, 1903

Tucetonella munda (SowerbY, 1903)コギッネガイ（岩川）

1903 Pectunculus mundus Sowerby, AMNH, (7) 12, p. 501.

1952 Tucetona shinkurosensis HATAI, NIINo \& KOTAKA JTUF, 39, (1) p. 109, f. 11, 12

1962 Tucetona (Tucetonella) planicostata HABE, CISJ, 2, app. p. 36, pl. 50, f. 5

1971 Tucetonella munda: KURODA \& HABE, SSSB, pp. 534 (Jap), 337 (Eng.), pl. 71, f. 18-20.

Loc. $\mathrm{S}$ (s) dp.

Dist. Honshu (north to Bōsō Peninsula) to Kyushu (Goto Islands).

Tucetonella hanzawai (NomURA \& ZINBO) ハンザワタマキガイ（黒田）

1934 Glycymeris hanzawai NomURA \& ZINBO, SRTIU, (2) 16 (2), 152, pl. 5, f. 3a, b

1961 Tucetona (Tucetonella) hanzawai: HABE, CISJ, 2, 112, pl. 50, f. 4

Loc. S (s) dp.

Dist. Honshu (north to Izu Islands) to Ryukyu Islands.

\section{Genus Tucetona IREDALEウチワガイ属}

1931 Tucetona Iredale RAM, 18, 201

1939 Tucetopsis IreDALE, GBRESR, 5(6), Moll. 1, 302. Type (OD): Pectunculus flabellatus

TENISON-WOODS

Tucetona amboinensis (GMELIN) クモエウチワガイ（黑田）

1791 Cardium amboinensis Gmelin, Syst. Nat., 13, (6), 3255

1843 Pectunculus pectiniformis: ReEve, Icon., sp. 11 
1938 Tucetona amboinensis extra IrEDALE, GBRESR, 5 (6), Moll. 1, 302, pl. 49, f. 3

1961 Tucetona (Tucetopsis) amboinensis: HABE, CISJ, 2, 111, pl. 49, f. 20

Loc. $\mathrm{S}$ (s) dp.

Dist. Honshu (north to Kii Peninsula)-Ryukyu. Indo-Pacific region (North Australia to Red Sea).

\section{Superfamily Limopsacea DALLオオシラスナガイ迢科 \\ Family Limopsidae DALL オオシラスナガイ科 \\ Genus Limopsis SASSI オオシラスナガイ属}

1827 Limopsis SASsI, Giorn Ligustico di Scienze, Letters, ed Arti, 1, 476. Type (M): Arca aurita BROCCHI

Limopsis tokaiensis YoKoYAMAオオシラスナガイ(岩川) :

トウカイシラスナガイ（滝・大山）

1910 Limopsis tokaiensis YoKoYAMA, JGST, 17, 1, pl. 9, f. 1-3; 3-7

1910 Limopsis tokaiensis elongata YoKоYAMA, JGST. 17, 1, pl. 9, f. 5-7

1914 Limopsis tajimae SOWERBY, AMNH, (8) 13, 445, p. 18, f. 3

1951 Limopsis uwadokoi OYAMA, MG., 23-24, 151, f. 8

1959 Limopsis tajimae emphatica KIRA, CISJ, 1, 112, pl. 44, f. 4

Loc. M S (s-m) tr.

Dist. Honshu to Kyushu and Japan Sea.

Limopsis obliqua A. ADAMs ナナメシラスナガイ（黒田）

1863 Limopsis obliqua A. AdAMs, PZSL (1862), 229

1953 Limopsis obliqua: HABE, ICJS, 1 (25), 201, pl. 19, f. 15, 16

1971 Limopsis obliqua: KURODA \& HABE, SSSB, 535 (J) 338 (E), pl. 71, f. 16, 17

Loc. $S$ (s-m) dp.

Dist. Honshu (Sagami Bay and Wakayama Prefecture).

Genus Empleconia DALL オリイレシラスナガイ属

1908 Empleconia Dall, BMCZ, 43, 393. Type (OD): Limopsis virginata DaLl

Empleconia cumingii (A. ADAMS) オリイレシラスナガイ（黒田）

1863 Limopsis cumingii A. ADAMS, PZSL (1862), 229

1912 Limopsis cumingii: LAMY, J. de C., 60, 118 (with tf.)

1922 Limopsis aureolata YoкоYАMA, JCSIUT, 44 (1), 194, pl. 17, f. 6, 7

1961 Limopsis (Empleconia) cumingii: HABE, CISJ, 2, 112, pl. 50, f. 6

Loc. M S (s-m) dp.

Dist. Honshu (north to Bōsō Peninsula) to Kyushu.

Genus Crenulilimopsis KuRODA \& HABE ナミジワシラスナガイ属

1971 Crenulilimopsis Kuroda \& HaBe, SSSB, 536 (J) 339 (E). Type (OD): Limopsis oblonga A. ADAMS

Crenulilimopsis oblonga (A. ADAMs)ナシジワシラスナガイ（黒田）

1860 Limopsis oblonga A. ADAMs, AMNH, (3) 5, 412

1863 Limopsis crenata A. ADAMs, PZSL (1862), 412

1931 Limopsis tumidula THIELE, Tiefsee, 21, 186, pl. 1, f. 26 
1961 Pectunculina oblonga: HABE, CISJ, 2, 112, pl. 50, f. 8

1971 Crenulilimopsis oblonga: KurodA \& HABE, SSSB, 537 (J) 339 (E), pl. 71, f. 13-15

Loc. M S (s-m) dp.

Dist. Honshu to Kyushu.

\section{Genus Oblimopa IREDALEシラスナガイ属}

1939 Oblimopa IREDALE, GBRESR, 5 (6), Moll. 1, 242

1939 Circlimopa IREdALE, GBRESR, 5 (6), Moll. 1, 243. Type (OD): Oblimopa macgillivrayi actaviva IREDALE

Oblimopa japonica (A. ADAMs) シラスナガイ（目八)

1863 Limopsis japonica A. ADAMs, PZSL (1862), 229

1863 Limopsis forskalii A. ADAMs, PZSL (1862), 230

1963 Limopsis woodwardii A. ADAMs, PZSL (1862), 231

1882 Limopsis woodwardii: DUNKER, Index, 237, pl. 16, f. 5, 6

1912 Limopsis multistriata: LAMY, J. de C., 60, 132

1953 Limopsis soyoae HaBe, Venus, 17 (3), 131, f. 7, 8

1959 Oblimopa forskalii: KIRA, CISJ, 1, 112, pl. 44, f. 1

Loc. M S (s-m) gl.

Dist. Honshu (north to Bōsō Peninsula) to Formosa.

\section{Genus Nipponolimopsis HABE マメシラスナガイ属}

1951 Nipponolimopsis HABE, GJS, 1, 45. Type (OD): Limopsis nipponica YoKoYama

Remark: According Moore's opinion (personal communication) this genus closely relates to Cratis HedLey, 1915 of the family Philobryidae.

Nipponolimopsis decussata (A. ADAMS) マメシラスナガイ（黒田）

1862 Cyrilla decussata A. Adams, AMNH, (3) 9, 295

1922 Limopsis nipponica YoKoYAMA, JCSIUT, 44 (1), 195, pl. 17, f. 16, 17

1930 Limopsis kinoshitai Kuroda, Venus, 1, App. 18, f. 24, 25

1931 Limopsis hilgendorfi THIELE, Tiefsee, 21, 184, pl. 1, f. 21, 21a

1961 Aspalima (Nipponolimopsis) decussata: HABE, CISJ, 2, 112, pl. 50, f. 7

Loc. M S (s-m) gl.

Dist. Honshu (north to Bōsō Peninsula) to Kyushu and Japan Sea.

Nipponolimopsis azumana YoKoYAMA マルシラスナガイ（波部）

1910 Limopsis azumana YoKoYAMA, JGST, 17, 3, pl. 9, f. 16-18

1910 Limopsis truncata YokoYAMA, JGST, 17, 4, pl. 9, f. 13, 14

1971 Nipponolimopsis azumana: KURoDA \& HABE, SSSB, 539 (J) 341 (E), pl. 71, f. 21, 22

Loc. $\mathrm{S}$ (s) tr.

Dist. Honshu (Sagami Bay to Kii Peninsula). 


\section{Order Mytiloida FÉRUSSAC イガイ目}

\section{Superfamily Mytilacea RAFINESQUE イガイ超科 \\ Family Mytilidae RAFINESQUE イガイ科 \\ Subfamily Mytilinae RAFINESQUE イガイ亜科}

\section{Genus Mytilus LinNaEUS イガイ属}

1758 Mytilus Linnaeus, Syst. Nat., 10, 645, 704 (ICZN Op. 94)

1900 Eumytilus IHERING, PMSL, 4, 86. Type (SD, GraY, 1847): Mytilus edulis LinNaEus

Mytilus edulis Linnaeus ムラサキイガイ(金丸)

1758 Mytilus edulis LinnaEus, Syst. Nat., 10, 705

1819 Mytilus galloprovincialis LAMARCK, AsV, 6, 126

1858 Mytilus edulis: ReEve, Icon., sp. 33

1959 Mytilus edulis: KIRA, CISJ, 1, 116, pl. 45, f. 19

Loc. N M S (r) int.

Dist. World-wide.

Mytilus coruscus Gould イガイ(目八)

1861 Mytilus coruscus Gould, PBSNH, 8, 38

1868 Mytilus crassitesta LischKe, MBL, 15, 221

1869 Mytilus crassitesta LisCHKE, JMC, 1, 151, pl. 11, f. 1, 2

1869 Mytilus dunkeri LiSCHKE, JMC, 1, 153, pl. 10, f. 7, 8

1959 Mytilus crassitesta KIRA, CISJ, 1, 116, pl. 45, f. 20

Loc. N M S (r) int.

Dist. Southern Hokkaido to Kyushu, Korea and Maritime Prov. of Siberia.

Genus Septifer RÉCLUZ クジャクガイ属

1848 Septifer RÉCluz, RZSC, 11, 275. Type (SD, StoliczKa, 1871): Mytilus bilocularis LINNAEUS

\section{Subgenus Septifer RÉCLUZ クジャクガイ亜属}

Septifer (Septifer) bilocularis (LinNaEUs) ・クジャクガイ（六介）

1758 Mytilus bilocularis Linnaeus, Syst. Nat., 10, 1705

1858 Mytilus pilosus ReEVe, Icon., sp. 35

1959 Septifer bilocularis: KIRA, CISJ, 1, 115, p1, 45, f. 10

1959 Septifer bilocularis pilosus: KIRA, CISJ, 1, 115, pl. 45, f. 9

Loc. N M S (r) int.

Dist. Ryukyu to Honshu (north to Bōsō Peninsula and Noto Peninsula). Widely ranging in the Indo-Pacific region.

Septifer (Septifer) excisus (WIFGMANN)シロインコガイ (平瀬)

1837 Tichogonia excisus WiEgmanN, AfN, 3 (1), 49

1857 Mytilus excisus: ReEve, Icon., sp. 13

1961 Septifer excisus: HABE, CISJ, 2, 113, pl. 50, f. 21

Loc. M S (r) int.

Dist. Ryukyu to Honshu (north to Izu Peninsula). Widely ranging in the central and western Pacific. 
Subgenus Mytilisepta HABE ムラサキインコガイ巠属

1951 Mytilisepta Habe, GJS, 1, 53. Type (OD): Tichogonia virgatus WiegmanN

Septifer (Mytilisepta) virgatus (WIEGMANN) ムラサキインコガイ（目八）

1837 Tichogonia virgatus WiEgMANN, AfN, $3(1), 49$

1853 Septifer crassus Dunker, ZfN, 86

1857 Mytilus crassus: ReEve, Icon., sp. 25

1959 Septifer (Mytilisepta) virgatus: KIRA, CISJ, 1, 115, pl. 45, f. 8

Loc. N M S (r) int.

Dist. Southern Hokkaido to Ryukyu Islands. Widely ranging in the IndoPacific region.

Septifer (Mytilisepta) keenae NomURAヒメイガイ（丹浦）

1936 Septifer keeni (sic) Nomura, Venus, 6 (4), 205, tf. 1-5

1961 Septifer (Mytilisepta) keenae: HABE, CISJ, 2, 114, pl. 50, f. 22

1971 Septifer (Mytilisepta) keenae: Kuroda \& HABE, SSSB, 544 (J) 344 (E), pl. 74, f. 3, 4, 9, 10

Loc. S (r) int.

Dist. Southern Hokkaido to Kyushu and Korea.

\section{Genus Trichomya IHERINGケガイ属}

1900 Trichomya IHERING, PMSL, 4, 87. Type (OD): Mytilius hirsutus LAMARCK

Trichomya hirsutus (LAMARCK) ケガイ (目八)

1819 Mytilus hirsutus LAMARCK, AsV, 6 (1), 120

1857 Mytilus hirsutus: REEVE, Icon., sp. 8

1959 Trichomya hirsuta: KIRA, CISJ, 1, 115, pl. 45, f. 15

Loc. N M S (r) int.

Dist. Honshu (north to Bōsō and Noto Peninsula). Widely ranging in the IndoPacific region.

\section{Subfamily Modiolinae KEEN ヒバリガイ亜科 \\ Genus Modiolus LAMARCK ヒバリガイ属}

1777 Volsella Scopoli, Introd. Hist. Nat., 397 (invalid, ICZN Op. 325)

1799 Modiolus LAMARCK, MSNHP, 87 (valid, ICZN Op. 325)

1801 Modiola LAMARCK, AsV, 113

1900 Eumodiolus Ihring, PMSL, 4, 87. Type (SD, GraY, 1847): Mytilus modiolus LinNaEus

\section{Subgenus Modiolus LAMARCKヒバリガイ亜属}

Modiolus (Modiolus) auriculatus (KRAUSS)リュウキュウヒバリガイ（杉谷）

1848 Modiola auriculata Krauss, S-Afr. Moll., 20, pl. 2, f. 4

1890 Modiola auriculata DUNKer, Cab., 8 (3), 96, pl. 29, f. 1, 2

1939 Modiolus agripedus IREDALE, GBRESR, 5 (6), 419, pl. 6, f. 21

1959 Modiolus agripedus: KIRA, CISJ, 1, 116, pl. 45, f. 17

Loc. N M S (r) int.

Dist. Honshu (north to Bōsō Peninsula). Widely ranging in the Indo-Pacific region. 
Modiolus (Modiolus) nipponicus (OYAMA) ヒバリガイ（目八）

1950 Volsella nipponica OYAMA, MG, 3, 225

1959 Modiolus nipponica: KIRA, CISJ, 1, 116, pl. 45, f. 16

Loc. N M S (r) int.

Dist. Honshu (north to Mutsu Bay) to Kyushu.

Modiolus (Modiolus) comptus (SoWERBY)ビロウドマクラガイ（黒田）

1915 Modiola compta Sowerby, AMNH, (8) 16, 168, pl. 10, f. 10

1961 Modiolus comptus: HABE, CISJ, 2, 113, pl. 50, f. 17

Loc. M S (s-m) gl.

Dist. Honshu (north to Bōsō Peninsula) to Kyushu.

Modiolus (Modiolus) margaritaceus (NoMURA \& HATAI) マメヒバリガイ（大山）

1950 Volsella margaritacea Nomura \& HATAI, SHKMRB, 19, 77, pl. 4, f. 2, 3

1961 Modiolus margaritaceus: HABE, CISJ, 2, 113, pl. 50, f. 20

1971 Modiolus margaritaceus: KurodA \& HABE, SSSB, 546 (J) 345 (E), pl. 117, f. 18

Loc. M S (s) gl.

Dist. Hokkaido to Kyushu.

Modiolus (Modiolus) metcalfei (HANLEY)・コケガラスガイ（岩川）

1843 Modiola metcalfei HanLey, Cat. Bivalve, 3, 235

1857 Modiola metcalfei: REEVE, Icon., sp. 16

1961 Modiolus metcalfei: HABE, CISJ, 2, 114, pl. 50, f. 26

Loc. $\mathrm{N}(\mathrm{g}) \mathrm{dp}$.

Dist. Honshu (north to Bōsō Peninsula). Widely ranging in the western Pacific.

Subgenus Fulgida LAMY サザナミマクラガイ亜属

1919 Fulgida Lamy, BMNHNP, 25 (2), 111. Type (M): Perna fulgida H. Adams=Modiola lignea REEVE

Modiolus (Fulgida) flavidus (DUNKER)サザナミマクラガイ（大山）

1857 Volsella flavida DUNKER, PZSL, 24, 19 (1856), 361

1858 Modiola flavida: ReEve, Icon., sp. 64

1961 Modiolus (Fulgida) flavidus: HABE, CISJ, 2, 114, pl. 50, f. 24

Loc. M S (s-m) dp.

Dist. Honshu (north to Bōsō Peninsula) to Kyushu.

*Modiolus (Fulgida) oyamai HABE sp. nov. コガラスマクラガイ（岩川）

Pl. 2, f. 3

Shell rather small, thin, transversely elongate and inflated. Umbo situated anteriorly and prominent beyond the dorsal margin. Ligament behind the umbo, black and on the short and straight posterior dorsal margin. Anterior margin roundedly curved and posterior margin gently curved at the posterodorsal area and rounded at the posterior end. Ventral margin incurved shallowly. Surface polished, brown at the anterior half while pale brown at the postrior half. Interior milky blue and pearly.

Length $21.0 \mathrm{~mm}$, height $9.8 \mathrm{~mm}$ and breadth $9.2 \mathrm{~mm}$ (Holotype conjoined specimen preserved in the National Science Museum, NSMT-Mo 58904)

Length $15.5 \mathrm{~mm}$, height $8.2 \mathrm{~mm}$ and breadth $4.5 \mathrm{~mm}$ (right valve from 
Tomioka, Amakusa Islands, Kyushu. NSMT-Mo 58904)

Type locality: Shionomisaki, Wakayama Pref., Honshu. S (s) dp

Distribution: Honshu (north to Bōsō Peninsula) to Kyushu.

Remarks: This new species is very closely related to Modiolus (Fulgida) flavidus (DUNKER, 1856) in general features, but has the more elongated shell with the shallowly incurved ventral margin. The specific name is dedicated to Dr. Katsura Oyama, Vice President of the Malacological Society of Japan who studied this family, Mytilidae and pointed out this new species.

Subgenus Modiolusia Yamamoto \& HABE カラスノマクラガイ亜属

1958 Modiolusia Yамамото \& Habe, BMBSA, 9 (1), 8. Type (OD): Modiola elongata SWATSNON

Modiolus (Modiolusia) elongatus (SWAINSON) カラスノマクラガイ (目八) ッヤガラスガイ（矢倉）

1821 Modiola elongata Swainson, Exot. Conch., 1, pl. 8

1857 Modiola elongata: ReEVE, Icon., sp. 4

1857 Modiola nitida ReEve, Icon., sp. 6

1890 Modiola rhomboidea Clessin, Cab., 8 (3), 103, pl. 26, f. 10 (non ReEve, 1857)

1891 Modiola sirahensis Jousseaume, Le Nat. 13, 222 (new name for Modiola rhomboidea CLESSIN, 1890)

1961 Modiolus (Modiolusia) nitidus: HABE, CISJ, 2, 114, pl. 50, f. 25

Loc. M S (s-m) dp.

Dist. Honshu (north to Mutsu Bay). Widely ranging in the central and west Pacific regions.

Subgenus Modiolatus JousseaUME ハンレイヒバリガイ亜属

1893 Modiolatus Jousseaume, Le Nat., (2) 7, 192. Type (OD): Mytilus plicatus GmeLIN

Modiolus (Modiolatus) hanleyi (DUNKER) ハソレイヒバリガイ（岩川）

1882 Modiola hanleyi DunKer, Index, 223, pl. 16, f. 3, 4

1890 Modiola hanleyi: CLessin, Cab., 8 (3), 95, pl. 26, f. 4, 5

1961 Modiolus hanleyi: HABE, CISJ, 2, 114, pl. 49, f. 23

Loc. N M (s-m) dp.

Dist. Honshu (north to Kii Peninsula) to Kyushu.

\section{Genus Limnoperna RocHEBRUNEカワヒバリガイ属}

1882 Limnoperna Rochebrune Bull. Soc. Philom. Paris, (7) 8, p. 102. Type (OD): Dreissena siamensis Morelet 1866

Limnoperna fortunei kikuchii HABE subsp nov. コウロエンカワヒバリガイ（波部）

Pl. 2, f. 2

Shell thin, rather small, thin, oblong, widing posteriorly, chestnut brown, shining Umbo situated near the anterior end and prominent beyond the dorsal margin. posterior dorsal margin long and straight. Ventral margin slightly incurved. Posterior margin obtuse and rounded at the posterior end. Surface weakly marked by the growth lines and run an obtuse ridge vanishing from the umbo to the postero ventral corner. Interior pearly and blue white on the anterior-ventral half and purple on the postero-dorsal half. 
Length $24.0 \mathrm{~mm}$, Height $11.8 \mathrm{~mm}$ and breadth $10.8 \mathrm{~mm}$ (Holotype specimen preserved in the National Science Museum, NSMT-Mo 58959)

Length $38.5 \mathrm{~mm}$ height $14.2 \mathrm{~mm}$ and breadth $11.6 \mathrm{~mm}$ (Paratype specimen NSMT-Mo 58960)

Length $24.7 \mathrm{~mm}$, height $10.8 \mathrm{~mm}$ and breadth $11.0 \mathrm{~mm}$ (paratype specimen NSMT-Mo 58961)

Type locality: Kōroen, Nishinomiya City, Hyōgo Pref., Honshu. N (s) int

Distribution: The eastern part of Setonaikai (Inland Sea of Japan) and Osaka Bay, Wakayama Harbor (Wakayama City), Ise Bay, Tokyo Bay, Honshu.

Remarks: This new species is common at the type locality together with Musculus (Musculista) senhousia (Benson). This is characterized by the smooth surface and the angularly protruded anterior margin beyond the umbo. L. fortunei (DUNKER) is closely related to this new subspecies but has the transversely elongated triangullar shells. Therefore this is a subspecies of that species in being the short quadrangular shell. The name is dedicated to Dr. Norio Kikuchi, Director of Nishinomyiya Kaisei Hospital who collected this interesting subspecies.

\section{Genus Hormomya MöRCHヒバリガイモドキ属}

1853 Hormomya Mörch, Yoldi, 2, 53. Type (SD, JUKes-Brown, 1905): Mytilus exustus LINNAUES

Hormomya mutabilis (GoulD)ヒバリガイモドキ（杉谷）

1856 Modiola curvata DUNKER, PZSL, 361

1858 Mytilus curvata: REEve, Icon., sp. 53

1861 Mytilus mutabilis Gould, PBSNH, 8, 38

1959 Hormomya mutabilis: KIRA, CISJ, 1, 114, pl. 45, f. 3

Loc. M S (r) int.

Dist. Honshu (north to Bōsō Peninsula). Widely ranging in the western Pacific.

$$
\text { Genus Exosiperna IREDALEオトヒメノユリカゴガイ属 }
$$

1929 Exosiperna Iredale, RAM, 17, 166. Type (OD): Arcoperna scapha Verco

Exosiperna kuroharai HABEオトヒメノユリカゴガイ（波部） Pl. 4, f. 7

1961 Exosiperna kuroharai HABE, Venus, 21 (2), 150, 154, f. 4

1977 Exosiperna kuroharai: HABE, SMJB/S, 55, pl. 6, f. 10

Loc. M S (s) dp.

Dist. Honshu (north to Kii Peninsula) to Kyushu.

\section{Genus Gregariella MontERosatoチデミタマエガイ属}

1883 Gregariella Monterosato, Natur. Sicil. 3, 90

1889 Botulina DALL, USNMB, 37, 38

1939 Tibialectus Iredale, GBRESR, 5 (6), Moll. 1, 424. Type (M): Modiola opifex SAY $=$ Modiolus sulcatus Risso

Gregariella coralliophaga (GMELIN) チデミタマエガイ（黒田）

1791 Mytilus coralliophagus GMELIN, Syst. Nat., 13, 3359

1847 Modiola divericata: PHLLIPPI, ZfM, 115

1851 Modiola divaricata: PHILIPPI. Abbild., 3, 21, pl. 2, f. 8

1961 Botulina coralliophaga: HABE, CISJ, 2, 113, pl. 51, f. 15 
Loc. N M S (bo) gl.

Dist. Honshu (north to Bōsō Peninsula). Widely ranging in the Indo Pacific region.

\section{Genus Trichomusculus IREDALEスジタマエガイ属}

1924 Trichomusculus Iredale, PLSNSW, 49, 196. Type (OD): Lithodomus barbatus REEVE

Trichomusculus semigranatus (REEVE)スジタマエガイ（岩川）

1858 Lithodomus semigranatus ReEve, Icon., sp. 28

1861 Modiolaria arcuata Gould, PBSNH, 8, 38

1971 Trichomusculus semigranatus: KURODA \& HABE, SSSB, 549 (J) 348 (E), pl. 117, f. 19

Loc. M S (bo) gl.

Dist. Honshu (north to Bōsō Peninsula). Widely ranging in the Indo Pacific region.

\section{Subfamily Crenellinae GRAY キザミガイ亜科}

\section{Genus Solamen IREDALE キサガイモドキ属}

1924 Solamen Iredale, PLSNSW, 49, 198. Type (M): Solamen rex Iredale

Solamen spectabilis A. ADAMSキサガイモドキ（黒田）；キヌブクロガイ（波部）

1862 Crenella spectabilis A. Adams, AMNH, (3) 9, 228

1907 Crenella diaphana DALL, SMC, 50, 171

1951 Solamen saccosericata HABE, GJS, 1, 48, f. 87, 88

1959 Solamen saccosericata: KIRA, CISJ, 1, 115, pl. 45, f. 14

1971 Solamen spectabilis: KuROdA \& HABE, SSSB, 540 (J) 342 (E), pl. 74, f. 7, 8

Loc. S (s-m) tr.

Dist. Honshu (north to Iwate Pref.) to Kyushu.

\section{Subfamily Musculinae IREDALE タマエガイ亜科 \\ Genus Musculus (RöDING) タマエガイ属}

1798 Musculus (RöDiNG), Bolten. 2, 156

1840 Lanistes SWaInson, Treat., 385 (non MonTForT, 1810)

1847 Lanistina Gray, PZSL, 15, 199. Type (SD, StoliczKa, 1871): Mytilus discors Linnaeus

Musculus pusio (A. ADAMs) チビタマエガイ（黒田）

1862 Modiolaria pusio A. Adams, AMNH, (3) 9, 229

1971 Musculus (Musculus) pusio: KURODA, \& HABE, SSSB, 550 (J) 349 (E), pl. 73, f. 11

Loc. N M (g) gl

Dist. Honshu (north to Bōsō Peninsula) to Kyushu and Japan Sea.

\section{Subgenus Modiolarca GraYタマエガイ亜属}

1843 Modiolarca Grax, Travels New Zealand, 2, 259

1959 Ryenella Fleming, TRSNZ, 87, 172. Type (M): Mytilus impactus HermanN

Musculus (Modiolarca) cupreus (GouLD)タマエガイ（丹浦）

1861 Modiolarca cuprea Gould, PBSNH, 8, 37

1861 Modiolaria quadrula Gould, PBSNH, 8, 38 
1941 Musculus neglectus Kuroda, MFSATIU, 22 (4), 196

1959. Musculus (Modiolarca) neglectus: KIRA, CISJ, 1, 114, pl. 45, f. 6

Loc. N M S (ep) int-gl.

Dist. Hokkaido to Formosa.

Subgenus Musculista YaMamoto \& HABE ホトトギスガイ坐属

1958 Musculista Yамамото \& HАBE, BMBSA, 9 (1), 9. Type (OD): Modiola senhousia BENSON

Musculus (Musculista) senhousia (BENSON)ホトトギスガイ（丹浦）

1842 Modiola senhousia BENSON, AMNH, 9, 489

1857 Modiola senhousii REEVE, Icon., sp. 22

1874 Modiola bellardiana Tapparone \& CANefri, Magenta, 144, pl. 4, f. 4, 4a, $1 \mathrm{~b}$

1959 Brachidontes (Arcuatula) senhousia: KIRA, CISJ, 1, 114, pl. 45, f. 1

Loc. N M S (s-m) int.

Dist. Hokkaido to Kyushu. Widely ranging in the western Pacific.

Musculus (Musculista) japonicus (DUNKER) ヤマホトトギスガイ（潜錦）

1857 Volsella japonica DUNKeR, PZSL, 24, (1856), 363

1857 Modiola japonica ReEve, Icon., sp. 26

1959 Brachidontes (Arcuatula) japonicus KIRA, CISJ, 1, 114, pl. 45, f. 4

Loc. N M S (s-m) int.

Dist. Honshu (north to Sagami Bay) to Kyushu.

Musculus (Musculista) perfragilis (DUNKER) ノジホトトギスガイ（波部）

1857 Volsella perfragilis DUNKER, PZSL, 24 (1856), 362

1857 Modiola perfragilis: REEvE, Icon., sp. 42

1961 Musculus (Musculista) perfragilis: HABE, CISJ, 2, 113, pl. 50, f. 16

1971 Musculus (Musculista) perfragilis: KURODA \& HABE, SSSB, 531 (J))350 (E), pl. 73, f. 6

Loc. N M S (s-m) dp.

Dist. Honshu (north to Bōsō Peninsula) to Kyushu.

Genus Amygdalum Megerle von MUHLFELDヌリッヤホトトギスガイ属

1811 Amygdalum Megerle von Muhlfeld, GNFBM, 5, 69. Type (M): Amygdalum dentriticum Megerle von MUHLFELD

Amygdalum peasei (NEWCOMB)ママボロシガイ（黒田）：ハネヌリッヤホトトギスガイ（黑田）

Pl. 4, f. 4

1870 Amygdalum peasei NewcomB, AJC, 5, 163, pl. 17, f. 2

1938 Volsella (Amygdalum) peasei: DALL, BARTSCH \& REHDER, BPBMB, 153, 45, p1. 8, f. 11-14

1971 Amygdalum plumeum Kuroda \& HABE, SSSB, 533 (J) 351 (E), pl. 73, f. 17

Loc. S (s-m) dp.

Dist. Honshu (north to Sagami Bay) to Kyushu and Hawaii.

Amygdalum watsoni (SMITH) ヌリッヤホトトギスガイ（平瀬）

1885 Modiola watsoni SMITH, Challenger, 13, 275, pl. 16, f. 5

1932 Modiolus (Amygdalum) watsoni: Prashad, Siboga, 53 C, 74, pl. 2, f. 32, 33

1959 Brachidontes (Amygdalum) watsoni: KIRA, CISJ, 1, 114, pl. 45, f. 5

1967 Amygdalum politum (Verril \& SMith, 1880): KNUdSEN, J. Murray, 11 (3), 269, tf. 14

Loc. N M S (s-m) tr.

Dist. Honshu (north to Sagami Bay). 
Amygdalum soyoae HABEユキゾラホトトギスガイ（黒田） Pl. 4, f. 3

1958 Amygdalum soyoae HABE, PSMBL, 6 (3), 21

1971 Amygdalum soyoae: KURODA, \& HABE SSSB, 553 (J) 351 (E), pl. 73, f. 7

Loc. S (s-m) tr.

Dist. Honshu (north to Sagami Bay), Shikoku and Kyushu.

Genus Idasola IREDALEキザミバマユイガイ属

1876 Idas Jeffreys (non Mulsant and Verreaux, 1875), AMNH, 4 (18), 428

1918 Idasala Iredale, PMSL, 11, 40 (new name for Idas JefFreys, 1876). Type (M): Idas argenteus JEFFREYS

*Idasola japonica HABEキザミバマユイガイ（波部）Ｐl. 4, f. 2

1976 Idasola japonica HABE, Venus, 35 (2), 37, pl. 1, f. 15, 16

Loc. S (s-m) tr.

Dist. Honshu (Enshu-nada to Kii Peninsula).

Genus Brachidontes SWAINSONスキゲヒバリガイ属

1840 Brachidontes Swainson, Treat., 384. Type (M): Modiola sulcata Lamarck, 1819 non $1805=$ Mytilus citrianus RöDING $=$ Arca modiolus LINNAEUs

Brachidontes setigera (DUNKER)スキゲヒバリガイ（黒田）

1856 Volsella setigera DUNKER, PZSL, 364

1857 Modiola setigera: ReEve, Icon., sp. 20

1857 Modiola subsulcata ReEve (non DunKer, 1856), Icon., sp. 47

1967 Brachidontes setigera: HABE \& Kosuge, KAI (Hoikusha), 127, pl. 47, f. 5

Loc. M (s-m) int.

Dist. Honshu (Kii Peninsula) to Formosa and the western Pacific.

\section{Genus Vignadula KURODA \& HABE クログチガイ属}

1971 Vignadula KURODA \& HABE, SSSB, 549 (J) 348 (E). Type (OD): Mytilus atratus Lischre

Vignadula atrata (LISCHKE) クログチガイ（目八）

1871 Mytilus atratus LischKe, M B L., 18, 44

1871 Mytilus atratus: LischKe, JMC, 2, 146, pl. 10, f. 4, 5

1871 Modiola aterrima DALL, AJC, 7, 154, pl. 14, f. 3

1861 Adula atrata: HABE, CISJ, 2, 113, pl. 50, f. 19

Loc. N M S (r) int.

Dist. Honshu (north to Bōsō Peninsula) to Kyushu and Korea.

Genus Terua DALL, BARTSCH \& ReHDER ヒラノマクラガイ属

1938 Terua Dall, Bartsch \& Rehder, BPBMB, 153, 57. Type (OD): Terua pacifica DalL, BARTSCH \& REHDER

Terua pacifica DALL, BARTSCH \& REHDER ヒラノマクラガイ（黒田）

1933 Myrina hiranoi Kuroda, Venus 4 (1), App. 136,f. 151, 152 (name and figures only)

1938 Terua pacifica DALL, Bartsch \& REHDER, BPBMB, 153, 58, pl. 11, f. 11-14

1977 Terua pacifica: HABE, Venus, 36 (1), 2, 7, pl. 2, f. 3-6

Loc. S (s-m) tr.

Dist. Honshu (north to Enshu-nada), Shikoku, Japan Sea and Hawaii. 
Terua crypta DALL, BARTSCH \& ReHDER ホソヒラノマクラガイ（波部） Pl. 1, f. 1

$$
\text { ナガマユイガイ(波部) }
$$

1938 Terua crypta Dall, BARTSCh \& RehDER, BPBMB, 153, 58, pl. 11, f. 5, 6

1977 Terua crypta: HABE, Venus 36 (1), 3, 7, pl. 1, f. 7, 8

Loc. $\mathrm{S}$ (s) tr.

Dist. Honshu (Wakayama Prefecture), Shikoku and Hawaii.

Terua iwaotakii HABE イトマユイガイ（黒田）

(1863 Modiola parasitica Deshayes, Reunion, 24, p1. 4, f. 1-3)

(1883 Lithophaga parasitica: Dunker, Cab., 8(3a), 24, pl. 5, f. 17, 18)

1958 Adula iwaotakii HABE, Venus, 20 (2), 174, 176, f. 5

1961 Adula iwaotakii: HABE, CISJ, 2, 113, pl. 50, f. 14

Loc. M S (bo) g1. [timbers deposited]

Dist. Honshu (north to Bōsō Peninsula) to Kyushu.

\section{Genus Botula MöRCHヌリマクラガイ属}

1853 Botula MörCH, Yoldi, 2, 55

1939 Botulopa Iredale, GBRESR, 5 (6), Moll. 1, 414. Type (SD, Dall, Bartsch \& ReHDer, 1938): Mytilus fuscus GMELIN

Botula silicula (LAMARCK) ヌリマクラガイ（黒田）

1819 Modiola cinnamonea LAMARCK, AsV, 6 (1), 114 non Link, 1807

1819 Modiola silicula LamarcK, AsV, 6 (1), 115

1939 Botulopa silicula infra IREDALE, GBRESR, 5 (6), Moll. 1, 415, pl. 6, f. 26

1961 Botula silicula: KIRA, CISJ, 1, 113, pl. 50, f. 13

Loc. M (bo) gl. [coral]

Dist. Honshu (north to Kii and Noto Peninsula) and Indo-Pacific region.

Subfamily Lithophaginae H. \& A. ADAMS イシマテガイ里科

Genus Lithophaga (RöDING)シギノハシガイ属

1798 Lithophaga RöDING, Bolten., 2, 156

1817 Lithodomus CuvIER, Reg. Anim., 2, 471

1811 Lithophagus Megerle von MuHzfeld, GNFBM, 5, 69. Type (M): Mytilus lithophagus LiNNAEUS

Lithophaga zitteliana DUNKER シギノハシガイ (丹浦)

1882 Lithophaga zitteliana Dunker, Index, 226, pl. 14, f. 1, 2, 8, 9

1959 Lithophaga zitteliana: KIRA, CISJ, 1, 115, pl. 45, f. 13

Loc. M S (bo) gl. [coral]

Dist. Honshu (north to Bōsō Peninsula) to Kyushu.

Lithophaga straminea (REEVE)ワライロシギノハシガイ（黒田）

1857 Lithophaga stramineus ReEve, Icon., sp. 11

1883 Lithophaga straminea: DunKer, Cab., 8 (3a) (1882), 6 pl. 2, f. 1, 2

Loc. M S (bo) gl. [coral]

Dist. Honshu (north to Kii Peninsula) to Kyushu.

Lithophaga teres (PHILIPPI) クロシギノハシガイ (平瀬)

1846 Modiola teres PHILIPPI, Abbild., 2, 148, pl. 1, f. 3 
1847 Modiola malayana PhILIPPI, ZfM, 117

1847 Modiola gracilis PhLLPPI, ZfM, 117 (non KLIPSTeIN, 1845)

1851 Modiola malayana: PhiLipPI, Abbild., 3, 19, pl. 2, f. 1

1966 Lithophaga teres: HABE \& Kosuge, SWC, 2, 130, pl. 48, f. 2

Loc. S (bo) gl. [coral].

Dist. Honshu (Kii Peninsula) to Ryukyu Islands. Western Pacific.

Subgenus Leiosolenus CARPENTER イシマテガイ亜属

1856 Leiosoleus CARPENTER, Mazatlan, 130. Type (M): Leiosolenus spatiosa CARPENTER

Lithophaga (Leiosolenus) curtus (LISCHKE) イシマテガイ（目八)

1874 Lithophagus curtus LischKe, JMC, 3, 111, pl. 9, f. 14-17

1959 Lithophaga (Leiosolenus) curta: KIRA, CISJ, 1, 115, pl. 45, f. 11

Loc. N M S (bo) int. [sand stone].

Dist. Honshu (north to Mutsu Bay) to Ryukyu Islands and Ogasawara Islands.

\section{Subgenus Labis DALLカクレイシマテガイ亜属}

1916 Labis Dall, PUSNM, 52, 405. Type (M): Lithophaga attenuata Deshayes

Lithophaga (Labis) erimitica KURODA \& HABEカクレイシマテガイ（胃田） Pl. 4, f. 5 1971 Lithophaga (Labis) erimitica KURODA \& HABE, SSSB, 554 (J) 352 (E), pl. 74, f. 11

Loc. M S (bo) gl. [coral].

Dist. Honshu (north to Bōsō Peninsula) to Kyushu.

\section{Subgenus Stumpiella SOOT-RYENキカイイシマテガイ亜属}

1955 Stumpiella Soot-Ryen, Rept. Allan Hancock Pacific Exped., 20 (1), 23. Type (OD): Lithophagus calculatus CARPENTER

Lithophaga (Stumpiella) lithura PILSBRYキカイイシマテガイ (平瀬) Pl. 4. f. 6

1905 Lithophaga lithura PILsBRy, PANSP, 57, 119, pl. 5, f. 37-39

Loc. M S (bo) gl. [coral].

Dist. Honshu (Kii Peninsula) to Ryukyu Islands.

\section{Subgenus Diberus DALL コンゴウイシマテガイ亜䝠}

1898 Diberus DaLl, TWFIP. 3 (4), 799

1939 Salebrolabis Iredale, GBRESR, 5 (6), Moll. 1, 417. Type (OD): Modiola palmula HANLEY

Lithophaga (Diberus) malaccana (REEVE) マラッカイシマテガイ（黑田)

1857 Lithodomus malaccana REEve, Icon., sp. 20

1932 Lithophaga levigata PrashaD, Siboga, 53 C, 78, pl. 2, f. 42, 43 (non Quoy \& Gaimard, 1835)

1939 Lithophaga divaricalx IREDALE, GBRESR, 5 (6), Moll. 420, pl. 6, f. 23

Loc.

Dist. Honshu (Kii Peninsula). Widely ranging in the Pacific region.

Subgenus Exodiberus IREDALE イワカワイシマテガイ亜属

1939 Exodiberus Iredale, GBRESR, 5 (6), Moll. 1, 417. Type (OD): Lithophaga calcifer IREDALE 
Lithophaga (Exodiberus) lima (LAMY) イワカワシギノハシガイ（黒田）

1919 Dactylus lima LAMY, BMHNP, 25, 257

1937 Lithophaga lima: LAMY, J. de C., 8 (2), 119

1959 Lithophaga (Leiosolenus) lima: KIRA, CISJ, 1, 115, p1. 45, f. 12

Loc. M S (bo) gl. [sand stone].

Dist. Honshu (Kii Peninsula) to Ryukyu Islands.

\section{Superfamily Pinnacea LEACHハボウキガイ超科 \\ Family Pinnidae LEACH ハボウキガイ科 \\ Genus Pinna LINNAEUS ハボウキガイ属}

1758 Pinna Linnaeus, Syst. Nat., 10, 707. Type (SD, Children, 1823): Pinna rudis Linnaeus

Subgenus Quantulopinna IREDALEイワカワハゴロモガイ里属

1939 Quantulopinna IREDALE, GBRESR, 5 (6), Moll. 1, 310. Type (OD): Quantulopinna delsa IREDALE

Pinna (Quantulopinna) muricata LinNAEUSイワカワハゴロモガイ（黑田）

1758 Pinna muricata Linnaeus, Syst. Nat., 10, 707

1837 Pinna semicostata CoNRAD, JANSP, 7, 245, pl. 20, f. 11

1939 Quantulopinna delsa IrEDALE, GBRESR, 5 (6), Moll. 1, 31 1, pl. 4, f. 16

1961 Pinna muricata: RosewATER, IPM, 1 (4), 188, pl. 141-145

1961 Pinna (Quantulopinna) muricata: HABE, CISJ, 2, pl. 2, f. 1

Loc. S (s-m) int.

Dist. Honshu (north to Kii Peninsula). Indo-Pacific region.

\section{Subgenus Cyrtopinna MöRCHハボウキガイ亜属}

1853 Cyrtopinna Mörch, Yoldi, 2, 51. Type (M): Pinna incurvata GMeLIN

Pinna (Cyrtopinna) bicolor GMELIN ハボウキガイ（六介）

1791 Pinna bicolor Gmelin, Syst. Nat., 13, 3366

1858 Pinna attenuata Revve, Icon., sp. 46

1961 Pinna bicolor: RoseWATER, IPM, 1 (4), 193, pl. 147-153

1961 Pinna attenuata: HABE, CISJ, 2, 117, pl. 52, f. 8

Loc. N M S (s-m) gl.

Dist. Honshu (north to Bōsō Peninsula). Indo-western Pacific region.

Remarks: Though the closely allied species Pinna (Cyrtopinna) incurvata GMELIN,

1791 had been reported from Shirahama (Seto) by Dr. T. Kuroda in 1930, it seems to be a form of this species.

\section{Genus Exitopinna IREDALEカラボウキガイ属}

1939 Exitopinna Iredale, GBRESR, 5 (6), Moll. 1, 315. Type (OD): Exitopinna deltodes ultra IREDALE

Exitopinna epica (JoUSSEAUME)カラボウキガイ (介志)

1894 Pinna epica JousseAume, Le Nat., 8 (16), 229

1953 Atrina (Servatrina) epica: HABE, ICJS, 1 (24), 194, pl. 25, f. 8

1953 Atrina (Servatrina) tenuis HABE, ICIS, 1 (24), 193, pl. 24, f. 3 
1953 Atrina (Servatrina) epica: HABE, PSMBL, 3 (2), 170, pl. 9, f. 1, 2

1960 Atrina (Servatrina) epica: HABE, CISJ, 2, 117, pl. 52, f. 9

Loc. M (s-m) gl.

Dist. Honshu (north to Izu Islands) to Shikoku.

\section{Exitopinna linguafelis (HABE) ヒシタイラギ（吉良）}

1953 Atrina (Servatrina) linguafelis HABE, ICJS, 1 (24), 194, pl. 25, f. 7

1961 Atrina (Servatrina) linguafelis: HABE, CISJ, 2, 117, pl. 52, f. 6

Loc. S (s) tr.

Dist. Honshu (north to Izu Islands) to East China Sea.

\section{Genus Atrina GRAY クロタイラギ属}

1847 Atrina Gray, PSZL, 199. Type (SD, Gray, 1847): Pinna nigra DillwyN

\section{Subgenus Atrina GRAY クロタイラギ鱼属}

Atrina (Atrina) vexillum (BORN) クロタイラギ（岩川)

1778 Pinna vexillum BorN, IMCV, 118

1780 Pinna vexillum BonN, Test. Vindob., 134, pl. 7, f. 8

1817 Pinna nigra Dillwyn, DCRS, 1, 325

1858 Pinna gouldii REEVE, Icon, sp. 63

1961 Atrina (Atrina) vexillum: RoseWATER, IPM, 1 (4), 204, f. 157, 158

1961 Atrina gouldi: HABE, CISJ, 2, 117, pl. 52, f. 7

Loc. M S (g) gl.

Dist. Honshu (Kii Peninsula). Indo-Pacific region.

\section{Subgenus Servatrina IREDALEタイラギ亜属}

1939 Servatrina Iredale, GBRESR, 5 (6), Moll. 1, 317. Type (OD): Pinna assimilis Reeve

Atrina (Servatrina) pectinata (LiNNAEUS) タイラギ（五介）

1767 Pinna pectinata LinNaEus, Syst. Nat, 12, 1160

1858 Pinna chemnitzii HANLEY, PZSL, 136

1841 Pinna chinensis Deshayes, Reg. Anim. Moll, pl. 85

1858 Pinna hanleyi ReEve, Icon, sp. 15

1858 Pinna japonica ReEve, Icon., sp. 47

1858 Pinna assimilis ReEve, Icon, sp. 59

1891 Pinna lischkeana Clessin, Cab(, 8 (1), 73, pl. 28, f. 1

1965 Atrina (Servatrina) pectinata: RosEWATER, IPM, 1 (4), 211, pl. 161-163

1971 Atrina (Servatrina) japonica KuRODA \& HABE, SSSB, 556 (J) 353 (E), pl. 76, f. 2-4

Loc. N M S (s-m) int.

Dist. Honshu (north to Bōsō Peninsula) to Kyushu. Western Pacific.

Atrina (Servatrina) penna (REEVE) 八ネタイラギ（黒田）

1858 Pinna penna ReEve, Icon., sp. 39

1932 Pinna penna Prashad, Siboga 53C, 137, pl. 4, f. 14, 15

1961 Atrina (Servatrina) penna: HABE, CISJ, 2, 117, pl. 52, f. 3

Loc. $\mathrm{N}$ (s) tr.

Dist. Honshu (north to Sagami Bay) to Indonesia.

*Atrina (Servatrina) kinoshitai HABE ミノタイラギ（吉良）

1953 Atrina (Servatrina) kinoshitai HABE, ICJS; 1 (24), 193, pl. 24, f. 4 
1959 Atrina (Servatrina) kinoshitai: KIRA CISJ, 1, 119, pl, 46, f. 11

Loc. M S (s) gl.

Dist. Honshu (north to Sagami Bay) to Kyushu.

*Atrina (Servatrina) lamellata HABEヒワダブキタイラギ（波部）

1961 Atrina (Servatrina) lamellata HABE, CISJ, 2, 117, App. 37, pl. 52, f. 5

Loc. M S (s-m) gl.

Dist. Honshu (Kii Peninsula) and Shikoku.

Atrina (Servatrina) teramachii HABEカワタイラギ（波部）

1953 Atrina (Servatrina) teramachii HABE, ICJS, 1 (24), 192, pl. 25, f. 5

1961 Atrina (Servatrina) teramachii: HABE, CISJ, 2, 52, 117, pl. 52, f. 4

Loc. S (s) tr.

Dist. Honshu (north to Sagami Bay) to East China Sea.

\section{Genus Streptopinna MARTENSカゲロウガイ属}

1880 Streptopinna Martens, Mollusken in Mobius, Beit. Meer. Mauritius Seychellen 318. Type (M): Pinna saccata Linnaeus

Streptopinna saccata (LINNAEUS)カゲロウガイ（介志）

1758 Pinna saccata Linnaeus, Syst. Nat., 10, 707

1858 Pinna saccata: ReEve, Icon., sp. 6

1959 Streptopinna saccata: KIRA, CISJ, 1, 119, pl. 46, f. 12

1961 Streptopinna saccata: RosewATER, IPM, 1 (4), 221, pl. 136, 167-171

Loc. N M S (r) int.

Dist. Honshu (north to Bōsō Peninsula) to Ryukyu Islands. Widely ranging in the Indo-Pacific region.

\section{Order Pterioida NeWELL ウグイスガイ目 \\ Suborder Pteriina NEWELLウグイスガイ亜目 \\ Superfamily Pteriacea GRAY ウグイスガイ超科 \\ Family Pteriidae GRAY ウグイスガイ科 \\ Genus Pteria SCOPOLIウグイスガイ属}

1777 Pteria Scopoli, Introd., 397

1791 Avicula BRUGUIÈre, EMHNV 1, pl. 177

1931 Austropteria Iredale, RAM, 18, 205. Type (M): Mytilus hirundo Linnaeus

Pteria brevialata (DUNKER) ウグイスガイ（六介）

1872 Avicula brevialata Dunker, Cab., 7 (3), 55, pl. 19, f. 3

1882 Avicula brevialata: DunKer, Index, 229, pl. 10, f. 3-5

1959 Pteria (Austopteria) brevialata: KIRA, CISJ, 1, 118, pl. 46, f. 5

Loc. M S (sy) gl. [golgotha].

Dist. Honshu (north to Bōsō Peninsula) to Ryukyu Islands. Western Pacific.

Pteria avicular (HoLTEN) ッバメガイ (介志)

1802 Mytilus avicular HoLten, Enum. Conchyl., 31 
1814 Avicula chinensis LeACH, Zool. Misc., 1, 86, pl, 38, f. 1

1819 Avicula crocea LAMARCK, AsV, 6 (1), 148

1872 Avicula crysellus DUNKER, Cab., 7 (3), 25, pl. 8, f. 4

1959 Pteria (Austropteria) crypsellus: KIRA, CISJ, 1, 118, pl. 46, f. 6

Loc. M S (sy) gl [golgotha].

Dist. Honshu (Kii Peninsula) to Ryukyu Islands. Western Pacific.

Pteria loveni (DUNKER) フクラスズメガイ（目八）

1872 Avicula loveni Dunker, Cab., 7 (3), 67, pl. 23, f. 6

1882 Avicula loveni: Dunker, Index, 229, pl. 10, f. 6

1959 Pteria (Austropteria) loveni: KIRA, CISJ, 1, 117, pl. 47, f. 4

Loc. M S (sy) gl [golgotha].

Dist. Honshu (north to Bōsō Peninsula) to Kyushu. Western Pacific.

Pteria coturnix (DUNKER) モンウグイスガイ（平瀬）

1872 Avicula coturnix DuNKER, Cab., 7 (3), 67, pl. 23, f. 4, 5

1882 Avicula coturnix: DUNKER, Index, 228, pl. 10, f. 1, 2

1961 Pteria (Austropteria) coturnix: HABE, CISJ, 2, 115, pl. 51, f. 9

Loc. M S (sy) gl [golgotha].

Dist. Honshu (Kii Peninsula) to Kyushu. Western Pacific.

Pteria tortirostris (DUNKER) コウライウグイスガイ（大山）

1848 Avicula tortirostris DUNKER, ZfM, 179

1857 Avicula iridescens ReEVE, Icon., sp. 48

1872 Avicula tortirostris: DUNKER, Cab., 7 (3), 37, p1. 11, f. 6

Loc. M S (sy) gl [golgotha].

Dist. Honshu (Kii Peninsula). Western Pacific.

Pteria dendronephthya HABEトゲトサカノウグイスガイ（波部）

1960 Pteria (Austropteria) dendronephthya HABE, PSMBL, 8 (2), 289

1961 Pteria (Austropteria) dendronephthya: HABE, CISJ, 2, 115, pl. 51, f. 10

Loc. M S (sy) gl. [Dendronephthya].

Dist. Honshu (north to Bōsō Peninsula) to Kyushu and China.

\section{Subgenus Magnavicula IREDALEマベガイ亜属}

1939 Magnavicula Iredale, GBRESR, 5 (6), Moll. 1, 331. Type (OD): Magnavicula bennetti IREDALE

Pteria (Magnavicula) penguin (RöDING) マベガイ（目八）

1798 Pinstada penguin RöDING, Bolten., 167

1819 Avicula macroptera LAMARCK, AsV, 6 (1), 147

1857 Avicula macroptera: ReEve, Icon., sp. 2

1939 Magnavicula bennetti IrEDALE, GBRESR, 5 (6), Moll. 1, 332,

1959 Pteria (Magnavicula) penguin: KIRA, CISJ, 1, 195, pl. 71, f. 9

Loc. $M$ (sy) gl [golgotha].

Dist. Honshu (Kii Peninsula) to Ryukyu Islands. Widely ranging in the Indo Pacific region.

\section{Genus Pterelectroma IREDALEシロチョウウグイスガイ属}

1939 Pterelectroma IredALE, GBRESR, 5 (6), Moll, 1, 332. Type (OD): Avicula zebra ReEvE 
Pterelectroma zebra (REEVE)シロチョウウグイスガイ（黒田）

1857 Avicula zebra REEVE, Icon., sp. 36

1932 Pteria tomlini Prashad, Siboga, 53C, 94, pl. 3, f. 3, 4

1959 Pterelectroma zebra tomlini: KIRA, CISJ, 1, 194, pl. 71, f. 6

1971 Pterelectroma zebra: Kuroda \& HABE, SSSB, 562 (J) 357 (E), pl. 75, f. 7-9

Loc. M S (sy) gl [Lytocarpia].

Dist. Honshu (north to Bōsō Peninsula) to Ryukyu Island. Tropic Pacific region.

\section{Genus Pinctada RöDINGアコヤガイ属}

1798 Pinctada RöDING, Bolten, 166

1901 Margaritifera JAMESON, PZSL, 372. Type (SD, IrEADLE, 1915): Mytilus margaritiferus LINNAEUS

Pinctada margaritifera (LinNaEUs)クロチウガィ（方言）

1758 Mytilus margaritiferus LrnNaeus, Syst. Nat., 10, 704

1857 Avicula margaritifera: REEVE, Icon., sp. 1

1961 Pinctada margaritifera: HABE, CISJ, 2, 116, pl. 51, f. 12

1961 Pinctada margaritifera: RANSON, MIRSNB, 6, 52, pl. 29-37

Loc. M S (r) int.

Dist. Honshu (Kii Peninsula) to Ryukyu Islands. Widely ranging in the Pacific region.

Pinctada maxima (JAMESON)シロチョウガイ (平攧)

1901 Pteria (Margaritifera) maxima JAMESON, PZSL, 377

1961 Pinctada maxima: RANson, MIRSNB, 67, pl. 40-42, f. 21-23

1966 Pinctada maxima: HABe \& Kosuge, SWC, 2, 135, pl. 50, f. 1

Loc. M (g) gl.

Dist. Honshu (Kii Peninsula) to Ryukyu Islands. Widely ranging in the eastern Indo-Pacific region.

Pinctada fucata martensii (DUNKER)アコヤガイ（目八） ベニコチョウガイ（黒田）

1850 Avicula fucata Gould, PBSNH, 3, 309

1857 Avicula fucata: ReEve, Icon., sp. 74

1872 Avicula japonica Dunker (non Dunker, 1852), Cab., 7 (3), 66, pl. 23, f. 2, 3

1872 Avicula martensii DUNKER, Cab., 7 (3), 79

1959 Pinctada martensii: KIRA, CISJ, 1, 117, pl. 46, f. 3

1959 Pinctada fucata: KIRA, CISJ, 1, 118, pl. 46, f. 7

1961 Pinctada martensii: RANSON, MIRSNB, 67, 25, pl. 12, 13

1971 Pinctada fucata martensii Kuroda \& HABE, SSSB, 563 (J) 358 (E), pl. 75, f. 4

Loc. N M S (r) int.

Dist. Honshu (north to Bōsō and Oga Peninsula) to Kyushu.

Pinctada chemnitzii (PHILIPPI) タイワンアオリガイ（黒田）

1849 Avicula chemnitzii PailipPI, ZfM, 19

1872 Avicula chemnitzii: DUNKER, Cab., 7 (3), 15, pl. 3, f. 5

1953 Pinctada shimizuensis S. K. WADA \& R. WAKA, Jour. Ocean. Soc. Japan, 8 $(3,4), 135$, f. $2 \mathrm{a}, \mathrm{b}$

1961 Pinctada chemnitzii: RANSON, MIRSNB, 6, 40, pl. 23, 24

1966 Pinctada chemnitzii: Habe \& Kosuge SWC, 2, 134, pl. 50, f. 5

Loc S (g) int

Dist. Honshu (north to Suruga Bay). Widely ranging in the tropic Pacific. 
Pinctada nigra (GouLD) ムラサキチョウガイ（黒田）

1851 Avicula nigra Gourd, PBSNH, 3, 309 (1850)

1872 Avicula (Mereagrina) scheepmakeri DuNKER, Cab., 7 (3), 6, pl. 1, f. 1

1872 Avicula (Mereagrina) tristis Dunker, Cab., 7 (3), 44, pl. 14, f. 3

1961 Pinctada scheepmakeri: HABE, CISJ, 2, 115, pl. 51, f. 8

1961 Pinctada nigra: Ranson, MIRSNB, 6, 29, pl. 14-16

Loc. S

Dist. Honshu (north to Suruga Bay). Widely ranging in the tropic Pacific.

Pinctada maculata (GouLD) ミドリアオリガイ（黑田）

1850 Avicula maculata Gould, PBSNH, 3, 309

1852 Avicula pica Gould (non PHILIPPI, 1849), USEE, 12, 443, pl. 39, f. 549, a, b.

1901 Margaritifera panasesae JAMESON, PZSL, 390, tf. 94

1959 Pinctada panasesae: KTRA, CISJ, 1, 117, pl. 46, f. 2

1961 Pinctada maculata: Ranson, MIRSNB, 6, 35, p1. 17-19.

Loc. M S (r) gl.

Dist. Honshu (Kii Peninsula) to Ryukyu Islands. Widely ranging in the tropic Pacific.

Genus Electroma STOLICZKA シマウグイスガイ属

1871 Electroma Stoliczka, P. Indica, 9 (6), 391. Type (M): Avicula smaragdina ReEve

Electroma ovata (QUOY \& GAIMARD)シマウグイスガイ（黑田）

(1791 Mytilus meleagridis BRUGUì̀e, EMHNV, pl. 157)

1817 Mytilus alacorvi DILLwYN, DCRS, 322

1834 Meleagrina ovata, QUOY \& GAIMARD, Astrolabe, 3, 459

1834 Meleagrina tongana Quoy \& GAIMARD, Astrolabe, 3, 460, pl. 77, f. 12-15

1857 Avicula smaragdina REEVE, Icon., sp. 45

1872 Avicula melaeagridis: DUNKER, Cab., 7 (3), 57, pl. 19, f. 5, 6

1961 Electroma ovata: HABE, CISJ, 2, 116, pl. 51, f. 11

Loc. S (r) gl.

Dist. Honshu (Kii Peninsula) to Ryukyu Islands. Widely ranging in the tropic Pacific.

\section{Family Isognomonidae WoODRING マクガイ科 \\ Genus Isognomon LIGHTFOOT マクガイ属}

1786 Isognomon Lightroot, Portland, 41, 52. Type (T): Ostrea perna Linnaeus

1786 Isognoma Lightfoot, Portland, 9, 4

\section{Subgenus Isognomon LIGHTFOOT マクガイ亜属}

1788 Metina Retzrus, Diss. Hist. Nat. p. 22,

Isognomon (Isognomon) ephippium (LINNAEUS) マクガイ（黒田）

1758 Ostrea ephippium LinnaUEs, Syst. Nat., 10, 700

1858 Perna ephippium: ReEve, Icon., sp. 8

1961 Isognomon (Melina) ephippium: HABE, CISJ, 2, 115, pl. 51, f. 3

Loc. $\mathrm{S}(\mathrm{r})$ int

Dist. Honshu (north to Kii Peninsula) to Ryukyu Islands. Widely ranging in the Indo Pacific region. 
Isognomon (Isognomon) legumen (GMELIN)シロアオリガイ（平瀬）

1791 Ostrea legumen GMeLIN, Syst. Nat., 13, 3339

1858 Perna linguaeformis ReEve, Icon., sp. 7

1959 Isognomon legumen: KrRA, CISJ, 1, 116, pl. 45, f. 25

1971 Isogonum legumen: KURODA \& HABE, SSSB, 559 (J) 355 (E), pl. 75, f. 5

Loc. N M S (r) int.

Dist. Honshu (north to Bōsō Peninsula) to Ryukyu Islands. Widely ranging in the Indo-Pacific.

Isognomon (Isognomon) perna (LINNAEUS) アオリガイ（介志 non 目八）

カイシアオリガイ（黒田）

1767 Ostrea perna LinNaEus, Syst. Nat., 12, 1149

1798 Isogonum marsupiale RöDING, Bolten., 2, 168

1819 Perna marsupium Lamarck, AsV, 6 (1), 141

1858 Perna marsupium: ReEve, Icon., sp. 15

1961 Isognomon marsupialis: HABE, CISJ, 2, 115, pl. 51, f. 1

Loc. N M S (r) int.

Dist. Honshu (north to Bōsō Peninsula) to Ryukyu Islands. Widely ranging in the Indo-Pacific.

Subgenus Parviperna IREDALE ヘリトリアオリガイ亜属

1939 Parviperna Iredale, GBRESR, 5 (6), Moll. 1, 322. Type (OD): Parviperna perexigua IREDALE

Isognomon (Parviperna) nucleus (LAMARCK) ヘリトリアオリガイ（平瀬）

1819 Perna nucleus LaMarck, AsV, 6 (1), 142

1850 Perna nana Gould, PBSNH, 3, 312

1858 Perna nucleus: Reeve, Icon., sp. 4

1869 Perna acutirostris Dunker, Mus. Godeffroy Cat., 4, 115 (name only)

1961 Isognomon (Parviperna) acutirostris: HABE, CISJ, 2, 115, pl. 51, f. 2

Loc. S (r) int.

Dist. Honshu (Kii Peninsula) to Ryukyu Islands. Widely ranging in the tropic Pacific.

\section{Family Vulsellidae H. \& A. ADAMS ホウオウガイ科}

\section{Genus Vulsella RöDINGホウオウガイ属}

1798 Vulsella RöDING, Bolten., 2, 156

1840 Reniella SwaInson, Treat., 386. Type(SD, Prashad, 1952, ICZN Op. 325): Mya vulsella LINNAEUS

Vulsella vulsella (LINNAEUS) ホウカウガイ（目八）

1758 Mya vulsella Linnaeus, Syst. Nat., 10, 671

1798 Vulsella major RöDING, Bolten., 2, 156

1819 Vulsella lingusta LAMARCK, AsV, 6 (1), 221

1959 Vulsella vulsella: KIRA, CISJ, 1, 117, pl. 45, f. 25

Loc. M S (sy) gl [sponge].

Dist. Honshu (north to Bōsô Peninsula) to Ryukyu Islands. Widely ranging in the Indo-Pacific region. 
Vulsella minor [RöDING] $\quad$ ヒメホウオウガイ（黒田） PI. 5, f. 8

1798 Vulsella minor RöDING, Bolten., 2, 156

1932 Vulsella minor: PraShad, Siboga, 53C, 89, pl. 2

Loc. M S (sy) gl [sponge attached to Composia retusa].

Dist. Honshu (Kii Peninsula) to Indonesia.

\section{Family Malleidae LAMARCK シュモクガイ科 \\ Genus Malleus LAMARCKシュモクガイ属}

1799 Malleus LAMARCK, MSHNP, 82

1817 Himantopoda Schumacher, ENSVT, 38, 109. Type (T): Ostrea malleus LinnaeUs

Malleus malleus LiNNAEUS クロシュミセンガイ (岩川)

1758 Ostrea malleus LinNaEUs, Syst. Nat., 10, 699

1819 Malleus vulgaris LAMARCK, AsV, 6 (1), 144

1858 Malleus vulgaris: REEve, Icon., sp. 5

1959 Malleus malleus: KIRA, CISJ, 1, 116, pl. 45, f. 24

Loc. S (r) gl.

Dist. Honshu (Kii Peninsula) to Ryukyu Islands. Western Pacific.

Malleus albus LAMARCK シュモクガイ (丹浦)

1819 Malleus albus LAMARCK, AsV, 6 (1), 144

1961 Malleus albus: HABE, CISJ, 2, 115, pl. 51, f. 7

Loc. M S (g) gl.

Dist. Honshu (north to Bōsō Peninsula) to Ryukyu Islands. Western Pacific.

\section{Genus Malvufundus DE GREGORIO ヒリョウガイ属}

1884 Fundella De GREGORIo, BSMI, 10, 72 (non ZELLER, 1848)

1885 Malvufundus De Gregorio, BSMI, 11, 122

1931 Parimalleus Iredale, RAM, 18, 205. Type (SD, Cox, 1969): Ostrea regula ForsKÅ

Malvufundus irregularis Jousseaume ヒリョウガイ（六介）

1894 Malvufundus irregularis JousseAume, Le Nat., 8, 228

1953 Malleus (Parimalleus) irregularis: HABE, PSMBL, 3 (2), 169, pl. 9, f. 3, 4

1961 Malleus (Parimalleus) irregularis: HABE, CISJ, 2, 115, pl. 51, f. 4

1971 Malleus (Malvufundus) irregularis: KuRODA \& HABE, SSSB, 560 (J) 356 (E), pl. 75, f. 6

Loc. N M S (r) int.

Dist. Honshu (north to Bōsō Peninsula) to Kyushu. Western Pacific.

\section{Superfamily Pectinacea RAFINESQUE イタヤガイ超科}

Family Pectinidae RAFINESQUE イタヤガイ科

Subfamily Amusiinae RIDEWOOD ッキヒガイ㑹科

Genus Amusium RöDING ッキヒガイ属

1798 Amusium RöDING, Bolten., 165 (Amussium auct. in error)

1831 Pleuronectes Bronn, Ergeb. Nat. Reisen, 2, 624 (non Linnaues, 1758), Type (SD, HeRRMANNSEN, 1846): Ostrea pleuronectes LinNAEUS 
Amusium japonicum (GMELIN) ツキヒガイ（群品）

1791 Ostrea japonica GMELIN, Syst. Nat., 13, 3317

1842 Pecten japonicus: SowerBY, Thes., 1, 55, pl. 15, f. 109

1959 Amusium japonicum: KIRA, CISJ, 1, 193, pl. 71, f. 1.

1964 Amusium japonicum: HABE, BNSM, 7, 5, pl. 2, f. $9,10$.

Loc. N M S (s-m) dp.

Dist. Honshu (north to Bōsō Peninsula) to Formosa.

Subfamily Propeamussinae ABBotT ワタゾコッキヒガイ巠科

Genus Propeamussium DE GREGORIO ワタゾコッキヒガイ属

1884 Propeamussium De Gregorio, Natura. Sicil., 3, 119. Type (OD): Pecten ceciliae De GREGORIO

\section{Subgenus Flavamussium OYAMA ワタン゙コッキヒガイ亜属}

1951 Flavamussium OYAMA, ICJS, 1 (13), 81. Type (OD): Amusium caducum E. A. SMITH

Propeamussium (Flavamussium) caducum (E. A. SMITH)ワタゾコッキヒガイ（黒田）

1885 Amussium caducum E. A. Sмiтн, Challenger, 13, 309, pl. 23, f. 1, 1a-c.

1932 Propeamussium nakazawai Kuroda, Venus, 3 (2), App. 87, f. 101, 102 (name and figure only)

1959 Parvamussium (Flavamussium) caducum: KIRA, CISJ, 1, 122, pl. 48, f. 15

Loc. M S (m) tr.

Dist. Honshu (north to Suruga Bay) to Indonesia.

\section{Subgenus Luteamussium OYAMAクラケッキヒガイ亜属}

1951 Luteamussium Oyama, ICJS, 1 (13), 82. Type (OD): Amussium sibogai DaUtzenberG \& BAVAY

Propeamussium (Luteamussium) sibogai (DAUTtZENBERG \& BAVAY)

クラゲッキヒガイ（黑田）

1904 Amussium sibogai Dautzenderg \& Bavay, J. de C., 52, 207, tf. 1-4

1912 Amussium sibogai: Dautzenderg \& Bavay, Siboga, 52b, 31, pl. 28, f. 1-4

1959 Luteamussium sibogai: KIRA, CISJ, 1, 122, pl. 48, f. 14

Loc. M S (m) tr.

Dist. Honshu (north to Enshu-nada) to Indonesia.

Subgenus Parvamussium SACCO オボロツキヒガイ兆虽

1897 Parvamussium SAcco, Bull. Mus. Zool. Torino, 12, 101

1939 Glyptamussium IREDALE, GBRESR, 5 (6), Moll. 1, 370

1944 Graptamussium OYama, Venus, 13, 252. Type (OD): Propeamussium duodecimlamellatum BRONN

Propeamussium (Parvamussium) rubrotinctum OYAMAオボロッキヒガイ（大山）

1951 Parvamussium (Parvamussium) rubrotincta OYAMA, ICJS, 1 (13), 81, pl. 13, f. 8, 9, 10

Loc. S (m) tr.

Dist. Honshu (north to Kii Peninsula) to Kyushu.

Genus Ctenamussium IREDALEクマドリッキヒガイ属

1929 Ctenamussium Iredalk, RAM, 17, 164. Type (OD): Amusium thetides HedLeY 
Ctenamussium scitulum (SMITH) クマドリッキヒガイ（黒田）

1885 Amissium scitulum Sмттн, Challenger., 13, 312, pl. 23, f. 4-4b.

1931 Propeamussium cmadritinctum Kuroda, Venus, 2 (6), App. 72, f. 81, 82

1961 Ctenamusium cmadritinctum: HABE, CISJ, 2, 118, pl. 53, f. 4

Loc. M S (s) dp.

Dist. Honshu (north to Bōsō Peninsula) to Indonesia.

\section{Genus Bathyamussium OYAMA ，ハナヤカッキヒガイ属}

1951 (Feb.) Bathyamussium OyAMA in HABE, GJS, 1, 7. Type (OD): Amussium jeffreysii SмIтH

Bathyamussium jeffreysii (SMITH)ハナヤカツキヒガイ（大山）

1885 Amusium jeffreysii Sмттн, Challenger., 13 (1), 310, pl. 23, f. 2, 2a-c.

1961 Bathyamussium jeffreysi: $\mathrm{H}_{\mathrm{ABE}}, \mathrm{CISJ}, 2,118$, pl. 53, f. 3

Loc. S (s-m) tr.

Dist. Honshu (north to Kii Peninsula) to Philippines.

Genus Polynemamussium HABE モトリニシキガイ属

1951 Polynemamussium Habe, GJS, 1, 72. Type (OD): Pecten intuscostatus Yokoxama

Polynemamussium intuscostatus (YoKoYAMA) モトリニシキガイ（黒田）

1921 Pecten intuscostatus YoкоYAMA, JCSIUT, 39 (6), 156, pl. 13, f. 9, 10

1922 Pecten tenuicostulatus YokoYama, JCSIUT, 44 (1), 184, pl. 17, f. 15

1961 Polynemamussium intuscostatum: HABE, CISJ, 2, 118, pl. 53, f. 5

1971 Polynemamussium intuscostatum: KURODA \& HABE, SSSB, 579 (J) 369 (E), pl. 79, f. 18-21

Loc. M S (s) dp.

Dist. Honshu (north to Tsuruga Strait) to Kyushu.

\section{Subfamily Chlamydinae KOROBKOV カミオニシキガイ里科}

\section{Genus Chlamys RöDINGカミオニシキガイ属}

1798 Chlamys RöDIng, Bolten., 2, 161. Type (SD, HeRmAnnsen, 1846): Pecten islandicus MÜLLER

\section{Subgenus Coralichlamys IREDALEサンゴナデシコガイ亜属}

1939 Coralichlamys Iredale, GBRESR, 5 (6), Moll. 1, 355. Type (OD): Coralichlamys acroporicola IREDALE

Chlamys (Coralichlamys) irregularis (SowERBY) ナデシコガイ（目八）

1842 Pecten irregularis Sowerby, Thes., 1, 69, pl. 13, f. 51, 52

1852 Pecten irregularis: REEVE, Icon., sp. 19

1882 Pecten irregularis: DunKer, Index. 240, pl. 11, f. 2, 15

1959 Chlamys irregularis: KIRA, CISJ, 1, 123, pl. 49, f. 3

Loc. N M S (g) int.

Dist. Honshu (north to Bōsō Peninsula) to Ryukyu Islands. Western Pacific.

Chlamys (Coralichlamys) lemniscata (REEvE)ワタゾコナデシコガイ（黒田）

1853 Pecten lemniscata ReEve, Icon., sp. 170 (corrected name on the index page)

1940 Chlamys perirregularis NomURA \& HATAI, SHKMRB, 19, 78, pl. 4, f. 15, 16 
1959 Pecten lemniscata: KIRA, CISJ, 1, 122, pl. 48, f. 9

1971 Chlamys (Chlamys) lemniscata: Kuroda \& HABE, SSSB, 570 (J) 363 (E), pl. 80, f. 1, 2.

Loc. M S (g) gl.

Dist. Honshu (north to Bōsõ Peninsula))to Kyushu. Western Pacific.

Chlamys (Coralichlamys) larvata (REEVE) リュウキュウナデシコガイ（平瀬）

1819 Pecten hybidus LAMARCK, AsV, 6 (1), 177 (non GMELIN, 1791)

1853 Pecten larvatus ReEve, Icon., sp. 158

1842 Pecten squamosus Sowerby, Thes., 1, 69, pl. 13, f. 48-50 (non Gmelin, 1791)

1926 Pecten (Chlamys) smegmata Dall, Naut., 40 (2), 67

1959 Chlamys larvata: KIRA, CISJ, 1, 123, pl. 48, f. 5

1960 Chlamys otanii Azums, Cat. Okinoshima, 102, pl. 2, f. 1, 2.

Loc. M S (g) gl.

Dist. Honshu (Kii Peninsula) to Kyushu. Widely ranging in the tropic Pacific.

Chlamys (Coralichlamys) jousseaumei BAVAYニクイロナデシコガイ（黒田）

1904 Chlamys jousseaumei BavaY, J. de C., 52, 203, pl. 6, f. 9, 10

1971 Chlamys (Chlamys) jousseaumei: KuRODA \& HABE, SSSB, 570 (J) 363 (E), pl. 79, f. 14, 15

Loc. M S (g) gl.

Dist. Honshu (north to Bōsō Peninsula) to Kyushu.

Chlamys (Coralichlamys) empressae KURODA \& HABE ミソノナデシコガイ (金丸)

1959 Chlamys mollita: KIRA, CISJ, 1, 121, pl. 8, f. 8 (non. REEVE, 1853)

1971 Chlamys empressae Kuroda \& HABE, SSSB, 571 (J) 363 (E), pl. 79, f. 9-11

Loc. M S (g) gl.

Dist. Honshu (north to Bōsō Peninsula) to Kyushu.

Chlamys (Coralichlamys) princessae KURODA \& HABE

ツヅレナデシコガイ（黒田・波部）

1971 Chlamys (Chlamys) princessae Kuroda \& HABE, SSSB, 571 (J) 364 (E), pl. 79, f. 16, 17

Loc. M S (g) gl.

Dist. Honshu (north to Sagami Bay) to Kyushu.

Subgenus Mimachlamys IREDALE ヒオウギガイ里属

1929 Mimachlamys Iredale, RAM, 17, 162. Type (OD): Pecten asperrimus LAMARcK

Chlamys (Mimachlamys) nobilis (REEvE) ヒオウギガイ（丹浦）

1852 Pecten nobilis Revve, Icon., sp. 3

1853 Pecten crassicostatus ReEve, Icon., sp. 64 (non Sowerby, 1842)

1882 Pecten crassicostatus: Dunker, Index, 239, pl. 13, f. 28

1959 Chlamys (Mimachlamys) nobilis: KIRA CISJ, 1, 124, p. 49, f. 12

1971 Chlamys (Mimachlamys) nobilis: KuRodA \& HaBE, SSSB, 572 (J) 365 (E), pl. 78, f. 1-3

Loc. N M S (r) gl.

Dist. Honshu (north to Bōsō Peninsula) to Ryukyu Islands. Western Pacific.

Chlamys (Mimachlamys) asperulata (ADAMS \& REEVE) ヒナノヒオウギガイ（矢含）

1842 Pecten rugosus Sowerby, Thes., 1, 66, pl. 19, f. 226 (non Lamarck, 1819)

1850 Pecten asperulata AdAms \& ReEve, Samarang, 74, pl. 21, f. 13

1853 Pecten rugosus: ReEve, Icon., sp. 144

1912 Chlamys pelseneeri DaUTZENBERG \& BAVAY, Siboga, 53b, 8

1959 Chlamys pelseneeri: KIRA, CISJ, 1, 122, pl. 48, f. 11 
Loc. N M S (st) gl.

Dist. Honshu (north to Bōsō Peninsula) to Ryukyu Islands.

Subgenus Azumapecten HABE アズマニシキガイ亜属

1977 Azumapecten Habe, SMJ B/S. 82. Type (OD): Pecten farreri Jones \& Preston

Chlamys (Azumapecten) farreri (JONES \& PRESTON) アズマニシキガイ（目八）

1861 Pecten laetus Gould, PBSNH, 8, 39 (non Gould, 1850)

1869 Pecten laetus: LischKE, JMC, 1, 169, pl. 12, f. 7, 8.

1904 Pecten farreri Jones \& Preston, PMSL, 6 (3), 149, tf.

1932 Chlamys farreri nipponensis KurodA, Venus, 3 (2), App. 91 (new name for Pecten laetus Gould, 1861)

1932 Chlamys farreri akazara KurodA, Venus, 3 (2), App. 92, f. 105

1959 Chlamys nipponensis: KIRA, CISJ, 1, 124, pl. 49, f. 11

1961 Pecten laetus: Johnson, USNMB, 239, 98, pl. 21, f. 8

Loc. S (g) gl.

Dist. Southern Hokkaido to East China Sea.

Chlamys (Azumapecten) squamata (GMELIN)＝シキガイ (丹浦)

1791 Ostrea squamata GMeLrs, Syst. Nat., 13, 3329

1842 Pecten squamatus: Sowerby, Thes., 1, 70, pl. 13, f. 57, 58

1959 Chlamys (Mirapecten) squamata: KIRA, CISJ, 1, 123, pl. 49, f. 3

1971 Chlamys (Chlamys) squamata: KURODA \& HABE, SSSB, 572 (J) 364 (E), pl. 80, f. 8-13

Loc. N M S (g) gl.

Dist. Honshu (north to Bōsō Peninsula) to East China Sea.

Genus Mirapecten DALL BARTSCH \& REHDER タエニシキガイ属

1938 Mirapecten Dall, BarTsch \& RehDER, BPBMB, 153, 84. Type (OD): Mirapecten thaanumi DALL, BARTSCeE \& ReHDER

Mirapecten mirificus (REEVE)タエニシキガイ（黒田）

1853 Pecten mirificus ReEve, Icon., sp. 104

1938 Mirapecten thaanumi Dall, BARTSCH \& REHDER, BPBMB, 153, 84, pl. 21, f. 7, 8.

1961 Chlamys (Mirapecten) mirifica: HABE, CISJ, 2, 118, pl. 53, f. 10

Loc. M S (r) gl.

Dist. Honshu (Kii Peninsula) to Ryukyu Islands. Widely ranging in the tropic Pacific.

\section{Genus Semipallium JoussEAUME ッッ゙レノニシキガイ属}

1928 Semipallium JousseaUme, BMNHNP, 34, 169

1939 Complicachlamys Iredale, GBRESR, 5 (6), Moll. 1, 346. Type (OD): Pecten tigris LAMARCK

Semipallium fulvicostum (ADAMS \& REEVE) ッッ゙レノニシキガイ（金丸）Ｐl. 1, f. 4

1850 Pecten fulvicosta Adams \& Reeve, Samarang, 74, pl. 21, f. 11

1853 Pecten luculentus ReEve, Icon., sp. 59

1953 Pecten dringi ReEve, Icon., sp. 152

1932 Pecten mollita var. Kanamaru, Venus 3 (5), 274, tf. 8 (non ReEve, 1853)

Loc. M S (st) gl.

Dist. Honshu (north to Izu Peninsula) to Ryukyu Islands. Western tropic Pacific. 
Semipallium tigris (LAMARCK) ニシキオウギガイ (平瀬)

1819 Pecten tigris LamarcK, AsV, 6 (1), 171

1842 Pecten tigris: SOWERBY, Thes., 1, 68, pl. 14, f. 95, 96

1961 Complicachlamys tigris: HABE, CISJ, 2, 118, pl. 53, f. 14

Loc. M (r) gl.

Dist. Honshu (Kii Peninsula) to Ryukyu Islands. Tropic Pacific.

Genus Bractechlamys IREDALE ナンカイニシキガイ属

1939 Bractechlamys IREDALE, GBRESR, 5 (6), Moll. 1,366. Type (OD): Bractechlamys evecta IREDALE

Bractechlamys quadrilirata (LISCHKE) タジマニシキガイ（矢倉）

1870 Pecten quadriliratas LisCHKE, M B L, 17, 29

1871 Pecten quadriliratus: LISCHKE, JMC, 2, 158, pl. 9, f. 5, 6

1959 Chlamys quadrilirata: KIRA CISJ, 1, 121, pl. 46, f. 6

Loc. M S (g) gl.

Dist. Honshu (Izu Peninsula) to Ryukyu Islands.

Bractechlamys aurantiaca (ADAMS \& REEVE)ナンカイニシキガイ（黒田）ＰI. 5, f. 1

1850 Pecten aurantiacus Adams \& ReEve, Samarang, 74, pl. 21, f. 12

1928 Pecten aurantiacus: YokoYama, RGSJ, 101, 93, pl. 13, f. 7, 8

Loc. M (g) gl.

Dist. Honshu (Kii Peninsula) to Ryukyu Islands. Tropic Pacific.

Bractechlamys schmeltzii (REEVE) ヤガスリヒヨクガイ (平瀬)

1860 Pecten schmeltzii Koвelt, Cat. Mus. Godeffroy., 4, 116

1892 Pecten schmeltzii: КовеLт, Cab., 7 (2), 272, pl. 71, f. 5, 6.

1959 Chlamys schmeltzii: KIRA, CISJ, 1, 122, pl. 48, f. 10

Loc. M S (g) int.

Dist. Honshu (Kii Peninsula) to Ryukyu Islands. Tropic Pacific.

\section{Genus Gloripallium IREDALEチサラガイ属}

1939 Gloripallium IREDALE, GBRESR, 5 (6), Moll. 1, 346, 357. Type. (OD): Ostrea pallium LINNAEUS

Gloripallium pallium (LINNAEUs) チサラガイ (田中)

1758 Ostrea pallium Linnaeus, Syst. Nat., 10, 697

1842 Pecten pallium: SowerBy, Thes., 1, 73, pl. 17, f. 148-150; pl. 18, f. 167

1959 Gloripallium pallium: KIRA CISJ, 1, 124, pl. 49, f. 10

Loc. S

Dist. Honshu (Kii Peninsula). to Ryukyu Islands. Widely ranging in the Indo Pacif ic.

Gloripallium speciosum (REEVE)オオシマヒオウギガイ（吉良）Ｐl. 1, f. 2

1853 Pecten speciosus ReEVE, Icon., sp. 112

1966 Gloripallium speciosa HABE \& KosUge, SWC, 1, 138, pl. 53, f. 1

Loc. M S (r) gl.

Dist. Honshu (Kii Peninsula) to Ryukyu Islands. Widely ranging in the Indo Pacific. 


\section{Genus Annachlamys IREDALE ニシキヒヨクガイ属}

1939 Annachlamys IreDALE, GBRESR, 5 (6), Moll. 1, 358. Type (OD): Annachlamys reevei (ADAMS \& REEVE)

Annachlamys reevei (ADAMS \& REEVE) ニシキヒヨクガイ (平瀬)

1850 Pecten reevei AdAms \& ReEve, Samarang, 73, pl. 21, f. 10

1853 Pecten reevei: ReEve, Icon., sp. 91

1912 Pecten (Aequipecten) reevei: Dautzenberg \& Bavay, Siboga, 53b, 18, pl. 27, f. $3,4$.

1959 Annachlamys reevei: KIRA, CISJ, 1, 195, pl. 71, f. 7

Loc. N M S (g) gl.

Dist. Honshu (north to Izu Islands). Widely ranging in the tropic Pacific.

Genus Cryptopecten DALL, BARTsch \& REHDER ヒョクガイ属

1938 Cryptopecten Dall, Bartsch \& ReHDer, BPBMB, 153, 347, 367. Type (OD): Cryptopecten alli DALL, BARTSCH \& ReHDER

Cryptopecten vesiculosus (DUNKER) ヒヨクガイ（六介）

1877 Pecten vesiculosus DUNKer, M B L, 24, 72

1882 Pecten vesiculosus: Dunker Index, 241, pl. 11. f. 1

1888 Pecten hastingsii Melvill, J. of C., 5, 278, pl. 2, f. 7

1959 Cryptopecten vesiculosus: KIRA, CISJ, 1, 123, pl. 49, f. 1

1971 Cryptopecten vesiculosus: KURODA \& HABE, SSSB, 574 (J) 366 (E), pl. 79, f. 1-7

Loc. N M S (s) gl.

Dist. Honshu (north to Bōsō Peninsula) to East China Sea.

Cryptopecten tissotii (BERNARDI) ヒラヒヨクガイ（黒田）

1858 Pecten tissotii Bernardr, J. de C., 7, 91, pl. 1, f. 2

1887 Pecten tissotii: Ковецт, Cab, 7 (2), 143, pl. 40, f. 5

Loc. M S (s) gl.

Dist. Honshu (Bōsō Peninsula) to Kyushu.

Remarks: This seems to be merely a form of C. vesiculosus (DUNKER)

Cryptopecten oweni (De GrEGORIO)ムムラクモヨクガイ（黒田） Pl. 5, f. 2

1842 Pecten pictus Sowerby (non Laborda, 1828 nec Deshayes, 1830), Thes, 1, 62, pl, 20, f. 233

1853 Pecten pictus: ReEve, Icon., sp. 116

1884 Pecten oweni De Gregorio, Nat. Sicil. Ann. Terzo, 5, 133

Loc. M S (st) int.

Dist. Honshu (Kii Peninsula) to Amami and Ryukyu Islands. Widely ranging in the tropic Pacific.

\section{Cryptopecten nux (REeVE) イササヒヨクガイ（吉良）}

1853 Pecten nux ReEve, Icon., sp. 143

1961 Cryptopecten nux: HABE, CISJ, 2, 118, pl. 53, f. 9

Loc. N M S (s) gl.

Dist. Honshu (north to Izu Peninsula) to Ryukyu Islands. Tropic Pacific.

\section{Cryptopecten inaequivalvis (SOWERBY) ウシジロヒョクガイ（黒田）}

1842 Pecten inaequivalvis Sowerby, Thes., 1, 50, p1. 19, 193-195

1852 Pecten inaequivalvis: REEVE, Icon., sp. 1

1959 Cryptopecten inaequivalvis: KIRA, CISJ, 1, 122, pl. 48, f. 13 
Loc. M S (s) dp.

Dist. Honshu (Kii Peninsula) to Ryukyu Islands. Tropic Pacific.

\section{Genus Decatopecten SoWERBYキンチャクガイ属}

1817 Pallium SCHUMACHER, ENSVT, 44, 120 (non SCHRÖTER, 1802)

1839 Decatopecten SowerBy, Conch. Man. 1, 37

1840 Decadopecten SwaInson, Theat., 388

1847 Dentipecten Gray, PZSL, 15, 200. Type (M): Ostrea plica LinNaEus

Decatopecten striatus (SCHUMACHER) キンチャクガイ（六介）

1817 Pallium striatum SCHUMACHER, ENSVT, 40, 120

1959 Decatopecten striatus: KIRA, CISJ, 1, 123, pl. 49, f. 6

Loc. N M S (g) gl.

Dist. Honshu (north to Bōsō Peninsula) to Indonesia.

Genus Volachlamys IREDALE ヤミノニシキガイ属

1939 Volachlamys Iredale, GBRESR, 5 (6), Moll. 1, 356. Type (OD): Pecten cumingii REEVE

Volachlamys hirasei hirasei (BAVAY) ヤミノニシキガイ（介志）

1.904 Chlamys hirasei BavaY, J. de C., 52 (3), 197, pl. 6, f. 1, 2

1904 Chlamys hirasei ecostata BavaY, J. de C., 52 (3), 198, pl. 6, f. 7, 8.

1959 Volachlamys hirasei: KIRA CISJ, 1, 124, pl. 49, f. 8

Loc. N (s-m) dp.

Dist. Honshu (Kii Peninsula) to Kyushu. Western Pacific.

Remark: In this form, the surface of shell is smooth, without any radial ribs.

Volachlamys hirasei awajiensis (PILSBRY)アワジチヒロガイ（岩川）

1905 Pecten awajiensis PILSBRy, PANSP, 121

1926 Pecten atsumiensis YokoYama, JFSIUT, (2) 1 (9), 375, pl. 43, f. 4, 5.

1959 Volachlamys hirasei awajiensis: KIRA, CISJ, 1, 124, pl. 49, f. 7

Loc. $N$ (s-m) dp.

Dist. Honshu (Kii Peninsula) to Kyushu.

Remark: This is the ribbed form of the preceding species.

\section{Genus Excellichlamys IREDALE チヒロガイ属}

1939 Excellichlamys Iredale, GBRESR, 5 (6), Moll. 1, 347, 366. Type (OD): Pecten spectabilis REEVE

Excellichlamys spectabilis (REEVE) チヒロガイ（六介）

1853 Pecten spectabilis REEve, Icon., sp. 128

1882 Pecten spectabilis: DuNKER. Index, 241, pl. 11, f. 12, 13

1959 Excellichlamys spectabilis: KIRA CISJ, 1, 123, pl. 49, f. 2

1971 Excellichlamys spectabilis: KURODA \& HABE, SSSB, 575 (J) 366 (E), pl. 79, f. 12, 13

Loc. M S (g) gl.

Dist. Honshu (north to Bōsō Peninsula) to Ryukyu Islands. Widely ranging in the Indo-Pacific.

\section{Genus Anguipecten DALL, BARTSCH \& REHDERナナソデキンチャクガイ属}

1938 Anguipecten Dall, BarTsch \& ReHDer, BPBMB, 153, 92. Type (OD): Anguipecten 
gregoryi DALL, BARTSCH \& REHDER $=$ Pecten superbus SOWERBY

Anguipecten superbus (SOWERBY)シュロノハキンチャクガイ（平瀬）Ｐl. 1, f. 3

1842 Pecten superbus Sowerby, Thes., 1, 62, pl. 12, f. 11

1853 Pecten superbus: ReEVe, Icon., sp. 107

1938 Anguipecten gregoryi DALL, BARTSCH \& REHDER, BPBMB, 153, 93, pl. 21, f. 5, 6.

1970 Anguipecten superbus: HABE, PSMBL, 2, 32, 2 figs.

Loc. M (s) gl.

Dist. Honshu (north to Kii Peninsula) to Ryukyu Islands, Hawaii Islands and the tropic Pacific.

\section{Subfamily Camptonectinae HABEハリナデシコガイ覀科}

\section{Genus Delectopecten STEWARTハリナデシコガイ属}

1930 Delectopectan Stewart, PANSP, Sp. Publ., 3, 118. Type(OD): Pecten (Pseudamussium) vancouverensis WHITEAVES

*Delectopecten macrocheiricola (HABE)ハリナデシコガイ（黒田）

1931 Pseudamussium (Palliolum) vitreum KuRodA, Venus, 3 (1), App. 81, f. 94, 95 (non GMeLIN, 1791)

1938 Pseudamussium (Palliolum) vitreum Hiro, AZJ, 17, 474, f. 5a-c

1951 Palliolum (Delectopecten) macrochericola HABE, GJS, 1, 80

1961 Palliolum (Delectopecten) macrocheiricola: HABE, CISJ, 2, 118, pl. 53, f. 7

1971 Palliolum (Delectopecten) macrocheiricola: KURODA \& HABE, SSSB, 576 (J) 367 (E), pl. 79, f. 22, 23

Loc. M S (sy) g1. [Macrocheira kaempferi]

Dist. Honshu to Kyushu.

\section{Subfamily Hemipectininae HABE イワォニシキガイ亜科 \\ Genus Hemipecten ADAMS \& REEvE イワオニシキガイ属}

1849 Hemipecten AdAMS \& REEve, PZSL, 16, 133

1886 Semipecten Fischer, M. de C., 945 (emend.). Type (M): Hemipecten forbesianus A. ADAMS \& REEVE

Hemipecten forbesianus ADAMS \& REEVE イワオニシキガイ（黒田）

1848 Hemipecten forbesianus ADAMs \& REEve, PZSL, 16, 133, pl. 1, f. 2

1849 Hemipecten forbesianus: ReEVE, Icon., sp. 1

1850 Hemipecten forbesianus: AdAMs \& REEVE, Samarang, 73, pl. 20, f. 1, 2.

1931 Hemipecten forbesianus: KURODA, Venus, 3 (1), 82, f. 96

Loc. S (r) gl.

Dist. Honshu (Kii Peninsula).

\section{Subfamily Pectininae RAfINESQUE イタヤガイ亜科}

\section{Genus Pecten MÜLLER イタヤガイ属}

1776 Pecten Müller, Zool. Donicae. Prod., 248

1853 Vola Mörch, Yoldi, 2, 59 (non Mulsant, 1850). Type (SD, Schmidt, 1818): Ostrea maxima LINNAEUS 


\section{Subgenus Oppenheimopecten TEPPERハナイタャガイ重属}

1922 Oppenheimopecten TePper, Foss. Cat. Anim., 15, 254. Type (OD): Pecten subbenedictus FONTANNES

Pecten (Oppenheimopecten) sinensis puncticulatus DUNKERハナイタヤガイ（六介）

1842 Pecten sinensis Sowerby, Thes., 1, 48, pl. 16, f. 120, 121.

1877 Pecten puncticulatus Dunker, M B L., 24, 71

1882 Pecten puncticulatus: Dunker, Index, 244, pl. 11, f. 10, 11

1959 Pecten (Notovola) puncticulatus: KIRA, CISJ, 1, 120, pl. 47, f. 1

1971 Pecten (Pecten) sinensis puncticulatus: KuRODA \& HABE, SSSB, 576 (J) 368 (E), pl. 81, f. 4,5 .

Loc. N M S (s) gl.

Dist. Honshu (north to Bōsō Peninsula) to Kyushu and Korea.

\section{Subgenus Notovola FINLEY イタヤガイ鱼属}

1926 Notovola FInLeY, TNZI, 57, p1. 451. Type (OD): Pecten novaelandiae ReEve

Pecten (Notovola) excavatus ANTONシナイタャガイ（岩川)

1839 Pecten excavatus ANTon, Verzeich, 19

1845 Pecten excavatus: PhILrpri, Abbild., 1, 201, pl. 2, f. 1

1852 Pecten excavatus: ReEve, Icon., sp. 33

1959 Pecten (Notovola) excavatus: KIRA, CISJ, 1, 120, pl. 47, f. 2

Loc. S (s) dp

Dist. Honshu (Kii Peninsula), Kyushu and China Seas.

Pecten (Notovola) albicans (SCHRÖTER) イタヤガイ（本和）

1802 Pecten albicans SCHRÖTER, AfZ, 3, 135

1842 Pecten laquetus Sowerby, Thes., 1, 46, pl. 15, f. 101

1944 Pecten albicans: ReHDER, Naut., 58, 54

1971 Pecten (Notovola) albicans KurOdA \& HABE, SSSB, 577 (J), 368 (E), pl. 81, f. 1-3

Loc. N M S (s) dp.

Dist. Southern Hokkaido to East China Sea.

\section{Subgenus Serratovola HABEシゼツホタテガイ亜属}

1951 Serratovola HABE, GJS, 1, 81. Type (OD): Pecten tricarinatus ANToN

Pecten (Serratovola) tricarinatus ANTONシゼツホタテガイ (波部)

1839 Pecten tricarinatus ANTon, Verzeich., 19, 707

1844 Pecten tricarinatus: PhILIPPI, Abbild., 1, 99, pl. 1, f. 4

1966 Serratovola tricarinatus: HABE \& Kosuge, SWC, 2, 139, pl. 53, f. 9

Loc. M (s) gl.

Dist. Honshu (north to Kii Peninsula). Western Pacific.

Pecten (Serratovola) asper SOWERBY ママダラシゼツホタテガイ（波部）

1842 Pecten asper Sowerby, Thes., 1, 50, pl. 19, f. 196, 197

1852 Pecten asper: REEVE, Icon., sp. 10

1966 Serratovola asper: HABE \& Kosuge, SWC, 2, 138, pl. 53, f. 2

Loc. M S (s) gl.

Dist. Honshu (north to Kii Peninsula). Western Pacific. 
Subfamily Patinopectininae MASUDA ホタテガイ亜科

Genus Patinopecten DALL ホタテガイ属

1898 Patinopecten Dall, TWFIP, 3, 695. Type (OD): Pecten caurius Gould

Subgenus Mizuhopecten MASUDA ホタテガイ亜属

1963 Mizuhopecten Masuda, TPPSJ (NS), 52, 151. Type (OD): Pecten tokyoensis Tokunaga

†Patinopecten (Mizuhopecten) tokyoensis (ToKUNAGA) トウキョウホタテガイ（鹿間）

モミジホタテガィ（吉良）

1906 Pecten tokyoensis ToKUnAGA, JCSIUT, 21 (2), 65, pl. 5, f. 1-10

1973 Patinopecten (Mizuhopecten) tokyoensis: OYAMA, PSJSP. P., 17, 86, pl. 35, f. 4, 7

Loc. M (s-m) tr.

Remarks: This is the extinct Pleistocene species and widely occurences around Japan at the bottom of about $200 \mathrm{~m}$ deep as the sea shore in the last gracial age.

\section{Family Spondylidae GRAY ウミギクガイ科}

Genus Spondylus LINNAEUS ウミギクガイ属

1758 Spondylus LinNaeus, Syst. Nat., 10, 690. Type (SD, SchmidT, 1818): Spondylus gaederopus LINNAEUS

\section{Subgenus Spondylus LiNNAEUSウミギクガイ亜属}

Spondylus (Spondylus) butleri (REEVE)カバトゲウミギクガイ（黒田）

1856 Spondylys butleri REEVE, Icon., sp. 14

1961 Spondylus butleri: HABE, CISJ, 2, 118, pl. 53, f. 16

Loc. M S (r) gl.

Dist. Honshu (Kii Peninsula). Western Pacific.

Spondylus (Spondylus) barbatus REEVEウミギクガイ（六介）

1847 Spondylus sinensis Sowerby, Thes., 1, 427, p1. 87, f. 32, 33 (non SchreIbers, 1793)

1856 Spondylus barbatus REEVE, Icon., sp. 16

1932 Spondylus japonicus Kuroda, Venus, 3 (3), 110

1962 Spondylus barbatus: KIRA, CISJ, 1, 126, pl. 50, f. 7

1971 Spondylus barbatus: Kuroda \& Habe, SSSB, 567 (J) 361 (E), pl. 80, f. 3, 4

Loc. N M S (r) gl.

Dist. Honshu (north to Bōsō Peninsula) to Kyushu. Western Pacific.

Spondylus (Spondylus) cruentus LISCHKEチリボタンガイ（目八）

1868 Spondylus cruentus LrschKe, M B L, 15, 221

1869 Spondylus cruentus: LiscHKe, JMC, 1, 172, pl. 12, f. 1-5

1962 Spondylus cruentus: KrRA, CISJ, 1, 126, pl. 50, f. 6

Loc. N M S (r) gl.

Dist. Honshu (north to Bōsō Peninsula) to Ryukyu Islands.

Remarks: This seems to be a form of Sp. barbatus REEvE.

Spondylus (Spondylus) anacanthus MAWEオオナデシコガイ（丹浦）

1823 Spondylus anacanthus MAWE, Linn, Syst. Conch., pl. 2, f. 3 
1847 Spondylus nudus Sowerby, Thes., 1, 430, pl. 48, f. 39, 40

1932 Spondylus anacanthus: Kuroda, Venus, 3 (3), App., 108, f. 117

1962 Spondylus anacanthus: KIRA, CISJ, 1, 125, pl. 50, f. 1

1971 Spondylus anacanthus: KURODA \& HABE, SSSB, 568 (J) 361 (E), pl. 80, f. 5, 6

Loc. M S (st) gl.

Dist. Honshu (north to Bōsō Peninsula) to Kyushu. Western Pacific.

Spondylus (Spondylus) versicolor SCHREIBERS ウニメンガイ (累田)

1793 Spondylus versicolor SchreIBERs, Vers. Couch. Linn. Syst., 159

1819 Spondylus aurantius LAMARCK, AsV, 6 (1), 192

1856 Spondylus aurantius: REEVE, Icon., sp. 10

1959 Spondylus versicolor: KIRA, CISJ, 1, 126, pl. 50, f. 5

Loc. M S (r) gl.

Dist. Honshu (north to Kii Peninsula) to Ryukyu Islands. Widely ranging in the tropic Pacific.

Spondylus (Spondylus) candidus LAMARCK ヤスリメンガイ（黒田）

1819 Spondylus candidus LamarcK, AsV, 6 (1), 188

1847 Spondylus candidus: Sowerby, Thes., 1, 429, pl. 1, f. 3-5

1959 Spondylus candidus: KIRA, CISJ, 1, 126, pl. 50, f. 3

Loc. M S (r) gl.

Dist. Honshu (Kii Peninsula) to Ryukyu Islands. Tropic Pacific.

Spondylus (Spondylus) nicobaricus SCHREIBERS ミヒカリメンガイ（黒田）

1793 Spondylus nicobaricus ScHReIBERs, Vers. Conch. Linn. Syst., 156

1819 Spondylus radians LAMARCK, AsV, 6 (1), 192

1847 Spondylus nicobaricus: SOWERBY, Thes., 1, 428, pl. 88

1856 Spondylus ocellatus ReEve, Icon., sp. 43

1959 Spondylus nicobaricus: KTRA, CISJ, 1, 125, p1. 50, f. 2

Loc. S (r) gl.

Dist. Honshu (Kii Peninsula) to Ryukyu Islands. Indo-Pacific.

Spondylus (Spondylus) setiger REEVEアズキイロメンガイ（黒田）

1856 Spondylus setiger ReEve, Icon., sp. 53

Loc. S (r).

Dist. Honshu (Kii Peninsula) to Ryukyu Islands. Tropic Pacific.

Spondylus (Spondylus) spinosus SCHREIBERS ～シロトゲウミギクガイ（黒田）

1793 Spondylus spinosus Schrerbers, Verg. Conch. Linn. Syst., 154

1819 Spondylus marisrubri RöDING, Bolten., 194

1847 Spondylus aculeatus Sowerby, Thes., 1, 419, pl. 85, f. 11, 13

1932 Spondylus spinosus: Kuroda, Venus, 3 (3), App. 109, f. 124

Loc. S (r) gl.

Dist. Honshu (Kii Peninsula) to Formosa. Western Pacific

Spondylus (Spondylus) squamosus SCHREIBERS メンガイ（岩川；オモテガイ（丹浦）

1793 Spondylus squamosus ScHreiBers, Vers. Conch. Linn. Syst., 157

1798 Spondylus ducalis RöDING, Bolten., 194

1856 Spondylus ducalis: ReEve, Icon., sp. 26

1965 Spondylus squamats (sci): HABE, NIEFJ, 2, 242, f. 907

Loc. S (r). 
Dist. Honshu (north to Kii Peninsula) and Ryukyu Islands. Widely ranging in the Indo Pacific region.

Subgenus Eleutherospondylos DUNKERショウジョウガイ悪属

1882 Eleutherospondylos Dunker, Index, 245. Type (OD): Spondylus regius LinNaEus

Spondylus (Eleutherospondylos) regius (LinNAEUs)ショウジョウガイ（宇久)

1758 Spondylus regius Linnaeus, Syst. Nat., 10, 690

1847 Spondylus regius: Sowerby, Thes., 1, 424, pl. 87, f. 30

1847 Spondylus cumingii Sowerby, Thes., 1, 425, pl. 89, f. 62

1959 Spondylus regius: KIRA, CISJ, 1, 127, pl. 50, f. 9

Loc. M S (r) gl.

Dist. Honshu (Kii Peninsula) to Indonesia.

Spondylus (Eleutherospondylos) serraticostus PRASHAD ショウキウミギクガイ（波部，林)

Pl. 5, f. 3

1932 Spondylus serraticosta Prashad, Siboga, 53c, 114, pl. 3, f. 10, 11

Loc. M S (r) gl.

Dist. Honshu (Kii Peninsula) to Indonesia.

Spondylus (Eleutherospondylos) sinensis ScHREIBERSダンドクメンガイ（黒田）

1793 Spondylus sinensis ScHreibers, Vers. Conch. Linn. Syst., 154. (non Sowerby, 1847)

1811 Spondylus difitatus PERry, Conchology, pl. 59, no. 3

1819 Spondylus costatus LAMARck, AsV, 6 (1), 180

1847 Spondylus petroselinum SowerBy, Thes., 1, 419, p1. 85, f. 6-10, 49.

1856 Spondylus foliaceus REEVE, Icon., sp. 25

1959 Spondylus sinensis: KIRA, CISJ, 1, 126, pl. 50, f. 4

Loc. M S (r) gl.

Dist. Honshu (Kii Peninsula) to Ryukyu Islands. Western Pacific.

Spondylus (Eleutherospondylos) erectospinosus HABEハリヤマウミギクガイ（波部)

1973 Spondylus erectospinosus HABE, BNSM, 16, 37, f. 1-2

Pl. 5, f. 4

1977 Spondylus erectospinosus: HABE, Taxa. HABE, 38, pl. 4, f. 10

Loc. M S (r) gl.

Dist. Honshu (Kii Peninsula) to South China Sea.

Subgenus Eltoperna IREDALE チイロメンガイ丳属

1939 Eltoperna Iredale, GBRESR, 5 (6), Moll. 1, 372, 377. Type (OD): Spondylus pacificus fortior IREDALE

Spondylus (Eltoperna) sanguineus DUNKERチイロメンガイ（黒田）

1852 Spondylus sanguineus DUNKER, ZfM, 55

1858 Spondylus sanguineus: DuNKER, NCM, 26, pl. 7, f. 4, 5

1866 Spondylus pacificus REEve, Icon., sp. 1

1959 Spondylus sanguineus: KrRA, CISJ, 1, 126, pl. 50, f. 8

1966 Spondylus (Eltoperna) sanguineus: HABE \& Kosuge, SWC, 2, 141, pl. 54, f, 2

Loc. $\mathrm{M} \mathrm{S}$ (r) gl.

Dist. Honshu (Kii Peninsula) to Ryukyu Islands. Widely ranging in the western Pacific. 


\section{Family Plicatulidae WATSON ネズミノテガイ科 \\ Genus Plicatula LAMARCKネズミノテガイ属}

1801 Plicatula LamarcK, AsV, 132. Type (SD, SchmidT, 1818): Spondylus plicatus Linnaeus

Plicatula simplex GouLDネズミノテガイ（目八）

1861 Plicatula simplex GouLD, PBSNH, 8, 39

1873 Plicatula simplex: Sowerby, Icon., sp. 2

1877 Plicatula cuneata DUNKER, M B L, 24, 73

1882 Plicatula cuneata: Dunker, Index, 246, pl. 11, f. 3

1964 Plicatula simplex: JoHNSON, USNMB, 239, 149, pl. 25, f. 4

1971 Plicatula simplex KuROdA \& HABE, SSSB, 566 (J) 360 (E), pl. 84, f. 6

Loc. M S (r) gl.

Dist. Honshu (north to Bōsō Peninsula) to Kyushu.

Plicatula australis LAMARCKカスリイシガキモドキ（黒田）

1819 Plicatula australis LAMARCK, AsV, 6 (1), 185

1873 Plicatula australis: SowerBy, Icon., sp. 10

1959 Plicatula australis: KIRA, CISJ, 1, 121, pl. 48, f. 2

1971 Plicatula australis: KunodA \& HABE, SSSB, 566 (E) 360 (E), pl. 84, f. 4

Loc. M S (st) gl.

Dist. Honshu (Bōsō Peninsula) to Ryukyu Islands. Tropic Pacific.

Plicatula horrida DUNKER イシガキモドキ(岩川)

1877 Plicatula rugosa DUNKER, M B L, 24, 73 (non LAMARCK, 1819)

1882 Plicatula horrida Dunker, Index, 247, pl. 11, f. 6, 7

1882 Plicatula irregularis DUNKER, Index. 261, pl. 11, f. 5

Loc. M S (r) gl.

Dist. Honshu (north to Bōsō Peninsula) to Kyushu.

Plicatula plicata (LINNAEUS)エダウネイシガキモドキ（黒田）

1767 Spondylus plicatus LinNaEus, Mus. Lud. Ulr., 511

1847 Plicatula ramosa Sowerby, Thes., 1, 435, pl. 90, f. 1-4

1853 Plicatula chinensis MörcH, Yoldi, 2, 61

1959 Plicatula chinensis: KIRA, CISJ, 1, 121, pl. 48, f. 4

Loc. S (r) gl.

Dist. Honshu (north to Bōsō Peninsula) to Ryukyu Islands. Widely ranging in the Indo Pacific.

\section{Genus Spiniplicatula HABEモグラノテガイ属}

1977 Spiniplicatula HABE, SMJ B/S, 95. Type (OD): Plicatula muricata SOWERBY

$$
\text { Spiniplicatula muricata (SoWERBY)モグラノテガイ（岩川) }
$$

1873 Plicatula muricata Sowerby, Icon., sp. 9

1882 Plicatula muricata: DuNKER, Index., 247, pl. 11, f. 4

1971 Plicatula muricata: KuRodA \& HABE, SSSB, 565 (J) 369 (E), pl. 84, f. 1-3

Loc. N M (g) gl.

Dist. Honshu (north to Bōsō Peninsula) to East China Sea. 


\section{Superfamily Dimyacea P. FISHER イシガキ超科 \\ Family Dimyidae P. FISHER イシガキ科 \\ Genus Dimya RoUAULT イシガキ属}

1850 Dimya Rouault, MSGF. (2) 3, 470

1937 Dimyarina Iredale, RAM, 19, 269. Type (M): Dimya deshayesiana Rounult

Dimya japonica HABE ギンパクイシガキ(波部)

1958 Dimya lima: HABe, PSMBL, 6, 262, pl. 1, f. 21 (non BARTSCH, 1913)

1971 Dimya japonica $\mathrm{H}_{\mathrm{ABE}}$, Veliger, 13 (4), 331, f. 9-19

1971 Dimya japonica: Kuroda \& HABE, SSSB, 563 (J) 359 (E), pl. 84, f. 9, 10

Loc. M S (g) gl.

Dist. Honshu (north to Bōsō Peninsula) to Kyushu and East China Sea.

Dimya filipina BARTSCHイシガキ（吉良）：ジミアガイ（黑田）

1913 Dimya flipina Bartsch, PUSNM, 45, 305, pl. 28, f. 1, 4

1928 Dimya radiata Kuroda, Venus, 1 (1), 14, pl. 1, f. 10, 11

1932 Dimya radiata takii Kuroda, Venus, 3 (3), App. 111, tf. 127, 128

1971 Dimya filipina: Kuroda \& HABE, SSSB, 564 (J) 359 (E), pl. 84, f. 7, 8

Loc. M S (g) gl.

Dist. Honshu (north to Sagami Bay) to Kyushu and Philippines.

\section{Superfamily Limacea RAFINESQUE ミノガイ超科 \\ Family Limidae RAFINESQUE ミノガイ科 \\ Genus Lima BRUGUrÈRE ミノガイ属}

1797 Lima Bruguike, EMHNV, 2, pl. 206. Type (T): Ostrea lima LinNaEus

Lima zushiensis YokoYama モクハチミノガイ (黒田)

1920 Lima zushiensis YoKoyama, JCSIUT, 39 (6), 148, pl. 12, f. 8

1927 Lima quantoensis YoкоуамA, JCSIUT, 39 (6) 150, pl. 12, f. 11a-c.

1959 Lima zushiensis: KIRA, CISJ, 1, 129, pl. 52, f. 8

1971 Lima zushiensis: KURODA \& HABE, SSSB, 581 (J) 370 (E), pl. 82, f. 1

Loc. M S (s) gl.

Dist. Honshu (north to Bōsō Peninsula) to Kyushu.

Lima vulgaris LINK ミノガイ (丹浦)

1807 Limaria vulgaris LINK, BNSUR, 157

1863 Lima sowerbyi Deshayes, Reunion, 30

1872 Lima squamosa: SOWERBY, Icon., sp. 10

1943 Lima sowerbyi nipponica OYAMA, CA., 1, 55, pl. 11, f. 2

1959 Lima sowerbyi: KIRA, CISJ, 1, 129, pl. 52, f. 9

1971 Lima sowerbyi nipponica: KURODA \& HABE, SSSB, 581(J) 371(E), pl. 83, f. 17, 18

Loc. N M S (r) gl.

Dist. Honshu (north to Bōsō Peninsula) to Kyushu. Widely ranging in the Indo Pacific. 
Lima vulgatula YoKoYAMA ロウイロミノガイ (大山)

1922 Lima vulgatula YoKоYAMA, JCSIUT, 44 (1), 179, pl. 17, f. 18, 19.

1943 Lima(Lima) fujitai OYAMA, CA, 61, pl. 12, f. 7

1959 Lima fujitai: KIRA, CISJ, 1, 128, pl. 52, f. 1

1971 Lima fujitai: Kuroda \& HABE, SSSB, 582(J) 371(E), pl. 83, f. 12

Loc. M (g) gl.

Dist. Honshu (north to Bōsō Peninsula) to Kyushu.

Lima tomlini PRASHAD マルミノガイ(大山)

1932 Lima tomlini Prashad, Siboga, 53c, 123, pl. 3, f. 29-31

1943 Lima tomlini: OуAMA, CA, 60, pl. 12, f. 9, 10 (not of 8)

1976 Lima tomlini: MASAHITO \& HABE, BNSM, (A) 2 (2), 88, f, 7, 8

Loc. $S$ (s) tr.

Dist. Honshu (north to Kii Peninsula) to Kyushu, Philippines, Indonesia and Arafura Sea.

Genus Acesta H. \& A. ADAMS オオ八ネガイ属

1858 Acesta H. \& A. AdAms, GRM, 2, 558. Type (M): Ostrea excavata FABricius

Acesta goliath (SOWERBY) オオハネガイ（岩川)

1883 Lima goliath SowERBY, PZSL, 30, pl. 7, f. 3

1943 Lima (Acesta) goliath: OYAMA, CA, 1, 39, pl. 3, f. la, b; pl. 14, f. 8

1959 Acesta goliath: KIRA, CISJ, 1, 129, pl. 52, f. 10

1971 Acesta (Acesta) goliath: KURODA \& HABE, SSSB, 583(J) 372(E), pl. 82, f. 2

Loc. M (g) gl.

Dist. Honshu (south to Kii Peninsula), southern Hokkaido and Japan Sea.

Acesta marissinica YAMASHITA \& HABE スダレオオ八ネガイ(山下・波部)

1969 Acesta (Callolima) marissinica YAMASHTA \& HABE, Venus, 27 (4), 133, pl. 8

Loc. (uncon firm)

Dist. Honshu (north to Kii Peninsula), East and South China Seas.

*Acesta citrina MASAHITO \& HABE ミカンオオ八ネガイ（正仁・波部）

1976 Acesta citrina MASAHITO \& HABE, BNSM, (A) 2 (2), 87, f. 1, 2

Loc. $\mathrm{S}$ (s) tr.

Dist. Honshu (Sagami Bay to Kii Peninsula).

\section{Subgenus Plicacesta VoKESスミス八ネガイ亜属}

1963 Plicacesta Vokes, Tulane Studies Geol., 1, 90. Type (OD): Lima smithi Sowerby

Acesta (Plicacesta) smithi (SowERBY) スミスハネガイ（黒田）

1888 Lima smithi, PZSL, 207, pl. 11, f. 12

1943 Acesta smithi: Oyama, CA, 1, 45, pl. 4, f. 3a, b; pl. 14, f. 11

1959 Acesta smithi: KIRA, CISJ, 1, 129, pl. 52, f. 7

1971 Acesta (Plicacesta) smithi: Kuroda \& HABE, SSSB, 583(J) 372(E), pl. 83, f. 3

Loc. S (s-m) tr.

Dist. Honshu (south to Kii Peninsula) and Japan Sea.

\section{Genus Ctenoides MöRCHハネガイ属}

1853 Ctenoides Mörch, Yoldi, 2, 56. Type (SD, STOLICZKA, 1871): Lima scabra BoRN 
Ctenoides ales (FINLEY) ウコンハネガイ（黒田）

1889 Lima alata HEDLey (non Roemer, 1836), RAM, 3, 84

1927 Lima ales FINLEY, TNZI, 57, 527

1943 Ctenoides ales: OYAMA, CA, 1, 66, pl. 13, f. 15; pl. 14, f. 18

1961 Ctenoides ales: HABE, CISJ, 2, 119, pl. 54, f. 10

Loc.

Dist. Bonin Islands and Honshu (north to Kii Peninsula) and Ryukyu Islands.

Widely ranging in the tropic Pacific.

Ctenoides annulata (LAMARCK) ミダレハネガイ（黒田）

1819 Lima anuulata LAMARCK, AsV, 6 (1), 157

1872 Lima annulata Sowerby, Icon, sp. 7

1943 Ctenoides annulata: OyAmA, CA, 1, 63, pl. 13, f. 1; pl. 14, f. 16

1961 Ctenoides annulatus(sic): Kira, CISJ, 1, 129, pl. 52, f. 5

Loc. S (st) gl.

Dist. Honshu (north to Kii Peninsula) to Ryukyu Islands. Widely ranging in the tropic Pacific.

Ctenoides lischkei (LAMY) ハネガイ（目八)

1877 Lima japonica DUNKer, M B L, 24, 70 (non Limatula japonica A. AdAMS, 1972)

1882 Lima japonica: Dunker, Index, 245, pl. 11, f. 8, 9

1885 Lima dunkeri SMITH, Challenger, 13, 291 (non HAGenow, 1842)

1930 Lima lischkei Lamy, J. de C., 74, 196

1959 Ctenoides japonica: KIRA CISJ, 1, 129, pl. 52, f. 6

Loc. N M S (st) gl.

Dist. Honshu (north to Bōsō Peninsula) to Kyushu. Indo Pacific.

Ctenoides suavis MASAHITO, KuRODA \& HABEテンニン八ネガイ（黒田）

1971 Ctenoides suavis MaSAHITo, Kuroda \& HABE, SSSB, 585(J) 374(E), pl. 83, f. 7, 8.

Loc. M S (st) gl.

Dist. Honshu (north to Sagami Bay) to Kyushu.

Ctenoides concentrica (SOWERBY) オオシマハネガイ (平瀬)

1888 Lima concentrica SoWerby, PZSL, 207, pl. 11, f. 20.

1914 Lima oshimensis Sowerby, AMNH, (8) 14, 38, pl. 2, f. 10.

1961 Ctenoides concentrica HABE, CISJ, 2., 119, pl. 54, f. 11.

Loc. M S (st) gl.

Dist. Honshu (north to Kii Peninsula) to Ryukyu Islands and Formosa.

\section{Genus Divarilima PoWELLタキハネガイ属}

1958 Divarilima Powell, Rec. Auck. Inst. Mus., 5, 72. Type (OD): Lima sydneyensis HedleY

Divarilima iwaotakii (HABE) タキハネガイ（波部） Pl. 5, f. 5

1961 Acesta iwaotakii Habe, Venus, 21, 419, 429, f. 3, 4

1971 Divarilima iwaotakii: Kuroda \& HABE, SSSB, 584(J) 373(E), pl. 83, f. 16

Loc. $\mathrm{S}(\mathrm{s}) \mathrm{dp}$.

Dist. Honshu (north to Bōsō Peninsula) to Kyushu.

Genus Limaria LrNK ユキミノガイ属

1807 Limaria LiNk, BNSUR, 3, 157 
1939 Promantellum IREDALE, GBRESR, 5(6), Moll. 1, 38. Type (SD, WINCKworTH, 1930): Limaria inflata LINK

Limaria basilanica (A. ADAMS \& REEVE) ユキミノガイ（目八); オオユキミノガイ（黒田）

1850 Lima basilanica A. AdAMs \& ReEve, Samarang, 75, p1. 21, f. 6

1850 Lima orientalis A. AdAMs \& ReEve, Samarang, 75, pl. 21, f. 7

1932 Lima(Lima) orientalis: Prashad, Siboga, 53c, 125, pl. 2, f. 3233

1943 Mantellum (Promantellum) basilanica: Oyama, CA, 1, 28, pl. 2, f. 3a, b; pl. 14, f. 3

1971 Limaria (Limaria) basilanica orientalis: KURODA \& HABE, SSSB, 587(J) 375(E), pl. 118, f. 2

Loc. N M S (g) dp.

Dist. Honshu (north to Bōsō Peninsula) to Ryukyu Islands. Widely ranging in the Indo-Pacific.

\section{Limaria orientalis (ADAMS \& REEVE) フクレユキミノガイ(波部)}

1850 Lima orientalis ADAMS \& REEVE, Samarang, 75, p1. 21, f. 7

1906 Lima hakodatensis ToKunAGA, JCSIUT, 21(2), 64, pl. 3, 27, a, b.

1961 Mantellum hakodatense: HABE, CISJ, 2, 119, pl. 54, f. 7

1971 Limaria(Limaria) hakodatensis: Kuroda \& HABE, SSSB, 586(J) 375(E), pl. 83, f. 13

Loc. M S (s) gl.

Dist. Southern Hokkaido to Kyushu, Korea and China. Western Pacific.

Limaria kawamurai MASAHITO \& HABEカワムラユキミノガイ(正仁・波部)

1972 Limaria (Limatulella) kawamurai MASAHITO \& HABE, Venus, 31 (1), 1, f. 1, 2

Loc. M S (s) gl.

Dist. Honshu (north to Enshu nada) to Shikoku.

\section{Subgenus Platilimaria HABEウスユキミノガイ亜属}

1977 Platilimaria HABE, SMJ B/S. 103. Type (OD): Lima hirasei PILSBRy

Limaria (Platilimaria) hirasei (PILSBRY) ウスユキミノガイ（岩川)

1901 Lima hirasei Pilsbry, PANSP, 52, 209, 402, pl. . 19, f. 12 (as Lima hians hirasei)

1919 Lima (Mantellum) dihiscens hirasei THIELE, Cab., 7 (2a), 32, pl. 5, f. 4

1943 Lima (Promantellum) hirasei: Oyama, CA, 1, 31, pl. 2, f. 5a, b; pl. 14, f. 3

1959 Promantellum hirasei: KIRA, CISJ, 1, 128, pl. 52, f. 4

1971 Limaria (Limaria) hirasei: KuROda \& HABE, SSSB, 586 (J) 374 (E), pl. 83, f. 9

Loc. N M S (s) dp.

Dist. Honshu (north to Bōsō Peninsula) to Kyushu.

Limaria (Platilimaria) fragilis (GMELIN)ヒラユキミノガイ(大山);

1791 Ostrea fragilis GMELIN, Syst. Nat, 13, 3332

エボシユキミノガイ（大山）

1843 Lima fragilis SOWERBY, Thes., 1, 86, pl. 22, f. 34-37

1843 Lima (Promantellum) kiiensis OYAMA: CA, 1, 34, pl. 2, f. 8a, b, pl. 14, f. 5

1959 Promantellum kiiense: KIRA, CISJ, 1, 128, pl. 52, f. 3

Loc. M S (s) dp.

Dist. Honshu (Kii Peninsula) to Kyushu. Tropic Pacific.

Subgenus Limatulella SACCo アマクサユキミノガイ巠属

1889 Limatulella SACCO, Moll. Terr. Terz. Piemonte e Liguria, 26. Type (OD): Lima loscombii SOWERBY 
Limaria (Limatulella) amakusaensis (HABE) アマクサユキミノガイ（波部）

1960 Mantellum amakusaense HABE, PSMBL, 8, 281

1961 Mantellum amakusaense: HABE, CISJ, 2, 119, pl. 54, f. 8

1971 Limaria (Limatulella) amakusaensis: KuRODA \& HABE, SSSB, 587 (J) 375 (E), pl. 83, f.15

Loc. $\mathrm{S}$ (s) dp.

Dist. Honshu (north to Sagami Bay) to Kyushu.

Genus Isolima IREDALE シラスナユキミノガイ属

1929 Isolima Iredale RAM 17, 116. Type (OD): Lima parvula Verco

Isolima limopsis (NomURA \& JINBO, 1934)シラスナユキミノガイ（大山）Ｐl. 5, f.6

1934 Lima (Limea) limopsis NomURA \& JiNBo, SRTIU., (2) 16, 154, pl. 5, f. 11a-b, 12a-b.

1976 Isolima limopsis MASAHITO \& HABE, BNSM, 2 (2), 88, f. 4, 5.

Loc. S (s) dp.

Dist. Honshu (north to Kii Peninsula) to Ryukyu Islands.

\section{Genus Limatula WooDユキバネガイ属}

1839 Limatula WoOD, AMNH, 3, 235. Type (SD, GraY, 1847): Pecten subauriculatus MONTAGU

Subgenus Limatula WoOD ユキバネガイ亜属

Limatula (Limatula) vladivostokensis (SCARLATO) ヒメユキバネガイ（黒田）

1855 Lima vladivostokensis SCARLATo, Atlas Invert. Par. East. Seas, 191, pl. 51, f. 4

1964 Limatula vladivostokensis: HABE, CISJ, 2, 119, pl. 54, f. 1

1971 Limatula (Limatula) vladiovostokensis: KURODA \& HABE, SSSB, 588 (J) 376 (E), pl. 83, f. 14

Loc. M S (s) dp.

Dist. Honshu (south to Kii Peninsula), Hokkaido, Saghalin and Japan Sea.

Limatula (Limatula) kurodai OYAMA クロダユキバネガイ（大山）

1943 Limatula kurodai OxamA, CA, 1, 21, pl. 1, f. 10; pl. 14, f. 1

1964 Limatula kurodai: HABE, CISJ, 2, 175, pl. 54, f. 6

1971 Limatula (Limatula) kurodai: KURODA \& HABE, SSSB, 583 (J) 376 (E), pl. 118, f. 3

Loc. M S (s) dp.

Dist. Honshu (north to Kashima nada) to Kyushu.

*Limatula (Limatula) nippona HABEコナユキバネガイ（波部)

1960 Limatula nippona; HABE, PSMBL, 8 (2), 282

1961 Limatula nippona; HABE, CISJ, 2, 119, pl. 54

Loc. $\mathrm{M}$ (s) gl.

Dist. Honshu (Kii Peninsula) to Amami Islands.

Subgenus Limatuletta FEMING ニッポンユキバネガイ巠属

1973 Limatuletta Fleming, Jour. Roy. Soc. N. Z., 8 (1), 73. Type (OD): Limatula japonica A. ADAMS

Limatula (Limatuletta) japonica A. ADAMS ニッポンユキバネガイ（黒田）

1863 Limatula japonica A. AdAms, PZSL, 509

1872 Lima japonica SOWERBY, Icon., sp. 21 
1943 Limatula (Limatula) japonica: OYAMA, CA, 1, 15, pl. 1, f. 7a, b, 8a, b. 1961 Limatula (Stabilima) japonica: HABE, CISJ, 2, 119, pl. 54, f. 3

Loc. M S (s) dp.

Dist. Southern Hokkaido to Kyushu.

\section{Subgenus Stabilima IREDALE，才オユキバホガイ更属}

1939 Stabilima IredAle, GBRESR, 5 (6), Moll. 1, 390. Type (OD): Stabilima tadena IredAlE

Limatula (Stabilima) bullata (BoRN)オオユキバネガイ（黒田）

1778 Lima bullata BorN, Mus Caes Vind,, 110, pl. 6, f. 8.

1943 Limatula (Limatula) strangei: OYAMA, CA, 1, 16, pl. 1, f. 9a, b, 10a, b. (non Sowerby, 1872)

1961 Limatula (Stabilima) strangei: HABE, CISJ, 2, 119, pl. 54, f. 4

1971 Limatula (Stabilima) strangei KuROdA \& HABE, SSSS, 589 (J), 377 (E), pl. 83, f. 10

Loc. M S (s) gl.

Dist. Honshu (north to Bōsō Peninsula) to Ryukyu Islands. Widely ranging in the Pacific region.

\section{Genus Notolimea IREDALEイイ゙ユキバネガイ属}

1924 Notolima IreDALE, PLSNSW, 49, 181. Type (OD): Lima australis SMITH

Notolima tosana (OYAMA) イボユキバネガイ（黒田）

1943 Limatula (Notolima) tosana Oyama, CA, 1, 11, pl. 1, f. 1a, b.

Loc. $\mathrm{S}$ (g) dp.

Dist. Honshu (Kii Peninsula), Shikoku, south to Yakushima.

Superfamily Anomiacea RAFINESQUE ナミマガシワガイ超科

$$
\begin{array}{cl}
\text { Family Anomiidae RAFINESQUE } & \text { ナミマガシワガイ科 } \\
\text { Subfamily Anomiinae RAFINESQUE } & \text { ナミマガシワガイ刺科 }
\end{array}
$$

Genus Anomia LinnaEUs ・ミマガシワガイ属

1758 Anomia Linnaeus Syst. Nat., 10, 700. Type (SD, Schmidt, 1818): Anomia ephippium LINNAUES

Anomia chinensis PHILIPPI ナミマガシワガイ（目八）

1849 Anomia chinensis PHILIPPI, ZfM, 5 (9) (1848), 130

1850 Anomia cytaeum GrAY, PZSL, 17 (197) (1849), 115

1907 Anomia lischkei DaUtZenberg \& FISCher, J. de C., 54 (1906), 210, pl. 5, f. 8-11

1920 Anomia nipponensis YoKoYAMA, JCSIUT, 39 (6), 146, pl. 11, f. 18

1929 Anomia nipponensis obsoletocostata GrabAU \& KING, Peitaiho, 165, pl. 2, f. 16

1928 Anomia cuticula Grabau \& KING, Peitaiho, 166, pl. 2, f. 17

1971 Anomia chinensis: Kuroda \& HaBe, SSSB, 590 (J) 378 (E), pl. 84, f. 12-14

Loc. N M S (r) gl.

Dist. Southern Hokkaido to South East Asia. 


\section{Subfamily Placunanomiinae BEU ナミマガシワガイモドキ亜科}

Genus Monia GRAY ナミマガシワガイモドキ属

1850 Monia Gray, PZSL, (1849), 121. Type (SD, Kobel, 1881): Anomia zelandica Gray in Dieffenbach

Monia umbonata (GouLD)シマナミマガシワガイモドキ（平瀨）

1861 Placunanomia umbonata GouLD, PBSNH, 8, 39

1961 Anomia radulina A. AdAMs, AMNH, (3) 8, 142

1861 Anomia pustulata A. AdAMs. AMNH, (3) 8, 142

1914 Placunanomia radiata SowERBY, AMNH, (8) 14(79), 38, pl. 2, f. 16

1922 Anomia sematana YoкоYAMA, JCSIUT, 44 (1), 177, pl. 14, f. 20, 21

1922 Anomia lunata Yokoyama, JCSIUT, 44 (1), 177, pl. 14, f. 22, 23

1971 Monia umbonata: KUroda \& HABE, SSSB, 591 (J) 378 (E), pl. 84, f. 15-17

Loc. N M S (g) gl.

Dist. Southern Hokkaido to Kyushu. Korea and China.

\section{Suborder Ostreina RAFINESQUEカキ亚目}

$$
\begin{array}{cc}
\begin{array}{c}
\text { Superfamily Ostreacea RAFINESQUE } \\
\text { Family Glyphaeidae VYALOV }
\end{array} & \text { ガッコ超科 } \\
\text { ベッガキ科 } \\
\text { Subfamily Pycnodonteinae STENZEL } & \text { ベッコウガキ互科 } \\
\text { Genus Neopycnodonte STENZEL } & \text { ベッコウガキ属 }
\end{array}
$$

1971: Neopycnodonte STENzel, Treat. Invert. Paleont., Moll. 6 (3-3), 1109. Type (OD): Ostrea cochlear PoLI

Neopycnodonte musashiana (YOKOYAMA) ベッコウガキ（目八）

1920 Ostrea musashiana YoкоуамA, JCSIUT, 39(6), 163, pl. 16, f. 1-5

1930 Ostrea hiranoi BAKER \& SPICER, San Diego, 6 (6), 175, pl. 18, f. 1-3

1961 Notostrea musashiana: HABE, CISJ, 2, 120, pl. 54, f. 16

1971 Pycnodonte musashiana: KuRODA \& HABE, SSSB, 592 (J) 379 (E), pl. 85, f. 1, 2.

Loc. M S (st) gl.

Dist. Southern Hokkaido to East China Sea.

*Neopycnodonte setoensis HABE チャワンガキ（波部）

1958 Ostrea sedea setoensis HABE, Venus, 19 (3-4), 178, 180

1977 Ostrea sedea setoensis: HABE, Taxa HABE, 110, pl. 1, f. 9

Loc. S (r) dp.

Dist. Honshu (Wakayama Pref.).

Remarks: This species seems to be a form of Neopycnodonte musashiana (YOKOYAMA).

\section{Genus Hyotissa STENZEL シャコガキ属}

1971 Hyotiss a Stenzel, Treat. Invert. Paleont., Moll. 6 (3-3), 1107. Type (OD): Mytilus hyotis LINNAEUS . 
Hyotissa hyotis (LINNAEUs)シャコガキ(岩川)

1758 Mytilus hyotis Linnaeus, Syst. Nat., 10, 704

1971 Ostrea sinensis GmeLin, Syst. Nat., 13, 3335

1870 Ostrea sinensis: SOWERBY, Icon., sp. 5

1870 Ostrea hyotis: SOWERBY, Icon., sp. 7

1959 Dendostrea hyotis: KIRA, CISJ, 1, 127, pl. 51, f. 2

Loc. S (r) gl.

Dist. Honshu (Kii Peninsula) to Ryukyu Islands. Indo-Pacific.

Genus Pretostrea IREDALEカキツバタガキ属

1939 Pretostrea IREDALE, GBRESR, 5 (6), Moll. 1, 397. Type (OD): Pretostrea bresia IREDALE

Pretostrea imbricata (LAMARCK)カカキツバタガキ（丹浦）

1819 Ostrea imbricata LAMARCK, AsV, 6 (1), 213

1871 Ostrea imbricata: SOWERBY, Icon., sp. 47

1961 Dendostrea imbricata: HABE, CISJ, 2, 120, pl. 54, f. 17

1971 Pretostrea imbricata: KuRODA \& HABE, SSSB, 593 (J) 380 (E), pl. 85, f. 8

Loc. N M S (st) gl.

Dist. Honshu (north to Bōsō Peninsula) to Kyushu. Western Pacific.

Pretostrea chemnitzii (HANLEY) ベニガキ（黒田）

1846 Ostrea chemnitzii HANLEY, PZSL (1845), 106

1871 Ostrea chemnitzii: SOWERBY, Icon., sp. 47

1971 Saxostrea chemnitzii: KuRoDA \& HABE, SSSB, 594 (J) 380 (E), p1. 85, f. 3-5

Loc. M S (r) gl.

Dist. Honshu (north to Bōsō Peninsula) to Ryukyu Islands. Tropic and western Pacific.

Pretostrea rosacea (DESHAYES) カノコガキ（平攧）

1836 Ostrea rosacea Deshayes, AsV, (2) 7, 236

1871 Ostrea rosacea: Sowerby, Icon., sp. 46

Loc. M S (st) gl.

Dist. Honshu (Sagami Bay) to Kyushu. Tropic Pacific.

$$
\begin{aligned}
\text { Family Ostreidae RAFINESQUE } & \text { イタボガキ科 } \\
\text { Subfamily Ostreinae RAFINESQUE } & \text { イタボガキ亜科 } \\
\text { Genus Crassostrea SACCO } & \text { マガキ属 }
\end{aligned}
$$

1897 Crassostrea SACCo, Moll Terr. Terz Piemonte e Liguria, 23, 15 (ICZN Op. 338)

1886 Gryphaea FisHer, M. de C., 927 (non LAMARCK, 1801)

1928 Dioeciostrea ORTON, Nature, 121 (3044), 320. Type (OD): Ostrea virginica GMELIN

\section{Crassostrea gigas (THUNBERG) マガキ（方言）}

1793 Ostrea gigas ThunBerg, Kungl. Vet. Acad. Nya. Handl., 14, 140

1861 Ostrea laperousii SchrencK, St. Petersburg, 4, 411

1862 Ostrea talienwhanensis Crosse, J. de C., 10, 149, pl. 6, f. 6

1959 Ostrea (Crassostrea) gigas: KIRA. CISJ, 1, 127, pl. 51, f. $3,8$.

Loc. N M S (r) int.

Dist. Saghalien to Formosa. Western Pacific. 
Crassostrea ariakensis (WAKIYA) スミノェガキ（藤田）

1869 Ostrea rivularis: LISCHKE, JMC, 1, 176, pl. 14, f. 2,3 (non Gould, 1861)

1929 Ostrea ariakensis WAKIYA, JJZ, 2, 363, pl. 9, f, 1, 2

1959 Ostrea (Crassostrea) rivularis: KIRA, CISJ, 1, 127, pl. 51, f. 6

Loc. M (g) gl.

Dist. Honshu (Kii Peninsula), Inland Sea of Japan and Ariake Bay, Kyushu.

Crassostrea nippona (SEKI) イワガキ (平瀬)

1929 Ostrea multistriata WAKIYA, JJZ, 2 (3), 365, pl. 10, f. 1, 2. (non HANLEY, 1846)

1930 Ostrea circumpicta S. HIRASE, JJZ, 3 (1), 42, tf. 56, 57, (non PIISBRY, 1904)

1934 Ostrea nippona SEKI, Venus, 4 (5), 276, tf. 1-5,

1959 Ostrea (Crassostrea) nippona: KIRA, CISJ. 1, 127, pl. 51, f. 5

1965 Crassostrea nippona: HABE, NIEFJ, 2, 245, no. 920

Loc. S (r) int.

Dist. Honshu (north to Mutsu Bay) to Kyushu.

Genus Saccostrea Dollfus \& DAUTZENBERG ，オ八グロガキ属

1920 Saccostrea Dollfus \& DAUTZENBERG, MSGF, 27, 471

1936 Saxostrea IreDAle, RAM, 19, 269. Type (M): Ostrea cucullata BorN

Saccostrea mordax (GouLD) オハグロガキ（黒田）

1850 Ostrea mordax GouLD, PBSNH, 3, 346

1871 Ostrea mordax: SowERBY, Icon., sp. 31

1930 Ostrea (Lopha) mordax: Kuroda, Venus, 2 (3), App. 51, f. 5

1939 Saxostrea amasa IREDALE, GBRESR, 5 (6), Moll. 1, 399, pl. 7, f. 8

1959 Saxostrea mordax: KIRA, CISJ, 1, 127, pl. 51, f. 4

Loc. M S (r) int.

Dist. Honshu (Kii Peninsula) to Ryukyu Islands. Tropic Pacific.

Saccostrea echinata (QuOY \& GAIMARD) ケガキ（目八）

1836 Ostrea echinata Quoy \& Gaimard, Astrolabe, 3, 455, pl. 76, f. $13,14$.

1836 Ostrea spinosa Deshayes, AsV, (2) 7, 237

1871 Ostrea spinosa: SowerBy, Icon., sp. 79

1961 Saxostrea echinata: HABE, CISJ, 2, 120, pl. 54, f. 13

1971 Saxostrea echinata: KurodA \& HABE, SSSB, 594 (J) 380 (E), pl. 85, f. 6, 7.

Loc. N M S (r) int.

Dist. Southern Hokkaido to Kyushu. Western Pacific.

\section{Genus Ostrea LinNAEUS イタボガキ属}

1758 Ostrea Linnafus, Syst. Nat., 10, 696. Type (SD, ICZN Op. 94): Ostrea edulis LinNaeus

Ostrea denselamellosa LischKE１タボガキ（目八）（渚丹）

1869 Ostrea denselamellosa LISCHKE, M B L, 16, 106

1869 Ostrea denselamellosa: LISCHKE, JMC, 1, 177, pl. 13, 14.

1871 Ostrea auriculata SowERBY, Icon., sp. 60

1871 Ostrea multicostata SOWERBY, Icon., sp. 61

1959 Ostrea denselamellosa: KIRA, CISJ, 1, 127, pl. 51

1971 Ostrea denselamellosa: KuRODA \& HABE, SSSB, 595 (J) 381 (E), pl. 85, f. 2, 3.

Loc. N M S (g) gl.

Dist. Honshu (north to Tokyo Bay) to East China Sea. 
Ostrea futamiensis SEKIクロヒメガキ（関）

1929 Ostrea futamiensis Sekr, Proc. Imp. Acad., 5 (10), 477, tf.

1930 Ostrea futamiensis: SEKI, Venus, 2 (1), 10, f. 1-13

Loc. $\mathbf{N}$ (g) int.

Dist. Honshu (north to Tokyo Bay) to Kyushu.

Ostrea circumpicta PILSBRY コケゴロモガキ（六介）

1904 Ostrea circumpicta PILSBRY, PANSP, 56, 559, pl. 11, f. 12, 13

1971 Ostrea circumpicta: KuRodA \& HABE, SSSB, 595 (J) 381 (E), pl. 84, f. 11

Loc. S (r) int.

Dist. Honshu (north to Mutsu Bay) to Kyushu, Korea and China.

\section{Genus Dendostrea SWAINSONワニガキ属}

1835 Dendostrea Swainson, Elem., Mod. Conch., 39

1839 Dendostraea Sowerby, Conch. Man., 1, 38, 75, 122

1840 Dendrostrea Swainson, Treat., 389. Type (SD, Herrmannsen, 1847): Ostrea folium LINNAEUS

Dendostrea folium (LinNAEUs)ワワガイ (介志)：ワニガキ（平瀬）

1758 Ostrea folium LinNaeus, Syst. Nat., 10, 699

1870 Ostrea folium: SOWERBY, Icon., sp. 40

1961 Dendostrea folium: HABE, CISJ, 2, 120, pl. 54, f. 14

Loc. N M S (r) gl.

Dist. Honshu (Kii Peninsula) to Formosa. Tropic and western Pacific.

Dendostrea crenulifera (SOWERBY) ノコギリガキ（黒田）

1871 Ostrea crenulifera Sowerby, Icon., sp. 67

1961 Dendostrea crenulifera: HABE, CISJ, 2, 120, pl. 54, f. 15

Loc. N M S (r) gl.

Dist. Honshu (north to Bōsō Peninsula) to Formosa. Tropic and western Pacific.

Dendostrea paulucciae (CROSSE)カモノアシガキ（黒田）

1870 Ostrea paulucciae Crosse, J. de C., 18, 108, pl. 2, f. 5

1871 Ostrea palmipes SOWERBY, Icon., sp. 56

1906 Ostrea irregularis ToKunAGA, JCSIUT, 21 (2), 68, pl. 4, f. 7a, b.

Loc. M (g) int.

Dist. Honshu (Kii Peninsula), Formosa and Philippines. 
Subclass Palaeoheterodonta NEWELL 古異菡巠綱

\author{
Order Unionoida STOLICZKA イシガイ日 \\ Superfamily Unionacea FLEMING イシガイ超科 \\ Family Unionidae FLEMING イシガイ科 \\ Subfamily Unioninae FLEMING イシガイ量科
}

Genus Unio Philipsson イシガイ属

1778 Unio PhILIPsson, Diss. Hist. Nat. Sist. Nova Test. Gen., 16 (ICZN, Op. 495)

1791 Limnaea PoLI, Test. Sicil, 1, Introd., 31

1815 Lymnium OKen, Lehrb. Nat., 3 (1), 236 invalid (ICZN, Op. 417, 495). Type (SD, TurTON, 1831): Mya pictorum LinNaEUs

\title{
Subgenus Nodularia CONRADイシガイ里属
}

1853 Nodularia CoNRAD, PANSP, 6, 267, 268

1901 Nodularidia Cockerell, Sciences, (N. S.) 13, 984. Type (OD): Unio douglasiae GrifFith \& PIDGEON

Unio (Nodularia) douglasiae GRIFFTth \& PIDGEON イシガイ（方言）

1834 Unio douglasiae Griffith \& PdDGron, Cuvier's Anim. Kingdom, 601, pl. 21, f. 2

1877 Unio nipponensis MARTENS, SBGNFB, 119

1879 Unio nipponensis: KoBelt, Senckenberg, 11, 422, pl. 12, f. 3

1931 Unio douglasiae nipponensis: KuRODA, Venus, 2 (5), App. 63, f. 70

1959 Lymnium douglasiae: KIRA, CISJ, 1, 171, pl. 63, f. 17

Loc. N M S (s).

Dist. Hokkaido to Kyushu, Korea and China.

\section{Genus Inversidens HAAS マツカサガイ属}

1911 Inversidens Cab., 9, 102.

1970 Pronodularia Starobogatov, Fauna Moll. Zoolgeogr. World, 66, 285. Type (OD): Unio brandti KoBeLT

Inversidens japanensis (LEA) マツカサガイ（目八）

1859 Unio japanensis LEA, PANSP, 3, 153 (1858)

1860 Unio japanensis: LEA, JANSP, 4, 244, pl. 36, f. 123

1879 Unio japanensis: KoBeLt, Senckenberg. 11, 423, pl. 12, f. 1, 2.

1893 Unio jokohamensis IHERING, Senckenberg, 18, 158.

1893 Unio haconensis IHERING, Senckenberg, 18, 161, f. 3

1911 Nodularia japanensis: HAAs, Cab., 9 (2), 85, pl. 8, f. 5-8

1911 Nodularia jokohamensis: HAAs, Cab., 9 (2), 87, pl. 8, f. 9-13

1911 Nodularia haconensis: HAAS, Cab., 9 (2), 89, pl. 9, f. 1-3

1931 Inversidens japanensis: KURODA, Venus, 2 (5), App. 64, f. 72

1959 Inversidens japanensis: KrRA, CISJ, 1, 171, pl. 63, f. 14

Loc. N M S (g).

Dist. Hokkaido to Kyushu. 


\section{Subfamily Anodontinae ORTMANN ドブガイ亜科 \\ Genus Anodonta LAMARCK ドブガイ属}

1799 Anodonta LAMARCK, MSHNP, 87 (ICZN, Op. 94, 561)

1886 Pteranodon Fisher, M. de C., 1003 (non MARsh, 1876). Type (Nom. Conser.): Mytilus cygneus LINNAEUs

\section{Subgenus Sinanodonta MODELLドブガイ亜属}

1945 Sinanodonta Modell, Jena Zeit. Med. Naturw. 78, p. 62. Type (OD): Symphynota woodiana LEA

Anodonta (Sinanodonta) woodiana (LEA) ドブガイ (通称)

1834 Symphynota woodiana LEA, Trans. Amer. Phil. Soc., 5, 42, pl. 5, f. 13

1870 Anodonta woodiana: SowerBy, Icon., sp. 149

1874 Anodonta japonica Clessin, Cab. 9 (1), 144, pl. 47, f. 3, 4

1875 Cristaria reiniana MARTENS, JDMG, 2, 136, pl. 3, f. 4

1877 Anodonta lauta MARTENs, SBGNFB, 117

1879 Dipsas reiniana: KoвELT, Senskenberg, 11, 432, pl. 12, f. 4; pl. 21, f. 2

1879 Anodonta lauta: Koвelt, Senckenberg, 11, 434, pl. 21, f. 1

1879 Anodonta japonica: KoBeLt, Senckenberg, 11, 436, pl. 22, f. 3

1879 Anodonta cellensis KoBelt, Senckenberg, 11, 435, pl. 22, f. 4 (non PfeIfFer, 1821)

1879 Anodonta kobelti IHERING, Senckenberg, 18, 152, f. 1

1893 Anodonta haconensis IHERING, Senckenberg, 18, 153, f. 3

1910 Anodontites lautus timens HAAS, AMNH, (8) 6, 499

1959 Anodonta lauta: KIRA, CISJ, 1, 171, pl. 63, f. 16

1959 Anodonta japonica: KIRA, CISJ, 1, 172, pl. 63, f. 19

Loc. N M S (m).

Dist. Hokkaido to Kyushu. Korea and China.

\section{Subclass Heterodonta NEUMAYR 異崡西綱 \\ Order Veneroida H. \& A.ADAMS マルスタレガイ目 \\ Superfamily Lucinacea Fleming ッキガイ超科

$\begin{array}{cc}\text { Family Lucinidae Fleming } & \text { ッキガイ科 } \\ \text { Subfamily Lucininae Fleming } & \text { ッキガイ两科 } \\ \text { Genus Codakia SCOPOLI } & \text { ッキガイ属 }\end{array}$

1777 Codakia ScopolI, Introd, 398

1817 Lentillaria ScHumacher, ENSVT, 49, 147

1930 Pexocodakia Iredale, RAM, 17, 389. Type (M): Venus orbicularis Linnaeus,

Codakia tigerina (LinNAEUs) ツキガイ (平瀬)

1758 Venus tigerina LinNaEus, Syst. Nat., 10, 688

1850 Lucina exasperata REEVE, Icon., sp. 4

1951 Codakia tigerina: HABE, GJS, 2, 130, f. 279, 180

1959 Codakia tigerina: KIRA, CISJ, 1, 133, pl. 53, f. 5

Loc. S (s) dp.

Dist. Honshu (Kii Peninsula) to Ryukyu. Indo-Pacific. 
Codakia paytenorum (IREDALE) ウラキッキガイ (平瀬)

1850 Lucina interrupta ReEve, Icon., sp. 5 (non Lamarck, 1818)

1937 Lentillaria paytenorum IREDALE, AZ, 8 (4), 240, pl. 16, f. 2 , 3.

1959 Codakia paytenorum: KIRA, CISJ, 1, 133, pl. 53, f. 3

Loc. M S (s) dp.

Dist. Honshu (Kii Peninsula) to Ryukyu Islands. Indo-Pacific.

\section{Genus Epicodakia IREDALEヒメッキガイ属}

1930 Epicodakia Iredale, RAM, 17, 390. Type (OD): Epicodakia consettiana Iredale

Epicodakia bella (CONRAD) ウミアサガイ（岩川）

1837 Lucina bella CONRAD, JANSP, 7, 254, p1. 19, f. 11

1904 Ctena bella delicatula PILSBRy, PANSP, 56, 555, pl. 41, f. 15, 16

1961 Ctena delicatula: HABE, CISJ, 2, 125, pl. 56, f. 23

Loc. N M S (s) int.

Dist. Honshu (north to Bōsō Peninsula) to Ryukyu Islands. Pacific.

Epicodakia divergens (PHILIPPI) ヒメッキガイ (平瀬)

1850 Lucina divergens PhILrpr, Abbild., 3, 103, pl. 2, f. 4

1959 Ctena divergens: KIRA, CISJ, 1, 133, pl. 53, f. 1

Loc. $S$ (s) int.

Dist. Honshu (Kii Peninsula) to Ryukyu Islands. Indo-West Pacific.

\section{Genus Pillucina PILSBRYウメノハナガイ属}

1921 Pillucina Pilsbry, PANSP, 72, 381. Type (OD): Pillucina spaldingi PILSBRY

Subgenus Pillucina PILsBRy ウメノハナガイ巠属

Pillucina pisidium (DUNKER) ウメノハナガイ（目八）

1860 Lucina pisidium DUNKER, M B L, 6, 277

1861 Lucina pisidium: DuNKeR, MJ 28, pl. 3, f. 9

1861 Lucina (Codakia) parvula Goutd, PBSNH, 8, 227

1961 Pillucina pisidium: HABE, CISJ, 2, 125, pl. 56, f. 20

1964 Lucina (Codakia) parvula: Johnson, USNMB, 239, 122, pl. 28, f. 5

1971 Pillucina (Pillucina) pisidium: Kuroda \& HABE, SSSB( 612 (J) 393 (E), pl. 118, f. 14

Loc. S (s-m) int.

Dist. Southern Hokkaido to Formosa.

*Pillucina (Pillucina) neglecta HABEコボレウメハハナガイ（波部)

1960 Pillucina neglecta HABE, PSMBL, 8 (2), 282, f. 7-9

Loc. M S (s-m) dp.

Dist. Inland Sea of Japan and Honshu (Wakayama Pref.).

Subgenus Sydrolina IREDALEアラウメノハナガイ要属

1930 Sydrolina IreDALE, RAM, 17 (9), 390. Type (OD): Sydrolina symbolis IrEDALE

Pillucina (Sydrolina) yamakawai (YoKoYAMA) アラウメノハナガイ（波部)

1920 Lucina yamakawai YoKoxama, JCSIUT, 39 (6), 135, pl. 10, f. 9

1938 Ctena transversa DALL, BARTSCH \& REHDER, BPBMB, 153, 131, pl. 35, f. 9-12

1961 Pillucina (Sydrolina) yamakawai: HABE, CISJ, 2, 125, pl. 56, f. 22 
1971 Pillucina (Sydrolina) yamakawai: KuRodA \& HABE, SSSB, 611 (J) 393 (E), pl. 118, f. 16

Loc. M.S (s-m) dp.

Dist. Honshu (north to Bōsō Peninsula) to Ryukyu Islands.

Subgenus Wallucina IREDALEチデミゥメノハナガイ亜属

1930 Wallucina IrEDALE, RAM, 17 (9), 390. Type (OD): Lucina jacksoniensis SMITH

Pillucina (Wallucina) striata (ToKUNAGA) チデミウメノハナガイ（黒田）

1882 Lucina contraria DuNKer, Index, 215, pl. 13, f. 12-14 (non DUNKER, 1846),

1906 Lasaea striata ToKUNAGA, JCSIUT, 21 (2), 53, pl. 3, f. 14a, b.

1938 Loripes (Wallucina) lamyi CHAVAN, J. de C., 82 (3), 227

1961 Pillucina (Wallucina) lamyi: HABE, CISJ, 2, 125, pl. 56, f. 24

1971 Pillucina (Wallucina) striata: KunODA \& HABE, SSSB, 611 (J) 392 (E), pl. 118, f. 15

Loc. M S (s-m) dp.

Dist. Honshu (north to Bōsō Peninsula) to Kyushu.

\section{Genus Bellucina DALLムッキウメノハナガイ属}

1901 Bellucina Dall, PUSNM, 23, 806. Type (OD): Parvilucina eucosmia DalL

Bellucina civica (YoKOYAMA) ムッキウメノハナガイ（黒田） Pl. 5, f. 7.

1927 Cardium civica YoкоYамA, JFSIUT, (2) 2 (4), 179, pl. 43, f. 3, 4.

1932 Dentilucina (Bellucina) hedleyi Prashad, Siboga, 53c, 163, pl. 5, f. 17, 18, 19, 20 (var. quadrata PRASHAD)

1961 Bellucina civica: HABE, CISJ, 2, 125, pl. 56, f. 19

1971 Bellucina civica: Kuroda \& HABE, SSSB, 612 (J) 394 (E), pl. 118, f. 17, 18

Loc. $S$ (s) dp.

Dist. Honshu (north to Bōsō Peninsula) to Kyushu and Indonesia.

$$
\begin{gathered}
\text { Subfamily Myrteinae CHAVAN } \\
\text { Genus Notomyrtea IREDALE } ＼mathrm{~ ワ タ キ ゙ ィ ゙ ィ ッ ト ゙ キ 無 科 ~}
\end{gathered}
$$

1924 Notomyrtea Iredale, PLSNSW, 49, 182, 206. Type (OD): Myrtea botanica HedleY

Notomyrtea soyoae HABEワタゾコッキガイ（波部）

1951 Notomyrtea soyoae HABE, GJS, 2, 134, f. 281, 282 (name only)

1953 Notomyrtea soyoae: HABE, Venus, 17 (3), 131, f. 8, 9

1961 Notomyrtea soyoae: HABE, CISJ, 2, 125, pl. 56, f. 29

1971 Notomyrtea soyoae: Kuroda \& HABE, SSSB, 613 (J) 394 (E), pl. 87, f. 13

Loc. M S (s) dp.

Dist. Honshu (north to Bōsō Peninsula) to Kyushu.

\section{Genus Lucinoma DALL ッキガイモドキ属}

1901 Lucinoma Dall, PUSNM, 23, 806. Type (OD): Lucina filosa Stimpson

\section{Lucinoma annulatum (REEVE) ッキガイモドキ（鳥羽)}

1850 Lucina annulata REeVE, Icon., sp. 17

1920 Lucina borealis: YoKоYAMA, JCSIUT, 39 (6), 135, pl. 10, f. 7 (non LINNAEUs, 1758)

1920 Woodia concentrica YoKoYAMA, JCSIUT, 39 (6), 141, pl. 11, f. 7a, b, c.

1959 Lucinoma annulata: KIRA, CISJ, 1, 133, pl. 53, f. 2

1971 Lucinoma annulata: KuRODA \& HABE, SSSB, 609 (J) 391 (E), p1. 87, f. 16 
Loc. $S$ (s) tr.

Dist. Southern Hokkaido to East China Sea.

Lucinoma spectabile (YoKoYAMA)オオツキガイモドキ（滰・大山）Ｐl. 5, f. 10.

1920 Lucina spectabilis YokoYama, JCSIUT, 39 (6), 134, pl. 10, f. 10-12

1973 Lucinoma (acutilineata forma?) spectabile: OYAMA, PSJSP, 17, 96, pl. 40, f. 5, 9, 10

Loc. M S (s) tr.

Dist. Honshu to Kyushu.

Remarks: This seems to be merely a form of the preceding species.

Lucinoma adamsianum HABEアダムスッキガイモドキ（大山）

1958 Lucinoma adamsiana HABE, PSMBL, 7 (1), 28

1961 Lucinoma adamsiana HABE, CISJ, 2, 125, pl. 56, f. 28

Loc. $S$ (s) tr.

Dist. Honshu (Bōsō Peninsula to Kii Peninsula) and southern part of Japan Sea.

Lucinoma yoshidai HABEヨシダツキガイモドキ(波部)Ｐ1. 5, f. 9.

1958 Lucinoma yoshidai HABE, PSMBL, 7, 27.

1961 Lucinoma yoshidai HABE, CISJ, 2, 125, pl. 56, f. 26.

1971 Lucinoma yoshidai KuROdA \& Habe, SSSB 609 (J.), 391 (E.), pl. 87, f. 17.

Loc. S (s) tr.

Dist. Honshu (south to Kii Peninsula) and Japan Sea.

\section{Genus Gonimyrtea MARWICKキヌハダッキガイモドキ属}

1929 Gonimyrtea MARwICK, TNZI, 59, 904, 912. Type (OD): Loripes concinnus HuTTON= Loripes icterica Lamy (non ReEve, 1850)

\section{Subgenus Alucinoma HABEキヌハダツキガイモドキ亜属}

1958 Alucinoma HABE, Venus, 19 (3-4), 181. Type (OD): Alucinoma soyoae HABE

Gonimyrtea (Alucinoma) soyoae (HABE)キヌハダツキガイモドキ(波部)

1958 Alucinoma soyoae HABE, Venus, 19 (3-4), 178, 181, tf. 5, 6

1961 Alucinoma soyoae: HABE, CISJ, 2, 125, pl. 56, f, 21

Loc. M S (s-m) gl.

Dist. Honshu (north to Kii Peninsula), Shikoku and the southern part of Japan

Sea.

Subfamily Milthinae CHAVANカブラッキガイ亜䅸

Genus Anodontia LINKカブラッキガイ属

1807 Anodontia Link, BNSUR, (3), 214. Type (OD): Venus edentula LinNaeus

Anodontia edentula (LINNAEUs)カブラッキガイ（黑田）

1758 Venus edentula LinNaEus, Syst. Nat., 10, 689.

1850 Lucina ovum ReEVE, Icon., sp. 21.

1850 Lucina pila ReEve, Icon., sp. 24.

1966 Anodontia edentula HABE \& Kosuge, SWC., 2, 150, pl. 57, f. 8.

Loc. S (s-m).

Dist. Honshu (north to Kii Peninsula) to Ryukyu Islands. Indo-Pacific regions. 
Anodontia stearnsiana OYAMA イセシラガイ (群品)

1895 Loripes bialata PILSBRy, CMMJ, 133, pl. 3, f. 13, 14 (non Lucina bialata BeLLARd, 1854)

1927 Loripes philippiana YokoYAMA, JFSIUT, (2) 1 (10), 434, pl. 50, f. 1, 2

1954 Lucina stearnsiana OYAMA. PSJSP, 2, 52, pl. 47, f. 1, 2

1959 Lucina stearnsiana: KIRA, CISJ, 1, 134, pl. 53, f. 7

Loc. N M S (s) dp.

Dist. Honshu (north to Mutsu Bay) to Ryukyu Islands.

\section{Genus Cavatidens IREDALE コカブラガイ属}

1930 Cavatidens Iredale, RAM, 17 (9), 391. Type (OD): Cavatidens omissa Iredale

*Cavatidens imajimai HABE sp. nov. コカブラガイ（波部）

P1. 2, fig 4.

Shell rather thin, white subquadrate in shape, with the broadly truncated posterior margin and obliquely placed straight anterior margin, rather inflated. Umbo small, slightly beaked forwards. Lunule small but distinctly and deeply excavated. Surface covered with a pale yellowish periostracum and marked rather weak growth lines and some distinctly impressed resting folds. Hinge edutulous and has a long internal ligament on the posterior dorsal portion. Anterior muscle scar elongate, white and posterior muscle scar ovate in shape. Pallial line continuous without a pallial sinus.

Length $14.7 \mathrm{~mm}$, height $12.5 \mathrm{~mm}$, breadth $3.6 \mathrm{~mm}$. (Holotype left valve preserved in the National Science Museum, NSMT-Mo 56857)

Type locality: Kushimoto, Wakayama Pref., Honshu. M S (s) dp.

Remarks: This is a first record of the genus Cavatidens from Japan. Cavatidens omissa Iredale 1930 from New South Wales, Australia, the type species of this genus closely related to this new species has the rounded and well inflated shell. This interesting species is named in honor of Dr. Minoru Imajima of the National Science Museum of Tokyo, who brought it to writer. $\mathrm{He}$ is a specialist of polycheate taxonomy.

\section{Subfamily Divaricellinae GILBERT七ワケッキガイ亜科 \\ Genus Divaricella MARTENSセワケツキガイ属}

1880 Divaricella MarTens, Mauritius u. Seychellen, 321. Type (M): Divaricella anguilifera MARTENS

Divaricella soyoae (HABE)七ワケッキガイ（波部)

1951 Divalucina soyoae HABE, GJS, 2, 133, f. 292 (name and figure only)

1952 Divaricella (Divalucina) soyoae: HABE, CISJ, 2, 125, pl. 56, f. 25

Loc. $\mathrm{M}$ (s) tr.

Dist. Honshu (Wakayama Pref.), Shikoku and Kyushu.

Family Thyasiridae DALL ハナシガイ科

Genus Conchocele GABBオウナガイ属

1866 Conchocele GABB, Geol. Surv. California Palaeont., 2, 27. Type (OD): Conchocele disjuncta GABB 
Conchocele bisecta (CONRAD) オウナガイ（黒田）

1849 Thyasira bisecta ConRaD, Rep. U. S. Exp., 10, 724, pl. 17, f. 10

1925 Thyasira bisecta nipponica YABE \& NoMURA, SRTIU, (2) 7 (4), 84, pl. 23, f. 8-10

1968 Conchocele bisecta disjuncta: HABE \& ITo, SWC, 1, 130, pl. 43, f. 17

Loc. S (m) tr.

Dist. Shikoku to Hokkaido and Japan Sea. Trans-Pacific distribution to Japan (Shikoku) from N. America (Oregon).

*Conchocele koyamai HABE sp nov. スマキオウナガイ（波部）

P1. 2, fig. 5.

Shell medium in size measuring $21.00 \mathrm{~mm}$ in length, inaequilateral, elongated posteriorly, thin, translucently white, weakly 21 convex. Umbo placed and howked anteriorly, small but prominent. Anterior margin slightly curved, concave and dorsal margin rather straight. Posterior and ventral margins gently curved. Lunule just below the umbo, smooth and broad, and bordering by the weak ridge. Surface with two strong the postero-ventral margin folds on the postero-dorsal area and one fold from the umbo to the antero-ventral corner, and many grooved striations and two obtuse ridges on the broad central area. Interior with densely set striations. Anterior muscle scar elongated oval in shape and placed on the fold. Posterior muscle scar rather small, ovate in shape and placed on the posterior folded area. Ligament long and narrow along the dorsal margin. No cardinal teeth on the hinge plate.

Length $21.0 \mathrm{~mm}$, height $18.0 \mathrm{~mm}$ and breadth $5.3 \mathrm{~mm}$ (Holotype left valve preserved in the National Science Museum, NSMT-Mo 58903)

Type locality: Off Cape Shionomisaki, Kii Peninsula, Honshu. M S (s) dp

Remarks: Conchocele bisecta CoNRAD, 1849, a closely allied species to this new species in shape in the young stage, has the large shell attaining $5 \mathrm{~cm}$ in length and $5 \mathrm{~cm}$ in height in fully grown speciemen, the surface of which is smooth and chalky even in the young stage.

\section{Genus Thyasira LAMARCK ハナシガイ属}

1818 Thyasira Lamarck, AsV, 5, 492 (in Synonym)

1822 Cryptodon TuRTon, Conch. Insul. Brit., 32, 121

1884 Prothyasira Iredale, RAM, 17, 393. Type (M): Tellina flexuosa MonTAGU

\section{Subgenus Thyasira LAMARCKハナシガイ巠属}

Thyasira (Thyasira) tokunagai KURODA \& HABEハナシガイ（黒田）

1906 Cryptodon flexuosus: Tokunaga, JCSIUT, 21 (2) 52 (non Montagu, 1803)

1927 Thyasira gouldii: Yokoyama, JFSIUT, (2) 1 (10), 433, pl. 1, f. 9 (non PHLIPPI, 1845)

1951 Thyasira (Thyasira) tokunagai KuRODA \& HABE, GJS, 1, 127, f. 266-268

1961 Thyasira tokunagai: HABE, CISJ, 2, 124, pl. 56, f. 17

1971 Thyasira (Thyasira) tokunagai: KuRODA \& HABE, SSSB, 607 (J) 390 (E), pl. 118, f. 7, 8

Loc. S (s) dp.

Dist. Hokkaido to Kyushu.

Subgenus Philis FISHER オトギノハナシガイ亜属

1861 Philis Fisher, J. de C., 9, 345. Type (M): Philis cumingii Fisher 
Thyrasira (Philis) cumingii FISCHER オトギノハナシガイ（波部）

1861 Philis cumingii Fisher, J. de C., 9, 346, pl. 16, f. 8, 9,

1951 Thyasira (Philis) kanamarui HABE, GJS, 1, 126, f. 263-265

1971 Thyasira (Philis) kanamarui: KURODA \& HABE, SSSB, 608 (J) 390 (E), pl. 118, f. 10

Loc. S (s) dp.

Dist. Honshu (north to Bōsō Peninsula) to Kyushu and Indonesia.

\section{Subgenus Maorithyas FLEMINGウスハナシガイ巠属}

1950 Maorithyas Fleming, TNZI, 78, 253. Type (OD): Maorithyas marama Fleming

Thyasira (Maorithyas) miyadii HABEウスハナシガイ (波部) Pl. 6, f. 1.

1951 Thyasira (Maorithyas) miyadii HABE, GJS, 1, 127, f. 272

Loc. $S$ (s) tr.

Dist. Honshu (Kii Peninsula).

\section{Genus Parathyasira IREDALEカワムラハナシガイ属}

1924 Parathyasira Iredale, RAM, 17, (9), 392. Type (OD): Parathyasira resupina Iredale

Parathyasira kawamurai (HABE)カワムラハナシガイ（波部）

1951 Thyasira (Parathyasira) kawamurai HABE, GJS, 1, 127, f. 262

1961 Thyasira (Parathyasira) kawamurai: HABE, CISJ, 2, 124, pl. 56, f. 16

1971 Thyasira (Parathyasira) kawamurai: KuRODA \& HABE, SSSB, 607 (J) 390 (E), pl. 118, f. 9

Loc. S (s) tr.

Dist. Honshu (Bōsō Peninsula to Kii Peninsula).

Parathyasira hexangulata (OKUTANI) ロッカクハナシガイ（奥谷）

1962 Thyasira (Parathyasira) hexangulata OKUTANI, BTRFRL, 32, 24, pl. 4, f. 7

Loc. $\mathrm{S}$ (s) tr.

Dist. Honshu (Sagami Bay to Kii Peninsula) to Kyushu.

\section{Genus Leptaxinus VERRILL \& BUSH マルハナシガイ属}

1898 Leptaxinus Verrill \& BUSH, PUSNM, 20, 796. Type (OD): Leptaxinus minutus Verrill $\&$ BusH

\section{Leptaxinus oyamai HABE マルハナシガイ（大山）}

1925 Thyasira trigonata YABE \& NOMURA, SRTU, (2) 7 (4), 94, pl. 23, f. 4a, b. (non YokoYAma, 1922)

1961 Leptaxinus rotundatus: HABE, CISJ (1) 2, 124, pl. 56, f. 14 (non YokoYAmA, 1926)

1962 Leptaxinus oyamai: HABE, CISJ, (2) 2, App. 46, pl. 56, f. 14

Loc. N M S (s) dp.

Dist. Honshu (north to Sagami Bay) to Kyushu.

\section{Family Ungulinidae H. \& A. ADAMS フタバシラガイ科 \\ Genus Cycladicama VALENCIENNES シオガマガイ属}

1954 Cycladicama Valenciennes, Voy, Pole Sud., 5, 116

1895 Joannisia Dalx, TWFIP. 3 (3), 546 (non Monterosato, 1884)

1895 Joannisiella DaLl, Naut., 9, 78. Type (OD): Cyrenoida oblonga HANLEY 
Cycladicama cumingii (HANLEY) シオガマガイ(六介) Pl. 6, f. 2.

1844 Cyrenoida cumingii HANLEX, Bivalv. Shells, 353, pl. 15, f. 5

1922 Diplodonta semiaspera Yokoyama, JCSIUT, 44 (1), 160, pl. 14, 15, f. 2 (non PhILIPPI, 1836)

1959 Joannisiella semiasperoides: KIRA, CISJ, 1, 132, pl. 52, f. 32 (non NoMURA, 1932)

1971 Cycladicama cumingii: Kuroda \& HABE, SSSB, 605 (J) 388 (E), pl. 87, f. 15

Loc. N M S (s-m) dp.

Dist. Honshu (north to Bōsō Peninsula) to Kyushu and Philippines. West Pacific.

Cycladicama coreensis (ADAMS \& REEVE) オウバイガィ（介志）

1850 Cyrenoida coreensis ADAMs \& REEve, Samarang, 80, pl. 24, f. 14

Loc. S (s) dp.

Dist. Honshu (north to Bōsō Peninsula) to Kyushu.

Remark: This seems to be as same as the preceding species.

Cycladicama lunaris (YoKOYAMA) マンゲツシオガマガイ（波部）

1927 Diplodonta lunaris YoKoYama, JFSIUT, (2) 20 (10), 433, pl. 50, f. 5, 6.

1961 Joannisiella lunaris: HABE, CISJ, 2, 124, pl. 56, f. 12

1971 Cycladicama lunaris: KuRODA \& HABE, SSSB, 605 (J) 388 (E), pl. 87, f. 13

Loc. M S (s-m) dp.

Dist. Honshu (north to Bōsō Peninsula) to Kyushu.

Cycladicama semiasperoides (NomuRA)ヒラシオガマガイ（滝・大们）

1920 Diplodonta semiaspera Yokoyama, JCSIUT, 39 (1), 131, pl. 10, f. 2, (non PHILIPPI, 1836)

1932 Diplodonta semiasperoides. Nomura, SRTIV, (2) 15, 78

1971 Cycladicama semiasperoides: KuRODA \& HABE, SSSB, 605 (J) 388 (E), pl. 87, f. 14

Loc. N M S (s) dp.

Dist. Southern Hokkaido to Honshu (Kii Peninsula).

*Cycladicama tsuchii YAMAMOTO \& HABEアツシオガマガイ(波部)

1961 Cycladicama tsuchii YaMAMOTo \& HABE, CISJ, 2, 124, pl. 56, f. 8

1971 Cycladicama tsuchii: Kuroda \& HaBE, SSSB, 606 (J) 389 (E), pl. 118, f. 5

Loc. S (s) dp.

Dist. Honshu to Kyushu and China.

Cycladicama nomurai HABE ノムラシオガマガイ (波部)；ヒラシオガマガイ（波部）

1920 Diplodonta semiaspera YoKoYAMA, JCSIUT, 39 (6), 131, pl. 10, f. 3

1960 Joannisiella nomurai HABE, PSMBL, 8, 290

Loc. M (g) gl.

Dist. Honshu (north to Bōsō Peninsula) to Kyushu.

Genus Felaniella DALL ウソシジミ属

1899 Felaniella Dall, J. of C., 9 (8), 244. Type (OD): Mysia (Felania) usta Gould

Felaniella sowerbyi KURODA \& HABE ウメノハナガイモドキ(平瀬)

1903 Felania minor Sowerby, AMNH, (7) 12, 501 (non DALL, 1900)

1952 Felaniella sowerbyi KuRODA \& HABE, Check, List, 20

1961 Felaniella sowerbyi: HABE, CISJ, 2, 124, pl. 56, f. 11

Loc. $\mathrm{N}$ (s) int. 
Dist. Honshu (Kii Peninsula) to Kyushu.

\section{Genus Diplodonta BRownフタバシラガイ属}

1831 Diplodonta Brown, Ergeb. Nat. Reisen, 2, 484. Type (SD, HerrmannSEN, 1846): Tellina rotundata MONTAGU

Diplodonta gouldi (YoKOYAMA)フタバシラガイ（黒田）

1920 Diplodonta gouldi YoKoYAMA, JCSIUT, 39 (6), 132, pl. 39, f. 11

1973 Diplodonta (Diplodonta) gouldi: OYAMA, PSJSP, 17, 94, pl. 39, f. 11

Loc. M (s) dp.

Dist. Southern Hokkaido to Honshu (south to Kii Peninsula).

Genus Phlyctiderma DALL ヤエウメノハナガイ属

1899 Phlytiderma Dall, J. of C., 9, 244. Type (OD): Diplodonta semiaspera PhILIPPI

Phlyctiderma japonicum (PILSBRY) ヤエウメノハナガイ（六介）

1895 Diplodonta japonica PILSBRy, CMMJ, 132, pl. 3, f. 7, 8

1959 Phlyctiderma japonicum: KIRA, CISJ, 1, 132, p1. 52, f. 30

1971 Phlyctiderma japonicum: KuRoda \& HABE, SSSB, 606 (J) 389 (E), pl. 118, f. 6

Loc. N M S (bo) gl.

Dist. Southern Hokkaido to Kyushu.

\section{Genus Minipisum HABE ミソハギガイ属}

1961 Minipisum HaBe, Venus, 21 (4), 420, 429. Type (M): Minipisum japonicum HABE

Minipisum japonicum HABE : シソハキガイ（波部） Pl. 6, f. 3.

1961 Minipisum japonicum HABE, Venus, 21 (4), 420, 429, tf. 5, 6.

1977 Minipisum japonicum: HABE, SMJ B/S, 136, pl. 26, f. 1, 2.

Loc. S (s) dp.

Dist. Honshu (Shima Peninsula and Kii Peninsula).

$$
\begin{array}{cc}
\begin{array}{c}
\text { Superfamily Chamacea LAMARCK } \\
\text { Family Chamidae LAMARCK }
\end{array} & \text { キクザルガイ超科 } \\
\text { Genus Chama LINNAEUS } & \text { キクザルガイ属 }
\end{array}
$$

1758 Chama Linnaues, Syst. Nat., 10, 691. Type (SD, Children, 1823; ICZN Op. 484): Chama lazarus LiNNAEUS

\section{Chama lazarus LINNAEUS ヒレインコガイ（黒田）}

1758 Chama lazarus LinNaeus, Syst. Nat., 10, 691

1961 Chama lazarus: HABE, CISJ, 2, 127, pl. 57, f. 17

1966 Chama lazarus: Habe \& Kosuge, SWC, 2, 156, pl. 60, f. 8

Loc. M S (r) gl.

Dist. Honshu (Kii Peninsula) to Ryukyu Islands. Indo-Pacific.

\section{Chama ambigua LISCHKE ヒトエギクガイ（目八）}

1870 Chama ambigua Lischke, M B L, 17, 27

1871 Chama ambigua: LischKe, JMC, 2, 128, pl. 8, f. 2

1959 Chama ambigua: KIRA, CISJ, 1, 134, pl. 53, f. 13 
Loc. M S (r) gl.

Dist. Honshu (north to Bōsō Peninsula) to Ryukyu Islands.

\section{Chama plinthota CoX シシガシラザルガイ（波部）}

1835 Chama imbricata BRODERIP, PZSL, 2 (24), 149 (non LAMARCK, 1801)

1927 Chama plinthota Cox, Zanzibar, 98

1961 Chama plinthota: HABE, CISJ, 2, 127, pl. 57, f. 16

Loc. $M$ (r) gl.

Dist. Honshu (Kii Peninsula) and East Africa.

\section{Chama brassica ReEVEシロザルガイ（黒田）}

1847 Chama brassica ReEve, Icon., sp. 31

1871 Chama imbricata LISCHKE, JMC, 2, 126, pl. 9, f. 4 (non BRODERIP, 1835)

1959 Chama brassica: KrRA, CISJ, 1, 135, pl. 53, f. 15

1966 Chama brassica: HABE \& Kosuge, SWC, 2, 156, pl. 60, f. 9.

Loc. S (r) int.

Dist. Honshu (Kii Peninsula) to Ryukyu Islands. Tropic Pacific.

Chama japonica LAMARCK キクザルガイ（目八）

1819 Chama japonica LAMARCK, AsV, 6 (1), 97

1846 Chama reflexa REEve, Icon., sp. 16

1846 Chama aspersa REEVE, Icon., sp. 24,

1846 Chama fibula REEve, Icon., sp. 27

1847 Chama jukesi ReEve, Icon., sp. 39

1959 Chama reflexa: KIRA, CISJ, 1, 134, pl. 53, f. 10

1950 Chama reflexa jukesi: KIRA, CISJ, 1, 134, pl. 53, f. 11

1971 Chama (Chama) reflexa: KuRodA \& HABE, SSSB, 616 (J) 396 (E), pl. 88, f. 1, 2.

Loc. N M S (r) gl.

Dist. Honshu (north to Bōsō Peninsula) to Ryukyu Islands. Indo-Pacific.

Chama fragum REEvEイチゴキクザルガイ（黒田）

1846 Chama fragum REEve, Icon., sp. 48

Loc. M S (r) gl.

Dist. Honshu (north to Bōsō Peninsula) to Ryukyu Islands. Widely ranging in the tropic Pacific.

Remarks: This may be a form of Chama japonica Lamarck.

\section{Chama isostoma CONRADカネッケザルガイ（黑田）}

1839 Chama isostoma CoNrad, JANSP, 7, 256

1846 Chama isostoma: REEVE, Icon., sp. 7

1959 Chama isostoma: KIRA, CISJ, 1, 135, pl. 53, f. 3

1966 Chama isostoma: HABE \& Kosuge, SWC ,2, 155, pl. 60, f. 3

Loc. S (r). gl.

Dist. Honshu (north to Izu Islands) to Ryukyu Islands. Widely ranging in the Pacific.

Chama divaricata ReEveシマザルガイ（黒田）

1846 Chama divaricata ReEve, Icon., sp. 20

Loc. S (r) gl.

Dist. Honshu (Kii Peninsula). Tropic Pacific. 
Chama dunkeri LISCHKEケイトウガイ (平瀬)

1870 Chama dunkeri LiscHKE, M B L, 17, 27

1871 Chama dunkeri: LISCHKE, JMC, 2, 128, pl. 8, f. 3, 4.

1959 Chama dunkeri: KIRA, CISJ, 1, 1134, pl. 53, f. 12

1971 Chama (Chama) dunkeri: Kuroda \& HaBE, SSSB, 617 (J) 397 (E), pl. 88, f. 6, 7

Loc. N M S (r) gl.

Dist. Honshu (north to Bōsō Peninsula) to Ryukyu Islands.

Chama semipurpurata LISCHKE ソメワケガシラガイ（平瀬）

1870 Chama semipurpurata LrschKE, M B L, 17, 27

1871 Chama semipurpurata: LisCHKE, JMC, 2, 130, pl. 8, f. 1

Loc. S (r). int.

Dist. Honshu (north to Boso Peninsula) to Ryukyu Islands.

\section{Genus Pseudochama ODHNER サルノカシラガイ属}

1917 Pseudochama OdHner, Kungl. Vet. Acad. Nya. Hand1., 52 (16), 28. Type (OD): Chama cristella LAMARCK

Pseudochama retroversa (LiSCHKE) サルノカシラガイ（目八）

1870 Chama retroversa LiscHKE, M B L, 17, 28

1871 Chama retroversa: LISCHKE, JMC, 2, 131, pl. 9, f. 1-3

1971 Chama (Pseudochama) retroversa: KURODA \& HABE, SSSB, 617 (J) 397 (E), pl. 88, f. 4

Loc. N M (r) gl.

Dist. Honshu (north to Boso Peninsula) to Formosa.

Pseudochama aurantia Kuroda \& HABEコガネザルガイ（黒田）

1971 Chama (Pseudochama) aurantia KURODA \& HABE, SSSB, 617 (J) 397 (E), pl. 88, f. 3

Loc. M (r) gl.

Dist. Honshu (north to Sagami Bay) and Shikoku.

Genus Amphichama HABE ウンモザルガイ属

1961 Amphichama HABE, CISJ, 2, 127. Type (M): Chama argentata KURODA \& HABE

Amphichama argentata (KURODA \& HABE) ウンモザルガイ（波部）

1958 Chama argentata KURODA \& HABE, PSMBL, 7 (1), 31

1971 Chama (Amphichama) argentata: KURODA \& HABE, SSSB, 618 (J) 398 (E), pl. 88, f. 5

Loc. M S (st) gl.

Dist. Honshu (north to Bōsō Peninsula) to Kyushu.

Superfamily Geleommtacea GRAY ウロコガイ超科

$$
\begin{array}{cc}
\text { Family Lasaeidae GRAY } & \text { チリハギガイ科 } \\
\text { Subfamily Lasaeinae GRAY } & \text { チリハギガイ承科 } \\
\text { Genus Lasaea BRown } & \text { チリハギガイ属 }
\end{array}
$$

1827 Lasaea Brown, Illust. Conch. Great. Britain, pl. 20, f. 17, 18. Type (M): Cardium rubrum MONTAGU = Venus adamsoni GEMLIN 
Lasaea undulata (Gould) チリハギガイ（矢倉） PI. 9, f. 1 .

1861 Kellia undulata Gould, PBSNH, 6, 34

1938 Lasaea nipponica KEEN, PMSL, 23 (1), 26, tf. A, B

1960 Kellia minutissima HABE, PSMBL, 8 (2), 283, f. 14, 15, 23, 24

1968 Kellia undulata: JoHNSON, USNMB, 163 , pl. 29 , f. 6

1971 Lasaea undulata: Kuroda \& HABE, SSSB, 630 (J) 406 (E), pl. 119, f. 8

Loc. N M S (r) int.

Dist. Honshu (north to Mutsu Bay), Shikoku, Kyushu, Okinawa and Hawaii.

\section{Genus Squillaconcha KURODA \& HABE コフジガイ属}

1971 Squillaconcha KURODA \& HABE, SSSB, 627 (J) 404 (E). Type (OD): Kellia subsinuata LISCHKE

Squillaconcha subsinuata (LISCHKE) コフジガイ（滝瞰）

1871 Kellia subsinuata LisCHKE, M B L, 18, 43

1871 Kellia subsinuata: LISCHKE, JMC, 2, 136, pl. 10, f. 1-3

1937 Pseudopythina subsinuata: HABE, CISJ, 2, 126, pl. 57, f. 2

1971 Squillaconcha subsinuata: KURODA \& HABE, SSSB, 627 (J) 404 (E), pl. 87, f. 12

Loc. M (sy) dp. [Squilla oratoria de Haan:]

Dist. Honshu (north to Bōsō Peninsula), Shikoku and Kyushu.

Squillachoncha sagamiensis (HABE)サガミコフジガイ（波部） Pl. 9, f. 2.

1961 Pseudopythina sagamiensis HaBe, Venus, 21 (2), 151, 154, tf. 5-8

1961 Pseudopythina sagamiensis: HABE, CISJ, 2, 126, pl. 57, f. 1

Loc. N S (s) dp.

Dist. Honshu (Sagami Bay and Wakayama Pref.)

\section{Genus Kellia TURTONコハクノッユガイ属}

1822 Kellia TurTon, Conch. Insularum Brit., 19, 56.

1839 Chironia DeshaYes, RZSC, 2, 357. Type (SD, ReCluz, 1844): Mya suborbicularis MONTAGU

Kellia japonica PILSBRY ドブシジミモドキ(岩川)

1894 Kellia japonica Pilsbry, CMMJ, 132, pl. 3, f. 18, 19

1904 Kellia japonica: PrLsBry, PANSP, 56, 558, pl. 41, f. 21

1961 Kellia japonica: HABE, CISJ, 2, 126, pl. 57, f. 7. .

1971 Kellia japonica: KurodA \& HABE, SSSB, 628 (J) 405 (E), pl. 119, f. 2

Loc. M S (g) gl.

Dist. Southern Hokkaido, Honshu, Shikoku and Kyushu.

Kellia porculus PILSBRY コハクノッユガイ（矢倉）

1904 Kellia porculus PlLSBRY, PANSP, 56, 558, pl. 41, f. 18-20

1961 Kellia porculus: HABE, CISJ, 2, 126, pl. 57, f. 6

1971 Kellia porculus: KuROdA \& Habe, SSSB, 628 (J) 405 (E), pl. 119, f. 3, 4.

Loc. M S (g) gl.

Dist. Honshu (north to Bōsō Peninsula), Shikoku and Kyushu.

Kellia subrotundata (DUNKER) スモモノハナガイ（黒田）

1882 Lepton subrotundatus DUNKER, Index, 219, pl. 14, f. 12, 13

1971 Kellia subrotundata: KURODA \& HABE, SSSB, 628 (J) 404 (E), pl. 119, f. 1 
Loc. $S$ (s) dp.

Dist. Honshu (north to Bōsō Peninsula), Shikoku and Kyushu.

Kellia tsujitai HABEツジタコハクノッユガイ（波部） P1. 9, f. 3.

1959 Kellia tsujitai HABE, PSMBL, 7 (2), 291, tf. 1, 2

1977 Kellia tsujitai: HABE, Taxa. HABE, 126, pl. 4, f. 9

Loc. $M$ (s-m) dp.

Dist. Honshu (Tanabe Bay, Wakayama Pref.) and Kyushu (Amakusa)

\section{Genus Platymysia HABEオキナノエガオガイ属}

1951 Platymysia HABE, GJS, 2, 136. Type (OD): Platymysia rugata HABE

Platymysia rugata HABEオキナノエガオガイ（波部）

1951 Platymysia rugata: GJS, 2, 136, tf. 294, 295

1961 Platymysia rugata: HABE, CISJ, 2, 126, pl. 57, f. 8

1977 Platymysia rugata: HABE, SMJ B/S, 144, pl. 27, f. 18, 19

Loc. $\mathrm{N}$ (s-m).

Dist. Honshu (north to Sagami Bay and Noto Peninsula), Shikoku and Kyushu.

Subfamily Melliterycinae IREDALE \& MCMICHAEL 八チミッガイ巠科

$$
\text { Genus Melliteryx IREDALE ハチミッガイ属 }
$$

1924 Melliteryx Iredale, PLSNSW, 49, 182, 207. Type (OD): Erycina acupuncta HedLeY

Melliteryx puncticulus (YoKOYAMA) 八チミッガイ（滰・大山）Ｐl. 9, f. 4.

1924 Lepton puncticulata YoKоYAMA, JCSIUT, 5 (1), 48, pl. 4, f. 8, 9

1960 Melliteryx puncticulata flexuosa HABE, PSMBL, 8 (2), 291, f. 4, 5.

1971 Melliteryx puncticulata: Kuroda \& HABE, SSSB, 629 (J) 406 (E), pl. 119, f. 7

Loc. N S (s) dp.

Dist. Honshu (north to Sagami Bay), Shikoku and Kyushu.

\section{Subfamily Borniolinae IRADALE \& MCMICHAEL セワケガイ巠科}

\section{Genus Byssobornia IREDALEセワケガイ属}

1936 Byssobornia Iredale, RAM, 19 (5), 275. Type (OD): Bornia filosa Hedley

Byssobornia adamsi (YoKoYAMA) セワケガイ（黒田）

1924 Galeomma adamsi YokoYama, JCSIUT, 45 (1), 47, pl. 4, f. 3, 4.

1971 Byssobornia yamakawai: Kuroda \& HABE, SSSB, 631 (J) 407 (E), pl. 119, f. 12, 13 (non YoKoyama, 1922)

1973 Byssobornia striatissima: OYAMA, PSJSP, 17, 97, pl. 41, f. 19, 21

Loc. M (s) dp.

Dist. Honshu (north to Sagami Bay), Shikoku and Kyushu.

$$
\text { Genus Borniola IREDALEケボリセワケガイ属Ｐl. 10, f. } 1 .
$$

1924 Borniola Iredale, PLSNSW, 49, 182, 207. Type (OD): Borniola lepida Hedley

Borniola yamakawai (YokoyAMA)ケボリセワケガイ（黒田・波部）

1922 Montacuta yamakawai YokоYAMA, JCSIUT, 44 (1), 158, pl. 9, f. 10 
1971 Borniola nipponica Kuroda \& HABE, SSSB, 631 (J) 407 (E), pl. 119, f. 14, 15 (non YokoYAMA, 1924)

1973 Scintillula yamakawai: OYAMA, PSJSP, 17, 97, pl. 41, f. 6a, 6 b.

Loc. $\mathrm{S}$ (s) dp.

Dist. Honshu (north to Sagami Bay), Shikoku and Kyushu.

Genus Paraborniola HABE マツモトウロコガイ属

1958 Paraborniola HABe, Venus, 19, 178, 182. Type (M): Paraborniola matsumotoi HABE

Paraborniola matsumotoi HABEマツモトウロコガイ（波部） Pl. 10, f. 2.

1958 Paraborniola matsumotoi HABE, Venus, 19, 178, 182, f. 3, 4

1971 Paraborniola matsumotoi: Kuroda \& HABE, SSSB, 632 (J) 408 (E), pl. 119, f. 16, 17

1977 Paraborniola matsumotoi: HABE, SMJ B/S, 142, pl. 26, f. 15, 16

Loc. M S (g) dp.

Dist. Honshu (north to Bōsō Peninsula and Oga Peninsula), Shikoku and Kyushu.

\section{Genus Borniopsis HABEケボリガイ属}

1959 Borniopsis HABE, PSMBL, 7 (2), 292. Type (OD): Borniopsis tsurumaru HABE

Borniopsis tsurumaru HABE ッルマルケボリガイ（波部）

1959 Borniopsis tsurumaru HABE, PSMBL, 7 (2), 292, tf. 10-12

1977 Borniopsis tsurumaru: HABE, SMJ B/S, 142, pl. 26, f. 19, 20

Loc.

Dist. Honshu (north to Mikawa Bay and Nanao Bay, Noto Peninsula) and Kyushu (Ariake Sea).

\section{Genus Radobornia DALL, BARTSCH \& REHDER チチノシタタリガイ属}

1938 Radobornia DALL, Bartsch \& Rehder, BPBMB, 153, 140. Type (OD): Radobornia araia DALl, Bartsch \& ReHDER

Radobornia bryani (PILSBRY)チチノシタタリガイ（黑田）

1921 Solecardia bryani PILSBRY, PANSP, 72, 324, f. 5

1938 Radobornia araia DALL, Bartsch \& Rehder, BPBMB, 153, 140, pl. 37, f. 5-7

1938 Radobornia bryani: DALL. Bartsch \& Rehder, BPBMB, 153, 140, pl. 37, f. 17, 18

1960 Nesobornia lactea KurodA, Cat. Okinawa, 82

1977 Radobornia bryani: HABE, Venus, 36, 3, pl. 1, f. 3-5

Loc. M S (s) dp.

Dist. Honshu (north to Kii Peninsula), to Ryukyu Islands and Hawaii.

$$
\text { Genus Hyalokellia HABE ヒョワガイ属 }
$$

1960 Hyalokellia HABE, PSMBL, 8 (2), 290. Type (M): Hyalokellia polita HABE

Hyalokellia polita HABEヒヨワガイ（波部), スキバガイ(波部)

1960 Hyalokellia polita HABE, PSMBL, 8 (2), 290

1977 Hyalokellia polita: HABE, Taxa. HABE, 98, pl. 1, f. 2

Loc. S (s)

Dist. Honshu (Kii Peninsula) and Kyushu (Amakusa). 


\section{Genus Lionelita JoussEAUMEキザミシタタリガイ属}

1888 Lionelita Jousseaume, MSZF, 1, 204. Type (OD): Erycina lionelita Deshayes

Lionelita denticulata (DESHAYES) キザミシタタリガイ（波部）

1856 Erycina denticulata Deshayes, PZSL (1885), 182

1888 Lionelita lionelita JousSEAUME, MSZF, 1, 204

1977 Lionelita denticulata: Habe, Venus, 36, (3), 8, pl. 2, f. 1, 2.

Loc. S (s) dp.

Dist. Honshu (Kii Peninsula) to Ryukyu Islands and Borneo.

Genus Kamelia KURODA \& HABE, ソバノミガイ属

1971 Kamekia KURODA \& HABE, SSSB, 633 (J.), 408 (E.). Type (OD) Kamekia pisiformis KURODA \& HABE, 1971

Kamekia pisiformis KuRODA \& HABEソバノミガイ(黒田・波部) Pl. 10, f. 3.

1971 Kamekia pisiformis KURODA \& HABE, SSSB, 633 (J.) 408 (E.), pl. 119, f. $20,21$.

Loc. S (s) dp.

Dist. Honshu (Sagami Bay to Kii Peninsula).

\section{Family Montacutidae CLARKブンブクヤドリガイ科 \\ Genus Montacuta TURTONブンブクヤドリガイ属}

1822 Montacuta Turton, Conch. Insularum Brit., 18, 58. Type (SD, Herrmannsen, 1846): Ligula substriata MONTAGU

Montacuta echinocardiophila HABEオカメブンブクヤドリガイ（波部）Ｐl. 10, f. 4.

1964 Montacuta echinocardiophila HABE, Venus, 23, 138

1977 Montacuta echinocardiophila: HABE, SMJ B/S. 144. pl. 27, f. 1

Loc. M (sy). [Echinocardium cordatum.]

Dist. Kyushu (west coast) and Inland Sea of Japan.

\section{Genus Montacutona YAMAMOTO \& HABE マルヤドリガイ属}

1959 Montacutona Yamamoto \& Habe, BMBSA, 9 (3), 93. Type (OD): Montacutona mutsuwanensis YAMAMOTO \& HABE

\section{Montacutona olivacea HABEムラサキマルヤドリガイ（波部）}

1959 Montacutona olivacea HABE, PSMBL, 7, 294, tf. 7-9

1977 Montacutona olivacea: HABE, SMJ B/S, 146, pl. 28, f. 1, 2.

Loc. M (m) dp.

Dist. Honshu (north to Shima Peninsula and Wakasa Bay) and Kyushu.

Montacutona japonica (YОКоYамA) マルヤドリガイ（波部） Pl. 11, f. 1.

1922 Montacuta japonica YokoYama, JCSIUT 44 (1), 157, pl. 9, f. 2, 3

1973 Mysella japonica: OYAMA, PSJSP, 17, 98, pl. 41, f. 10, 11

Loc. N M S (s) dp.

Dist. Honshu (north to Bōsō Peninsula) and Kyushu.

Montacutona tanakai HABE タナカマルヤドリガイ（波部）

1959 Montacutona tanakai HABE, PSMBL, 7 (2), 293, tf. 3, 4. 
Loc. $M(s-m) d p$.

Dist. Honshu (Tanabe Bay, Wakayama Pref.) and Kyushu (Ariake Sea).

$$
\text { Genus Eolepton OYAMA コデマリガイ属 }
$$

1973 Eolepton OYAMA, PSJSP, 17, 93. Type (OD): Montacula crassa YoKoYAMA

\section{Eolepton crassa (YoKoyama) コデマリガイ（大山）}

1927 Montacuta? crassa Yokoyama, JFSIUT, (2) 1 (10), 432, pl. 49, f. 4, 5

1973 Eolepton crassa: OYAMA, PSJSP, 17, 93, pl. 41, f. 15-17

1977 Eolepton crassa: HABE, SMJ B/S. 146, pl. 28, f. 3, 4

Loc. M (s) dp.

Dist. Honshu (Tanabe Bay, Wakayama Pref.) and Kyushu.

\section{Genus Fronsella LASERONハナビラガイ属}

1956 Fronsella LASERON, RAM, 24 (2), 9. Type (OD): Fronsella adipata LASERON

Fronsella ohshimai HABE ハナビラガイ (波部)

1958 Fronsella ohshimai HABE, Venus, 20, 174, 177, tf. 1, 2

1961 Fronsella ohshimai: HABE, CISJ, 2, 126, pl. 57, f. 3

Loc. S (sy). [Sipunculus nudus LinNaeus.]

Dist. Honshu (Wakayama Pref.) and Kyushu (Amakusa and Mogi, Nagasaki Pref.).

Fronsella fujitaniana (YоKоYAMA）フジタニコハクノッュガィ（大山） Pl. 11, f. 2.

1927 Kellia fujitaniana YoKoYAMA, JFSIUT, (2) 1 (10), 431, p1. 48, f. 17, 18

1977 Fronsella fujitaniana: HABE, SMJ B/S. 146, p1. 27, f. 4

Loc. S (sp) dp.

Dist. Honshu (north to Mutsu Bay) and Kyushu.

Genus Nipponomysella YaMaMoto \& HABE マルヘノジガイ属

1959 Nippinomysella YаMAMOTO \& HABE, BMBSA, 9 (3), 92. Type (OD): Montacuta oblongata YOKOYAMA

Nipponomysella oblongata (Yокоуама) マルヘノジガイ (澺) Pl. 11, f. 3.

1922 Montacuta oblongata ҮокочамА, JCSIUT, 44 (1), 157, p1. 73, f. 9, 10

1971 Nipponomysella oblongata: KuRODA \& HABE, SSSB, 635 (J) 410 (E), pl. 120, f. 2

1977 Nipponomysella oblongata: HABE, SMJ B/S. 147. pl. 28, f. 5

Loc. M S (sy). [bristle star]

Dist. Honshu (north to Mutsu Bay), Shikoku and Kyushu.

Nipponomysella subtruncata (YoKоYамA)スジホシムシヤドリガイ（波部）P1. 11, f. 4.

1927 Montacuta subtruncata YokoYAMA, JFSIUT, (2) 1 (10), 432, pl. 48, f. 8

1973 Nipponomysella subtruncata: OYAMA, PSJSP, 17, 98, pl. 41, f. 5

Loc. M S (sy). [Sipunculus nudus LinNAEUs]

Dist. Honshu (north to Kii Peninsula) and Kyushu.

*Nipponomysella obesa HABEフクレマルヘノジガイ（波部） Pl. 12, f. 1.

1960 Nipponomysella obesa HABE, PSMBL, 8 (2), 285, tf. 4-6

Loc. M S (s) dp.

Dist. Honshu (Tanabe Bay, Wakayama Pref.). 
*Nipponomysella tanabensis $\mathrm{HABE}$ タナベヤドリガイ（波部）

1960 Nipponomysella tanabensis HABE, PSMBL, 8 (2), 284, tf. 10-11

Loc. $\mathrm{M}$ (s) dp.

Dist. Honshu (Tanabe Bay, Wakayama Pref.).

\section{Genus Callomysia HABEマツイガイ属}

1951 Callomysia HABE, GJS, 2, 137. Type (OD): Callomysia matsuii HABE

Callomysia matsuii HABEマッイガイ（波部）

1951 Callomysia matsuii HABE, GJS, (2), 137, tf. 313

1977 Callomysia matsuii: HABE, SMJ B/S, 148, pl. 27, f. 6, 7

Loc. M (s) dp.

Dist. Honshu (north to Shima Peninsula) and Kyushu.

Genus Nipponomontacuta YAMAMOTO \& HABE イソギンチャクヤドリガイ属

1961 Nipponomontacuta YAMAMoto \& HABE, PSMBL, 9 (2), 265. Type (M): Nipponomontacuta actinariophila YамAMOTO \& HABE

*Nipponomontacuta actinariophila YAMAMOTO \& HABE イソギンチャクヤドリガイ（波部）

P1. 12. f. 3.

1961 Nipponomontacuta actinariophila Yамамото \& HABE, PSMBL, 9 (2), 265, f. 1, 2

1971 Nipponomontacuta actinariophila: KURODA \& HABE, SSSB, 635 (J) 410 (E), pl. 119, f. 11

Loc. S (sy). [Halcampella maxima HeRTwIG]

Dist. Honshu (north to Sagami Bay) and Kyushu (west coast).

Family Mysellidae IREDALE \& MCMICHAEL タナベガイ科

Genus Mysella ANGASタナベガイ属

1877 Mysella ANGAS, PZSL, 176. Type (M): Mysella anomala ANGAS

*Mysella tanabensis $\mathrm{HABE}$ タナベガイ(波部)

1960 Mysella tanabensis HABE, PSMBL, 8 (2), 284

Loc. $S(s) d p$.

Dist. Honshu (Tanabe Bay, Wakayama Pref.). and Kyushu (Tomioka, Amakusa)

*Mysella nipponica HABE sp. nov. ニホンタナベガイ（波部） Pl. 2, fig. 6.

Shell small, thin and fragile, equivalve, roundly trigonal in shape, rather inflated. Posterior dorsal margin gently curved and longer than the rather straight anterior dorsal margin. Umbo directed forwards. Surface covered with a pale ashy colored periostracum and marked concentric growth lines. Interior ashy white and translucent. Right valve with two weak cardinal teeth. Left valve with two stout cardinal teeth. Anterior and posterior lateral teeth week and apart from cardinal teeth. Ligament internal below and behind the umbo and between the two cardinal teeth. Anterior and posterior adductor muscle scar large and ovate in shape. Pallial line entire.

Length $9.4 \mathrm{~mm}$, height $6.4 \mathrm{~mm}$ and breadth $4.0 \mathrm{~mm}$. (Holotype conjoined valves preserved in the National Science Museum, NSMTMo 58963) 
Type locality: Sakai Harbor of Minabe, Wakayama Pref., Honshu., among the spines of Spondylus barbatus REEVE. S (s) dp.

Remarks: Mysella tanabensis HABE is an ally to this new species but has the rather flat valve with the small cardinal teeth. This species has been collected by Mr. Tsutomu Tanaka of Uchita-Cho, Naga County.

\section{Genus Curvemysella HABE ヘノジガイ属}

1959 Curvemysella HABE, PSMBL, 7 (2), 294. Type (OD): Pythina paula A. AdAms

Curvemysella paula A. ADAMS ヘノジガイ (平瀬)

1856 Pythina paula A. AdAMs, PZSL, 24, 47

1856 Pythina peculiaris A. ADAMs. PZSL, 24, 47

1885 Montacuta paula: SмrтH, Challenger, 203, pl. 12, f. la, b 1

1959 Curvemysella paula: HABE, PSMBL, 7 (2), 294, tf. 5, 6

Loc. N M S (sy). gl. [shells occupaid by helmet crab]

Dist. Honshu (north to Mutsu Bay), Shikoku and Kyushu. Widely ranging in the Indo Pacific region.

\section{Genus Thecodonta A. ADAms コメガイ属}

1864 Thecodonta A. Adams, AMNH, (3) 13, 308. Type (M): Thecodonta sieboldii A. Adams 1864.

1938 Kona Dall, BARTSch \& Rehder BPBMB, 153, 143.

Thecodonta sieboldii A. AdAMs コメガイ（胃田・波部） Pl. 12, f. 2.

1864 Thecodonta sieboldii A. AdAms, AMNH, (3) 13, 308.

1961 Thecodonta sieboldii HABE, Prof. Makiyama Mem. Vol. 193, pl. 1, f. 6.

1971 Kona oryzaeformis KuRODA \& HABE, SSSB, 633 (J.), 409 (E.), pl. 119, f. 22, 23.

Loc. S (s) dp.

Dist. Honshu (north to Kashima Nada) to Kyushu.

\section{Family Galeommatidae GraY ウロコガイ科}

\section{Genus Galeomma SowERHY in Turton, 1825 キザミウロコガイ属}

1825 Galeomma Sowerby in Turton, ZJ., 2 (7), 381. Type (SD, Gray 1847): Galeomma turtoni SOWERBY

*Galeomma purpurea HABE sp. nov. ウスムラサキキザミウロコガイ（波部）

Pl. 3, fig. 1.

Shell pale purple in color, thin, inaequilateral, transversely elongated elliptical in outline, slightly narrowing to the roundly curved anterior margin and with a straight anterior and posterior dorsal margins. Ventral margin rather straight and widely gaping. Surface with densely set minute radiating scales all over, causing denticulation at the anterior, posterior and ventral margins, and a groove running from the umbo to the middle of ventral margin. Hinge without teeth, Ligament internal, small, just behind the umbo. Interior silvery white. Anterior and posterior muscle scars small. Pallial line entire.

Length $11.8 \mathrm{~mm}$, height $5.6 \mathrm{~mm}$, breadth $2.4 \mathrm{~mm}$. (Holotype left valve preserved in the National Science Museum, NSMT-Mo 56845) 
Type Locality: Minabe, Wakayama Pref., Honshu. M (s-m) gl.

Remarks: This species is somewhat related to Ephippodentina murakamii Kuroda, 1945 from Wakayama Pref. but the latter species has the white lunate shell with small but distinct cardinal teeth. This is first record of the real Galeomma species from Japan.

\section{Genus Lepirodes FISCHER ウロコガイ属}

1887 Lepirodes Fischer, M. de C., 1031

1899 Paralepida Dale, PUSNM, 21, 875. Type (M): Galeomma formosa Deshayes

*Lepirodes takii (KURODA)ウロコガイ（黒田） Pl. 12, f. 4.

1945 Galeomma (Paralepida) takii KurodA, Venus, 14, 39, pl. 2, f. 9-12

1961 Ephippodonta murakamii: HaBe, (non Kuroda, 1945) CISJ, 2, 126, pl. 57, f. 12

Loc. M S (st) gl.

Dist. Honshu (Wakayama Pref.), Shikoku and Kyushu.

\section{Genus Galeommella HABEオウギウロコガイ属}

1958 Galeommella HABE, Venus, 20, 174, 178. Type (M): Galeommella utinomii HABE

Galeomella utinomii $\mathrm{HABE}$ オウギウロコガイ（波部） PI. 13, f. 3.

1958 Galeommella utinomii HABE, Venus, 20, 174, 178, f. 3

1961 Galeommella utinomii: HABE, CISJ, 2, 126, pl. 57, f. 11

Loc. $S$ ( $\mathrm{s}-\mathrm{m})$ int.

Dist. Honshu (north to Sagami Bay and Noto Peninsula), Shikoku and Kyushu.

\section{Genus Ephippodontina KURODA ウスモノウロコガイ属}

1945 Ephippodontina KURODA, Venus, 14, 41. Type (M): Ephippodonta (Ephippodontina) murakamii KURODA

Ephippodonta murakamii (KURODA)ウスモノウロコガイ（黒田） Pl. 13, f. 1 .

1945 Ephippodonta (Epippodontina) murakamii Kuroda, Venus, 14, 41, pl. 2, f. 7, 8

1960 Ephippodonta murakamii: AraKawa, Venus, 21, 50, pl. 7, 8

Loc. M S (st) gl.

Dist. Honshu (Kii Peninsula) to Kyushu.

$$
\text { Genus Pseudogaleomma HABE ニッポンマメアゲマキガイ属 }
$$

1964 Pseudogaleomma HABE, SWC, 2, 185. Type (OD): Galeomma japonica A. ADAMS

Pseudogaleomma japonica (A. ADAMs) マメアゲマキガイ (平瀬)

$$
\text { ニッポンマメアゲマキガイ（波部） }
$$

1864 Galeomma japonica A. AdAMs, AMNH, (3) 9 (51), 228

1904 Solecardia vitrea japonica PILsBry, PANSP, 56, 558, pl. 41, f. 6

1964 Pseudogaleomma japonica: HABE, SWC, 2, 185, pl. 57, f. 13

1971 Pseudogaleomma japonica: KuRoDA \& HABE, SSSB, 636 (J) 410 (E), pl. 120, f. 11, 12

Loc. M S (g) int.

Dist. Honshu (north to Bōsō Peninsula and Tobishima Island) to Kyushu.

Genus Yamamotolepida HABE ヤマモトウロコガイ属

1976 Yamamotolepida HABE, Venus, 35, 38. Type (OD): Yamamotolepida semicircula HABE 
*Yamamotolepida semicircula HABEヤマモトウロコガイ（波部） Pl. 13, f. 2.

1976 Yamamotolepida semicircula HABE, Venus, 35, 38, pl. 1, f. 13, 14

Loc. N M S (st) gl.

Dist. Honshu (Wakayama Pref.) and Kyushu (Ōita Pref.).

\section{Genus Scintilla DESHAYESマメアゲマキガイ属}

1856 Scintilla Deshayes, PZSL, 23, 173. Type (SD, StoliczKa, 1871): Scintilla philippinensis Deshayes

*Scintilla violescens KURODA \& IW. TAKIイナズママメアゲマキガイ（黒田）

1961 Scintilla violescens Kuroda \& Iw. TAKr, Venus, 21, 141

1961 Scintilla violescens AraKaWA, Venus, 21, 143, tf. 1-5b

1971 Scintilla ione Kuroda \& HABE, SSSB, 637 (J) 411 (E), pl. 120, f. 5, 6

Loc. N M S (s-m) dp.

Dist. Honshu (north to Sagami Bay) to Kyushu.

*Scintilla nitidella HABEッヤマメアゲマキガイ（波部）

1961 Scintilla vitrea HABE, CISJ, 2, 127, pl. 57, f. 14 (non QUoY \& GAIMARD, 1832)

1962 Scintilla nitidella HABE, CISJ, (2) 2, 46, pl. 57, f. 14

1971 Scintilla nitidella: Kuroda \& HABE, SSSB, 637 (J) 411 (E), pl. 120, f. 7

Loc. M S (s-m) dp.

Dist. Honshu (Sagami Bay to Kii Peninsula).

Scintilla timorensis DESHAYES イオウノシタタリガイ（黒田）

1856 Scintilla timorensis DESHAYES, PSZL (1855), 23, 174

1874 Scintilla timoreusis: REEve, Icon., sp. 14

Loc. M S (g) int.

Dist. Honshu (north to Kii Peninsula) to Ryukyu Islands. Widely ranging in the western Pacific.

Scintilla solidula DESHAYESカタマメアゲマキガイ（黑田）

1856 Scintilla solidula DeSHAYES, PZSL, 23 (1855), 174

1862 Scintilla solidula: Sowerby, Thes., 3, 178, pl. 235, f. 48,49

Loc. M S (g) int.

Dist. Honshu (north to Kii Peninsula). Tropic Pacific.

Genus Scintillona FINLEYオオブンブクヤドリガイ属

1926 Scintillona FINLEY, TNZI, 57, 465. Type (OD): Spaniorinus zealandica ODHER

Scintillona stigmatica (PILSBRY)オオブンブクヤドリガイ（山本・波部）Ｐl. 13, f. 4.

1921 Solecardia stigmatica PILSBRy, PANSP, 72 (1920), 325, f. 7

1938 Scintillona stigmatica: DALL, Bartsch \& Rehder, BPBMB, 153, 145, pl. 38, f. 5-8, tf. 21

1974 Scintillona stigmatica: Yамамото \& HaBE, Venus, 33, 116, tf. 1

Loc. M (sy). [Brissus latecarinatus (LESKE).]

Dist. Honshu (Kii Peninsula) to Kyushu and Hawaii.

Genus Sagamiscintilla HABEマルマメアゲマキガイ属

1971 Scintillorbis Kuroda \& HABE, SSSB, 638 (J) 412 (E), (non DALL, 1899)

1975 Sagamiscintilla HABE, Venus, 34 (1) 183 . Type (OD): Scintillorbis opalinus Kuroda \& HABE 
Sagamiscintilla thalassemicola HABE ミドリユムシャドリガイ（波部）

1962 Achasmea thalassemicola HABE, Venus, 22, 117, f. 1-3

Loc. $M$ (sy) int. [Thalassema mucosum IKEDA]

Dist. Honshu (Kii Peninsula) and west coast of Kyushu (Amakusa).

\section{Superfamily Cyamiacea PHILIPPI ノミハマグリ超科}

Family Sportellidae DaLL イソカゼガイ科

Genus Basterotia HoRNESイソカゼガイ属

1859 Basterotia HoRNes, Abh. Geol. Reichsanst Wien, 4, 40

1850 Eucharis Récluz, J. de C., 1, 164, 167. (non LatreILle, 1804). Type (M): Basterotia corbuloides HORNES

Basterotia gouldii (A. AdAMs) イソカゼガイ (滝餀)

1864 Eucharis gouldii A. ADAMs, AMNH, (3) 13, 309

1882 Eucharis gouldii: DUNKER, Index, 178, pl. 9, f. 8, 9

1961 Anisodonta (Fulcrella) gouldii: HABE, CISJ, 2, 123, pl. 56, f. 6

1971 Basterotia gouldii: KURODA \& HABE, SSSB, 626 (J) 403 (E), pl. 118, f. 22, 23

Loc. N M S (s-m) dp.

Dist. Honshu (north to Bōsō Peninsula) to Kyushu.

Basterotia stimpsoni (A. ADAMs) ソヨカゼガイ（波部）

1864 Eucharis stimpsoni A. ADAMs, AMNH, (3) 13, 310

1961 Anisodonta (Basterotia) stimpsoni: HABE, CISJ, 2, 123, pl. 56, f. 5

1971 Basterotia stimpsoni: KurodA \& HABE, SSSB, 626 (J) 403 (E), pl. 118, f. 21

Loc. N S (s-m) dp.

Dist. Honshu (north to Bōsō Peninsula) to Kyushu.

\section{Genus Anisodonta DESHAYESケッ゙メガイ属}

1858 Anisodonta Deshayes, Bassin de Paris, 1, 542. Type (M): Anisodonta complanatum DESHAYES

Anisodonta angulata (H. ADAMS) ケッ゙メガイ (滝嚴)

1870 Eucharis angulata $\mathrm{H}$. ADAMs, PZSL, 789, pl. 48, f. 3

1938 Anisodonta angulata: DALL, BARTSCH \& ReHDER, BPBMB, 153, 125, pl. 34, f. 5, 6.

Loc. S

Dist. Honshu (north to Bōsō Peninsula) to Kyushu and Hawaii.

Superfamily Carditacea FLEMING トマヤガイ超科

Family Carditidae FLEMING トマヤガイ科

Subfamily Carditinae FLEMING トマヤガイ亜科

Genus Cardita BRUGUIÈREトマヤガイ属

1792 Cardita Bruguì̀re, EMHNV, 1 (2), 401 . Type (SD, Gray, 1847): Chama calyculata LINNAEUS 


\section{Cardita leana DUNKER トマヤガイ（目八）}

1860 Cardita leana Dunker, M B L, 6, 223

1860 Cardita cumingiana DUNKER, M B L, 6, 223

1861 Cardita leana: DunkeR, MJ, 29, pl. 3, f. 17

1861 Cardita cumingiana: DunKer, MJ, 29, pl. 3, f. 18

1959 Cardita leana: KIRA, CISJ, 1, 130, pl. 52, f. 16

1971 Cardita leana: Kuroda \& HABE, SSSB, 600 (J) 385 (E), pl. 87, f. 7

Loc. N M S (st) int.

Dist. Honshu (north to Tsugaru Strait) to Formosa.

Cardita nodulosa LAMARCK モモイロトマヤガイ（黒田）

1819 Cardita nodulosa LAMARCK, AsV, 6 (1), 25

1843 Cardita rufescens REEve, Icon., sp. 19

1959 Cardita nodulosa: KIRA, CISJ, 1, 130, pl. 52, f. 15

1971 Cardita nodulosa: Kuroda \& HABE, SSSB, 601 (J) 385 (E), p1. 87, f. 8

Loc. M (g) gl.

Dist. Southern Hokkaido to Kyushu. Widely ranging in the tropic Pacific.

Cardita kyushuensis (OKUTANI) =クイロトマヤガイ（波部・小菅）

$$
\text { トゲナガフミガイ (舆谷) }
$$

1963 Glans kyushuensis OKUTAN, PS, 17 (1), 88, f. 21

1967 Cardita kyushuensis: Habe \& Kosuge, Kai (Hoikusha), 142, pl. 53, f. 10

Loc. S (r) dp.

Dist. Honshu (north to Kii Peninsula) to Kyushu.

\section{Subfamily Venericardiinae CHAVAN マルフミガイ亜科}

\section{Genus Megacardita SACCO フミガイ属}

1889 Megacardita SAcco, Moll. Terr. Terz Piemonte e Liguria, 27, 9. Type (SD): Venericardia jouanneti BASTEROT

Megacardita ferruginosa (ADAMS \& REEVE) フミガイ（目八）

1850 Cardita ferruginosa Adams \& ReEve, Samarang, 76, pl. 21, f. 21

1913 Cardita kiiensis SowERBY, AMNH, (8) 12, 238, pl. 3, f. 14

1920 Venericardia cipangoana YoKоYAMA, JCSIUT, 39 (6), 137, pl. 11, f. $2 \mathrm{a}-\mathrm{c}$

1959 Venericardia (Megacardita) (sic.) ferruginosa: KIRA, CISJ, 1, 130, pl. 52, 18

1971 Megacardita ferruginosa: Kunoda \& HABE, SSSB, 602 (J) 386 (E), pl. 87, f. 5

Loc. N M S (g) gl.

Dist. Honshu (north to Bōsō Peninsula) to Kyushu and Indonesia.

\section{Genus Cyclocardia CoNRADマルフミガイ属}

1867 Cyclocardia Conrad, AJC, 3, 191. Type (SD, StoliczKa, 1871): Cardita borealis ConRAD

Cyclocardia ferruginea (CLESSIN)クロマルフミガイ（滝庸）

1888 Cardita ferruginea CLessin, Cab., 10 (1), 17, pl. 6, f. 11

1920 Venericardia ferruginea: Yoкоумма, JCSIUT, 39 (6), 139, pl. 11, f. 3, 4

1961 Venericardia (Cyclocardia) ferruginea: HABE, CISJ, 2, 122, pl. 55, f. 13

Loc. $S$ (m) tr.

Dist. Southern Hokkaido to Kyushu. 


\section{Genus Glans Megerle von MüHLFELD ヒラセフミガイ属}

1811 Glans Megerle von MüHLfeld, GNFBM, 5, 68. Type (SD, Herrmennsen, 1846): Chama trapezia LinNaeus

Glans hirasei (DALL) ヒラセフミガイ（岩川)

1918 Venericardia hirasei DALI, PUSNM, 54 (2234), 234

1932 Cardita pelseneeri Prashad, Siboga, 53c, 147, pl. 5, f. 1, 2

1959 Glans hirasei: KIRA, CISJ, 1, 130, pl. 52, f. 20

Loc. M S (g) tr.

Dist. Honshu (Kii Peninsula) to Kyushu and Indonesia.

Glans millegrana (NomURA \& ZiNBO) ハナフミガイ（波部)

1934 Venericardia millegrana NomURA \& ZiNBo, SRTIU, (2) 16 (2), 154, pl. 5, f. 13,14

1934 Venericardia quadriangulata NomURA \& ZINBO, SRTIU, (2) 16 (2), 155, pl. 5, f. 15, 16

1961 Glans millegrana: HABE, CISJ, 2, 122, pl. 55, f. 15

Loc. M S (s-m) dp.

Dist. Honshu (Kii Peninsula) to Ryukyu Islands.

\section{Genus Pleuromeris CoNRAD ヒナフミガイ属}

1867 Pleuromeris ConRad, AJC, 3, 12. Type (OD): Pleuromeris decemcostata ConRaD

Pleuromeris pygmaea KURODA \& HABEヒナフミガイ (㲛)

1903 Cardita abbreviata SOWERBY, AMNH, (7) 12, 500 (non CONRAD, 1841)

1951 Venericardia (Pleuromeris) pygmaea KURODA \& HABE, ICJS, 1 (13), 86

1961 Venericardia (Pleuromeris) pygmaea: HABE, CISJ, 2, 122, pl. 55, f. 11

1971 Pleuromeris pygmaea: KURODA \& HABE, SSSB, 603 (J) 387 (E), pl. 87, f. 9

Loc. M S (st) gl.

Dist. Honshu (north to Bōsō Peninsula) to Kyushu.

Subfamily Carditellinae IrEDale \& MCMichaEL ケシザルガイ亜科

Genus Carditella SMITHケシザルガイ属

1881 Carditella SMITH, PZSL, 42. Type (SD, DALL, 1903): Carditella pallida SMITH

$$
\text { Subgenus Carditellona IREDALEケシザルガイ亜属 }
$$

1936 Carditellona Iredale, RAM, 19, 272. Type (OD): Carditella angasi SMtTH

Carditella (Carditellona) hanzawai (NomURA)ケシザルガイ（波部）

1933 Cardium (Cerastoderma) hanzawai NomURA, SRTIU, (2) 16 (1), 79, pl. 3, f. 18a, b, 19a, b

1952 Carditellona hanzawai: HABE, ICJS, 1 (18), 128, pl. 18, f. 17, 18

1961 Carditellona hanzawai: HABE, CISJ, 2, 123, pl. 56, f. 2

Loc. M (s) dp.

Dist. Honshu (Kii Peninsula) to East China Sea and Japan Sea.

Genus Carditellopsis IREDALE，ケシフミガイ属

1936 Carditellopsis Iredale, RAM, 19, 272. Type (OD): Carditella elegantula TATE \& MAY

Carditellopsis toneana (YoKоYAMA) ケシフミガイ（波部)

1922 Venericardia toneana YokoYAmA, JCSIUT, 44, (1), 163, pl. 13, f. 17, 18 
1952 Carditellopsis toneana: HABE, ICJS, 1 (18), 128, pl. 18, f. 16

1961 Carditellopsis toneana: HABE, CISJ, 2, 123, pl. 56, f. 1

1971 Carditellopsis toneana: Kuroda \& HABE, SSSB, 604 (J) 387 (E), pl. 118, f. 4

Loc. M S (s) dp.

Dist. Honshu (north to Bōsō Peninsula) to Kyushu and Japan Sea.

\section{Superfamily Astartacea D’ORBIGNY エゾシラオガイ造科 \\ Family Astartidae D’ORBIGNY エゾシラオガイ科 \\ Genus Tridonta SCHUMACHERエゾシラオガイ属}

1817 Tridonta Schumacher, ENSVT, 49, 146. Type (M): Tridonta boroalis Schumacher

Tridonta bennettii (DALL) コエゾシラオガイ（黒田）

1903 Astarte bennettii DALL, PUSNM, 26, 946, pl. 63, f. 6

1935 Astarte aomoriensis Nomura \& HaTAI, SHKMRB, 6, 115, pl. 9, f. 5.

1952 Astarte (Tridonta) bennettii: HABE, ICJS, 1(22), 163, pl. 23, f. 1.

1961 Astarte (Tridonta) bennettii: HABE, CISJ, 2, 121, pl. 55, f. 7.

Loc. S (s) tr.

Dist. Honshu (south to Kii Peninsula), Hokkaido to Alaska and Japan Sea.

Remark: This is a doubtful record from Wakayama Pref.

\section{Superfamily Crassatellacea FÈRUSSAC モシオガイ超科}

$$
\begin{array}{cl}
\text { Family Crassatellidae FÈRUSSAC } & \text { モシオガイ科 } \\
\text { Subfamily Crassatellinae FÈRUSSAC } & \text { モシオガイ亜科 }
\end{array}
$$

Genus Eucrassatella IREDALEモシオガイ属

1924 Eucrassatella IreDAle, PLSNSW, 49, 181, 202. Type (OD): Crassatella kingicola LAMARCK

Subgenus Nipponocrassatella KURODA \& HABEモシオガイ亜属

1971 Nipponocrassatella KuRoDA \& HABE, SSSB, 598 (J) 383 (E). Type (OD): Crassatella nana A. ADAMs \& ReEve

Eucrassatella (Nipponocrassatella) nana (ADAMs \& REEVE) スダレモシオガイ（介志）

1850 Crassatella nana Adams \& ReEve, Samarang, 81, pl. 23, f. 2

1866 Crassatella nana: Koвelt, Cab., 10 (1a), 15, pl. 6, f. 3

1866 Crassatella loebbeckei KoBelt, Cab., 10 (1a), 18, pl. 2, f. 6

1959 Crassatellites nanus: KIRA, CISJ, 1, 130, pl. 52, f. 13

Loc. N M S (s) gl.

Dist. Honshu (north to Bōsō Peninsula) to Kyushu and South China Sea.

Eucrassatella (Nipponocrassatella) japonica (DUNKER) モシオガイ（目八）

1882 Crassatella japonica Dunker, Index, 220

1889 Crassatella japonica: SowerbY, JLSLZ, 20, 399, pl. 25, f. 19

1895 Crassatellites heteroglyptus PILsBry, CMMJ, 135 (new name for Crassatella japonica SOWERBY, 1889 non DUNKER, 1882)

1959 Crassatellites japonicus: KirA, CISJ, 1, 129, pl. 52, f. 12

1971 Eucrassatella (Nipponocrassatella) japonica: KuRODA \& HABE, SSSB, 598 (J), 383 (E), pl. 87 , f. 4 
Loc. N M S (s) gl.

Dist. Honshu (north to Bōsō Peninsula) to Kyushu.

Eucrassatella (Nipponocrassatella) sagamiensis KURODA \& HABE

サガミモシオガイ（黒田・波部）

1971 Eucrassatella (Nipponocrassatella) sagamiensis KURODA \& HABE, SSSB, 598 (J) 383 (E), pl. 87 , f. 1

Loc. $S$ (s). dp.

Dist. Honshu (Sagami Bay and off Kushimoto).

Genus Indocrassatella CHAVANワタゾコモシオガイ属

1952 Indocrassatella Chavan, Chaies Geol. 14, 119. Type (OD): Crassatella indica SMrtH

Indocrassatella oblongata (YoKOYAMA) ワタゾコモシオガイ（波部)

1920 Crassatella oblongata YokочамA, JCSIUT, 39, (6), 143, pl. 11, f. 8, 9

1961 Crassatina oblongata: HABE, CISJ, 2, 122, pl. 55, f. 9

1971 Crassatina oblongata KURODA: \& HABE, SSSB, 599 (J) 384 (E), pl. 87, f. 2, 3

Loc. M S (s) gl.

Dist. Honshu (north to Bōsō Peninsula) to Kyushu.

\section{Superfamily Cardiacea LAMARCK ザルガイ超科 \\ Family Cardiidae LAMARCK ザルガイ科 \\ Subfamily Trachycardiinae STEWART ザルガイ亜科 \\ Genus Vasticardium IREDALE ザルガイ属}

1927 Vasticardium Iredale, RAM, 16, 75. Type (OD): Vasticardium nebulosum Iredale

Vasticardium arenicola (REEVE) キヌザルガイ（目八）

1845 Cardium arenicolum(sic) REEVE, Icon., sp. 78

1959 Vasticardium arenicola: KIRA, CISJ, 1, 139, pl. 55, f. 10

1971 Vasticardium arenicola: Kuroda \& HABE, SSSB, 619 (J) 398 (E), pl. 89, f. 1, 2.

Loc. N M S (s) dp.

Dist. Honshu (north to Bōsō Peninsula) to Kyushu. Tropic Pacific.

Vasticardium beauforti (PRASHAD) - - ソバカスザルガイ（波部)

1932 Cardium beauforti Prashad, Siboga, 53c, 270, pl. 6, f. 27, 28

Loc. S (s) dp.

Dist. Honshu (north to Izu Peninsula) and Shikoku to Indonesia.

Vasticardium burchardi (DUNKER) ザルガイ（目八）

1877 Cardium burchardti (sic) DUNKER, M B L, 24, 67

1882 Cardium burchardi: Dunker, Index, 210, pl. 18, f. 4-6

1959 Vasticardium burchardi: KIRA, CISJ, 1, 135, pl. 53, f. 16

1971 Vasticardium burchardi: KuRODA \& HABE, SSSB, 620 (J) 399 (E), pl. 89, f. 4

Loc. N M S (s) gl.

Dist. Honshu (north to Bōsō Peninsula) to Kyushu. 
Vasticardium enode (SowERBY) ナガザルガイ（岩川）

1841 Cardium enode Sowerby, PZSL, 8 (92) (1840), 108

1841 Cardium enode: Sowerby, Conch. Illust., f. 51

1845 Cardium enode: ReEve, Icon., sp. 73

1959 Vasticardium enode: KIRA, CISI, 1, 135, pl. 53, f. 17

1971 Vasticardium enode: Kuroda \& HABE, SSSB, 620 (J) 399 (E), pl. 89, f. 3

Loc. N M S (s) gl.

Dist. Honshu (north to Bōsō Peninsula) to Kyushu. Widely ranging in the Indo-Pacific.

Vasticardium transcendens (MeLVILL \& STANDEN, 1899) ッッ゙レザルガイ〔桜井・波部)

$$
\text { ヒガノコザルガイ（黒田） Pl. 1, f. } 5 .
$$

1899 Cardium (Trachycardium) transcendens MeLvill \& StANDen, JLSLZ, 27, 191, pl. 2, f. 21.

1928 Cardium perstriatum KURODA, Cat. Shell Moll. Amami-Oshima, p. 11.

1966 Laevicardium pulcherrimum SAKURAI \& HABe, Venus, 24 (4), pp. 293, 295.

1977 Laevicardium pulcherrimum: HABE, Taxa HABE, 99, pl. 1, f. 5.

Loc. S (s) dp.

Dist. Honshu (north to Wakayama Pref.), Amami and Ryukyu Islands. Tropic and Western Pacific.

\section{Subfamily Fraginae STEWART オオヒシガイ亜科 \\ Genus Fragum RöDING オォヒシガイ属}

1798 Fragum RöDNG, Bolten, 189

1840 Hemicardium Swainson, Treat., 373 (non SchweIGger, 1820). Type (T): Cardium fragum LINNAEUS

Fragum bannoi (OTUKA) ヒシガイ（六介）

1909 Hemicardium (Fragum) carinata LJNGE, Del. Kgl. Vidersk, Selsk., (7) 5 (3), 261, pl. 5, f. 20 (non BRonN, 1832)

1937 Corculum (Fragum) bannoi OTUKA, Venus, 7 (3), 138, tf. 54a, b

1959 Fragum bannoi: KrRA, CISJ, 1, 136, pl. 54, f. 11

1961 Fragum carinatum: HABE, CISJ, 2, 127, pl. 57, f. 18

Loc. S (s) int.

Dist. Honshu (north to Bōsō Peninsula) to Formosa and the Western Pacific.

\section{Genus Lunulicardia GRAY モクハチアオイガイ属}

1853 Lunulicardia Gray, AMNH, (2) 11, 41

1879 Opisocardium Bayle, J. de C., 27, 35. Type (M): Cardium retusum Linnaeus

Lunulicardia retusa (LINNAEUs) モクハチアオイガイ（岩川)

1767 Cardium retusum Linnaeus, Syst. Nat. 12, 1121

1845 Cardium retusum: ReEve, Icon., sp. 103

1959 Lunulicardia retusa: KIRA, CISJ, 1, 137, p1. 54, f. 16

Loc. M S (s) int.

Dist. Honshu (north to Kii Peninsula). Widely ranging in the Indo-Pacific.

\section{Genus Afrocardium ToMLIN ベニバトガイ属}

1931 Afrocardium Tomun, Ann. Natal. Mus., 6, 449. Type (OD): Fragum (Afrocardium) shepstonense TomLIN 
Afrocardium ebaranum (YoKOYAMA)ベニバトガイ (目八)

(1845 Cardium carditaeforme ReEve, Icon., sp. 127)

1927 Cardium ebaranum YoKoYama, JFSIUT, (2) 1 (10), 430, pl. 48, f. 15

1959 Afrocardium ebaranum: KIRA, CISJ, 1, 136, pl. 54, f. 5

1971 Afrocardium ebaranum: KunODA \& HABE, SSSB, 625 (J) 402 (E), pl. 88, f. 10

Loc. M S (s) int.

Dist. Honshu (north to Bōsō Peninsula) to Kyushu.

Afrocardium infantile (NomuRA \& ZINBO)マルル゙ニバトガイ（波部） Pl. 1, f. 7.

1831 Cardium radula ThieLE \& JACKel, Tiefsee, 21 (1), 227, pl. 4, f. 99 (non BroderIP \& SOWERBy, 1826 nec Deshayes, 1832)

1834 Cardium (Trachycardium) infantile NomURA \& ZINBO, SRTIU, (2) 16, 156, pl. 5, f. 17a, b

1977 Laevicardium (Trachycardium) thielei Fischer-PIETTE, MMNHN (A), 101, 55

Loc. M S (s) dp.

Dist. Honshu (north to Izu Islands) to Kyushu and Indian Ocean.

Genus Microfragum HABE キヌヒシガイ属

1952 Microfragum Habe, GJS, 148. Type (OD): Cardium festivum DeshayeXs

Microfragum festivum (DESHAYES) キヌヒシガイ（活部）

1855 Cardium festivum DeSHAyes, PZSL, (1854), 332

1932 Cardium (Ctenocardia ?) festivum: Prashad, Siboga, 53c, 279, pl. 6, f. 29-32

1959 Microfragum festivum: KIRA, CISJ, 1, 136, pl. 56, f. 10

Loc. S (s) tr.

Dist. Honshu (north to Kii Peninsula) to Ryukyu Islands and Indonesia.

Genus Trigoniocardia DALL ハナザルガイ属

1900 Trigoniocardia TWFIP, 3 (5), 1075. Type (OD): Cardium graniferum BRODERIP \& SOWERBY

Trigoniocardia fornicata (SOWERBY)ハナザルガイ（黒田）

1841 Cardium fornicatum SOWERBY PZSL (1840), p. 110

1841 Cardium fornicatum SOWERBy, Conch. Illust., 7, f. 50a, b

1850 Cardium adamsi Adams \& ReEve, Samarang, 77, pl. 22, f. 2

1966 Trigoniocardia adamsi HABE \& Kosuge, SWC, 2, 153, pl. 59, f. 3.

Loc. M S (s) dp.

Dist. Honshu (north to Kii Peninsula) to Ryukyu Islands. Tropical Pacific region.

\section{Subfamily Protocardiinae KEEN キンギョガイ巠科 \\ Genus Frigidocardium HABEアアサヒザルガイ属}

1951 Frigidocardium HaBe, GJS, (2), 152 (Erigidocardium in error), 278. Type (OD): Cardium eos KUROdA

Frigidocardium torresi (SMITH)アサヒザルガイ（黑田）

1885 Cardium (Fragum) torresi SMITH, Challenger, 164, pl. 8, f. 4-4b

1929 Frigidocardium eos: KuRoDA, Venus, 1, 93, pl. 3, f. 3

1959 Frigidocardium eos: KIRA, CISJ, 1, 136, pl. 54, f. 7

1971 Frigidocardium eos: Kuroda \& HABE, SSSB, 622 (J) 401 (E), pl. 88, f. 13 
Loc. N M S (s) dp-tr.

Dist. Honshu (north to Philippines).

Frigidocardium exasperatum (SOWERBY)シモオキザルガイ（金丸）

1841 Cardium exasperatum Sowerby, PZSL, 8 (92) (1840), 106

1845 Cardium exasperatum: ReEve, Icon., sp. 107

1959 Frigidocardium exasperatum: KIRA, CISJ, 1, 136, pl. 54, f. 8

Loc. N M S (s) dp-tr.

Dist. Honshu (Kii Peninsula) to the Western Pacific.

Genus Trifaricardium KURODA \& HABEオトヒメザルガイ属

1951 Trifaricardium KURODA \& HABE, GJS, 2, 152. Type (OD): Cardium nomurai KURODA \& $\mathrm{HABE}$

Trifaricardium nomurai KURODA \& HABEオトヒメザルガイ（黒田）

ミカワリザルガイ（吉良）

1933 Cardium cancellatum NomurA, SRTIU, (2) 16 (1), 81, pl. 3, f. 91, b (non GMELIN, 1791)

1951 Trifaricardium nomurai KURODA \& HABE, ICJS, 1 (13) , 86

1959 Trifaricardium nomurai: KIRA, CISJ, 1, 136, pl. 54, f. 6. .

Loc. N M S (s) tr.

Dist. Honshu (Kii Peninsula) to Shikoku.

Genus Microcardium THIELE サクライキンギョガイ属

1951 Microcardium ThIELE, Weicht. 3, 878. Type (SD, KeEN, 1937): Cardiumperamabile DALL

Microcardium sakuraii HABEサクライキンギョガイ（波部）

1961 Nemocardium (Microcardium) sakuraii HABE, Venus, 21 (2), 152, f. 9

1961 Nemocardium (Microcardium) sakuraii: HABE, CISJ, 2, 127, pl. 57, f. 27

Loc. M S (s) tr.

Dist. Honshu (Kii Peninsula) to Shikoku.

\section{Genus Nemocardium MEEK キンギョガイ属}

1876 Nemocardium MeeK, Rep. U. S. Geol. Surv. (HAYden), 9, 167. Type (SD, SACCO, 1889): Cardium semiasperum DeshaYes

Nemocardium bechei (REEVE) キンギョガイ（六介）

1947 Cardium bechei ReEve, PZSL, 25

1882 Cardium bechei: Dunker, Index, 212, pl. 15, f. 1-3

1959 Nemocardium bechei: KIRA, CISJ, 1, 137, pl. 54, f. 18

1971 Nemocaraium bechei: Kuroda \& HABE, SSSB, 623 (J) 401 (E), pl. 88, f. 8

Loc. N M S (s) gl.

Dist. Honshu (north to Bōsō Peninsula) to Kyushu. Widely ranging in the Indo-West Pacific.

\section{Genus Discors DEshaYES ギンギョガイ属}

1858 Discors Deshayes, Basin. Paris, 1, 553, 569. Type (T): Cardium discors Lamarck

\section{Subgenus Lyrocardium MEEK ギンギョガイ亜属}

1876 Lyrocardium MeEk, Rep. U. S. Geol. Surv. (HAYdeN), 9, 173. Type (SD, Dall, 1900): Cardium lyratum SOWERBY 
Discors (Lyrocardium) lyrata (SowERBY) ギンギョガイ（群品）

1841 Cardium lyratum SowerBy, PZSL, 8, (1840), 109

1845 Cardium lyratum: ReEve, Icon., sp. 12

1959 Discors lyratum: KIRA, CISJ, 1, 137, p1. 54, f. 19

Loc. M S (s) gl.

Dist. Honshu (north to Bösō Peninsula) to Kyushu. Widely ranging in the Indo-Pacific.

\section{Genus Keenaea HABEシマキンギョガイ属}

1951 Keenaea Habe, GJS, (2), 152. Type (OD): Cardium samarangae MakiYama,

Keenaea samarangae (MAKIYAMA)シマキンギョガイ（黒田）

1840 Cardium modestum Adams \& Reeve, Samarang, 77, pl. 22, f. 6 (non Philrppi, 1849)

1872 Cardium adamsi Tryon, AJC, 7, 267 (non Adams \& ReeVE, 1850)

1934 Cardium samarangae MAKIYAMA, MSCKIU, (B), 10 (2), 143

1959 Nemocardium (Keenaea) samarangae: KIRA, CISJ, 1, 136, pl. 54, f. 8, 9

1971 Nemocardium (Keenaea) samarangae: KurodA \& HABE, SSSB, 624 (J) 402 (E), pl. 88, f. 11,12

Loc. M (s) tr.

Dist. Honshu (north to Bōsō Peninsula) to East China Sea.

$$
\begin{array}{cc}
\text { Subfamily Laevicardiinae KEEN } & \text { マクラザルガイ亜科 } \\
\text { Genus Laevicardium SWAINSON } & \text { マクラザルガイ属 }
\end{array}
$$

1840 Laevicardium Swainson, Treat., 373. Type (SD, Stolrczka, 1871): Cardium oblongum GMELIN

Laevicardium biradiatum (BRUGurèrE)スベリザルガイ（悬田）

1789 Cardium biradiatum BRUGUìere, EMHNV, 1, 231

1845 Cardium biradiatum: ReEve, Icon., sp. 49

1959 Laevicardium biradiatum: KIRA, CISJ, 1, 137, pl. 53, f. 5

1966 Laevicardium biradiatum: HABE \& KosUGE, SWC, 2, 154, pl. 59, f. 11

Loc. S (s) tr.

Dist. Honshu (Kii Peninsula) to Ryukyu Islands. Widely ranging in the IndoPacific.

Laevicardium rubropictum HABE \& Kosugeアアフザルガイ（波部・小管） Pl. 1, f. 6. 1966 Laevicardium rubropictum HABE \& KosUGE, SWC, 2, 153, pl 59, f. 2

Loc. M S (s) dp.

Dist. Honshu (north to Kii Peninsula) to Ryukyu Islands. Widely ranging in the tropic Pacific.

Remarks: According to Wilson \& Stevenson's paper (1978) this species is as same as Laevicardium biradiatum BRUGUIÉRE, 1789, while Reeve's figure of Cardium biradiatum is quite different from this species.

\section{Genus Clinacardium KEEN イシカゲガイ属}

1936 Clinocardium KeEn, San Diego, 8 (17), 119. Type (OD): Cardium nuttallii ConRAD 


\section{Subgenus Keenocardium KAFANOV イシカゲガイ里属}

1974 Keenocardium Kafanov, Zool. Zh., 53 (10), 1468. Type (OD): Cardium californiense Deshayes

Clinocardium (Keenocardium) buellowi (ROLLE) イシカゲガイ（六介）

1896 Cardium buellowi Rolse, M B L. 114, tf. c

1898 Cardium vulva JousseAume, Le Nat., 12, 81

1959 Clinocardium buellowi: KIRA, CISJ, 1, 138, pl. 55, f. 3

1971 Clinocardium californiense buellowi: KuRODA \& HABE, SSSB, 622 (J) 400 (E), pl. 88, f. 9

Loc. $\mathrm{N}$ (s) dp.

Dist. Southern Hokkaido to Kyushu.

\section{Genus Fulvia GRAY トリガイ属}

1853 Fulvia Gray, AMNH, (2) 11, 40. Type (M): Cardium apertum Bruguière

Fulvia undatopicta (PILSBRY) マダラチゴトリガイ（岩川)

1904 Cardium hungerfordi undatopictum PILSBRY, PANSP, 56, 556, pl. 40, f. 14, 15

1904 Cardium hungerfordi stigmaticum PILSBRY, PANSP, 56, 556, pl. 41, f. 13, 14

1959 Laevicardium undatopictum: KTRA, CISJ, 1, 135, pl. 54, f. 2

1971 Laevicardium undatopictum: KURODA \& HABE, SSSB, 621 (J) 399 (E), pl. 89, f. 5, 6

Loc. N M S (s) dp.

Dist. Honshu (north to Bōsō Peninsula) to Kyushu. Widely ranging in the Indo Pacific.

Fulvia mutica (REEVE) トリガイ (目八)

1844 Cardium mutica Reeve, Icon., sp. 32

1860 Cardium japonicum DUNKER, M B L. 6, 223

1861 Cardium japonicum: DunkeR, MJ, 28, pl. 3, f. 16

1904 Cardium annae Pilsbry, PANSP, 56, 557, pl. 40, f. 20

1959 Fulvia mutica: KirA, CISJ, 1, 194, pl. 71, f. 2

1971 Fulvia mutica: KuRODA \& HaBe, SSSB, 621 (J) 400 (E), pl. 89, f. 7

Loc. N M S (s-m) dp.

Dist. Honshu (north to Mutsu Bay) to the west Pacific.

Fulvia australis (SOWERBY) ボタンガイ（六介）

1841 Cardium australe Sowerby, PZSL, 8 (1840), 105

1845 Cardium australe: REEVE, Icon., sp. 97

1845 Cardium pulchrum REEVE, Icon., sp. 98

1959 Fulvia australis: KIRA, CISJ, 1, 136, pl. 54, f. 4

Loc. N M S (s-m) dp.

Dist. Honshu (Kii Peninsula) to Kyushu. Widely ranging in the Indo-Pacific.

Fulvia aperta (BRUGUIÈRE) エマイボタンガイ（黒田）

1789 Cardium apertum BRUGUİRE, EMHNV, 1, 226

1817 Cardium rugata: Dillwyn, DCRS., 1, 125

1844 Cardium rugatum: REEVE, Icon., sp. 63

1959 Fulvia aperta: KIRA, CISJ, 1, 135, pl. 54, f. 3

Loc. N M S (s) dp.

Dist. Honshu (north to Bōsō Peninsula) to Kyushu. Widely ranging in the Indo-Pacific. 
Fulvia hungerfordi (SOWERBY) チゴトリガイ（岩川）

1901 Cardium hungerfordi SowerBY, J. of M., 8, 108, pl. 9, f. 5

1904 Cardium hungerfordi: PrLsBry, PANSP, 56, 555, pl. 41, f. 17

1959 Fulvia hungerfordi: KIRA, CISJ, 1, 135, pl. 54, f. 1

Loc. N M S (m) dp.

Dist. Honshu (north to Bōsō Peninsula) to Kyushu and China.

\title{
Superfamily Mactracea LAMARCKバカガイ超科
}

\author{
Family Mactridae LAMARCK バカガイ科 \\ Subfamily Mactrinae LAMARCK バカガイ亜科 \\ Genus Mactra LINNAEUS バカガイ属
}

1767 Mactra Linnaeus, Syst. Nat., 12, 1125. Type (SD, Fleming, 1818): Cardium stultorum LINNAEUS

1778 Trigonella DA CosTA, Brit. Conch., 196.

1929 Colorimactra IRLDALE, MQM, 9, 268.

\section{Subgenus Mactra LINNAEUSバカガイ亜属}

Mactra (Mactra) chinensis PHILIPPI バカガイ（目八）

1846 Mactra chinensis PHLIPPI, Abbild., 2, 73

1854 Mactra sulcataria ReEve, Icon., sp. 5

1904 Mactra carneopicta PiLSBRy, PANSP, 56, 550, pl. 39, f. 1-3

1959 Mactra sulcataria: KIRA, CISJ, 1, 150, pl. 58, f, 10

1971 Mactra chinensis: Kuroda \& HABE, SSSB, 668 (J) 435 (E), p1. 95, f. 6

Loc. N M S (s-m) int.

Dist. Hokkaido to Kyushu, Korea and China.

Mactra (Mactra) ornata GRAYベニハマグリ（六介）

1837 Mactra ornata GRAY, AMNH, 1, 371

1854 Mactra ornata: ReEve, Icon., sp. 58

1959 Mactra ornata: KIRA, CISJ, 1, 150, pl. 58, f. 7

1971 Mactra ornata: KURODA \& HABE, SSSB, 669 (J) 435 (E), pl. 95, f. 11

Loc. M S (s) dp.

Dist. Honshu (north to Bōsō Peninsula) to Kyushu. Widely ranging in the Indo-West Pacific region.

Mactra (Mactra) nipponica KURODA \& HABEチゴバカガイ（黒田）

1971 Mactra nipponica KURODA \& HABE, SSSB, 668 (J) 436 (E), pl. 96, f. 1, 2

Loc. $M$ (s-m) dp.

Dist. Honshu (north to Bōsō Peninsula) to Kyushu.

Mactra (Mactra) veneriformis REEVEシオフキガイ（六介）

1854 Mactra veneriformis REEVE, Icon., sp. 2

1854 Mactra quadriangularis ReEVE, Icon., sp. 3

1858 Mactra bonneaui Bernard, J. de C., 7, 92, pl. 2, f. 2

1871 Mactra veneriformis zonata LischKE, JMC, 2, 121, pl. 9, f. 7, 8.

1928 Trigonella quadriangularis ventricosa Grabau \& King, Peitaiho, 190, pl. 6, f. 53

1959 Mactra veneriformis: KIRA, CISJ, 1, 151, pl. 58, f. 12 
Loc. N M S (s-m) int.

Dist. Honshu (north to Bōsō Peninsula) to Kyushu, Korea and China.

Mactra (Mactra) maculata GMELINリュウキュウバカガイ（岩川）

1771 Mactra maculata GMELIN, Syst. Nat., 13, 3260

1854 Mactra maculata: ReEve, Icon., sp. 56

1961 Mactra maculata: HABE, CISJ, 2, 133, pl. 60, f. 8

Loc. $\mathbf{S}$ (s) dp.

Dist. Honshu (north to Kii Peninsula) to Ryukyu Islands. Widely ranging in the Indo-Pacific region.

\section{Subgenus Telemactra IREDALEヒメバカガイ亜属}

1929 Telemactra IredAle, MQM, 9, 268. Type (OD): Mactra obesa REEVE

Mactra (Telemactra) iridescens KURODA \& HABEニシミナトガイ（黒田）

1958 Mactra iridescens KURODA \& HABE, PSMBL, 7 (1), 48

1961 Mactra iridescens: HABE, CISJ, 2, 135, pl. 60, f. 6

Loc. M S (s) dp.

Dist. Honshu (Kii Peninsula) to Yakushima, south of Kyushu.

\section{Genus Coelomactra DALLアリソガイ属}

1895 Coelomactra Dall, TWFIP, 3, 875. Type (OD): Mactra violacea GMeLIN

Coelomactra antiquata (SPENGLER) アリソガイ (目八)

1802 Mactra antiquata SPENGLeR, SNS, 5 (2), 102

1854 Mactra cornea REEVE, Icon., sp. 75

1871 Mactra spectabilis LisCHKE, M B L., 18, 149

1871 Mactra spectabilis: LisCHKE, JMC, 2, 120, pl. 11, f. 1, 2

1959 Coelomactra antiquata: KIRA, CISJ, 1, 151, pl. 58, f. 11

1971 Coelomactra antiquata: Kuroda \& HABE, SSSB, 669 (J) 436 (E), pl. 95, f. 9

Loc. N M S (s) dp.

Dist. Honshu (north to Bōsō Peninsula) to Kyushu, Korea and China.

Genus Mactrinula GRAY ソリタママキガイ属

1853 Mactrinula Gray, AMNH, (2) 11, 41. Type (M): Mactra plicaria GraY=Mactra plicataria LINNAEUS

Mactrinula dolabrata (REEVE) ソリタママキガイ（黒田）

1854 Mactra dolabrata ReEVE, Icon., sp. 107

1961 Mactrinula dolabrata: HABE, CISJ, 2, 133, pl. 60, f. 5

Loc. N M (s-m) dp.

Dist. Honshu (north to Bōsō Peninsula) to Indonesia.

\section{Genus Mactrotoma DALLヒナミルガイ属}

1894 Mactrotoma Dall, Naut., 8, 26. Type (OD): Mactra fragilis GMELIN

Mactrotoma depressa (SPENGLER) ヒナミルガイ（滰皦）

1802 Mactra depressa SPENGLER, SNS, 5 (2), 118

1971 Mactra depressa: KuRoda \& HABE SSSB, 669 (J) 437 (E), pl. 95, f. 6 (not of 5) 
Loc. $\mathrm{N}$ (s-m) dp.

Dist. Honshu (north to Bōsō Peninsula) to Kyushu, South-East Asia and Indonesia.

\section{Genus Micromactra DALLワカミルガイ属}

1894 Micromactra Dale, Naut., 8, 40. Type (OD): Mactra californica ConRaD

Micromactra angulifera (REEVE)ワカミルガイ（滝嗾）

1854 Mactra àngulifera ReEve, Icon., sp. 83

1961 Mactra (Micromactra) angulifera: HABE, CISJ, 2, 133, pl. 60, f. 7

Loc. $\mathrm{N}(\mathrm{m}) \mathrm{dp}$.

Dist. Honshu (north to Bōsō Peninsula) to Kyushu and South-East Asia.

\section{Genus Oxyperas MöRCHホクロガイ属}

1853 Oxyperas Mörch, Yoldi., (2), 4. Type (M): Mactra triangularis LAMARCK non MoNTAGU = Mactra lentiginosa GouLD

\section{Subgenus Oxyperas MöRCHホクロガイ粑属}

Oxyperas (Oxyperas) bernardi (PILSBRY)ホクロガイ (平瀨)

1904 Spisula bernardi PILSBRY, PANSP, 56, 550, pl. 39, f. 4-6

1959 Oxyperas bernardi: KiRA, CISJ, 1, 150, pl. 58, f. 8

1971 Oxyperas bernardi: KURODA \& HABE, SSSB, 670 (J) 437 (E), pl. 95, f. 5, 6

Loc. N M S (s-m) dp.

Dist. Honshu (north to Bōsō Peninsula) to Formosa.

Subgenus Pseudoxyperas SACCo オオシマホクロガイ亜属

1901 Pseudoxyperas SAcco. Moll Terr. Terz. Piemonte e Liguria, 29, 26. Type (OD): Pseudoxy peras proaspera $\mathrm{SACCO}$.

Oxyperas (Pseudoxyperas) aspersa (SOWERBY)オオシマホクロガイ（黒田）

1825 Mactra aspersa SowERBY, Tankerville, app. 2.

1966 Oxyperas (Pseudoxyperas) aspersa: HABE, SWC, 2, 165, pl. 65, f. 5.

Loc. M (s-m) gl.

Dist. Honshu (Kii Peninsula), Amami and Ryukyu Islands. Indo-Pacific region.

\section{Subfamily Lutrariinae H. \& A. ADAMS オオトリガイ亜科}

\section{Genus Lutraria LAMARCKオオトリガイ属}

1799 Lutraria LamarCK, MSHNP. Type (T): Mya lutraria Linnaeus

\section{Subgenus Psammophila BROWNオオトリガイ亚属}

1827 Psammophila Brown, Conch. Great Brit., pl. 12, f. 1. Type (M): Lutraria solenoides LAMARCK $=$ Mya oblonga GMELIN

Lutraria (Psammophila) maxima Jonasオオトリガイ（六介）

1844 Lutraria maxima JoNAS, ZfM, 34

1854 Lutraria maxima: REEVE, Icon., sp. 11 
1961 Lutraria maxima: HABE, CISJ, 2, 134, pl. 60, f. 12

1971 Lutraria (Psammophila) maxima: KuRODA \& HABE, SSSB, 671 (J) 439 (E), pl. 69, f. 2

Loc. N M S (s) int.

Dist. Honshu (north to Bōsō Peninsula) to Kyushu, China and South-East Asia.

Lutraria (Psammophila) sieboldii REEVEヒラカモジガイ（介志）

1854 Lutraria sieboldtii (sic) REEVE, Icon., sp. 15

1861 Lutraria lucida Gould, PBSNH, 8, 29

1920 Lutraria radiata YoKoYAMA, JCSIUT, 39 (6), 110, pl. 7, f. 11

1959 Lutraria sieboldii: KIRA, CISJ, 1, 150, pl. 58, f. 5

1964 Lutraria lucida: JoHnson, USNMB, 239, 104, pl. 28, f. 7

1971 Lutraria (Psammophila) sieboldii: Kuroda \& HaBe, SSSB, 672 (J) 439 (E), pl. 97, f. 7, 8

Loc. N M S (s) dp.

Dist. Honshu (north to Bōsō Peninsula) to Kyushu and South East Asia.

Lutraria (Psammophila) arcuata (REEVE) カモジガイ（介志）

1854 Lutraria arcuata ReEve, Icon., sp. 6

1959 Lutraria arcuata: KIRA, CISJ, 1, 150, pl. 57, f. 6

Loc. N M S (s-m) dp.

Dist. Honshu (north to Bōsō Peninsula) to Kyushu, China and South-East Asia.

Remarks: The preceding species seems to be a form of this species.

\section{Genus Tresus GRAY ミルクイガイ属}

1853 (Jan.) Tresus Gray, AMNH, (2) 11, 42. Type (M): Lutraria maxima MidDendorfF non JONAs = Schizothaerus keenae KuRODA \& HABE

1853 (Feb.) Schizothaerus ConRAD, PANSP, 6, 199. Type (M): Lutraria nuttalli CONRAD

Tresus keenae (KuRODA \& HABE) ミルクイガイ (丹浦)

1849 Lutraria maxima Middendorff (non Jonas, 1844 nec ReEve, 1845), Malac. Rossica., 3, 582 , pl. 19, f. 1-4

1852 Schizothaerus keenae KURODA \& HABE, Check., 50

1959 Schizothaerus keenae: KIRA, CISJ, 1, 150, pl. 58, f. 9

1971 Tresus keenae: KurODA \& HABE, SSSB, 671 (J) 438 (E), pl. 96, f. 1

Loc. N M S (s-m) dp.

Dist. Saghalien to Kyushu, Korea and Maritime Prov. of Siberia.

\section{Genus Meropesta IREDALEユキガイ属}

1856 Merope H. \& A. AdAMs, GRM, 2, 382 (non Newman, 1838).

1929 Meropesta IREDALE, MQM, 9, 268. Type (SD, StolicZKA, 1861): Mactra nicobarica GMELIN

Meropesta nicobarica (GMELIN)ユキガイ（渚錦）シロマスオガイ（岩川）

1791 Mactra nicobarica, Gmedrn, Syst. Nat., 13, 3261

1854 Mactra aegyptica ReEve, Icon., sp. 112

1959 Sandella (Meropesta) nicobarica: KIRA, CISJ, 1, 150, pl. 58, f. 4

1971 Meropesta nicobarica: KURODA \& HABE, SSSB, 673 (J) 439 (E), pl. 95, f. 4

Loc. N M S (s) int.

Dist. Honshu (north to Bōsō Peninsula) to Kyushu. Widely ranging in the Indo-Pacific region. 


\section{Subfamily Pteropsellinae KEENヤチヨノ八ナガイ亜科}

\section{Genus Raeta GRAY ヤチョノハナガイ属}

1853 Raeta GraY, AMNH, (2) 11, 43. Type (M): Mactra campechiensis GraY=Mactra plicatella LAMARCK

\section{Subgenus Raetina DALL ヤチョノハナガイ亜属}

1898 Raetina DALL, TWFIP, 3 (4), 882. Type (OD): Labiosa (Raetina) indica DALL

Raeta (Raetina) pellicula (REevE) ヤチヨノハナガイ（黒田）

1854 Mactra pellicula REEVE, Icon., sp., 124

1922 Raeta magnifica Yoкочама, JCSIUT, 44, (1), 132, pl. 8, f. 12, 13

1932 Raeta fragilis Grabau \& King, Peitaiho, 190, pl. 7, f. 266

1961 Raeta (Raetina) pellicula (sic) HABE, CISJ, 2, 133, pl. 60, f. 9

Loc. $\mathbf{N}$ (m) dp.

Dist. Honshu (north to Bōsō Peninsula) to Kyushu, China and South-East Asia.

\section{Genus Raetellops HABEチョノハナガイ属}

1952 Raetellops Habe, GJS, 3, 197. Type (OD): Poromya pulchella A. Adams \& Reeve

Raetellops pulchella (AdAMs \& ReEve) チョノハナガイ（群品）

1850 Poromya pulchella ADAMs \& REEVE, Samarang, 83, pl. 23, f. 1

1895 Raeta yokohamensis PILSBRY, CMMJ, 119, pl. 3, f. 4, 5

1922 Raeta elliptica Yокочама, JCSIUT, 44 (1), 131, pl. 8, f. 7

1959 Raeta (Raetellops) pulchella: KIRA, CISJ, 1, 149, pl. 58, f. 1

1971 Raetellops pulchella: KuRODA \& HABE, SSSB, 670 (J) 437 (E), pl. 95, f. 7, 8

Loc. N M S (s-m) dp.

Dist. Hokkaido to Kyushu. Korea, China and South-East Asia.

\section{Family Mesodesmatidae GRAY チドリマスオガイ科 \\ Subfamily Mesodesmatinae GRAY チドリマスオガイ亜科}

Genus Atactodea DALL イソハマグリ属

1895 Atactodea DALI, PMSL, 1, 213. Type (OD): Mactra glabrata GMELrN=Mactra striata GMELIN

Atactodea striata (GMELIN) イソハマグリ（目八）

1791 Mactra striata GmeLIN, Syst. Nat., 13, 3257

1791 Mactra glabrata GmeLrN, Syst. Nat., 13, 3258

1854 Mesodesma striata: ReEVE, Icon., sp. 10

1959 Atactodea striata: KIRA, CISJ, 1, 149, pl. 57, f. 33

Loc. N M S (s) int.

Dist. Honshu (north to Bōsō Peninsula) to Kyushu. Widely ranging in the Indo-Pacific region.

\section{Genus Donacilla BLAINVILLEチドリマスオガイ属}

1819 Donacilla Blainville, DSN, 13, 429. Type (M): Donacilla lamarckii PhILIPPI= Amphideoma donacilla LAMARCK 
Donacilla picta DUNKER チドリマスオガイ (丹浦)

1877 Donacilla picta Dunker, M B L., 24, 64

1882 Donacilla picta: DUnKer, Index, 175, pl. 7, f. 7-10

1961 Donacilla picta: HABE, CISJ, 2, 133, pl. 60, f. 3

Loc. N M S (s) int.

Dist. Honshu (north to Bōsō Peninsula and Noto Peninsula) to Ryukyu Islands.

\section{Subfamily Davilinae DALL ナミノコマスオガイ亜科 \\ Genus Davila GRAY ナミノコマスオガイ属}

1853 Davila GraY, AMNH, 2 (11), 44. Type (M): Davila polita GraY=Mesodesma planum HANLEY

Davila plana (HANLEY) ナミノコマスオガイ（黒田）

1843 Mesodesma planum HANLEY, PZSL, 102

1851 Mesodesma munda Gould, PBSNH, 3, 217

1853 Davila picta GrAY, AMNH, (2) 11, 44

1854 Mesodesma crassula ReEve, Icon., sp. 26

1959 Davila crassula: KIRA, CISJ, 1, 149, pl. 57, f. 31

Loc. S (s) int.

Dist. Honshu (Wakayama Pref.) to Ryukyu Islands. Indo-Pacific region.

\section{Subfamily Ervilinae DALL ハマチドリガイ亜科 \\ Genus Coecella GRAYクチバガイ属}

1853 Coecella Gray, AMNH, 2 (11), 43. Type (M): Coecella horstfieldii GraY

(Caecella auct in error.)

Coecella chinensis (DESHAYES) クチバガイ（目八）

1855 Coecella zebuensis Deshayes, PZSL (1854), 334

1855 Coecella chinensis Deshayes, PZSL (1854), 334

1855 Coecella convexa DeShaYes, PZSL (1854), 334

1859 Coecella lata DeshaYes, PZSL (1854), 334

1869 Coecella chinensis: LISCHKE, JMC, 1, 133, pl. 10, f. 5, 6

1920 Ervilia otsuensis YoKoYAMA, JCSIUT, 39 (6), 109, pl. 17, f. 21, 22

1959 Coecella chinensis: KrRA, CISJ, 1, 149, pl. 57, f. 32

1971 Coecella chinensis: Kuroda \& HABE, SSSB, 666 (J) 434 (E), pl. 95, f. 3

Loc. N M S (g) int.

Dist. Southern Hokkaido to Kyushu, Korea, China and Philippines.

Genus Spondervilia IREDALE ハマチドリガイ属

1930 Spondervilia IreDALe, RAM, 17, 402. Type (OD): Ervilia australis ANGAS =Ervilia bisculpta GouLD

Spondervilia bisculpta (GouLD) ハマチドリガイ（黒田）

1861 Ervilia bisculpta GouLD, PBSNH, 8, 28

1861 Ervilia livida GouLD, PBSNH, 8, 28

1862 Ervilia japonica A. ADAMS, AMNH, (3) 9 (51), 224

1877 Ervilia australis ANGAS, PZSL, 175, pl. 26, f. 21

1938 Ervilis (Spondervilia) ambla DALL, Bartsch \& Rehder, BPBMB, 153, 44, pl. 44, f. 5-8

1971 Spondervilia bisculpta: KuRODA \& HABE, SSSB, 667 (J) 434 (E), pl. 121, f. 1 
Loc. M S (s) dp.

Dist. Honshu (north to Bōsō Peninsula) to Kyushu, South East Asia, Northern Australia and Hawaii.

\section{Genus Rochefortina DALL ハコベノハナガイ属}

1924 Rochefortina DALL, PBSW, 37, 88. Type (OD): Rochefortina semele DALL=Ervilia sandwichensis SMTTH

Rochefortina sandwichensis (SMIT)ハコベノハナガィ（大山） Pl. 6, f. 8.

1885 Ervilia sandwichensis SMITH, Challenger, 13 (1), 81, pl. 25, f. 5, 5a, b

1924 Rochefortina semele DALL, PBSW, 37, 88

1938 Spondervilia sandwichensis: DALL, Bartsch \& Rehder, BPBMB, 153, 169, pl. 44, f. 9-12

1953 Spondervilia rubra LASERON, RAM, 23 (2) 52, f. 42, 42a, 42b

Loc. M S (s) dp.

Dist. Honshu (Kii Peninsula), Hachijo Island, Gotō Islands, North Australia and Hawaii.

\section{Family Cardiliidae FISCHER キサガイ科 \\ Genus Cardilia DESHAYES キサガイ属}

1835 Cardilia Deshayes, AsV, (2), 6, 448. Type (SD, Herrmannsen, 1846): Isocardia semisulcata LAMARCK

Cardilia semisulcata (LAMARCK) キサガイ（丹浦）ゾウガイ（岩川）

1819 Isocardia semisulcata LAMARCK, AsV, 6 (1), 32

1873 Cardilia semisulcata: SOWERBY, Icon., sp. 1

1882 Cardilia semisulcata: DUNKER, Index, 213, pl. 8, f. 1-3

1951 Cardilia semisulcata: HABE, ICJS, 1 (15), 98, tf. 16-19

1961 Cardilia semisulcata: HABE, CISJ, 2, 134, pl. 60, f. 13

1971 Cardilia semisulcata: KuRODA \& HABE, SSSB, 673 (J) 440 (E), pl. 97, f. 11, 12

Loc. N M S (s) dp.

Dist. Honshu (north to Bōsō Peninsula and Noto Peninsula) to Kyushu, South Asia and North Australia.

\section{Superfamily Tellinacea de BLAINVILLE ニッコウガイ超科}

Family Donacidae FLEMING フジノハナガイ科

\section{Genus Chion SCOPOLIフジノハナガイ属}

1777 Chion Scopoli, Introd,, 308. Type (M): Donax denticulatus Linnaeus

\section{Chion semigranosus (DUNKER)フジノハナガイ（群品）}

1877 Donax semigranosus DUNKeR, M B L., 24, 68

1881 Donax proximus BerTIN, NAMHNP, 100, pl. 3, f. 2a-c

1882. Donax semigranosus: Dunker, Index, 193, pl. 7, f. 14-16.

1959 Chion semigranosum (sic): KIRA, CISJ, 1, 151, pl. 58, f. 14

Loc. N M S (s) int.

Dist. Honshu (north to Bōsō Peninsula and Oga Peninsula) to Kyushu.

Genus Tentidonax IREDALE キュウシュウナミノコガイ属

1930 Tentidonax Iredale, RAM, 17, 398. Type (OD): Donax verninus Hedley . . 
Tentidonax kiusiuensis (PILSBRY)キュウシュウナミノコガイ（岩川）

1901 Donax kiusiuensis Pilsbry, PANSP, 53, 207, 400, pl. 20, f. 19

1927 Donax paululus YoкоYAMA, JCSIUT, (2) 1 (10), 455, pl. 52, f. 6

1928 Tellina trigonoides Grabau \& KING, Peitaiho, 183, pl. 7, f. 42

1961 Chion (Tentidonax) kiusiuensis: HABE, CISJ, 2, 134, pl. 60, f. 14.

Loc. N S (s) dp.

Dist. Southern Hokkaido to Formosa and China.

Genus Latona SCHUMACHER ナミノコガイ属

1917 Latona SchUMACHeR, ENSVT, 52, 156. Type (M): Latona variabilis Schumacher non SAY $=$ Donax cuneata LINNAEUS

Latona cuneata (LINNAEUS) ナミノコガイ（目八）

1758 Donax cuneatus Linnaeus, Syst. Nat., 10, 683

1791 Donax bicolor GMelin, Syst. Nat., 13, 3265

1818 Donax australis LAMARCK, AsV, 5, 548

1854 Donax cuneatus: REEVE, Icon., sp. 13

1959 Latona cuneata: KIRA, CISJ, 1, 151, pl. 5, f. 15

1971 Latona cuneata: KurodA \& HABE, SSSB, 174 (J) 440 (E), pl. 97, f. 3, 4

Loc. N M S (s) int.

Dist. Honshu (north to Bōsō Peninsula and Noto Peninsula) to Ryukyu. Widely ranging in the Indo-Pacific region.

\section{Family Tellinidae BLAINVULE ニッコウガイ科 \\ Subfamily Tellininae BLAINVILLE ニッコウガイ鳏科}

Genus Tellinella MöRCH ニッコウガイ属

1853 Tellinella Mörch, Yoldi., 2, 13

1887 Eutellina Fischer, M. de C., 1147

1934 Tellinarius Salisbury, PMSL, 21, 78, 90. Type (SD, StoliczKA, 1870): Tellina virgata

LINNAEUS,

Tellinella staurella (LAMARCK) ヒメニッコウガィ (平瀬)

1798 Tellina cruciata SPENGLER, SNS, 4 (2), 83

1818 Tellina staurella LAMARCK, AsV, 5, 522

1918 Tellina exculta hawaiensis PILSBRY, PANSP (1917), 332

1959 Tellinella staurella: KIRA, CISJ, 1, 150, pl. 60, f. 24

1969 Tellina (Tellinella) staurella: Boss, BMCZ, 138 (4), 95, pl. 2, f. 2 ; pl. 3, f. 1, 2; pl. 4, f. 2

Loc. S (s) dp.

Dist. Honshu (Kii Peninsula) to Ryukyu Islands. Widely ranging in the IndoPacific region.

Tellinella radians (DESHAYES)コニッコウガイ（黒田）

1855 Tellina radians DeShaYes, PZSL (1854), 22 (282), 366

1859 Tellina radians SOWERBY, Icon., sp. 335

1878 Tellina gibba BERTIN, NAMHNP, 1 (2), 264, pl.9, f. 8

1955 Tellina gibba: HABE, Venus, 18 (4), 242, tf. 4, 5

1961 Tellinella radians: HABE, CISJ, 2, 138, p1. 62, f. 17

Loc. S (s) dp.

Dist. Honshu (north to Kii Peninsula) to Ryukyu Islands. 
Tellinella pulcherrima (SOWERBY) サメハダヒノデガイ（平瀬）

1825 Tellina pulcherrima Sowerby, Tankerville, App. 3, pl. 1, f. 1

1867 Tellina pulcherrima: SOWERBY, Icon., sp. 108

1959 Tellinella pulcherrima: KIRA, CISJ, 1, 108, pl. 60, f. 16

1971 Tellinella pulcherrima: KURODA \& HABE, SSSB, 694 (J) 456 (E), pl. 100, f. 4

Loc. M S (s) dp.

Dist. Honshu (north to Bōsō Peninsula) to Ryukyu Islands. Widely ranging in the tropic Pacific region.

Tellinella asperrima (HANLEY) ウロコヒノデガイ（黒田）

1844 Tellina asperrima HANLEY, PZSL, 12 (134), 59

1867 Tellina asperrima: SOWERBY, Icon., sp. 198

Loc. S (s) dp.

Dist. Honshu (Kii Peninsula) to Ryukyu Islands. Widely ranging in the tropic Pacific region.

Tellinella patagiata (PRASHAD)ヒナザクラガイ（波部） Pl. 1, f. 9.

1932: Tellina (Tellina) patagiata Prashad, Siboga, 53c, 182, pl. 5, f. 34, 35

Loc. S (s) dp.

Dist. Honshu (Kii Peninsula) to Ryukyu Islands and Indonesia.

Tellinella crucigera (LAMARCK) ヒノデガイ (平㖽)

1818 Tellina crucigera LAMARCK, AsV, 6,

1850 Tellina tithonia GouLd, PBSNH, 3, 252

1866 Tellina crucigera: ReEve, Icon., sp. 47

1959 Tellinella philippii: KIRA, CISJ, 1, 159, pl. 60, f. 21 (non PHILIPPI, 1844)

1978 Tellinella tithonia: HABE, Venus, 37 (4), 219. tf. 14, 165

Loc. M S (s) dp.

Dist. Honshu (Wakayama Pref.) to Ryukyu Islands. Widely ranging in the tropic Pacific.

\section{Genus Pharaonella LAMY ベニガイ属}

1918 Pharaonella Lamy, BMNHNP, 24, 31. Type (T): Tellina pharaonis HANLEY

Pharaonella sieboldii DESHAYES ベニガイ（六介）

[1846 Tellina vulsella HANLEY in SOWERBY, Thes., 1, 235, pl. 61, f. 162, 163]

1855 Tellina sieboldii DeshaYes, PZSL (1854), 22 (282), 368

1878 Tellina perrieri BERTIN, NAMHNP, (2) 1, 255, pl. 8, f. 6a, b

1903 Tellina consanguinea SowERBY, AMNH, (7), 12, 500

1950 Pharaonella perrieri: KIRA, CISJ, 1, 158, pl. 60, f. 14

1955 Tellina perrieri: HABE, Venus, 18 (4), 241, tf. 1-3

1971 Pharaonella perrieri: KuRODA \& HABE, SSSB, 694 (J) 456 (E), p1. 100, f. 6, 7

Loc. N M S (s) int.

Dist. Honshu (north to Tsuruga Strait) to Kyushu.

Pharaonella perna (SPENGLER)ダイミョウガイ（六介）

1798 Tellina perna SPENGLeR, SNS, 4 (20), 79

1844 Tellina pharaonis HANLEY, PZSL, 12 (139), 148

1959 Pharaonella perna: KIRA, CISJ, 1, 159, pl. 60, f. 23

1969 Tellina (Pharaonella) perna: Boss, BMCZ, 138 (4), 116, pl. 8, f. 1-4 
Loc. N M S (s) int.

Dist. Honshu (north to Kii Peninsula) to Ryukyu Islands. Widely ranging in the Indo-Pacific region.

\section{Genus Tellinides LAMARCK ヘラサギガイ属}

1818 Tellinides Lamarck, AsV, 5, 535. Type (M): Tellinides timorensis LamarCK

Tellinides ovalis SoWERBY ヒラザクラガイ (平瀬)

1825 Tellinides ovalis SOWERBY, Tankerville, App. 3

1867 Tellina ovalis: Sowerby, Icon., sp. 105

1871 Tellina gratiosa RöMER, Cab., 10 (4), 170, pl. 34, f. 10-12

1938 Angulus hawaiensis DALL, Bartsch \& Rehder, BPBMB, 153, 198, pl. 50, f. 9-12

1959 Tellinides ovalis: KIRA, CISJ, 1, 158, pl. 60, f. 11

1971 Tellinides ovalis: KuRoda \& HABE, SSSB, 693 (J) 455 (E), pl. 100, f. 8

Loc. N M S (s) dp.

Dist. Honshu (north to Bōsō Peninsula) to Ryukyu Islands. Widely ranging in the Indo-Pacific region.

Tellinides coccinea SowERBYヒカンザクラガイ（黒田）：エンジザクラガイ（黒田）

1869 Tellina coccinea Sowerby, Icon., sp. 107

Loc. S (s) dp.

Dist. Honshu (Wakayama Pref.)

Remarks: This species seems to be merely a color variety of the preceding one.

Genus Angulus Megerle von MüHLFELD クモリザクラガイ属

1811 Angulus Megerle von Mühlfeld, GNFBM, 5, 47. Type (SD, Gray, 1847): Tellina lanceolata GMELIN

Angulus vestalioides (YoKоуамA) クモリザクラガイ（黒田）

1871 Tellina vestalis RöMER, Cab., 10 (4), 174, pl. 35, f. 9-11 (non HANLEY, 1844)

1920 Tellina vestalioides Yokoyama, JCSIUT, 39 (6), 114, pl. 7, f. 25

1959 Angulus vestalioides: KIRA, CISJ, 1, 158, pl. 60, f. 12

1971 Angulus vestalioides: KuRODA \& HABE, SSSB, 693 (J) 455 (E), pl. 99, f. 9

Loc. N M S (s-m) dp.

Dist. Southern Hokkaido to Kyushu, China and South East Asia.

Genus Omala ScHUMACHERクサビザクラガイ属

1817 Omala Schumacher, ENSVT, 43, 128. Type (M): Omala inaequivalvis Schumacher Tellina hyalina GMELIN

Omala hyalina (GMELIN)クサビザクラガイ（黒田)

1791 Tellina hyalina GMELIN, Syst. Nat. 13, 3236

1867 Tellina opalina SOWERBY, Icon., sp. 58

1871 Tellina hyalina: RöMER, Cab., 10 (4), 172, pl. 35, f. 5-8

1977 Omala hyalina: HABE, SMJ B/S, 195, p1. 38, f. 3, 4

Loc. S (s).

Dist. Honshu (north to Kii Peninsula) and Shikoku. Tropic Pacific

Genus Arcopella THIELE ハッヒザクラガイ属

1934 Arcopella ThIele, Weicht, 2 (3), 914. Type (M): Tellina balaustina LinNaEUs 
Arcopella isseli (H. ADAMs) ハッヒザクラガイ（黒田）

1870 Tellina isseli $\mathrm{H}$. ADAMS, PZSL, 790, pl. 48, f. 10

1961 Arcopella isseli: HABE, CISJ, 2, 136, pl. 61, f. 28

1971 Arcopella isseli: KuRODA \& HABE, SSSB, 685 (J) 449 (E), pl. 99, f. 20

Loc. M S (s) dp.

Dist. Honshu (north to Bōsō Peninsula) to Kyushu. Widely ranging in the Western Pacific, and Persian Gulf.

\section{Genus Clathrotellina THIELEアミメザクラガイ属}

1934 Clathrotellina ThIele, Weicht. 3, 917. Type (M): Tellina pretiosa DeshaYes non

EICHWALD $=$ Tellina pretium SALISBURY

Clathrotellina pretium (SALISBURY) アミメザクラガイ（黒田）

1855 Tellina pretiosa Deshayes (non EICHWALD, 1830), PZSL (1854), 360

1867 Tellina costata SOWERBY (non THUNBerg, 1815), Icon., sp. 194

1934 Tellina pretium SALISBURY, PMSL, 21 (2), 86

1961 Clathrotellina pretium: HABE, CISJ, 2, 136, pl. 61, f. 26

Loc. N M S (s) dp.

Dist. Honshu (north to Bōsō Peninsula) to Kyushu and China.

Clathrotellina carnicolor (HANLEY)ヒラセザクラガイ（岩川）

1846 Tellina carnicolor Hanley, Thes., 1, 263, pl. 56, f. 15

1867 Tellina corbis: Sowerby, Icon., sp. 127 (non Brown, 1831)

1904 Tellina hirasei PILSBRY, PANSP, 56, 554, pl. 41, f. 3

1959 Clathrotellina carnicolor: KIRA, CISJ, 1, 155, pl. 59, f. 15

Loc. N M S (s) dp.

Dist. Honshu (north to Kii Peninsula), Shikoku and Kyushu. Widely ranging in the tropic Indo-Pacific region.

\section{Subfamily Arcopagiinae イチョウシラトリガイ亜科 \\ Genus Merisca OALL イチョウシラトリガイ属}

1900 Merisca Dall,PUSNM, 23, 299. Type (OD): Tellina crystallina Wood=Tellina cristallina SPENGLER

\section{Subgenus Pistris THIELE１チョウシラトリガイ亚属}

1918 Pristis Lamy (non Link, 1790), BMNHNP, 24, 29

1934 Pristis THIELE, Weicht., 3, 917

1936 Pristipagia Iredale, RAM, 19, 281. Type (T): Tellina pristis Lamarck

Merisca (Pistris) capsoides (LAMARCK)イチョウシラトリガイ (平瀬);

ヌノメイチョウシラトリガイ（黑田）

1818 Tellina capsoides LAMARCK, AsV, 5, 531

1818 Tellina pristis LAMARCK, AsV, 5, 531

1855 Tellina diaphana DeshaYes, PZSL (1854), 22, 364

1860 Tellina siamensis MARTENS, PZSL, 18

1868 Tellina capsoides: SOwERBY, Icon., sp. 183

1868 Tellina diaphana: Sowerby, Icon., sp. 183

1901 Tellina (Merisca) pristiformis PILSBRY, PANSP, 53, 400, pl. 19, f. 8

1959 Arcopagia (Merisca) diaphana: KIRA, CISJ, 1, 160, pl. 60, f. 25 
1971 Merisca diaphana: Kuroda \& HABE, SSSB, 687 (J) 450 (E), pl. 121, f. 6

1969 Tellina (Serratima) capsoides: Boss, BMCZ, 138 (4), 113, pl. 6, f. 4; pl. 8, f. 5, 6; pl. 14, f. 3

Loc. $\mathrm{N} M \mathrm{~S}$ (s-m) int.

Dist. Honshu (north to Mutsu Bay) to Ryukyu Islands. Widely ranging in the Indo Pacific region.

Merisca (Pistris) subtruncata (HANLEY)ユウヒザクラガイ（黒田）

1844 Tellina subtruncata HANLEY, PZSL, 149

1865 Tellina subtruncata: SOWERBY, Icon., sp. 241

1906 Tellina ojiensis TokUNAGA, JCSIUT, 21 44, pl. 2, f. 34

1934 Tellina subtruncata: SAliSbury, PMSL, 21, 85, pl. 11, f. 2, 3

1961 Arcopagia (Merisca) subtruncata:HABE, CISJ, 2, 137, pl. 62, f. 8

1971 Merisca subtruncata: KuRodA \& HABE, SSSB, 688 (J) 451 (E), pl. 99, f. 21

Loc. N M S (s-m) dp.

Dist. Honshu (north to Mutsu Bay) to Kyushu. Widely ranging in the Western Pacific region.

Merisca (Pistris) tokunagai IKEBEトクナガシラトリガイ（池辺）

1936 Arcopagia (Merisca) tokunagai IkeBE, Venus, 6 (4), 203, f. 3

1961 Arcopagia (Merisca) tokunagai: HABE, CISJ, 2, 137, pl. 62, f. 11

1971 Merisca tokunagai: Kuroda \& HABE, SSSB, 688 (J) 451 (E), pl. 99, f. 11

Loc. M S (s-m) tr.

Dist. Honshu (north to Kashima Nada and Wakasa Bay) to Kyushu.

Merisca (Pistris) margaritina (LAMARCK)アコヤザクラガイ（黒田）

1818 Tellina margaritina LAMARCK, AsV, 5, 525

1867 Tellina margaritina: SOWERBY, Icon., sp. 116

1905 Tellina sericostata TokuNAGA, JCSIUT, 21, 43, pl. 2, f. 32a, b

1961 Arcopagia (Merisca) margaritina: HABE, CISJ, 2, 137, pl. 62, f. 10

1971 Merisca margaritina Kuroda \& HABE, SSSB, 687 (J) 450 (E), pl. 99, f. 8

Loc. M S (s-m) dp.

Dist. Honshu (north to Mutsu Bay) to Kyushu. Widely ranging in the western Pacific region.

Genus Quidnipagus IREDALE リュウキュウシラトリガイ属

1929 Quidnipagus Iredale, MQM, 9, 266. Type (OD): Cochlea palatum MarTiN (n. n)= Tellina palatam IREDALE

Quidnipagus palatam IREDALE リュウキュウシラトリガイ（岩川）

1778 Tellina rugosa BORN IMCV, 18 (non PENNANT, 1777)

1780 Tellina rugosa: BoRN, TMCV, 29, pl. 2, f. 3, 4

1918 Tellina (Tellina) rugosa obtusa LAMY, BMNHNP 24, 29 (non SowerBY, 1868)

1929 Quidnipagus palatam IREDALE, MQM, 9, 266

1959 Quidnipagus palatam: KIRA, CISJ, 1, 159, pl. 60, f. 19

1969 Tellina (Quidnipagus) palatam: Boss, BMCZ, 138 (4), 109, pl. 7, f. 1, 2

Loc. S (s) dp.

Dist. Honshu (north to Kii Peninsula) to Ryukyu Islands. Widely ranging in Indo Pacific region.

\section{Genus Arcopaginula LAMY ゴイシザラガイ属}

1918 Arcopaginula LAMY, BMNHNP, 24, 168. Type (M): Tellina inflata GMELIN 
Arcopaginula inflata (GMELIN)ゴイシザラガイ（黒田）

1791 Tellina inflata Gmelin, Syst. Nat., 13, 3230

1843 Tellina hippoidea PhiLIPpI, Abbild,, 1, 72, pl. 1, f. 3

1846 Tellina striatula HANLEY, Thes., 1, 255, pl. 61, f. 175 (non Lamarck, 1818)

1866 Tellina striatula: SowerbY, Icon., sp., 34

1961 Arcopaginula inflata: HABE, CISJ, 2, 137, pl. 62, f. 7

Loc. M (s-m) dp.

Dist. Honshu (north to Kii Peninsula) to Ryukyu Islands. Widely ranging in the Indo Pacific region.

\section{Genus Cadella DALL, BARTSCH \& REHDERクサビザラガイ属}

1938 Cadella DALL, BARTSCH \& REHDER, BPBMB, 153, 196. Type (OD): Tellina lachiogramma MeLVILL

Cadella hoshiyamai KURODAホシヤマナミノコザラガイ (黑田);

$$
\text { マルクサビザラガイ (波部) }
$$

(1938 Cadella mauia DaLL, Bartsch \& Rehder, BPBMB, 153, 197, pl. 50, f. 1-4)

1960 (Oct.) Arcopagia (Cadella) hoshiyamai Kuroda, Cat. Okinawa, 83

1960 (Dec.) Cadella narutoensis HABE, PSMBL, 8, (2), 292, f. 1

1961 Cadella narutoensis: HABE, CISJ, 2, 136, pl. 61, f. 25

1971 Cadella narutoensis: KurodA \& HABE, SSSB, 689 (J) 451 (E), pl. 99, f. 18

Loc. M S (s) dp.

Dist. Honshu (north to Bōsō Peninsula) to Ryukyu Islands.

\section{Cadella delta (YoKOYAMA) クサビザラガイ（黒田）}

1922 Tellina delta Yокочама, JCSIUT, 44, (1), 141, pl. 10, f. 8-10

1961 Cadella delta: HABE, CISJ, 2, 136, pl. 61, f. 27

Loc. N S (s) dp.

Dist. Honshu (north to Bōsō Peninsula) to Kyushu.

Cadella semen (HANLEY) ナミノコザラガイ（黒田）

1845 Tellina semen HANLEY, PZSL (1844), 12 (141), 164

1867 Tellina semitorta Sowerby, Icon., sp. 221

1909 Tellina semitorta: LYNGE, Siam., 199, pl. 3, f. 43-46

1938 Cadella oahuana DaLL, Bartsch \& Rehder, BPBMB, 153, 197, pl. 50, f. 5, 8

1961 Cadella semitorta: HABE, CISJ, 2, 136, pl. 61, f. 22

1969 Tellina (Cadella) semen: Boss, BMCZ, 138 (4), 136, pl. 14, f. 5; pl. 15, f. 1-4

Loc. N M S (s) dp.

Dist. Honshu (north to Kii Peninsula) to Ryukyu Islands. Widely ranging in the tropic Pacific.

\section{Genus Quadrans BERTINトゲウネガイ属}

1878 Quadrans BERTIN, NAMHNP (92) 1, 265. Type (SD, DALL, 1900): Tellina gargadia LINNAEUS

\section{Quadrans spinosa (HANLEY)トゲウネガイ (平瀬)}

1844 Tellina spinosa HANLEY, PZSL, 12 (1844), 148

1867 Tellina spinosa: SowERBY, Icon., sp. 206

1961 Arcopagia (Quadrans) spinosa: HABE, CISJ, 2, 136, pl. 61, f. 29

1971 Quadrans spinosa: KurodA \& HABE, SSSB, 686 (J) 450 (E), pl. 99, f. 19

Loc. M S (s) int. 
Dist. Honshu (north to Bōsō Peninsula and Noto Peninsula) to Ryukyu Islands. Widely ranging in the tropic Pacific.

\section{Genus Pinguitellina IREDALEウラキヒメザラガイ属}

1927 Pinguitellina IreDale, RAM, 16, 76. Type (OD)：Tellina robusta HANLeY

Pinguitellina pinguis (HANLEY)：ガキヒメザラガイ（黒田）

1845 Tellina pinguis HANLEY, PZSL (1844), 12, 63

1868 Tellina pinguis SOWERBY, Icon., sp. 172

1939 Pinguitellina nucella DaLl, BARTSCH \& ReHder, BPBMB, 153, 195, p1. 49, f. 9-12

1961 Pinguitellina nux: HABE, CISJ, 2, 136, pl. 61, f. 20 (non HANLEY, 1844)

1977 Pinguitellina pinguis: $\mathrm{H}_{\mathrm{ABE}}, \mathrm{SMJ} \mathrm{B} / \mathrm{S}, 203$, pl. 41, f. 9-10

Loc. M S (s) int.

Dist. Honshu (north to Kii Peninsula) to Ryukyu Islands. Widely ranging in the tropic Pacific.

Pinguitellina nux (HANLEY)サッマヒメザラガイ（黑田)；サッマザラガイ（黒田）

1845 Tellina nux HANLEY, PZSL (1844), 12, 62

1868 Tellina nux: Sowerby, Icon., sp. 76

Loc. M S (s) int.

Dist. Honshu (north to Kii Peninsula) to Ryukyu Islands. Widely ranging in the tropic Indo Pacific.

\section{Genus Punipagia IREDALEゴシキヒメザラガイ属}

1930 Punipagia Iredale, RAM, 17, 398. Type (OD): Tellina subelliptica Sowerby non Meek \& HAXDEN $=$ Tellina hypelliptica SALISBURY

Punipagia radiatolineata (YOKоYAMA) ゴシキヒメザラガイ（黒田）

1868 Tellina subelliptica SOWERBY, Icon., sp. 220 (non MeEK \& HAYDEN 1864)

1924 Tellina radiatolineata YokоуамA, JCSIUT, 45, (1), 41, pl. 11, f. 18, 19

1937 Tellina hypelliptica SALISBURY, PMSL, 21 (2), 89

1961 Punipagia hypelliptica: HABE, CISJ, 2, 136, pl. 61, f. 23

Loc. N M S (s) int.

Dist. Honshu (north to Sagami Bay) to Ryukyu Islands. Widely ranging in the Pacific region.

\section{Genus Loxoglypta DALL, BARTSCH \& REHDERシボリザクラガイ属}

1939 Loxoglypta Dall, BarTsCh \& Rehder, BPBMB, 153, 192. Type (OD): Tellina obliquilineata CONRAD

Loxoglypta clathrata (DESHAYES)シボリザクラガイ（介志）

1835 Tellina clathrata DESHAYES, AsV, (2) 6, 208

1835 Tellina rhomboides QuoY \& Gaimard, Astrolabe, 3, 502, pl. 81, f. 4-7 (non Gmelin, 1791)

1850 Tellina (Angulus) lauta Gound, PBSNH, 3, 253

1850 Tellina (Angulus) compta GouLd, PBSNH, 3, 253

1867 Tellina clathrata: SOWERBY, Icon. sp. 114

1959 Jactellina (Loxoglypta) lauta: KIRA, CISJ, 1, 157, pl. 60, f. 10

1971 Loxoglypta clathrata: KURODA \& HABE, SSSB, 692 (J) 454 (E), pl. 100, f. 1

Loc. N M S (s) int. 
Dist. Honshu (north to Bōsō Peninsula) to Ryukyu Islands. Widely ranging in the Indo Pacific.

\section{Genus Elliptotellina CossManN チデミヒメザラガイ属}

1866 Elliptotellina Cossmann, Ann. Soc. Malac. Belg., 21, 71. Type (OD): Donax tellinella LAMARCK

Elliptotellina euglypta (GouLD) チデミヒメザラガイ（黒田）

1861 Tellina euglypta GouLd, PBSNH, 8, 28

1904 Tellina fabrefacta PrLSBRY, PANSP, 56, 555, pl. 41, f. 14, 15

1961 Elliptotellina euglypta: HABE, CISJ, 2, 136, pl. 61, f. 18

1964 Tellina euglypta: JoHNson, USNMB, 239, 72, pl. 25, f. 1

1971 Elliptotellina euglypta: KURODA \& HABE, SSSB, 686 (J) 449 (E), pl. 121, f. 5

Loc. M S (s) int.

Dist. Honshu (north to Bōsō Peninsula), Shikoku and Kyushu. Widely ranging in the South East Asia and North Australia.

\section{Genus Semelangulus IREDALE コメザクラガイ属}

1924 Semelangulus Iredale, PLSNSW, 49, 212. Type (OD): Tellina tenuilirata Sowerby

Semelangulus tokubeii HABE コメがクラガイ（黑田）

1961 Semelangulus tokubeii HABE, CISJ, 2, App. 39, pl. 61, f. 19

1971 Semelangulus tokubeii: KunODA \& HABE, SSSB, 689 (J) 452 (E), pl. 99, f. 17

Loc. N M S (s) int.

Dist. Honshu (north to Bōsō Peninsula), Shikoku and Kyushu.

Semelangulus miyatensis (YoKoYAMA) ニクイロザクラガイ (滝篇)

1920 Tellina miyatensis YoKoyama, JCSIUT, 29 (6), 115, pl. 7, f. 18

1961 Semelangulus miyatensis: HABE, CISJ, 2, 136, pl. 61, f. 24

Loc. N M S (s-m) int.

Dist. Honshu (north to Tokyo Bay) to Kyushu.

\section{Genus Moerella FISCHERモモノハナガイ属}

1851 Donacilla Gray, LBACBM, 7, 39 (non Philippi 1836)

1856 Moera H. \& A. ADAMs, GRM, 2, 396 (non HüBNER, 1819)

1887 Moerella Fischer, M. de C., 1174. Type (M): Tellina donacina LinNaeus, 1758

Moerella jedoensis (LISCHKE) —モノ八ナガイ（丹浦); エドザクラガイ（平瀬）

1872 Rellina jedoensis LischKE, M B L., 19, 106

1874 Tellina jedoensis: LisCHKE, JMC, 3, 92, pl. 9, f. 1-3

1959 Moerella jedoensis: KIRA, CISJ, 1, 157, pl. 60, f. 5

1971 Moelella jedoensis: Kuroda \& HABE, SSSB, 690 (J) 452 (E), pl. 100, f. 2

Loc. N M S (s-m) int.

Dist. Honshu (north to Bōsō Peninsula) to Kyushu, Korea and China.

Moerella rutila (DUNKER) ユウシオガイ（目八）

1860 Tellina rutila DUNKER, M B L., 6, 236

1861 Tellina rutila: DuNKeR, MJ, 27, pl. 3, f. 6

1928 Tellina (Tellinedes) planissima peitaihoensis GrabaU \& KING, Peitaiho, 180, pl. 5, f. 37

1959 Moerella juvenilis: KIRA, CISJ, 1, 157, pl. 60, f. 9 (non HANLEY, 1844)

1971 Moerella rutila: Kuroda \& HABE, SSSB, 690 (J) 452 (E), pl. 99, f. 14 
Loc. N M S (s-m) int.

Dist. Honshu (north to Mutsu Bay) to Kyushu, Korea and China.

Moerella nishimurai KURODA \& HABE ニシムラザクラガイ（黒田）

1958 Moerella nishimurai KURODA \& HABE, PSMBL, 7 (1), 45

1961 Moerella nishimurai: HABE, CISJ, 2, 137, pl. 62, f. 5

Loc. N S (s) int.

Dist. Honshu (north to Bōsō Peninsula and Oga Peninsula) to Kyushu.

Moerella iridescens (BENSON)テリザクラガイ（岩川）

1842 Sanguinolaria iridescens Benson. AMNH, 9, 490

1844 Tellina carnea PhILIPPI, ZfM, 162

1869 Tellina iridescens: SOWERBY, Icon., sp. 129

1961 Moerella iridescens: HABE, CISJ, 2, 137, pl. 62, f. 3

Loc. $\mathrm{N}$ (s-m) int.

Dist. Honshu (north to Bōsō Peninsula) to Kyushu, Korea, China and South East Asia.

Genus Bathytellina KURODA \& HABEワダッミザクラガイ属

1958 Bathytellina KURODA \& HABE, PSMBL, 7 (1), 45. Type (OD): Bathytellina citrocarnea Kuroda \& Habe

Bathytellina citrocarnea KURODA \& HABEワダッミザクラガイ（黒田）

1958 Bathytellina citrocarnea KURODA \& HABE, PSMBL, 7 (1), 48

1961 Bathytellina citrocarnea: HABE, CISJ, 2, 137, pl. 62, f. 2

1971 Bathytellina citrocarnea: KURODA \& HABE, SSSB, 692 (J) 454 (E), pl. 100, f. 3

Loc. M S (s) tr.

Dist. Honshu (north to Bōsō Peninsula) to Kyushu.

Bathytellina abyssicola (HABE)フゲンザクラガイ（波部)

1958 Fabulina abyssicola HABE, PSMBL, 7 (1), 46

1961 Bathytellina abyssicola: HABE, CISJ, 2, 137, pl. 62, f. 1

Loc. S (s) tr.

Dist. Honshu (north to Kii Peninsula) to Kyushu and the southern part of Japan Sea.

\section{Genus Nitidotellina SCARLAToサクラガイ属}

1961 Nitidotellina SCARLato, SMS, 8, 85. Type (OD): Tellina nitidula DunKER

Nitidotellina nitidula (DUNKER) サクラガイ（介譜)

1860 Tellina nitidula DUNKeR, M B L., 6, 236

1861 Tellina nitidula: DUNKER, MJ, 27, pl. 3, f. 14

1871 Tellina nitidula: LrschKe, JMC, 2, 113, pl. 10, f. 10, 11

1959 Fabulina nitidula: KIRA, CISJ, 1, 157, pl. 60, f. 7

1971 Nitidotellina nitidula: Kuroda \& HABE, SSSB, 690 (J) 453 (E), pl. 99, f. 13

Loc. N M S (s-m) int.

Dist. Southern Hokkaido to Kyushu, Korea and China.

Nitidotellina iridella (MARTENS)カバザクラガイ（群品）

1865 Tellina iridella MARTENS, AMNH, (3) 16, 431 
1871 Tellina iridella: LISCHKE, JMC, 2, 114, pl. 10, f. 8, 9

1959 Fabrina iridella: KIRA, CISJ, 1, 157, pl. 60, f. 6

1971 Nitidotellina iridella: KURODA \& HABE, SSSB, 691 (J) 453 (E), pl. 99, f. 15

Loc. N M S (s-m) int.

Dist. Honshu (north to Bōsō Peninsula) to Formosa.

Nitidotellina soyoae HABEソウヨウザクラガイ（東); キザクラガイ（波部）

1958 Fabulina soyoae HABE, PSMBL, 7 (1), 45

1961 Fabulina soyoae: HABE, CISJ, 2, 137, pl. 62, f. 4

Loc. S (s) tr.

Dist. Honshu (north to Enshu nada) to Kyushu and the southern part of Japan Sea.

Nitidotellina pallidula (LISCHKE) ハッザクラガイ（群品）

1871 (Jan.) Tellina pallidula LischKe, M B L., 18, 42

1871 Tellina pallidula: LisCHKE, JMC, 2, 114, pl. 10, f. 6, 7, 7a

1959 Fabulina pallidula: KIRA, CISJ, 1, 157, pl. 60, f. 8

1971 Nitidotellina pallidula: KuRODA \& HABE, SSSB, 691 (J) 453 (E), pl. 99, f. 16

Loc. M S (s-m) int.

Dist. Honshu (north to Mutsu Bay) to Kyushu, China and South East Asia.

Nitidotellina minuta (LISCHKE) ウズザクラガイ（黒田）

1872 Tellina minuta LISCHKE, M B L., 19, 106

1874 Tellina minuta: LischKe, JMC, 3, 94, pl. 9, f. 4-6

1959 Fabulina minuta: KrRA, CISJ, 1, 157, pl. 60, f. 4

1971 Nitidotellina minuta: KURODA \& HABE, SSSB, 691 (J) 454 (E), pl. 99, f. 12

Loc. N M S (s) int.

Dist. Southern Hokkaido to Kyushu, Korea and China.

Subfamily Strigillinae IREDALE \& MCMICHAELオガタザラガイ里科

Genus Aeretica DALL オガタザラガイ属

1900 Aeretica DALL, TWFIP, 3 (5), 108. Type (OD): Tellina senegarensis HANLEY non GMELIN = Strigilla polyaula $x$ TOMLIN \& SHACKLEPORD

Aeretica tomlini (SMITH)オガタザラガイ（黒田）

1846 Tellina splendida HANLEY Thes., 1, 259, pl. 56, f. 36 (non ANTON, 1839)

1866 Tellina splendida: ReEve, Icon., sp. 38

1915 Strigilla tomlini SмIтH J. of C., 14 (11), 339

1961 Strigilla (Aeretica) tomlini: $\mathrm{H}_{\mathrm{ABE}}$, CISJ, 2, 137, pl. 62, f. 9

Loc. N M S (s) int.

Dist. Honshu (north to Kii Peninsula) to Philippines.

\section{Subfamily Macominae CLESSINシラトリガイ亜科 \\ Genus Heteromacoma HABEシラトリガイモドキ属}

1952 Heteromacoma Habe, GJS, (3), 218

1959 Sinomacoma Yamamoto \& Habe, BMBSA 9 (3), 102. Type (OD): Tellina irus HANLeY

Heteromacoma irus (HANLEY)シラトリガイモドキ (平瀬)

1845 Tellina irus HANLEY, PZSL (1844), 166

1863 Fragilia yantaiensis Crosse \& Debeaux, J. de C., 11, 75, 255, pl. 9 , f. 2 
1868 Tellina irus: Sowerby, Icon., sp. 295

1882 Lucina corrugata DuNKer, Index, 216, pl. 8, f. 9-11

1934 Tellina irus: SALISBURY, PMSL, 21, 85, p1, 12, f. 7, 8

1959 Heteromacoma oyamai KIRA, CISJ, 1, 155, pl. 59, f. 21

1959 Heteromacoma yantaiensis: KIRA, CISJ, 1, 156, pl. 59, f. 22

1971 Heteromacoma irus: Kuroda \& HABE, SSSB, 695 (J) 456 (E), pl. 100, f. 10

Loc. N M S (g) int.

Dist. Southern Hokkaido to Kyushu, Korea and China.

\title{
Genus Macoma LEACHシラトリガイ属
}

1819 Macoma LeACH, Voy, Discov. Baffin's Bay, App. 2, 62. Type (M): Macoma tenera LEACH $=$ Macoma calcarea GMELIN

\author{
Subgenus Macoma LEACHシラトリガイ亜属 \\ Macoma (Macoma) praetexta (MARTENS) オ才モモノハナガイ（岩川） \\ 1865 Tellina praetexta MARTENs, AMNH, (3) 16, 430 \\ 1872 Tellina praetexta: LISCHKE, JMC, 2, 113, pl. 10, f. 14 \\ 1959 Macoma praetexta: KIRA, CISJ, 1, 155, pl. 59, f. 16 \\ 1971 Macoma praetexta: KURODA \& HABE, SSSB, 666 (J) 457 (E), pl. 99, f. 6 \\ Loc. N M S (s) int. \\ Dist. Honshu (north to Bōsō Peninsula) to Kyushu. \\ Macoma (Macoma) contabulata (DESHAYES)サビシラトリガイ（黒团） \\ 1854 Tellina contabulata DESHAYES, AsV, 5 (2), 356 \\ 1867 Tellina contabulata: Sowerby, Icon., sp. 311 \\ 1934 Tellina contabulata: SALISBURY, PMSL, 21, 85, pl. 12, f. 6 \\ 1950 Macoma anser OYAMA, MG, 3 (6), 227 \\ 1959 Macoma anser: KIRA, CISJ, 1, 155, pl. 59, f. 20 \\ 1971. Macoma contabulata: Kuroda \& HABE, SSSB, 697 (J) 458 (E), pl. 100, f. 12 \\ Loc. N (m) int. \\ Dist. Honshu (north to Mutsu Bay) to Kyushu, Korea and China.
}

Macoma (Macoma) tokyoensis MAKIYAMA ゴイサギガイ（目八）

1865 Tellina dissimilis MARTENS, AMNH, (3) 16, 430 (non DeshaYes, 1854)

1927 Macoma tokyoensis MAKIYAMA, MCSKIU, (B) 2 (1), 50

1959 Macoma tokyoensis: KIRA, CISJ, 1, 155, pl. 59, f. 19

Loc. M S (m) int.

Dist. Honshu (north to Mutsu Bay) to Kyushu.

Macoma (Macoma) incongrua (MARTENS)ヒメシラトリガイ（黒田；

1865 Tellina incongrua MARTENS, AMNH(3), 16, 430

1959 Macoma incongrua: KIRA, CISJ 1, 155, pl. 59, f. 18

1971 Macoma incongrua: Kuroda \& HaBe, SSSB, 695 (J) 457 (E), pl. 100, f. 11

Loc. N M S (m) int.

Dist. Kurile, Hokkaido, Honshu, Shikoku, Kyushu, Korea and China.

Macoma (Macoma) calcarea (GMELIN)ケショウシラトリガイ（黒田)；

$$
\text { ヨーロッパシラトリガイ（鹿間） }
$$

1791 Tellina calcarea GMELIN, Syst. Nat., 13, 3236

1867 Tellina calcarea: SoWERBY, Icon., sp. 151 
1961 Macoma calcarea: HABE, CISJ, 2, 133, pl. 62, f. 13

1967 Macoma orientalis SCARLATo, Tr. Zool. Inst., 42, 122, f. 105

1971 Macoma calcarea: KuRoda \& HABE, SSSB, 696 (J) 459 (E), pl. 100, f. 9

Loc. N M S (s-m) dp.

Dist. Hokkaido to Shikoku. Widely ranging in North Atlantic, North Pacific and Arctic Seas.

Subgenus Psammacoma DALL アワジチガイ亜属

1900 Psammacoma Dall, PUSNM, 23 (1210), 292. Type (OD): Psammotaea candida LAMARCK

Macoma (Psammacoma) candida (LAMARCK)アアワジチガイ（矢倉）

1818 Psammotaea candida LAMARCK, AsV, 5, 517

1914 Macoma awajiensis SowerBy, AMNH, (8) 14, 38, pl. 2, f. 11

1959 Macoma (Psammacoma) awajiensis: KrrA, CISJ, 1, 154, pl. 59, f. 13

Loc. N M (s-m) dp.

Dist. Honshu (north to Bōsō Peninsula and Noto Peninsula) to Kyushu and China.

\section{Genus Rexithaerus TRYONサギガイ属}

1869 Rexithaerus Tryon, AJC, 4 (5), Suppl., 104. Type (SD, DALL, 1900): Tellina secta CONRAD

Rexithaerus sectior OYAMA サギガイ（目八）

1854 Tellina japonica Deshayes, PZSL, 356 (non THUNBERG, 1815)

1950 Macoma (Rexithaerus) sectior OYAMA, MG, 3 (6), 229

1971 Rexithaerus sectior: KuRODA \& HABE, SSSB, 697 (J) 458 (E), pl. 100, f. 5

Loc. N S (s-m) dp.

Dist. Saghalien to Formosa and China.

Genus Psammotreta DALLアオサギガイ属

1900 Psammotreta Dall, PUSNM, 23, 292. Type (OD): Tellina aurora HANLeY

Subgenus Pseudometis LAMY アオサギガイ亜属

1918 Pseudometis LAMY. BMNHNP, 24, 170. Type (SD, SALISBURY, 1934); Tellina truncata Philippi non LINNAEUS $=$ Tellina praerupta SALISBURY

Psammotreta (Pseudometis) praerupta (SALISBURY)アアサギガイ（岩川）

1843 Tellina truncata PhildppI, Abbild., 1 (3), 71, pl. 5, f. 2 (non Linnaeus, 1767)

1934 Tellina praerupta SALIBURY, PMSL, 21, (2), 90

1961 Macoma (Pseudometis) praerupta: HABE, CISJ, 2, 138, pl. 62, f. 15

1964 Psammotreta (Pseudometis) papyracea keenae HABE, SWC, 2, 202, pl. 62, f. 15

Loc. N M S (s-m) dp.

Dist. Honshu (north to Bōsō Peninsula and Noto Peninsula) to Kyushu. Western Pacific.

Subgenus Tellinimactra LAMYタイワンシラトリガイ栜属

1918 Tellinimactra LAMY, BMNHNP, 24, 169. Type (OD): Tellina edentula SPENGLeR 
Tellinimactra edentula (SPENGLER)タイワンシラトリガイ（黒田）

1791 Tellina angulata GMELIN, Syst. Nat., 13, 3229 (non LinNaEus, 1767)

1798 Tellina edentula SPENGLER, SNS, 4 (2), 96

1977 Tellinimactra edentula: HABE, SMJ B/S, 212, pl. 41, f. 10-12

Loc. $M$ (s-m) dp.

Dist. Honshu (Wakayama Pref). Formosa and South East Asia.

Remark: This species is doubtfully recorded.

\section{Family Semelidae STOLICZKA アサジガイ科 \\ Genus Semele ScHUMACHERアサジガイ属}

1817 Semele Schumacher, ENSVT, 53, 165

1818 Amphidesma LAMARCK, AsV, 5, 489. Type (M): Semele reticulata $\mathrm{SCHUMACHER}=$ Tellina proficua PUlteney

Semele zebuensis (HANLEY) アサジガイ (介志)

1844 Amphidesma zebuensis HANLEY, PZSL, 12, 17

1850 Amphidesma exarata AdAMs \& ReEve, Samarang, 81, pl. 24, f. 9

1853 Amphidesma exarata: ReEVE, Icon., sp. 1

1853 Amphidesma duplicata: REEve, Icon., sp. 14

1853 Amphidesma zebuensis: REEve, Icon., sp. 25

1861 Semele duplicatum GouLD, PBSNH, 8, 27

1868 Semele gouldi Tryon, AJC, 4, App. 120

1959 Semele zebuensis: KuRA, CISJ, 1, 152, pl. 58, f. 19

1971 Semele zebuensis: Kuroda \& HaBe, SSSB, 682 (J) 446 (E), pl. 99, f. 1

Loc. N M S (s) dp.

Dist. Honshu (north to Bōsō Peninsula) to Kyushu, China and Philippines.

Semele cordiformis (HoLTEN) フルイガィ（介志）

1803 Tellina cordiformis HoLTEN, Enumeratio (1802), 10

1853 Amphidesma sinensis ReEve, Icon., sp. 28

1854 Amphidesma sinensis: A. AdAMs, PZSL, 21 (1853), 95

1959 Semele cardiformis (sic): KIRA, CISJ, 1, 152, pl. 58, f. 18

Loc. N (s-m) dp.

Dist. Honshu (north to Bōsō Peninsula and Noto Peninsula) to Kyushu. Widely ranging in the West Pacific.

Semele carnicolor (HANLEY)サメザラガイモドキ（平瀬）

1845 Amphidesma carnicolor HANLEY, PZSL (12), 162

1853 Amphidesma carnicolor: REEVE, Icon., sp. 6

1861 Semele alveata GouLd, PBSNH, 8, 27

1959 Semele carnicolor: KIRA, CISJ, 1, 152, pl. 58, f. 17

Loc. S (g) int.

Dist. Honshu (north to Kii Peninsula) to Ryukyu Islands. Widely ranging in the West Pacific.

Semele obscura DESHAYES シワサメザラガイ（波部）

1863 Amphidesma obscura DesHaYEs, Reunion, 9, pl. 28, f. 16, 17

1863 Amphidesma burbonica Deshayes, Reunion, 9, pl. 28, f. 18, 19

1928 Semele tita DALL, BARTSCH \& REHDER, BPBMB, 153, 177, pl. 45, f. 1-4,

1961 Semele australis: HABE, CISJ, 2, 135, pl. 61, f. 10 (non Sowrrby) 
1964 Semele tita: Habe \& Kosuge, SWC, 2, 198, pl. 61, f. 10

Loc. S (g) int.

Dist. Honshu (north to Kii Peninsula) to Ryukyu Islands, Hawaii and Reunion.

\section{Genus Iacra H. \& A. ADAMS ヨセギザクラガイ属}

1856 Iacra $\mathrm{H}$, \& A. AdAMS, GRM, 2, 409

1861 Strigillina DunKer, M B L., 8, 43. Type (M): Scrobicularia (Iacra) seychellarum A.

ADAMS

Iacra japonica (A. ADAMS) ヨセギザクラガイ（黒田）

(1856 Scrobicularia (Iacra) seychellarum A. ADAMs, GRM, 2, 409)

1864 Iacra japonica A. ADAMS, AMNH, (3) 13, 308

1977 Iacra japonica: HABE, SMJ B/S, 215, pl. 45, f. 1, 2

Loc. N S (s-m) int.

Dist. Honshu (north to Bōsō Peninsula) to Formosa.

\section{Genus Leptomya A. AdAMSナノハおガイ属}

1864 Leptomya A. Adams, AMNH, (3) 13, 208. Type (SD, Stoliczka, 1871): Neaera cochlearis HiNDs

Leptomya cuspidariaeformis HABEコチョウシャクシガイ（黒田）

1852 Leptomya cuspidariaeformis HABE, GJS, (2), 209, f. 505, 507

1961 Leptomya cuspidariaeformis: HABE, CISJ, 2, 135, pl. 61, f. 8

1971 Leptomya cuspidariaeformis: KuroDA \& HABE, SSSB, 683 (J) 447 (E), pl. 99, f. 2

Loc. N S (s-m) dp.

Dist. Honshu (north to Bōsō Peninsula) to Kyushu.

*Leptomya minuta HABE：ジンコチョウシャクシガイ（波部） Pl. 6, f. 5.

1960 Leptomya minuta HABE, PSMBL, 8 (2), 286, f. 1-3

1977 Leptomya minuta: HABE, SMJ B/S, 216, pl. 45, f. 14, 15

Loc. M (s-m) dp.

Dist. Honshu (Tanabe Bay, Wakayama Pref.).

Leptomya adunca (GouLD) ナノハナガイ（黒田）

1861 Scrobicularia (Capsa) adunca Gould, PBSNH, 8, 28.

1890 Cumingia (Thyella) stimpsoni DALL, TWFIP., 5, 999.

1978 Leptomya adunca HaBE, Venus, 37, 99, f. 3, 4.

Loc. $M \quad(s-m)$ dp.

Dist. Honshu (north to Kii Peninsula) to Ryukyu Islands.

Genus Leptomyaria HABE チビコチョウシャクシガイ属

1960 Leptomyaria HABE, PSMBL, 8 (2), 287. Type (OD): Leptomyaria trigona HABE

Leptomyaria trigona HABEチビコチョウシャクシガイ（波部）

1960 Leptomyaria trigona HABE, PSMBL, 8 (2), 287, f. 21, 22

1977 Leptomyaria trigona: $\mathrm{HABE}, \mathrm{SMJ}$ B/S, 216, pl. 45, f. 5, 6

Loc. M S (s-m) dp.

Dist. Honshu (Tanabe Bay, Wakayama Pref.) and Kyushu (Tomioka, Amakusa). 


\section{Genus Theora H. \& A. ADAMS シズクガイ属}

1856 Theora H. \& A. ADAMs, GRM, 2, 369

1864 Endopleura A. AdAms, AMNH (3) 13, 209. Type (SD, Sroliczka, 1871): Neaera lata HiNDS

Theora fragilis (A. ADAMS) シズクガイ (平瀬)

(1844 Neaera lata Hinds, PZSL,)

1855 Neaera fragilis A. ADAMS, PZSL, 226

1861 Theora lubrica Gound, PBSNH, 8, 24

1861 Theora nitida Gould, PBSNH, 8, 24

1882 Theora lubrica: DUNKER, Index, 181, pl. 7, f. 20-22

1961 Theora lata: HABE, CISJ, 2, 136, pl. 61, f. 7

1964 Theora nitida: Johnson, USNMB, 239, 116, pl. 24, f. 3

1964 Theora lubrica: JoHnson, USNMB, 239, 104, pl. 25, f. 5

1971 Theora lubrica: KuRODA \& HABE, SSSB, 682 (J) 447 (E), pl. 121, f. 2

1977 Theora fragilis: HABE, SMJ B/S. 218, pl. 44, f. 11, 12

Loc. M. S (m) dp.

Dist. Southern Hokkaido, Honshu, Shikoku, Kyushu, China and South East Asia.

\section{Genus Abra LAMARCKリュウグウザクラガイ属}

1818 abra Lamarck, AsV, 5, 492. Type (SD, Herrmannsen, 1846): Mactra tenuis Montagu

Abra soyoae HABEシラトリリュウグウザクラガイ（波部）；ソウヨウリュウグウザクラガイ(東)

1958 Abra soyoae HABE, PSMBL, 7 (1), 41, pl. 1, f. 12, 13

1961 Abra soyoae: HABE, CISJ, 2, 135, pl. 61, f. 9

Loc. S (s-m) tr.

Dist. Honshu (north to Bōsō Peninsula) to Kyushu.

Abra profundorum japonica OKUTANIマサリリュウグウザクラガイ（黑田）

(1885 Semele (Abra) profundorum Sмттн, Challenger, 13, 88, pl 5, f. 5--56)

1968 Abra profundorum: OKUTANI (non SMITH), BTRFRL, 56, 21, f. 2

1975 Abra profundorum japonica OKUTANI, BTRFRL, 82, 71, pl. 3, f. 3, 4

Loc. $\mathrm{S}(\mathrm{m})$ tr.

Dist. Honshu (north to Izu Islands) to Kyushu and Ogasawara

Abra fujitai HABEリュウグウザクラガイ（黒田）

1958 Abra fujitai HABE, PSMBL, 7 (1), 42, pl.

1961 Abra fujitai: HaBE, CISJ, 2, 135, pl. 61, f. 11

1971 Abra fujitai: KURODA \& HABE, SSSB, 684 (J) 448 (E), pl. 121, f. 3

Loc. S (s-m) tr.

Dist. Honshu (north to Bōsō Peninsula) to Kyushu.

Abra kyurokusimana NOMURA \& HATAI ツボミリュウグウザクラガイ（波部）

1940 Tellina kyurokusimana NoMURA \& HATAI, SHKMRB, 19, 85, pl. 4, f. 16 a-c

1961 Abra kyurokusimana: HABE, CISJ, 2, 135, pl. 61, f. 12

Loc. S (s-m) tr.

Dist. Honshu (north to Sagami Bay) to Kyushu and Japan Sea.

Abra kurodai HABEウラシマザクラガイ（波部）

1961 Abra kurodai HABE, Venus, 21, 153, 156, tf. 1, 2 
1961 Abra kurodai: CISJ, 2, 135, pl. 61, f. 13

1971 Abra kurodai: KURODA \& HABE, SSSB, 684 (J) 447 (E), p1. 99, f. 5

Loc. S (s-m) tr.

Dist. Honshu (north to Sagami Bay) and Shikoku.

Remarks: It seems to be the fully grown specimens of Abra fujitai HaBE.

\section{Genus Abrina HABEシロバトガイ属}

1951 Abrina HABE, GJS, (3), 210. Type (OD): Abra kanamarui KurodA=Macoma lunella Gould

Abrina lunella GouLDシロバトガイ（金丸）；コバトガイ（波部）

1861 Macoma lunella Gould, PBSNH, 8, 29

1933 Macoma hakushatonensis NomURA, SRTIU, (2) 16 (1), 103, pl. 1, f. 6a, b

1951 Abra kanamarui KURODA: Venus, 16, 71, tf. 3-6

1961 Abrina lunella: HABE, CISJ, 2, 136, pl. 61, f. 15

1964 Macoma lunella: JoHNson, USNMB, 239, 105, pl. 27, f. 1

1971 Abrina lunella: KuRODA \& HABE, SSSB, 684 (J) 448 (E), pl. 121, f. 4

Loc. N M S (s-m) dp.

Dist. Honshu (north to Sagami Bay) to Formosa.

Abrina s-kinoshitai KURODA \& HABEキノシタシロバトガイ（波部）

マミレリュウグウザクラガイ（黑田）

1958 Abrina skinoshitai KURODA \& HABE, PSMBL, 7 (1), 42

1961 Abrina skinoshitai: HABE, CISJ, 2, 136, pl. 61, f. 16

1971 Abrina skinoshitai: KuRODA \& HABE, SSSB, 685 (J) 448 (E), pl. 99, f. 3

Loc. S (s-m) tr.

Dist. Honshu (north to Kashima Nada) to Kyushu.

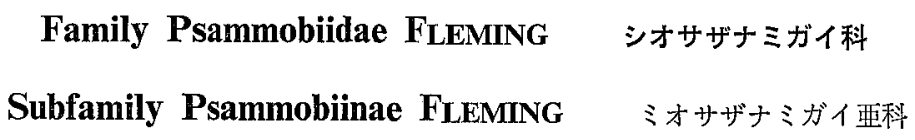

\section{Genus Gari SCHUMACHERシオサザナミガイ属}

1817 Gari Schumacher, ENSVT, 44, 131. Type (M): Gari vulgaris Schumacher=Solen methystus. Wood

Gari truncata (LINNAEUS)シオサザナミガイ（丹浦）

1758 Tellina gari LinNaeus, Syst. Nat., 10, 674 (reject. by ICZN)

1767 Tellina truncata Linnaeus, Syst. Nat., 12, 1113

1849 Psammobia bipartita PHILIPPI, ZfM, 5 (11) (1848), 166

1857 Psammobia caerulescens ReEve (non LamarCK, 1818), Icon., sp. 60

1959 Gari truncata: KIRA, CISJ, 1, 153, pl. 59, f. 1

1971 Gari truncata: KunOdA \& HABE, SSSB, 676 (J) 441 (E), pl. 98, f. 4

Loc. N M S (s) dp.

Dist. Honshu (north to Bōsō Peninsula) to Kyushu. Widely ranging in the Indo-West Pacific.

Gari anomala (DEHSYAYES) ウスベニマスオガイ（岩川）

1855 Psammobia anomala DeshaYes, PZSL, 22 (1854), 32

1857 Psammobia anomala: RevVe, Icon., sp. 5

1961 Gari anomala: HABE, CISJ, 2, 135, pl. 61, f. 4 
1971 Gari anomala: KuRODA \& HABE, SSSB, 676 (J) 442 (E), pl. 97, f. 13

Loc. N M (s-m) dp.

Dist. Honshu (north to Bōsō Peninsula) to Kyushu. Widely ranging in the Western Pacific.

Gari hosoyai HABE ヒノデアシガイ(波部)

1958 Gari hosoyai HABE, PSMBL, 7 (1), 40

1961 Gari hosoyai: HABE, CISJ, 2, 135, pl. 61, f. 1

1971 Gari hosoyai: KURODA \& HABE, SSSB, 676 (J) 442 (E), p1. 97, f. 14

Loc. N S (s) dp.

Dist. Honshu (north to Bōsō Peninsula) to Kyushu.

Gari sibogai PRASHAD ホソアシガイ(波部)

1932 Gari sibogai PRASHAD, Siboga, 53, 303, pl. 7, f. 3, 4

1961 Gari sibogai: HABE, CISJ, 2, 135, pl. 61, f. 3

1971 Gari sibogai: KURODA \& HABE, SSSB, 677 (J) 442 (E), pl. 97, f. 15, 16

Loc. S (s) dp.

Dist. Honshu (north to Bōsō Peninsula) to Kyushu and Indonesia.

Gari maculosa (LAMARCK) アシガイ（目八）

1818 Psammobia maculosa LAMARCK, AsV, 5, 513

1855 Psammobia ornata DESHAYES, PZSL, 22 (1854), 323

1856 Psammobia ornata: REEVE, Icon., sp. 26

1959 Gari maculosa: KIRA, CISJ, 1, 153, pl. 59, f. 6

1971 Gari maculosa: Kuroda \& HABE, SSSB, 677 (J) 442 (E), pl. 97, f. 9

Loc. N M S (s) gl.

Dist. Honshu (north to Bōsō Peninsula) to Kyushu. West Pacific.

Genus Psammobia LAMARCK ミヒカリマスオガイ属

1818 Psammobia Lamarck, AsV, 5, 511. Type (SD): Tellina feroensis (sic) (forvensis) Gmeurn

Psammobia radiata (PHLIPPI)：ミヒカリマスオガイ（黒田）

1844 Psammobia radiata PHILPPI, Abbild., 1, 194, pl. 2, f. 5

1856 Psanmobia amethyastina REEVE, Icon., sp. 19

1959 Psammocola radiata: KIRA, CISJ, 1, 153, pl. 59, f. 2

1971 Gobraeus radiatus: KuRODA \& HABE, SSSB, 678 (J) 443 (E), p1. 97, f. 10

Loc. N M S (s) dp.

Dist. Honshu (north to Bōsō Peninsula), to Kyushu. South East Asia and Nicobar Islands.

Genus Grammatomya DALL ハスメヨシガイ属

1898 Grammatomya Dall, PANSP, 57. Type (M): Psammobia squamosa LAMARCK

*Grammatomya kurodai HABE sp. nov. クロダヨシガイ（新称）

Pl. 3, fig. 5 .

Shell rather solid, elongated oval in shape, rounded at the anterior margin and roundly truncated and slightly gaping at the posterior margin, rather compressed. Umbo slightly behind the middle of dorsal margin, low and slightly backwards. Surface basically white to light yellow with purplish red broad and narrow 
radiating rays and sculptured with the radiating ribs of 9-12 in number from the umbo to the crenulated posterior margin and oblique folds from the anterodorsal margin to the postero-ventral margin besides the weak growth lines. Right valve with two bifid cardinal teeth of equal size and left valve with one large cardinal tooth. No lateral teeth. Inside of shell white with purplish hue. Pallial sinus deep and broad extending to a point below the umbo. Its lower border confused with the pallial line. Anterior and ventral margins smooth. Posterior margin crenulated by the endings of radial ribs on the surface.

Length $26.8 \mathrm{~mm}$, height $13.3 \mathrm{~mm}$ and breadth $6.4 \mathrm{~mm}$ (Holotype conjoined valves preserved in the National Science Museum, NSMT-Mo 58965)

Length $31.0 \mathrm{~mm}$, height $15.0 \mathrm{~mm}$ and breadth $4.3 \mathrm{~mm}$ (Paratype right valve, NSMT-Mo 58966)

Length $26.8 \mathrm{~mm}$, height $13.0 \mathrm{~mm}$ and breadth $3.6 \mathrm{~mm}$ (Paratype left valve, NSMT-Mo 58967)

Type Locality: Kochi Pref., Shikoku M S (s-m) gl.

Distribution: Shikoku up to Enshu-Nada, Honshu.

Remarks: Grammatomya squamosa (LAMARCK 1818) is an ally to this new species but has the oblique folds on the anterior portion gradually growing to the radial ribs on the posterior portion without a distinct border as in this new species. The new species is dedicated of Dr. Tokubei Kuroda, Honorary President of the Malacological Society of Japan.

\section{Genus Asaphis MODEER リュウキュウマスオガイ属}

1793 Asaphis Modeer, Kungl. Vet-Acad. Nya. Handl., 14, 176

1797 Capsa Bruguiere, ENHNV, 2, pl. 231. Type (M): Venus deflorata LinNaEus

Asaphis dichotoma (ANTON) リュウキュウマスオガイ（岩川）

1838 Sanguinolaria dichotoma ANTon, Verzeich. Conchyl, 4

1856 Capsa deflorata ReEve, Icon., sp. 1 (non LinNaEus, 1758)

1959 Asaphis dichotoma: KIRA, CISJ, 1, 153, pl. 59, f. 4

Loc. S (g) int.

Dist. Honshu (north to Kii Peninsula) to Ryukyu Islands. Widely ranging in the Indo-Pacific.

\section{Genus Kermadysmea PoWELL, 1958 スダレマスオガイ属}

1958 Kermadysmea Powell Rec. Auckland Inst. \& Mus., 5, 73. Type (OD): Kermadysmea galatheae Powent

Kermadysmea nishimurai HABE 1976 スダレマスオガイ（波部） Pl. 6, f. 9.

1976 Kermadysmea nishimurai HABE, Venus, 35 (2), 39, 42, pl. 1, f. 12.

Loc. S (s) dp.

Dist. Izu Jslands to Kii Peninsula, Honshu.

Genus Heteroglypta MARTENS ウチトミガイ属

1880 Heteroglypta Martens, Maurit. u. Seych., 331. Type (M): Psammobia contraria DESHAYES

Heteroglypta contraria (DESHAYES)ウチトミガイ（黒田）

1863 Psammobia contraria Deshayes, Reunion, 11, pl. 28, f. 20, 21 
1934 Heteroglypta nipponica Kuroda, Venus, 4 (6), inside of cover.

1961 Heteroglypta nipponica: HABE, CISJ, 2, 135, pl. 61, f. 7

Loc. M S (bo) gl.

Dist. Honshu (north to Sagami Bay) to Kyushu. Widely ranging in the IndoWest Pacific.

\section{Genus Psammotaea LAMARCK マスオガイ属}

1818 Psammotaea LAMARCK, AsV, 5, 516

1855 Capsella Deshayes, PZSL, 22 (1854), 347 (non Gray, 1851). Type (SD, ChiLdREN, 1823): Psammotaea donacina LAMARCK

Psammotaea elongata (LAMARCK) マスオガイ（六介）

1818 Psammotaea elongata Lamarck, AsV, 5, 514

1818 Psammotaea violacea LAMARcK, AsV, 5, 517

1959 Psammotaea elongata: KIRA, CISJ, 1, 153, pl. 59, f. 3

Loc. M S (m) int.

Dist. Honshu (north to Kii Peninsula) to Ryukyu Islands. Widely ranging in the Indo-Pacific.

Psammotaea virescens (DeShAYES) オチバガイ（六介）

1855 Capsella virescens Deshayes, PZSL, 22 (1854) 349

1855 Capsella tenuis DeSHAYES, PZSL, 22 (1854), 349

1872 Soletellina moesta LisCHKE, M B L. 19, 107

1874 Soletellina moesta: LISCHKE, JMC, 3, 99, pl. 8, f. 4-6

1959 Psammotaea virescens: KIRA, CISJ, 1, 154, pl. 59, f. 7

Loc. N M S (m) int.

Dist. Honshu (north to Tokyo Bay) to Kyushu. Widely ranging in the Western Pacific.

Psammotaea minor (DESHAYES)ハザクラガイ（黒田）

1855 Capsella minor DeshaYes, PZSL, 22 (1854), 347

1857 Capsella minor: ReEve, Icon., sp. 9

1959 Psammotaea minor: KIRA, CISJ, 1, 154, p1. 59, f. 8

Loc. N M S (s-m) int.

Dist. Honshu (north to Bōsō Peninsula) to Kyushu. Ranging in the Western Pacific.

\section{Subfamily Sanguinolariinae GRANT \& GALEムラサキガイ亜科}

$$
\text { Genus Hiatula MoDEER ムラサキガイ属 }
$$

1793 Hiatula Modeer, Kungl. Vet-Acad. Nya. Hand1., 14, 178

1824 Soletellina Blainville, DSN, 32, 350. Type (SD, Stoliczka, 1871): Solen diphos LINNAEUS

Hiatula diphos (LINNAEUS) ムラサキガイ（六介）

1771 Solen diphos LinNaeus, Mantissa., 544

1818 Solen violaceus LAMARCK, AsV, 5, 455

1857 Soletellina cumingiana ReEve, Icon., sp. 4

1857 Soletellina adamsii REEVE, Icon., sp. 5

1959 Soletellina diphos: KIRA, CISJ, 1, 154, p1. 59, f. 11 
Loc. N M S (m) int.

Dist. Honshu (north to Bōsō Peninsula) to Kyushu. Widely ranging in the Western Pacific.

Hiatula atrata (REEVE)アケボノキヌタガイ（黒田）

1857 Soletellina atrata REeVE, Icon., sp. 14

1971 Hiatula atrata: HaBe \& Kosuge, SWC, 2, 196, pl. 60, f. 10

Loc. S. (s) int.

Dist. Honshu (north to Bōsō Peninsula) to Philippines and China.

Hiatula boeddinghausi (LISCHKE)フジナミガイ（六介）

1870 Soletellina boeddinghausi LischKe, M B L., 17, 26

1871 Soletellina boeddinghausi: LISCHKE, JMC, 2, 118, pl. 9, f. 9

1959 Soletellina boeddinghausi: KIRA, CISJ, 1, 154, pl. 59, f. 2

1971 Hiatula boeddinghausi: KURODA \& HABE, SSSB, 678 (J) 443 (E), pl. 98, f. 1

Loc. N M S (s) int.

Dist. Honshu (north to Bōsō Peninsula) to Kyushu.

\section{Genus Nuttallia DALL イソシジ属}

1900 Nuttallia DaLl, PANSP, 58. Type (OD): Sanguinolaria nuttallii CONRAD

Nuttallia japonica (REEVE) イソシジ（目八）

1857 (Jan.) Soletellina japonica REEve, Icon., sp. 16

1874 Soletellina olivacea: LISCHKE, JMC, 3, 98, pl. 8, f. 7-12

1953 Nuttallia olivacea: Krra, Venus, 17, 147, tf. 1a, 2a

1959 Nuttallia olivacea: KIRA, CISJ, 1, 154, pl. 59, f. 9

1971 Nuttallia olivacea: KuRoda \& HaBE, SSSB, 679 (J) 444 (E), pl. 98, f. 2

1978 Nuttallia japonica: Roтн, Venus, 37 (4), 223, tf. 1-4

Loc. N M (s-m) int.

Dist. Southern Hokkaido to Kyushu, Korea and China.

Nuttallia obscura (REEve) ワスレイソシジミ（吉良）

1857 (Jan.) Soletellina obscura ReEve, Icon., sp. 21

1857 (June) Psammobia olivacea JAY, Rep. Jap. Exp. Com. perry. 292, pl. 1, f. 8, 9

1953 Nuttallina japonica: KIRA, Venus, 17, 147, tf. 1b, 2b

1953 Nuttallia solida KrRA, Venus, 17, 149, tf. 1c, 2c

1959 Nuttallia solida: KIRA, CISJ, 1, 154, pl. 59, f. 10 （アッイソシジミ 吉良）

1978 Nuttallia obscura: Rотн, Venus, 37 (4), 225, tf. 5-8

1978 Nuttallia olivacea: Roth, Venus, 37 (4), 226, tf. 9, 10

Loc. S (s-m) int.

Dist. Honshu (Kii Peninsula) to Kyushu.

\section{Family Solecurtidae d'ORBIGNY キヌタアゲマキガイ科 \\ Genus Solecurtus BLAINVILLEキヌダアゲマキガイ属}

1824 Solecurtus BLATNvilLe, DSN, 32, 351

1835 Macha OKEN, Allg. Naturges., 5 (1), 298. Type (SD, DesHAYES, 1829): Solen strigillatus LINNAEUS

Solecurtus divaricatus (LISCHKE) キヌタアゲマキガイ (岩川)

1869 Macha divaricata LisCHKE, M B L., 16, 108 
1869 Macha divaricata: LISCHKEk, JMC, 1, 142, pl. 10, f. 1,2

1882 Macha divaricata: Dunker, Index, 175, pl. 7, f. 26

1959. Solecurtus dunkeri KIRA, CISJ, 1, 152, pl. 58, f. 22

1959 Solecurtus divaricatus: KIRA, CISJ, 1, 153, pl. 58, f. 23

1971 Solecurtus divaricatus: KuRODA \& HABE, SSSB, 680 (J) 444 (E), pl. 99, f. 7

Loc. N M S (s) dp.

Dist. Honshu (north to Bōsō Peninsula) to Formosa.

Solecurtus consimilis KURODA \& HABE ッヤキヌタアゲマキガイ（波部）

1961 Solecurtus consimilis KURODA \& HABE, CISJ, 2, App. 39, p1. 61, f. 6

1971 Solecurtus consimilis: Kuroda \& HaBe, SSSB, 680 (J) 445 (E), pl. 99, f. 6

Loc. M S (s) dp.

Dist. Honshu (north to Bōsō Peninsula) to Kyushu.

Solecurtus sagamiensis KURODA \& HABEヤワラキヌタアゲマキガイ（黒田・波部）

Pl. 6, f. 6.

1971 Solecurtus sagamiensis KURODA \& HABE, SSSB, 680 (J) 445 (E), pl. 98, f. 5

Loc. M S (s) dp.

Dist. Honshu (north to Bōsō Peninsula) to Kyushu.

Solecurtus wilsoni TRYONウスキヌタアゲマキガイ（黒田）

1870 Solecurtus wilsoni TrYoN, AJC, 6, 23, pl. 1, f. 2

1961 Solecurtus wilsoni: HABE, CISJ, 2, 135, pl. 61, f. 5

Loc. N S (s-m) dp.

Dist. Honshu (north to Enshu nada) to Kyushu and East China Sea.

Solecurtus rhombus (SPENGLER)シロキヌタアゲマキガイ（黒田） Pl. 6, f. 7.

1794 Solen rhombus SPENGLER, SNS, 3 (2), 102

1874 Solecurtus exaratus SoWERBY Icon., sp. 1

Loc. N M (s) dp.

Dist. Honshu (north to Kii Peninsula) to Ryukyu Islands. Tropical Pacific.

\section{Genus Azorinus RÉCLUZ ズソグリアゲマキガイ属}

1844 Azor Brown, Illust. Conch. Great. Britain, (2), 113 (non Sowerby, 1824)

1869 Azorinus Récluz, Actes Soc. Linn. Bordeaux, 27, 67

1930 Zozia WINkworth, PMSL, 19, 15 (new name for Azor). Type (M): Solen coarctatus GMELIN

Azorinus abbreviatus (GouLD)ズングリアゲマキガイ（岩川）

[1791 Solen coarctatus GMELIN, Syst. Nat., 13, 3227]

[1793 Solen emarginatus SPENGLER, SNS, (3) 7, 105]

1818 Solen constrictus LAMARCK, AsV, 5, 455

1861 Solecurtus abbreviatus GouLd, PBSNH, 8, 26

1908 Novaculina andamanensis Preston, RIAM, 2, 109, pl. 16, f. 40

1959 Azorius minutus KIRA, CISJ, 1, 152, pl. 58, f. 20

1959 Azorius abbreviatus: KIRA, CISJ, 1, 152, pl. 58, f. 21

1971 Azorinus abbreviatus: KURODA \& HABE, SSSB, 681 (J) 446 (E), pl. 98, f. 6

Loc N M S (s-m) dp.

Dist. Honshu (north to Bōsō Peninsula) to Kyushu, Korea and China. Widely ranging in the Indo-West Pacific region. 


\section{Superfamily Solenacea LAMARCK マテガイ超科 \\ Family Solenidae LAMARCK マテガイ科 \\ Genus Solen LINNAEUS マテガイ属}

1758 Solen Linnadus, Syst. Nat., 10, 672

1806 Solenarius Dumeril, ZA, 168. Type (SD, Schumacher, 1817): Solen vagina LinnaEus

Subgenus Solen LINNAEUS マテガイ亜属

Solen (Solen) strictus GouLD ママテガイ (目八)

[1818 Solen corneus LAMARCK, AsV, 5, 451]

1861 Solen strictus Gould, PBSNH, 8, 26

1861 Solen gracilis Gould, PBSNH, 8,28 (non Sowerby 1844 nec PhitrpPI, 1847)

1867 Solen gouldi ConRAD, AJC, 3, App. 28

1882 Solen gouldi: DunkeR, Index, 173, pl. 4, f. 1

1888 Solen incertus Clessin, Cab., 11 (3), 31, pl. 12, f. 8

1928 Solen corneus pechiliensis Grabau \& KInG, Peitaiho, 186, pl. 6, f. 47

1959 Solen gouldi: KIRA, CISJ, 1, 161, pl. 61, f. 7

1964 Solen corneus: HABE, BNSM, 7 (1), 8, pl. 1, f. 4

1971 Solen (Solen) strictus: KuRoda \& HABE, SSSB, 699 (J) 458 (E), pl. 101, f. 2, 3

Loc. N M S (s) int.

Dist. Southern Hokkaido to Kyushu, Korea and China.

Solen (Solen) gordonis YoroYAMAアアカマテガイ（岩川）

1920 Solen gordonis YoKoYama, JCSIUT, 39 (6), 111, pl. 7, f. 23

1959 Solen gordonis: KIRA, CISJ, 1, 161, pl. 61, f. 9

1964 Solen gordonis: HABE, BNSM, 7, 12, pl. 1, f. 3

1971 Solen gordonis: KuRODA \& HABE, SSSB, 699 (J) 459 (E), pl. 101, f. 4,

Loc N M S (s) dp.

Dist. Honshu (north to Bōsō Peninsula) to Kyushu and Korea.

Solen (Solen) grandis DUNKERオオマテガィ（岩川）

1861 Solen grandis DUNKER, PZSL, 418

1871 Solen grandis: Dunker, Nov. Conch., 71, pl. 24, f. 5

1928 Solen beckii: Grabau \& King, Peitaiho, 186, pl. 6, f. 46 (non PHILIPPI, 1847),

1959 Solen grandis: KIRA, CISJ, 1, 161, pl. 61, f. 8

1964 Solen grandis: HABE, BNSM, 7, 11, pl. 1, f. 5

1971 Solen (Solen) grandis: KuRODA \& HABE, SSSB, 699 (J) 460 (E), pl. 101, f. 1

Loc. N S (s) dp.

Dist. Honshu (north to Bōsō Peninsula) to Kyushu, Korea and China.

*Solen (Solen) kurodai HABEダンダラマテガイ（黒田）

1962 Solen intermedia: KIRA, CISJ, 1, 161, pl. 61, f. 2 (non PHILIPPI, 1843)

1864 Solen kurodai HABE, BNSM, 7, 10, pl. 1, f. 13

1971 Solen (Solen) kurodai: KuRodA \& HABE, SSSB, 699 (J) 460 (E), pl. 101, f. 7

Loc. N M S (s) int.

Dist. Honshu (north to Bōsō Peninsula) to Kyushu.

Solen (Solen) sloani HANLEY リュウキュウマテガイ (平瀬)

1843 Solen sloani HANLeY, Cat. Bivalve, 12, 336, pl. 11, f. 18

1847 Solen versicolor PHILIPPI, Abbild., 3, 43, pl. 2, f. 3 
1959 Solen sloani: KIRA, CISJ, 1, 161, pl. 61, f. 2

1964 Solen sloani: HABE, BNSM, 7, 12, pl. 1, f. 6

Loc. M S (s) dp.

Dist. Honshu (north to Kii Peninsula) to Ryukyu Islands. Widely ranging in the Indo-Pacific region.

\section{Subgenus Ensisolen HABE エゾマテガイ亜属}

1977 Ensisolen HaBE, SMJ B/S, 228. Type (OD): Solen krusensterni SchrencK

Solen (Ensisolen) krusensterni SCHRENCKエゾマテガイ (岩川)

1869 Solen krusesnterni SCHRENCK, Reise, 2, 194, pl. 25, f. 9-12

1959 Solen krusensterni: KIRA, CISJ, 1, 161, pl. 61, f. 3

1964 Solen krusensterni: HABE, BNSM, 7, 14, pl. 1, f. 1

1971 Solen (Solenarius) krusensterni: KURODA \& HABE, SSSB, 700 (J) 460 (E), pl. 101, f. 5

Loc. N M (s) dp.

Dist. Sakhalien to Kyushu and Korea.

Solen (Ensisolen) roseomaculatus PILSBRY バラフマテガイ (岩川)

1901 Solen roseomaculatus PiLSBRY, PANSP, 53, 399, pl. 19, f. 13

1959 Solen roseomaculatus: KIRA, CISJ, 1, 161, pl. 61, f. 4

1964 Solen roseomaculatus: HABE, BNSM, 7, 15, pl. 1, f. 7

1971 Solen (Solenarius) roseomaculatus: KURODA \& HABE, SSSB, 700 (J) 461 (E), pl. 101, f. 9-11

Loc. N M S (s) int.

Dist. Honshu (north to Bōsō Peninsula and Noto Peninsula) to Kyushu. Widely ranging in the Indo-Western Pacific region.

Solen (Ensisolen) luzonicus DUNKER ヒナマテガイ（吉良）

1861 Eusis luzonicus DUNKER, PZSL, 421

1874 Solen luzonicus: Sowerby, Icon., sp. 24

1959 Solen luzonicus: KIRA, CISJ, 1, 160, pl. 61, f. 1

1964 Solen luzonicus: HABE, BNSM, 7, 15, pl. 1, f. 8

1971 Solen (Solenarius) luzonicus: KURODA \& HABE, SSSB, 701 (J) 461 (E), pl. 101, f. 6, 8

Loc. M S (s) tr.

Dist. Honshu (north to Bōsō Peninsula) to Philippines.

\section{Family Cultellidae DAvIS ユキノアシタガイ科}

\section{Genus Siliqua MÜHLFELD ミゾガイ属}

1811 Siliqua MüHLFELD, GNFBM, 5, 44

1815 Aulus OKEN, Lehrb. Nat., 3 (1), 8, 225 (Invalid)

1841 Machaera Gould, Mass., 32 (non Cuvier, 1832). Type (M): Solen radiatus LinnaEus

Siliqua pulchella (DUNKER) ミン゙ガイ（六介）

1852 Aulus pulchellus DuNKER, ZfM, 58

1888 Machaera pulchella: Clessin, Cab., 11 (3), 65, pl. 20, f. 6

1959 Siliqua pulchella: KrrA, CISJ, 1, 162, pl. 61, f. 13

1965 Siliqua pulchella: HABE, Venus, 23, 194, pl. 13, f. 8, 9

1971 Siliqua pulchella: KuRODA \& HABE, SSSB, 701 (J) 461 (E), pl. 102, f. 3

Loc. N S (s) dp.

Dist. Honshu (north to Bōsō Peninsula) to Kyushu, Korea and North China. 
Siliqua japonica (DUNKER)シボリミゾガイ（黒田)

1862 Aulus japonicus Dunker, PZSL (1861), 426

1874 Cultellus japonicus: SowerBy, Icon., sp. 5

1959 Siliqua japonica: KIRA, CISJ, 1, 162, pl. 61, f. 12

1965 Siliqua japonica: HABE, Venus, 23 (4), 194, pl. 13, f. 10, 11

Loc. $N$ (s) dp.

Dist. Honshu (north to Kii Peninsula) to Kyushu.

\section{Genus Ensiculus H. ADAMS タカノハガイ属}

1860 Ensiculus H. Adams, PZSL, 2, 369. Type (OD): Solen cultellus Linnaeus

Ensiculus cultellus (LINNAEUS) タカノ八ガイ（六介）

1758 Solen cultellus Linnaeus, Syst. Nat., 10, 673

1862 Cultellus australis DuNKER, PZSL (1861), 423

1862 Cultellus lividus DUNKer, PZSL (1861), 423

1862 Cultellus marmoratus DUNKer, PZSL (1861), 423

1874 Cultellus cultellus: SOWERBX, Icon., sp. 23

1877 Cultellus philippianus DuNKER, M B L., 24, 68

1882 Ensiculus philippianus: Dunker, Index, 174, pl. 7, f. 23

1882 Ensiculus marmoratus: DUNKER, Index, 174, pl. 7, f. 24

1888 Cultellus aspersus Clessin, Cab., 11 (3), 45, pl. 14, f. 3

1906 Cultellus (Ensiculus) maculatus Preston, J. of M., 12, 8, pl. 2, f. 36

1936 Ensiculus hilaris IREDALE, RAM, 19, 284, pl. 20, f. 15

1959 Ensiculus philippianus: KirA, CISJ, 1, 161, pl. 61, f. 6

1965 Ensiculus cultellus: HABE, Venus, 23 (4), 195, pl. 13, f. 1

1971 Ensiculus philippianus: KurOdA \& HABE, SSSB, 702 (J) 462 (E), pl. 102, f. 2

Loc. N M S (s) int.

Dist. Honshu (north to Bōsō Peninsula) to Kyushu and Korea. Widely ranging in the Indo Pacific region.

\section{Genus Phaxas GRAYユキノアシタガイ属}

1852 Phaxas Gray, Syn., 259, 262. Type (M): Solen pellucidus PenNant

Phaxas attenuatus (DUNKER)ユキノアシタガイ（六介）

(1862 Cultellus hanleyi DunkeR, PZSL (1861), 421)

1862 Cultellus attenuatus DunKer, PZSL (1861), 422

1862 Cultellus attenuatus: DunKER, Nov. Conch (1861), 72, pl. 24, f. 4

1874 Cultellus attenuatus: SowerBy, Icon., sp. 8

1959 Phaxas attenuatus: KIRA, CISJ, 1, 162, pl. 61, f. 11

1965 Cultellus (Culterensis) attenuatus: HABE, Venus, 23 (4), 195, pl. 13, f. 5

1971 Culterensis attenuatus: KunodA \& HABE, SSSB, 701 (J) 462 (E), pl. 102, f. 1

Loc. N M S (s-m) dp.

Dist. Honshu (north to Bōsō Peninsula) to Philippines and China.

Superfamily Dreissenacea Gray in TURTONマゴコロガイ超科

Family Dreissenidae Gray in TURTON マゴコロガイ科

Genus Peregrinamor SHOJIマゴコロガイ属

1838 Peregrinamor Sнол, Venus, 8, 124. Type (M): Peregrinamor ohshimai SHon 
Peregrinamor ohshimai SHOJI マゴコロガイ (庄司)

1938 Peregrinamor ohshimai SHon, Venus, 8, 124, pl. 3, 4

1954 Peregrinamor ohshimai: HABE, ICJS, 1 (21), 160, pl. 22, f. 22-24

Loc. S (sy). [Urogebia major (de HAAN)]

Dist. Wakayama Pref., Honshu, Inland Sea of Japan (Setonaikai) and northern

Kyushu.

\section{Superfamily Arcticacea NEWTON アイスランドガイ超科 \\ Family Kelliellidae FISCHER ケシハマゲリ科 \\ Genus Alvenius CONRADケシトリガイ属}

1865 Alvenius ConRaD, AJC, 1, 138. Type (M): Alvenius minutus ConRAD

Alvenius ojianus (YoKoYAMA) ケシトリガイ（黑田）

1927 Kellia(?) ojiana YoKoYaMA, JFSIUT, (2) 1 (10), 432, pl. 50, f. 7, 8

1951 Alvenius ojianus: HABE, GJS, 2, 116, f. 223-5

Loc. M S (s-m) dp.

Dist. Honshu (north to Tokyo Bay) to Kyushu.

\section{Family Trapeziidae LAMY フナガタガイ科}

Genus Trapezium Megerle-von MüHLFELDフナガタガイ属

1811 Trapezium Megerle von MüHLFELD, GNFBM, 5, 68

1817 Libitina SCHUMACHER, ENSVT, 54, 168

1819 Cypricardia Lamarck, AsV, 6, 27. Type (SD, StewarT, 1930): Chama oblongum

LINNAEUS

Subgenus Trapezium Mergerle von MüHLFELDフフガタガイ平属

Trapezium (Trapezium) bicarinatum (ScHUMACHER)フナガタガイ（黒田）

1817 Libitina bicarinata Schumacher, ENSVT, 169

1818 Cypricardia angulata LAMARCK, AsV, 6, 28

1843 Cypricardia angulata: ReEve. Icon., sp. 2

1954 Trapezium (Trapezium) bicarinatum: SoleM, PMSL, 31 (2), 68, p1. 5, f. 8-13

1959 Trapezium bicarinatum: KIRA, CISJ, 1, 131, pl. 52, f. 25

Loc. M S (r) int.

Dist. Honshu (Kii Peninsula) to Ryukyu Islands. Widely ranging in the Indo-Pacific.

Trapezium (Trapezium) oblongum (LINNAEUS)スエヒロフナガタガイ（黒田）

1785 Chama oblonga LinNaues, Syst. Nat., 10, 692

1797 Cardita carinata Bruguière, EMHNV, 1, 409, pl. 234, f. 2a, b

1819 Cypricardia guinaica LAMARCK, AsV, 6 (1), 28

1811 Trapezium perfectum Megerle von MüHFELD, GNFBM, 5, 68

1843 Cypricardia guinaica: ReEve, Icon., sp. 13

1954 Trapezium (Trapezium) oblongum: Solem. PMSL, 31 (2), 66, pl. 5, f. 3-7

1959 Trapezium oblongatum (sic.): KIRA, CISJ, 1, 131, p1. 52, f. 26

Loc. S (r) int.

Dist. Honshu (Kii Peninsula) to Ryukyu Islands. Widely ranging in the IndoPacific. 


\section{Subgenus Neotrapezium HABEタガソデガイモドキ亜属}

1951 Neotrapezium HABE, GJS, 1, 119. Type (OD): Cardita sublaevigata LAMARCK

Trapezium (Neotrapezium) sublaevigatum (LAMARCK)タカソデガイモドキ（黒田）

1819 Cardita sublaevigatum LaMARCK, AsV, 6 (1), 26

1843 Cypricardia vellicata REEVE, Icon., sp. 7

1954 Trapezium (Neotrapezium) sublaevigatum: Solem, PMSL, 31 (2), 71, pl. 6, f. 4, 5, 8, 9, $13-15$

1959 Trapezium (Neotrapezium) sublaevigatum: KIRA, CISJ, 1, 132, pl. 52, f. 28

Loc. N M S (r) int.

Dist. Honshu (north to Bōsō Peninsula) to Ryukyu Islands. Widely ranging in the Indo Pacific.

Trapezium (Neotrapezium) liratum (REEVE) ウネナシトマヤガイ（平瀬）

1843 Cypricardia lirata ReEve, Icon., sp. 1

1905 Trapezium japonicum PILSBRY, PANSP, 57, 119, pl. 5, f. 34-36

1905 Trapezium japonicum delicatum PILSBRY, PANSP, 57, 120, pl. 5, f. 44

1922 Trapezium nipponicum YoKoYAMA. JCSIUT, 44 (1), 167, pl. 13, f. 1a, $1 \mathrm{~b}$

1922 Trapezium ventricosum YoKoYAмA, JCSIUT, 44 (1), 168, pl. 13, f. 1a, b

1954 Trapezium (Neotrapezium) liratum: Solen, PMSL, 31 (2), 73, pl. 6, f. 1-3, 6, 7, 10

1959 Trapezium (Neotrapezium) japonicum: KIRA, CISJ, 1, 132, pl. 52, f. 29

Loc. $\mathrm{M} \mathrm{N}$ (st) int.

Dist. Honshu to Kyushu. Widely ranging in the Indo Pacific.

\section{Genus Coralliophaga BLAINVILLEタガソデガイ属}

1824 Coralliophaga BLAINVILLE, DSN, 32, 343

1854 Lithophagella GRAY, AMNH, (2) 14, 21. Type (M): Chama coralliophaga GMELIN

Coralliophaga coralliophaga (GMELIN) タガソデガイ（目八）

1791 Chama coralliophaga GMELIN, Syst. Nat., 13, 3305

1843 Cypricardia coralliophaga: ReEve, Icon., sp. 12

1959 Coralliophaga coralliophaga: KIRA, CISJ, 1, 132, pl. 52, f. 27

Loc. M S (bo) gl.

Dist. Honshu (north to Mutsu Bay) to Kyushu. Indo-Pacific and West Indies.

\section{Superfamily Glossacea GRAY コウホネガイ迢科 \\ Family Glossidae GRAY コウホネガイ科}

Genus Meiocardia H. \& A. ADAMS コウホネガイ属

1857 Meiocardia H. \& A. Adams, GRM, 2, 461. Type (SD, Martens, 1870; StolrczKa, 1870): Chama moltkiana GMELIN

Meiocardia moltkiana (GMELIN)カノコシボリコウホネガイ（黑田）

1791 Chama moltkiana GMELIN, Syst. Nat., 13, 3303

1845 Isocardia moltkiana: REEVE, Icon., sp. 1

1882 Isocardia sanguinomaculata DUNKER, Index, 213

1959 Meiocardia moltkiana sanguinomaculata: KIRA, CISJ, 1, 194, pl. 71, f. 5

1971 Meiocardia moltkiana: Kuroda \& HABE, SSSB, 614 (J) 395 (E), pl. 87, f. 11

Loc. M S (g) dp. 
Dist. Honshu (north to Bōsō Peninsula) to Ryukyu Islands. West Pacific.

Meiocardia tetragona (ADAMS \& REEVE)コウホネガイ（丹浦）

1850 Isocardia tetragona. AdAMs \& ReEve, Samarang, 76, pl. 22, f. 1

1959 Meiocardia tetragona: KIRA, CISJ, 1, 131, pl. 52, f. 24

1971 Meiocardia tetragona: KuRODA \& HABE, SSSB, 614 (J) 395 (E), pl. 87, f. 10

Loc. M S (s) gl.

Dist. Honshu (north to Bōsō Peninsula) to Ryukyu Islands. Central and West Pacific.

Meiocardia lamarckii (REEVE)テリコウホネガイ（黑田）

1845 Isocardia lamarckii REEVE, Icon., sp. 5

1959 Meiocardia lamarckii: KIRA, CISJ, 1, 131, pl. 52, f. 23

Loc. M S (s) tr.

Dist. Honshu (north to Bōsō Peninsula) to Kyushu. West Pacific.

\section{Family Vesicomyidae DALLオトヒメハマグリ科 \\ Genus Callogonia DALL ウラシマハマグリ属}

1889 Callogonia DALL, BMCZ, 18, 440. Type (M): Callocardia (Callogonia) leeana DALL

*Callogonia hayashii HABE ウラシマハマグリ（波部） Pl. 7, f. 8.

1976 Callogonia hayashii HABE, Venus, 35 (2), 40, 43, pl. 1, f. 5, 6

Loc. S (s) tr.

Dist. Honshu (Wakayama Pref.).

Genus Waisiuconcha BEETSオトヒメハマグリ属

1942 Waisiuconcha BeETs, Leidsche Geol. Med., 315, 316. Type (OD): Waisiuconcha alberdinae BeETS

Waisiuconcha katsuae (KURODA) オトヒメハマグリ（黒田）

1952 Vesicomya katusuae KURODA, Venus, 17 (1), 4, f. 5 9

1961 Vesicomya katsuae: HABE, CISJ, 2, 123, pl. 56, f. 3

1962 Vesicomya nakai OKUTAN, BTRFRL, 32, 22, pl. 3, f. 1; pl. 4, f. 1, 1a

Loc. S (s-m) tr.

Dist. Honshu (Wakayama Pref.) to Kyushu.

\section{Superfamily Corbiculacea GRAY シジミ超科 \\ Family Corbiculidae GRAY シジミ科 \\ Subfamily Corbiculinae GRAY シジミ亜科 \\ Genus Corbicula MEGERLE von Mühlfeld ヤマトシジミ属}

1811 Corbicula Megerle von Mühlfeld GNFBM, 5, 56

1818 Cyrena Lamarck, AsV, 5, 551. Type (SD, by Gray, 1847): Tellina fluminalis Müller

Subgenus Corbicula MEgeRLE von Muhlfeld. ヤマトシジミ亜属

Corbicula (Corbicula) japonica PRIMEヤマトシジ（岩川)

1864 Corbicula japonica PrIme, Ann. Lyc. Nat. Hist. N. Y., 8, 68, f. 15 
1877 Corbicula biformis REINHARDT, SBGNFB, 70

1877 Corbicula transversa MARTENs, SBGNFB, 120

1877 Cyrena yokohamensis SowERBY, Icon., sp. 55

1878 Corbicula fuscata atrata REINHARDT, JDMG, 5, 191

1878 Corbicula martensii Clessin, Cab., 9 (3), 196, p1. 38, f. 17, 18

1878 Corbicula reiniana Clessin, Cab., 9, (3), 196, pl. 39, f. 8, 9

1878 Corbicula doenitziana Clessin, Cab., 9 (3), 197, pl. 39, f. 4

1878 Corbicula ovalis ReINHARdT, JDMG, 5, 192, pl. 5, f. 5 (non PRIME, 1864)

1879 Cyrena biformis: KoBelt, Extramar, 154, pl. 21, f. 3

1879 Cyrena martensii: KoBELT, Extramar., 157, pl. 20, f. 5

1879 Cyrena transversii: KoBELT, Extramar., 156, pl. 21, f. 2

1879 Cyrena pexata: KoBelt, Extramar., 156, pl. 20, f. 2 (non PRIME, 1864)

1901 Corbicula sadoensis PILsBRY, PANSP, 53, 406

1907 Corbicula nipponensis PILSBRY, AZJ, 6 (3), 159, pl. 7, f. 3, 4

1907 Corbicula nipponensis delicata PILSBRY, AZJ, 6 (3), 160, pl. 7, f. 11, 12

1922 Corbicula sandaeformis YokoYama, JCSIUT, 44 (1), 165, pl. 13, f. 14, 15

1922 Corbicula kobelti YokoYama, JCSIUT, 44 (1), 166, pl. 13, f. 18, 19

1938 Corbicula japonica: Kuroda, Venus, 8 (1), 34, f. 1

1959 Corbicula japonica: KJRA, CISJ, 1, 170, pl. 63, f. 10

1977 Corbicula japonica: HABE, Chiribotan, 9 (7), 151, pl. 15, lower 4f.

Loc. N M S (g) .

Dist. Hokkaido to Kyushu.

\section{Subgenus Corbiculina DALL マシジミ曲属}

1903 Corbiculina DALL, PBSW, 16, 6

1903 Corbiculina Dall, TWFIP, 3 (6), 1449. Type (OD): Corbicula angasi PRIME

Corbicula (Corbiculina) leana PRIME マシジミ (岩II)

1864 Corbicula leana Prime Ann. Lyc. Nat. Hist. N. Y., 8, 68, f. 14

1877 Corbicula straminea MARTENs, SBGNFB, 70

1878 Corbicula pexata ReINHARd, JDMG, 5, 193, pl. 5, f. 6 (non Prime, 1864)

1879 Cyrena straminea: KoвeLt, Extramar, 155, pl. 20, f. 4

1901 Corbicula awajiensis PILsBry, PANSP, 53, 407

1907 Corbicula orthodonta PILSBRY, AZJ, 6 (3), 156, pl. 7, f. 1, 2, 5, 6

1907 Corbicula awajiensis: PILSBRY, AZJ, 6 (3), 159, pl. 7, f. 13, 14

1938 Corbicula leana: KurodA, Venus, 8 (1), 35, f. 9

1959 Corbicula (Corbiculina) leana: KTRA, CISJ, 1, 174, pl. 63, f. 11

1977 Corbicula leana: HABE, Chirbotan, 9 (7), 151, pl. 15, upper 4 f.

Loc. N M S (g).

Dist. Honshu to Kyushu.

\section{Family Pisidiidae GRAY マメシジミ科 \\ Genus Sphaerium SCOPOLI トブシジミ属}

1777 Sphaerium Scopoli, Introd., 397 (Valid by ICZN, Op. 94)

1798 Cyclas BRUGUìre, EMHNV, 2, pl. 301

1807 Musculina LINK, BNSUR, 3, 152

1872 Calyculina Clessin, M B L., 19, 159. Type (ICZN, Op. 94): Tellina cornea Linnaeus

Sphaerium lacustre japonicum (WeSTERLUND) トブシジミ（平瀬）(目八)

1774 Tellina lacustris MüLler, Verm. Terr. Fluv. Anim., 2, 204

1883 Calyculina japonica WesterLund, M B L. 15, 58 
1883 Calyculina japonica: WeSTERLund, Vega, 4, 216

1895 Sphaerium heterodon PrLSBRX, CMMJ, 159, pl. 3, f. 15-17

1901 Sphaerium inutilis PILSBRY, PANSP, 53, 406

1933 Sphaerium japonicum: MoRI, Venus, 4 (3), 153, f. 1A

1933 Sphaerium japonicum inutile: MoRI, Venus, 4 (3), 155, f. 1C

1933 Sphaerium biwaense: MoRI, Venus, 4 (3), 154, f. 1B

1937 Sphaerium japonicum okinawaense: MoRI, Venus, 7 (4), 170, f. 1a-d

1961 Sphaerium japonicum: HABE, CISJ, 2, 121, pl, 55, f. 3

Loc. N S (m).

Dist. Hokkaido to Okinawa main Island.

\section{Superfamily Veneracea RAFINESQUE マルスダレガイ超科 \\ Family Veneridae RAFINESQUEママルスダレガイ科 \\ Subfamily Venerinae RAFINESQUEマルスダレガイ量科}

Genus Venus LINNAEỤS 、ルスダレガイ属

1758 Venus Linnaeus, Syst. Nat., 10, 684. Type (SD, Gray, 1847, ICZN, 1950): Venus verrucosa LinNaeus

\section{Subgenus Ventricolaria KEEN マルスダレガイ亜属}

1954 Ventricolaria KeEn, J of P., 26, 218. Type (OD): Venus rigida DillwyN

Venus (Ventricolaria) toreuma GoULDマルスダレガイ (平瀬); シコロガイ (丹浦)

1850 Venus toreuma GouLd, PBSNH, 3, 277

1852 Venus toreuma: Gould, USEE, 12, 419, pl. 37, f. 537a

1853 Venus jukesii Deshayes, Cat. Conch. Brit. Mus, 100

1853 Venus sculpta DeshaYes, PZSL, 3

1938 Venus hawaiensis DALL, BARTSCH \& REHDER, BPBMB, 153, 164, pl. 42, f. 13-15

1959 Venus toreuma: KIRA, CISJ, 1, 142, pl. 56, f. 15

1971 Venus (Ventricolaria) toreuma: KuRODA \& HABE, SSSB, 658 (J) 428 (E), pl. 93, f. 11

Loc. N M S (g) gl.

Dist. Honshu (north to Bōsō Peninsula) to Kyushu. Widely ranging in the tropic Pacific.

\section{Subgenus Ventricoloidea SACCo ビノスガイモドキ巠属}

1900 Ventricoloidea SACco, Moll. Terr. Terz. Piemonte e Liguria 28, 31. Type (OD): Cytherea multilamella LAMARCK $=$ Venus nux GMELIN

Venus (Ventricoloidea) foveolata (SoWERBY) ビノスガイモドキ（平瀬)

1853 Venus foveolata Sowerby, Thes., 730, pl. 154, f, 46

[1926 Chione cassinaeformis YoKoYAMA (in part, JFSIUT, (2) 1 (9), 352, pl. 39, f. 8-9

1959 Venus (Ventricola) foveolata: KIRA, CISJ, 1, 145, pl. 56, f. 16

1971 Venus (Ventricoloidea) foveolata: KuRODA \& HABE, SSSB, 659 (J) 428 (E), pl. 93, f. 2

Loc. N M S (s-m) dp.

Dist. Honshu (north to Bōsō Peninsula) to Kyushu and East China Sea.

\section{Genus Antigona ScHUMACHER サッマアサリ属}

1817 Antigona SchUMACHER, ENSVT, 51, 154

1853 Omphaloclathrum Mörch, Yoldi, 2, 24. Type (M): Antigona lamellaris SchUMACHER 
Antigona lamellaris SCHUMACHER サッマアサリ（目八）

1817 Antigona lamellaris SCHUMACHER, ENSVT, 155, pl. 14, f. 2a, b

1853 Venus nodulosa SoweRBY, Thes., 708, pl. 153, f. 16

1959 Antigona lamellaris: KIRA, CISJ, 1, 142, pl. 56, f. 10

1971 Antigona lamellaris: KURODA \& HABE, SSSB, 660 (J) 429 (E), pl. 90, f. 8

Loc. N M S (s) dp.

Dist. Honshu (north to Bōsō Peninsula) to Kyushu. Widely ranging in the Pacific region.

Genus Periglypta JuKkS-BRownEヌノメガイ属

1914 Periglypta JUKES-Browne PMSL, 11, 72. Type (OD): Venus puerpera LINNAEUs

Subgenus Periglypta JuKES-BRownEヌノメガイ重属

Periglypta (Periglypta) lacerata (HANLEY)イササメガイ（丹浦）

1845 Venus lacerata HANLEY, PZSL, 1844, 12, 161

1853 Venus lacerata: SowERBY, Thes., 2, 704, p1. 152, f. 3, 4

1863 Venus lacerata: ReEve, Icon., sp. 18

Loc. S (s) dp.

Dist. Honshu (north to Bōsō Peninsula) to Kyushu. Widely ranging in the tropic Pacific region.

Remarks: This species seems to be a variant of Periglypta puerpera LinNaeus

Periglypta (Periglypt) puerpera LinNaEUS ヌノメガイ（目八）

1771 Venus puerpera LinNaeus, Mantissa, 2, 545

1842 Venus listeri HANLEY, Cat. Bivalve, 110

1863 Venus puerpera: ReEve, Icon., sp. 1

1959 Periglypta puerpera: KrRA, CISJ, 1, 142, pl. 56, f. 12

Loc. S (s).

Dist. Honshu (north to Kii Peninsula) to Kyushu. Widely ranging in the Indo Pacific region.

Periglypta (Periglypta) clathrata (DeSHAYES)オオヌノメガイ（黒田）

1854 Venus clathrata DeshaYes, PZSL, 21 (1853), 3

1864 Venus clathrata: REEVE, Icon., sp. 3

1959 Periglypta clathrata: KIRA, CISJ, 1, 142, pl. 56, f. 13

Loc. M S (s) gl.

Dist. Honshu (Kii Peninsula) to Ryukyu Islands. Widely ranging in the Pacific region.

Periglypta (Periglypta) reticulata (LinNAEUS) アラヌノメガイ（岩川)

1758 Venus reticulata LinNaEUs, Syst. Nat., 10, 687

1818 Venus corbis Lamarck, AsV, 5, 595

1852 Venus fischeri RḱCLUZ, J. de C., 3, 411, pl. 12, f. 9

1853 Venus reticulata: Sowerdy, Thes., 2, 106, pl. 153, f. 11-13

1864 Venus reticulata: ReEve, Icon., sp. 34

1938 Periglypta edmondsoni DALL, BARTSCH \& REHDER, BPBMB, 153, 162, pl. 43, f. 1-4

1959 Periglypta reticulata: KIRA, CISJ, 1, 142, pl. 56, f. 13

Loc. M S (s) gl.

Dist. Honshu (Kii Peninsula) to Ryukyu Islands. Widely ranging in the Indo Pacific region. 


\section{Subgenus Tigammona IREDALEヨロイガイ巠属}

1930 Tigammona Iredale, RAM, 17, 396. Type (OD): Tigammona persimile Iredale

Periglypta (Tigammona) chemnitzii (HANLEY) ヨロイガイ (浄五)

1844 Venus chemnitzii HANLEY, PZSL, 160

1853 Venus crispa DeSHAYES, PZSL, 2

1863 Venus crispa: ReEve, Icon., sp. 31

1863 Venus chemnitzii: REEVE, Icon., sp. 32

1904 Cytherea crispa amicata Pilsbry, PANSP, 56, 551, pl. 39, f. 10, 11

1930 Tigammona persimile IrEdALE, RAM, 17, 396, pl. 62, f. 1, 2

1945 Periglypta (Tigammona) fischeri: KIRA (non RÉcLUZ, 1852), CISJ, 1, 142, pl. 56, f. 11

Loc. M S (s) gl.

Dist. Honshu (Kii Peninsula) to Ryukyu Islands. Widely ranging in the Indo Pacific region.

Periglypta (Tigammona) langfordi (KURODA)ヒガノコヌノメガイ（黒田）

1945 Antigona langfordi KurodA, Venus, 14, 32, tf. 1-2

Loc. M S (s) dp.

Dist. Honshu (Kii Peninsula) to Ryukyu Islands.

Remarks: This may be a young form of Tigammona chemnitzii (HANLEY)

Subfamily Chioninae FrIZZELLカノコアサリ亜科

Genus Anomalocardia SCHUMACHERタイワンオモイバガイ属

1817 Anomalocardia SchUmacher, ENSVT, 44, 134. Type (OD): Anomalocardia rugosa SCHUMACHER $=$ Venus flexuosa LINNAEUS

Subgenus Anomalodiscus DALLシオヤガイ覀属

1902 Anomalodiscus DALL, PUSNM, 26, 359. Type (OD): Cytherea squamosa LAMARCK $=$ Venus squamosa LINNAEUS

Anomalocardia (Anomalodiscus) squamosa (LINNAEus)シオヤガイ（平瀬）

1758 Venus squamosa LinNaEus, Syst. Nat., 10, 688

1860 Venus roemeri DUNKER, M B L. 6, 240

1861 Venus roemeri: DUNKER, MJ, 26, pl. 3, f, 10

1959 Anomalodiscus squamosus: KIRA, CISJ, 1, 147, pl. 57, f. 16

Loc. N. M S (m) int.

Dist. Honshu (Kii Peninsula) to Kyushu. Widely ranging in the Western Pacific region.

\section{Genus Callanaitis IREDALEユメハマグリ属}

1917 Callanaitis Iredale, PMSL, 12, 322, 329. Type (OD): Venus yatei GraY

Callanaitis hiraseana KURODAユメハマグリ（黒田）

1930 Callanaitis hiraseana KuRODA, Venus, 2 (1), 2, tf. 1

1959 Callanaitis hiraseana: KIRA, CISJ, 1, 147, pl. 57, f. 19

Loc. M S (s) dp.

Dist. Honshu (Kii Peninsula) to Yakushima. 


\section{Genus Placamen IREDALE ハナガイ属}

1923 Placamen Iredale, RAM, 14, 248. Type (OD): Venus placida PHILIPPI

\section{Placamen tiara (DILLWYN) ハナガイ（六介）}

1817 Venus tiara Dillwyn, DCRS, 1, 162

1846 Venus foliacea PHILIPI, Abbild., 2 (4), 107, pl. 5, f. 1

1959 Placamen tiara: KIRA, CISJ, 1, 147, pl. 57, f. 20

1971 Placamen tiara: Kuroda \& Habe, SSSB, 660 (J) 429 (E), pl. 95, f. 8, 9

Loc. N M S (s) dp.

Dist. Honshu (north to Bōsō Peninsula and Noto Peninsula) to Ryukyu Islands. Widely ranging in the Indo-Pacific region.

\section{Genus Glycydonta COTTONカノコアサリ属（ニシキアサリ属）}

1936 Glycydonta Cotton, RAM, 5, 503. Type (OD): Venus marica Linnaeus

Glycydonta marica (LINNAEUs)カノコアサリ（黑田），ニシキアサリ（目八）

1758 Venus marica LinNaeus, Syst. Nat., 10, 685

1863 Chione marica: ReEve, Icon., sp. 104

1954 Leukoma japonica KIRA, [CISJ], 1, (ed. 1), 163, pl. 57, f. 17

1959 Leukoma japonica: KIRA, CISJ, 1, 147, pl. 57, f. 17

1959 Leukoma marica: KIRA, CISJ, 1, 147, pl. 57, f. 18

1971 Glycydonta marica japonica: KURODA \& HABE, SSSB, 662 (J) 430 (E), p1. 93, f. 4

Loc. M S (s) int.

Dist. Honshu (north to Bōsō Peninsula) to Ryukyu Islands. Widely ranging in the tropic Pacific region.

\section{Genus Veremolpa IREDALEヒメカノコアサリ属}

1930 Veremolpa Iredale, RAM, 17, 397. Type (OD): Veremolpa ethic Iredale

Veremolpa mindanensis (SMITH)アデヤカヒメカノコアサリ（波部）

1885 Venus (Chione) mindanensis SMITH, Challenger, 130, pl. 3, f. 4, 4a, b

1920 Chione minuta Yoxoyama, JCSIUT, 39, (6), 122, pl. 8 f. 14

1932 Chione (Tomoclea) mindanensis: PRASHAD, Siboga, 53c, 354, pl. 6, f. 21,22

1961 Veremolpa minuta: HABE, CISJ, 2, 131, pl. 59, f. 2

1961 Veremolpa mindanensis: HABE, CISJ, 2, 131, pl. 59, f. 4

1971 Veremolpa mindanensis: KuRODA \& HABE, SSSB, 661 (J) 430 (E), pl. 120, f. 9

Loc. M S (s) dp.

Dist. Honshu (north to Bōsō Peninsula) to Kyushu. Widely ranging in the IndoPacific region.

Veremolpa micra (PILSBRY) ヒメカノコアサリ（岩川)

1904 Chione micra PILSBRY, PANSP, 56, 552, pl. 41, f. 4, 5

1961 Veremolpa micra: HABE, CISJ, 2, 131, pl. 59, f. 3

1971 Veremolpa micra: KuRODA \& HABE, SSSB, 661 (J) 430 (E), pl. 93, f. 10. .

Loc. M S (s-m) int.

Dist. Honshu (north to Bōsō Peninsula) to Kyushu.

Velemolpa costellifera A. AdAMs \& REEvEチリメンカノコアサリ（黒田）;

ヒゼンカノコアサリ（岩川）

1850 Venus costellifera A. AdAms \& Reeve, Samarang, 79, p1. 21, f. 18 
1904 Chione hizenensis PrLSBRY, PANSP, 56, 553, pl. 41, f. 1, 2

1961 Veremolpa costellifera: HABE, CISJ, 2, 131, p1. 59, f. 6

Loc. N M S (s) int.

Dist. Honshu (north to Kii Peninsula) to Ryukyu Islands. Widely ranging in the tropic Pacific.

Veremolpa laevicostata (KURODA)オオギカノコアサリ（黒田） Pl. 7, f. 3.

1960 Tomoclea laevicostata KURODA, Cat. Okinawa, 82

Loc. M S (s) int.

Dist. Honshu (Kii Peninsula) to Kyushu Islansd.

Genus Protothaca DALLオニアサリ属

1902 Protothaca DALL, PUSNM, 26, 364. Type (OD): Venus thaca Molina

Subgenus Notochione Hertlein \& StRong オニアサリ再属

1948 Notochione Hertlein \& Strong, Zoologica 33 (4), 186. Type (OD): Venus columbiensis SOWERBY

Protothaca (Notochione) jedoensis (LisCHKE)オニアサリ（目八）

1874 Venus jedoensis LISCHKE, JDMG, 1, 57

1874 Venus jedoensis: LischKE, JMC, 3, 84, pl. 7, f. 1-9

1901 Venus hirasei PrLSBRY, PANSP, 53, 205, 400, pl. 19, f. 1; pl. 20, f. 20

1959 Protothaca jedoensis: KIRA, CISJ, 1, 143, pl. 56, f. 18

1971 Notochione jedoensis: KURODA \& HABE, SSSB, 662 (J) 431 (E), pl. 93, f. 3

Loc. N M S (g) int.

Dist. Southern Hokkaido to Kyushu, Korea and North China.

$$
\text { Subgenus Novathaca HABEヌノメアサリ亜属 }
$$

1951 Novathaca HABE, GJS, (2), 180. Type (OD): Chione euglypta Sowerby

Protothaca (Novathaca) schencki (NomURA) メオニアサリ（黑田）

1937 Protothaca schencki Nomura., SHKMRB, 13, 8, pl. 3, f. 2a, b

1961 Protothaca (Novathaca) schencki: HABE, CISJ, 2, 132, pl. 59, f. $14 \quad J_{\mathrm{ABE}}$

Loc. M S (s-m) int.

Dist. Honshu to Kyushu.

\section{Subfamily Circinae DALL シラオガイ亜科}

Genus Gafrarium RöDING ホソスジイナミガイ属

1798 Gafrarium Röding, Bolten, 2, 176. Type (SD, Dall, 1902): Venus pectinata LinNaEus

Gafrarium pectinatum (LINNAEUS) ホソスジイナミガイ (平瀬)

1758 Venus pectinata Linnaeus, Syzt. Nat., 10, 689

1851 Circe pectinata: SowerBy, Thes., 2, 649, pl. 137, f. 1-3

1863 Circe pectinata: REEve, Icon., sp. 20

1959 Gafrarium pectinatum: KIRA, CISJ, 1, 145, pl. 57, f. 4

Loc. S (s) int.

Dist. Honshu (Kii Peninsula) to Ryukyu Islands. Widely ranging in the Indo Pacific region. 
Gafrarium dispar (DILLwYN) 1ナミガイ（五介）

1817 Venus dispar DILLwYN DCRS, 1, 199

1851 Circe dispar: SowerBy, Thes., 2, 650, pl. 137, f. 10, 11; pl. 163, f. 53, 54

1853 Circe tranversa Deshayes, Cat. Couch. Brit. Mus, 1, 90

1961 Gafrarium dispar: HABE, CISJ, 2, 129, pl. 58, f. 9

1971 Gafrarium dispar: KuRODA, SSSB, 639 (J) 413 (E), p1. 90, f. 10

Loc. N M S (g) int.

Dist. Honshu (north to Bōsõ Peninsula) to Ryukyu Islands. Widely ranging in the Indo-Pacific region.

Gafrarium divaricatum (GMELIN) ケマンガイ（介志）

1791 Venus divaricata GMELIN, Syst. Nat., 13, 3277

1851 Circe aequivoca Sowerny, Thes., 2, 650, pl. 137, f. 12-15

1863 Circe divaricata: REEVE, Icon, sp. 23

1959 Gafrarium divaricatum: KIRA, CISJ 1, 145, pl. 57, f. 6

Loc. N M S (g) int.

Dist. Honshu (north to Shima Peninsula), Shikoku and Kyushu. Widely ranging in the tropic Pacific region.

\section{Genus Circe SchUMACHERシラオガイ属}

1817 Circe SCHUMACHER, ENSVT, 152. Type (M): Circe violacea SCHUMACHER=Venus scripta LINNAUES

Circe scripta (LinNAEUS)シラオガイ（六介）

1758 Venus scripta LINNAEUs, Syst. Nat., 10, 189

1824 Venus stutzeri Donovan, Naturalists Repositiry, 3, pl. 78

1851 Circe scripta: Sowerby, Thes., 2, 651, pl. 38, f. 38-40

1854 Circe personata DeshaYes, PZSL, 21 (1853), 6

1863 Circe divaricata: REeve, Icon., sp. 23

1959 Circe stutzeri: KIRA, CISJ, 1, 146, pl. 57, f. 7

1971 Circe scripta: Kuroda \& HaBe, SSSB, 640 (J) 413 (E), pl. 90, f. 6

Loc. N M S (s) int.

Dist. Honshu (north to Bosō Peninsula and Noto Peninsula) to Kyushu. Widely ranging in the Indo-Pacific region.

Circe intermedia (REEVE) アッシラオガイ（黑田）

1863 Circe intermedia REEve, Icon., sp. 26

1959 Circe intermedia: KIRA, CISJ, 1, 146, pl. 57, f. 8

Loc. M S (s) int.

Dist. Honshu (north to Shima Peninsula) to Kyushu. Widely ranging in the Pacific region.

$$
\text { Subgenus Redicirce IREDALE トモシラオガイ亜属 }
$$

1936 Redicirce IREDAle, RAM, 19, 276. Type (OD): Redicirce mistura IrEDALE

Circe (Redicirce) sulcata GRAY トモシラオガイ（黒田）

1838 Circe sulcata GRAY, Analyst., 8, 307

1851 Circe plebeja Sowerby, Thes., 2, 653, pl. 138, f. 33-37

1863 Circe sulcata: ReEve, Icon., sp. 16

1961 Circe (Redicirce) sulcata: HABE, CISJ, 2, 129, pl. 58, f. 2 
Loc. M S (s) dp.

Dist. Honshu (north to Suruga Bay) to Kyushu. Widely ranging in the tropical Pacific.

\section{Genus Crenocirce HABE コキザミシラオガイ属}

1960 Crenocirce HABE, PSMBL, 8 (2), 286. Type (OD): Dorisca (Crenocirce) picta HABE

Crenocirce picta (HABE) ヨキザミシラオガイ（波部）

1960 Dorisca (Crenocirce) picta HABE, PSMBL, 8 (2), 286, f. 1-3

1961 Circe (Crenocirce) picta: HABE, CISJ, 2, 129, pl. 58, f. 3

Loc. M S (s) dp.

Dist. Honshu (Kii Peninsula) to Ryukyu Islands.

Genus Dorisca DALL, BARTsCh \& REHDER チゴシラオガイ属

1939 Dorisca Dall, Bartsch \& ReHDER BPBMB 153, 157, 159. Type (OD): Dorisca cookei

DALL, BARTSCH \& ReHDER

Dorisca nana (MelvILL) チゴシラオガィ（黒田）

1898 Circe nana Melvill, Proc. Manchester Lit. Phila. Soc., 43 (2), 31, pl. 2, f. 9

1931 Circe salamensis THIELE \& JACKeL, Tiefsee, 21 (1), 233, pl. 4, f. 106

1932 Circe nana: Prashad, Siboga, 53c, 227, pl. 6, f. 13, 14

1934 Gafrarium (Circe) hanzawai NoMURA \& ZINBO, SRTIU, (2) 18 (3), 245, pl. 11, f. 14, 15

1961 Circe (Redicirce) nana: HABE, CISJ, 2, 129, pl. 58, f. 5

1971 Circe (Redicirce) nana: KuRoda \& HABE, SSSB, 640 (J) 413 (E), pl. 90, f. 3

Loc. M S (s) dp.

Dist. Honshu (north to Bōsō Peninsula) to Kyushu and Indonesia.

GenuS Microcirce HABE ミジンシラオガイ属

1951 Microcirce Habe, GJS, 2, 160. Type (OD): Meretrix gordonis YoKoYama

Microcirce dilecta (GoulD) シジンシラオガイ (波部)

1861 Gouldia dilecta GouLD, PBSNH, 8, 32

1927 Meretrix gordonis Yoxoyama, JFSIUT, (2) 1 (10), 429, pl. 48, f. 13, 14

1964 Gouldia dilecta: JoHNson, USNMB, 239, 68, p1. 29, f. 4

1971 Microcirce dilecta: Kuroda \& HABE, SSSB, 641 (J) 414 (E), pl. 120, f. 13, 14

Loc. M S (s) dp.

Dist. Southern Hokkaido, Honshu to Kyushu.

Subfamily Pitarinae STEWART ユウカゲハマグリ亜科

Genus Pitar RöMER ユウカゲハマグリ属

1857 Pitar RöMer, Krit, Unters. Venus, 15

1862 Caryatis RöMER, M B L., 9, 58, 60 (non HüBNER, 1819). Type (M): Venus tumens GMeLIN

Subgenus Pitarina JUKES-BRoWNEユウカゲハマグリ亞属

1913 Pitarina Jukes-Browne, PMSL, 10, 346. Type (OD): Cytherea citrina LamarcK

Pitar (Pitarina) japonicum KURODA \& KAWAMOTO ウスハマグリ（目八）

1956 Pitar (Agriopeda) japonicum Kuroda \& Kawamoto, Cat. Yamaguchi, 89, f. 1-4

1961 Pitar (Agriopoma) japonica: HABE, CISJ, 2, 130, pl. 58, f. 12 
1971 Pitar (Pitarina) japonicum: Kuroda \& HABE, SSSB, 642 (J) 415 (E), pl. 50, f. 11

Loc. N M S (s) dp.

Dist. Honshu (north to Bōsō Peninsula) to Kyushu.

Pitar (Pitarina) lineolatum (SowERBy)ガンギハマグリ（黒田）

1854 Cytherea lineolata SowerBy, Thes., 2, 786, pl. 168, f. 214, 215

1961 Pitar (Pitarina) lineaolatum: HABE, CISJ, 2, 130, pl. 58, f. 12

1971 Pitar (Pitarina) lineolatum: KurodA \& HABE, SSSB, 641 (J) 414 (E), pl. 90, f. 16

Loc. N M S (s) dp.

Dist. Honshu (north to Bōsō Peninsula) to Formosa. Widely ranging in the West Pacific.

Pitar (Pitarina) noguchii HABEシロウスハマグリ（波部）

1958 Pitar (Agriopoma) noguchii: HABE, PSMBL, 7 (1), 34

1961 Pitar (Agriopoma) noguchii: HABE, CISJ, 2, 129, pl. 58, f. 6

1971 Pitar (Pitarina) noguchii: KurodA \& HABE, SSSB, 642 (J) 415 (E), pl. 120, f. 15

Loc. N M S (s) dp.

Dist. Honshu (north to Bōsō Peninsula) to Kyushu.

Pitar (Pitarina) sulfureum PILSBRYイオウハマグリ（平瀬）

1904 Pitar sulfurea PllsBry, PANSP, 56, 553, pl. 39, f. 7-9

1961 Pitar (Pitarina) sulfreum (sci): HABE, CISJ, 2, 130, pl. 58, f. 13

Loc. N M (m) int.

Dist. Honshu (north to Bōsō Peninsula) to Formosa.

Pitar (Pitarina) pellucidum (LAMARCK) オミナエシハマグリ（六介）

1818 Cytherea pellucida LAMARCK, AsV, 5, 571

1863 Dione pellucida: ReEve, Icon., sp. 48

1959 Pitar (Pitarina) pellucida: KIRA, CISJ, 1, 146, pl. 56, f. 11

Loc. S (s) int.

Dist. Honshu (Kii Peninsula) to Ryukyu Islands. Widely ranging in the Indo Pacific region.

Pitar (Pitarina) affine (GMELIN) ムラクモハマグリ（黒田）

1791 Venus affinis GmeLIN, Syst. Nat., 13, 3278

1818 Cytherea laeta Lamarck, AsV, 5, 567

1851 Cytherea inflata Sowerby, Thes., 2, 637, pl. 133, f. 127

1863 Dione laeta: REEve, Icon., sp. 35

1867 Venus affinis: Römer, Venus, 1, 105, pl. 28, f. 3, 3a-c; pl. 33, f. 6, 7

1959 Pitar (Pitarina) affine: KIRA, CISJ, 1, 146, pl. 57, f. 10

1971 Pitar (Pitarina) variegatum KURODA \& HABE, SSSB, 643 (J) 416 (E), pl. 90, f. 13, 14

Loc. N M S (s) dp.

Dist. Honshu (north to Bōsō Peninsula) to Kyushu. Widely ranging in the Pacific region.

Pitar (Pitarina) nipponicum (KURODA \& HABE)スナカムリハマグリ（黒田・波部）

1971 Pitar (Pitarina) nipponicum KuRodA \& HABE, SSSB, 643 (J), 415 (E) pl. 90, f. 15

Loc. M (s) dp.

Dist. Honshu (north to Bōsō Peninsula) to Kyushu. 
Genus Hyphantosoma DALLマダライオウハマグリ属

1902 Hyphantosama Dall, PUSNM, 26, 354. Type (OD): Cytherea carbosea GuPPY

Hyphantosoma limatulum (SOWERBY)マダライオウハマグリ（平瀬）

1851 Cytherea limatula Sowersy, Thes., 2, 460, pl. 136, f. 200, 201

1977 Hyphantosoma limatulum: HABE, Venus, 36 (1), 6, 9, pl. 1, f. 9

Loc. $\mathrm{N}$ M (s) dp.

Dist. Honshu (Wakayama Pref.) to Ryukyu Islands. Widely ranging in the Western Pacific.

\section{Genus Costellipitar HABEチデミマメハマグリ属}

1851 Costellipitar HABE, GJS, 2, 162. Type (OD): Caryatis chordata RöMER

Costellipitar chordatum (RöMER) チヂミマメハマグリ（滝悠）

1867 Caryatis chordata RöMER, Venus., 1, 125, pl. 33, f. 2

1940 Pitar fukaurensis NomuRA \& HaTAI, SHKMRB, 19, 83, pl. 4, f. 10a, b

1961 Pitar (Costellipita) chordatum: HABE, CISJ, 2, 129, pl. 58, f. 1

1971 Costellipitar chordatum: Kuroda \& HABE, SSSB, 644 (J) 416 (E), pl. 120, f. 16

Loc. M S (s) dp.

Dist. Honshu (north to Iwate Pref.) to Kyushu.

Subfamily Lioconchinae IREDALE \& MCMICHAEL マルオミナエシガイ亜科

Genus Lioconcha MöRCHマルオミナエシガイ属

1853 Lioconcha Mörch, Yoldi., (2), 26. Type (SD, StoliczKA, 1870): Venus castrensis LINNAEUS

Subgenus Lioconcha MöRCHマルオミナエシガイ巠属

Lioconcha (Lioconcha) fastigiata (SOWERBY) サラサガイ（六介）

1851 Cytherea fastigiata SOWERBy, Thes., 2, 643, pl. 135, f. 155-159 (not of 157)

1959 Lioconcha fastigiata: KIRA, CISJ, 1, 145, pl. 57, f. 1

Loc. S (s) int.

Dist. Honshu (north to Kii Peninsula) to Ryukyu Islands. Widely ranging in the Indo Pacific region.

Lioconcha (Lioconcha) ornata DILlwYNムラヤマサラサガイ（黒田）Ｐl. 1, f. 8.

1817 Venus ornata Dillwyn, Descript. Cat., 1, 184

1818 Cytherea picta LAMARCK, AsV, 563

1863 Circe picta: ReEve, Icon., sp. 42

1868 Venus (Cytherea, Lioconcha) picta RöMER, Venus, 1, 164, pl. 45, f. 3, 3a-c

Loc. M S (s) dp.

Dist. Honshu (Sagami Bay) to Ryukyu Islands. Widely ranging in the West Pacific.

Lioconcha (Lioconcha) castrensis LINNAEUS マルオミナエシガイ (平瀬)

1758 Venus castrensis LiNnAEUs, AsV, 10, 687

1863 Circe castrensis: ReEve, Icon., sp. 28 
1959 Lioconcha castrensis: KIRA, CISJ, 1, 145, pl. 57, f. 3

Loc. S (s) int.

Dist. Honshu (north to Kii Peninsula) to Ryukyu Islands. Indo-Pacific.

$$
\text { Subgenus Sulcilioconcha HABE イナズマスダレガイ重属 }
$$

1951 Sulcilioconcha HABE, GJS, (2), 158. Type (OD): Cytherea philippinarum HaNLEY

Lioconcha (Sulcilioconcha) dautzenbergi PRASHADナルカミスダレガイ（黒田）

Pl. 7, f. 2.

1932 Hysteroconcha (Lamelliconcha) dautzenbergi PrASHAD, Siboga, 53c, 217, pl. 6, f. 11, 12

1961 Lioconcha (Sulcilioconcha) philippinarum: HABE, CISJ, 2, 129, pl. 58, f. 10

Loc. S (s) dp.

Dist. Honshu (north to Wakayama Pref.) to Ryukyu Islands and Indonesia.

$$
\text { Genus Callocardia A. AdAMs オフクハマグリ属 }
$$

1864 Callocardia A. Adams, AMNH, (3), 13, 307. Type (M): Callocardia guttata A. Adams

Callocardia guttata A. ADAMS オフクハマグリ（波部）

1864 Callocardia guttata A. ADAMs, AMNH, (3), 13, 307

1888 Cytherea hungerfordi SOWERBY, PZSL, 212, pl. 11, f. 4

1952 Callocardia guttata: Kuroda, Venus, 17, 1, pl. 1, tf. 1-4

1977 Callocardia guttata: HABE, Venus, $36(1), 6,9$, pl. 2, f. 15, 16

Loc. S (s-m) dp.

Dist. Honshu (Kii Peninsula) to Kyushu (Korean Strait) and South China Sea (off Hong Kong).

$$
\begin{aligned}
\text { Subfamily Samarangiinae KEEN } & \text { コンゴウハマグリ棘科 } \\
\text { Genus Samarangia DALL } & \text { コンゴウハマグリ属 }
\end{aligned}
$$

1902 Samarangia DaLL, PUSNM, 26, 361

1945 Petroderma Kuroda, Venus, 14 (1-4), 29. Type (OD): Venus quadrangularis Adams \& REEVE

Samarangia quadrangularis (ADAMS \& REEVE)コンゴウハマグリ（黑田） Pl. 7, f. 1.

1850 Venus quadrangularis ADAMs \& REEVE, Samarang, 79, pl. 24, f. 7

1864 Venus quadrangularis: REEVE, Icon., sp. 129

1942 Samarangia quadrangularis: ClenCH, Naut., 55, 73, pl. 6, f. 1-4

1945 Petroderma thaanumi Kuroda, Venus, 14 (1-4), 29, pl. 1, f. 1-4

1966 Samarangia quadriangularis: HABE \& KosuGE, SWC, 2, 164, pl. 64, f. 12

Loc. M (s) gl.

Dist. Honshu (Wakayama Pref.) to Ryukyu Islands and Philippines.

$$
\begin{gathered}
\text { Subfamily Dosiniinae DESHAYES } \\
\text { Genus Dosinorbis DALL カガミガイ业科 } \\
\text { ヒナガイ属 }
\end{gathered}
$$

1902 Dosinorbis Dall, PUSNM, 26, 347. Type (OD): Artemis bilunulata Gray

$$
\text { Subgenus Dosinorbis DALL ヒナガイ亜属 }
$$

Dosinorbis (Dosinorbis) bilumulatus (GRAY) ヒナガイ（目八)

1838 Artemis bilunulata GrAY, Analy Jour., 24, 309 
1850 Artemis bilunulata: ReEVe, Icon., sp. 22

1859 Dosinia (Dosinorbis) bilunulata: KIRA, CISJ, 1, 141, pl. 56, f. 8

1971 Dosinorbis (Dosinorbis) bilunulatus: KURODA \& HABE, SSSB, 648 (J) 420 (E), pl. 91 , f. 2

Loc. N M S (s) dp.

Dist. Honshu (north to Bōsō Peninsula) to Kyushu.

\section{Subgenus Phacosoma JUKES-BRoWnEカガミガイ巠属}

1912 Phacosoma Jukes-Browne, PMSL, 10, 100. Type (OD): Artemis japonica ReEve

Dosinorbis (Phacosoma) japonicus (REEVE)カガミガイ（目八）

1850 Artemis japonica ReEve, Icon., sp. 17

1859 Dosinia (Phacosoma) japonica: KIRA, CISJ, 1, 141, p1. 56, f. 7

1971 Dosinorbis (Phacosoma) japonic (sic): Kuroda \& HABE, SSSB, 649 (J) 421 (E), pl, 91, f. 1

Loc. N M S (s) dp.

Dist. Southern Hokkaido to Kyushu, Korea and China.

Dosinorbis (Phacosoma) troscheli (LISCHKE) マルヒナガイ（岩川)

1873 Dosinia troscheli LISCHKE, M B L., 21, 24

1874 Dosinia troscheli: LischKe, JMC, 3, 89, pl. 8, f. 1-3

1971 Dosinorbis (Phacosoma) troscheli: KuRoDa \& HABE, SSSB, 649 (J) 420 (E), pl. 91, f. 3, 4

Loc. N M S (s) dp.

Dist. Southern Hokkaido to Kyushu, Korea and China.

Dosinorbis (Phacosoma) hayashii HABE ミカガミガイ (黒田)

1976 Dosinorbis hayashii HaBE, Venus, 35 (2), 41, pl. 1, f. 17, 18

Loc. S (s) int.

Dist. Honshu (Wakayama Pref. to Aichi Pref.)

Dosinorbis (Phacosoma) roemeri (DUNKER) アアツガミガイ（河本・黑田 non 黑田・波部）

1862 Dosinia biscocta: RöMer (non ReEve, 1850), Dosinia, 59, pl. 16, f. 3, a, b

1863 Dosinia roemer: DUNKER, NCM, 2,43, pl. 13, f. 7-9

1956 Dosinia (Phacosoma) biscocta Kawamoto \& TANabe, Cat. Yamaguchi, 75, pl. 24, f. 222,223

1961 Dosinia (Phacosoma) biscocta: HABE, CISJ, 2, 130, pl. 58, f. 16

1967 Dosinia (Asa) malecocta Fischer-PIetTI \& DeLmas, MMNHN (A) 47 (1), 42

Loc. N. (s).

Dist. Honshu (Wakayama Pref.) and Inland Sea of Japan (Seto Naikai)

Dosinorbis (Phacosoma) pubescens (PHILIPPI) ヒメカガミガイ（目八)

1847 Cytherea pubescens PHILIPI, Abbild., 3, 36, pl. 8, f. 3

1850 Artemis serica ReEVe, Icon., sp. 36

Loc. M S (s) dp.

Dist. Honshu (Wakayama Pref. and Awaji) (fide Kuroda)

Genus Dosinella DALL ウラカガミガイ属

1902 Dosinella Dall, PUSNM, 26, 347. Type (OD): Cytherea angulosa PHILIPPI

Dosinella corrugata (REEVE) ウラカガミガイ (目八)

1850 Artemis corrugata ReEve, Icon., sp. 24

1850 Artemis penicillata REEVE, Icon., sp. 32

1855 Dosinia trailli A. Adams, PZSL, 223 
1961 Dosinea (Dosinella) angulosa: Habe (non PhiLIPPI, 1847), CISJ, 2, 132, pl. 59, f. 8

1967 Dosinia (Dosinella) corrugata: Fischer-Pierte \& Delmas, MMNHN (A) 47 (1), 74, pl. 13 , f. 1-3

1967 Dosinia (Dosinella) trailli: Fischer-PietTe \& Delmas, MMNHN (A) 47 (1), 75, pl. 13, f. 4-12

Loc. N M (s-m) dp.

Dist. Honshu to Kyushu, Korea and China.

Dosinella orbiculata (DUNKER) オトヒメカガミガイ（大山）

1877 Dosinia orbiculata DUNKER, M B L., 24, 69

1882 Dosinia orbiculata: Dunker, Index, 204, pl. 8, f. 12-14

1961 Dosinia (Dosinisca) orbiculata: Oyama, Venus, 21, 288, tf. 7, 8

1971 Dosinella orbiculata: KURODA \& HABE, SSSB, 650 (J) 421 (E), pl. 91, f. 5

Loc. N (s).

Dist. Honshu (north to Sagami Bay) to Kyushu.

Dosinella subalata (SMITH)タマカガミガイ（滰篁）

1916 Dosinia subalata SMIrH, PMSL, 12, 17, pl. 1, f. 11-13

1961 Dosinia (Dosinisca) subalata: Oyama, Venus, 21, 288, f. 5, 6

1971 Dosinella subalata: KuRODA \& HABE, SSSB, 650 (J) 422 (E), pl. 120, f. 17, 18

Loc. M S (s) dp.

Dist. Honshu (north to Bōsō Peninsula) to Philippines.

\section{Genus Pardosinia IREDALEウスカガミガイ属}

1929 Pardosinia Iredale, MQM, 9, 264. Type (OD): Pardosinia colorata Iredale

Pardosinia amphidesmoides (REEvE) ウスカガミガイ（黒田）

1850 Artemis amphidesmoides REEVE, Icon., sp. 48

1862 Dosinia erythraea: RöMER, Dosinia, 36, pl. 7, f. 1

1961 Dosinia (Pardosinia) amphidesmoides: HABE, CISJ, 2, 131, pl. 59, f. 9

Loc. M S (s) dp.

Dist. Honshu (north to Shima Peninsula) to Kyushu.

\section{Genus Bonartemis IREDALE オイノカガミガイ属}

1929 Bonartemis IrEDALE, MQM, 9, 265. Type (OD): Bonartemis stabilis IrEDALE=Venus histrio GMELIN

Bonartemis histrio histrio (GMELIN) オイノカガミガイ（平瀕）

1791 Venus histrio Gmelin, Syst. Nat., 13, 3287

1838 Artemis variegata GraY, Analyst., 8, 309

1850 Artemis variegata: ReEVE, Icon., sp. 33

1860 Dosinia historio: RöMER, Dosinia, 33, pl. 6, f. 2, 3

1961 Dosinia (Bonartemis) histrio: HABE, CISJ, 2, 132, pl. 59, f. 13

Loc. S (s) dp.

Dist. Honshu (Wakayama Pref.) to Ryukyu Islands. Widely ranging in the Pacific region.

\section{Bonartemis histrio iwakawai OYAMA \& HABE：サザメガイ（六介）}

1791 Venus histrio GMeLIN, Syst. Nat., 13, 3287

1961 Dosinia (Bonartemis) iwakawai, OYAMA \& HABE, CISJ, 2, 131, App. 38, pl. 59, f. 11

1971 Bonartemis histrio iwakawai: KunodA \& HABE, SSSB, 651 (J) 422 (E), pl. 91, f. 7 
Loc. N M S (s) dp.

Dist. Honshu (north to Bōsō Peninsula) to Kyushu.

Bonartemis juvenilis (GMELIN) ワカカガミガイ（黒田）

1791 Venus juvenilis GmeLIN, Syst. Nat., 13, 3287

1850 Artemis juvenilis: ReEVE. Icon., sp. 5

1850 Artemis laminata ReEVE, Icon., sp. 41

1961 Dosinia (Bonartemis) juvenilis: HABE, CISJ, 2, 130, pl. 58, f. 17

Loc. S (s) int.

Dist. Honshu (Kii Peninsula) to Ryukyu Islands. Widely ranging in the tropic Pacific region.

\section{Subfamily Tapetinae H. \& A. ADAMS リニウキュウアサリ互科}

Genus Paphia RöDING スダレガイ属

1798 Paphia Rödng, Bolten, (2), 175. Type (SD, DalL, 1902): Paphia alapapillionis RöDING

$=$ Venus rotundata LiNNAEUS

\section{Subgenus Paphia RöDINGスダレガイ刺属}

Paphia (Paphia) euglypta (PHILIPPI)スダレガイ（六介）

1847 Tapes euglypta PhiLIPPI, ZfM, 87

1848 Tapes euglypta: PhilipPI. Abbild., 3, 76, pl. 7, f. 3

1874 Tapes euglypta: LisCHKe, JMC, 3, 80, pl. 6, f. 8-11

1959 Paphia euglypta: KIRA, CISJ, 1, 144, pl. 56, f. 25

1971 Paphia euglypta: KurOda \& HABE, SSSB, 652 (J) 423 (E), pl. 92, f. 2

1971 Paphia lischkei Fischer-Piette \& Metivier, MMNHN, (A) 71, 43

Loc. N M S (s) dp.

Dist. Southern Hokkaido to Kyushu.

Paphia (Paphia) schnelliana (DUNKER)オオスダレガイ (平瀬)

1866 Tapes schnelliana Dunker, Nov. Conch., 75, pl. 25, f. 7-9

1874 Tapes schnelliana: LisCHKE, JMC, 3, 80, pl. 6, f. 1-4

1959 Paphia schnelliana: KIRA, CISJ, 1, 144, pl. 56, f. 26

1971 Paphia schnelliana: Kuroda \& HABE, SSSB, 653 (J) 423 (E), pl. 92, f. 7

Loc. N M (s) dp.

Dist. Honshu (north to Bōsō Peninsula) to Kyushu.

Paphia (Paphia) amabilis (PHILIPPI) サッマアカガイ（目八）

1847 Tapes amabilis PHILIPPI, ZfM, 90

1849 Tapes amabilis: PhILtPPI, Abbild., 3, 75, pl. 7, f. 2

1874 Tapes amabilis: LischKE, JMC, 3, 82, pl. 6, f. 5-7

1959 Paphia amabilis: KIRA, CISJ, 1, 144, pl. 56, f. 29

1971 Paphia amabilis: Kuroda \& Habe, SSSB, 653 (J) 424 (E), pl. 92, f. 1

Loc. N M S (s) dp.

Dist. Honshu (north to Bōsō Peninsula) to Formosa.

Paphia (Paphia) vernicosa (Gould) アケガイ（目八)

1861 Tapes vernicosa Gould (non ReEve, 1864), PBSNH, 830

1877 Tapes greeffei Dunker, M B L., 24, 73

1882 Tapes greeffei: Dunker, Index, 207, pl. 8, f. 15-17

1959 Paphia vernicosa: KIrA, CISJ, 1, 144, pl. 56, f. 28 
1964 Tapes vernicosa: Johnson, USNMB, 239, 166, pl. 25, f. 3

1971 Paphia vernicosa: Kuroda \& HABE, SSSB, 653 (J) 424 (E), p1. 92, f. 5, 6

Loc. N M S (s) dp.

Dist. Southern Hokkaido to Kyushu.

Paphia (Paphia) exarata (PHILIPPI) ヒメスダレガイ（目八）

1846 Venus exarata PHurpri, Abbild., 2, 109, p1. 5, f. 6

1961 Paphia exarata: HABE, CISJ, 2, 132, pl. 59, f. 12

1971 Paphia exarata: Kuroda \& HABE, SSSB, 653 (J) 424 (E), pl. 92, f. 3

Loc. N S (s) dp.

Dist. Honshu (north to Bōsō Peninsula) to Kyushu.

\section{Subgenus Neotapes KuRODA \& HABE イヨスダレガイ亜属}

1971 Neotapes KuRODA \& HABE, SSSB, 654 (J) 424 (E). Type (OD): Venus undulata BorN

Paphia (Neotapes) undulata (BoRN) イヨスダレガイ（六介）

1778 Venus undulata BoRN, IMCV, 54

1780 Venus undulata: BorN, TMCV, 67

1852 Tapes rimosa SowerBy, Thes., 2, 682, pl. 146, f. 29

1959 Paphia (Paratapes) undulata: KIRA, CISJ, 1, 144, pl. 56, f. 24

1971 Paphia (Neotapes) undulata: Kuroda \& HABE, SSSB, 654 (J) 425 (E), pl. 92, f. 4

Loc. N M S (s-m) dp.

Dist. Honshu (north to Bōsō Peninsula and Noto Peninsula), Shikoku to

Kyushu. Widely ranging in the Indo-West Pacific region.

\section{Genus Tapes Megerle von MüHLFELD リュウキュウアサリ属}

1811 Tapes Megerle von MüHLFELD, GNFBM, 5, 51. Type (M): Venus literata LinNAEUS

Tapes platyptycha (PILSBRY) スリガハマガイ（群品）

1901 Tapes platyptycha PILSBRY, PANSP, 206, 400, pl. 19, f. 5

1959 Tapes platyptycha: KIRA, CISJ, 1, 144, pl. 56, f. 27

1971 Tapes platyptycha: KuROdA \& HABE, SSSB, 655 (J) 425 (E), pl. 94, f. 1

Loc. M S (s) dp.

Dist. Honshu (north to Sagami Bay) to Ryukyu Islands.

\section{Genus Ruditapes ChIAMENTI アサリ属}

1857 Amygdala Römer, Venus, 16 (non Gray, 1825)

1900 Ruditapes chiamenti, Riv. Ital. Sci. Nat. Siena, 20, 13. Type (SD, DaLL, 1902); Venus decussata LINNAEUS

Ruditapes philippinarum ADAMS \& REEVE アサリ (目八)

1850 Venus philippinarum AdAms \& ReEve, Samarang, 79, pl. 32, f. 10

1853 Tapes japonica DEsHAYES, PZSL, 10

1853 Tapes violacea Deshayes, PZSL, 10

1864 Tapes semidecussata REEVE, Icon., sp. 67

1870 Tapes ducalis RöMER, M B L. 17, 9

1871 Tapes ducalis: RöMER, Venus, 2, 81, pl. 28, f. 3

1938 Paphia bifurcata QuAYre, Jour. Fish. Res. Board Canada, 4 (1), 53, f. c, f

1959 Venerupis (Amygdala) japonica: KIRA, CISJ, 1, 144, pl. 56, f. 23

1971 Ruditapes philippinarum: KURODA \& HABE, SSSB, 655 (J) 425 (E), pl. 94, f. 8-10 
Loc. N M S (g) int.

Dist. Saghalien to Formosa, Korea and China.

Ruditapes variegata (SOWERBY) ヒメアサリ (平滇)

1852 Tapes variegata Sowerby, Thes., 2, 196, pl. 151, f. 133-138

1959 Venerupis (Amygdala) variegata: KIRA, CISJ, 1, 143, pl. 56, f. 22

1971 Ruditapes variegata: KURODA \& HABE, SSSB, 656 (J) 426 (E), pl. 94, f. 2

Loc. N M S (g) int.

Dist. Honshu (north to Bōsō Peninsula) to Ryukyu Islands. Widely ranging in the Pacific.

\section{Genus Gomphina MöRCHフキアゲアサリ属}

1853 Gomphina Mörch, Yoldi, (2), 19. Type (M): Venus undulosa LAMARcK

Gomphina neastartoides (YOKOYAMA) キタノフキアゲアサリ（波部）

1922 Venus neastartoides YoKoYAMA, JCSIUT, 44 (1), 149, pl. 11, fi. 9, 10.

1961 Gomphina neastartoides: HABE, CISJ, 2, 131, pl. 59, f. 5

1971 Gomphina neastartoides: KurodA \& HABE, SSSB, 656 (J), 426 (E), pl. 90, f. 4

Loc. $\mathrm{S}$ (s) dp.

Dist. Honshu (north to Bōsō Peninsula) to Ryukyu Islands.

Subgenus Macridiscus DALL オキアサリ亜属（コタマガイ亜属）

1902 Macridiscus DALL, PUSNM, 26, 336, 359. Type (OD): Donax aequilatera SowERBY

Gomphina (Macridiscus) aequilatera (SowERBY)オキアサリ（目八） コタマガイ（目八）

1825 Donax aequilatera SOWERBY, Tankerville, 12

1853 Venus donacina SoWerby (non GMeLin, 1791), Thes., 2, 739, pl. 159, f. 165-7

1853 Venus veneriformis Sowerby, Thes., 2, 739, pl. 159, f. 168 (non LAMARCK, 1818)

1853 Venus aequilatera: SOWERBY, Thes., 2 739, pl. 159, f. 169

1861 Gomphina melanaegis RöMER, M B L., 7, 157

1862 Gomphina melanaegis: DUNKER, Nov. Conch., 40, pl. 12, f. 12, 13

1874. Comphina melanaegis: LischKe, JMC, 3, 86, pl. 7, f. 10, 11

1959 Gomphina (Macridiscus) veneriformis: KIRA, CISJ, 1, 143, pl. 56, f. 20

1959 Gomphina (Macridiscus) melanaegis: KIRA, CISJ, 1, 143, pl. 56, f. 21

1971 Gomphina (Macridiscus) veneriformis: KURODA \& HABE, SSSB, 657 (J) 427 (E), pl. 90, f. 12

Loc. N M S (s) dp.

Dist. Southern Hokkaido to Kyushu, Korea and China.

\section{Genus Irus ScHMIDT マッカゼガイ属}

1818 Irus Schmid, Natur. f. Schulen, 2, 647. Type (T): Donax irus Linnaeus

Irus mitis (DESHAYES) マッカゼガイ（六介）

1854 Venerupis mitis Deshayes, PZSL (1853), 5

1882 Vererupis semipurpurea DUNKER, Index, 208

1959 Irus mitis: KIRA, CISJ, 1, 148, pl. 57, f. 26

1971 Irus mitis: Kuroda \& HABE, SSSB, 657 (J) 427 (E), pl. 94, f. 13

Loc. N M S (bo) int.

Dist. Honshu to Kyushu. Widely ranging in the tropical Pacific. 
Irus macrophyllus (DESHAYES) 八ネマツカゼガイ（岩川）

1854 Venerupis macrophyllus Deshayes, PZSL, (1853) 12, pl. 18, f. 8

1959 Irus macrophyllus: KIRA, CISJ, 1, 148, pl. 57, f. 24

Loc. N M S (bo) int.

Dist. Honshu (Kii Peninsula) to Kyushu. Widely ranging in the tropic Pacific.

Irus ishibashianus KIRA オキナマツカゼガイ（黒田）

1952 Irus ishibashianus KURODA \& HABE, Checklist, 21 (n.n.)

1959 Irus ishibashianus: KIRA, CISJ, 1, 148, pl. 57, f. 25

1971 Irus ishibashianus: Kuroda \& HABE, SSSB, 658 (J) 427 (E), pl. 94, f. 15

Loc. M S (bo) int.

Dist. Honshu (north to Bōsö Peninsula) to Kyushu.

Subfamily Callistinae HABE \& KOSUGE マッヤマワスレガイ亜科

Genus Callista POLIマツャマワスレガイ属

1791 Callista Poli Testa Sicil., 1, 30. Type (SD, Meek, 1876): Venus chione Linnaeus

Callista chinensis (HoLten) マッヤマワスレガイ（目八）

1803 Venus chinensis Holten, Enumaratio (1802), 20

1817 Venus pacifica Dillwyn, Descrip. Cat., 1, 175 (non Röding, 1798)

1855 Cytherea chinensis SOWERBY, Thes., 2, 624, pl. 131, f. 80, 81

1861 Chione roscida Gould, PBSNH, 8, 31

1864 Chione chinensis: ReEve, Icon., sp. 4

1959 Callista chinensis: KIRA, CISJ, 1, 141, p1. 56, f. 3

1971 Callista chinensis: Kuroda \& HABE, SSSB, 644 (J) 417 (E), pl. 90, f. 9

Loc. N M S (s) dp.

Dist. Honshu (north to Bōsō Peninsula) to Formosa, Korea and China.

Callista pilsbryi HABE コマッヤマワスレガイ（岩川）

1960 Callista pilsbryi HABE, PSMBL, 8 (2), 291

1960 Callista amamiensis HABE, PSMBL, 8 (2), 291

1961 Callista pilsbryi: HABE, CISJ, 2, 130, pl. 58, f. 14

1971 Callista pilsbryi: KuRODA \& HABE, SSSB, 645 (J) 417 (E), pl. 90, f. 2, 5

1977 Callista amamiensis: HABE, Taxa HABE, 22, 172, pl. 1, f. 4

Loc. M S (s) dp.

Dist. Honshu (north to Bōsō Peninsula) to Ryukyu Islands.

Callista politissima KURODA 一ヌリッヤハマグリ（黑田） Pl. 7, f. 5.

1945 Callista politissima Kuroda, Venus, 14 (1-4), 35, pl. 1, f. 5, 6

1967 Callista politissima: HABE, \& Kosuge, Kai (Hoikusha), 150, pl. 56, f. 11

Loc. M S (s) dp.

Dist. Honshu (Kii Peninsula) to Ryukyu Islands.

Genus Saxidomus CONRADウチムラサキガイ属

1837 Saxidomus ConRaD, JANSP, 7, 249. Type (M): Saxidomus nuttallii ConRAD

Saxidomus purpurata (SoWERBY) ウチムラサキガイ（目八）

1852 Tapes purpurata SowHERBY, Thes., 2, 692, pl. 150, f. 124, 125

1959 Saxidomus purpuratus: KrRA, CISJ 1 142, pl. 56, f. 9

1971 Saxidomus purpuratus: Kuroda \& HABE, SSSB, 645 (J) 416 (E), p1. 93, f. 5-7 
Loc. N M S (g) int.

Dist. Hokkaido to Kyushu and Korea.

\section{Subfamily Sunettinae STOLICZKA ワスレガイ亜科 \\ Genus Cyclosunetta E. FISCHER ワスレガイ属}

1891 Sunettina Jousseaume, Le Nat., (2) ,5, 208 (non PFeIFFer, 1869)

1939 Cyclosunetta E. Fischer, BMNHNP, (2), 11, 146. Type (T): Sunettina sunettina JousSEAUME $=$ Sunetta comtempta $\mathrm{SMITH}$

Cyclosunetta mentrualis (MeNkE) ワスレガイ (六介)

1843 Cytherea excavata HANLEY (non MoRTON, 1834), PZSL (1842), 123

1843 Cytherea menstrualis MeNKE, Moll. Nov. Holl., 43

1848 Cytherea menstrualis: PHILIPPI, Abbild., 2, 96, pl. 3, f. 3

1851 Meröe excavata: Soweriy, Thes., 2, 610, pl. 126, f. 13, 14

1864 Meröe magnifica REEVE, Icon., sp. 9

1959 Sunetta (Cyclosunetta) menstrualis: KIRA, CISJ, 1, 146, pl. 57, f. 13

1971 Cyclosunetta menstrualis: KurOdA, \& HABE, SSSB, 646 (J) 418 (E), pl. 91, f. 8

Loc. N M (s) int.

Dist. Honshu (north to Bōsō Peninsula) to Kyushu. Widely ranging in the tropic Pacific region.

Cyclosunetta concinna DUNKER シマワスレガイ（黒田）：イソワスレガイ（吉良）

1865 Sunetta concinna DunkeR, Nov. Conch., 1, 84, p1. 25, f. 4-6

1969 Sunnetta (Cyclosunetta) concinna: KrRA, CISJ, 1, 146, pl. 57, f. 14

Loc. S (s) dp,

Dist. Honshu (Kii Peninsula) to Ryukyu Islands. Western Pacific.

\section{Genus Sunettina PFEIfFER ベニワスレガイ属}

1870 Sunettina Pferffer, Cab., 11 (1), 86. Type (M): Cytherea solanderii GraY

Sunettina solanderii (GRAY) ベニワスレガイ（黑田）

1825 Cytherea solanderii Gray, Ann. Phil., 25, 137

1864 Meröe solanderii ReEve, Icon., sp. 10

1959 Sunetta (Sunettina) solanderii: KIRA, CISJ, 1, 147, pl. 57, f. 15

1971 Sunettina solanderii: Kuroda \& HABE, SSSB, 647 (J), 419 (E) pl. 90, f. 1

Loc. M S (s) dp.

Dist. Honshu (north to Sagami Bay) to Kyushu and South East Asia.

\section{Subfamily Meretricinae FISCHER ハマグリ互科}

Genus Meretrix LAMARCK ハマグリ属

1799 Meretrix LAMARCK, MSHNP, 85

1806 Cytherea LamarcK, AMHNP, 7 (38), 132 (non Fabricius, 1792). Type (T): Venus meretrix LINNAEUS

Meretrix lisoria (RöDING) 八マグリ（目八）

1798 Venus lusoria RöDing, Bolten., 1, 180

1817 Meretrix formosa LAMARCK, AsV, 6, 299

1851 Cytherea lusoria: SOWERBy, Thes. 2, 620, p1. 128, f. 40-42

1851 Cytherea formosa: Sowerby, Thes., 2, 620, pl. 129, f. 47 
1864 Cytherea formosa: REEVE, Icon., sp. 14

1864 Cytherea lusoria: ReEve, Icon., sp. 20

1864 Cytherea zonaria: REEve, Icon., sp. 9

1959 Meretrix lusoria: KIRA, CISJ, 1, 140, pl. 56, f. 1

1971 Meretrix lusoria: KuRodA \& HABE, SSSB, 647 (J) 419 (E), pl. 94, f. 3-6

Loc. N M S (s-m) int.

Dist. Southern Hokkaido to Formosa, Korea and China.

Meretrix lamarckii DeshaYeS チョウセンハマグリ（目八）

1853 Meretrix lamarckii Deshayes, Cat. Conch. Brit. Mus, 39

1864 Cytherea lamarckii: ReEve, Icon., sp. 8

1959 Meretrix lamarckii: KIRA, CISJ, 1, 141, pl. 56, f. 2

1971 Meretrix lamarckii: Kuroda \& HABE, SSSB, 648 (J) 419 (E), pl. 93, f. 1

Loc. N M S (s-m) int.

Dist. Honshu (north to Bōsō Peninsula) to Formosa, Korea and China.

\section{Subfamily Clementinae FrIZZELL フスマガイ亜科}

\section{Genus Clementia GRAY フスマガイ属}

1842 Clementia Gray, Syn. 44, 75. Type (SD GRAY, 1847): Venus papyracea GmeLIN

Clementia vatheleti MABILLE フスマガイ (介志)

1901 Clementia vatheleti Mabille, Bull. Soc. Philom. Paris, (8) 3, 57

1961 Clementia vatheleti: HABE, CISJ, 2, 132, pl. 59, f. 19

1971 Clementia vatheleti: Kuroda \& HABE, SSSB, 663 (H) 431 (E), pl. 94, f. 7

Loc. N M S (s-m) int.

Dist. Honshu (north to Bōsō Peninsula) to Kyushu and Korea.

\section{Subfamily Cyclininae FrIzzEL オキシジミ亜科 \\ Genus Cyclina DESHAYES オキシジミ属}

1850 Cyclina Deshayes, Traité Elémént. Conch., 1 (2), 62

1908 Eocyclina DALl, BMCZ, 43, 241. Type (SD DALL, 1902): Venus sinensis GMELIN

Cyclina sinensis (GMELIN) オキシジ（目八）

1791 Venus sinensis GMELIN, Syst. Nat., 13, 3285

1850 Artemis chinensis ReEve, Icon., sp. 6

1855 Artemis orientalis SowerBy, Thes., 2, 661, pl. 141, f. 79

1959 Cyclina sinensis: KIRA, CISJ, 1, 141, pl. 56, f. 5

1971 Cyclina siennsis: Kuroda \& HABE, SSSB, 652 (H) 422 (E), pl. 91 , f. 6

Loc. N M S (m) int.

Dist. Honshu (north to Sagami Bay) to Kyushu, Korea, China, Formosa and

South East Asia.

\section{Family Petricolidae Deshayes イワホリガイ科 \\ Genus Petricola LAMARCK イワホリガイ属}

1801 Petricola LAMARck, AsV, 5, 121

1853 Naranio Gray, AMNH, (2) 11, 38. Type (SD SCHMIDT, 1818): Petricola costata LaMaRCK

$=$ Venus lapicida GMELIN 
Petricola divergens (GMELIN) イワホリガイ (平瀬)

1791 Venus divergens Gmeun, Syst. Nat. 13, 3269

1818 Petricola lucinalis Lamarck, AsV, 5, 45

1853 Naranio radiata GRAY, AMNH, 11, 38

1854 Petricola divericata: SOWERBY, Thes., 2, 776, pl. 166, f. 24, 25

1951 Petricola divergens: HABE, ICJS, 1 (15), 96, pl. 15, f. 6, 11

1959 Petricola divergens : KIRA, CISJ, 1, 148, pl. 57, f. 28

Loc. N M S (bo) g1.

Dist. Honshu (north to Kii Peninsula) to Ryukyu Islands. Widely ranging in the Indo-Pacific region.

\section{Genus Claudiconcha FISCHERセミアサリ属}

1887 Claudiconcha Fischer, M. de C., 187. Type (M): Venus monstrosa GMeLIN

Claudiconcha monstrosa (GMELIN)ヌノメセミアサリ（黑田）

1791 Venus monstrosa Gmelin, Syst., Nat. 13, 3280

1854 Venerupis monstrosus: SowERBY, Thes., 2, 768, pl. 164, f. 13

1934 Petricola monstrosa: Kunoda, Venus, 4 (6), 188, f. 20-22

1951 Claudiconcha monstrosa: HABE, ICJS, 1 (15), 99, pl. 15, f. 10

1959 Claudiconcha monstrosa: KIRA, CISJ, 1, 149, pl. 57, f. 30

Loc. S (g) int.

Dist. Honshu (Kii Peninsula) Amami and Ryukyu Islands. Widely ranging in the Indo-Pacific region.

Claudiconcha japonica (DUNKER) セミアサリ（介志）

1882 Petricola japonica Dunker, Index, 209, pl, 9, f. 4-6

1923 Petricola japonica circumdata LAMY, J. de C., 67, 356, with f.

1934 Petricola japonica: Kuroda, Venus, 4 (6), 188, f. 17-19

1951 Claudiconcha japonica: HABE, ICJS, 1 (15), 99, pl. 15, f. 12-14

1959 Claudiconcha japonica: KIRA, CISJ, 1, 148, pl. 57, f. 29

1971 Claudiconcha japonica: KURODA \& HABE, SSSB, 664 (J) 432 (E), pl. 94, f. 14

Loc. N M S (bo) int.

Dist. Honshu to Kyushu.

\section{Genus Petricolirus HABE シオッガイ属}

1951 Petricolirus Habe, ICJS, 1 (15), 95. Type (OD): Petricola aequistriata Sowerby

Petricolirus aequistriatus (SOWERBY) シオッガイ（目八）

1874 Petricola aequistriata SowerBy, Icon., sp. 19

1951 Petricolirus aequistriatus: HABE, ICJS, 1 (15), 96, pl. 15, f. 4, 5

1959 Petricolirus aequistriatus: KIRA, CISJ, 1, 148, pl. 54, f. 27

1971 Petricolirus aequistriatus: Kuroda \& HABE, SSSB, 665 (J) 433 (E), pl. 94, f. 11

Loc. $\mathrm{N}$ M (bo) int.

Dist. Honshu (north to Bōsō Peninsula) to Kyushu.

$$
\text { Genus Pseudoirus HABEチヂミイワホリガイ属 }
$$

1951 Pseudoirus Habe, ICJS, 1 (15), 96. Type (OD): Venerupis mirabilis Deshayes

Pseudoirus mirabilis (DEsHAYES)チデミイワホリガイ（黒田）

1853 Venerupis mirabilis Deshayes, Cat. Conch. Brit. Mus, 207 
1854 Venerupis mirabilis: SowERBY, Thes., 2, 766, pl. 165, f. 24

1874 Venerupis mirabilis: SOWERBY, Icon., sp. 4

1951 Pseudoirus mirabilis: HABE, ICJS, 1 (15), 96, pl. 15, f. 9, tf. 15

1961 Ruppellaria (Pseudoirus) mirabilis: HABE, CISJ, 2, 133, pl. 60, f. 2

1971 Petricola (Pseudoirus) mirabilis: Kuroda \& HABE, SSSB, 665 (J) 433 (E), pl. 94, f. 11

Loc. M S (bo) gl.

Dist. Southern Hokkaido to Kyushu.

\title{
Genus Rupellaria Fleuriau DE BELLEVUEクシケガイ属
}

1802 Rupellaria Fleuriau DE Bellevue, Jour. de Phys., 54, 347. Type (M): Venus lithophaga RETZIUS

Rupellaria fabagella (LAMARCK) クシケガイ (群品) Pl. 7, f. 4.

1818 Petricola fabagella Lamarck, AsV, 5, 505

1854 Petricola chinensis SOWERBY, Thes., 2, 768, pl. 164, f. 13

1901 Petricola cyclas PiLsBry, PANSP, 53, 204, 400, pl. 19, f. 3, 4

1901 Petricola cyclas sculpturata PILSBRY, PANSP, 53, 205, 400, pl. 1, f. 7

1951 Ruppellaria fabagella: HABE, ICJS, 1 (15), 96, pl. 15, f. 7, 8

Loc. M S (bo) gl.

Dist. Honshu (north to Bōsō Peninsula) to Kyushu. Widely ranging in the Indo Pacific region.

\section{GenuS Lajonkairia DESHAYES チヂミガイ属}

1854 Lajonkairia Deshayes, Cat. Couch. Brit. Mus. (2), 217. Type (T): Venerupis decussata PHILIPPI=Lajonkairia lajonkairii PAYRAUdEAU

Lajonkairia divaricata (LISCHKE) チデミガイ（六介）

1872 Lucinopsis divaricata LrsCHKE, M B L., 19, 188

1874 Lucinopsis divaricata: LischKe, JMC, 3, 90, pl. 7, f. 12-14

1951 Lajonkairia divaricata: HABE, ICJS, 1 (15), 95, pl. 15, f. 1-3

1961 Lajonkairia divaricata: HABE, CISJ, 2, 133, p]. 60, f. 1

1971 Lajonkairia divaricata: KuRODA \& HABE, SSSB, 664 (J) 432 (E), pl. 120, f. 20

Loc. N S (s) dp.

Dist. Honshu (north to Bōsō Peninsula) to Kyushu.

\author{
Order Myoida STOLICKZKA オォノガイ目 \\ Suborder Myina STOLICZKa オオノガイ亜目 \\ Superfamily Myacea LAMARCK オオノガイ超科 \\ Family Myidae LAMARCK オオノガイ科 \\ Subfamily Myinae LAMARCKオオノガイ亜科 \\ Genus Mya LinnaEus オオノガイ属
}

1758 Mya Linnaeus, Syst. Nat. 10, 670. Type (SD Chlldren, 1822, ICZN Op. 94): Mya truncata LINNAEUS 


\section{Subgenus Arenomya WINCKWORTHオオノガイ亜属}

1930 Arenomya Winckworth, PMSL, 19, 15. Type (OD): Mya arenaria LINNAeus

Mya (Arenomya) arenaria oonogai MAKIYAMA オオノガイ（目八）

1758 Mya arenaria LinNAeus, Syst. Nat., 10, 670

1935 Mya oonogai MakiYama, Warera-no-Kōbutsu, 4 (3), 39, f.

1957 Mya japonica oonogai: FUJE, JFSHU, (4) 9 (4), 403

1959 Mya (Arenomya) arenaria oonogai: KIRA, CISJ, 1, 163, pl. 61, f. 22

Loc. N S (m) int.

Dist. Hokkaido to Kyushu and Korea.

\section{Subfamily Cryptomyinae HABE ヒメマスオガイ髙科 \\ Genus Cryptomya CoNRAD ヒメマスオガイ属}

1848 Cryptomya ConRad, PANSP, 4, 121. Type (M): Sphaenia californica CONRAD

Cryptomya busoensis YoKoYAMAヒメマスオガィ（黑田）

1922 Cryptomya busoensis YoKoYAMA, JCSIUT, 44 (1), 125, pl. 7, f. 1, 2

1961 Cryptomya busoensis: HABE, CISJ, 2, 140, pl. 63, f. 13

1971 Cryptomya busoensis: KURODA \& HABE, SSSB, 704 (J) 463 (E), pl. 121, f. 7

Loc. N S (s) dp.

Dist. Hokkaido to Kyushu.

\section{Genus Venatomya IREDALE クシケマスオガイ属}

1930 Venatomya Iredale, RAM, 17 (9), 403. Type (OD): Sphaenia elliptica A. AdAMs

Venatomya truncata (Gould)クシケマスオガイ（黑田）

1861 Cryptomya truncata Gould, PBSNH, 8, 24

1927 Cryptomya tachibanensis YokoYama, JFSIUT, (2) 1 (10), 454, pl. 52, f. 5

1951 Venatomya truncata: HABE, ICJS, 1 (12), 75, pl. 12, f. 12-14

1961 Venatomya truncata: HABE, CISJ, 2, 140, pl. 63, f. 12

1965 Venatomya truncata: KuRODA \& HABE, NIEFJ, 2, 299, f. 1134

Loc. N S (m) int.

Dist. Honshu (Ise Bay) to East China Sea.

\section{Genus Distugonia IREDALEオフクマスオガイ属}

1936 Distugonia IrEDALE, RAM, 19 (5), 283. Type (OD): Distugonia inopinata Iredale

Distugonia decurvata (A. AdAMs) オフクマスオガイ（黒田）

1851 Sphaenia decurvata A. ADAMS, PZSL, 18 (1850), 88

1875 Mya decurvata: SOWERBY, Icon., sp. 11

1951 Distugonia decurvata: HABE, ICJS, 1 (12), 75, pl. 12, f. 15, 16

1961 Distugonia decurvata: HABE, CISJ, 2, 140, pl. 63, f. 11

Loc. N M S (s) dp.

Dist. Honshu (Kii Peninsula) to Kyushu. Western Pacific.

\section{Genus Sphaenia TURTON イジケガイ属}

1822 Sphaenia Turron, Conch. Ins. Brit., 17, 36. Type (SD Gray, 1847): Sphaenia binghami TURTON 
Sphaenia coreanica HABE イジケガイ（黒田）

1951 Sphaenia coreanica HABE, ICJS, 1 (12), 76, tf. 3-4

1961 Sphaenia coreanica: HABE, CISJ, 2, 140, pl. 63, f. 10

1971 Sphaenia coreanica: Kuroda \& HABE, SSSB, 704 (J) 463 (E), pl. 121, f. 8

Loc. M S (st) gl.

Dist. Honshu (north to Bōsō Peninsula) to Kyushu and Korea.

\section{Genus Paramya CONRAD ハマカゼガイ属}

1861 Paramya ConRAD, PANSP, 13, 232. Type (M): Myalina subovata ConRAD

Paramya recluzii (A. ADAMS) ハマカゼガイ（波部）

1864 Eucharis recluzii A. ADAMs, AMNH, (3) 13, 310

1920 Basteriotia trapezium YoKoYAMA, JCSIUT, 39 (6), 108, pl. 7, f. 8, 9

1961 Anisodonta recluzii: HABE, CISJ, 2, 123, pl. 56, f. 7

1971 Anisodonta recluzii: KuRodA \& HABE, SSSB, 625 (J) 403 (E), pl. 118, f. 20

Loc. N M S (s-m) dp.

Dist. Honshu (north to Tokyo Bay) to Kyushu.

\section{Family Corbulidae LAMARCK クチベニガイ科 \\ Subfamily Corbulinae LAMARCK クチベニガイ亜科 \\ Genus Anisocorbula IREDALE ッマベニガイ属}

1930 Anisocorbula Iredale, RAM, 17, 404. Type (OD): Corbula macgillivrayi Sмтtн

Anisocorbula scaphoides (HINDS)ッマベニガイ（介志）

1843 Corbula scaphoides HiNDs, PZSL, 11, 56

1844 Corbula scaphoides: REEVE, Icon., sp. 24

1929 Corbula tosana YoKоYAMA, SR GSJ, 104, 15, pl. 8, f. 1

1961 Anisocorbula scaphoides: HABE, CISJ, 2, 139, p1. 63, f. 1

1961 Anisocorbula tosana: HABE, CISJ, 2, 139, pl. 63, f. 2

1971 Anisocorbula scaphoides: KURODA \& HABE, SSSB, 706 (J) 465 (E), pl. 102, f. 13

Loc. N M S (s) dp.

Dist. Honshu (north to Bōsō Peminsula) to Ryukyu Islands. Western Pacific.

Anisocorbula venusta (GouLD) クチベニデガイ（矢倉）

1861. Corbula venusta Gould, PBSNH, 8, 25

1949 Anisocorbula venusta: HABE, ICJS, 1 (1), 3, pl. 1, f. 16

1961 Corbula venusta: JoHnson, USNMB, 239, 165, pl. 23, f. 6

1965 Anisocorbula venusta: HABE, ICJS, 1 (1), 3, pl. 1, f. 16

1965 Anisocorbula venusta: Kuroda \& HABE, SSSB, 707 (J) 465 (E), pl. 102, f. 15

Loc. M S (s) int.

Dist. Southern Hokkaido to Kyushu and Korea. Western Pacific.

Anisocorbula nipponica HABE イナカクチベニガイ（波部）

1961 Anisocorbula nipponica HABE, CISJ, 2, 139, App. 40, pl. 63, f. 5

1971 Anisocorbula nipponica: KURODA, \& HABE, SSSB, 707 (J) 465 (E), pl. 102, f. 14

Loc. M (s) gl.

Dist. Honshu (north to Bōsō Peninsula) to Kyushu. 
Anisocorbula modesta (REEVE)ヒナクチベニガイ（黒田）

1843 Corbula modesta ReEve, Icon., sp. 14a, b.

1843 Corbula modesta: HINDS, PZSL, 11, 57

1949 Anisocorbula modesta: HABE, ICJS, 1 (1), 3, p1. 1, f. 15

Loc. M S (s) int.

Dist. Honshu (Kii Peninsula) to Ryukyu Islands.

Genus Minicorbula HABE チビクチベニガイ属

1977 Minicorbula HABE, SMJ B/S, 283. Type (OD): Anisocorbula minitissima HABE

*Minicorbula minitissima (HABE) チビクチベニガイ（波部)

1949 Anisocorbula minitissima HABE, ICJS, 1 (1), 4, pl. 1, f. 6

1971 Anisocorbula minitissima: KURODA \& HABE, SSSB, 708 (J) 466 (E), pl. 121, f. 10

Loc. M (s) dp.

Dist. Honshu (north to Bōsō Peninsula) to Kyushu.

\section{Genus Varicorbula GRANT \& GALE コダキガイ属}

1931 Varicorbula Grant \& Gale, San Diego, 1, 422. Type (OD): Corbula gibba Olivi

Varicorbula rotalis (HINDS) コダキガイ (互瀬)

1843 Corbula rotalis HINDs, PZSL, 11, 56

1844 Corbula rotalis: REEVE, Icon., sp. 34

1860 Corbula bifrons A. ADAMs, AMNH, (3) 5, 412

1922 Corbula substriata YoKоYAMA, JCSIUT, 44 (1), 125, pl. 7, f. 3

1961 Varicorbula bifrons: HABE, Dr. MaKiYAMA Mem. Vol., 193, pl. 1, f. 5; pl. 4, f. 17

1961 Varicorbula bifrons: HABE, CISJ, 2, 139, pl. 63, f. 3

1971 Varicorbula rotalis: KuRODA \& HABE, SSSB, 705 (J) 464 (E), pl. 121 , f. 9

Loc. M S (s) dp.

Dist. Honshu (north to Bōsō Peninsula) to Formosa.

\section{Genus Solidicorbula HABEクチベニガイ属}

1949 Solidicorbula HABE, ICJS, 1 (1), 2. Type (OD): Corbula erythrodon LAMARCK

Solidicorbula erythrodon (LAMARCK) クチベニガイ（目八）

1818 Corbula erythrodon LAMARCK, AsV, 5, 496

1848 Corbula erythrodon: REEVE, Icon., sp. 4

1949 Solidicorbula erythrodon: HABE, ICJS, 1 (1), 2, f. 7-9

1959 Solidicorbula erythrodon: KiRA, CISJ, 1, 163, pl. 61, f. 19

1971 Solidicorbula erythrodon: KuRODA \& HABE, SSSB, 705 (J) 464 (E), pl. 102, f. 12

Loc. N M S (s) int.

Dist. Honshu (north to Bōsō Peninsula) to Kyushu. Western Pacific.

\section{Superfamily Hiatellacea GRAY キヌマトイガイ超科 \\ Family Hiatellidae GRAY キヌマトイガイ科 \\ Genus Hiatella Bosc キヌマトイガイ属}

1801 Hiatella Bosc, Hist. Nat. Coq., 3, 120.

1802 Saxicava Fleiuriau, Jour. de Phys., 54, 354, 349. Type (SD Winckworth, 1932):

Hiatella monoptera $\mathrm{BOSC}=$ Mya arctica LINNAEUS 


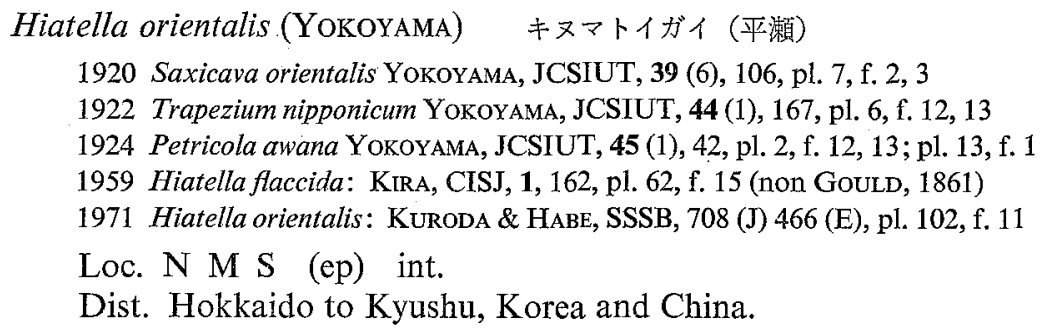

\section{Genus Panopea MENaRd ナミガイ属}

1799 Glycymeris Lamarck, MSHNP, 83 (non da Costa, 1778)

1807 Panopea Menard, AMHNP, 9, 50. Type (SD Fleming, 1817): Panopea faujasi MENARD $=$ Mya glycymeris BORN

Panopea japonica (A. ADAMS) ナミガイ (目八)

1850 Panope japonica A. ADAMs, PZSL, 170, pl. 6, f. 5

1861 Panopea fragilis GouLd, PBSNH, 8, 25

1952 Panope tyosiensis Ozaki, BNSM, 4 (1), 135, pl. 11, f. 5

1959 Panope japonica: KiRA, CISJ, 1, 162, pl. 61, f. 16

1971 Panopea japonica: KurodA \& HABE, SSSB, 709 (J) 467 (E), pl. 102, f. 10

Loc. N S (s-m) dp.

Dist. Hokkaido to Kyushu.

\section{Superfamily Gastrochaenacea GRAY ックエガイ超科 \\ Family Gastrochaenidae GRAY ックエガイ科 \\ Genus Gastrochaena SPENGLER ックエガイ属}

1783 Gastrochaena SPENGLeR, Nye Saml. K. Dansk. Vid. Selsk. Skirft., 2, 172

1788 Chaena PhiLIPsson, Diss. Nova. Testa. Gen., 19

1829 Rocellaria BlatNville, DSN, 57, 244. Type (SD Childrenm, 1822): Gastrochaena cuneiformis SPENGLER

\section{Subgenus Gastrochaena SPENGLER ツクエガイ平属}

Gastrochaena (Gastrochaena) cuneiformis SPENGLER ツクエガィ（六介）

1783 Gastrochaena cuneiformis Spengler, Nye. Saml. K. Densk. Vid. Selsk. Skirft., 2, 180, pl. 1, f. 8-11

1830 Fistulana gigantea DESHAYSE, EMHNV, 2 (2), 142

1882 Gastrochaena grandis DUNKER, Index, 171, pl. 14, f. 10, 11 (non DESHAYES, 1830)

1959 Rocellaria cuneiformis: KIRA, CISJ, 1, 166, pl. 62, f. 14

Loc. M S (bo) gl.

Dist. Honshu (north to Izu Peninsula) to Ryukyu Islands. Indo-West Pacific.

$$
\text { Subgenus Cucurbitula GouLDチビックエガイ西属 }
$$

1861 Cucurbitula Gound, PBSNH, 8, 22. Type (M): Chaena lagenula Gould

Gastrochaena (Cucurbitula) cymbium SPENGLER チビックエガイ（六介）

1783 Gastrochaena cymbium Spengler, Nye. Saml. K. Dansk. Vid. Selsk. Skirft, 2, 180, pl. 1, f. $12-17$ 
1818 Fistulana lagenula LAMARCK, AsV, 5, 436

1861 Chaena lagenula Gould, PBSNH, 8, 22

1878 Gastrochaena lagenula: SowerBy, Icon., sp. 18

1961 Rocellaria (Cucurbitula) cymbium: HABE, CISJ, 2, 140, pl. 63, f. 15

1971 Cucurbitula cymbium: Kuroda \& HABE, SSSB, 711 (J) 468 (E), pl. 102, f. 19

Loc. N M S (sy) int [Cripeasperoida, etc]

Dist. Honshu (north to Bōsō Peninsula) to Kyushu and China. Indo West Pacific.

\section{Genus Spengleria TRYON サヤガイ属}

1862 Spengleria Tryon, PANSP, 472, 485. Type (SD Stoliczka, 1871): Gastrochaena mytiloides LAMARCK

Spengleria mytiloides (LAMARCK) サヤガイ（黑田）

1818 Gastrochaena mytiloides LAMARcK. AsV, 5, 447

1863 Gastrochaena retzi DESHAYES, Reunion, 7, pl. 28, f. 1-3

1878 Gastrochaena retzi: SOWERBY, Icon., sp. 6

1878 Gastrochaena mytiloides: SowERBY, Icon., sp. 12

1959 Spengleira mytiloides: KIRA, CISJ, 1, 166, pl. 62, f. 33

Loc. M S (bo) dp.

Dist. Honshu (Kii Peninsula) to Ryukyu Islands. Indo West Pacific.

\section{Genus Eufistulana EAMES コッ゙ッガイ属}

1789 Fistulana Bruguière, EMHNV, 1, 712 (non MÜLLER, 1776)

1951 Eufistulana EAmEs, Philos. Trans. Roy. Soc. London, (B) 627, 235, 445. Type: Gastrochaena mumia SPENGLER

Eufistulana grandis (DESHAYES) コッ゙ッガイ (平瀬)

1855 Chaena grandis DeshaYes, PZSL, 22 (1854), 230

1878 Fistulana grandis: SowerBy, Icon., sp. 3

1927 Vermetus ebaranus YoKoyama, JFSIUT, (2) 1 (10), 414, pl. 46, f. 15-17

1944 Gastrochaena grandis: Iw. Taki, Venus, 13, 115, tf. 3-6, 6-14

1961 Gastrochaena grandis: HABE, CISJ, 2, 140, pl. 63, f. 14

1971 Eufistulana grandis: Kuroda \& HABE, SSSB, 710 (J) 467 (E), pl. 102, f. 16-18

Loc. N M (s) dp.

Dist. Honshu (north to Bōsō Peninsula) to Kyushu and South East Asia.

\section{Suborder Pholadina H. \& A. ADAMS ニオガイ覀目}

\section{Superfamily Pholadacea LAMARCK ニオガイ超科 \\ Family Pholadidae LAMARCK ニオガイ科 \\ Subfamily Pholadinae LAMARCK ニオガイ亜科}

Genus Barnea Risso ニオガイ属

1826 Barnea Risso, HNEM, 4, 376

1887 Holopholas Fischer, M. de C., 1133. Type (OD): Barnea spinosa Risso=Pholas candida LINNAEUS 


\section{Subgenus Anchomasa LEACHニオガイ巠属}

1852 Anchomasa LeACH, Syn., 250, 253. Type (OD): Anchomasa pennatiana LEACH= Pholas parvus PenNant

Barnea (Anchomasa) manilensis (PHILIPPI) ニオガイ（群品)

1847 Pholas manilensis PHILIPPI, ZfM, 72

1848 Pholas manilensis: PHILrpPr. Abbild., 3, 51, pl. 1, f. 2

1849 Pholas fragilis: SOWERBY, PZSL, 17, 161

1872 Pholas fragilis: SOWERBY, Icon., sp. 8

1895 Pholas manilensis inornata PILSBRY, CMMJ, 116

1959 Barnea (Anchomasa) manilensis: KIRA, CISJ, 1, 168, pl. 62, f. 23

1960 Barnea (Anchomasa) elongata THANG \& LI, AZS, 12 (1), 68, f. 5

1961 Barnea (Anchomasa) manilensis inornata: HABE, CISJ, 2, 141, pl. 63, f. 16

1971 Barnea (Anchomasa) manilensis inornata: Kuroda \& HABE, SSSB, 712 (J) 469 (E), pl. $102, \mathrm{f} .6$

Loc. N M S (bo) int.

Dist. Hokkaido to Kyushu. Western Pacific.

\section{Subgenus Umitakea HABEウミタケガイ亜属}

1952 Umitakea HABE, GJS, 3, 241. Type (OD): Pholadomya japonica YoкоYAMA

Barnea (Umitakea) dilatata (SOULEYET) ウミタケガイ (目八)

1843 Pholas dilatata SOULEYET, RZSC, 6, 76, 176

1849 Pholas latissima Sowerby, Thes., 2 (10), 489, pl. 103, f. 15, 16

1920 Pholadomya japonica YoKoYAMA, JCSIUT, 39 (6), 106, pl. 6, f. 30, 31

1959 Barnea (Umitakea) japonica: KTRA, CISJ, 1, 168, p1. 62, f. 22

Loc. N (s-m) dp.

Dist. Honshu (north to Bōsō Peninsula) to Kyushu. Western Pacific.

\section{Genus Zirfaea GRAY ニオガイモドキ属}

1942 Zirfaea Gray, Syn. 44, 76. Type (SD Gray, 1847): Pholas crispata Linnaeus

Zirfaea subconstricta (YoKoYAMA) ニオガイモドキ（平瀬）

1849 Pholas constricta Sowerby, Thes., 2, 489, pl. 104, f. 27, 28 (non PhilipPI, 1829)

1924 Pholas subconstricta Yокочама, JCSIUT, 45 (1), 38, pl. 2, f. 13

1924 Jouannetia yabei YoKоYамA, JCSIUT, 45 (1), 38, pl. 2, f. 11

1959 Zirfaea subconstricta: KIRA, CISJ, 1, 167, pl. 62, f. 21

1960 Zirfaea minor THANG \& Li, AZS, 12, 71, f. 8

Loc. N M S (bo) int.

Dist. Hokkaido to Kyushu. Western Pacific.

\section{Subfamily Martesinae GRANT \& GALE, 1931 カモメガイモドキ亜科}

\section{Genus Martesia SowERBY カモメガイモドキ属}

1824 Martesia Sowerby, Gen. Rec. Foss. Shells, 23, 2, 4

1898 Martesiella VeERILL \& BUSH, PUSNM, 20, 777

1936 Hiata ZETEK \& MCLEAN, Naut., 49, 111

1943 Mesipholas Iw. TAKI \& Habe, Venus, 14, 109

1945 Diplolax Bartsch \& ReHDER, SMC, 104 (41), 10. Type (OD): Pholas clavata LamarcK $=$ Pholas striata LINNAEUs 
Martesia striata (LINAEUS)カモメガイモドキ（矢倉）

1758 Pholas striata LiNNAEUs, Syst. Nat., 10, 669

1924 Pholas striata: Sowerby, Thes., 2, 494, pl. 104, f. 40-42, pl. 105, f. 43, 44

1924 Pholas cupula YoKoYAMA, JCSIUT, 45 (1), 37, pl. 2, f. 15

1927 Martesia striata tokyoensis Yokoyama, JFSIUT, (2) 1 (10), 428, pl. 48, f. 2, 3

1932 Martesia pulchella YoKoyama, JFSIUT, (2) 3 (6), 288, pl. 2, f. 5

1938 Martesia hawaiensis DALL, Bartsch \& ReHDER, BPBMB, 153, 205, pl. 52, f. 1-7

1945 Mesopholas nucicola Iw. TAKI \& HABE, Venus, 14, 110

1945 Mesopholas intusgranosa Iw. TAKт \& HABE, Venus, 14, 110

1959 Martesia cupula: KIRA, CISJ, 1, 167, pl. 62, f. 16

Loc. M (bo) dp.

Dist. World wide.

\section{Genus Penitella Valenciennes カモメガイ属}

1846 Penitella Valenciennes, Voy. Venus Atlas Moll., 24

1851 Navea Gray, AMNH, (2) 8, 381, 385

1960 Monoplax Thang \& Li, AZS, 13, 72. Type (SD Grant \& Gale, 1931): Penitella conradi VALENCIENNES

Penitella kamakurensis (YoKOYAMA) カモメガイ(目八)

1922 Jouannetia kamakurensis Yокочама, JCSIUT, 44 (1), 120, pl. 6, f. 10

1959 Pholadidea (Penitella) kamakurensis: KIRA, CISJ, 1, 167, pl. 62, f. 18

1960 Pholadidea (Monoplax) dolichothyra THANG \& LI, AZS, 12, 71, f. 9

1960 Pholadidea (Monoplax) acutithyra THANG \& LI, AZS, 12, 73, f. 10

1971 Penitella kamakurensis: Kuroda \& HABE, SSSB, 713 (J) 469 (E), p1. 102, f. 8

Loc. N S (bo) int.

Dist. Southern Hokkaido to Kyushu.

\section{Genus Parapholas ConRAD モモガイ属}

1848 Parapholas Conrad, PANSP, 4, 121. Type (OD): Pholas californica ConRAD

Parapholas quadrizonata (SPENGLER) モモガイ (群品)

1792 Pholas quadrizonata SPENGLER, SNS, 2 (1), 93

1849 Pholas incii Sowerby, Thes., 2, 491, pl. 105, f. 45, 46

1882 Pholas piriformis DunKeR, Index, 171, pl. 14, f. 7

1959 Parapholas quadrizonata: KIRA, CISJ, 1, 167, pl. 62, f. 19

1971 Parapholas quadrizonata: KuRODA \& HABE, SSSB, 713 (J) 470 (E), p. 102, f. 9

Loc. M S (bo) int.

Dist. Honshu (north to Bōsō Peninsula) to Ryukyu Islands. Indo Pacific.

\section{Subfamily Jouannetiinae TRYON スズガイ亜科 \\ Genus Nettastomella CARPENTERヨコヤマスズガイ属}

1864 Netastoma Carpenter, RBAAS, (1863), 637 (non Netastoma RafiNesque, 1815)

1865 Nettastomella CARPENTER, PZSL, 202. Type (M): Netastoma darwinii CARPENTER (nOn SOWERBY)

Nettastomella japonica (YoKoYAMA) ヨコヤマスズガイ（滝・波部）

1920 Jouannetia japonica YoKoYAMA, JCSIUT, 39 (6), 105, pl. 7, f. 1

1955 Nettastomella japonica: Iw. TAKI \& HABE, ICJS, 2 (2), 14, pl. 2, f. 3, 4

1961 Nettastomella japonica: HABE, CISJ, 2, 141, pl. 63, f. 17 
1971 Nettastomella japonica: Kuroda \& HABE, SSSB, 714 (J) 471 (E), pl, 102, f. 5

Loc. N M S (bo) int.

Dist. Hokkaido to Kyushu.

\section{Genus Jouannetia Des MoULINSスズガイ属}

1828 Jouannetia Des Moulins, BHNLB, 2 (12), 244. Type (OD): Jouannetia semicaudata Des Moulins

\section{Subgenus Jouannetia Des MouLINSスズガイ亚属}

Jouannetia (Jouannetia) cumingii (SowERBY) スズガィ（目八）

1849 Triomphalia cumingii SowERBY, Thes., 2, 502, pl. 106, f. 56, 57

1850 Triomphalia cumingii: SowerBy, PZSL, 17 (1848), 161, pl. 5, f. 3

1959 Jouannetia cumingii: KIRA, CISJ, 1, 167, pl. 62, f. 17

Loc. M S (bo) int. . [Coral]

Dist. Honshu (Kii Peninsula) to Ryukyu Islands. Indo-Pacific.

\section{Subgenus Pholadopsis CoNRAD トゲスズガイ巠属}

1849 Pholadopsis CONRAD, PANSP, 4, 156

1849 Triomphalia Sowerby, Thes., 2 (10), 500. Type (OD): Pholadopsis pectinata CONRAD

Jouannetia (Pholadopsis) globulosa (Quoy \& GAImARD) トゲスズガイ（矢倉）

1835 Pholas globulosa Quoy \& GaImard, Astrolabe, 3, 549

1849 Triomphalia globosa Sowerby, Thes., 2, 501, p1. 106, f. 54, 55

1955 Jouannetia (Triomphalia) globulosa: Iw. TAKI \& HABE, ICJS, 2 (2), 15, pl. 2, f. 7, 8

1959 Jouannetia (Triomphalia) globulosa: KiRA, CISJ, 1, 167, pl. 62, f. 15

1971 Jouannetia (Pholadopsis) globulosa: KurodA, \& HABE SSSB, 714 (J) 470 (E), pl. 102, f. 7

Loc. M S (bo) int. [Soft rock]

Dist. Honshu (north to Bōsō Peninsula) to Ryukyu Islands. Indo-Pacific.

\section{Family Xylophaginidae PURCHON キクイガイ科}

Genus Xylophaga TURToN チョウチョウキクイガイ属

1822 Xylophaga TURTON, Conch. Insularum Brit., 253. Type (OD): Teredo dorsalis Turton

Xylophaga indica SMITH ·ョウチョウキクイガイ（滝・波部）

1904 Xylophaga indica SMrTH, AMNH, (17) 79, 7

1950 Xylophaga japonica Iw. TAKì \& HABE, ICJS, 1 (7), 45, tf. 6, 7

1977 Xylophaga indica: HABE, SMJ B/S. 294. pl. 62, f. 1, 2

Loc. S (bo) gl. [timbers deposited]

Dist. Honshu (Wakayama Pref.), Shikoku and Indian Ocean.

\section{Genus Neoxylophaga Iw. TAKI \& HABE キクイガイ属}

1950 Neoxylophaga Iw. TAKI \& HABE, ICJS, 1 (7), 46. Type (OD): Xylophaga rikuzenica Iw. TAKI \& HABE

Neoxylophaga rikuzenica Iw. TAKI \& HABE キクイガイ(滝・波部)

1945 Xylophaga rikuzenica Iw. TAKI \& HABE, Venus, 14, 112

1950 Neoxylophaga rikuzenica: Iw. TAKr \& HABE, ICJS, 1 (7), 46, tf. 4, 5

1977 Neoxylophaga (Neoxylophaga) rikuzenica: HABE, SMJ B/S. 295, pl. 62, f. 3, 4 
Loc. M S (bo) gl. [timbers deposited]

Dist. Honshu (Rikuzen to Wakayama Pref.) and Japan Sea.

Genus Mesoxylophaga HABEテラマチキクイガイ属

1977 Mesoxylophaga Habe, SMJ B/S. 295. Type (OD): Neoxylophaga teramachii Iw. TAKI \& HABE

Mesoxylophaga teramachii (Iw. TAKI \& HABE)テラマチキクイガイ（滝・波部）

Pl. 7, f. 6.

1950 Neoxylophaga teramachii Iw. TAKI \& HABE, ICJS, 1 (7), 46, tf. 3

1971 Neoxylophaga teramachii: KURODA \& HABE, SSSB, 715 (J) 471 (E), pl. 121, f. 11

Loc. S (bo) tr. [timbers deposited]

Dist. Honshu (north to Sagami Bay) and Shikoku.

Genus Metaxylophaga Iw. TAKI \& HABE イノリキクイガイ属

1950 Metaxylophaga Iw. TAKI \& HABE, ICIS, 1 (7), 47. Type (OD): Metaxylophaga supplicata TAKI \& HABE

Metaxylophaga supplicata Iw. TAKI \& HABE イノリキクイガイ（滝・波部）

Pl. 7, f. 7.

1950 Metaxylophaga supplicata Iw. TAKI \& HABE, ICJS, 1 (7), 47, tf. 1, 2

1965 Xylophaga wolffi KNUDSEN, Galathea, 5, 187, 29, 30

1977 Metaxylophaga supplicata: HABE, SMJ B/S, 295, pl. 62, f. 5, 6

Loc. S (bo) gl. [timbers deposited]

Dist. Honshu (Wakayama Pref.), Shikoku and Sulu Sea.

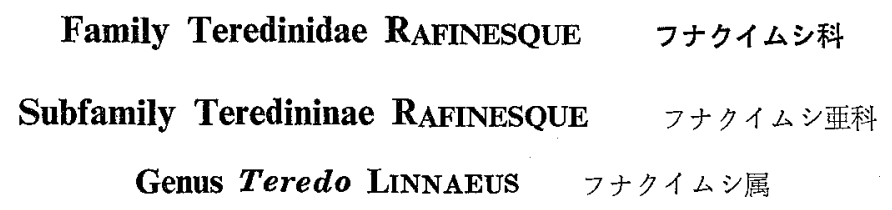

1758 Teredo Linnaeus, Syst. Nat., 10, 651. Type (SD ICZN Op. 94): Teredo navalis Linnaeus

Teredo navalis LINNAEUS フナクイムシ (通称)

1758 Teredo navalis Linnaeus, Syst. Nat., 10, 651

1893 Teredo japonica Clessin, Cab., 11, 78

1929 Teredo sinensis Roch, Hamburg, 44, 13, pl. 2, f. 11

1929 Teredo japonica Kuronuma, Venus, 2, 297, pl. 8, f. 1; pl. 9, f. 9-11

1961 Teredo japonica: HABE, CISJ, 2, 141, pl. 63, f. 22

1971 Teredo navalis japonica: Kunoda \& HABE, SSSB, 716 (J) 472 (E), pl. 121, f. 12, 13

Loc. M S (bo) gl. [timbers]

Dist. Worldwide.

\section{Genus Lyrodus GouLDヤッフナクイムシ属}

1870 Lyrodus Gould, Binney's Rep. Invert. Mass., (2), 34. Type (OD): Teredo chlorotica GOULD

Lyrodus pedicellatus (QUATRFFAGUS) ヤツフナクイムシ(滝)

1849 Teredo pedicellata QuATREFAGUS, ASNZ, (3) 11, 26, pl. 1, f. 2

1927 Teredo siamensis BARTSCH, J. Siam Soc. N. H. Suppl., 7, 59, p. 6, f. 2-5, 9, 11 
1929 Teredo yatsui Moll Hamburg, 44, 10, pl. 1, f. 5

1931 Teredo yatsui: Kuronuma, Venus, 2, 298, pl. 8, f. 2; pl. 9, f. 12-15

1961 Lyrodus yatsui: HABE, CISJ, 2, 141, pl. 63, f. 21

1966 Teredo (Teredo) pedicellatus: TuRnER, Cat. Teredinidae, 116, pl. A.C.D.E. 4B

1971 Lyrodus siamensis: KuRODA \& HABE, SSSB, 717 (J) 472 (E), pl. 121, f. 14

Loc. N M S (bo). [timbers.]

Dist. Hokkaido to Formosa. Indo-West Pacific.

Genus Nototeredo BARTSCH, 1923 キラフナクイムシ属

1923 Nototeredo BARTSCH, PBSW, 36, 100

1937 Phylloteredo Roch, Hamburg, 22, 169. Type (OD): Teredo edax HEDLEY

Nototeredo edax (HEDLEY) オオシマフナクイムシ（滝・波部）

1895 Teredo edax HEDLEY, PLSNSW, 19, 501, pl. 32, f. 1-5.

1928 Teredo hydei Sivickis, Philippine, 37 (3), 294, pl. 3

1928 Teredo appendicullaria Sivickis, Philippine, 37 (3), 295, pl. 3, f. 16

1945 Psiloteredo (Psiloteredo) pentagonalis Iw. TAKI \& HABE, Venus, 14, 114

1952 Psiloteredo (Psiloteredo) pentagonalis HABE, GJS, 3, 251, f. 674

1960 Psiloteredo septa Mawatari \& Kitamura, Misc. Rep. Res. Inst. Nat. Res. Tokyo, 52, 53 72,75 , tf. $8 \mathrm{a}-\mathrm{d}$, pl. 5 , f. 3-12

1961 Psiloteredo pentagonalis HABE, CISJ, 2, 141, pl. 63, f. 20

Loc. $\mathrm{M}(\mathrm{bo})$.' [timbers]

Dist. Honshu (Kii Peninsula) to Kyushu. Central and West Pacific.

Nototeredo kirai (Iw. TAKI \& HABE) キラフナクイムシ（滰・波部）

1945 Psiloteredo (Phylloteredo) kirai Iw. TAKr \& HABE, ,Venus, 14, 114

Loc. M (bo) gl. [timbers deposited]

Dist. Tosa Bay, Shikoku.

Genus Teredothyra BARTSCH グンバイフォクイムシ属

1921 Teredothyra BARTSCH, PBSW, 34, 26

1927 Ungoteredo BARTSCH, BUSNM, 11 (2) 544

1945 Idioteredo Iw. TAKI \& HABE, Venus 14, 115. Type (OD): Teredo (Teredothyra) dominicensis BARTSCH

Teredothyra smithi (BARTSCH) グンバイフナクイムシ（滝・波部）

1927 Teredothyra smithi BarTSCH, USNMB, 11 (2), 540, pl. 53, f. 6, 7; pl. 56, f. 4; pl. 59, f. 10-12

1927 Teredo (Teredothyra) radcliffei BARTSCH, USNMB, 11 (2), 542, pl. 53, f. 11, 14; pl. 56, f. 5 ; pl. 59, f. 8,9

1945 Kuphus (Idioteredo) smithi kiiensis Iw. TAKI \& HABE, Venus, 14, 115

1953 Idioteredo smithi: HABE, GJS, 253, f. 680

Loc. M (bo). [timbers deposited]

Dist. Honshu (Kii Peninsula) to Philippines.

Genus Teredora BARTSCH コチョウフナクイムシ属

1921 Teredora BARTSCH, PBSW, 34, 26

1847 Malleolus Gray, PZSL, 15, 188 (non RAFINesque, 1815). Type (OD): Teredo malleolus TURTON

Teredora princesae (SIVICKIS) コチョウフナクイムシ（滝・波部）

1928 Teredo princesae Sivickis, Philippine., 37 (3), 291, pl. 2, f. 11 
1931 Teredo diederichsen Rocн, Hamburg, 44, 6, pl. 1, f. 2

1931 Teredo spaerki Roch, AfZ, 22 (13), 15, pl. 2, f. 4

1938 Teredo gregoryi DALL, BARTSCH \& ReHDER, BPBMB, 153, 212, pl. 55, f. 1-5

1952 Teredo aurita HABE, GJS, 253, f. 663, 664, 678, 679 (non HedLEY, 1899)

1961 Teredo aurita: HABE, CISJ, 2, 141, pl. 63, f. 18

Loc. S (bo) [coconut]

Dist. Honshu (north to Bōsō Peninsula) to Philippines.

\section{Genus Uperotus GUETTARDボロウチワフナクイムシ属}

1770 Uperotus GuetTard, Mem. Diff. Sci., 3, 126

1936 Glumebra Iredale, Destr. Timber. Mar. Organisms, 12, 42. Type (SD Lamy, 1927):

Teredo clava GMELIN

Uperotus clavus (GMELIN)ボロウチワフナクイムシ（波部）

1791 Teredo clava GmeLin, Syst. Nat., 13, 3748

1936 Glumebra elegans IREDALE, Destr. Timber Mar. Organisms, 12,

1953 Glumebra shionomisakiensis HABE, Venus, 17, 132, 140, f. 13-15

Loc. $\mathrm{S}$ (bo) [coconut]

Dist. Honshu (Kii Peninsula). Indo-Pacific.

\section{Subfamily Bankiinae TURNER オオフナクイムシ亜科}

\section{Genus Bankia GRAYオオフナクイムシ属}

1842 Bankia Gray, Syn. 44, 76

1847 Xylotrya GraY, PZSL, 188. Type (SD GRAY, 1847): Teredo bipalmulata LAMARCK

Subgenus Bankiaオオフナクイムシ亜属

Bankia (Bankia) bipalmulata (Lamarck) オオフナクイムシ（滝・波部）

1801 Teredo bipalmulata LAMARCK, AsV, 6, 129

1928 Bankia rubra SIvICKIs, Philippine., 37, 288, pl. 1, f. 6

Loc. M (bo) [t!mbers]

Dist. Honshu (north to Bōsō Peninsula) to Kyushu. Indo-Pacific.

Subgenus Lyrodobankia MoLL ヤセオオフナクイムシ属

1941 Lyrodobankia MoLL, SBGNFB, 200

1946 Bankiopsis Clench \& Turner, Johnsonia, 2 (1), 11, 16. Type (SD Turner, 1966):

Nausitora kamiyai $\mathrm{RoCH}$

Bankia (Lyrodobankia) carinata (GRAY) ヤセオオフナクイムシ（澺・波部）

1827 Teredo carinata GraY, Philos. Mag., 2 (n.s.), 411

1887 Teredo carinata: SowerBy, Thes., 5, , pl. 469, f. 15

1928 Bankia oryzaformis Sivicks, Philippine., 37, 286, pl. 1, f. 2

1929 Nausitora orientalis Roch, Hamburg, 44, 17, pl. 2, f. 15

1929 Nausitora kamiyai RoTH, Hamburg, 44, 17, pl. 2, f. 16

1929 Bankia kuronuii RocH, Hamburg, 44, 19, pl. 2, f. 10

1931 Bankia nakazawai Kuronuma, Venus, 2, 296, pl. 8, f. 8: pl. 9, f. 32-34

1952 Bankia (Bankiella) oryzaformis: HABE, GJS, 249, f. 683

Loc. M (bo) [timbers]

Dist. Honshu (north to Bōsō Peninsula) to Kyushu. Indo-Pacific. 
Subfamily Zachsiinae HABE ネムグリガイ黒科

Genus Zachsia BulatofF \& RJABTSCHIKoFFネムグリガイ属

1933 Zachsia Bulatoff \& RJabtschikoff, Zool. Anz., 104, 165. Type (SD Habe, 1952):

Zachsia zenkewitschi BULATOFF \& RJABTSCHIKOFF

Zachsia zenkewitschi BULATOFF \& RJABTSCHIKOFFネムグリガイ（滝・波部）

1933 Zachsia zenkewitschi BulatofF \& RJABtschIKoff, Zool. Anz., 104, 165, f. 1-5, 7-10, 12

1933 Zachsia lignaui Bulatoff \& RJABTschikoff, Zool. Anz., 104, 165, f. 6

1977 Zachsia zenkewitschi: HABE, SMJ B/S, 301, pl. 63, f. 21

Loc. M (bo). [Zostera]

Dist. Kyushu to Honshu and Siberia.

\section{Subclass Anomalodesmacea DALL 異勒带亜綱}

\section{Order Pholadomyoida NEWELLウミタケガイモドキ目}

\section{Superfamily Pholadomyacea GRAY ウミタケガイモドキ超科}

Family Pholadomyidae GRAY ウミタケガイモドキ科

Genus Pholadomya SowERBY ウミタケガイモドキ属

1823 Pholadomya Sowerby, Gen. Rec. Fossil Shells, 1, pl. 19. Type (SD Gray, 1847): Pholadomya candida SOWERBY

Pholadomya pacifica DALLウミタケガイモドキ（黒田）

1906 Pholadomya japonica Dall, Naut., 22, 115, 142

1907 Pholadomya pacifica DALL, SMC, 50 (2), 172

1925 Pholadomya pacifica: Dall, PUSNM, 66 (17), 24, pl, 29, f. 8, 9

1961 Pholadomya pacifica: HABE, CISJ, 2, 143, pl. 64, f. 13

1971 Pholadomya pacifica: Kuroda \& HaBe, SSSB, 718 (J) 473 (E), pl. 121, f. 17

Loc. S (s-m) tr.

Dist. Southern Hokkaido to Shikoku.

\section{Genus Nipponopanacca HABEッギノウミタケガイモドキ属}

1977 Nipponopanacca HABE, SMJ B/S. 303. Type (OD): Pholadomya (Panacca) sakuraii HABE

*Nipponopanacca sakuraii (HABE)ツギノウミタケガイモドキ（波部）

1958 Pholadomya (Panacca) sakuraii HABE, Venus, 20, 179, f. 4

1977 Nipponopanacca sakuraii: HABE, SMJ B/S, 303, f. 3

Loc. S (s-m) tr.

Dist. Honshu (Kii Peninsula). 


\section{Superfamily Pandoracea RAFINESQUEーネリガイ超科}

\section{Family Lyonesiidae FISCHER サザナミガイ科}

\section{Genus Lyonsia TURTONサザナミガイ属}

1822 Lyonsia Turton, Conch. Inst. Brit. Dithyra, 35. Type (M): Mya striata MonTAgu $=$ Mya norvegica GMELIN

\section{Lyonsia ventricosa GoULDサザナミガイ（目八）}

1861 Lyonsia ventricosa GouLd, PBSNH, 8, 23

1874 Lyonsia ventricosa: LISCHKE, JMC, 3, 103, pl. 9, f. 13

1882 Lynosia praetenuis DUNKER, Index, 180, pl. 7, f. 13

1861 Lyonsia ventricosa: HABE, CISJ, 2, 142, p1. 64, f. 1

1971 Lyonsia ventricosa: KuRODA \& HABE, SSSB, 723 (J) 477 (E), pl. 103, f. 18

Loc. N M S (s) dp.

Dist. Southern Hokkaido to Kyushu and Maritime Prov. of Siberia.

\section{Lyonsia kawamurai HABEカワムラサザナミガイ（波部）}

1952 Lyonsia kawamurai HABE, ICJS, 1 (21), 154, pl. 22, f. 1

1964 Lyonsia kawamurai: HABE, CISJ, 2, 142, pl. 64, f. 6

1971 Lyonsia kawamurai: Kuroda \& HABE, SSSB, 723(J) 477 (E), pl. 103, f. 19

Loc. S (s) dp.

Dist. Honshu (north to Bōsō Peninsula) to Kyushu.

Remarks: This species seems to be a form of Lyonsia ventricosa Gould.

*Lyonsia tanakai HABE sp. nov. シンテイサザナミガイ（波部）

Pl. 3, fig. 2.

Shell thin and fragile, pearly, pale yellowish white, translucent, transversely inaequilateral, elongated ovale in shape, rounded at the anterior margin, and gently curved at the ventral margin. Left valve a little larger and more convex than the right valve. Umbo a little anterior from the middle of dorsal margin. Surface with coarse radiating lines of about 40 in number. Periostracum on the surface light brown, often with agglutinated sand grains along the radial lines at the marginal area. Ligament internal and behind the umbo, with a supporting white lithidesma. Hinge without teeth. Interior of shell glassy and pearly with the external ribbing showing throught. Pallial sinus deep, extending beyond the umbo.

Length $21.2 \mathrm{~mm}$, height $13.5 \mathrm{~mm}$, breadth $9.7 \mathrm{~mm}$. (Holotype specimen preserved in the National Science Museum NASMT-Mo 56851)

Length $18.2 \mathrm{~mm}$, height $11.8 \mathrm{~mm}$, breadth $8.8 \mathrm{~mm}$. (Paratype specimen preserved in the National Science Museum, NSMT-Mo 55405)

Type Locality: Off Minabe, Wakayama Pref., Honshu at about $200 \mathrm{~m}$ deep

Distribution: Honshu (Enshu-Nada to off Kii Peninsula) S (s) dp.

Remarks: Lyonsia kawamurai HABE is a closely allied species to this new species, but has apparently densely set radial lines on the subquadrate oval shell. This species is named in honor of Mr. Tsutomu Tanaka of Uchita-cho, Naga County who collected this deep sea Lyonsia for the writer's disposal. 


\section{Genus Allogramma DALL リュゥグウオビクイガイ属}

1903 Allogramma DALL, TWFIP, 3 (6), 1514. Type (OD): Lyonsia formosa JeFFREYS

Allogramma oahuensis DALLリュウグウオビクイガイ（波部）Ｐl. 8, f. 5.

1913 Lyonsia (Allogramma) oahuensis DALL, PUSNM, 45 (2002), 594

1939 Allogramma oahuensis DALI, BARTSCH \& REHDER, BPBMB. 153, 215, pl. 56, f. 9-12

1962 Lyonsia (Allogramma) oahuensis: OKUTANI, BTRFRL, 32, pl. 3, f. 4

Loc. S (m) tr.

Dist. Honshu (Izu Islands to off Kii Peninsula) and Hawaii.

\section{Genus Bentholyonsia HABEワタゾコオビクイガイ属（波部）}

1952 Bentholyonsia HABE, GJS, 3, 257. Type (M): Allogramma (Bentholyonsisa) teramachii HABE

Bentholyonsia teramachii (HABE)ワタゾコオビクイガイ（波部）

1952 Allogramma (Bentholyonsia) teramachii $\mathrm{HABE}, \mathrm{GJS}, 3,257$

1961 Allogramma (Bentholyonsia) teramachii: HABE, CISJ, 2, 142, pl. 64, f. 3

1971 Bentholyonsia teramachii: KuRODA \& HABE, SSSB, 725 (J) 478 (E), pl. 121, f. 18

Loc. S (s) tr.

Dist. Honshu (north to Bōsō Peninsula) to Shikoku.

Genus Agriodesma DALLオビクイガイ属

1909 Agriodesma DALL, PUSNM, 37, 284. Type (OD): Lyonsia saxicola BATRD

Agriodesma navicula (ADAMS \& REEVE)オビクイガイ (丹浦)

1850 Lyonsia navicula ADAMS \& REEvE, Samarang, 83, pl. 23, f. 11

1895 Entodesma truncatissima PILsBRY, CMMJ, 137, pl. 3, f. 11, 12

1922 Entodesma (Agriodesma) naviculoides YoKoyAmA, JCSIUT, 44 (1), 170, pl. 6, f. 11

1959 Entodesma navicula: KirA, CISJ, 1, 163, p.1 61, f. 20

1959 Entodesma naviculoides: KIRA, CISJ, 1, 163, pl. 61, f. 21

1971 Agriodesma navicula: KURODA \& HABE, SSSB, 724 (J) 478 (E), pl. 103, f. 17

Loc. N M S (ep) gl.

Dist. Hokkaido to Kyushu and Borneo (?).

\section{Family Pandoridae RAFINESQUEネリガイ科}

\section{Genus Pandorella ConRad ネリガイ属}

1863 Pandorella CONRAD, PANSP (1862), 572.

1864 Kennerlia CARPENTER, PZSL, 602. Type (M): Pandorella arenosa CONRAD

\section{Pandorella otukaki (HABE)ネリガイ（五介）}

1952 Pandora (Kennerlia) otukai HABE, ICJS, 1 (18), 124, pl. 18, f. 11, 12

1961 Pandora (Kennerlia) otukai: HABE, CISJ, 2, 142, pl. 64, f. 6

1971 Pandora (Pandorella) otukai: Kuroda \& HABE, SSSB, 719 (J) 474 (E), p.1 103, f. 21

Loc. N M S (s) dp.

Dist. Honshu (north to Bōsō Peninsula) to Kyushu.

Pandorella pseudobilirata NomuRA \& HATAIウスネリガイ（波部）

1874 Pandora delicatula Sowerby, Icon., sp. 17 (non CARPENTER, 1864)

1940 Pandora (Kennerleyia) pseudobilirata Nomura \& HATAI, SHKMRB, 19, 87, pl. 4, f. 15a, b 
1952 Pandora (Kennerleyia) pseudobilirata: HABE, ICJS, 1 (18), 124, p1. 18, f. 9

1961 Pandora (Kennerlia) pseudobilirata: HABE, CISJ, 2, 142, pl. 64, f. 5

1971 Pandora (Pandorella) pseudobilirata: KURODA \& HABE, SSSB, 720 (J) 474 (E), pl. 103, f. 21

Loc. S (s) tr.

Dist. Honshu (north to Bōsō Peninsula), Shikoku and Kyushu.

Pandorella carinata (PRASHAD)ワタゾコネリガイ（吉良）

1932 Calopodium (Kennerlia) carinatum PRASHAD, Siboga 53c, 323, pl. 7, f. 15-18

1952 Pandora (Kennerlia) nipponica HABE, ICJS, 1 (18), 125, pl. 18, f. 6

1961 Pandora (Kennerlia) nipponica: HABE, CISJ, 2, 142, pl. 64, f. 4

Loc. $\mathrm{S}$ (s) tr.

Dist. Honshu (Kii Peninsula) to Kyushu and Indonesia.

\section{Family Myochamidae BRONN ミッカドカタビラガイ科}

Genus Myadora GRAY ミッカドカタビラガイ属

1840 Myadora Gray, AMNH. (1) 4 (15), 306. Type (OD): Pandora brevis Sowerby

Myadora fluctuosa GoulD ミッカドカタビラガイ（平瀬）

1861 Myadora fluctuosa Gould, PBSNH, 8, 23

1880 Myadora proxima SMrTH, PZSL, 586, pl. 53, f. 8a, b

1882 Myadora triangularis DUNker, Index, 181, pl. 7, f. 11, 12, 17, 18

1950 Myadora japonica HABE, ICJS, 1 (4), 27, pl. 4, f. 4-6

1961 Myadora japonica: HABE, CISJ, 2, 143, pl. 64, f. 9

1961 Myadora fluctuosa: HABE, CISJ, 2, 143, pl. 64, f. 10

1971 Myadora fuctuosa: Kuroda \& HABE, SSSB, 725 (J) 478 (E), pl. 103, f. 11

1971 Myadora japonica: KuRODA \& HABE, SSSB, 726 (J) 479 (E), pl. 103, f. 12

Loc. M S (s) dp.

Dist. Honshu (north to Bōsō Peninsula) to Kyushu and Korea.

Myadora reeveana (SMITH) コカタビラガイ（岩川）

1880 Myadora reeveana SMITH, PZSL, 586, pl. 53, f. 10, 10a

1950 Myadora reeveana: HABE, ICJS, 1 (4), 25, pl. 4, f. 18

1961 Myadora reeveana: HABE, CISJ, 2, 143, pl. 64, f. 11

Loc. M S (s) dp.

Dist. Honshu (Bōsō Peninsula to north limit) to Kyushu and Korea.

Myadora quadrata SMITHテラマチカタビラガイ（波部）Ｐl. 8, f. 3.

1899 Myadora quadrata SMITH. AMNH, (7) 4, 250

1931 Myadora valdiviae THELE \& JACKeL, Tiefsee., 21 (1), 85, pl. 4, f. 121

1950 Myadora termachii HABE, ICJS, 1 (4), 26, pl. 4, f. 14-16

1967 Myadora quadrata: KNUDSEN, John Murray Exped., 11 (3), 276, pl. 2, f. 19, 20

Loc. S, (s) tr.

Dist. Honshu (Kii Peninsula) to Shikoku and Indian Ocean.

Genus Myadoropsis HABEヤマザキスエモノガイ属

1960 Myadoropsis HABE, PSMBL 8 (2), 293. Type (OD): Thracia transmontana YoKoYAMA

Myadoropsis transmontana (YoKOYAMA)ヤマザキスエモノガイ（黒田） Pl. 8, f. 2.

1922 Thracia transmontana YoKoYAMA, JCSIUT, 44 (1), 172, pl. 14, f. 13, 14 
1961 Myadoropsis transmontana: HABE, CISJ, 2, 142, pl. 64, f. 8

1971 Myadoropsis transmontana: KuRODA, \& HABE SSSB, 726 (J) 479 (E), pl. 103, f. 7

Loc. M S (s) dp.

Dist. Honshu (north to Bōsō Peninsula), Shikoku and Kyushu.

Myadoropsis dissimilis HABEヒロヤマザキスエモノガイ（波部）

1960 Myadoropsis dissimilis HABE, PSMBL, 8, (2) p. 293

1961 Myadoropsis dissimilis $\mathrm{HABE}, \mathrm{CISJ}, 2$, p. 142, pl. 64, f. 7

Loc. S (s) dp.

Dist. Honshu (north to Kii Peninsula) to Kyushu,.

\section{Family Periplomatidae DALLリユウグウハゴロモガイ科}

Genus Periploma SCHUMACHER リュウグウハゴロモガイ属

1817 Periploma Schumacher, ENSVT, 115. Type (M): Periploma inaequivalvis SchumaCher - Corbula margaritacea LAMARCK

Periploma otohimeae OzAKIリュゥグウハゴロモガイ（黒田）

1958 Periploma ovata Kuroda \& Horikoshi, Venus, 17, 19, f. (non d'OrbignY, 1846)

1958 Periploma otohimeae OzAKI, BNSM, 4 (1), 120

1961 Periploma otohimeae: HaBe, CISJ, 2, 144, pl. 64, f. 19

1971 Periploma otohimeae: Kuroda \& Habe, SSSB, 722 (J) 476 (E), pl. 103, f. 2

Loc. $M \quad(m)$ tr.

Dist. Honshu (north to Sagami Bay) to Kyushu, East China Sea and Yellow Sea.

\section{Family Laternulidae HEDLEY オキナガイ科 \\ Genus Laternula RöDINGオキナガイ属}

1798 Laternula RöDING, Bolten., 155

1818 Anatina LAMARCK, AsV, 5, 462

1952 Laternulina HABE, GJS, 3, 266. Type (SD GRAY, 1847): Laternula anatina RödING

$=$ Solen anatinus LINNAEUS

\section{Subgenus Laternula RöDINGオキナガイ互属}

Laternula anatina (LinNAEUs)オキナガイ（丹浦）

1758 Solen anatinus LinNaEus, Syst., Nat., 10, 673

1863 Anatina flexuosa ReEve, Icon., sp. 2

1863 Anatina subrostrata: ReEve, Icon., sp. 6

1872 Anatina japonica Lischke, M B L., 19, 107

1874 Anatina japonica: LischKe, JMC, 3, 101, pl. 9, f. 7-10

1959 Laternula (Laternulina) flexuosa: KIRA, CISJ, 1, 166, pl. 62, f. 11

1971 Laternula (Laternula) flexuosa: KURODA \& HABE, SSSB, 722 (J) 476 (E), pl. 103, f. 5

Loc. N M S (s-m) dp.

Dist. Honshu (north to Bōsō Peninsula) to Kyushu. Indo-Pacific region.

Laternula boschasina (REEVE)コオキナガイ（岩川）

1863 Anatina boschasiana ReEve, Icon., sp. 13

1901 Anatina impura PILSBRY, PANSP, 53, 208, 402, pl. 19, f. 9

1961 Laternula (Laternulina) impura: HABE, CISJ, 2, 144, pl. 64, f. 21

1971 Laternula (Laternulina) impura: KURODA \& HABE, SSSB, 721 (J) 475 (E), pl. 103, f. 1 
Loc. N M S (s-m) int.

Dist. Honshu (north to Bōsō Peninsula) to Kyushu. Indo-West Pacific region.

\section{Subgenus Exolaternula HABE ソトオリガイ雨属}

1977 Exolaternula HaBe, SMJ B/S, 286. Type (OD): Anatina limicola ReEve

Laternula (Exolaternula) navicula (REEVE) ソトオリガイ（丹浦）

1863 Anatina navicula ReEve, Icon., sp. 11

1863 Anatina limicola ReEve, Icon., sp. 27

1895 Anatina kamakurana PILSBRY, CMMJ, 138, pl. 3, f. 2, 3

1928 Anatina peichiliensis Grabau \& KING, Peitaiho, (2), 193, pl. 7, f. 58

1959 Laternula limicola: KIRA, CISJ, 1, 166, pl. 62, f. 12

1971 Laternula limicola: KuRoDA \& HABE, SSSB, 721 (J) 475 (E), pl. 103, f. 4

Loc. N M S (s-m) dp.

Dist. Saghalin to Kyushu. Indo-Pacific region.

\section{Family Thracidae STOLICZKA スエモノガイ科}

Genus Cyathodonta CoNRADスナゴスエモノガイ属

1849 Cyathodonta CONRAD, PANSP, 4, 155. Type (M): Cyathodonta undulata CONRAD

Cyathodonta granulosa (ADAMS \& REEVE)スナゴスエモノガイ（黒田）Ｐl. 8, f. 1 .

1850 Thracia granulosa ADAMs \& ReEve, Samarang, 82, pl. 23, f. 16

1961 Cyathodonta granulosa: HABE, CISJ, 2, 143, pl. 64, f. 16

1971 Cyathodonta granulosa: Kuroda \& HABE, SSSB, 727 (J) 480 (E), pl. 103, f. 3

Loc. N M S (s-m) dp.

Dist. Honshu (north to Bōsō Peninsula) to Kyushu and East China Sea.

\section{Genus Eximiothracia IREDALEシナヤカスエモノガイ属}

1924 Eximiothracia Iredale, PLSNSW, 49 (3), 199. Type (OD): Thracia speciosa AnGas

Eximiothracia concinna (GouLD) シナヤカスエモノガイ（黒田）

1861 Thracia concinna Gould, PBSNH, 8, 23

1961 Cyathodonta (Eximiothracia) concinna HABE, CISJ, 2, 143, p1. 64, f. 14

1971 Eximiothracia concinna KURODA \& HABE, SSSB, 727 (J) 480 (E), pl. 103, f. 6

Loc. $\mathrm{N}$ (s) int.

Dist. Honshu (north to Bōsō Peninsula) to Kyushu.

*Eximiothracia koyamai HABE sp. nov. コヤマスエモ/ガイ（波部）

Pl. 3 fig. 4.

Shell small, thin and fragile, white to light yellow, elongated ovate in shape with the rounded anterior margin and widely truncated and slightly gaping posterior margin, rather compressed, inequivalve, right valve a little convex than the left, and inequilateral, the anterior dorsal margin weakly curved and longer than the straight posterior. Umbo low and somewhat posterior from the middle of dorsal margin. Surface with densely and minutely granulated all over, especially on the postero-dorsal area, and an obtuse ridge running from the umbo to the postero-ventral corner. Ligament small, external and just behind the umbo. Resilium on the white small chondrophore immediately behind the umbo. 
Pallial sinus deep extending nearly to the middle of the shell length.

Length $23.3 \mathrm{~mm}$, height $13.0 \mathrm{~mm}$ and breadth $4.0 \mathrm{~mm}$. (Holotype right valves preserved in the National Science Museum, NSMT-Mo 58970)

Length $22.7 \mathrm{~mm}$, height $14.2 \mathrm{~mm}$ and breadth $4.0 \mathrm{~mm}$. (Paratype left valve from the Sagami Bay, Honshu, NSMT-Mo 58971)

Type Locality: off Kushimoto, Kii Peninsula, Honshu at about $50 \mathrm{~m}$.

$\mathrm{S}$ (s) dp.

Distribution: off Kii Peninsula to Sagami Bay, Honshu.

Remarks: Exmiothracia concinna GouLD, 1861 is a closely related species to this new species, but has the white and thin shell without an obtuse ridge from the umbo to the postero-ventral corner, and with the umbo a little posterior from the middle of the dorsal margin and the rather short posterior truncation.

\section{Genus Trigonothracia YaMaMoto \& HaBE ノムラスエモノガイ属}

1959 Trigonothracia Yамамото \& HaBe, BMBSA, 9 (3), 117. Type (OD): Thracia (Trigonothracia) nomurai YАMАMOTO \& HАBЕ

Trigonothracia pusilla (GouLD) /ムラスエモノガイ(山本・波部)

1861 Thracia pusilla GoulD, PBSNH, 8, 23

1959 Thracia (Trigonothracia) nomurai Yамамото \& HABE, BMBSA, 9 (3), 117, pl. 12, f. 9, 10

1961 Thracia (Trigonothracia) pusilla: HABE, CISJ, 2, 143, pl. 64, f. 15

1961 Thracia pusilla: JoHnson, USNMB, 239, 136, pl. 26, f. 7

Loc. S (s) dp.

Dist. Honshu (north to Mutsu Bay) to Kyushu.

Genus Ixartia GRAY ママツカゼスエモノガイ属

1852 Ixartia Gray, Syn., 272. Type (M): Mya distorta MontaGU

Ixartia cuneolus (REEV) マッカゼスエモノガイ（黒田）

1861 Thracia cuneola ReEve, Icon., sp. 2

Loc. $\mathrm{N}$ (s) dp.

Dist. Honshu (Kii Peninsula) and Philippines.

Genus Thracidora IREDALEワタゾコスエモノガイ属

1924 Thracidora Iredale, PLSNSW, 49 (3), 200. Type (OD): Thraciopsis arenosa Hedley

Thracidora japonica HABE ワタゾコスエモノガイ（波部）

1952 Thracidora japonica HABE, GJS, 3, 264, f. 702-704 (name and figures only)

1961 Thracidora japonica: HABE, CISJ, 2, 144, pl. 64, f. 18

Loc. $S$ (s) tr.

Dist. Honshu (north to Sagami Bay) and Shikoku.

Superfamily Clavagellacea D’ORBIGNY 八マユウガイ超科

Family Clavagellidae D’ORBIGNY ハマユウガイ科

Genus Nipponoclava SMITH ッッガキ属

1976 Nipponoclava SMITH, JMSA, 3, 197. Type (OD): Aspergillum giganteum Sowerby 
Nipponoclava gigantea (SOWERBY) ツッガキ(六介)

1888 Aspergillum giganteum SOWERBY, PZSL, 290

1895 Aspergillum giganteum: PILSBRY, CMMJ, 136, pl. 3, f. 1

1926 Aspergillum giganteum: YokoYama, JFSIUT, (2) 1 (9), 368, pl. 42, f. 1, 2

1954 Brechites yokoyamai SHIKAMA. SRYNU, (2) 3, 63, pl. 3, f. 1a-d

1959 Penicillus (Warnea) giganteus: KIRA CISJ, 1, 168, pl. 62, f. 25

1976 Nipponoclava giganta: Smirh, JMSA, 3, 197, f. 16-17

Loc. M S (s) gl.

Dist. Honshu (north to Bōsō Peninsula) to Kyushu.

\section{Genus Bryopa GRAYサンゴッツガキ属}

1847 Bryopa Gray, PZSL, 15, 188. Type (OD): Clavagella aperta SOWERBY

Bryopa lata (BRODERIP) サンゴッッガキ（仲嶺・波部）

1834 Clavagella lata BRoDerIP, PZSL, 2, 116.

1835 Clavagella elongata BroderiP, Trans. Zool. Soc. London, 1, 265. pl. 35, f. 1-4.

1834 Clavagella lata: Broderip, Tran. Zool. Soc. London, 1, 265, pl. 30, f. 8-16.

1873 Clavagella lata: ReEve, Icon. sp. 3.

1873 Clavagella elongata: ReEve, Icon, sp. 7.

1974 Clavagella (Bryopa) lata: AppukuTTAN, JMSA, 3, 19, pl. 4.

1976 Clavagella (Bryopa) lata: SMrTH, JMSA, 3, 192, pl. f. 8.

1980 Bryopa lata: NAKAMINE \& HABE, Chiribotan, 11, 39, pl. 4, f. 2, 3.

Loc. M (s-m) gl.

Dist. Ryukyu Islands and Kii Peninsula, Honshu. S. India, Philippines.

\section{Genus Clavagella LAMARCKクビタテツツガキ属（新称）}

1818 Clavagella LAmARCK, AsV, 430, Type (SD. Children, 1923): Clavagella echinata LAmARCK

*Clavagella japonica HABE sp. nov. クビタテッツガキ（波部）

Pl. 3, fig. 3.

Right valve free, small, thin and fragile, roundly ovate and with growth lines and its interior smooth and polished and pearly. Left valve fused on the inside of the swollen anterior portion of tube. Mantle line with a deep posterior sinus. No cardinal teeth on the hinge plate and ligament small, external and situates behind the anteriorly placed umbo.

Tube rather long, calcareous, white, thick and solid, and slightly compressed laterally in section owing to the weak dorsal and ventral ridges. Tube attaches by the anterior end with the small siphonal tubules to the substrata and stands up from the substrata vertically and is usually covered by the fouling organisms.

Free right valve: length $10.5 \mathrm{~mm}$, height $9.2 \mathrm{~mm}$ and breadth $1.2 \mathrm{~mm}$ (type specimen preserved in the National Science Museum, NSTM-Mo 58900)

Attached left valve: length $10.2 \mathrm{~mm}$ and height $9.8 \mathrm{~mm}$ (type specimen) Tube: length $26.0 \mathrm{~mm}$. diam. of the anterior portion: dorso-ventral $10.3 \mathrm{~mm}$ and lateral $10.0 \mathrm{~mm}$, diam. of the posterior portion: dorso-ventral $8.0 \mathrm{~mm}$ and lateral $6.1 \mathrm{~mm}$. (type specimen)

Tube: length $29.5 \mathrm{~mm}$, diam. of the anterior portion: dorso-ventral 12.7 $\mathrm{mm}$ and breadth $10.3 \mathrm{~mm}$, diam. of the posterior portion: dorso-ventral $6.7 \mathrm{~mm}$ and lateral $5.7 \mathrm{~mm}$ (paratype specimen no. 1. NSMT-Mo 58901)

Tube: length $27.4 \mathrm{~mm}$. diam. of the anterior portion: dorso-ventral 14.3 
$\mathrm{mm}$ and breadth $8.6 \mathrm{~mm}$, diam. of the posterior portion: dorso-ventral $6.8 \mathrm{~mm}$ and lateral $5.9 \mathrm{~mm}$ (paratype specimen no. 2. NSMT-Mo 58902)

Type locality: Tsuga Koza-chō, Wakayama Pref., Honshu at 40-50m deep S (s-m) gl.

Remarks: This new species is an ally to Clavagella torresi Smith, 1885 from the northern Queensland, Australia, but has the smaller shell than the latter and very weak longitudinal ridges on the tube.

\section{Genus Stirpuliniola KuRODA \& HABE ハマユウガイ属}

1971 Stirpuliniola Kuroda \& Habe, SSSB, 729 (J) 481 (E). Type (OD): Clavagella ramosa DUNKER

*Stirpuliniola ramosa (DUNKER)ハマユウガイ（丹浦）

1882. Clavagella ramosa DUNKER, Index, 172, pl. 16, f. 1, 2

1959 Clavagella (Stirpulina) ramosa: KrRA,CISJ, 1, 168, pl. 62, f. 24

1971 Stirpuliniola ramosa: Kuroda \& HABE, SSSB, 729 (J) 481 (E), pl. 103, f. 20

Loc. M S (s) gl.

Dist. Honshu (north to Bōsō Peninsula) to Kyushu.

\section{Superfamily Poromyacea DALL スナメガイ超科}

\section{Family Verticordiidae STOLICZKA オトヒメゴコロガイ科 \\ Genus Verticordia SoWERBYオトヒメゴコロガイ属}

1844 Verticordia SOWERBY, MCGB, 7, 67. Type (M): Verticordia cardiformis Sowerby

$$
\text { Subgenus Spinosipella IREDALEウズマキゴコロガイ互属 }
$$

1930 Spinosipella IredAle, RAM, 17 (9) 388. Type (OD): Verticordia ericia HedLeY

Verticordia (Spinosipella) deshayesiana (FISCHER) ウズマキゴコロガイ（黒田）

1862 Verticordia deshayesiana Fischer, J. de C., 10, 35, pl. 5, f. 10, 11

1862 Verticordia japonica A. ADAMS, AMNH, (3) 9, 224

1952 Verticordia (Spinosipella) japonica: Kuroda, Venus, 17, 10, pl. 1, f. 10, 11

1959 Vericordia (Spinosipella) japonica: HABE, CISJ, 2, 145, pl. 65, f. 7

1965 Spinosipella japonica: Kuroda \& HaBE, NIEFJ, 2, 305, f. 1159

1971 Spinosipella deshayesiana: Kuroda \& HaBE, SSSB, 731 (J) 482 (E), pl. 103, f. 26

Loc. M S (s) dp.

Dist. Honshu (north to Bōsō Peninsula) to Kyushu and southern part of Japan

Sea. Indonesia and Hawaii.

\section{Genus Haliris DALLウネシゲゴコロガイ属}

1886 Haliris DALl, BMCZ, 12, 287. Type (OD): Verticordia fischeriana DALL

Haliris multicostatus (A. ADAMS) ウネシゲゴコロガイ（黒田）

1862 Verticordia multicostata A. ADAMs, AMNH, (3) 9, 224

1952 Verticordia (Haliris) multicostata: KuRODA, Venus, 17, 10, p1. 1, f. 4, 5

1961 Haliris multicostatus: HaBe, Dr. MakiYama's Mem. Vol., 193, pl. 1, f. 13; p1. 2, f. 19, 20

1971 Haliris multicostatus: KURODA \& HABE, SSSB, 731 (J) 482 (E), pl. 121, f. 21

Loc.

Dist. Honshu (north to Bōsō Peninsula) to Kyushu. 


\section{Subgenus Setaliris IREDALEヒシオトヒメゴコロガイ車属}

1930 Setaliris IREDALE, RAM, 17, 388. Type (OD): Verticordia setosa HeLDEY

Haliris (Setaliris) pygmaea KURODAヒナノオトヒメゴコロガイ（黒田）

1952 Verticordia (Heliris) pygmaea KuRoDA, Venus, 17 (1), 10, pl. 1, f. 6, 7

1952 Verticordia (Heliris) makiyamai HABE in Kuroda, Venus, 17 (1), 10, pl. 1, f. 8,9

Loc. $\mathrm{S}$ (s) dp.

Dist. Honshu (Kii Peninsula) and Shikoku.

\section{Genus Policordia DALL, BARTSCH \& REHDER ムスメゴコロガイ渭}

1938 Policordia Dall, Bartsch \& Rehder, BPBMB, 153, 219. Type (OD): Policordia diomedea DALL, BARTSCH \& REHDER

Policordia pilula (PRASHAD) ムスメゴコロガイ（東)

1932 Lyonsiella pilula Prashad, Siboga, 53c, 325, pl. 7, f. 23, 24

1961 Lyonsiella japonica HABE, CISJ, 2, 145, App., 41, pl. 65, f. 6 (キゴコロガイ 波部)

1962 Lyonsiella media OKUTANI, BTRFRL, 32, pl. 3, f. 9; pl. 4, f. 3 (マメキゴコロガイ 奥谷)

Loc. $\mathrm{S}$ (s) tr.

Dist. Honshu (north to Izu Islands) to Indonesia.

Genus Euciroa DALL ギンスナゴガイ属

1881 Euciroa DALL, BMCZ, 9, 106. Type (OD): Verticordia elegantissima DALL

Euciroa crassa THIELE \& JAECKEL ギンスナゴガイ（吉良）

1931 Euciroa crassa THIELE \& JAECKeL, Tiefsee 21, 248, pl. 5, f. 130

1952 Euciroa cistagemma KuRODA, Venus, 17, 14, pl. 1, f. 16-18

1961 Euciroa cistagemma: HABE, CISJ, 2, 145, pl. 65, f. 8

Loc. S (s) tr.

Dist. Honshu (north to Kii Peninsula) to Shikoku. Indian Ocean 183-1463 m deep.

Genus Acreuciroa THIELE \& JAECKELオナガキンスナゴガイ属

1933 Acreuciroa THIELE \& J J $\mathrm{J}_{\mathrm{AECKEL}}$, Thiefsee 21, 250. Type (OD): Euciroa (Acreuciroa) rostrata THIELE \& JAECKEL

Acreuciroa rostrata (THIELE \& JAECKEL)オナガギンスナゴガイ（声良）

1931 Euciroa (Acreuciroa) rostrata THIELE \& JACKEL, Tiefsee 21, 249, pl. 5, f. 132

1952 Euciroa (Acreuciroa) teramachii KuRoDA, Venus, 17, 15, pl. 1, f. 19-20

1959 Euciroa (Acreuciroa) teramachii: KIRA, CISJ, 1, 165, p1. 62, f. 9

Loc. $\mathrm{S}$ (s) tr.

Dist. Honshu (Kii Peninsula) to Indonesia.

\section{Family Poromyidae D $\mathbf{D}_{\mathrm{ALL}}$ スナメガイ科 \\ Genus Poromya FoRBES スナメガイ属}

1844. Poromya Forbes, RBAAS, (1843), 191. Type (M): Poromya anatinoides ForBes= Corbula granulata NYST \& WESTENDROP 


\section{Polomya flexuosa YoKoYamaスナメガイ（黒田）}

1922 Poromya flexuosa Yoкоуама, JCSIUT, 44 (1), 173, pl. 14, f. 15, 16

1959 Poromya flexuosa: KiRA, CISJ, 1, 165, pl. 62, f. 7

1965 Poromya flexuosa: HABE, NIEFJ, 2, 305, f. 1160

1971 Poromya flexuosa: KunOda \& HABE, SSSB, 732 (J) 483 (E), pl. 103, f. 27, 28

Loc. M S (s) dp.

Dist. Honshu (north to Bōsō Peninsula) to Kyushu.

Poromya hayashii HABEヨコナガスナメガイ（波部）

1958 Poromya hayashii $\mathrm{HABE}$, Venus, 29 (2), 175, 180

1961 Poromya hayashii: HABE, CISJ, 2, 144, pl. 65, f. 1

Loc. S (s) dp.

Dist. Honshu (north to Enshu-nada) and Kyushu.

\section{Genus Cetoconcha DALLビイドロスナメガイ属}

1886 Cetoconcha Dall, BMCZ, 12, 280. Type (OD): Lyonsia bulla DaLL

\section{Cetoconcha japonica HABE ，ダイオウスナメガイ（波部）}

1952 Cetoconcha japonica HABE, ICJS, 1 (21), 159, pl. 22, f. 2-4

1961 Cetoconcha japonica: HABE, CISJ, 2, 145, pl. 64, f. 4

1971 Cetoconcha japonica: KURODA \& HABE, SSSB, 733 (J) 483 (E), pl. 103, f. 24

Loc. $\mathrm{S}$ (s) dp.

Dist. Hokkaido to Shikoku.

Cetoconcha eximia (PELSENEER)ビイドロスナメガイ（黒田）

1911 Poromya eximia PelseneEr, Siboga, 53a, 78, pl. 26, f. 3, 4

1932 Poromya (Cetoconcha) eximia: PRASHAD, Siboga, 53c, 327, pl. 7, f. 31,32

1952 Cetoconcha eximia intermedia ICJS, 158, pl. 21, f. 18, 19

1959 Cetoconcha eximia intermedia: KTRA, CISJ 1, 165, pl. 62, f. 8

1971 Cetoconcha eximia intermedia: KURODA \& HABE, SSSB, 733 (J) 483 (E), pl. 103, f. 25

Loc. S (s) tr.

Dist. Honshu (north to Bōsō Peninsula) to Indonesia.

\section{Family Cuspidariidae DALL シャクシガイ科 \\ Genus Cuspidaria NARDo シャクシガイ属}

1840 Cuspidaria NARdo, Rev. Zool., 30: Atti Rium. Sci. Ital., 1 (1839), 202

1834 Neaera Gray, in Griffith \& Pidgron, Cuvier's Anim. Kingdom, 12, pl. 22 (non

Robineau-Desvomy, 1830). Type (M): Cuspidaria types Nardo, 1840=Tellina cuspidata OLrvi, 1792

Cuspidaria chinensis (GRIFFITH \& PIDGEON)サザナミシャクシガイ（吉良）

1834 Neaera chinensis Griffith \& Pidgeon, Cuvier's Anim. Kingdom, 12, pl. 2, f. 5

1961 Cuspidaria chinensis: HABE, CISJ, 2, 146, pl. 65, f. 18

Loc. N M (s-m) tr.

Dist. Honshu (north to Bōsō Peninsula) to East China Sea.

Cuspidaria hindsiana (A. ADAMs) ツギノシャクシガイ（黒田）

1864 Neaera hindsiana A. ADAMs, AMNH, (3) 13, 207

1948 Neaera hindsiana: KurodA, Venus, 15, 8, pl. 2, f. 19 
1959 Cuspidaria hindsiana: KIRA, CISJ, 1, 165, pl. 62, f. 4

1961 Cuspidaria hindsiana kagoshimensis HABE, CISJ 2, 146, App. 41, pl. 65, f. 14

1971 Cuspidaria hindsiana: Kuroda \& HABE, SSSB, 734 (J) 485 (E), pl. 103, f. 15

Loc. $\mathrm{M}$ (s-m) tr.

Dist. Honshu (north to Bōsō Peninsula and Noto Peninsula) to Kyushu.

\section{Cuspidaria steindachneri STURANY シャクシガイ (平瀬)}

1901 Cuspidaria steindachneri STURANY, DAWW, 69, 26, pl. 1, f. 5-9

1948 Cuspidaria hirasei KurodA, Venus, 15, 10, pl. 6, f. 3

1959 Cuspidaria hirasei: KIRA, CISJ, 1, 165, pl. 62, f. 5

Loc. $\mathrm{S}$ (s-m) dp.

Dist. Honshu (Kii Peninsula) to Kyushu.

Cuspidaria nobilis (A. ADAMs) オオシャクシガイ（平瀬)

1864 Neaera nobilis A. ADAMs, AMNH, (3) 15, 207

1959 Cuspidaria nobilis: KIRA, CISJ, 1, 165, pl. 62, f. 6

1961 Cuspidaria consimilis HABE, CISJ, 2, 146, App. 42, p. 65, f. 21 (キバシシャクシガイ波部)

1965 Cuspidaria nobilis: KURODA \& HABE, NIEFJ, 2, 306, f. 1164

1971 Cuspidaria nobilis: KuRODA \& HABE, SSSB, 734 (J) 484 (E), pl. 103, f. 24

Loc. M S (s-m) dp.

Dist. Honshu (north to Bōsō Peninsula) to Kyushu and Japan Sea.

Cuspidaria teramachii KURODA テラマチシャクシガイ（黒田）

1948 Cuspidaria termachii KuRODA, Venus, 15, 14, pl. 1, f. 7

Loc. S (s-m) tr.

Dist. Honshu (Wakayama Pref.) and Shikoku.

Cuspidaria nasuta (A. ADAMs)ホソエシャクシガイ（吉良）テングシャクシガイ（黒田）

1864 Neaera nasuta A. ADAMS, AMNH, (3) 13, 207

1948 Neaera nasuta: KurodA, Venus, 15, 13, pl. 2, f. 18

Loc. $\mathrm{M}$ (s-m) tr.

Dist. Honshu (Wakayama Pref.), Shikoku and Kyushu.

Cuspidaria dautzenbergi PRASHAD ハトムネシャクシガイ（波部）

1932 Myonera dautzenbergi Prashad, Siboga, 53c, 334, pl. 7, f. 51

1968 Cuspidaria (Myonera) dautzenbergi: OKUTANI, BTRFRL, 56, 19, pl. 1, f. 7

1976 Myonera dautzenbergi: OKutanr, Chiribotan, 9 (3), 44, pl. 5, f. 4

Loc. $\mathrm{S}(\mathrm{m})$ tr.

Dist. Honshu (north to Sagami Bay) and Indonesia.

Cuspidaria macrorhynchus SMITH エナガシャクシガイ（吉良）

1895 Cuspidaria macrorhynchus SMITH, AMNH, (6) 16, 12, pl. 2, f. 5, 5a

1940 Cuspidaria suganumai Nomura, ROWJ, 12, 101, pl. 2, f. 13a,)b

1948 Cuspidaria suganumai: KurodA, Venus, 15, 12, pl. 1, f. 1

1959 Cuspidaria suganumai: KIRA, CISJ, 1, 164, pl. 62, f. 2

1965 Cuspidaria suganumai: KuRodA \& HABE, NIEFJ, 2, 306, f. 1163

1967 Cuspidaria macrorhynchus :KNUDSEN, John Murray Exped., 11 (3), 315, pl. 3, f. 14, 15, tf. $37 \mathrm{a}, \mathrm{b}$

1971 Cuspidaria suganumai: Kuroda \& HABE, SSSB, 734 (J) 484 (E), pl. 103, f. 10

Loc. M S (s-m) tr.

Dist. Honshu (north to Rikuchū Coast) to Indonesia and Indian Ocean. 
Cuspidaria japonica KURODAタマシャクシガイ（吉良）

1948 Cuspidaria japonica Kuroda, Venus, 15, 14, pl. 1, f. 7

1959 Cuspidaria japonica: KIRA, CISJ, 1, 164, pl. 62, f. 3

Loc. M S (s-m) tr.

Dist. Honshu (Kii Peninsula) and Shikoku.

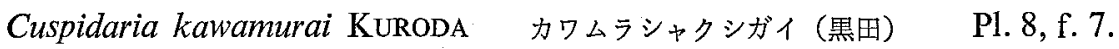

1932 Cuspidaria gigantea Prashad, Siboga, 53c, 329, pl. 7, f. 38 (non Verrill, 1884)

1948 Cuspidaria kawamurai Kuroda, Venus, 15, 11, pl. 1, f. 4

1961 Cuspidaria kawamurai: HABE, CISJ, 2, 145, pl. 65, f. 13

Loc. S (s-m) tr.

Dist. Honshu (Kii Peninsula) to Indonesia.

Genus Leiomya A. ADAMS シャクシガイダマシ属

1864 Leiomya A. AdAms, AMNH (3) 13, 208. Type (M): Neaera adunca Gould

*Leiomya tanabensis (HABE)タナベシャクシガイダマシ（波部改称）Ｐl. 8, f. 6.

[1861 Neaera adunca GouLd, PBSNH, 8, 24]

1960 Plectodon (Pseudoneaera) tanabensis HABE, PSMBL, 8 (2), 288, tf. 18

Loc. $\mathrm{M} \cdot(\mathrm{S}-\mathrm{m}) \mathrm{dp}$.

Dist. Honshu (Wakayama Pref.) and Kyushu (Ariake and Kagoshima Bay).

Genus Pseudoneaera StuRANY シャタシガイモドキ属

1901 Pseudoneaera StUrany, DAWW, 69 (1900), 265. Type (M): Pseudoneaera thaumasia STURANY

Pseudoneaera semipellucida (KURODA)シャクシガイモドキ（黒田）

1948 Cuspidaria (Pelctodon) semipellucida KuRODA, Venus, 15, 24, pl. 2, f. 10, 10a

1948 Cuspidaria (Pseudoneaera) iridella Kuroda, Venus, 15, 25, pl. 2 , f. 9

1961 Plectodon (Pseudoneaera) iridella: HABE, CISJ, 2, 145, pl. 65, f. 9

1971 Pseudoneaera semipellucida: Kuroda \& Habe, SSSB, 737 (J) 484 (E), p. 103, f. 8

Loc. M S (s-m) dp.

Dist. Honshu (north to Bōsō Peninsula) to Kyushu.

\section{Genus Cardiomya A. ADAMS ヒメシャクシガイ属}

1864 Cardiomya A. AdAms, AMNH, (3) 13, 208. Type (M): Neaera gouldiana Hinds

$$
\text { Subgenus Cardiomya A. ADAMs ヒメシャクシガイ亜属 }
$$

Cardiomya (Cardiomya) gouldiana (HINDS) ヒメシャクシガイ (平瀬)

1843 Neaera gouldiana HINDS, PZSL, 11, 77

1948 Neaera gouldiana septentrionalis KURODA, Venus, 15, 18, pl. 2, f. 12

1961 Cardimya gouldiana septentrionalis: HABE, CISJ, 2, 145, pl. 65, f. 10

1965 Cardiomya gouldiana: KURODA \& HABE, NIEFJ, 2, 306, f. 1161

1971 Cardiomya gouldiana septentrionalis: KURODA \& HABE, SSSB, 735 (J) 485 (E), pl. 103, f. 13

1972 Cardiomya lindbergi SCARLATo, Tr. Zool. Inst. 52, 126, f. 14-16.

Loc. M S (s-m) dp.

Dist. Honshu (north to Bōsō Peninsula) and Japan Sea to Indonesia. 
$\begin{array}{ll}\text { Cardiomya (Cardiomya) tosaensis (KuRODA) } & \begin{array}{l}\text { トヒメシャクシガイ (黒田); } \\ \text { サガミヒメシャクシガイ（桜井・奥谷） }\end{array}\end{array}$

1948 Cuspidaria (Cardiomya) tosaensis KuRoda, Venus, 15, 18, pl. 2, f. 13

1964 Cuspidaria (Cardiomya) sagamiensis OKUTANI \& SAKURAI, BNSM, 7, 21, pl. 1, f. 2

1971 Cardiomya sagamiensis: KurodA \& HABE, SSSB, 736 (J) 486 (E), pl. 103, f. 14

Loc. M (s-m) gl.

Dist. Honshu (north to Bōsō Peninsula), Shikoku and Kyushu.

\section{Subgenus Kurodamya OKUTANI \& SAKURAIカイシャクシガイ亜属}

1961 Kurodamya OKutani, \& SAKurai, BNSM, 7, 25, 27. Type (OD): Cuspidaria (Cardiomya) fortisculpta KURODA

Cardiomya (Kurodamya) alcocki (SMITH) カイシャクシガイ（黒田）

1884 Cuspidaria alcocki SMrTH, AMNH, (6) 14, 170, pl. 5, f. 8

1901 Cuspidaria (Cardiomya) potti Sturany, DAWW 69, 264, pl. 1, f. 10-16

1932 Cuspidaria (Cardiomya) persculpta PRASHAD, Siboga, 53c, 332, pl. 7, f. 44

1932 Cuspidaria (Cardiomya) multicarinata PRASHAD, Siboga, 53c, 332, pl. 7, f. 45, 46

1932 Cuspidaria (Cardiomya) semicostata Prashad, Siboga, 53c, 333, pl. 7, f. 47, 48

1948 Cuspidaria (Cardiomya) fortisculpta KuRoda, Venus, 15, 20, pl. 2, f. 16a, b

1965 Cardiomya (Kurodamya) fortisculpta: HABE, NIEFJ, 2, 306, f. 1162

Loc. S (s-m) tr.

Dist. Honshu (Kii Peninsula) to Indonesia and Indian Ocean.

Genus Myonera DALL タテヒダシャクシガイ属

1886 Myonera Dall, BMCZ, 12, 302. Type (OD): Neaera paucistriata DALL

Myonera dautzenbergi PRASHAD タテヒダシャクシガイ（波部） Pl. 8, f. 4. コウモリシャクシガイ（鹿間 non 黒田）

1932 Myonera dautzenbergi Prashad, Siboga, 53c, 334, pl. 7, f. 51

1938 Cuspidaria (Myonera) dispar DALL, BARTSCH \& REHDER, BPBMB, 153, 225, pl. 58, f. 5-7

1962 Cuspidaria (Myonera) dispar: OKUTANI, BTRFRL, 32, 37, pl. 3, f. 11

Loc. S (s-m) tr.

Dist. West coast of Kyushu. Hawaii and Indonesia.

Genus Rengea KURODA \& HABE レンゲシャクシガイ属

1971 Rengea KURODA \& HABE, SSSB, 736 (J) 486 (E). Type (DO): Myonera fluctuosa KURODA

Rengea caduca (SMITH) Vンゲシャクシガイ（吉良)

1894 Cuspidaria caduca SMITH, AMNH, (6) 14, 170, pl. 5, f. 9, 10

1948 Myonera fluctuosa KurodA, Venus, 15, 25, pl. 2, f. 20

1959 Myonera fluctuosa: KIRA, CISJ, 1, 164, pl. 62, f. 1

1971 Rengea fluctuosa: KuRODA \& HABE, SSSB, 736 (J) 486 (E), pl. 103, pf. 9

Loc. S (s-m) tr.

Dist. Honshu (north to Boso Peninsula) to Indonesia. 


\section{Genus Plectodon CARPENTER ヒナノシャクシガイ属}

1864 Plectodon Carpenter, Suppl. Rep. Brit. Assoc. Adv. Sci., 611, 638. Type (OD): Plectodon scaber CARPENTER

\section{Plectodon ligulus (YOKOYAMA) ヒナノシャクシガイ (滝)}

1922 Cuspidaria ligula Yoкочама, JCSIUT, 44 (1), 169, pl. 14, f. 3, 4

1961 Plectodon ligula: HABE, CISJ, 2, 145, pl. 65, f. 11

1971 Plectodon ligulus: KuRODA \& HABE, SSSB, 737 (J) 487 (E), pl. 121, f. 22, 23

Loc. M (s-m) gl.

Dist. Honshu (north to Bōsō Peninsula and Toyama Bay) to Kyushu. 


$$
\begin{gathered}
\text { P L A T E } \\
\text { O F } \\
\text { B I V A L V I A }
\end{gathered}
$$




\section{PLATE 1}

1 Terua crypta DALL, BARTSCH \& REHDER

$$
\text { ホソヒラノマクラガイ（波部）（ナガマユイガイ） } 52.9 \times 13.2 \mathrm{~mm} \quad \text { p. } 52
$$

2 Gloripallium speciosum (REEVE)

$$
\text { オオシマヒオウギガイ（声良） } 45.5 \times 44.7 \mathrm{~mm} \quad \text { p. } 66
$$

3 Anguipecten superbus (SOWERBY)

$$
\text { シュロノハキンチャクガイ (平瀬) } 47.2 \times 48.8 \mathrm{~mm} \quad \text { p. } 69
$$

4 Semipallium fulvicostum (ADAMs \& REEVE)

$$
\text { ツッ゙レノニシキガイ (金丸) } 42.1 \times 48.4 \mathrm{~mm} \text { p. } 65
$$

5 Vasticardium transcendens (Melvill \& STANDEM)

ツッ゙レザルガイ（兴牛・波部）（ヒガノコザルガイ） $21.7 \times 25.6 \mathrm{~mm} \mathrm{p.} 111$

6 Laevicardium rubropictum HABE \& KOSUGE

$$
\text { アカフザルガイ (波部・小望) } 28.7 \times 32.4 \mathrm{~mm} \text { p. } 114
$$

7 Afrocardium infantile (Nomura \& ZINBO)

$$
\text { マルベニバトガイ (波部) } 8.0 \times 8.6 \mathrm{~mm} \text { p. } 112
$$

8 Lioconcha (Lioconcha) ornata DILLWYN

$$
\text { ムラヤマサラサガイ（黒间） } 33.8 \times 29.0 \mathrm{~mm} \text { p. } 159
$$

9 Tellinella patagiata (PRASHAD)

$$
\text { ヒナザクラガイ (波部) } 9.6 \times 5.8 \mathrm{~mm} \quad \text { p. } 124
$$


PLATE 1

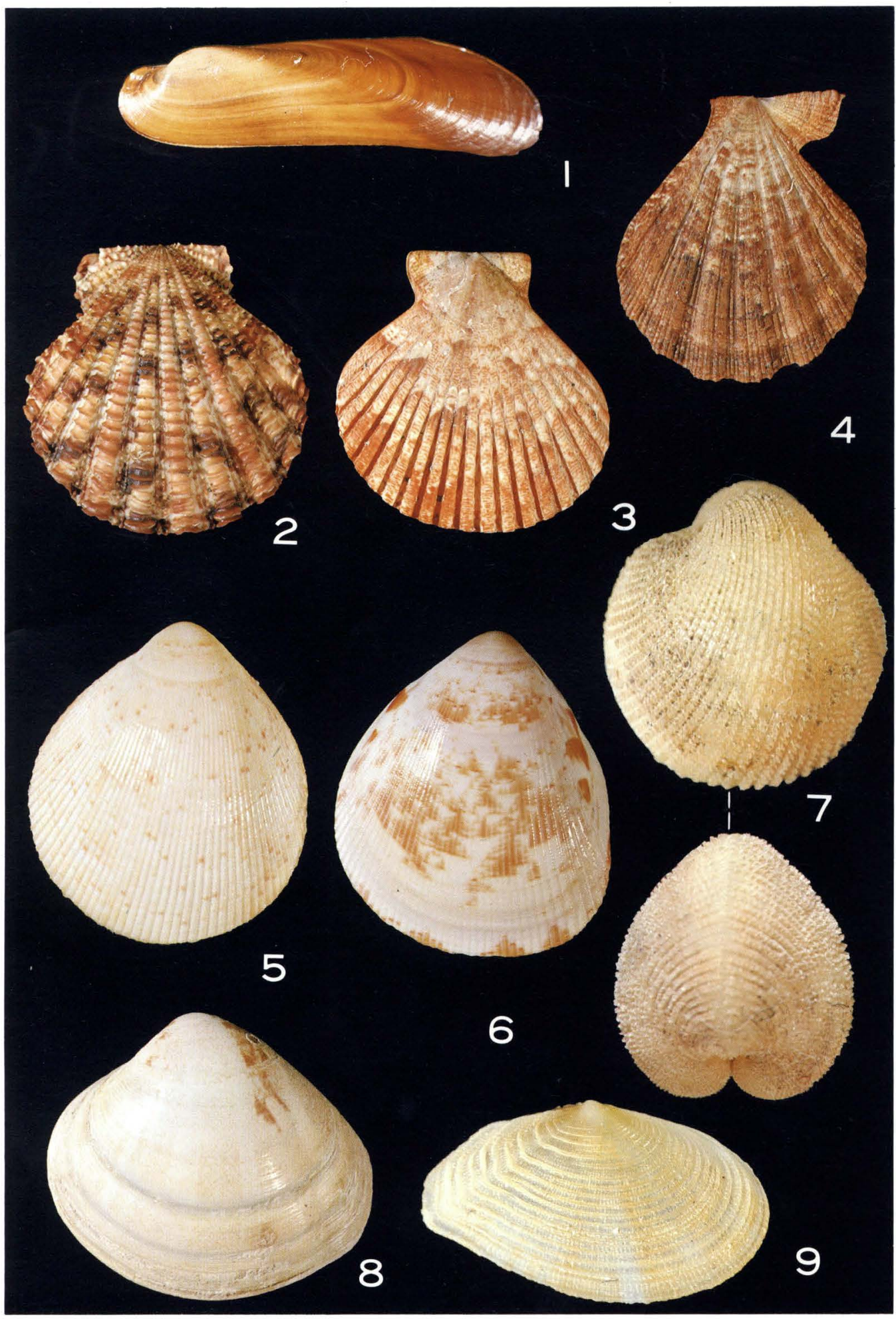




\section{PLATE $\cdot 2$}

1 Arcopsis minabensis HABE sp. nov.

$$
\text { チョビヒゲミミエガイ（波部） } 6.5 \times 4.5 \times 3.5 \mathrm{~mm} \text { p. } 38
$$

2 Limnoperna fortunei kikuchii HABE subsp. nov.

$$
\text { コウロエンカワヒバリガイ（波部） } 24.0 \times 11.8 \times 10.8 \mathrm{~mm} \quad \text { p. } 47
$$

3 Modiolus (Fulgida) oyamai HABE sp. nov.

$$
\text { コガラスマクラガイ (岩川) } 21.0 \times 9.8 \times 9.2 \mathrm{~mm} \quad \text { p. } 46
$$

4 Cavatidens imajimai HABE sp. nov.

$$
\text { コカブラガイ (波部) } 14.7 \times 12.5 \times 3.6 \mathrm{~mm} \text { (left valve) p. } 90
$$

5 Conchocele koyamai Haвe sp. nov.

$$
\text { スマキオウナガイ（波部) } 21.0 \times 18.0 \times 5.3 \mathrm{~mm} \text { (left valve) p. } 91
$$

6 Mysella nipponica HABE sp. nov.

$$
\text { ニホンタナベガイ (波部) } 9.4 \times 6.4 \times 4.0 \mathrm{~mm} \text { p. } 102
$$


PLATE 2

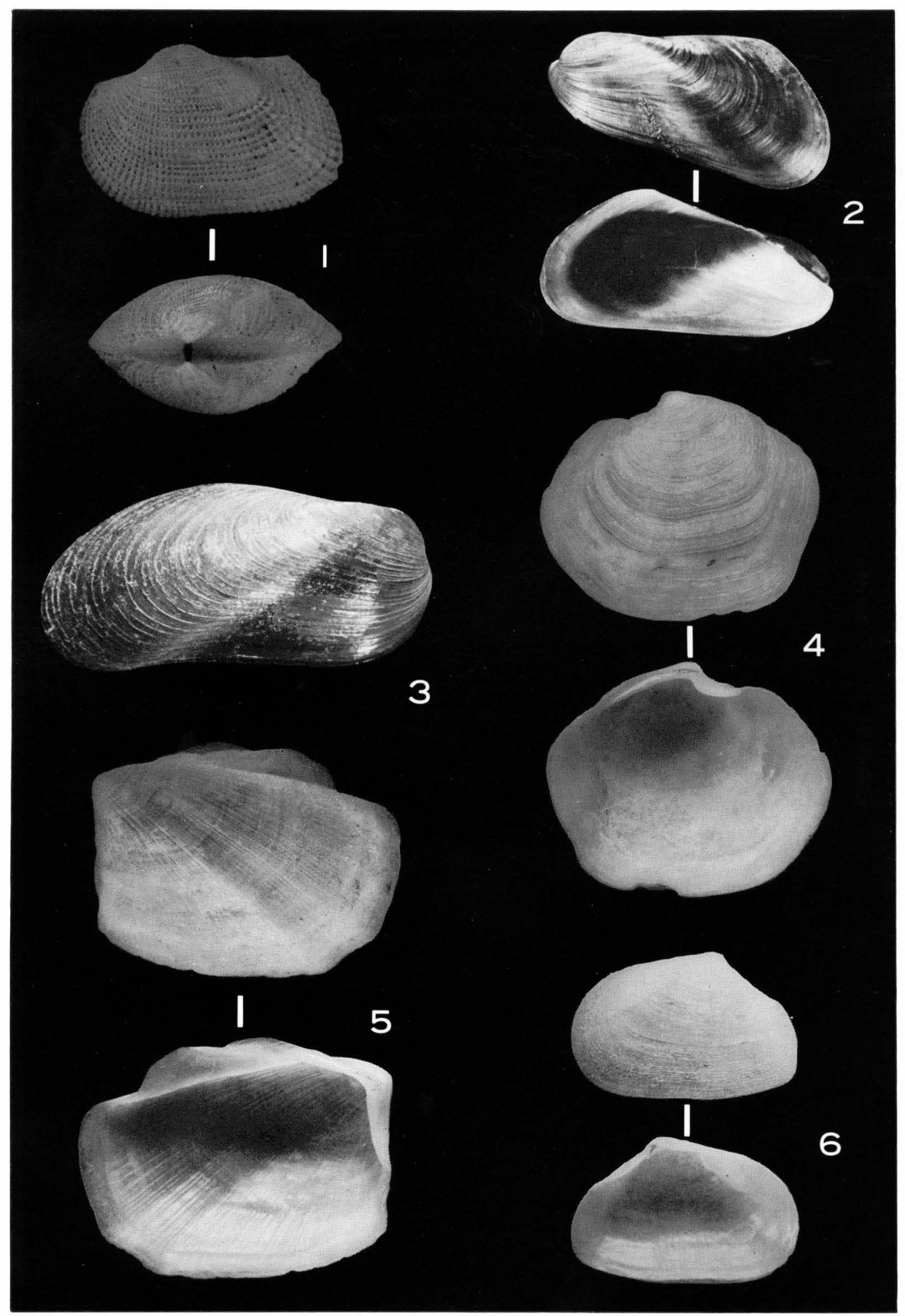




\section{PLATE 3}

1 Galeomma purpurea HABE sp. nov.

ウスムラサキキザミウロコガイ（波部） $11.8 \times 5.6 \times 2.4 \mathrm{~mm}$

(left valve) p. 103

2 Lyonsia tanakai HABE sp. nov.

シンテイサザナミガイ（波部） $21.2 \times 13.5 \times 9.7 \mathrm{~mm}$ (left valve) p. 183

3, 6 Clavagella japonica HABE sp. nov.

クビタテッッガキ (波部) $26 \mathrm{~mm}$ (tube) and $10.5 \times 9.2 \times 1.2 \mathrm{~mm}$

(right valve) p. 189

4 Eximiothracia koyamai HABE sp. nov.

コヤマスエモノガイ（波部） $22.7 \times 14.2 \times 4.0 \mathrm{~mm}$ (left valve) p. 187

5 Grammatomya kurodai HABE sp. nov.

クロダヨシガイ（波部） $26.8 \times 13.3 \times 6.4 \mathrm{~mm}$ p. 139 
PLATE 3

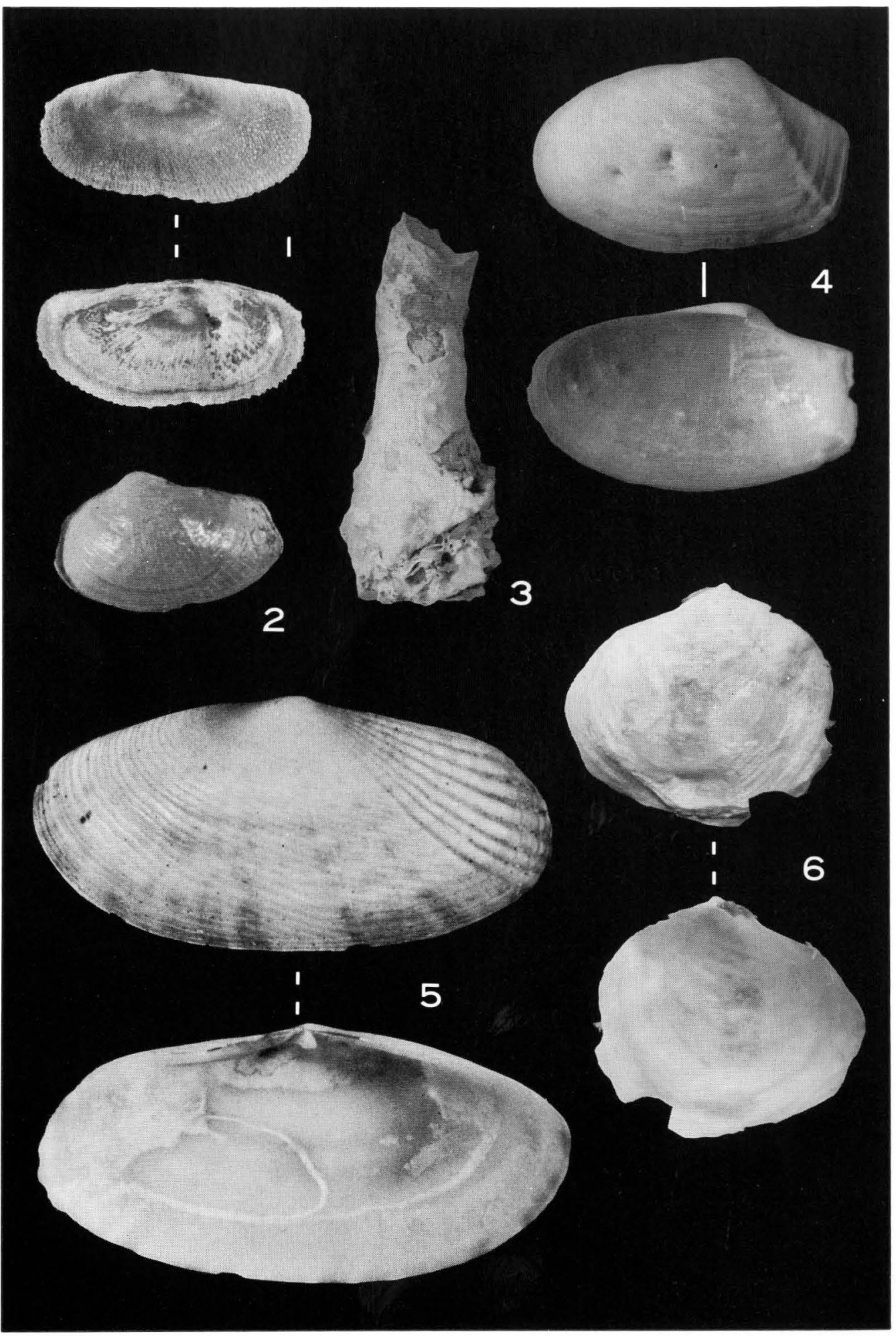




\section{PLATE 4}

1 Barbatia (Ustularca) hachijoensis HATAI, NiINo \& KoTAKA

p. 33 オオハナエガイ（黑田） $33.8 \times 17.0 \mathrm{~mm}$

2 Idasola japonica $\mathrm{HABE}$

$$
\text { キザミバマユイガイ（波部） } 14.2 \times 4.3 \mathrm{~mm} \quad \text { p. } 51
$$

3 Amygdalum soyoae HABE

$$
\text { ユキゾラホトトギスガイ（黒田） } 18.0 \times 10.0 \mathrm{~mm} \quad \text { p. } 51
$$

4 Amygdalum peasei (NewCOMB)

$$
\begin{aligned}
& \text { マボロシガイ（黒田）(ハネヌリッヤホトトギスガイ) } \\
& 21.8 \times 10.4 \mathrm{~mm} \quad \text { p. } 50
\end{aligned}
$$

5 Lithophaga (Labis) erimitica KURODA \& HABE

$$
\text { カクレイシマテガイ (黑四) } 33.2 \times 10.9 \mathrm{~mm}
$$

p. 53

6 Lithophaga (Stumpiella) lithura PILSBRY

$$
\text { キカイイシマテガィ (平瀬) } 14.8 \times 6.5 \mathrm{~mm}
$$

p. 53

7 Exosiperna kuroharai HABE

$$
\text { オトヒメノュリカゴガイ（波部） } 3.8 \times 2.4 \mathrm{~mm}
$$

p. 48

8 Bathyarca japonica HABE

$$
\text { ダイコクワタゾコエガイ（波部） } 12.4 \times 10.5 \mathrm{~mm}
$$

p. 35 
PLATE 4

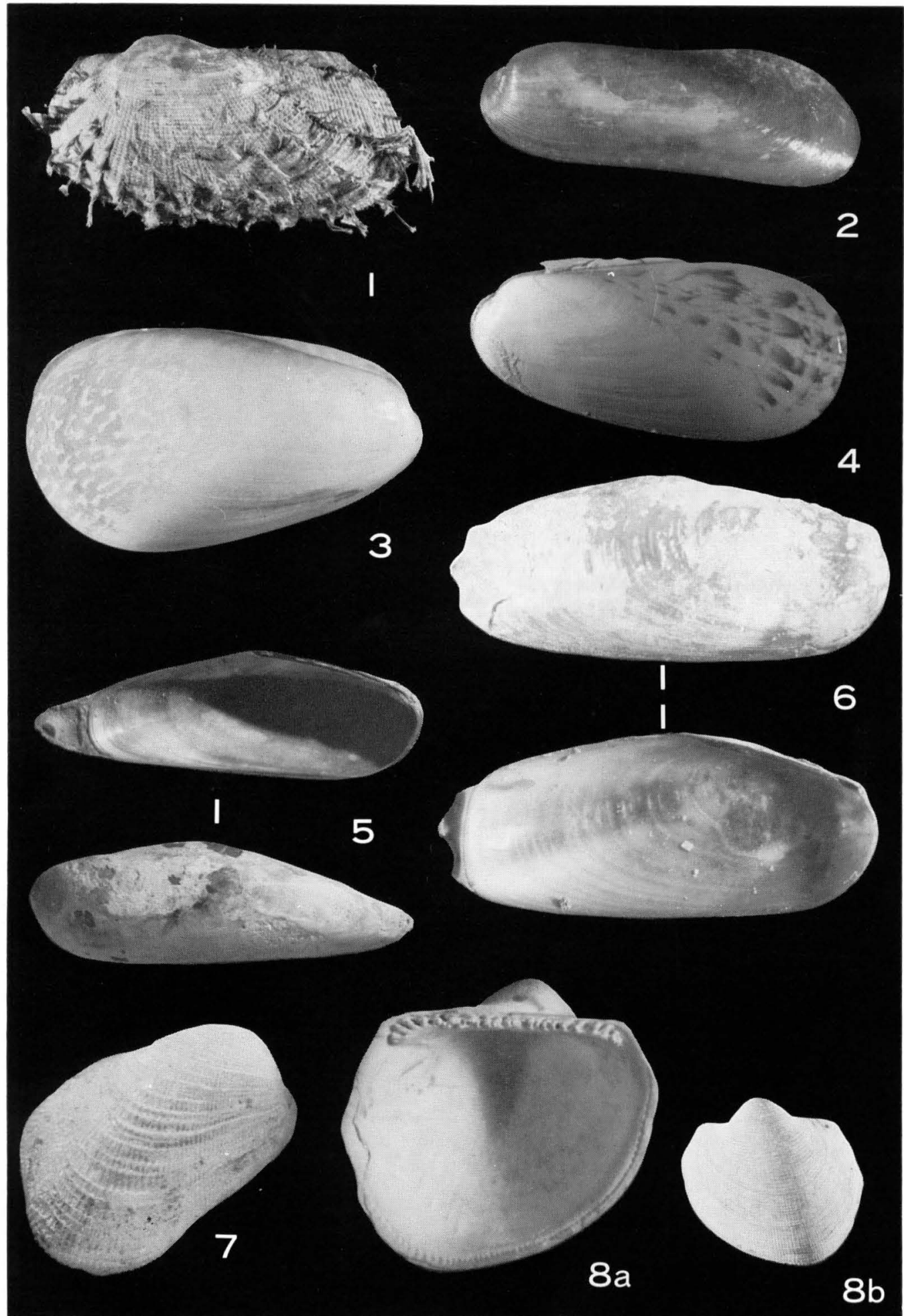




\section{PLATE 5}

1 Bractechlamys aurantiaca (ADAMs \& REEVE)

$$
\text { ナンカイニシキガイ (黒田) } 50.2 \times 45.5 \mathrm{~mm} \text { p. } 66
$$

2 Cryptopecten oweni (DE GREGORIO)

$$
\text { ムラクモヒヨクガイ（黑田） } 19.3 \times 19.4 \mathrm{~mm} \quad \text { p. } 67
$$

3 Spondylus (Eleutherospondylos) serraticostus PRASHAD

$$
\text { ショウキウミギクガイ（波部・林） } 55.9 \times 54.1 \mathrm{~mm} \text { p. } 73
$$

4 Spondylus (Eleutherospondylos) erectospinosus HABE

$$
\text { ハリヤマウミギクガイ（波部） } 31.4 \times 36.5 \mathrm{~mm} \quad \text { p. } 73
$$

5 Divarilima iwaotakii (HABE)

$$
\text { タキハネガイ(波部) } 7.3 \times 7.8 \mathrm{~mm}
$$

6 Isolima limopsis (NomURA \& ZINBO)

$$
\text { シラスナュキミノガイ（大山） } 3.2 \times 3.8 \mathrm{~mm} \quad \text { p. } 79
$$

7 Bellucina civica (YокоYама)

$$
\text { ムツキウメノハナガイ（黑田） } 4.9 \times 4.5 \mathrm{~mm} \quad \text { p. } 88
$$

8 Vulsella minor [RöDING]

$$
\text { ヒメホウオウガイ（黒田） } 10.8 \times 21.4 \mathrm{~mm} \quad \text { p. } 61
$$

9 Lucinoma yoshidai HABE

$$
\text { ヨシダッキガイモドキ(波部) } 20.0 \times 18.0 \mathrm{~mm} \text { p. } 89
$$

10 Lucinoma spectabile (YoKоYAMA)

$$
\text { オオッキガイモドキ（滝・大山） } 66.0 \times 64.0 \mathrm{~mm} \text { p. } 89
$$


PLATE 5

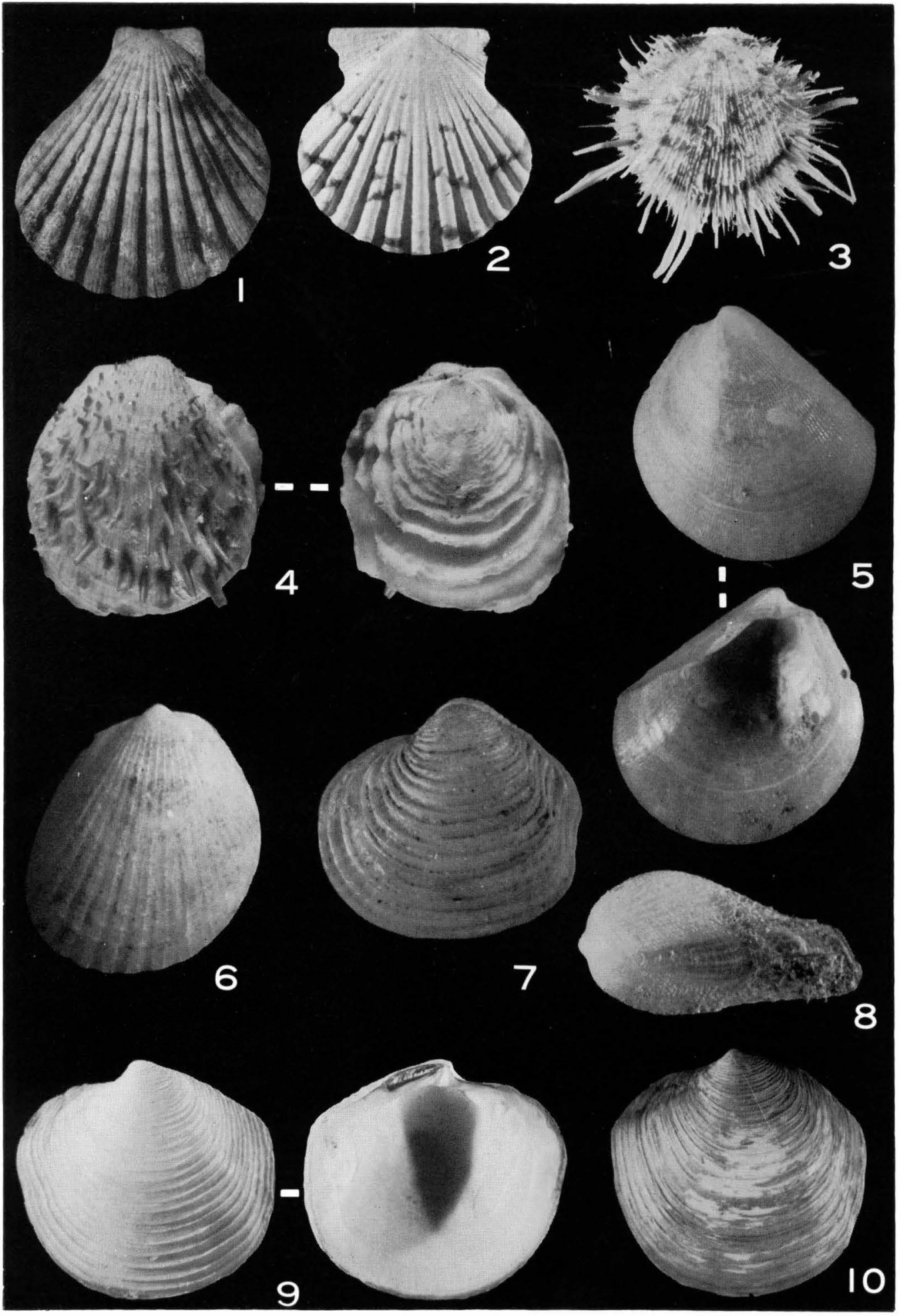




\section{PLATE 6}

1 Thyasira (Maorithyas) miyadii $\mathrm{HABE}$

$$
\text { ウスハナシガイ（波部） } 14.5 \times 13.4 \mathrm{~mm} \text { p. } 92
$$

2 Cycladicama cumingii (HANLEY)

$$
\text { シカガマガイ（六介） } 42.7 \times 39.1 \mathrm{~mm} \text { p. } 93
$$

3 Minipisum japonicum $\mathrm{HABE}$

$$
\text { ミソハギガイ (波部) } 3.1 \times 2.7 \mathrm{~mm} \text { p. } 94
$$

4 Iacra japonica (A. ADAMs)

$$
\text { ヨセギザクラガイ (黒田) } 6.9 \times 6.0 \mathrm{~mm} \quad \text { p. } 136
$$

5 Leptomya minuta HaBE

$$
\text { ミシンコチョウシャクシガイ（波部） } 3.6 \times 2.4 \mathrm{~mm} \text { p. } 136
$$

6 Solecurtus sagamiensis Kuroda et HaBE

$$
\text { ヤワラキヌタアゲマキガイ（黒田・波部） } 33.0 \times 14.5 \mathrm{~mm} \quad \text { p. } 143
$$

7 Solecurtus rhombus (SPENGLER)

$$
\text { シロキヌタアゲマキガイ（黒田） } 45.4 \times 23.6 \mathrm{~mm} \quad \text { p. } 143
$$

8 Rochefortina sandwichensis (SMITH)

$$
\text { ハコベノハナガィ (大山) } 4.1 \times 3.3 \mathrm{~mm}
$$

9 Kermadysmea nishimurai HABE

$$
\text { スダレマスオガイ（波部） } 16.0 \times 4.6 \mathrm{~mm} \text { p. } 140
$$


PLATE 6

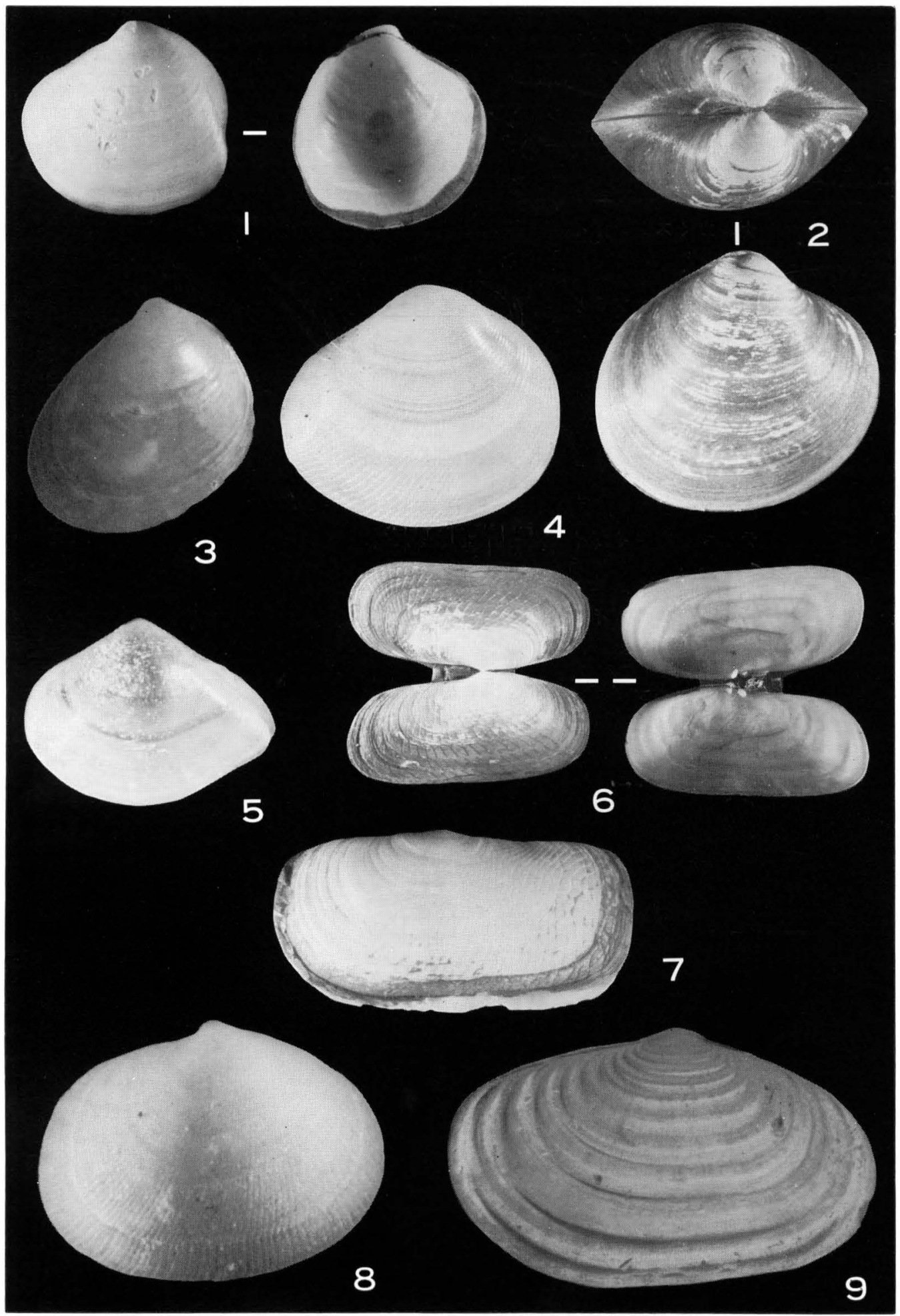




\section{PLATE 7}

1 Samarangia quadrangularis (ADAMs \& REEvE)

$$
\text { コンゴウハマグリ（黒田） } 66.5 \times 63.0 \mathrm{~mm} \text { p. } 160
$$

2 Lioconcha (Sulcilioconcha) dautzenbergi PRASHAD

$$
\text { ナルカミスダレガイ（黒田） } 13.6 \times 12.2 \mathrm{~mm} \text { p. } 160
$$

3 Veremolpa laevicostata (KURODA)

$$
\text { オオギカノコアサリ（黑田） } 14.5 \times 11.0 \mathrm{~mm} \text { p. } 155
$$

4 Rupellaria fabagella (LAMARCK)

$$
\text { クシケガイ (群品) } 25.5 \times 20.5 \mathrm{~mm} \quad \text { p. } 170
$$

5 Callista politissima KURODA

$$
\text { ヌリッヤハマグリ（黑田） } 46.9 \times 35.3 \mathrm{~mm} \text { p. } 166
$$

6 Mesoxylophaga teramachii (Iw. TAKI \& HABE)

$$
\text { テラマチキクイガイ（滰・波部） } 10.0 \times 9.4 \mathrm{~mm} \text { p. } 179
$$

7 Metaxylophaga supplicata $\mathrm{Iw}$. TAKI \& HABE

$$
\text { イノリキクイガイ（滝・波部） } 13.8 \times 13.5 \times 13.0(\mathrm{w}) \mathrm{mm} \quad \text { p. } 179
$$

8 Callogonia hayashii HABE

$$
\text { ウラシマハマグリ（波部） } 18.0 \times 15.0 \mathrm{~mm} \text { p. } 149
$$


PLATE 7

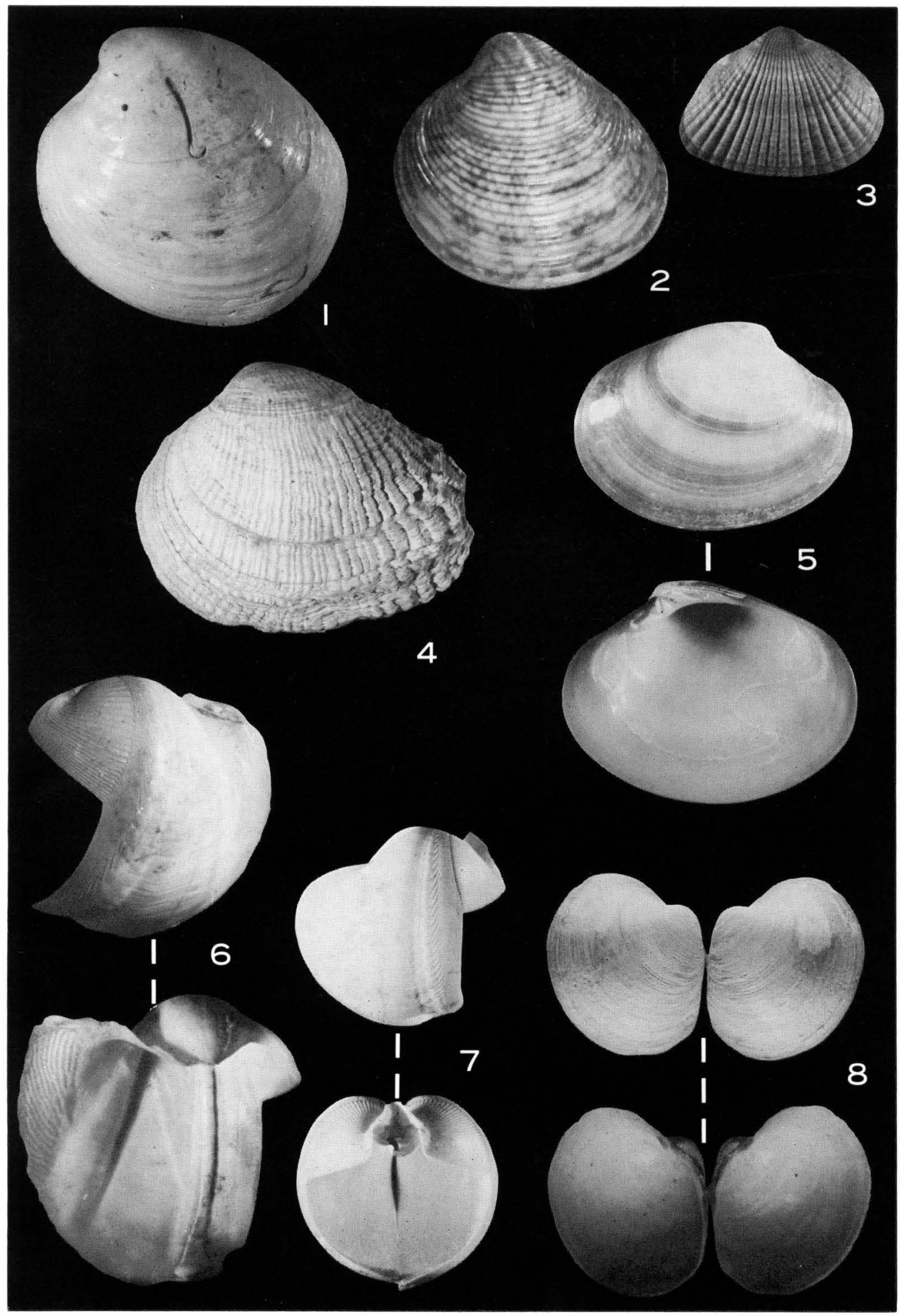




\section{PLATE 8}

1 Cyathodonta granulosa (ADAMS \& ReEve)

$$
\text { スナゴスエモノガイ（黒田） } 30.5 \times 22.0 \mathrm{~mm}
$$

2 Myadoropsis transmontana (YoKOYAMA)

$$
\text { ヤマザキスエモノガイ（黒田） } 6.6 \times 3.9 \mathrm{~mm} \text { p. } 185
$$

3 Myadora quadrata SMITH

$$
\text { テラマチカタビラガイ（波部） } 15.2 \times 11.7 \mathrm{~mm} \quad \text { p. } 185
$$

4 Myonera dautzenbergi PRASHAD

$$
\text { タテヒダシャクシガイ（波部） } 8.6 \times 6.6 \mathrm{~mm} \quad \text { p. } 195
$$

5 Allogramma oahuensis DALL

$$
\text { リニウグウオビクイガイ（波部） } 20.3 \times 13.0 \mathrm{~mm} \quad \text { p. } 184
$$

6 Leiomya tanabensis (HABE)

$$
\text { タナベシャクシガイダマシ（波部） } 5.8 \times 4.3 \mathrm{~mm} \quad \text { p. } 194
$$

7 Cuspidaria kawamurai KURODA
カワムラシャクシガイ（黑田） $35.7 \times 31.1 \mathrm{~mm}$
p. 194 
PLATE 8

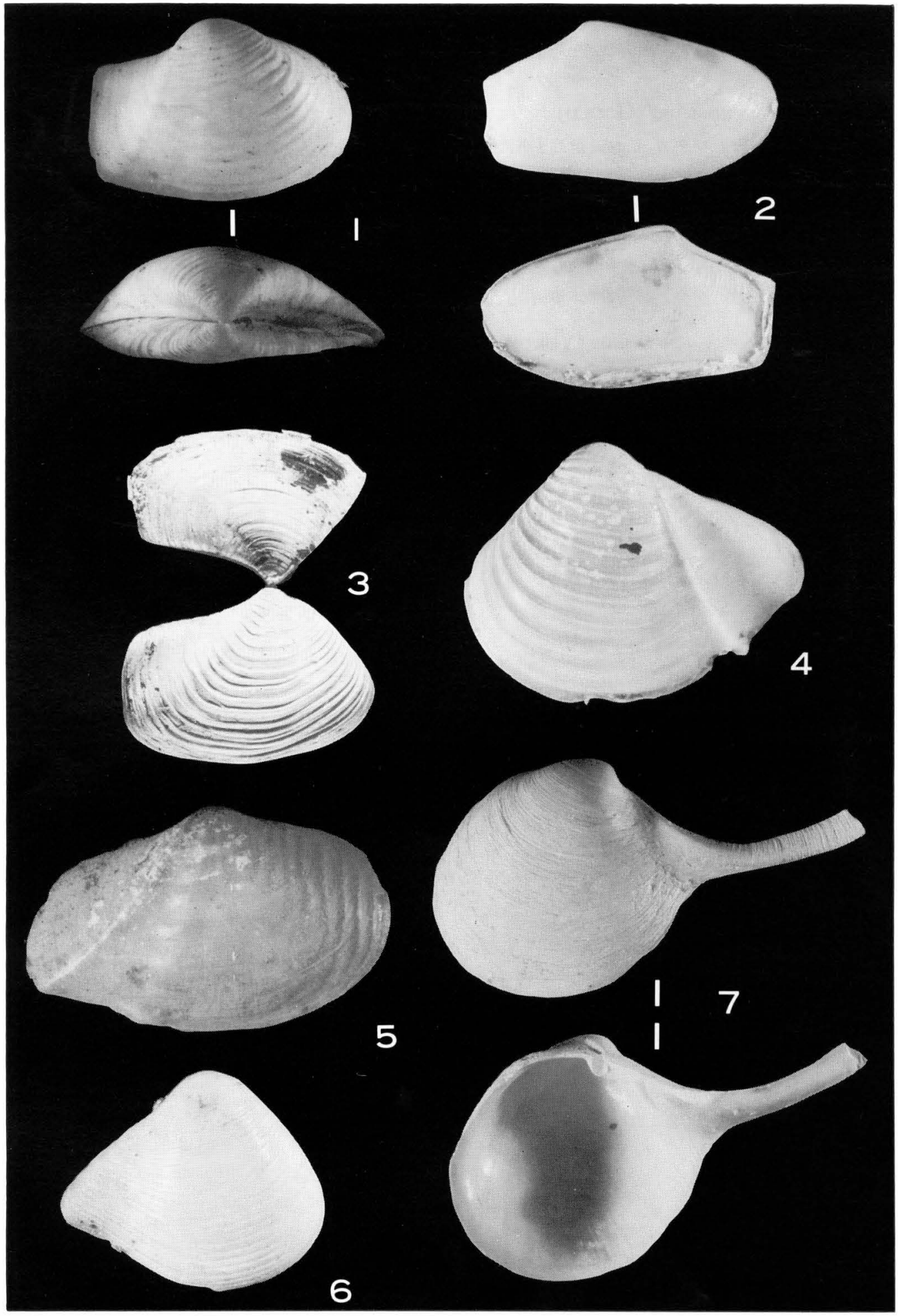




\section{PLATE 9}

1 Lasaea undulata (Gould)

$$
\text { チリハギガイ(矢倉) } 1.8 \times 1.5 \mathrm{~mm} \quad \text { p. } 97
$$

2 Squillaconcha sagamiensis (HABE)

$$
\text { サガミコフジガイ (波部) } 19.4 \times 10.7 \mathrm{~mm} \quad \text { p. } 97
$$

3 Kellia tsujitai HABE

$$
\text { ツジタコハクノッユガイ（波部） } 5.5 \times 4.3 \mathrm{~mm} \quad \text { p. } 98
$$

4 Melliteryx puncticulus (YoKoYAMA)

$$
\text { ハチミッガイ（滝・大山） } 9.7 \times 6.1 \mathrm{~mm}
$$

p. 98 
Plate 9

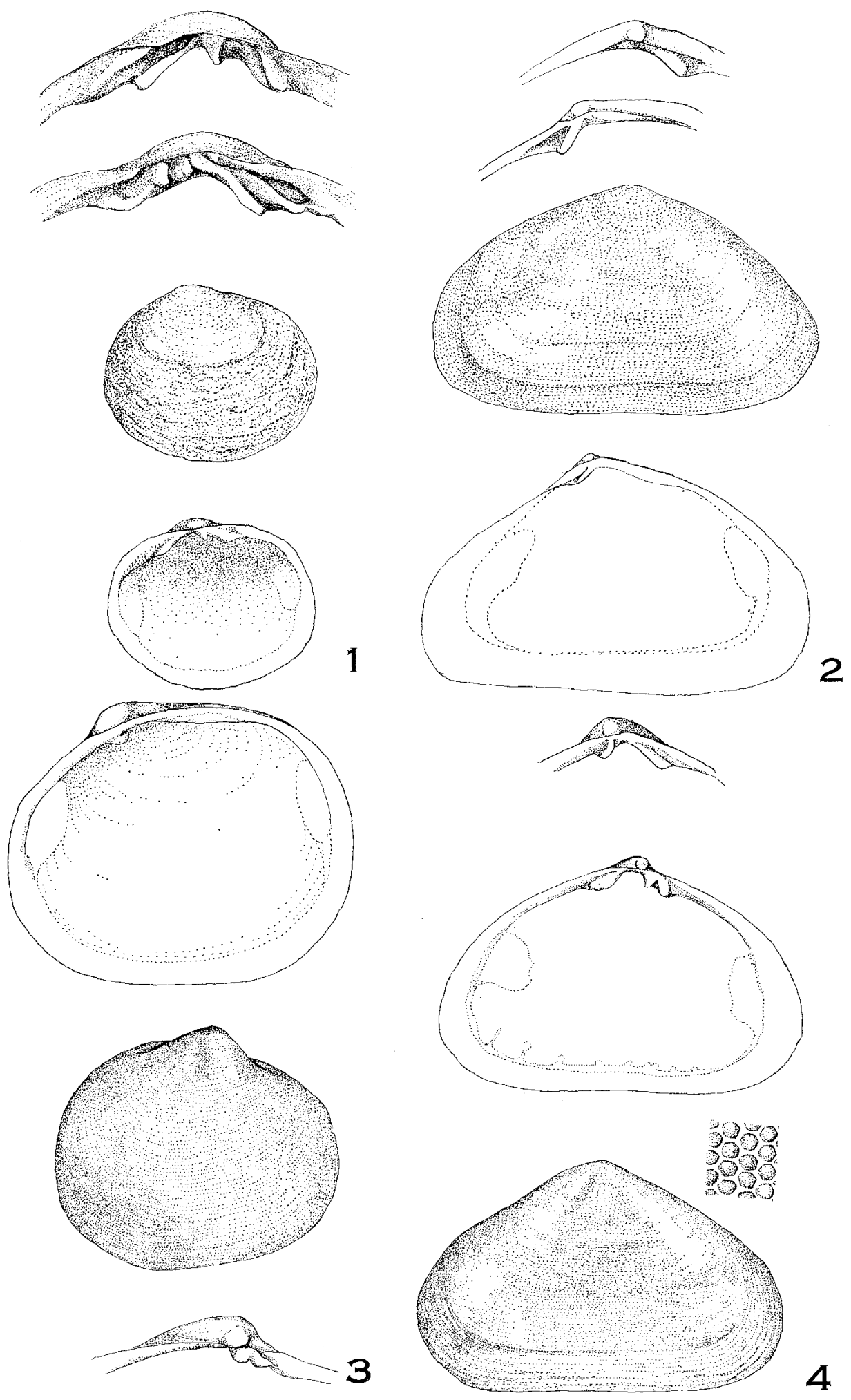


PLATE 10

1 Borniola yamakawai (YoKoYAMA)

$$
\text { ケボリセワケガイ（黒田・波部） } 10.7 \times 7.8 \mathrm{~mm} \quad \text { p. } 98
$$

2 Paraborniola matsumotoi $\mathrm{HABE}$

$$
\text { マツモトウロコガイ（波部） } 7.6 \times 4.0 \mathrm{~mm} \text { p. } 99
$$

3 Kamekia pisiformis KURODA \& $\mathrm{HABE}$

$$
\text { ソバノミガイ（黒田・波部） } 8.8 \times 6.0 \mathrm{~mm} \text { p. } 100
$$

4 Montacuta echinocardiophila HABE

$$
\text { オカメブンブクヤドリガイ（波部） } 2.7 \times 1.9 \mathrm{~mm} \text { p. } 100
$$




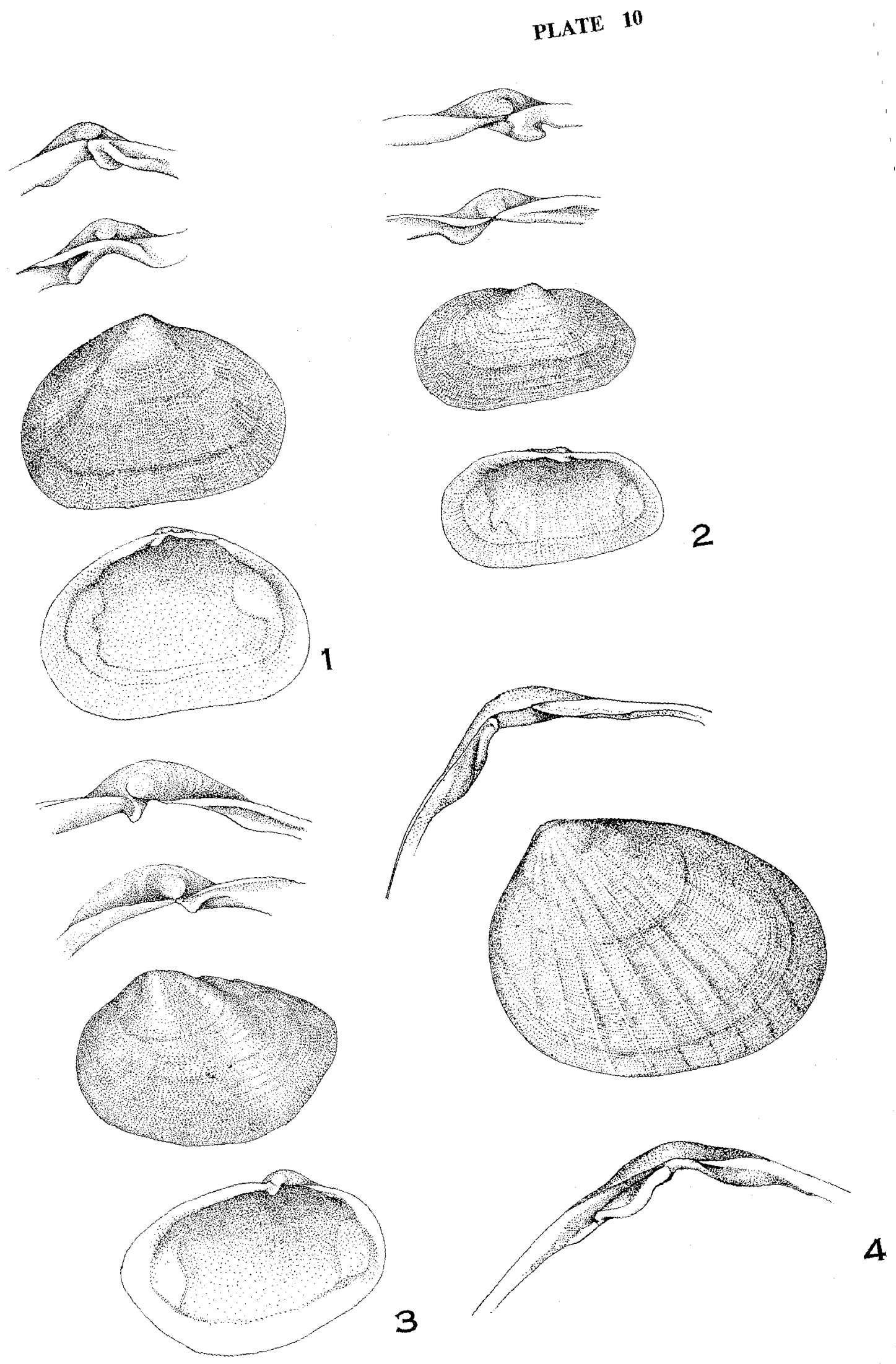




\section{PLATE 11}

1 Montacutona japonica (YокоYАMA)

$$
\text { マルヤドリガイ（波部） } 3.8 \times 2.9 \mathrm{~mm}
$$

p. 100

2 Fronsella fujitaniana (ҮокочАма)

$$
\text { フジタニコクノッュガイ（大山） } 6.9 \times 4.0 \mathrm{~mm} \text { p. } 101
$$

3 Nipponomysella oblongata (YoKoYAMA)

$$
\text { マルヘノジガイ(澈澱) } 4.5 \times 2.4 \mathrm{~mm}
$$

p. 101

4 Nipponomysella subtruncata (YоKоYAMA)

$$
\text { スジホシムシャドリガイ（波部） } 3.6 \times 2.7 \mathrm{~mm}
$$

p. 101 


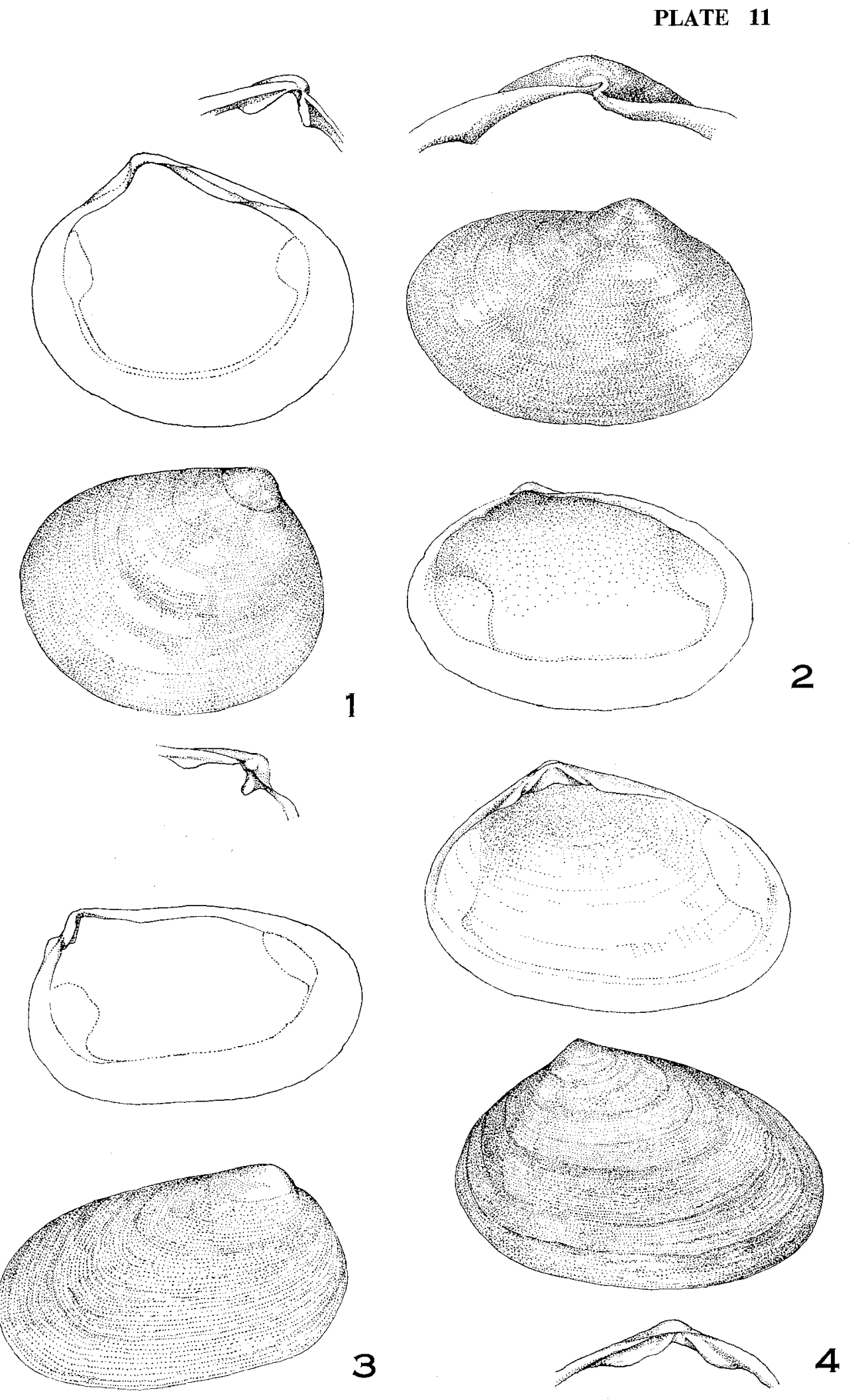




\section{PLATE 12}

1 Nipponomysella obesa $\mathrm{HABE}$

$$
\text { フクレマルヘノジガイ（波部） } 7.5 \times 4.6 \mathrm{~mm}
$$

p. 101

2 Thecodonta sieboldii A. ADAMs

$$
\text { コメガイ (黒田・波部) } 4.4 \times 3.1 \mathrm{~mm}
$$

p. 103

3 Nipponomontacuta actinariophila Yамамото \& HABE

$$
\text { イソギンチャクヤドリガイ（波部） } 7.0 \times 5.0 \mathrm{~mm} \text { p. } 102
$$

4 Lepirodes takii (KURODA)

$$
\text { ウロコガイ（黒田） } 13.1 \times 6.6 \mathrm{~mm}
$$

p. 104 


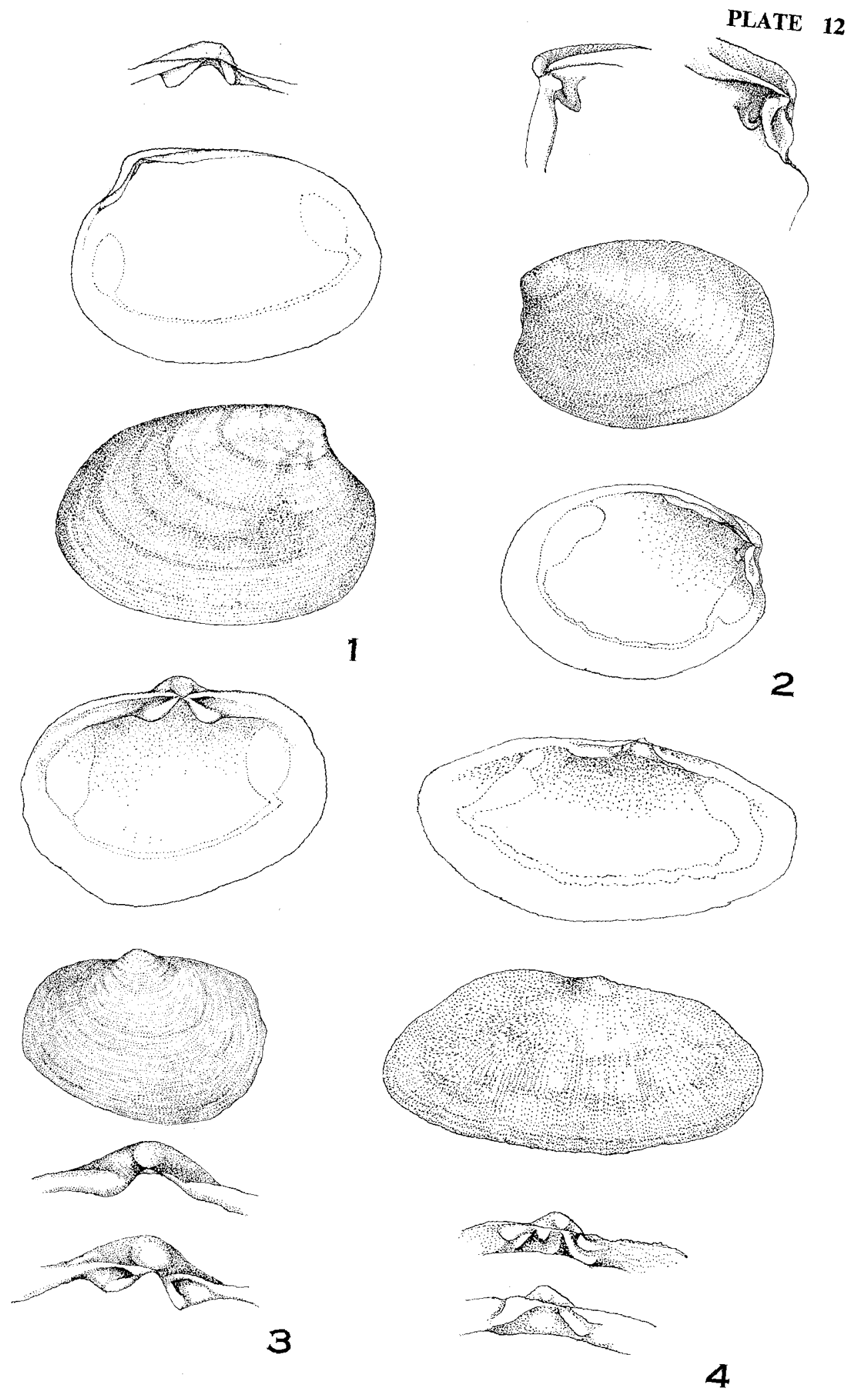




\section{PLATE 13}

1 Ephippodonta murakamii (KURODA)

$$
\text { ウスモノウロコガイ（黒田） } 12.0 \times 6.7 \mathrm{~mm}
$$

p. 104

2 Yamamotolepida semicircula HABE

$$
\text { ヤマモトウロコガイ（波部） } 7.9 \times 5.6 \mathrm{~mm}
$$

p. 105

3 Galeomella utinomii $\mathrm{HABE}$

$$
\text { オウギウロコガイ（波部） } 4.5 \times 2.9 \mathrm{~mm}
$$

p. 104

4 Scintillona stigmatica (PILSBRY)

$$
\text { オオブンブクヤドリガイ（山本・波部） } 6.6 \times 3.7 \mathrm{~mm}
$$

p. 105 
PLATE 13
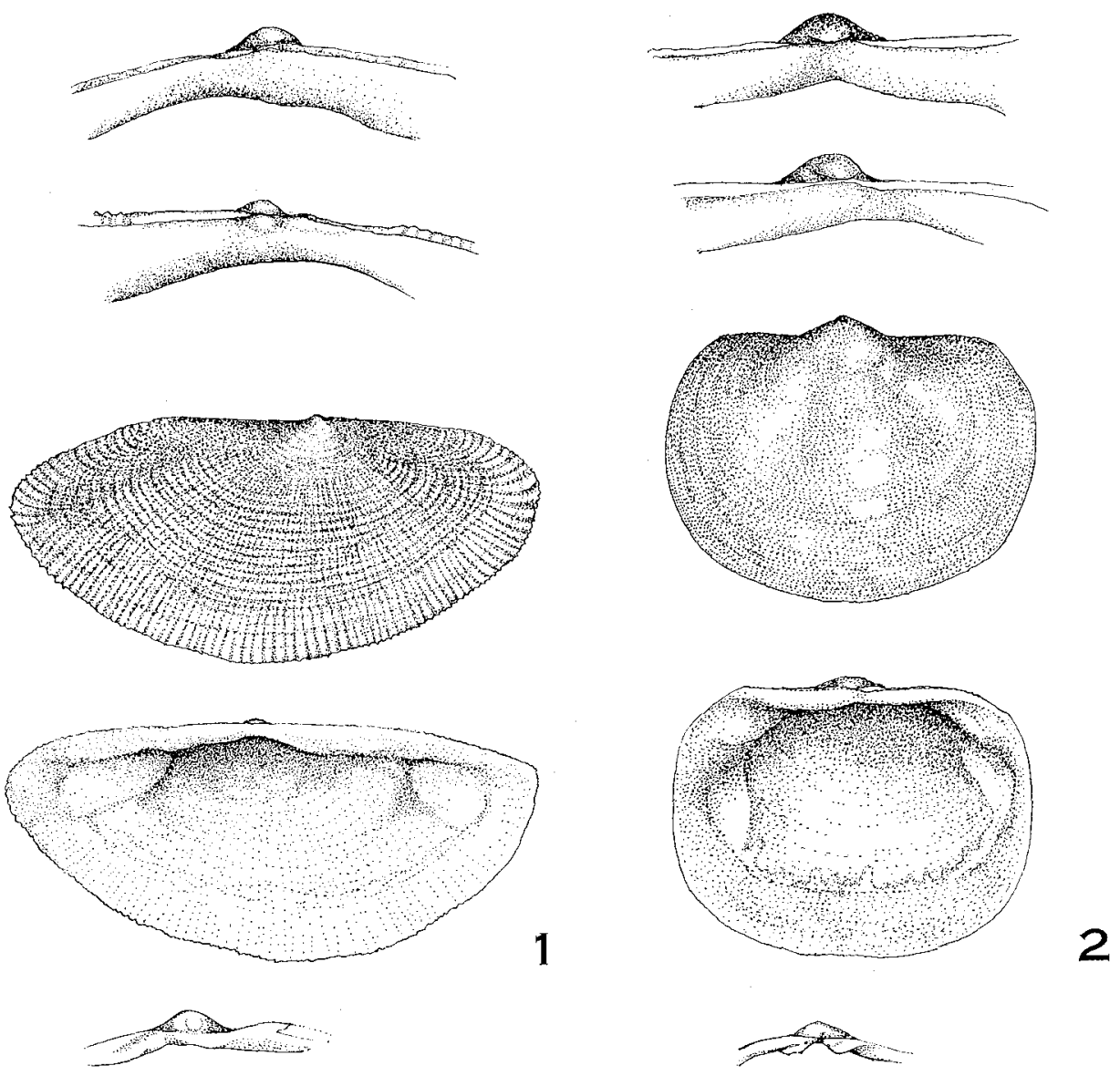

1
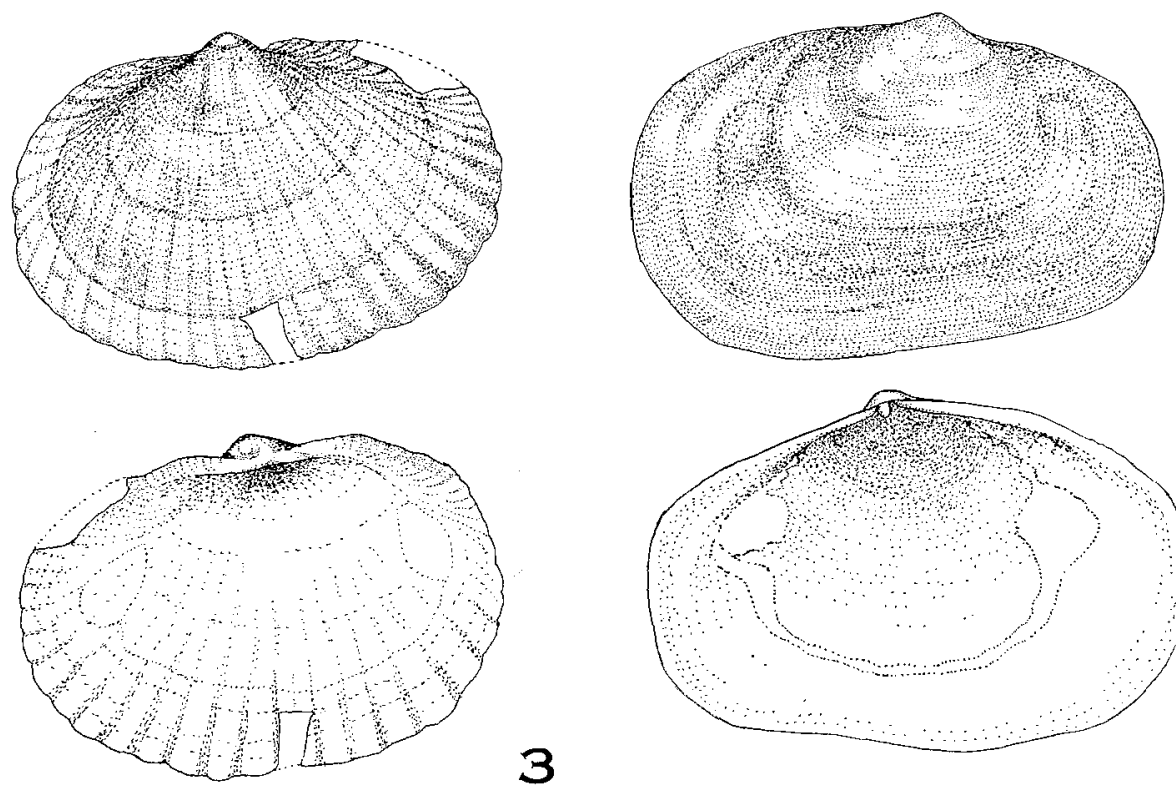

3

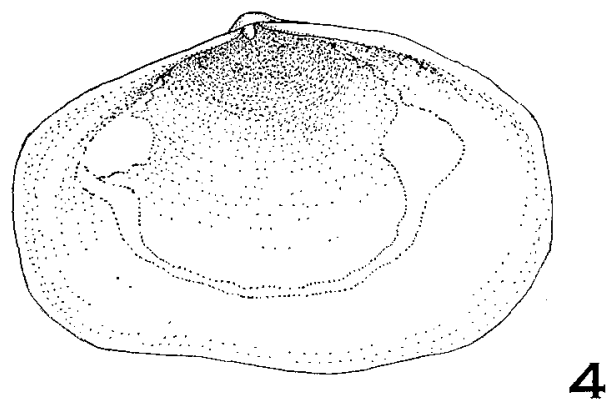




\title{
S C A P H O P O D A
}

\author{
prepared by Tadashige $\mathrm{H}_{\mathrm{ABE}}$
}

\section{Class Scaphopoda 掘足綱}

\section{Order Dentalioida PALMER ッノガイ目 \\ Family Dentaliidae GRAY ッノガイ科 \\ Subfamily Dentaliinae GRAY ゾウゲッノガイ亜科 \\ Genus Dentalium LINNAEUs ゾウゲッノガイ属}

1758 Dentalium LinNaEUs, Syst. Nat., 10, 785.

1811 Dentalia Perry, Conchology, pl. 52. Type (SD Montfort, 1810): Dentalium elephantinum LINNAEUS

\section{Subgenus Paradentalium CoTTON \& GODFREY †カドッノガイ亜属}

1933 Paradentalium Cotton \& Godfrey, S. Aust. Nat., 14, 139. Type (OD): Dentalium intercalatum GouLD

Dentalium (Paradentalium) octangulatum DonovaNヤカドツノガイ（岩川）

$$
\text { ムカドッノガイ (岩川) }
$$

1804 Dentalium octangulatum Donovan, Nat. Hist. Brit. Shells, 5, pl. 162

1818 Dentalium octogonum LaMarCK, AsV, 5, 344

1859 Dentalium hexagonum Gould, PBSNH, 7, 166

1860 Dentalium sexcostatum Sowerby, Thes. 3,103 , pl. 223, f. 11

1877 Dentalium japonicum DUNKER, M B L., 24, 68

1882 Dentalium japonicum: Dunker, Index, 153, pI. 5, f. 2

1879 Dentalium yokohamense WATSON, JLSLZ, 14, 517

1886 Dentalium yokohamense: WATson, Challenger, 15, 11, pl. 2, f. 1

1959 Dentalium (Paradentalium) octangulatum: KIRA, CISJ, 1, 80, pl. 41, f. 7

1964 Dentalium hexagonum: JoHnson, USNMB, 139, 88, pl. 22, f. 4

1964 Dentalium (Paradentalium) octangulatum: HABE, Fauna, 8, pl. 1, f. 1, 2

1971 Dentalium '(Paradentalium) octangulatum: Kuroda \& HABE, SSSB, 486 (J) 305 (E), pl. 65, f. 10,11

Loc. N M S (s) dp.

Dist. Southern Hokkaido to Kyushu. Indo-West Pacific.

$$
\text { Genus Fissidentalium FISCHER ヤスリッノガイ属 }
$$

1885 Fissidentalium Fischer, M. de C. 17, 94. Type (M): Dentalium ergasticum FISCHER

$$
\text { Subgenus Fissidentalium FISCHER ヤスリッノガイ巠属 }
$$

Fissidentalium (Fissidentalium) yokoyamai (MAKIYAMA) ヤスリッノガィ（黒田）

1920 Dentalium complexum YoKoyama, JCSIUT, 39 (6), 101, pl. 6, f. 27 (non DaLL, 1895)

1931 Dentalium yokoyamai MakiYama, MCSKIU, (B) 7 (1), 44, pl. 1, f. 1 .

1959 Dentalium (Fissidentalium) yokoyamai: KIRA, CISJ, 1, 106, pl. 40, f. 12 
1964 Fissidentalium (Fissidentalium) yokoyamai: HABE, Fauna, 12, pl. 1, f. 10

Loc. N M S (s-m) dp.

Dist. Honshu (Kii Peninsula) and Shikoku.

Fissidentalium (Fissidentalium) lima KURODA \& HABEトクサッノガイ（黑田）

1963 Fissidentalium (Fissidentalium) lima KurodA \& HABE, BNSM, 6, 260, pl. 37, f. 15

1964 Fissidentalium profundorum HABE, Fauna, 13, pl. 1, f. 15; pl. 5, f. 59 (non SмITH, 1894)

Loc. S (s) tr.

Dist. Honshu (Kii Peninsula) and Shikoku.

Fissidentalium (Fissidentalium) kawamurai KURODA \& HABEカワムラッノガイ（黒田）

1961 Fissidentalium kawamurai Kunoda \& Habe, CISJ, 2, 106, App., 34, pl. 47, f. 14

1963 Fissidentalium (Fissidentalium) kawamurai: HABE, Fauna, 14, pl. 1, f. 15

1971 Fissidentalium (Fissidentalium) kawamurai: KuRODA \& HABE, SSSB, 487 (J) 306 (E), pl. $65, \mathrm{f} .1$

Loc. $S$ (s) tr.

Dist. Honshu (north to Bōsō Peninsula) to Shikoku.

Subgenus Pictodentalium KIRA in HABE ニシキッノガイ亜属

1959 Pictodentalium KIRA, CISJ, 1, 105 (nom. nudum)

1963 Pictodentalium KirA, in Habe, BNSM, 6, 255. Type (OD): Dentalium (Pictodentalium) formosum hirasei KIRA

Fissidentalium (Pictodentalium) formosum (ADAMS \& REEVE) ニシキノッノガイ (平瀬)

1850 Dentalium formosum AdAMs \& ReEve, Samaramg, 71, pl. 5, f. 1a, b

1860 Dentalium formosum: SowERBY, Thes., 3, 102, pl. 223, f. 2

1897 Dentalium formosum: PilsBry \& Sharp, M. of C., 17, 2, pl. 1, f. 9-11

1913 Dentalium festivum SowERBy, PMSL, 11, 8, tf.

1959 Dentalium (Pictodentalium) formosum hirasei KIRA, ICJS, 1, 105, pl. 40, f. 11

1964 Fissidentalium (Pictodentalium) formosum: HABE, Fauna, 15, pl. 1, f. 4, 12; pl. 4, f. 22

Loc. M S (s) dp.

Dist. Honshu (Kii Peninsula) to Philippines and New Caledonia.

Fissidentalium (Pictodentalium) vernedei (SoWERBY) マルッノガイ（目八）

1860 Dentalium vernedei SOWERBY, Thes., 3, 101, pl. 223, f. 3

1897 Dentalium vernedei: PILSBry \& SharP, M. of C., 17, 80, pl. 3, f. 35,43

1959 Dentalium (Fissidentalium) vernedei: KrRA, CISJ, 1, 106, pl. 40, f. 13

1964 Fissidentalium (Pictodentalium) vernedei: HABE, Fauna, 16, pl. 1, f. 9; pl. 4, f. 30, 31

Loc. N (s-m) dp.

Dist. Honshu (north to Bōsō Peninsula) to Kyushu and China.

Subgenus Compressidentalium HABE ヒラッノガイ两属

1963 Compressidentalium HABE, BNSM, 6, 260. Type (OD): Dentalium hungerfordi PILSBRY $\&$ SHARP

Fissidentalium (Compressidentalium) hungerfordi (PILSBRY \& SHARP)

ヒラッノガイ（岩川）

1888 Dentalium compressum SoWERBY, PZSL, 569, p.1 28, f. 18 (non WATSON, 1879)

1897 Dentalium hungerfordi PiLSBRy \& Sharp. M. of C., 17, 84, pl. 6, f. 83

1906 Dentalium compressiusculum BoISSEVAIN, Siboga, 45, 33, p. 6, f. 12

1957 Dentalium (Fissidentalium) hungerfordi: KIRA, CISJ, 1, 105, pl. 40, f. 10 
1964 Fissidentalium (Compressidentalium) hungerfordi: HABE, Fauna, 17, pl. 1, f. 14; pl. 4, f. 25,26

Loc. $M$ (s-m) dp.

Dist. Honshu (north to Bōsō Peninsula) to Kyushu, China and Indonesia.,

\section{Genus Lentigodentalium HABEカスリッノガイ属}

1963 Lentigodentalium HABe, BNSM, 6, 258. Type (OD): Dentalium variabile DeshaYeS

Lentigodentalium variabile (DESHAYES)カスリッノガイ（黑田）

1825 Dentalium variabile DeshaYes, MSHNP, 2, 367, pl. 16, f. 30

1860 Dentalium variabile: SOWERBY, Thes., 3, 101, p1. 224, f. 30

1897 Dentalium belcheri: SOWERBY, Icon., sp. 1

1963 Dentalium (Lentigodentalium) variabile: HABE, BNSM, 6, 258, tf. 53, 54

1964 Lentigodentalium variabile: HABE, Fauna, 10, pl. 5, f. 53, 54

Loc. M S (s) dp.

Dist. Honshu (Kii Peninsula) to Philippines and India.

\section{Genus Spadentalina HABEッメッノガイ属}

1963 Spadentalina HABE, BNSM, 8, 264. Type (OD): Dentalium tubiforme BoISSEVAIN

Spadentalina tubiformis (BorSSEVAIN) ツメッノガイ（波部)

1906 Dentalium tubiforme Boissevain, Siboga, 45, 19, pl. 6, f. 5

1963 Spadentalina tubiforme (Sic): HABE, BNSM, 8, 264, f. 9-11

Loc. S (s) dp.

Dist. Honshu (north to Kii Peninsula) and Shikoku and Indonesia.

\section{Subfamily Antaliinae STOLICZKA ッノガイ亜科 \\ Genus Antalis H. \& A. ADAMS ッノガイ属}

1854 Antalis H. \& A. AdAMS, GRM, 457

1778 Dentale Da Costa, HNTB, 24

1846 Antalis Herrmannsen, Indicis, Gen. Malak. 63

1847 Entalis GRAY, PZSL, 158

1894 Entaliopsis Newton R HARRIS, PMSL, 66

1896 Antale SACCo, Bull. Mus. Zool. Torino, 11, 98. Type (ICZN Op, 361): Dentalium entalis LiNNAEUS

Antalis tibana (NoMURA) ミガキマルッノガイ（岩川）

1940 Dentalium tibanum NomurA, ROWJ, 12, 101, pl. 1, f. 11, 11a

1961 Antalis indicum tibanum: HABE, CISJ, 2, 105, pl. 47, f. 9

1971 Antalis tibanum: KURODA \& HABE, SSSB, 488 (J) 307 (E), pl. 65, f. 6, 7

Loc. M (s) dp.

Dist. Honshu (north to Bōsō Peninsula) to Kyushu.

Antalis weinkauffi (DUNKER) ッノガイ (目八)

1877 Dentalium weinkauffi DunKer, M B L., 24, 68

1882 Dentalium weinkauffi: DunKer, Index, 153, pl. 5, f. 1

1959 Dentalium (Dentale) weinkauffi: KIRA, CISJ, 1, 105, pl. 40, f. 6

1963 Antalis weinkauff: HABE, BNSM, 6, 261, pl. 36, f. 30, tf. 27

1963 Antalis septentrionalis KURODA \& HABE, in HABE, BNSM, 6, 262, pl. 38, f. 34, tf. 15-17 
1971 Antalis weinkauff: KuRodA \& HABE, SSSB, 488 (J) 307 (E), pl. 65, f. 12, 13

Loc. M S (s-m) dp.

Dist. Honshu to Kyushu.

\section{Genus Striodentalium HABEムチッノガイ属}

1964 Striodentalium HABE, Fauna, 22. Type (OD): Dentalium rhabdotum PILSBRY

Striodentalium rhabdotum (PILSBRY) ムチッノガイ（黑田・菊池）

1905 Dentalium rhabdotum PILSBRy, PANSP, 57, 116, pl. 5, f. 45-47

1933 Dentalium (Antalis) rhabdotum: Kuroda \& KIKUCH, Venus, 4, 8, pl. 1, f. 1, 2

1961 Antalis rhabdotum: HABE, Fauna, 22, pl. 2, f. 17, 18

1971 Striodentalium rhabdotum: Kuroda \& HABE, SSSB, 489 (J) 307 (E), pl. 65, f. 4, 5

Loc. M (m) tr.

Dist. Honshu to East China Sea.

\section{Genus Graptacme PILSBRY \& SHARP ヒナッノガイ属}

1897 Graptacme PILSBRY, \& Sharp M. of C., 17, 85. Type (OD): Dentalium eboreum ConRAD

Graptacme buccinula (GOULD) ヒメナガツノガイ (平瀬)

1859 Dentalium buccinulum Gould, PBSNH, 7, 166

1872 Dentalium buccinulum: SoWERBY, Icon., sp. 30

1897 Dentalium buccinulum: PILSBRy \& Sharp, M. of C., 17, 14, pl. 5, f. 74-76; pl. 6, f. 84

1935 Dentalium motidukii OtUKA, Bull. Earthq. Res. Inst., 8, 879, pl. 45, f. 89

1961 Graptacme buccinulum: HABE, CISJ, 2, 105, pl. 47, f. 8

1964 Graptacme buccinulum: HABE, Fauna, 25, pl. 2, f. 9-11; pl. 4, f. 3

1971 Graptacme buccinulum: KURODA \& HABE, SSSB, 489 (J) 308 (E), pl. 65, f. 22, 23

Loc. M S (s) dp.

Dist. Honshu (north to Bōsō Peninsula) to Ryukyu Islands and China.

\section{Family Laevidentalidae PALMER ミガキッノガイ科 \\ Genus Laevidentalium CossMaNN ミガキッノガイ属}

1888 Laevidentalium Cossmann, Ann. Soc. Malac. Belg., 23, 7. Type (OD): Dentalium incertum DesHAYES

Laevidentalium langfordi HABE ミガキッノガイ（黒田）

1963 Laevidentalium langfordi HABE, BNSM, 6, 268, p1. 38, f. 22

1964 Laevidentalium langfordi: HABE, Fauna, 35, pl. 2, f. 22

1971 Laevidentalium langfordi: KurodA \& HABE, SSSB, 491 (J) 309 (E), pl. 65, f. 30, 31

Loc. M (s-m) tr.

Dist. Honshu (north to Bōsō Peninsula) to Ryukyu Islands.

\section{Genus Fustiaria STOLICZKA サケッノガイ属}

1868 Fustiaria Stoliczka, P. Indica, 2, 439. Type (SD Pilsbry \& SharP, 1897): Dentalium circinatum SOWERBY

Fustiaria nipponica (YoKoYAMA)サケッノガイ（黒田）；シゾナガッノガイ（平瀬）

1922 Dentalium nipponicum YokoYama, JCSIUT, 44 (1), 119, pl. 6, f. 7

1927 Dentalium nagoense DaLL, PUSNM, 70 (2668), 1

1931 Dentalium (Fustiaria) numatai S. HIRASE, J. of C., 19, 139, pl. 3, f. 11 
1964 Fustiaria nipponica: HABE, Fauna, 27, pl. 2, f. 6-8; pl. 3, f. 8, 11; pl. 4, f. 1, 2 1971 Fustiaria nipponica: Kuroda \& HABE, SSSB, 490 (J) 308 (E) pl. 116, f. 15, 16

Loc. S (s) tr.

Dist. Honshu (north to Kashima Nada) to Philippines, China, Indonesia and India.

\section{Family Episiphonidae CHISTIKOV ロウソクッノガイ科 \\ Genus Episiphon PILSBRY \& SHARP ロウソクッノガイ属}

1897 Episiphon Pilsbry \& Sharp, M. of C., 17, 117. Type (SD, Suter, 1913): Dentalium sowerbyi GUILDNG

Episiphon yamakawai (YoKoYAMA) ロウソクッノガイ（黒田・菊池）

1927 Dentalium yamakawai YокочамA, JFSIUT, (2) 1 (10), 427, pl. 48, f. 6

1933 Dentalium (Episiphon) (subrectum) makiyamai KURODA \& KIKUCHI, Venus, 4, 11, pl. 1, f. 8

1961 Episiphon makiyamai: HABE, CISJ, 2, 105, pl. 47, f. 7

1964 Episiphon makiyamai: HABE, Fauna, 28, pl. 2, f. 1

1971 Episiphon makiyamai: KURODA \& HABE, SSSB, 491 (J) 309 (E), pl. 65, f. 26, 27

Loc. S (s-m) dp.

Dist. Nothern most of Honshu to Ryukyu Islands.

Episiphon candelatum KIRAワロウソクッノガイ (吉良)

1959 Dentalium (Episiphon) candelatum KIRA, CISJ, 1, 105, pl. 40, f. 5

Loc. S (s-m) dp.

Dist. Honshu (Kii Peninsula) to Shikoku.

\section{Family Calliodentaliidae CHISTIKoV サフランッノガイ科}

\section{Genus Calliodentalium HABEサフランツノガイ属}

1964 Calliodentalium HABE, Fauna, 30. Type (OD): Dentalium crocinum Dall

Calliodentalium crocinum (DALL) サフランッノガイ（黒田）

1907 Dentalium crocinum DaLL, SMC, 50 (2), 169

1925 Dentalium crocinum: Dall, PUSNM, 66 (17), 16, pl. 29, f. 5

1959 Dentalium (Laevidentalium) crocinum: KIRA, CISJ, 1, 105, pl. 40, f. 9

1964 Calliodentalium crocinum: HABE, Fauna, 31, pl. 2, f. 2; pl. 4, f. 28, 29

Loc. $\mathrm{N}$ (s-m) dp.

Dist. Honshu (north to Bōsō Peninsula) to Kyushu and China.

\section{Family Gadilinidae CHISTIKov シラサヤツノガイ科 \\ Genus Gadilina FoRESTI シラサヤツノガイ属}

1895 Gadilina ForestI, BSMI, 19, 259. Type (M): Dentalium triquetrum BRocCHI

Gadilina corusca (PILSBRY)シラサヤッノガイ（黑田； 女トモノッノガイ（黒田・菊池）

1905 Dentalium coruscum PILsBry, PANSP, 57, 117, pl. 5, f. 42, 43

1906 Dentalium pachypleurum BoISSEvalN, Siboga, 45, 51, pl. 5, f. 21, 22

1933 Dentalium (Laevidentalium) coruscum: KuRODA \& KIKUCHI, Venus, 4, 10, pl. 1, f. 7

1963 Laevidentalium coruscum: HABE, BNSM, 6, 268, pl. 38, f. 29, 35, tf. 28, 29 
1964 Gadilina coruscum: HABE, Fauna, 32, p1. 2, f. 15, 26, 29

Loc. $S$ (m) tr.

Dist. Honshu (north to Enshu-Nada) to Shikoku and Indonesia.

\section{Family Omniglyptidae CHISTIKOV ハリッノガイ科 \\ Genus Omniglypta KuRoDA \& HABE ハリッノガイ属}

1953 Omniglypta Kuroda \& Habe, GJS, 4, 296. Type (OD): Dentalium cerinum PILSBRY

Omniglypta cerina (PILSBRY) ハリッノガイ（黒田）

1905 Dentalium cerinum PILSBRY, PANSP, 57, 117, pl. 5, f. 40, 41

1906 Dentalium tracheatum Borssevain, Siboga, 45, 56, pl, 4, f. 22

1953 Omniglypta cerina: HABE, GJS, 4, 296, f. 753, 754

1961 Omniglypta cerina: HABE, CISJ, 2, 106, pl. 47, f. 13

1971 Omniglypta cerina: KURODA \& HABE, SSSB, 492 (J) 310 (E), pl. 65, f. 24, 25

Loc. $\mathrm{S}(\mathrm{m})$ tr.

Dist. Honshu (north to Iwate Pref.) to Kyushu.

\section{Order Siphonodentalioida Palmer クチキレッノガイ目}

\section{Family Siphonodentaliidae SIMROTH クチキレッノガイ科 \\ Genus Entalinopsis HABE ユキツノガイ属}

1957 Entalinopsis Habe, PSMBL, 6, 132. Type (OD): Dentalium nivosum KURoda \& KiKUCHI

Entalinopsis intercostata (BoISSEVAIN)ユキッノガイ（黒田・㐘池）

1907 Dentalium intercostatum BoIssevain, Siboga, 45, 14, pl. 6, f. 4, tf. 11

1933 Dentalium nivosum KURODA \& KIKUCHI, Venus, 4, 7, pl. 1, f. 9, 10

1940 Dentalium tugaruense NomURA \& HATAI, SHKMRB, 19, 73, pl. 13, f. 4

1961 Entalinopsis nivosum: HABE, CISJ, 2, 105, pl. 47, f. 3

1964 Entalinopsis intercostatus: HABE, Fauna, 40, pl. 2, f. 24, 25; pl. 5, f. $63-65$

1971 Entalinopsis intercostatus: KURODA \& HABE, SSSB, 492 (J) 310 (E), pl. 65, f. 20, 21

Loc. S (s) tr.

Dist. Honshu to Kyushu and Indonesia.

Entalinopsis habutae (KURODA \& KIKUCHI)ハブタエッノガイ（黒田・菊池）

1933 Dentalium habutae KuRODA \& KikUCH, Venus, 4, 3, pl. 1, f. 12, 13, tf. 3, 4

1961 Entalinopsis habutae: HABE, CISJ, 2, 105, pl. 47, f. 4

1971 Entalinopsis habutae: KURODA, \& HABE SSSB, 493 (J) 311 (E), pl. 65, f. 8, 9

Loc. S (s-m) dp.

Dist. Honshu to Kyushu.

Genus Entalina MonTerosato ミカドッノガイ属

1872 Entalina Monterosato, Noiz. Conch. 27. Type (OD): Dentalium tetragonum BrocchI

Entalina quadrangularis BoISSEVAIN ミカドッノガイ（黒田・木下）

1906 Entalina quadrangularis Borssevain, Siboga, 45, 62, pl. 6, f. 73-75, 85, 86

1959 Entalina majestica KIRA, CISJ, 1, 105, pl. 40, f. 3

1964 Entalina quadrangularis: HABE, Fauna, 39, pl. 3, f. 6; pl. 4, f. 20, 21 
Loc. $S(s-m-m)$ tr.

Dist. Honshu (north to Sagami Bay) to Shikoku and Indonesia.

\section{Genus Megaentalina HABE オキナミカドッノガイ属}

1963 Megaentalina HABE, BNSM, 6, 272. Type (OD): Megaentalina teramachii KuRoDA \& HABE

Megaentalina mediocarinata (BoISSEVAIN) オキナミカドッノガイ（黑田）

1906 Entalina mediocarinata Boissevain, Siboga, 45, 63, pl. 6, f. 70-72, 87, 88

1963 Megaentalina teramachii KurodA \& HABE, BNSM, 6, 273, pl. 38, f. 12, 13

1964 Megaentalina mediocarinata: HABE, Fauna, 42, pl. 2, f. 12, 13

Loc. $S$ (s) tr.

Dist. Honshu (Kii Peninsula) to Shikoku and Indonesia.

\section{Genus Siphonodentalium M. SARS クチキレッノガイ属}

1859 Siphonodentalium M. SARS, Forh, Viden (1858), 52. Type (M): Dentalium vitreum M. SARS nOn GMELIN = Siphonodentalium lobatum SOWERBY

Siphonodentalium japonicum HABE ニッポンクチキレッノガイ(波部)

1960 Siphonodentalium japonicum HABE, PSMBL, 8, 294

1964 Siphonodentalium japonicum: HABE, CISJ, 2, 105, pl. 47, f. 1

1971 Siphonodentalium japonicum: Kuroda \& HABE, SSSB, 494 (J) 311 (E), pl. 116, f. 19, 20

Loc. S (s) dp

Dist. Honshu (north to Iwate Pref.) to Kyushu.

\section{Genus Pulsellum StoLICZKA ヒゲッノガイ属}

1868 Pulsellum StoliczKa, P. Indica, 2, 441

1878 Siphoentalis G. O. SARs, Moll. Reg. Arct. Norvez, 104

1896 Siphonodentalis Clessin, Cab., 8 (5), 30. Type (SD Cossmann, 1888): Siphonodentalium lofotense M. SARS

Pulsellum hige HABEヒゲッノガイ（黒田）

1963 Pulsellum hige HaBE, BNSM, 6, 273, tf. 47, 48

1964 Pulsellum hige: HABE, Fauna, 43, pl. 5, f. 47, 48

1971 Pulsellum hige: Kuroda \& HaBe, SSSB, 494 (J) 311 (E), p1. 116, f. 17, 18

Loc. S (s-m) dp.

Dist. Southern Hokkaido to Kyushu.

\section{Family Cadulidae GRANT \& GALE ハラブトッノガイ科}

Genus Dischides JEFFREYS フタマタッノガイ属

1867 Dischides JEFFREYS, AMNH, (3) 20, 251. Type (M): Dentalium bifissum S. WOOD $=$ Cadulus polisus $\mathrm{S}$. WooD

Dischides belcheri (PILSBRY \& SHARP) フタマタッノガィ (矢倉)

1897 Cadulus belcheri Pilsbry \& Sharp, M. of C., 17, 145, pl. 26, f. 84-87

1959 Dischides belcheri: KIRA, CISJ, 1, 104, pl. 40, f. 1

1964 Dischides belcheri: HABE, Fauna, 50, pl. 5, f. 34

Loc. S (s) dp.

Dist. Honshu (north to Bōsō Peninsula) to Formosa. 
Genus Polyschides PILSBRy \& SHARPオオ八ラブトッノガイ属 (ダイオウハラブトツノガイ属)

1897 Polyschides Pilsbry \& Sharp, M. of C., 17, 146. Type (OD): Cadulus tetraschistus WATSON

\section{Subgenus Platyschides HENDERSON ハラブトッノガイ鱼属}

1920 Platyschides Henderson, USNMB, 111, 104. Type (OD): Cadulus grandis VerRILLL

Polyschides (Platyschides) virginalis (BoISSEVAIN) ユミハリハラブトッノガイ（黒田）

1906 Cadulus (Gadila) virginalis Borssevarn, Siboga, 45, 72, pl. 6, f. 60-64

1959 Gadila novilunata KIRA, CISJ, 1, 104, pl. 40, f. 2

1964 Platyschides virginalis: HABE, Fauna, 49, pl. 5, f. 40-42

Loc. S (s) tr.

Dist. Honshu (Kii Peninsula) to Shikoku and Indonesia. 


\title{
Class Cephalopoda CUVIER, 1798 頭足綱
}

\section{Subclass 1. Protocephalopoda GRIMPE, 1922 ${ }^{1)}$ 原始頭足亜綱 \\ (= Tetrabranchiata OwEN, 1836 四鰓類) \\ (= Ectocochlia SchwARTZ, 1894 外款頪) \\ (= Tomochonia HAECKEL, 1896)}

\author{
Order 1. Nautiloidea ZITTEL, 1881 オゥムガイ目 \\ Suborder 8. Nautilida AGASSIZ, 1847 オウムガイ里目 \\ Family Nautilidae BLAINVILLE, 1825 オウムガイ科 \\ Genus Nautilus LINNAEUS, 1758 オウムガイ属
}

Nautilus pompilius LinNaEus, 1758 オウムガイ（鷥鵡介, 六百介品）

Nautilus pompilius Linnaeus, 1758, p. 708; ReEve, 1861, pl. 2; Küster, 1868, p. 9, p.1 2, f. 2;

Tryon, p. 214, pl. 99, f. 506; Fischer, 1882, p. 335, frontispiece; COOKe, 1895, p. 392, f.

252; Pelseneer, 1906, 304, f. 270; Iredale, 1944, p. 295; STenzel, 1957, p. 1135; Voss,

1963, p. 19; Stenzel, 1964, p. 59; Hamada, 1965, p. 193, txf. 1; Hamada, 1977, p. 155;

HAMADA, 1978, p. 135.

This species inhabits nearly the whole tropical West Pacific from the central and southern regions of the Philippine Islands to Australia; empty shells may be carried north by the warm Kuroshio Current and drifted ashore along the Japanese coasts for their buoyancy. MrNato (1965) recorded a shell thus stranded ashore at Migusa of Shirahama-cho, Wakayama Prefecture (Nanki-Seibutsu, vol. 7, no. 1) (HAMADA, 1965, p. 194).

\section{Subclass 2. Metacephalopoda GRIMPE, 1922 ${ }^{2)}$ 後生頭足巠綱 \\ (= Dibranchiata OWEN, 1836 二鰓類) \\ (= Sepiodea AGassiz, 1847) \\ (= Coleoidea BATHER, 1888 鞘形類) \\ (=Endocochlia Schwartz, 1894 内殼類)}

1,2) Grimpe (1922) introduced two new group names as subclasses of the Class Cephalopoda. Formerly, Cephalopoda had been divided into two subclasses, namely Tetrabranchiata and Dibranchiata, according to the number of gills. Tetrabranchiata contains the orders Nautiloidea and Ammonoidea. Nautiloidea consists of a single genus, Nautilus which is represented by only four extant species, all others being extinct. The soft body of the Nautilus has been studied anatomically in detail. It has four gills and many other physiological characteristics, primitive when compared to those of common cuttlefish, squid and devilfish, all of these have two gills. However, it is impossible to learn the real characters of the soft body in the extinct groups, of which the number of species may attain as many as ten thousand species or more. With this in mind, the new names proposed by Grimpe for the two subclasses are regarded as appropriate and have been adopted. 


\title{
Order 1. Decembrachiata WINCKWORTH, ${ }^{3)} 1932$ 十腕目 \\ ( = Decapoda LEACH, 1818 十脚類, non Decapoda \\ Latreille, 1812 (an Order in Class Crustacea)) \\ (= Decabrachia C. R. BoETTGER, 1952 十腕類)
}

\section{Suborder 2. Sepioidea NAEF, 1916 コウイカ东目}

\author{
Superfamily Sepiacea THIELE, 1934 コウイカ超科 \\ Family Spirulidae OWEN, 1836 トグロコウイカ科 \\ Genus Spirula LAMARCK, 1799 トグロコウイカ属
}

Spirula spirula (LinNAEUS, 1758) トトグロコウイカ（滝）

Nautilus Spirula LinnaEus, 1758, p. 710, no. 239.

Spirula spirula SUTER, 1913, p. 1047; (1915) pl. 63, f. 17, pl. 69, f. 5; MACPherson \& GABRIEL, 1962, p. 407.

Spirula australis LaMARCK, 1816, pl. 465, f. 5; ChUn, 1910, p. 413; (1914), pls. 64-73.

Spirula péronii LamarCK, 1822, p. 601; TrYoN, 1879, p. 205, pl. 96, f. 467-469, pl. 105, f. 585.

Spirulea protopyros PÉRon, 1804, p. 30, f. 4.

Spirula reticulata HuXLeY \& PeLSENEER, 1895, p. 46.

Range: Atlantic Ocean (Greater Antilles; Canary Islands; Madeira; Capetown) Indian Ocean (South India; Nicobar Islands; Sumatra); Bali.

Pacific Ocean (Philippines (Cebu; Mindanao); New Guinea; Sulawesi (= Celebes); Fiji; Norfolk Island; North Island, New Zealand).

The animal lives in the deep 200-1750 m layers. The empty shells may be carried afloat by the Kuroshio Current along the Japanese coasts.

Minato reported an empty shell stranded at Migusa, Shirahama-cho in 1965 and on the beach of Tsubaki and also on the northwest shore of the Seto Marine Biological Laboratory of Kyoto University, Shirahama-cho (1978).

\footnotetext{
Family Sepiidae KEFERSTEIN, 1866 コウイカ科

Genus Sepia LinnaEus, 1758 コウイカ属

Subgenus Acanthosepion RocHEBRUNE, 1884 アミミンコウイカ恋属

1. Sepia (Acanthosepion) pharaonis EHRENBERG, 1831 トラフコウイカ（滰）

Sepia pharaonis EHRENBERG, 1831, p. (?); ADAM \& ReEs, 1966, p. 22, pl. 8, f. 38-43; pl. 41, f. 240. Sepia rouxii FÉRUSSAC et ORBIGNY, 1841, p. 271, pl. 19.

Sepia torosa ORTMANN, 1888, p. 652, pl. 23, f. 2.

Sepia framea ORTMANN, 1891, p. 675 , pl. 46 , f. 2.

Sepia singalensis GOODRICH, 1896, p. 3, pl. 1, f. 4-8.

Sepia koettlizi Hoyle \& STANDEn, 1901, p. 1, pl, 1.
}

3) "Decembrachiata is proposed as a substitute for the cephalopod order Decapoda, LEACH 1817 , to avoid confusion with the crustacean order Decapoda, which is in current use and dates from Latreille 1812. For symmetry, Octopoda is altered to Octobrachiata."

(From WINCKWORTH, 1932, p. 251) 
Sepia formosa Berry, 1912, p. 420, f. 2, pl. 9, f. 2 (female); SASAKI, 1929, p. 165, f. 165, 166, pl. 30, f. 9-11.

Crumenasepia hulliana IREDALE, 1926, p. 239, pl. 35, f. 1-2.

Sepia tigris SASAKI, 1929, p. 168, f. 167, pl. 28, f. 13-16 (male).

Crumenasepia ursulae CotTon, 1929, p. 90, pl. 15, f. 3-4.

Sepia sinope Voss, 1962, p. 3 (non Gray, 1849).

Location: Seto (a stranded shell).

Distribution: Tokyo Bay (Probably error or market); Kagoshima Bay; Formosa.

Indo-Pacific from Japan west to the Red Sea and east to Australia.

Habitat: Benthic, sublittoral-upper shelf; tropical-warm temperate.

2. Sepia (Acanthosepion) lycidas GRAY, 1849 カミナリイカ(佐々木)

$\left[\begin{array}{l}\text { モンゴウイカ (紋甲鳥䁍)・ } \\ \text { ギッチョイカ(愛媛県方言) } \\ \text { コブイカ (大和本草)・マルイチ(方言) }\end{array}\right]$

Sepia lycidas Gray, 1849, p. 103; Hoyle, 1886, p. 24; Adam \& ReEs, 1966, p. 8, pl. 3, f. 12-15, pl. 41, f. 236; Voss \& Williamson, 1971, p. 27, pl. 5, f. 4, 7, 9 ; Nishimura \& Suzuki, 1971, p. 144, pl. 53, f. 1; OKUTANI, 1975, p. 6.

Sepiella lycidas RocheBrune, 1884, p. 89; AdAM, 1944, p. 225.

Sepia subaculeata SASAKI, 1914, p. 609, pl. 12, f. 6-7; SASAKI, 1929, p. 173, pl. 16, f. 13-14, pl. 17, f. 1, textf. 104; TAKI, 1965, p. 312.

Location: Seto.

Distribution: Honshu (west of Bōsō Peninsula); Inland Sea; Kyushu; Pusan, southern Korea; Canton (type-locality), South China; Hong Kong; Sarawak, Borneo (ADAM \& ReEs).

Habitat: Benthic; sublittoral; tropical-warm temperate.

$$
\begin{gathered}
\text { Subgenus Platysepia NAEF, } 1923 \text { コウイカ覀属 } \\
\text { (= Fiscisepia Iredale, 1926) }
\end{gathered}
$$

3. Sepia (Platysepia) esculenta HOYLE, 1885 コウイカ(本草縌目啓蒙)

[八リイカ (三才図会)]

Diphtherosepion dabryi (pars) RochEBRUNE, 1884, p. 81.

Sepia esculenta HoYle, 1885a, p. 188; HoYLe, 1885c, p. 291 ; HoYLE, 1886, p. 129, pl. 17, f. 1-5, pl. 18, f. 1-6; SASAKI, 1929, p. 175, pl. 1, f. 5; Voss, 1963, p. 28, pl. $2 a+b$, pl. textf. $2 a+b$;

TAKI, 1965, p. 312; ADAM \& REes, 1966, p. 18, pl. 6, f. 30-33, pl. 43, f. 263; Voss \&

Williamson, 1971, p. 33, pl. 6, textf. 5, 7b, 11; OKUTANI, 1975, p. 10, 210.

Sepia hoylei OrTmanN, 1888, p. 650, pl. 22, f. 5, pl. 23 , f. 1.

Platysepia esculenta NAEF, 1923, p. 546.

Location: Seto.

Distribution: Honshu (type-location: Yokohama Market; west of Toyama Bay and Ibaraki Prefecture); Inland Sea; Shikoku; Kyushu; southern Korea; Philippine Islands; China (Shantung, Kingsu); Hong Kong.

Habitat: Benthic; sublittoral; subtropical-warm temperate.

Subgenus Doratosepion RochEBRUNE, 1884 ハリイカ棘属

4. Sepia (Doratosepion) peterseni APPELLöF, 1886 シシイカ(佐々木) 
Sepia peterseni APPELLÖF, 1886, p. 23, pl. 2, f. 1-6, pl. 3, f. 21; IKEDA, 1891, p. 292; WÜLKER, 1910, p. 14; SASAKI, 1920, p. 194; SASAKI, 1929, p. 195, pl. 18, f. 10-11, pl. 19, 27, textf. 109. Sepia (Doratosepion) peterseni BERRY, 1912, p. 423; SASAKI, 1914, p. 618; TAKI, 1965, p. 313.

Location: Kii Suido (SASAKI, 1920: Albatross)

Station 4961. Two males. 34-09'15" N., 134-56'40' E., $33 \mathrm{fms} .(=60 \mathrm{~m}$.)

Station 4963. Two males. 34-06'15" N., 134-57'50" E., $40 \mathrm{fms} .(=73 \mathrm{~m}$.)

Distribution: Honshu (west of Bōsō Peninsula and Toyama Bay); Shikoku; Kyushu, 59-72 m. (SASAKI, 1929: TAKI, 1965)

Habitat: Benthic; sublittoral; temperate.

5. Sepia (Doratosepion) kobiensis HoYLE, 1885 ヒメコウイカ（山本）

Sepia andreanoides Hoyle, 1885a, p. 193; HoYLe, 1885c, p. 297; HoYle, 1886, p. 139, pl. 21, f. 11-19, pl. 22, f. 11.

Sepia kobiensis Hoyle, 1885a, p. 195; Hoyle, 1885c, p. 300; HoYle, 1886, p. 142, pl. 18, f. 7-14, textf. 7; APPellöF, 1886, p. 20, pl. 3, f. 7; SASAKI, 1929, p. 203; TAKI, 1965, p. 313; AdAM \& ReEs, 1966 , p. 71 , pl. 19, f. 116-120, pl. 43, f. 255, pl. 44, f. 264; Voss \& WiLliamson, 1971, p. 40, textf. 5d, 14; Nishimura \& SUzuKi, 1971, p. 53, f. 3.

Sepia kobiensis var. typica SASAKI, 1929, p. 204, pl. 19, f. 1-4, textf. 111.

Sepia kobiensis var. sagamiana SASAKI, 1929, textf. 112-114.

Sepia kobiensis var. toyamensis SASAKI, 1929, p. 209, pl. 19, f. 8-12, textf. 115.

Sepia kobiensis var. beppuana SASAKI, 1929, p. 211, pl. 19, f. 13-15, textf. 116.

Sepia kobiensis var. crassa SASAKI, 1929, p. 213, pl. 19, f. 16-18.

Sepia kobiensis var. albatrossi SASAKI, 1929, p. 214, pl. 19, f. 19-26, textf. 117.

Location: Seto.

Distribution: Southern Hokkaido; Honshu; Shikoku; Inland Sea; Kyushu; South Korea (Kyosai-tō, Chinkai, Ryūsō-tō, all in Keishō-Nando (YAMAмoto, 1942). (巨済島, 鎮海, 竜草島 (慶尚南道))

Habitat: Benthic; sublittoral; tropical-cool temperate.

Note: Adam \& Rees (1966, p. 13) have written "We agree with Sasaki (1929, p. 203) that $S$. andreanoides and $S$. kobiensis cannot be separated specifically, and although $S$. andreanoides has page-priority, we have to follow Sasaki (1929, p. 206) who described S. andreanoides as a variety of S. kobiensis."

6. Sepia (Doratosepion) appelloefi WÜLKER, 1910 サガミコウイカ（澺）

Sepia appelloefi WüLKDr, 1910, p. 14, pl. 2, f. 8, pl. 3, f. 15-18; SASAKI, 1929, p. 184, pl. 17, f. 10-12, textf. 106; ADAM \& ReEs, 1966, p. 60, pl. 17, f. 101, 103, pl. 43, f. 259.

Location: Sakai, Minabe-cho.

Distribution: Honshu (Misaki, Sagami Sea, 58 fms. (=104.4 m) (Albatross))

Kyushu (Krusenstern Strait, 59 fms. (=106.2 m) (Albatross) ); west of Amakusa Islands, $53 \mathrm{fms} .(=95.4 \mathrm{~m})$ (Albatross); Kagoshima Prefecture; off SataMisaki, 152 fms. (=273.6 m) (Albatross)).

Habitat: Benthic; upper to lower shelf; temperate: $95-273 \mathrm{~m}$. 


\section{Genus Metasepia HoYLE, 1885 ハナイカ属}

7. Metasepia tullbergi (APPELLÖF, 1886) ハナイカ(長崎方言)

Sepia tullbergi APPELLÖF, 1886, p. 26, pl. 2, f. 7-14.

Sepia (Metasepia) tullbergi OrTMANN, 1888; p. 656; AdAM \& ReEs, 1966, p. 116; Voss \& Williamson, 1971, p. 42, textf. 6, 15, pl. 10.

Metasepia tullbergi SASAKI, 1929, p. 217;, pl. 18, f. 16-19, textf. 118; TAKI, 1965, p. 313; ANON. 1974, p. 18; OKUTANi, 1975, pl. 22, 23, p. 155, 247.

Location: Seto.

Distribution: Honshu (west of Tokyo Bay); Kyushu (Nagasaki; Kagoshima); Formosa (Akōchō); Hong Kong.

Habitat: Benthic; littoral-sublittoral; warm temperate.

\section{Genus Sepiella GRAY, 1849 シリヤケイカ属}

8. Sepiella japonica SASAKI, 1929 シリヤケイカ（山陽地方方言）

$\left[\begin{array}{l}\text { マイカ (山口県)・シリクサリ，ツベクサリ（広島県 } \\ \text { 向島町)・ハリナシコウイカ (矢倉) }\end{array}\right.$

Sepiella japonica SASAKI, 1929, p. 219, pl. 18, f. 20-23, pl. 19, f. 28, textf. 170; YASUMoto, 1938, p. 45, f. 1-2; ADAM, 1939, p. 110, f. 15A; TAKI, 1965, p. 314; AdAM \& REes, 1966, p. 128; pl. 39, f. 228-231; Voss \& Williamson, 1971, p. 44, pl. 11, f. 6, 16.

?Sepiella ovata Pfeffer, 1884, p. 14, f. 18, 18a.

?Sepiella obtusata PFefrer, 1884, p. 15, f. 19, 19a.

?Diphtherosepion dabryi (pars) ROCHEBRUNe, 1884, p. 81.

?Sepia sinensis FérussaC \& ORbiGny, 1835, pl. 9, f. 1-2.

?Sepia (Sepiella) sinensis GRAY, 1849, p. 106.

Sepiella maindroni HoYLE, 1886, p. 149, pl. 22, f. 1-10.

Sepiella inermis Joubin, 1879, p. 103; OKUTANI, 1975, p. 22.

Location: Northern Wakayama Prefecture.

Distribution: Honshu (west of Tokyo and Toyama Bays); Shikoku; Inland Sea; Kyushu; eastern, southern and western Korea (YАмамото, 1942); Hong Kong, Chefoo, Shantung, Chekiang, China （山東省芝罱・浙江省）.

Habitat: Benthic; sublittoral; warm temperate.

\section{Family Sepiadariidae FISCHER, 1882 ミミイカダマシ科 \\ Genus Sepiadarium STEENSTRUP, 1881 ミミイカダマシ属}

9. Sepiadarium kochii STEENSTRUP, 1881 ミミイカダマシ(佐々木)

Sepiadarium kochii SteEnStrup, 1881, p. 214, pl. 1, f. 1-10; APPellöF, 1898, p. 593, pl. 32, f. 9-10, pl. 33, f. $14,19,21$, pl. 24, f. 23, 25, 27; HoYLE, 1904, p. 198; SASAKI, 1914, p. 597;

SASAKr, 1929, p. 152, pl. 15, f. 18-19; CotTON \& Godfrey, 1940, p. 408; AdAM, 1954, p. 124; TAKI, 1965, p. 315; Voss \& WILLIAMSON, 1971, p. 51, textf. 18, pl. 13.

Sepiadarium nipponianum BERRY, 1932, p. 42.

Location: Seto.

Distribution: Honshu; Inland Sea; Kyushu.

Hong Kong; Indo-Pacific from Japan east to Australia and west to India (SASAKI, 1929); Amboina (Brock); West Australia (Robson); Andamans 
(GOODRICH); Sri Lanka ( $=$ Ceylon) (GoOdRICH); India (STEENSTRUP).

Habitat: Benthic; sublittoral; tropical-warm temperate.

Note: Berry (1932) established S. nipponianum on Japanese specimens but Adam (1954) did not admit his new species, saying that the characters enumerated by Berry for this species are variable according to the state of conservation of specimens.

\section{Family Sepiolidae (LEACH, 1817) KEFERSTEIN, 1866 ダンゴイカ科（方言）}

Subfamily Rossiinae (APPELLÖF, 1898) NAEF, 1912 ボウズイカ亜科

\section{Genus Rossia OWEN, 1835 ボウズイカ属}

10. Rossia pacifica BERRY, 1911 ボウズイカ(佐タ木)

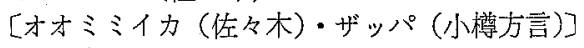

Rossia pacifica BerRY, 1911, p. 591; BERRY, 1912, p. 290, pls. 41-42, f. 1-4, pl. 44, f. 1, 5 ; SASAKI, 1929, p. 154, pl. 16, f. 3-6, textf. 92-94; TAKI, 1965, p. 314; AKIMUSHKIN, 1965, p. 156; OKUTANI, 1975, p. 28.

Location: Sakai, Minabe-cho.

Distribution: Aleutian Islands; Sakhalin; Hokkaido; Honshu (north of Oki Islands and Chōshi); Korea (Pusan, South Kyonseng; Chumunjin, Kangwen; North Hamgyeng).

Habitat: Benthic; sublittoral-bathyal; cool temperate-subarctic.

11. Rossia mollicella SASAKI, 1920 ワタゾコボウズイカ(滝)

Rossia mollicella SASAKI, 1920, p. 189, pl. 25, f. 1; SASAKI, 1929, p. 157, pl. 16, f. 7-10, textf. 95-97.

Location: Off Kii (=Wakayama Prefecture).

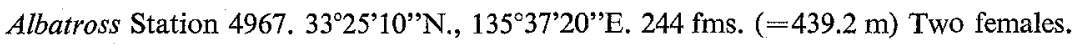

Station 4972 . $33^{\circ} 25^{\prime} 45^{\prime}$ NN., $135^{\circ} 33$ 'E. 440 fms. (=792 m) One male.

Distribution: Honshu. Station 5051. Off Kinka-san. 38 $11^{\prime}$ N., $142^{\circ} 12^{\prime}$ E. 399 fms. (=718.2 m.) One female.

Habitat: Benthic; bathyal; endemic to Japan.

\section{Subfamily Sepiolinae HoYLE, 1904 ダンゴイカ亜科 \\ Genus Euprymna StEenstRUP, 1887 : ミイカ属}

12. Euprymna morsei (VeRRILL，1881） ミミイカ（東京・神奈川県・庇児島県）

[ダンゴイカ (富山畨)・ヒドコイ力 (長崎県)]

Inioteuthis morsei VerRILl, 1881, p. 417, footnote; APPELLöF, 1889, p. 15, pl. 2, f. 15-16, pl. 3, f. 16, 19-20, 23; HoyLe, 1886, p. 112, pl. 14, f. 1-9; OrTMANN, 1888, p. 647, 665, pl. 21,

f. 7, pl. 22, f. 3; GoODrich, 1896, p. 3; JoUBIN, 1897, p. 101; JouBin, 1902, p. 97, f. 11-12. Sepiola bursa PfefFer, 1884, p. 6, f. 6.

Euprymna morsei SteenstruP, 1887, p. 66, 89; Hoyle, 1904, p. 26; Hoyle, 1904a, p. 198; HoYLE, 1905, p. 981; BERRY, 1909, p. 418; BERRY, 1912, p. 408, pl. 6, f. 1-2; WülKer, 1910, p. 9, pl. 1, f. 9, pl. 3, f. 23-24, pl. 4, f. 40; NAEF, 1912, p. 247; SASAKI, 1914, p. 247; 
SASAKI, 1920, p. 187; SASAKI, 1927, p. 1255, f. 2415 ; SASAKI, 1929, p. 146, pl. 15, f. 14, textf. 89; SASAKT, 1947, p. 1019, f. 2887; YAMAMOTO, 1942, p. 129; ADAM, 1954, p. 125, f. 1; Voss, 1963, p. 56; TAKI, 1960, p. 30, pl. 20, f. 11; TAKI, 1965, p. 314; DoNG, 1963, p. 145; OKUTANI, 1967, p. 7; OKuTANI, 1973, p. 92; Nishimura \& Suzuki, 1971, p. 144, pl. f. 5.

Euprymna similis SASAKI, 1913, p. 249, 1 f. (in Japanese); SASAKr, 1914, p. 591, pl. 11, f. 5-8; SASAKI, 1920, p. 187.

Euprymna berryi SASAKI, 1929, p. 143, pl. 15, f. 12-13, textf. 88; YамAMOTO, 1942, p. 129; DonG, 1963, p. 144; Voss \& Williamson, 1971, p. 48, pl. 12, f. 12-13, textf. 88.

Location: Seto.

Distribution: Hokkaido (Teshio; Takashima; Oshoro; Kikonai, Oshima Province, southwest of Hakodate; Hakodate); Honshu (Tohoku District; Kanto District; Tokyo Bay; Kanagawa Prefecture; Noto Peninsula); Inland Sea; Kyushu; South Korea; Formosa; northern to southern Chinese coasts; Philippine Islands; Indonesian Sea; Andaman Islands; Maldive Archipelago; Funafuti Island; Rangiora, South Island, New Zealand.

Habitat: Benthic-nektonic; littoral-sublittoral; tropical-cool temperate.

Note: Adam (1954, p. 128) stated that, of the genus, only Euprymna morsei was found in the Indonesian Sea, and that E. berryi was synonymous with $E$. morsei, adding, "Chez les mâles de Poeloe Weh, la disposition des ventouses des bras sessiles est celle d'Euprymna morsei $\in t$ les ventouses tentaculaires sont à peine plus hautes que larges (fig. 1D). Chez les mâles, provenant de la Baie de Sagami (Japon) et de Koer, la disposition des ventouses des bras sessiles est la même que chez Euprymna berryi; par contre, les ventouses tentaculaires sont aussi longues que large (fig. 1E). A l'heure actuelle, il n'est impossible de faire la révision, cependant très nécessaire, du genre Euprymna. Provisioirement j'ai classé tout la materiel sous le nom d'Euprymna morsei: E. stenodactyla, dont je n'ai pu retrouver le type, est par trop peu connu."

Voss (1963 p. 52) described, in addition to Euprymna stenodactyla, "?E. morsei" and "Unidentified Euprymna" in his "Cephalopods of the Philippine Islands". According to him, the identification of the specimens of Euprym$n a$ seems very difficult. Dong (1963) described E. berryi and E. morsei along the Chinese coasts, while Voss \& Williamson (1971) described only E. berryi in their "Hong Kong Cephalopods".

Okutani $(1967,1973)$ synonymized berryi with morsei.

In these circumstances, the distribution area of Euprymna morsei will extend very wide as seen in the following localities that are arranged from north to south; Hokkaido-Honshu, Shikoku, Kyushu-Korea-Chinese coasts-Hong Kong-PhilippinesIndonesian Seas-Maldive Archipelago-Andaman Islands-Funafuti, Ellice IslandsRangiroa, South Island. Throughout the whole range, the distance between the Maldive Archipelago in the west and Funafuti of the Ellice Islands in the east is about $8800 \mathrm{~km}$ and that from Teshio, Hokkaido in the north to Rangiroa, South Island in the south attains to about $10,600 \mathrm{~km}$. 


\section{Family Idiosepiidae APPELLöF, 1898 ヒナイカ科 \\ Genus Idiosepius SteEnStRUP, 1881 ヒナイカ属 \\ Subgenus Idiosepion P.FISCHER, 1882 ヒナイカ亜属}

13. Idiosepius (Idiosepion) pygmaeus paradoxus (ORTMANN, 1888)

$$
\begin{aligned}
& \text { ヒナイ力 (雛鳥賊) (三才図会) } \\
& \text { [ヒメイ力 (佐々木)] }
\end{aligned}
$$

Idiosepius pygmaeus STEENSTRUP, 1881, p. 219; WüLKER, 1910, p. 22; SASAKT, 1914, p. 599; SASAKI, 1920, p. 191; BERRY, 1921, p. 355, textf. 64, chart 11; SASAKI, 1923, p. 209, textf. $1-3$.

Microteuthis paradoxa ORTMANN, 1888, p. 649, pl. 22, f. 4; JoubIN, 1902, p. 105, f. 15.

Idioteuthis paradoxa BERRY, 1912, p. 405; BERRY, 1921, p. 359, textf. 65; SASAKI, 1927, p. 1254, f. 2414; SASAKI, 1929, p. 133, pl. 15, f. 1-3, textf. 78-79; Yамамото, 1942, p. 128; SASAKI, 1947, p. 1019 , f. 2886.

Idiosepius pygmaeus paradoxus GrimPe, 1931, p. 167; SUGANo, 1974, p. 30.

Idiosepius (Idiosepion) pygmaeus paradoxus TAKI, 1965, p. 314, with fig.; NATSUKARI, 1970, p. 15 , pls. $1-4$.

Location: Hatakejima, Tanabe Bay, sea-weed pool.

Distribution: Southern Hokkaido; Honshu; Inland Sea; Kyushu; southern and western Korea.

Habitat: Benthic (Sessile on sea-grass and sea-weed); littoral; temperate.

\section{Superfamily 2 Loliginacea THIELE, 1934 ジンドウイカ超科}

(= Myopsida OrBIGNY, 1845 閉眼類)

\section{Family Loliginidae LESUEUR, 1821 ジンドウイカ科 \\ Genus Loligo SCHNEIDER, 1784 ジンドウイカ属}

14. Loligo japonica HoYLE, 1885 ジンドウイカ(佐々木)

$$
\left[\begin{array}{l}
\text { ボウズイカ（越中方言）・ヒイ力 (鳥取方言) ・ } \\
\text { コイカ (方言) }
\end{array}\right]
$$

Loligo japonica HoYle, 1885, p. 187; HoYLE, 1886, p. 157, pl. 24, f. 7-15; SASAKr, 1914, p. 602, pl. 14, f. 1-6, textf. 60-62; SASAKI, 1929, p. 112, pl. 14, f. 1-6, textf. 60-62; TAKI, 1965, p. 316; OKUTAN, 1975, p. 30.

Loligo tetradinamia ORTMANN, 1888 , p. 659 , pl. 23 , f. $4 \mathrm{a}-4 \mathrm{k}$, pl. 25 , f. 1.

Location: Seto.

Distribution: Hokkaido; Honshu; Inland Sea; Shikoku; northern Kyushu; Korea; northern China.

Habitat: Nektonic, neritic, warm to cool temperate; endemic.

15. Loligo edulis edulis HOYLE, 1885 ケンサキイカ（方言）

$$
\left[\begin{array}{l}
\text { メヒカリイカ (方言)・ゴトウイカ }(\text { 方言 }) \cdot \\
\text { マワシッコ（幼動物の方言） }
\end{array}\right]
$$

Loligo edulis HOYLe, 1885, p. 186; Hoyle, 1885a, p. 281; HoYLe, 1886, p. 152; pl. 23, SASAKI, 1920, p. 184; Voss, 1963, p. 67, textf. 11a-e; Voss \& WilliaMson, 1971, p. 57, pls. 14-16, textf. 19, 20, 22.

Loligo kensaki Wakrya \& M. IshiKaWA, 1921, p. 283, pl. 1, f. 9, textf. 4; M. IshIKaWA, 1932, p. 441 , f. B. 
Loligo edulis forma nagasakiensis SASAKI, 1929, p. 107, pl. 13, f. 1-4, textf. 57-59.

Loligo edulis edulis OKUTANI, 1975, p. 46.

Location: Kii-Suido. Station 4963 (Albatross), Two young specimens. $34^{\circ}$ 06'15" N., 134 57'50" E. 40 fms. (=73 m.) Seto (by dragnet).

Distribution: Honshu; Shikoku; Kyushu; southern Korea; Formosa; Southern China; Philippines.

Habitat: Nektonic; neritic; tropical-warm temperate.

16. Loligo uyii WAKIYA \& M. ISHIKAWA, 1921 ウイジンドウイカ(滰)

Loligo uyii WakTYA \& M. IshIKaWA, 1921, p. 286; OKUTANI, 1967, p. 8; OKUTANI, 1973, p. 94.

Location: Kii Peninsula and Kagoshima ( 2 females at each location: WAKIYA \& M. IsHIKAWA, 1921).

Distribution: unknown.

Habitat: Nektonic; warm temperate.

\section{Genus Sepioteuthis BLAINVILLE, 1824 アオリイカ属}

17. Sepioteuthis lessonina LESSON, 1830 アオリイカ（倭漢三才図会・東京, 越中方言）

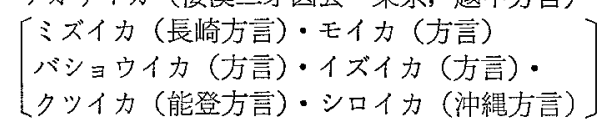

Sepioteuthis lessoniana Lesson, 1830, p. 244; ORBIGNy et Férussac, 1839, p. 302, pl. 1, pl. 6, f. 9-14; TrYon, 1879, p. 152, pl. 62, f. 212, pl. 64, f. 213; AppellöF, 1886, p. 32, pl. 1, f. 11, pl. 3, f. 11-15; WüLKer, 1910, p. 11, 26, pl. 3, f. 28, pl. 4, f. 29, 31; BERRY, 1912, p. 401, pl. 6, f. 3, 5; SASAKI, 1914, p. 606; SASAKI, 1920, p. 185; SASAKI, 1929, p. 127, textf. 74-77, pl. 14, f. 15-17, pl. 29, f. 8-9; ADAM, 1939, p. 21, pl. 1, f. 1-2, tables 1-3; Voss, 1963, p. 77, textf. 13; TAKI, 1965, p. 317; Voss \& Williamson, 1971, p. 66, pl. 20, textf. 19, 20, 26; OKUTANI, 1967, p. 7; NishimuRa \& SUZUKI, 1971, p. 144, pl. 53, f. 7; OKUTANI, 1975, p. 58.

Location: Seto (by dragnet: fished)

Distribution: Hokkaido; Honshu; Shikoku; Inland Sea; Kyushu. Southern Korea; Southern China; Indo-Pacific from Japan to North Australia; from Hawaii to India; Red Sea.

Habitat: Benthic-nektonic; littoral-shelf; tropical-cool temperate.

\section{Superfamily 3 Architeuthacea THIELE, 1934 ダイオウイカ超科 (佐々末)} (=Oegopsida ORBIGNY, 1839 開眼類)

\section{Family Enoploteuthidae PFEFFER, 1900 ギンボシイカ科（滰）}

Subfamily Abraliinae ホタルイカモドキ亚科（山本）

$$
\text { Genus Wataseniac IsHIKAWA, } 1914 \text { ホタルイカ属 }
$$

18. Watasenia scintillans (BERRY, 1911) ホタルイカ（越中方言）

$$
\left[\begin{array}{l}
\text { マッイカ, コイカ(越中方言) } ・ \\
\text { ベニイカ, グミイカ }(\text { 相模小田原方言 })
\end{array}\right]
$$

Abraliopsis scintillans BerRy, 1911, p. 93; BERRY, 1912, p. 424, pl. 7, 8, 9, f. 1-6. Abraliopsis (Nepioteuthion) nishikawae Pfefrer, 1912, p. 149. 
Watasea scintillans IsHIKAWA, 1913, p. 162, 6 figs.; Watasenia IsHIKAWA, 1914, p. 336 (non Watasea Jordan \& SNYDer, 1901); SASAKI, 1914, p. 75, pl. 1-2; SASAKI, 1929, p. 249 , f. 9-10, textf. 124; TAKI, 1965, p. 317; OKUTANi, 1967, p. 9.

Location: Seto.

Distribution: Sea of Okhotsk; Hokkaido: Honshu (Pacific coast from Aomori Prefecture to Wakayama Prefecture, Japan Sea from Aomori Prefecture to Yamaguchi Prefecture); eastern coast of Korea.

Habitat: Planktonic; bathypelagic,endemic (warm-cool temperate).

\section{Family Histioteuthidae VERRILL, 1881 クラゲイカ科（滰） Genus Histioteuthis ORBIGNY, 1841 クラゲイカ属}

19. Histioteuthis corona inermis (TAKI, 1964) ヨ゙マフイカ(滝)

Calliteuthis corona Voss \& Voss, 1962, p. 191, f. 5a-f. $6 \mathrm{bd}$.

Calliteuthis inermis TAKI, 1964, p. 287, pl. 1, f. 3-4, textf. 21-33; OKUTANI, 1967, p. 11.

Histioteuthis inermis Voss, Nancy A., 1969, p. 852.

Location: Off Oshima, Wakayama Prefecture, $250 \mathrm{fms}$. (=450 m.). 29th February, 1970, by E. TSUCHIDA.

Distribution: Pacific coast of Honshu and Shikoku (Suruga Bay, Tosa Bay, $600 \mathrm{~m}$. (Sōyō-maru). Sagami Bay, 600 m. (Sōyō-maru)).

Habitat: Planktonic; bathypelagic; endemic (?).

\section{Family Ommastrephidae STEENSTRUP, 1857 アカイカ科 (方言)}

\section{Subfamily Todarodinae スルメイカ亜科}

\section{Genus Todarodes SteEnSTRUP, 1880 スルメイカ属}

20 Todarodes pacificus SteEnstrup, 1880

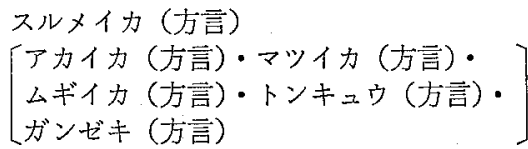

Todarodes pacificus STEENSTRUP, 1880, p. 83, 90, 1 fig. (pars); HoyLe, 1886, p. 34, 163, 207, 219, 227, pl. 28, f. 1-5.

Ommatostrephes sloanei pacificus PfeFFeR, 1912, p. 456, pl. 34, f. 3-6.

Ommastrephes pacificus AppelzöF, 1886, p. 35, pl. 3, f. 8-10.

Ommastrephes sloanei pacificus SASAKI, 1920, p. 199; SASAKI, 1929, p. 279, pl.23, f. 1-6, textf. 133-134.

Todarodes pacificus OKutani, 1962, p. 92; Taki, 1965, p. 319; OKUTANI, 1967, p. 12; Voss \& WiLliamson, 1971, p. 72, textf. 20, 27, 29, pl. 22; OKutant, 1975, p. 104; Okutant, 1977, p. 142.

Location: Seto (caught April, 1930).

Distribution: Hong Kong; Korea (Yamamoto, 1942); Japan; Japan Sea; Okhotsk Sea; west coast of Canada (Mercer, 1968).

Habitat: Nektonic; epipelagic; endemic (warm to cool temperate).

Note: This species migrates along the Pacific and Japan Sea coasts from southern 
Kyushu to the Okhotsk Sea. The southernmost record is from $26^{\circ} 23.8^{\prime}$ N. (near Okinawa) and the northernmost one from $57^{\circ} \mathrm{N}$. in the Okhotsk Sea.

\section{Subfamily Ommastrephinae STEENSTRUP, 1857 アカイカ亜科}

\section{Genus Hyaloteuthis GRAY, 1849 シラホシイカ属}

21. Hyaloteuthis pelagica (BosC, 1802) シラホシイカ(滝)

Sepia pelagicus Bosc, 1802, p. 46, pl. 1, f. 1-2.

Ommastrephes pelagicus ORBIGNY et FérussaC, 1839, p. 348, Calmar, pl. 18, f. 1-2, Ommastrephes, pl. 1, f. 17-18; SteEnstrup, 1880, p. 76, 80, 84, 1 fig.

Ommastrephes (Hyaloteuthis) pelagicus GraY, 1849, p. 63; TRYON, 1879, p. 181, pl. 82, f. 374.

Hyaloteuthis pelagicus PfeFfer, 1912, p. 462, f. 5-8; SASAKI, 1929, p. 286, pl. 23, f. 12-18, textf. 137-138; REHDER, 1940, p. 71.

Location: Seto (one specimen).

Distribution: Hachijo Island; southern Kyushu; Bonin (Ogasawara) Islands (SASAKI, 1929); Marshall Islands (Voss, 1954); Atlantic (Clarke, 1966).

Habitat: Nektonic; epipelagic; tropical.

Note: Teuthis (Gr. calamary or squid, Loligo vulgaris) is a feminine noun; thus the species name "pelagicus" should be altered to "pelagica".

Genus Ommastrephes ORBIGNY, 1839 アカイカ属

22. Ommastrephes bartrami (LESUEUR, 1821) アカイカ（方言)

$$
\left[\begin{array}{l}
\text { バカイカ }(\text { 東京、相模方言 }) ・ \\
\text { ムラサキイカ (方言 }) \\
\text { メダマ }(\text { 東京市場方言 })
\end{array}\right]
$$

Loligo bartrami LeSUEUR, 1821, p. 90, pl. 2, f. 1a-f.

Ommastrephes bartrami ORBIGNY in ORBIGNY et FérussaC, 1839, p. 347, Loligo, pl. 2, pl. 21,

f. 5, Ommastrephes, pl. 2, f. 11-12; TRYoN, 1879, p. 180, pl. 80, f. 361-362; JATTA, 1896, p. 64, pl. 10, f. 1-16; OKUTANI, 1975, p. 114; OKUTANi, 1977, p. 154.

Stenoteuthis pteropus VeRRILL, 1880, p. 223.

Stenoteuthis bartrami SASAKI, 1929, p. 289, pl. 1, f. 8, pl. 24, f. 1-3, textf. 139.

Location: Seto.

Distribution: From Kyushu north to southern Hokkaido (frequently fished for together with Todarodes pacificus). Indo-Pacific Ocean; Atlantic Ocean.

Habitat: Nektonic; epipelagic; cosmopolitan (tropical-warm temperate).

\section{Family Thysanoteuthidae KEFERSTEIN, 1866 ソデイカ科}

\section{Genus Thysanoteuthis TrosCHEL, 1857 ソデイカ属}

23. Thysanoteuthis rhombus Troschel, 1857 ソデイカ(方言)

$$
\begin{aligned}
& \text { 「オオトビイカ(佐々木)。 } \\
& \begin{array}{l}
\text { チョウセンイカ(平户方言) : } \\
\text { タルイカ, ハコイカ, 力サイ力, アカイカ } \\
\text { (古言) }
\end{array}
\end{aligned}
$$

Thysanoteuthis rhombus TROSCHEL, 1857, p. 70, pl. 4, f. 12, pl. 5, f. 1-4; TRYoN, 1879, p. 167, 
pl. 72, f. 285-287; JATTA, 1896, p. 56, pl. 9, f. 1-13, textf. 54; SASAKI, 1929, p. 301, pl. 24, f. 6-8, textf. 141; Voss \& ERDMAN, 1959, p. 23; NisHimURA, 1966, p. 327, textf. 1-6; OKUTANI, 1975, p. 120; OKUTAN, 1977, p. 160.

Thysanoteuthis nuchalis PFEFFER, 1912, p. 531.

Thysanoteuthis elegans TroscheL, 1857, p. 74, pl. 4, f. 10-11.

Location: Tsunashirazu, in Tanabe Bay (stranded).

Distribution: Sagami Bay; east of Honshu; Japan Sea.

Yellow Sea; South Africa; Mediterranean Sea; North Atlantic; Caribbean Sea (Voss \& ERdman, 1959).

Habitat: Nektonic; epipelagic, tropical-warm temperate.

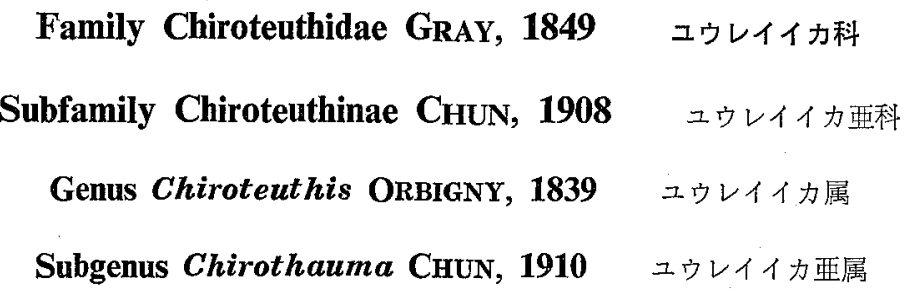

24. Chiroteuthis (Chirothauma) imperator CHUN, 1910 ユゥレイイカ（方言）

[ミズイカ (相模方言)]

Chiroteuthis imperator ChUn, 1910, p. 240, 241, 281, pl. 38, pl. 39, f. 1-10, pl. 40, f. 2-5, 7, pl. 41, pl. 42, f. 1-4, pl. 43, pl. 44, f. 3, 6-16; SASAKI, 1929, p. 305, pl. 24, f. 9-10, textf. 142; INABA, 1963, p. 164; Voss, 1963, p. 136, textf. 30a.

Location: Seto (stranded); Tanabe Bay (2 specimens).

Distribution: Pacific coast of Japan extending from Kyushu to Sagami Bay, 1501265 m. (SASAKI, 1929, and Sōyō-maru); Inland Sea (INABA, 1963). IndoMalayan Sea (Amboina, Philippines, Bay of Bengal, Arabian Sea (Voss, 1963).

Habitat: Planktonic; meso- to bathypelagic; tropical to warm temperate.

Family Cranchiidae GRAY, 1849 サメハダホウズキイカ科（佐々木）

Subfamily Cranchiinae PFEFfER, 1912 サメハダホウズキイカ益科（佐々木）

Genus Liocranchia PFEFFER, 1884 ホウズキイカ属(佐々木)

25. Liocranchia valdiviae CHUN, 1906 ホウズキイカ(佐々木)

Liocranchia valdiviae CHUN, 1906, p. 84; CHUN, 1910, p. 337, pl. 48, f. 3-4, pl. 51, f. 1-4, 8-14, pl. 60, f. 7-11; PfefFer, 1912, p. 675; SASAKI, 1920, p. 203; SASAKI, 1929, p. 336, pl. 26, f. $17-19$, pl. 27 , f. 5-6.

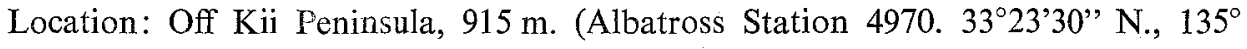
36'30" E. 500 fms. (=900 m.) Aug. 30, 1906.

Distribution: Indian Ocean.

Habitat: Planktonic; bathypelagic; tropical-warm temperate. 


\title{
Family Bathothaumatidae GRIMPE, 1922 メナガイカ科 \\ Genus Bathothauma CHUN, 1906 メナガイカ属
}

26. Bathothauma lyromma CHUN, 1906 メナガイカ(滝)

Bathothauma lyromma CHUN, 1906, p. 86; CHUN, 1910, p. 305, 389, pl. 57, f. 1-2, pl. 58, f. 6-7 (English translation, 1975 (Israel) ), p. 240, p. 303, pl. 56, f. 9, pl. 57, f. 1-2, pl. 58, f. 6-7; AllaN, 1945, p. 337, pl. 25, f. 18; Voss, 1963, p. 153, f. 34; OKUTANI, 1967, p. 15.

Location: Off Shionomisaki, $150 \mathrm{~m}$. (Sōyō-maru: OKUTANI)

Distribution: Eastern Mindanao, Philippine Islands, 695 m. (Voss, 1963); Tasmania (Allan, 1945); North Atlantic (Chun, 1913; Joubin, 1920). West of Cape Verde (Guinea Stream), $3000 \mathrm{~m}$. (CHun, 1906); 36 05'30" N. 9³0" (400 km west of Gibraltar), 3660 m. (JoubIN); off Bermuda (Voss); Eastern Pacific (HoYle).

Habitat: Planktonic; epi- to bathypelagic; tropical-temperate.

\section{Order 2. Vampyromorphae GRIMPE, 1917 コウモリダコ目}

\author{
Family Vampyroteuthidae THIELE, 1915 コウモリダコ科 \\ $(=$ Watasellidae SASAKI, 1920)
}

\section{Genus Vampyroteuthis CHUN, 1903 コウモリダコ属}

27. Vampyroteuthis infernalis CHUN, 1903 コウモリダコ(滝)

Vampyroteuthis infernalis CHUN, 1903, p. 88; PICKFORD, 1946, p. 1; PICKFORD, 1949, p. 1.

Melanoteuthis lucens JouBIN, 1912, p. 1, textf. 1-12.

Cirroteuthis macrope BERRY, 1911, p. 589.

Hymenoteuthis macrope BERRY, 1912, p. 372, pl. 32, f. 1-3, textf. 1; THIELE, 1911 (1914, in

CHUN), p. 534, pl. 90, 91, f. 1-5, textf. 67-70.

Melanoteuthis schmidti JouniN, 1929, p. 372, f. 3-6.

Melanoteuthis beebei RoBson, 1929, p. 469, textf. 1-8.

Danateuthis schmidti JouBIN, 1929, p. 373, f. 3-6.

Retroteuthis pacifica JouBIN, 1929, p. 383, textf. 13-16.

Hansenoteuthis lucens JouBIN, 1929, p. 388, textf. 17-23.

Melanoteuthis anderseni JouBIN, 1931, p. 170, f. 1-5.

Watasella nigra SASAKI, 1920, p. 168, pl. 23, f. 1; SASAKI, 1929, p. 4, pl. 1, f. 1-4, pl. 7, f. 1-2, textf. 1-2.

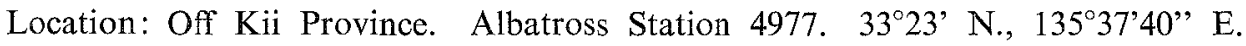
depth 544 fms. (=995.5 m.). Aug. 31, 1906. 1 specimen.

Distribution: Pacific Ocean; Indian Ocean; Atlantic Ocean.

Note: A number of new genera and species had been proposed for this species. Pickford $(1946,1949)$ summarized the results of re-examination of 75 specimens reported already and defined the species as follows, the postembryo, two-finned, up to the mantle-length $8-9 \mathrm{~mm}$; larva, four-finned, mantle-length $25-30 \mathrm{~mm}$; adult form, two-finned, mantle-length $40-50 \mathrm{~mm}$. The distribution of this species is world wide though predominating in the 
deep water of tropical and subtropical oceans and quite absent in the Mediterranean Sea. In the Indo-Pacific, it ranges from 1500 to $2500 \mathrm{~m}$ vertically. It seems to be stenothermal, being found mainly in the range of $2.0^{\circ}-5.9^{\circ} \mathrm{C}$.

\section{Order 3. Octobrachiata WINCKWORTH, 1932 八腕目 (= Octopoda LEACH, 1818 八脚類)}

$$
\begin{aligned}
& \text { Suborder 1. Cirrata GRIMPE, } 1916 \text { 有触毛垔目 } \\
& \text { (= Pteroti ReINHARdT \& Prosch, } 1846 \text { 有翼類) } \\
& \text { (= Lioglossa (LÜTKEN, 1882) HoYLE, } 1886 \text { 滑舌類) } \\
& \text { (= Cirroteuthoidea BERRY, } 1920 \text { ヒゲダコ類) } \\
& \text { (= Metapinnata SASAKI, } 1929 \text { 後翼類) } \\
& \text { (= Cirromorpha RoBson, } 1932 \text { 類触毛類) } \\
& \text { (= Cirroteuthacea THIELE, } 1934 \text { ヒゲダコ類) }
\end{aligned}
$$

\section{Superfamily Cirroteuthacea THIELE, 1934 ヒゲダコ超科（佐タ木）}

\section{Family Opisthoteuthidae VERRILL，1896 メンダコ科（相模方言）}

\section{Genus Opisthoteuthis VERRIL, 1883 メンダコ属}

\section{*28. Opisthoteuthis japonica TAKI, 1962 センベイダコ(滝)}

Opisthoteuthis japonica $\dot{T}_{A K I}, 1962$, p. 794; TAKI, 1963, p. 74. pl, 3, textf. 20-22. (type and paratype specimens, males)

Location: Off Minabe-cho, Wakayama Prefecture; depth $152 \mathrm{~m}$.

Distribution: Unknown.

Habitat: Benthic; warm to temperate.

\section{Suborder 2. Incirrata GRIMPE, 1916 無触毛互目 \\ (= Apteri ReINHARDT \& Prosch, 1846 無翼類) \\ (= Trachyglossa (LÜTKEN, 1882) HoYLE, 1886 糙舌類) \\ (= Polypodoidea, NAEF, 1921 マダコ類) \\ (= Apinna SASAKI, 1929 無翼類)}

\section{Superfamily 1. Bolitaenacea THIELE, 1934 フクロダコ超枓（滰） (=Ctenoglossa NAFF, 1921 櫛舌類)}

\section{Family Bolitaenidae CHUN, 1911 フクロダコ科 \\ Genus Japetella HoYLE, 1885 ナツメダコ属 \\ (= Chunella SASAKI, 1920, non KÜKENTHAL, 1902)}

29. Japetella diaphana HoYLE, 1885 ナツメダコ（滝）

Japetella diaphana Hoyse, 1885, p. 232; RoBson, 1932, p. 332.

Eledonella diaphana HoYLE, 1886, p. 107, pl. 9, f. 3-6.

Bolitaena diaphana CHUN, 1911, p. 21.

Chunella diaphana SASAKI, 1920, p. 171; SASAKI, 1929, p. 14, pl. 8, f. 1-4. 
Location: Off Kii. Albatross Station 4956. $32^{\circ} 32^{\prime}$ N., $130^{\circ} 25^{\prime}$ E. $720 \mathrm{fms}$. (= $1317.6 \mathrm{~m}$.)

Distribution: Sagami Bay. Pacific Ocean; Indian Ocean; Atlantic Ocean.

Habitat: Bathypelagic; tropical-temperate.

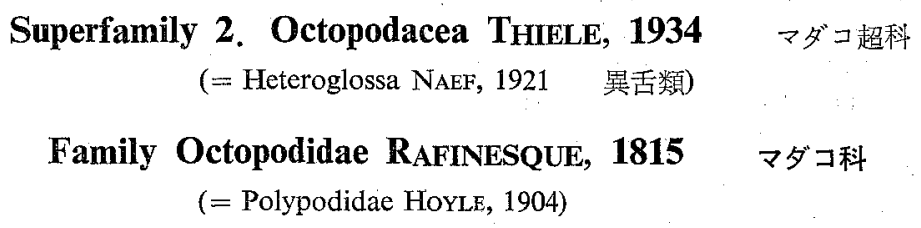

\section{Subfamily Octopodinae GRIMPE, 1921 マダコ亚科}

Genus Octopus LAMARCK, 1798 マダコ属

(= Polypus Hoyle, 1901 (non Polypus SchneIder, 1784 (non binom.))

30. Octopus vulgaris CuviER, 1797 マダコ (池田作次郎)

Octopus vulgare Cuvier, 1797, p. 380 (Cf. HeMmiNG, 1958, p. 10)

Octopus vulgaris LamarcK, 1799, p. 18; Férussac et OrbignY, 1840, p. 26, pl. 2, 3, 3 bis, pl. 8,

f. 2-3, pl. 11-15, pl. 29, f. 6; APPelLöF, 1886, p. 7, pl. 13; JATTA, 1896, p. 212, pl. 4, f. 1 , pl. 7, f. 9, pl. 22, . 2-10, pl. 23, f. 1-4.

Octopus octopodia TRYON, 1879, p. 113, pl. 23, f. 3-4, pl. 24, f. 5-7.

Polypus vulgaris HoYLe, 1907, p. 35 ; PFefFer, 1908, p. 20, f. 11-13; BERRY, 1912, p. 386; SASAKr, 1929 , p. 35 , pl. 4, f. 1 , pl. 9 , f. 1-3, pl. 29 , f. 1, textf. $11-12$ :

Octopus (Octopus) vulgaris RoBson, 1929, p. 57, pl. 1, f. 1, textf. 6-7.

Location: Wakanoura (BERRY); Seto (with dragnet: angled).

Distribution: Honshu (southwest of Sendai and Toyama Bay); Inland Sea; Shikoku;

Kyushu.

Korea (western and southern coasts); Lüta Peninsula; Formosa. Indo-Pacific

(India; Mauritius; Timor; Red Sea); Northeast Atlantic (Haiti, Cuba, Bahia); South Africa; Mediterranean Sea.

Habitat: Littoral; benthic; tropical-cool temperate.

31. Octopus berenice GRAY, 1814 マメダコ(佐々木)

Octopus berenice GraY, 1849, p. 11.

Polypus parvus SASAKI, 1917, p. 365; SASAKI, 1920, p. 171; SASAKI, 1929, p. 38, pl. 3, f. 15-17, pl. 9, f. 4-10, textf. 13-14.

Octopus (Octopus) berenice RoBson, 1929, p. 84, pl. 2 , f. 1.

Octopus rugosus ADAM, 1934, p. 16, textf. 8; ADAM, 1954, p. 169, pl. 1. f. 7, pl. 2, f. 4-5.

Location: Tonda-mura.

Distribution: Honshu (Misaki; Shimizu-minato); Shikoku; Kyushu (Kagoshima, Akune, Satsuma).

South China Sea (Tambelan Island (ADAM, 1954)); Sunda Islands (Celebes, Java, Amboina, Soemba, etc. ADAM, 1954).

Habitat: Littoral; benthic; tropical-temperate. 
32. Octopus minor (SASAKI, 1920)

$$
\begin{aligned}
& \text { テナガダコ（三才㘡会） } \\
& {\left[\begin{array}{l}
\text { アンガダコ(大和本草) ・アシナガイイダコ(池田)・ } \\
\text { シャクシダコ (池田) }
\end{array}\right]}
\end{aligned}
$$

Octopus macropus HoYle, 1886, p. 95, non Risso, 1826; OrTMANN, 1888, p. 643, pl. 21, f. 3;

Sakujiro IKEDA, 1890, p. 526 (in Japanese). Joubiv, 1897, p. 99; SASAKI, 1920, p. 180.

Octopus cuvieri APPELlÖF, 1886, p. 6, pl, 1, f. 6.

Polypus macropus BERRY, 1912, p. 390; WüLKeR, 1910, p. 8.

Polypus macropus var. minor SASAKI, 1920, p. 181.

Polypus variabilis SASAKI, 1927, p. 1251, f. 2407.

Polypus variabilis var. typicus SASAKI, 1929, p. 90, pl. 1, f. 9, pl. 3, f. 21, pl. 21, f. 13-14, textf. 47-48.

Octopus minor TAKI, 1965, p. 323; OKUTANI, 1975, p. 167 (with fig.), p. 236.

Location: Wakanoura (BERRY, 1912).

Distribution: Sakhalin (Albatross); Hokkaido; Honshu; Inland Sea; Shikoku; Kyushu.

Korea (western and southern coasts): Lüta Peninsula (遼東半島); Liaoning (遼寧省); China; Formosa.

Habitat: Littoral; benthic, temperate-cool temperate.

Note: This species is not found in Hong Kong.

Remarks: Sasaki (p. 93, 1929) writes that the differences between O. macropus and the present species are as follows:

"According to the excellent illustrated description done by Jatta (1896), in Octopus macropus,

(1) the funnel organ is distinctly and nicely W-shaped and not double V-shaped as in the present variety,

(2) the hectocotylized part is small, being shorter than one-twentieth the entire length of the arm and it appears to be quite different in structure from that of the present variety, tapering to a pointed extremity and its copulatory groove not characteristically spacious,

(3) the suckers are more numerous and much more closely packed together and those on the hectocotylized arm number 70 pairs, viz. about twice as many as the maximum number in the variety in question.

These differences have at last compelled me to separate these two species."

33. Octopus luteus (SASAKI, 1929) サメハダテナガダコ（滰）

[Towpow (泥婆); Ancgion (紅鱆)]

Polypus luteus SASAKI, 1929, p. 45, pl. 28, f. 6-9, pl. 29, f. 4-5, textf. 160.

Octopus luteus ARAKAWA, 1962, p. 176, textf. 1-7.

Octopus macropus luteus TAKI, 1965, p. 323, f. 1212; TAKI, 1967, p. 20.

Location: Kada; Seto (by prawn net: diving)

Distribution: Southern coast of Shikoku (ARAKAwA, 1962); Takara-jima, Tokara Islands.

Tainan market (Masamitsu Oshima coll.); Pescadores, Formosa (SASAKI, 1929). 
Habitat: Littoral; benthic; subtropical-temperate.

34. Octopus sasakii TAKI, 1942 マッバダコ(私山方言)

Polypus globosus SASAKI, 1929, p. 97, pl, 12, f. 21-22; Not Octopus globosus APPELLöF, 1886, p. 7, pl. 1, f. 4-5; Cf. RoBson, 1929, p. 93.

Octopus sasakii TAKI, 1942, p. 75, textf. 5, 15.

Location: Seto.

Distribution: Honshu (Middle and southern regions); Inland Sea (Mukaishima, Hiroshima Prefecture); Shikoku (Mitsu, Ehime Prefecture); Kyushu (Nagasaki).

Habitat: Littoral; benthic; temperate.

35. Octopus aegina GraY, 1849 スナダコ（佐々木）

Octopus Aegina GraY, 1849, p. 7.

Octopus kagoshimensis Ortmann, 1888, p. 644, p1. 21, f. 2a, $2 \mathrm{~b}$.

Polypus granulatus SASAKI, 1929, p. 40, pl. 3, f. 18, pl. 9, f. 11-13, pl. 29, f. 2-3; non LAMARCK, 1799.

Octopus aegina RoBson, 1929, p. 113, pl. 5, f. 1, textf. 31-32; Voss \& Williamson, 1971, p. 80, textf. 32, pl. 24; ADAM, 1962, p. 55.

Octopus rugosus ADAM, 1942, non Bosc, 1792.

Octopus rugosus TAKI, 1944, p. 280, textf. 10 (form of penis).

Location: Seto (Ezura: by prawn net; angled).

Distribution: Honshu (south of Tohoku District; southwest from Tokyo Bay; Hachijō Island); Kyushu (Nagasaki and Kagoshima Prefectures).

Formosa; Indian Ocean; Tropical regions of Pacific and Atlantic Oceans.

Habitat: Littoral; benthic; tropical-temperate.

Note: This species has not been recorded from the Philippines (Voss, 1963).

36. Octopus ocellatus GRAY, 1849 イイダコ(大和本草)

Octopus fang-siao ORBTGNY, 1840, p. 170; TRYON, 1879, p. 126.

Octopus sinensis ORBIGNY, 1840, p. 68, pl. 9.

Octopus ocellatus GraY, 1849, p. 15; RoBson, 1929, p. 120, pl. 3, f. 1 ,pl. 7, f. 2, textf. 33-35, 37A; DONG, 1963, p. 150.

Octopus areolatus ORBIGNY, 1840, p. 65; BeRRY, 1912, p. 393; HoYLe, 1886, p. 86, p1. 3, f. 6-7; RoBson, 1929, p. 122, textf. 36-37.

Polypus fang-siao var. typicus SASAKI, 1929, p. 49, pl. 10, f. 1-3, textf. 18-19.

Polypus fang-siao var. etchuanus SASAKI, 1929, p. 53, pl. 3, f. 19-20, pl. 10, f. 4-10.

Location: Wakanoura, Kii (Berry, 1912, as Polypus areolatus (de HAAN, 1838), one female)

Distribution: Japan; China.

Habitat: Littoral; benthic; temperate.

Remarks: Orbigny (1840) established Octopus fang-siao and Octopus sinensis based on the descriptions in Terajima's "Encyclopaedia" (Wakan Sansai Zue, 1713). Thus, the description was too brief and far from of a scientific significance, Sasaki (1929) adopted the name "fang-siao" for the octopod that had been known as "Ii-dako" in Japan. The taxonomic treatment 
should be based on the specimens caught in the natural habitat but never be cited from the figures or descriptions seen in old-day printings. Therefore, the usage of "fang-siao" is inappropriate.

There are two names for the present species: ocellatus GRAY (1849) and areolatus ORBIGNY (1840) or O. fang-siao var. typicus and fang-siao var. etchuanus SASAKI (1929). However, the difference between these two species or varieties is very slight, as Sasaki confessed that he created "etchuanus" "with a great deal of hesitation". Robson (1929) showed the difference between these two in detail, but I am of the opinion that his material should be treated as one species, as the localities are not limited in these, but covering widely both the Japanese and Chinese seas.

\section{Octopus membraceus QuOY et GAIMARD, 1832 コッブイイダコ（澺, 改称) \\ [コツブダコ(佐々木)]}

Octopus membranaceus QuoY et GAIMARD, 1832, p. 89, pl. 6, f. 5; ORBIGNY, 1840, p. 43, pl. 10, f. 4, pl. 28, f. 1-4; TrYon, 1879, p. 124, pl. 28, f. 20-21, pl. 29, f. 28, pl. 38, f. 57; BROCK, 1887, p. 609, 612; ORTMANN, 1888, p. 662; ODHNER, 1917, p. 12, 70; ROBSON, 1929, p. 125; Voss, 1963, p. 159, textf. 36; Voss \& Williamson, 1971, p. 84, textf. 34, pl. 26.

Amphioctopus membranaceus FISCHER, 1882, p. 333.

Polypus membranaceus BerRY, 1912, p. 397.

Polypus ovulum SASAKI, 1917, p. 364; SASAKI, 1926, p. 100, 116; SASAKI, 1929, p. 55, pl. 10, f. 11-15, textf. 21-22.

Octopus pulcher BROCK, 1887, p. 607; RoBson, 1929, p. 125.

Octopus ovulum DonG, 1963, p. 151, pl, 4, f. 4.

Location: Kada, 11. April 1933, Male and female; Tōshima, Seto. One female. May, 1929.

Distribution: Japan.

China; Hong Kong; Philippines; Indonesia; Port Dorey, New Guinea; Australia; Indian Ocean; Red Sea; East Africa.

Habitat: Littoral; benthic; tropical-temperate.

Remark: The present species resembles $O$. ocellatus rather closely but these two may be separable from each other at the level of species by the following characters:

\begin{tabular}{l|l|l}
\hline \multicolumn{1}{c|}{ Characteristic } & \multicolumn{1}{c|}{ O. ocellatus } & \multicolumn{1}{c}{ O. membranaceus } \\
\hline maximum body length & $300 \mathrm{~mm}$ & $320 \mathrm{~mm}$ (Voss \& WiLdiamson) \\
number of demibranches & $7-8$ & $8-9$ \\
index of specially & $9.5-30.0$ & $6.4-10.0$ \\
$\begin{array}{l}\text { enlarged sucker } \\
\text { length of matured ovum } \\
\text { range of distribution }\end{array}$ & $13 \mathrm{~mm}$ & $3 \mathrm{~mm}$ \\
& Japan and China & $\begin{array}{l}\text { Indian Ocean and Indonesian } \\
\text { Seas; Philippines; China; Japan }\end{array}$ \\
\hline
\end{tabular}




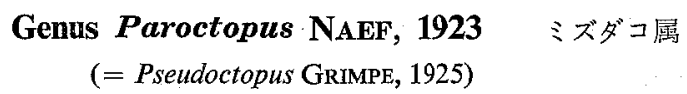

38. Paroctopus dofleini dofleini (WüLKER, 1910) ミズダコ(方言)

[マダコ (北海道方言)]

Polypus dofleini WüLKER, 1910, p. 7, pl. 2, f. 1-2, pl. 3, f. 10.

Polypus hongkongensis SASAKI, 1920, p. 177 (partim).

Polypus pustulosus SASAKI, 1920, pl. 176, p. 23, f. 5.

Polypus madokai BeRRY, 1921, p. 353; SASAKI, 1929, p. 71, pl. 5, f. 2-3, pl. 11, f. 9, textf. 34.

Octopus (Octopus) dofleini Rosson, 1929, p. 130, textf. 40; AKIMUSHKIN, 1963, p. 130; AKIMUSHKIN, 1965, p. 118.

Octopus dofleini Үамамото, 1942, p. 127.

Octopus dofleini dofleini Pickrord, 1964, p. 51; OKutan, 1975, p. 168, 270.

Location: Seto (angled in deep waters).

Distribution: Bering Sea; Alaska; Hokkaido; Honshu (Japan Sea coast; Pacific coast)

Korea (eastern coast) (Pusan, Ryōho, Chumunshin, Chosen, Reishin)

(釜山，良浦，注文津，長箭，霊津）

Hong Kong; Off Sri Lanka (AdAM, 1939).

Habitat: Littoral; benthic, tropical-boreal.

\section{Genus Callistoctopus TAKI, 1964 シマダコ属（滰）}

39. Callistoctopus arakawai TAKI, 1964 シマダコ(滝)

Callistoctopus arakawai TAKI, 1964, p. 292, pls. 2-3, textf. 34-41; TAKI, 1965, p. 324; OKUTAN, 1975, p. 169, 219.

Location: Seto (by gillnet; angled).

Distribution: Honshu (Ōsatsu-machi, Toba City, Mie Prefecture); Kyushu (Kamaemachi, Minami Amabe Gun, Ōita Prefecture; Tomioka, Reihoku-cho, Amakusa Gun, Kumamoto Prefecture; Amami Ōshima, Kagoshima Prefecture); Hachijo Island, Tokyo.

Habitat: Littoral; benthic; tropical-temperate.

40. Callistoctopus cyanea (GRAY, 1849) ワモンダコ (滰)

Octopus cyanea GraY, 1849, p. 15; TrYon, 1879, p. 124; RoBson, 1929, p. 94, textf. 21-23.

Octopus cyanea marmoratus HoYLE, 1885, p. 227; HoYLe, 1886, p. 85, pl. 6.

Octopus horsti Jounin, 1898, p. 21.

Polypus marmoratus SASAKI, 1929, p. 47, pl. 1, f. 10, pl. 5, f. 4, pl. 9, f. 19-24, textf. 17.

Octopus cyaneus ADAM, 1945, p. 6; ADAM, 1954, p. 171.

Octopus tonganus HoYLE, 1885, p. 225; HoYLE, 1886, p. 83, pl. 8, f. 1-2 (pars).

Callistoctopus magnocellatus TAKI, 1964, p. 298, p1. 4, f. 1-2, textf. 42-46; TAKI, 1965, p. 324;

OKutani, 1975, p. 169, 283.

Location: Seto (angled).

Distribution: Shikoku (Minamiuwa-gun, Ehime Prefecture); Bonin Islands.

Pacific Ocean (Taihoku, Formosa; Hawaii; Fiji Islands; Solomon Islands; Amboina); Indian Ocean (Madras; Coetivy Atoll; Sri Lanka; Burma; Zanzibar); Red Sea; Australia. 
Habitat: Littoral; benthic; tropical-temperate.

Remarks: Adam (1945; 1954) changed the specific name of the present species to "cyaneus" of the meaning of blue, but this change seems inappropriate, as Gray (1849) had proposed already a number of woman's names from Greek mythology, for the species of the genus Octopus, such as aegina, berenice, cephea, cyanea and geryonea.

\section{Genus Hapalochlaena RoBson, 1929 ヒョウモンダコ属（滝）}

41. Hapalochlaena maculosa (HoYLE, 1883)ヒョウモンダコ(滝)

$$
\text { [ヒョウダコ(佐々木) 改名)] }
$$

Octopus pictus Brock, 1882, p. 603, pl. 37, f. 3 (preoccupied by BlAINVILLE, 1828, p. 8)

Octopus maculosus HoYLe, 1883, p. 319, p1. 6.

Octopus pictus var. fasciata HoYLE, 1886, p. 94, pl. 8, f. 3.

Octopus robustus BROCK, 1887, p. 317.

Polypus fasciatus SASAKI, 1929, p. 58, pl. 11, f. 1-3.

Hapalochlaena maculosa RoBson, 1929, p. 211, textf. 87-88; ADAM, 1954, p. 182, textf. 33 B, 34.

Location: Seto (collected on the rocky shores).

Distribution: Honshu (Misaki; Sagami Bay; Tokyo market).

Pacific Ocean (Ternate, Molucca Sea, Indonesia; Australia (Port Jackson;

Queensland; New South Wales; South Australia); Indian Ocean (Sri Lanka).

Habitat: Littoral; benthic; warm temperate.

42. Berrya hoylei (BERRY, 1909) ヤワハダダコ(滝)

$$
\text { Genus Berrya ADAM, } 1939 \text { ヤワハダダコ属（滰） }
$$

Polypus hoylei BerRy, 1909, p. 407, f. 1; Berry, 1914, p. 296, pl. 47, f. 1, pl. 48, f. 2-4; MASSY, 1916, p. 207.

Octopus hoylei: hoylei var. annae RoBson, 1929, p. 219, textf. 89.

Berrya hoylei ADAM, 1939, p. 100, pl. 1, f. 2-3, textf. 22-23; TAKI, 1967, p. 24.

Hapaloctopus albidus TAKI, 1963, p. 68, pl. 2, textf. 15-19.

Location: Kinanshō, off Shiono-misaki, Wakayama Prefecture $\left(136^{\circ}-136^{\circ} 30^{\prime}\right.$ E., $33^{\circ}$ N., depth-ca. $710 \mathrm{~m}$.) from the stomach of Alepisaurus borealis (GILL) (Mizu-uo in Japanese) TAKI, 1963, Specimen B).

Distribution: Japan-Off Ashizurizaki, 153-227 m. in Tosa Bay, Kochi Prefecture (TAKI, 1963. Specimen A); Nagasaki (Hokkaido University).

Hawaiian Islands (BERRY); Persian Gulf, 25 fms. (=46 m.) (MASSY); Arabian

Sea $130 \mathrm{fms}$. (=238 m.) (MASSY); South of Sri Lanka, 52-68 fms. (=95124 m.) (MASSY).

Habitat: Planktonic; bathypelagic; tropical-warm temperate.

\section{Subfamily Bathypolypodinae RoBSON, 1932 ワタゾコダコ刺科（滝）}

\section{Genus Benthoctopus GRIMPE, 1921 チヒロダコ属（滝）}

43. Benthoctopus profundorum RoBSON, 1932 チヒロダコ(滰)

Octopus januarii HoYLe, 1885, p. 229 (partim); HoYLE, 1886, p. 97, pl. 7, f. 1-4. 
Polypus januarii SASAKI, 1920, p. 172; SASAKI, 1929, p. 61, pl. 4, f. 3, pl. 11, f. 4, textf. 22-23.

Benthoctopus profundorum RoBson, 1932, p. 237, pl. 4, f. 1, textf. 33, 42.

Location: Off Kii, Wakayama Prefecture. Albatross Station 4973.

$33^{\circ} 24^{\prime} 15^{\prime \prime}$ N., $135^{\circ} 30^{\prime} 30^{\prime \prime}$ E. One young female. $600 \mathrm{fms} .(=1098 \mathrm{~m}$.) Aug. 30, 1906.

Distribution: Off Yokohama ( $34^{\circ} 37^{\prime}$ N., $140^{\circ} 32^{\prime}$ E.) in $1875 \mathrm{fms} .(=3375 \mathrm{~m}$.) blue mud (HoYLE); Bungo Suido, in 437 fms. (=786.6 m.) (SASAKI); Hyūga Nada (SASAKI).

Aleutian Islands, $54^{\circ} 33^{\prime} \mathrm{N}, 178^{\circ} 44^{\prime}$ E., in $584 \mathrm{fms}$ (=1051.2 m.) (SASAKI); Bay of Bengal (Goodrich); Andaman Sea, 13 $27^{\prime}$ N., 93 $3^{\circ} 14^{\prime}$ E., in 405 fms. $\left(=729\right.$ m.) (MASSY); Same area, $10^{\circ} 21^{\prime}$ N. $92^{\circ} 46^{\prime}$ E., in 279 fms. $(=502.2$ m.) (MASSY); Rio de Janeiro (Steenstrup); Brazil (Hoyle).

Habitat: Bathypelagic; tropical-warm temperate.

44. Benthoctopus abruptus (SASAKI, 1920) キシュウチヒロダコ（奥谷)

Polypus abruptus SASAKI, 1920, p. 173; SASAKI, 1929, p. 68, pl. 6, f. 1, textf. $29-31$.

Benthoctopus abruptus RoBson, 1932, p. 241, textf. 43; OKUTANI, 1975, p. 169, 201.

Location: Off Kii, (Wakayama Prefecture), 587 fms. (=1074 m.), Albatross Station 4969, 33 $23^{\prime} 40^{\prime \prime}$ N., $135^{\circ} 33^{\prime}$ E. One male and female. Aug. 29, 1906.

Distribution: From Sagami Bay to Kii Peninsula, 300-750 m. in depth (Okutani).

Habitat: Bathypelagic; temperate.

Superfamily 3. Argonautacea THIELE, 1934 アオイガイ超科

Family Alloposidae VERRILL, 1881 カンテンダコ科 (滝)

Genus Alloposus VERRILL, 1880 カンテンダコ属（滝）

45. Alloposus mollis VerRILL, 1880 カンテンダコ(滝)

Alloposus mollis VerriLL, 1880, p. 394; VerRILL, 1881, p. 113, pl. 4, f. 4, pl. 8; RobSON, 1932, p. 215.

Location: Off Tonda-mura (either caught in a driftnet, or drifted ashore).

Distribution: North Atlantic, East; Azores (surface), $37^{\circ} 10^{\prime} \mathrm{N} ., 11^{\circ} 48^{\prime} \mathrm{W}$. (surface), $42^{\circ} 45^{\prime}$ N., $22^{\circ} 16^{\prime} \mathrm{W}$., (surface); $41^{\circ} 32^{\prime} 20 \mathrm{~W} ., 43^{\circ} 20^{\prime} \mathrm{W}$., (surface)

(JoubIN) ;West. 100-115 miles south of Newport, Rhode Island (225-487 fms. $(=405-876.6 \mathrm{~m}$.) ), off the mouth of Chesapeake Bay (300 fms. $=540$ m.), South of Nantucket Island $(1346-1735 \mathrm{fms} .=2422.8-4823 \mathrm{~m}$.), Off Newport, Rhode Island, 506-360 fms. (=910.8-648 m.); Martha's Vineyard, 310-715 fms. (=558-1287 m.); Delaware Bay (197 fms.=354.6 m.) (VerriLl); North Atlantic (Hoyle); Pailolo Channel, Hawaiian Islands, (286-290 fms. $=514.8-522 \mathrm{~m}$.) (BERRY).

Habitat: Epi to bathypelagic; temperate. 


\section{Family Tremoctopodidae TRYON, 1879 ムラサキダコ科（佐々木） \\ Genus Tremoctopus delle CHIAJE, 1830 ムラサキダコ属（佐々ホ）}

46. Tremoctopus violaceus delle CHIAJE, 1830 ムラサキダコ(佐々末)

Tremoctopus violaceus delle CHIAJE, 1830, pl. 70, 71; delle CHIAJE, 1840, p. 6; Kölliker, 1849, p. 67 ; KeFERSTEIN, 1866, p. 1449; TRYON, 1879, p. 130; pl. 43, WülKER, 1910, p. 5 ; SASAKI, 1929, p. 29, pl. 8, f. 17-19, textf. 9-10; RoBSon, 1932, 206; OKUTANi, 1975, p. 169, 273.

Location: Seto.

Distribution: Honshu (Misaki, Sagami Bay; Kyoto Prefecture); Kyushu (Nagasaki Prefecture).

Warm seas in general (Pacific Ocean; Atlantic Ocean, Mediterranean Sea).

Habitat: Epipelagic; tropical-temperate.

\section{Family Ocythoidae GRAY, 1847 アミダコ科（佐々木） \\ Genus Ocythoe RAFINESQUE, 1814 ： アミダコ属（佐々木）}

47. Ocythoe tuberculata RAFINESQUE, 1814 アミダコ（佐々木）[イボダコ(佐々木)] Ocythoe tuberculata RafinesQUe, 1814, p. 29; STEENSTRUP, 1880, p. 104; JaTta, 1896, p. 198, p. 198, pl. 6, 7, 19; WüKLER, 1910, p. 4; SASAKI, 1929, p. 26, pl. 3, f. 13-14, pl. 8, f. 12-16, textf. 8; RoBson, 1932, p. 201.

Hectocotylus octopodis VÉRANY, 1851, pl. 41.

Location: Seto (drifted ashore).

Distribution: Honshu (Misaki, Kanagawa Prefecture; Tokyo Bay; Shimane Prefecture).

Pacific Ocean; California; northeastern Atlantic Ocean; Mediterranean Sea.

Habitat: Epipelagic; temperate.

Note: This species has not been recorded from the Red Sea, the Indian Ocean nor

South Africa.

\section{Family 4 Argonautidae RAFINESQUE, 1815 アオイガイ科}

Genus Argonauta LINNAEUS, 1758 アオイガイ属

48. Argonauta argo LiNNAEUS, 1758 アオイガイ(浦の錦)

[カイダコ(三才図会)]

Argonauta argo LinNAEUs, 1758, p. 708; KüsteR, 1868, p. 4, pl. 1, f. 1; LISChKe, 1869, p. 29;

TRYON, 1879, p. 138, pls. 47-49; Hoyle, 1886, p. 69; SUTER, 1913, p. 1066; NAEF, 1928,

p. 281, pls. 32-37; GrIMPE, 1928, p. 77, pls. 3-5; SASAKI, 1929, p. 23, pl. 3, f. 8-12, textf. 7;

RoBson, 1932, p. 181, textf. 26; OKUTANI, 1975, p. 170, 171.

Location: Seto (shells only drifted ashore).

Distribution: Hokkaido. (Yuwanai): Honshu (Mutsu Bay, Aomori Prefecture;

Toyama Prefecture; Tango, Kyoto Prefecture); Kyushu (Kagoshima Prefecture)

Pacific Ocean (Amboina; Tahiti; Cocos Island; Lower California and Panama;

New Zealand; Australia); Red Sea; Atlantic Ocean (Madeira; Azores; 
Portugal; New Jersey; Florida; Cuba; Venezuela; Cape of Good Hope)

Habitat: Surface dweller; circumtropical, subtropical, temperate.

49. Argonauta hians [LightFoOT, 1786] タコブネ（群分品鯟）

[フネダコ(佐々ホ)]

Argonauta hians Lightroot, 1786, p. 44; Tryon, 1879, p. 133, 136, pl. 46; SASAKI, 1920, p. 171;

SASAKI, 1929, p. 20, pl. 3, f. 3-6, pl. 8, f. 9-10, textf. 6; RoBSON, 1932, p. 192; REHDER, 1967, p. 12, 29; OKUTANI, 1975, p. 170, 230.

Location: Seto (drifted ashore).

Distribution: Honshu (Mutsu Bay, Aomori Prefecture; Enshü Nada, Shizuoka Prefecture); Kyushu (Kagoshima City, Kagoshima Prefecture; Okinawa Prefecture).

China. "found almost everywhere in tropical and subtropical waters" (RoBson, 1932, p. 193).

Habitat: Surface dweller; circumtropical, subtropical, temperate.

50. Argonauta boettgeri Maltzin, 1881

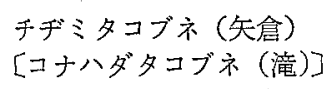

Argonauta boettgeri MALTZAN, 1881, p. 163, pl. 6, f. 7; SASAKr, 1929, p. 22, pl. 3, f. 7, pl. 8, f. 11; Robson, 1932, p. 195; YaGURA, 1932, p. 61; TAKI, 1932, pp. 209, 357; OKUTANI, 1975, pp. 170, 231.

Location: Seto (shell only, drifted ashore)

Distribution: Honshu (Misaki, Kanagawa Prefecture); Kyushu (Kagoshima Prefecture, "Albatross")

Pacific Ocean (China Sea; Masbate, Philippines; Hawaiian Islands; Andaman Islands; Australia; New Zealand); Indian Ocean (Chagos Islands).

Habitat: Surface dweller; tropical-temperature.

51. Argonauta nouryi LoROIS, 1852 チビタコブネ (平類信太郎)

Argonauta nouryi LoRoIs, 1852 (January), p. 10, pl. 1, f. 5; TRYON, 1879, p. 138, pl. 50, f. 126-127. Argonauta nouryi Robson, 1932, p. 198, pl. 2, f. 2, pl. 3, f. 1; Shintaro Hrrase, 1934, pl. 128, f. 4.

Argonauta gruneri DUNKER, 1852 (March), p. 48.

Location: Wakayama Prefecture.

Distribution: Pacific Ocean (Marquesas; Gulfs of California and Panama, off Acapulco, Peru)

Habitat: Surface dweller; tropical-temperate.

Note: Shintarō Hirase (1934) included the present species in his book of Japanese shells, illustrated in natural colors, but without showing locality of the specimen figured. Later 1947 Drs. K. Oyama and M. Horikoshi supplied such a list of localities to his book. According to this list Argonauta nouryi had been found in Province Kii (=Wakayama Prefecture). 


\section{Remarks on the distribution of Cephalopods \\ in Wakayama Prefecture}

Fifty-one species of cephalopods have hitherto been known from Wakayama Prefecture.

Of these, (1) the species from temperate waters are the most abundant:

Sepia esculenta Sepia peterseni Sepia kobiensis Sepia appelloefi

Sepia lycidas Metasepia tullbergi Sepiella japonica

Sepiadarium kochii Euprymna morsei Idiosepius pygmaeus paradoxus

Loligo japonica Loligo uyii Histioteuthis corona inermis

Liocranchia valdiviae Octopus vulgaris Octopus berenice

Octopusminor Octopus luteus Octopus sasakii Octopus aegina

Octopus ocellatus Octopus membranaceus Ocythoe tuberculata.

(2) The warm Kuroshio Current brings the south sea elements into the waters along the coast of the prefecture, so that tropical and subtropical cephalopods are involved here.

Sepia pharaonis Sepioteuthis lessoniana Loligo edulis edulis

Todarodes pacificus Ommasrephes bartrami Callistoctopus arakawai

Callistoctopus cyanea Hapalochlaena maculosa Berryahoylei*

Japetella diaphanaa Alloposus mollis Tremoctopus violaceus

Argonauta argo Argonauta hians Argonauta boettgeri

Argonauta nouryi

(3) Northern species are only rarely found:

Rossia pacifica . Rossia mollicella Watasenia scintillans

Paroctopus dofleini dofleini Benthoctopus profundorum

Benthoctopus abruptus.

These species show a rather wide distribution and are caught in deep water of low temperature.

(4) The occurrence of Vampyroteuthis infernalis is noteworthy. A single specimen was obtained in August 1906 by the United States "Albatross" at a depth of $999.5 \mathrm{~m}$ : a larva of a total length of $25 \mathrm{~mm}$, a ventral length of mantle $8.3 \mathrm{~mm}$, maximum breadth $8 \mathrm{~mm}$. As shown by Pickford (1946), this species shows a world wide distribution in the range from 1000 to $7000 \mathrm{~m}$ deep in tropical and subtropical seas.

(5) Species of the genus Argonauta are surface-dwellers, and A. argo and $A$. hians have respectively a world-wide distribution. A. boettgeri and $A$. nouryi are rather rarely known and only from stranded shells.

(6) At present, Opisthoteuthis japonica is endemic to the waters off Wakayama Prefecture, though in future some more specimens may be found in the depths of the

* This species was obtained from the contents of the stomach of Alepisaurus borealis caught at about $710 \mathrm{~m}$ deep of Kinan-shō. 
neighbouring waters. Probably this might be an endemic species in the subtropical water of Japan.

(7) Stranding ashore of shells of Nautilus pompilius and Spirula spirula along the coast of Wakayama Prefecture is noticeable, too. In these species, the shell maintains buoyancy after the death of animals. Thus, the shells may be carried by the warm Kuroshio current northerly to the coast of Wakayama Prefecture. The original localities of these two species may be found around the southern islands (for example Cebu and its neighbouring islands) of the Philippine Islands.

\section{References}

Adam, William. 1934. Cephalopoda. Résultats scient. voy. aux Indes Oriental. Néerl. de LL. AA.RR. le Prince et la Princesse Léopold de Belgique. vol. 2, fasc. 16: 1-28, text-figs. 1-15.

1939. The Cephalopoda in the Indian Museum, Calcutta. Records Ind. Mus., 41: 61-110, pls. 1-2, text-figs. 1-28.

1939a. Cephalopoda. Partie 1. Le genre Sepioteuthis Blainville, 1824. Siboga-Expeditie, Monogr. 55a (Livr. 134): 1-33, tabl. 1-3, pl. 1.

1939b. Cephalopoda. Partie 2. Révision des espèces indo-malaises du genre Sepia Linné, 1758. III. Révision du genre Sepiella (Gray) Steenstrup, 1880. Ibid., Monogr. 55b (Livr. 135): 35-122, tabl. 1-7, pls. 1-4.

1945. Cephalopods from Dr. Sixten Bock's expedition to the South Pacific Islands. Arkiv för Zoologi, vol. 37A: 1-25, pls. 1-2, text-figs. 1-11.

1954. Cephalopoda. Partie III. Céphalopodes à l'exclusion des genres Sepia, Sepiella, et Sepioteuthis. Siboga-Expeditie, Monogr. 55c (Livr. 144): 121-194, tables 1-4, pls. 1-4.

1964. Considérations sur la systématique des Sepiidae (Cephalopoda). Zool. Mededelingen, 39: $263-278$.

Adam, W. \& Ress, W. J. 1966. A review of the Cephalopod family Sepiidae. Sci. Report John Murray Exped. 1933-34. vol. 11:1-165, pls. 1-46, text-fig. 1.

Akimushkin, I. I. 1963. Golovonogie mollyuski morei SSSR. Institute of Oceanology: Academy of the U.S.S.R. pp. 1-234. (English translation) 1965. Cephalopods of the seas of the U.S.S.R. pp. 1-223. Jerusalem.

Allan, Joyce. 1945. Planktonic Cephalopod larvae from the eastern Australian coast. Records Austral. Mus., 21: 317-350, pls. 24-27.

Appellöf, A. 1886. Japanska Cephalopoder. Kongl. Sevnska Vetenskaps-Akademiens-Handlingar, 21 : $1-40$, pls. $1-3$.

Brock, J. 1887. Indische Cephalopoden. Zool. Jahrb. Abt. Syst. Bd. 2: 591-613, pl. 16.

Berry, S. Stillman. 1909. Diagnoses of new Cephalopods from the Hawaiian Islands. Proc. U.S. Nat. Mus., 37: 407-419, 9 text-figs.

1911. Preliminary notices of some new Pacific Cephalopods. Proc. U.S. Nat. Mus., 40: 589594.

1912. A catalogue of Japanese Cephalopoda. Proc. Acad. Nat. Sci. Philadelphia, 1912: 380444, pls. 5-9.

1912a. A review of the Cephalopods of Western North America. Bull. Bur. Fisher. 30: 269336, pls. 32-54, text-figs. $1-18$.

1914. The Cephalopoda of the Hawaiian Islands. Bull. Bur. Fisher. 32: 257-362, pls. 45-55.

1921. A review of the Cephalopod genera Sepioloidea, Sepiadarium, and Idiosepius. Records S. Austral. Mus. 1: 347-364, text-figs. 64-67.

1921a. Notes on some Japanese Cephalopods.-A review of Sasaki's 'Albatross' report. Ann. Mag. Nat. Hist. ser. 9, 8: 351-353.

1932. Cephalopods of the genera Sepioloidea, Sepiadarium and Idiosepius. Philipp. J. Sci. 47: 39-53, pl. 1 .

Bosc, L. A. 1802. Histoire naturelle des Coquilles. Paris. 
Chun, Carl. 1903. Aus den Tiefen des Weltmeeres: G. Fischer, Jena.

1914. Die Cephalopoden. II. Myopsida: Octopoda. Wissen. Ergeb. d. Deutschen TiefseeExpedition auf dem Dampfer "Valdivia" 1898-1899. Band 18, Teil 2: 405-552.

Clarke, M. R. 1966. A review of the systematics and ecology of oceanic squids. Advances in Mar. Biol. 4: 93-323, text-figs. 1-59, tables 1-6.

Cooke, A. H. 1895. Molluscs. Cambridge Nat. Hist. vol. 3. London.

Cotton, B. C. \& Godfrey, F. K. 1940. The Mollusca of South Australia. Part II. Cephalopoda, pp. 342-465, textfigs. $365-450$. Adelaide.

Cuvier, G. L. C. F. D. 1797. Tabl. élém. histoire naturelle des animaux. Paris.

Dong, Zheng-Zhi (董 正之) 1963. A preliminary taxonomic study of the Cephalopoda from the Chinese waters. Studia Marina Sinica, No. 4: pp. 125-155, English summary, pp. 156-157, pls. 1-4.

Dunker, W. 1852. Argonauta gruneri. Zeits. Malakozoologie, vol. 9, p. 48.

Ekman, Sven. 1935. Tiergeographie des Meeres. 542 pp. Akadem. Verlagsgesel. Leipzig. (English translation) 1953. Zoogeography of the sea. 417 pp. Sidgwick \& Jackson, Ltd., London.

Fischer, Paul. 1882. Manuel de conchyliologie. 1369 pp., 23 pls. Paris.

Férussac, A. E. et d'Orbigny, A. 1835-1848. Histoire naturelle générale et particulière des Céphalopodes acètabulifères vivants et fossiles. Paris.

Goodrich, E. A. 1896. Report on a collection of Cephalopoda from the Calcutta Museum. Trans. Linn. Soc. London, ser. 2, vol. 7: 1-24, 5 pls.

Gray, J. E. 1849. Catalogue of the Mollusca in the collection of the British Museum. Part I. Cephalopoda antepedia. $164 \mathrm{pp}$. London.

Grimpe, G. 1922. Systematische Übersicht der europäischen Cephalopoden. Sitz. ber. naturf. Ges. Leipzig. Bde, 45-48: 36-52.

1931. Teuthologische Mitteilungen. XIII. Ueber die Cephalopoden der Sunda-Expedition Rensch. Zool. Anzeiger, 95: 149-174, txfigs. 1-14.

Hamabe, Mototsugu (浜部 基次) 1965. Studies on the embryology and ecology of Todarodes pacificus in the Japan Sea. pp. 1-147, pls. 1-3, tables 1-15. (In Japanese)

Hamada, Takashi (浜田 隆士) 1965. Post-mortem drift of Nautilus. Venus, 24 (3): 181-198, pl. 20, txfigs. 1-13. (Eng. sum. p. 197)

1977. A supposition of the area of life in Nautilus. Natural Science and Museum, 44 (4): 155-157. (In Japanese)

Hamada, T. et al. 1978. Recent advancements in rearing experiment of Nautilus in Japan. Venus, 37 (3): 131-136.

Hemming, F. 1958. Official list of specific names in zoology. First instalment, pp. 1-206. London.

Hirase, Shintaro (平瀬信太郎) 1934. A collection of Japanese shells with illustrations in natural colours. Matsumura Sanshōdō, Tokyo. 14 pp., 129 pls., 217 pp.

Hoyle, W. F. 1885. Diagnoses of new species of Cephalopods collected during the cruise of $\mathbf{H} . \mathbf{M}$. S. Challenger. Part I. The Octopoda. Ann. Mag. Nat. Hist. ser. 5, 15: 222-236.

1885a. Diagnoses of new species of Cephalopods collected during the cruise of H. M. S. Challenger. Part II. The Decapoda. Ibid. ser. 5, 16: 181-203.

1885b. Preliminary report on the Cephalopoda collected by H. M. S. "Challenger" Part I. The Octopoda. Proc. Roy. Soc. Edinb. 13: 94-114.

1885c. Preliminary report on the Cephalopoda collected by H. M. S. "Challenger." Part II. The Decapoda. Ibid. 13: 281-310.

1886. Report on the Cephalopods collected by H. M. S. Challenger during the years 1873-76. Report Sci. Res. Voy. Challenger 1873-76. Zoology, vol. 16: 1-245, map 1, txfigs. 1-10.

Ikeda, Sakujiro (池田作次郎) 1890-91. A list of Japanese Cephalopoda in the Zoological Institute of Imperial University. Zool. Mag. Tokyo, vols. 2-3. (In Japanese)

Inaba, Akihiko (ed.) (稻葉 明彦) 1963. Fauna and flora of the Inland Sea of Seto. 352.pp., 6 pls. Mukaishima Marine Biological Station of Hiroshima University.

Iredale, Tom. 1926. The biology of North-West Islet, Capricorn Group. D. Marine Mollusca. Australian Zoologist, 4: 238-240. 
1944. Australian pearly Nautilus. Australian Zoologist, 10 (3): 294-298.

Ishikawa, Chiyomatsu (不川下代松) 1913. Einige Bemerkungen über den leuchtenden Tintenfisch, Watasea n. gen. (Abraliopsis der Autoren) scintillans Berry, aus Japan. Zool. Anzeiger, 43: 162-172, 6 figs; Correction, Watasenia, Ibid., p. 336. 1914.

Ishikawa, Masashi (石)li 香) 1933. On the useful species of the genus Loligo in Japan. Report on the Advance of Science in Japan, 8: 440-444, 2 text-figs. (In Japanese)

Jatta, G. 1896. Cefalopodi. Fauna und Flora des Golfes von Neapèl, Monogr. 23, 1-268, 21 pls., 64 text-figs.

Joubin, L. 1897. Observations sur divers Céphalopodes. Troisième note: Céphalopodes du Musée Polytechnique de Moscou. Bu1l. Soc. Zool. France, 22: 96-104.

1902. Revision des Sepiolidae. Mém. Soc. Zool. France, 15: 80-145, 38 text-figs.

1912. Études préliminaires sur les Céphalopodes recueillis au cours des croisières de S.A.S. le Prince de Monaco. Ire Note: Melanoteuthis lucens nov. gen. et sp. Bull. Inst. Océanogr. Monaco, No. 220: 1-14, 12 text-figs.

1929. Notes préliminaires sur les Céphalopodes des croisières du Dana (1921-22). Octopodes, Ire partic. Ann. Inst. Océanogr. Paris, 6: 363-394.

1939. Les Octopodes de la croisières du "Dana" 1921-22. Dana-Report, No. 11: 1-49.

Küster, H. C. 1868. Die Gattungen Argonauta, Nautilus und Spirula. Martini und Chemnitz: System. Conchylien-Cabinet, 1:3-15, pls. 1-4.

Kuroda, Tokubei (黑田 徳米) 1929. Shells of Province Kii. Venus, 1: 123-127. (In Japanese)

Lamarck, J. B. 1798. Bull Soc. Phil. Paris, vol. 2, p. 129.

1799. Mémoire sur les genres de la sèche .... Mém. Soc. Hist. Nat. Paris, vol. 1, p. 1.

Lesson, R. P. 1830. Zoologie du voyage autour du monde sur la "Coquille" par Duperrey. Paris.

Lesueur, C. A. 1821. Descriptions of several new species of cuttle-fish. J. Acad. Nat. Sci. Philadelphia , 2: 86-101.

Lightfoot, John. 1786. A catalogue of the Portland Museum, lately the property of the Duchess Dowager of Portland, deceased, which will be sold at auction, by Mr. Skinner and Co. London, viii $+194 \mathrm{pp}$.

Linnaeus, Carolus. $\quad$ 1758. Systema naturae per regna tria naturae. 824 pp. Stockholm.

Massy, A. L. 1916. The Cephalopoda of the Indian Museum. Records Ind. Mus. 12: 185-247, pls. 23-24.

Meyer, W. T. 1906. Die Anatomie von Opisthoteuthis depressa (Ijima und Ikeda). Zeits. wiss. Zool, 85: 183-269, pls. 11-16, 16 text-figs.

Naef, Adolf. 1921. Die Cephalopoden. Fauna und Flora des Golfes von Neapel und der angrenzenden Meeres-abschnitte. 35. Monogr. Teil 1, Lfg. 1: 1-148, text-figs. 1-62.

1923. Die Cephalopoden. Ibid. Teil 1, Bd. 1, Lfg. 2, xiv+149-863, text-figs. 63-471.

1928. Die Cephalopoden. Ibid. Teil 1, Bd. 2. Embryologie, 1-357, text-figs. 1-138, pls. $1-37$.

Natsukari, Y. (夏获 豊) 1970. Egg-laying behavior, embryonic development and hatched larva of the pygny cuttlefish, Idiosepius pygmaeus paradoxus Ortmann. Bull. Fac. Fisheries, Nagasaki Univ., No. 30: 15-25, pls. 1-4.

Nishimura, S. (西村 三郎) 1966. Notes on the occurrence and biology of the oceanic squid, Thysanotenthis rhombus Troschel, in Japan. Publ. Seto Mar. Biol. Lab. 14: 327-349, 6 text-figs.

1968. Glimpse of the biology of Argonauta argo Linnaeus (Cephalopoda: Octopodida) in Japanese waters. Ibid. 16:61-70.

1968a. A preliminary list of the pelagic Cephalopoda from the Japan Sea. Ibid. 16: 71--83.

Odhner, N. H. 1917. Results of Dr. E. Mjöberg's Swedish scientific expeditions to Australia 1910-1913. 17. Mollusca. K. Svenska Vet.-Ak. Hand1. 52 (16): 1-115, pls. 1-3.

d'Orbigny, A. et Férussac, A. de 1840. Histoire naturelle ...des Céphalopodes acétabulifères. vol. $1: 1-240$.

Okutani, Takashi (舆谷 喬司) 1960. Argonauta boettgeri preys on Cavolinia tridentata. Venus, 21: 39-41, text-figs. 1-3. (In Japanese)

1962. Scientific name of Surume-ika (Todarodes pacificus Steenstrup, 1880). Venus, 22: 92-93. (In Japanese) 
1967. Preliminary catalogue of Decapodan Mollusca from Japanese waters. Bull. Tokai Reg. Fisheries Res. Lab. No. 50:1-16.

1973. Ecology of the Cephalopoda. Lectures on Oceanology, vol. 9 (Ocean Ecology): 79-125, text-figs. 1-23. (In Japanese)

1974. Problems in studies on early life history of oceanic squids. Kaiyo Kagaku, 6: 61-66. (In Japanese)

1975. Shells, II (Bivalves: Land Shells: Squids, Octopods and others). Gakken Picture Books for Junior and High School Pupils. Edited by T. Habe. Plates 1-170, pp. 171-294. Gakushu Kenkyusha, Ltd. Tokyo. (In Japanese)

1977. A monograph of the useful squids and cuttlefishes of the world. Revised edition, 165 pp. The Tōwa Electrical Machine Manufacturing Co., Ltd. Tokyo.

1980. Useful and latent cuttlefish and squids of the world. pp. 1-66. Published from the National Cooperative Association of Squid Processors for the 15th Anniversary of its Foundation.

Ortmann, A. 1888. Japanische Cephalopoden. Zool. Jahrb. Abt. System. 3: 639-670, pls. 20-25.

Oyama, Katsura \& Horikoshi, Masuoki (大山 桂 - 堀越 増與) 1947. The locality of the specimens of Hirase's "A collection of Japanese shells with illustrations in natural colours." Report of the Third Department (Geology and Mineralogy), Research Institute of Natural Resources, Miscellaneous Series, No. 1. 22 pp. (mimeographed)

Pelseneer, Paul. 1906. Mollusca. Lankester (ed.) A treatise on zoology, Part 5. London.

Pfeffer, Georg. 1884. Die Cephalopoden des Hamburger Naturhistorischen Museums. Abhandl. Naturwiss. Verein Hamburg, 8: 63-90, 3 pls.

1912. Die Cephalopoden der Plankton-Expedition. Zugleich eine monographische Übersicht der Oegopsiden Cephalopoden. Ergebn. Plankton-Expedition d. Humboldt-Stiftung. Bd. 2, F.a, 1-815, Atlas, pls. 1-48. Kiel und Leipzig.

Pickford, Grace E. 1946. Vampyroteuthis infernalis Chun. An archaic Cephalopod. 1. Natural history and distribution. Dana-Report, 29: 1-40, text-figs. 1-8.

1949. Ibid. II. External anatomy. Tbid. 32: 1-132, pls. 1-9, text-figs. 42-75.

1950. The Vampyromorpha (Cephalopoda) of the Bermuda Oceanographic Expeditions. Zoologica (New York), 35: 87-95, text-figs. 1-8.

1952. The Vampyromorpha of the Discovery Expeditions. Discovery Reports, 26: 197-210, text-figs. $1-4$.

1957-59. Vampyromorpha. Galathea Report, 1: 243-253, text-figs. 1.

1964. Octopus dofleini (Wülker), the giant octopus of the North Pacific. Bull. Bingham Oceanogr. Collection, Peabody Mus. Nat. Hist. Yale Univ. 19: 1-70.

Pilsbry, H. A. 1895. Catalogue of the marine Mollusks of Japan. Detroit.

Quoy, J. R. C. et Gaimard, J. P. 1832. Voyage de l'Astrolabe, pendant les années 1826-29, sous le commendement de M. J. Dumont d'Urville. Zoologie. Tome 2. Paris.

Reeve, L. A. 1861. Monograph of the genus Argonauta. Conchologia Iconica, vol. 17: 1-5, pls. 1-4. London.

Rehder, H. A. 1940. Hyaloteuthis pelagicus (Bosc) in the North Pacific. Nautilus, 54: 71-72.

1967. Valid zoological names of the Portland Catalogue. "Proc. U.S. Nat. Mus. 121: 1-51.

Robson, Guy C. 1929. A monograph of the recent Cephalopoda based on the collection in the British Museum (Natural History). Part 1. Octopodinae. vol. 1: 1-236, 7 pls. 88 textfigs. London.

1929. On the rare abyssal Octopod, Melanoteuthis beebei. Proc. Zool. Soc. London, 1929: $469-486$.

1931. On the phylogeny of the Octopoda. Archivio Zoologica Italiano, 16: 1118-1121.

1932. Ibid. Part 2. The Octopoda. 1-359, 6 pls. 79 text-figs.

Rochebrune, A. T. de 1884. Étude monographique de la famille des Sepiadae. Bull. Soc. philomath. Paris, sér. 7, 8: 74-122, pls. 3-6.

Roper, C. F. E., Young, R. E. \& Voss, G. L. 1969. An illustrated key to the families of the Order Teuthoidea (Cephalopoda). Smiths. Contrib. to Zool. 13: 1-16, pls. 1-16.

Sasaki, Madoka (佐々木 望) 1914. Notes on Japanese Myopsida. Annot. Zool. Japon. 8; $587-629$, pls. $11-12$. 
1916. Notes on Oegopsid Cephalopods found in Japan. Ibid. 9: 89-120, p1. 3.

1917. Notes on the Cephalopoda. (1) On the male of Amphitretus pelagicus Hoyle: (2) Diagnoses of four new species of Polypus. Ibid. 9: 361-367, 2 text-figs.

1918. On Inioteuthis inioteuthis (Naef) and Sepiola birostrata, n.sp. Zool. Mag. 30: 235-236. (In Japanese)

1920. Report of Cephalopods collected during 1906 by the United States Bureau of Fisheries Steamer "Albatross" in the north western Pacific. Proc. U.S. Nat. Mus., 57: 163-203, pls. 23-26.

1926. Cuttlefishes and octopuses. In Okazaki, T. (ed.): Fundamental studies of zoological materials for a national sixth year grade science textbook. Bun'yōsha. pp. 85-122, pl. 1. (In Japanese)

1927. Cephalopoda. In S. Uchida: Figuraro de Japanaj Bestoj. pp. 1245-1266. Hokuryūkan \& Co., Ltd. Tokyo (In Japanese)

1929. A monograph of the Dibranchiate Cephalopods of the Japanese and adjacent waters.

J. Coll. Agr. Hokkaido Imp. Univ. Supplementary No. vol. 20: 1-357, 30 pls., 159 text-figs.

1947. Cephalopoda. In S. Uchida: Illustrated Encylopedia of the Fauna of Japan. pp. 1012-1027. Hokuryūkan \& Co., Ltd. Tokyo (In Japanese)

Schilder, F. A. 1956. Lehrbuch der allgemeinen Zoogeographie. 150 pp. G. Fischer, Jena.

Sttenstrup, Japetus. 1881. Sepiadarium og Idiosepius, to nye Slägter af Sepiernes familie. Danske Vid. Selsk. Skrift. ser. 6, 1: 213-242, 1 pl.

Sugano, Tōru (菅野 徽) 1974. “Hime-ika". Shore animals of Miura Peninsula, No. 10. Asahi-Larousse Weekly: Encyclopaedia of World Animals, No. 181, p. 30. (In Japanese)

Stenzel, H. B. 1957. Nautilus. Hedgpeth, Treat. Mar. Ecol. Paleoecol. vol. 1. Ecology, pp. 1135-1141.

1964. Living Nautilus. Moore (ed.) Treat. Invert. Paleont. Part K, Mollusca 3, CephalopodaNautiloidea, pp. 59-93.

Taki, Iwao (滰 膂) 1932. Notes on shells (4). (18) Argonauta boettgeri Maltzan. Venus, 3: 209-212, text-figs. 3, 5. (In Japanese)

1936. Notes on shells (6). (30) On the generic name of Octopus (non Polypus). Venus, 4: 38-41. (In Japanese)

1936. Notes on shells (7). (39) The specific name of "Ii-dako". (40) The specific name of "Tenaga-dako." Venus, 6: 49-55. (In Japanese)

1942. The Mollusea of the Inland Sea of Japan (1). Venus, 12: 64-80, text-figs. 1-15.

1944. Studies on Octopus (2). Sex and the genital organ. Venus, 13 (5-8): 267-310, pls. 8-11. (In Japanese)

1949. A draft of the catalogue of the Cephalopoda of Wakayama Prefecture. Nanki-seibutsu, 1: 47-55 (mimeographed) (In Japanese)

1963. On four newly known species of Octopoda from Japan. J. Fac. Fish. Anim. Husb. Hiroshima Univ. 5: 57-83, pls. 1-5.

1964. On eleven new species of Cephalopoda from Japan, including two new species of Octopodinae. Ibid. 5: 277-330, pls. 1-7.

1965. Cephalopoda. Y. K. Okada-S. Uchida-T. Uchida (ed.): New Illustrated Encyclopedia of the Fauna of Japan, vol. 2: 307-326. Hokuryükan, Tokyo. (In Japanese)

1967. Taki, Iwao \& Igarashi, Takao (五十媇 孝夫) A list of Cephalopod specimens in the Fisheries Museum, Faculty of Fisheries, Hokkaido University (Sasaki Collection and specimens collected by the Marine Zoological Laboratory). Contrib. No. 7: 1-27. Hakodate, Hokkaido. (In Japanese)

Thiele, Johannes. 1935. Classis Cephalopoda. In Handbuch d. system. Weichtierkunde, 2: 948-995, text-figs. 868-893. G. Fischer, Jena.

Troschel, H. 1857. Bemerkungen über die Cephalopoden von Messina. Arch. f. Naturg. 23: $41-76$.

Tryon, G. W. Jr. 1879. Cephalopoda. Manual of Conchology, 1: 1-316, pls. 1-111. Philadelphia.

Vérany, J. 1851. Mollusques méditerranéens ....

Verrill, A. E. 1880-81. The Cephalopods of the north-eastern coast of America. Part 2. The 
smaller Cephalopods, including the squids and the octopi, with other allied forms. Trans. Connecticut Acad. Sci. 5: 259-446, pls. 26-56.

1896. The Opisthoteuthidae. Amer. J. Sci. 2: 74-80, text-figs. 1-7.

Volsøe, A., Knudsen, J. \& Rees, W. 1962. The Cephalopod papers of Japetus Steenstrup. 330 pp. Danish Science Press, Ltd. Copenhagen.

Voss, G. L. 1963. Cephalopods of the Philippine Islands. Bull. U.S. Nat. Mus. 234: 1-180, 4 pls., 36 text-figs.

1967. The biology and bathymetric distribution of deep-sea Cephalopods. Proc. Intern. Confer. Trop. Oceanogr. Stud. Trop. Oceanogr. Miami, 5: 511-535.

1971. Cephalopods collected by the R/V John Elliott Pillsbury in the Gulf of Panama in 1967. In Bayer, F. M. \& Voss, G. L. (ed.): Studies in Tropical American Mollusks, 1-34, textfigs. 1-6, tables 1-12.

1977. Classification of recent Cephalopods. In Nixon, M. \& Messenger, J. B. 1977. The biology of Cephalopods. Appendix II, 575-579.

Voss, G. L. \& Erdman, D. S. 1959. Thysanoteuthis rhombus, large Cephalopod new to the eastern Atlantic. Nautilus, 73:23-25.

Voss, G. L. \& Williamson, G. R. 1971. Cephalopods of Hong Kong. 138 pp., 35 pls., 68 textfigs. Hong Kong Government Press.

Wakiya, Yojiro \& Ishikawa, Masashi (脇谷 洋次郎- 石川 昌) 1921. On the Myopsida of the Cephalopods of Japan, with descriptions of four new species. Dobutsugaku Zasshi, 33: 279-292, pls. 1-2. (In Japanese)

Winckworth, R. 1932. The British marine Mollusca. J. Conch. 19: 211-252.

Wülker, Gerhard 1910. Über japanische Cephalopoden. Beiträge zur Kenntnis der Systematik und Anatomie der Dibranchiaten. Abhandl. II. Klasse d. K. Bayer. Akad. Wiss. III. Suppl. Bd. 1: 1-72, pls. 1-5.

Yagura, Wasaburo (矢倉 和三郎) 1913. Photographs of Japanese Shells. 1-80 pls. Maiko Conchological Museum. (In Japanese)

1932. A Catalogue of the Molluscan Shells of Hyōgo Prefecture. Revised Edition. 63+6+8 pp. 5 pls. Kōnan Kairui-sō, Nishinomiya. (In Japanese)

Yamamoto, Takaharu (山本 孝治) 1940. Mortal influence of salinity on some Cephalopod larvae. Botany and Zoology, 8: 1879-1882, text-fig. 1. (In Japanese)

1941. Observations on the habits of the squid larvae, Sepia esculenta Hoyle, with special reference to the change of colour pattern on the mantle. Ibid. 9: 349-353, text-figs. 1-3. (In Japanese)

1941. The breeding habits of Octopus ocellatus Gray, with observations on the hatched young. Ibid. 9: 9-14, text-figs. 1-11. (In Japanese)

1941. Some observations on the embryonal development of the eggs of Octopus ocellatus Gray. Ibid. 9: 234-243, text-figs. 1-7. (In Japanese)

1942. On the distribution of Cephalopods in Korea. Venus, 11: 126-133. (In Japanese)

1942. On the ecology of Octopus variabilis typicus (Sasaki) with special reference to its breeding habits. Venus, 12: 9-20,text-figs. 1-10. (In Japanese)

Yasumoto, Koichiro (安元 - 幸一郎) 1938. Sex-correlated patterns on the dorsal side of a cuttlefish, Sepiella japonica Sasaki. Venus, 8: 45-47, text-figs. 1-2. (In Japanese) 